NISTIR 8263

\title{
Thermophysical Properties of Polyol Ester Lubricants
}

Thomas J. Bruno

Tara J. Fortin

Marcia L. Huber

Arno Laesecke

Eric W. Lemmon

Elisabeth Mansfield

Mark O. McLinden Stephanie L. Outcalt

Richard A. Perkins

Kimberly N. Urness

Jason A. Widegren

This publication is available free of charge from:

https://doi.org/10.6028/NIST.IR.8263

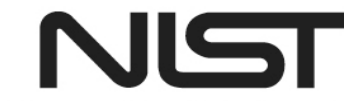

National Institute of Standards and Technology U.S. Department of Commerce 
NISTIR 8263

\title{
Thermophysical Properties of Polyol Ester Lubricants
}

\author{
Thomas J. Bruno \\ Tara J. Fortin \\ Marcia L. Huber \\ Arno Laesecke \\ Eric W. Lemmon \\ Elisabeth Mansfield \\ Mark O. McLinden \\ Stephanie L. Outcalt \\ Richard A. Perkins \\ Kimberly N. Urness \\ Jason A. Widegren \\ Applied Chemicals and Materials Division \\ Material Measurement Laboratory
}

This publication is available free of charge from:

https://doi.org/10.6028/NIST.IR.8263

August 2019

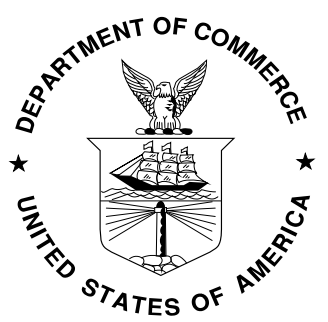

U.S. Department of Commerce Wilbur L. Ross, Jr., Secretary

National Institute of Standards and Technology Walter Copan, NIST Director and Undersecretary of Commerce for Standards and Technology 
Certain commercial entities, equipment, or materials may be identified in this document in order to describe an experimental procedure or concept adequately. Such identification is not intended to imply recommendation or endorsement by the National Institute of Standards and Technology, nor is it intended to imply that the entities, materials, or equipment are necessarily the best available for the purpose.

National Institute of Standards and Technology Interagency or Internal Report 8263

Natl. Inst. Stand. Technol. Interag. Intern. Rep. 8263, 222 pages (August 2019)

This publication is available free of charge from:

https://doi.org/10.6028/NIST.IR.8263 


\begin{abstract}
This report summarizes the results of work performed for the Naval Air Warfare Center Aircraft Division by the National Institute of Standards and Technology (NIST), Applied Chemicals and Materials Division on the properties of polyol ester lubricants under interagency agreement number N0042115IP00008. The work includes purity analysis and assessment, decomposition studies, experimental measurements on density, isobaric heat capacity, speed of sound, vapor pressure, viscosity, thermal conductivity, and distillation curve measurements. Models were developed for the thermodynamic properties, viscosity, and thermal conductivity that represent these properties to nearly within their experimental uncertainty for three pure polyol ester base fluids (pentaerythritol tetrapentanoate (POE5), pentaerythritol tetraheptanoate (POE7), pentaerythritol tetranonanoate (POE9), and a fully qualified lubricant meeting military specification MIL-PRF-23699.
\end{abstract}

\title{
Key words
}

Correlation, density, equation of state, heat capacity, lubricants, speed of sound, thermal conductivity, thermal decomposition, thermophysical property, vapor pressure, viscosity 


\section{Table of Contents}

1. Accomplishments and New Findings ......................................................................... 1

2. Purity Analysis of Polyol Ester Base Oils and MIL-PRF-23699 Oil............................. 2

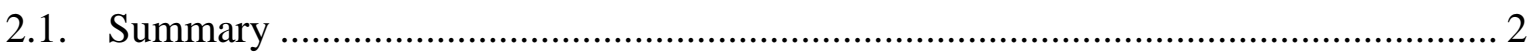

2.2. GC-FID and GC-MS Analysis .................................................................. 2

3. Thermal Decomposition Kinetics of Polyol Ester Lubricants .......................................... 5

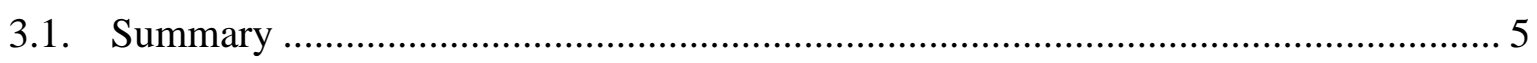

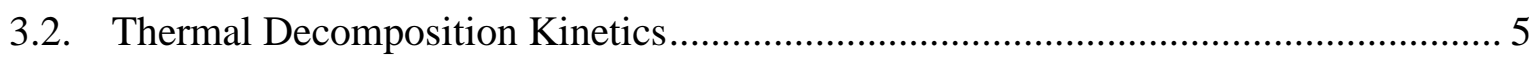

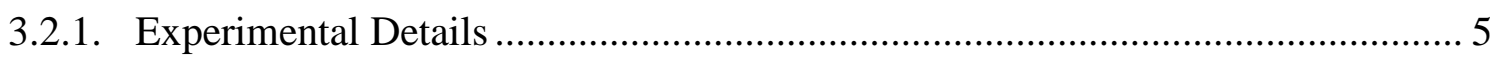

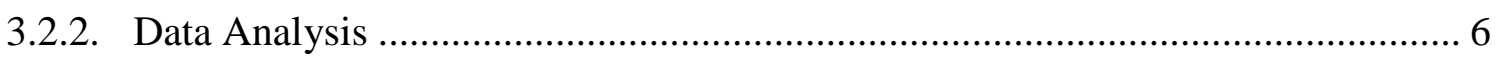

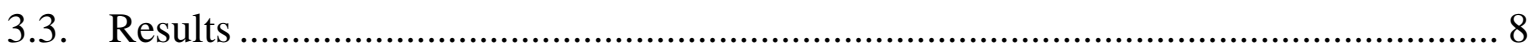

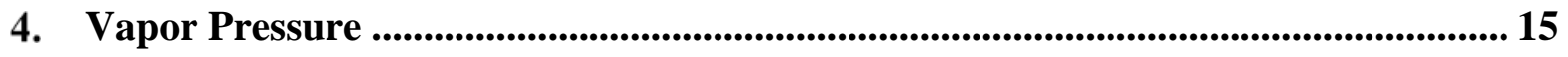

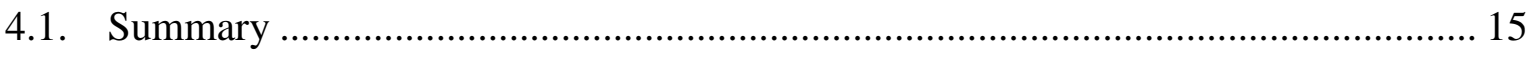

4.2. Vapor Pressure of POE5 ................................................................................. 15

4.2.1. Apparatus Description .................................................................................. 15

4.2.2. Measurements............................................................................................... 16

4.2.3. Instrument Modifications .............................................................................. 17

4.2.4. Temperature Measurements .............................................................................. 18

4.2.5. Mass of Carrier Gas..................................................................................... 18

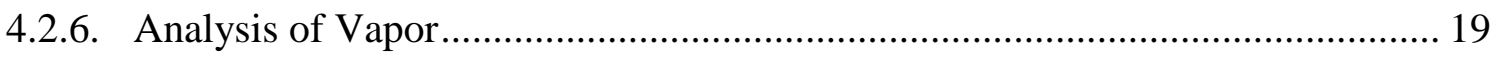

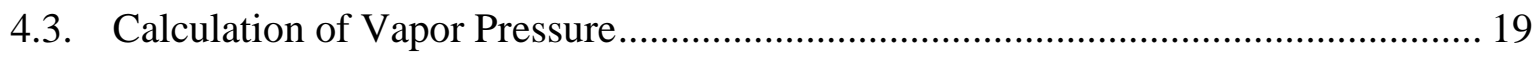

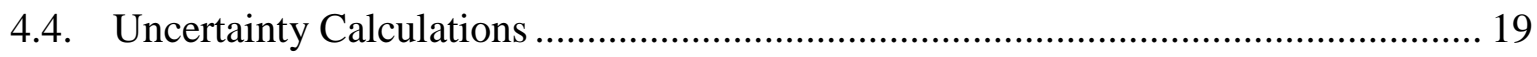

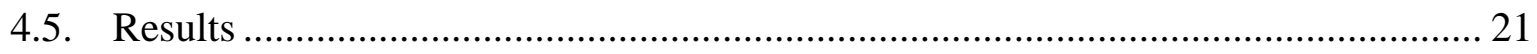

5. Isobaric Specific Heat Capacity .................................................................................... 23

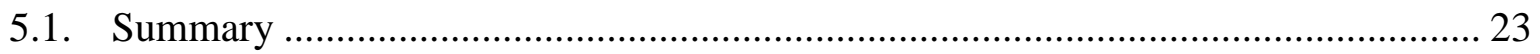

5.2. Experimental Methods ................................................................................... 23

5.2.1. Instrument Calibration................................................................................ 24

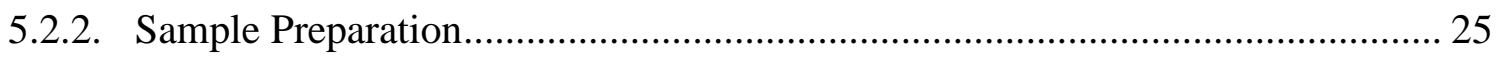

5.2.3. Measurement Procedure ............................................................................... 26

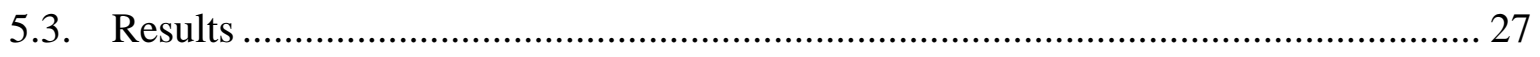

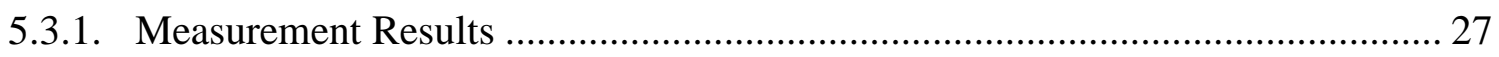

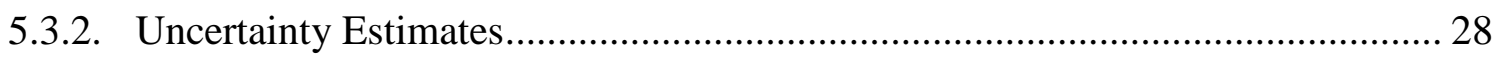

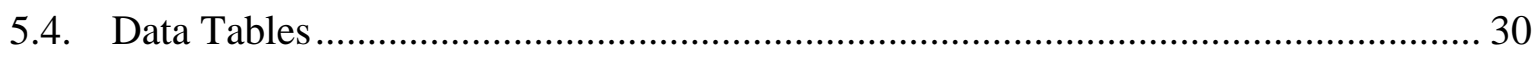




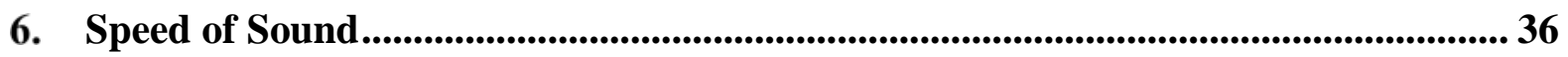

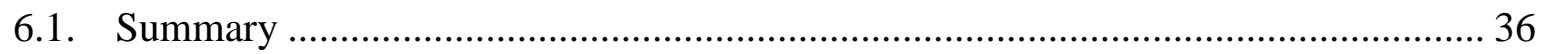

6.2. Principle of the Measurement............................................................................... 36

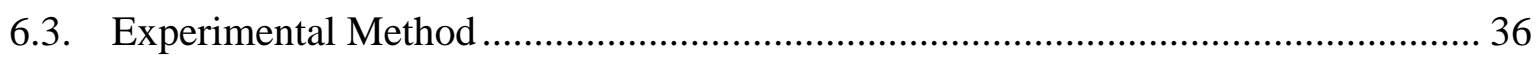

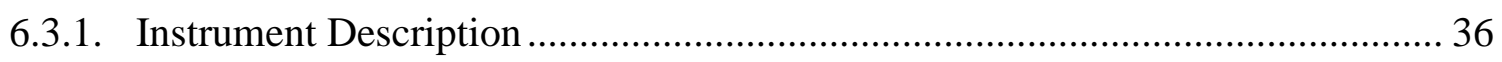

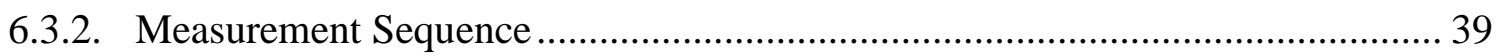

6.4. Calibration of Path-Length Difference and Measurement Uncertainties ................... 40

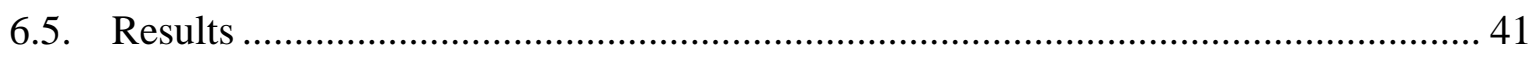

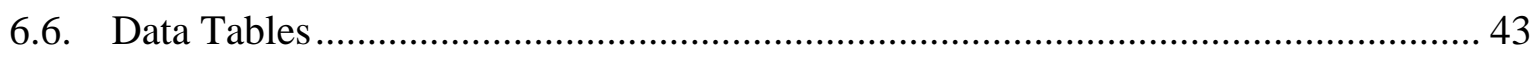

7. Compressed-Liquid Density ............................................................................................ 47

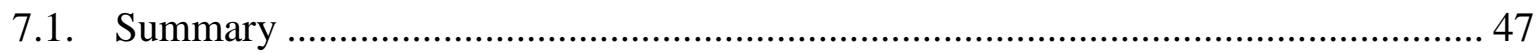

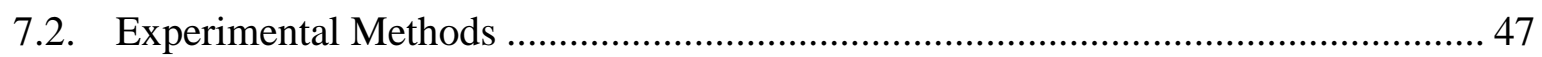

7.3. Determination of Uncertainty.................................................................................. 48

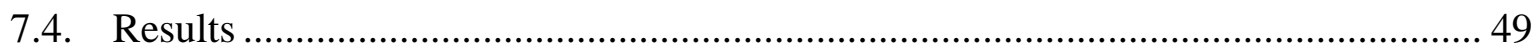

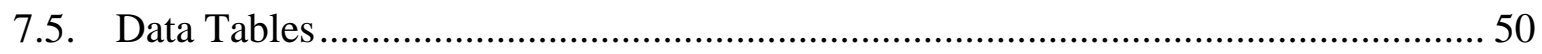

8. Ambient-Pressure Density and Speed of Sound ............................................................... 54

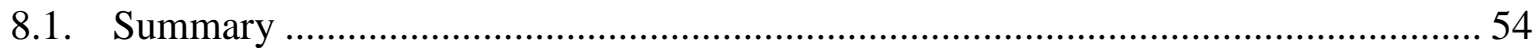

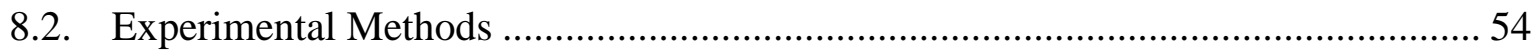

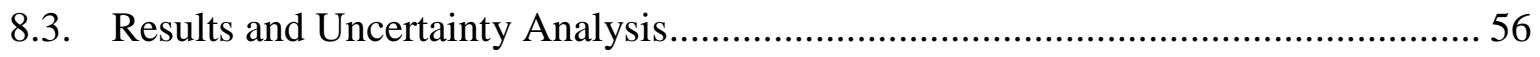

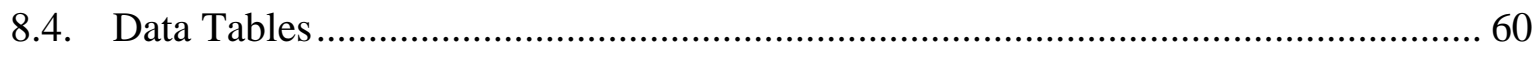

9. Thermal Conductivity ....................................................................................................... 63

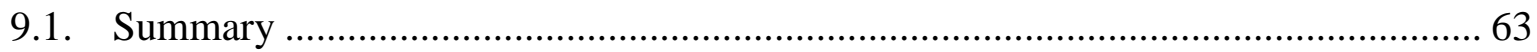

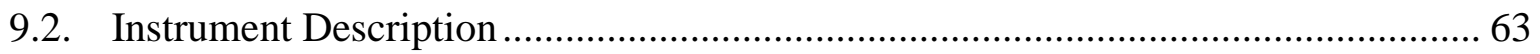

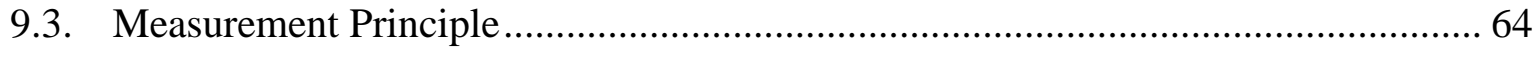

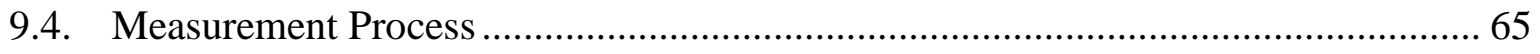

9.4.1. Catalytic Alumina Support/ Insulator Problem .................................................... 66

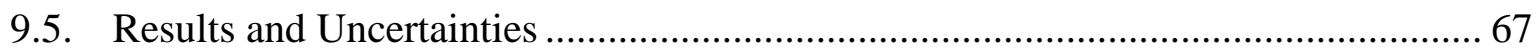

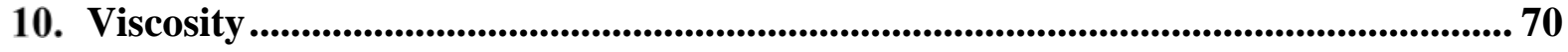

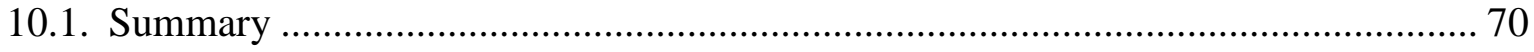

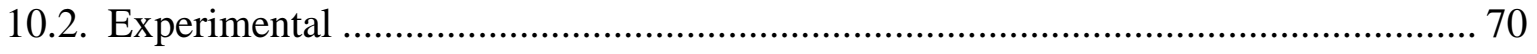

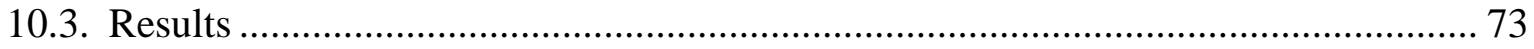

11. Advanced Distillation Curve of MIL-PRF-23699........................................................... 77

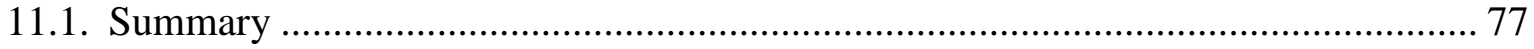

11.2. Advanced Distillation Curve Applied to MIL-PRF-23699........................................ 77 


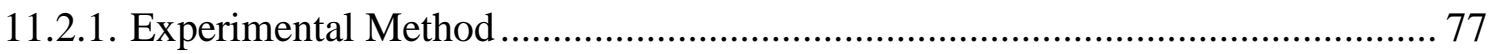

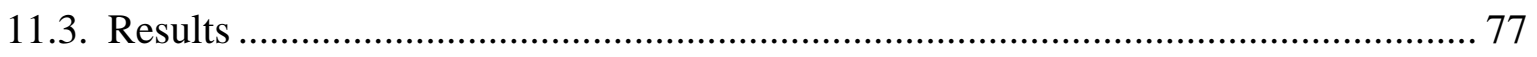

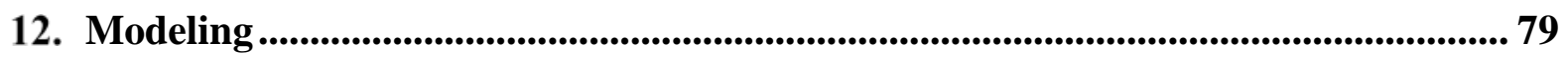

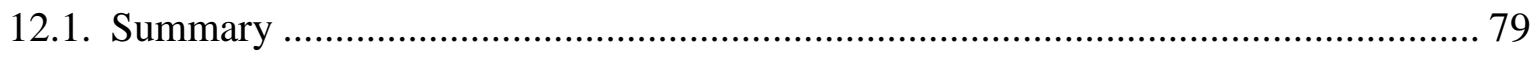

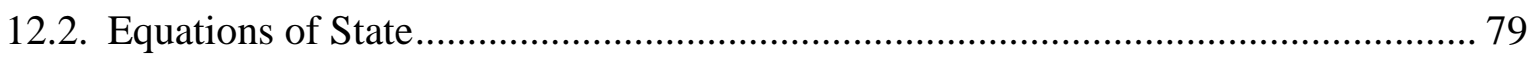

12.2.1. Functional Form of the Equation of State ....................................................... 82

12.2.2. Experimental Data and Comparisons to the Equation of State ........................... 87

12.2.3. Extrapolation Behavior................................................................................... 94

12.2.4. Estimated Uncertainties of Calculated Properties ............................................... 99

12.2.5. Calculated Values............................................................................................ 99

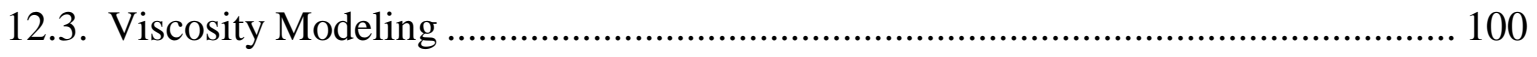

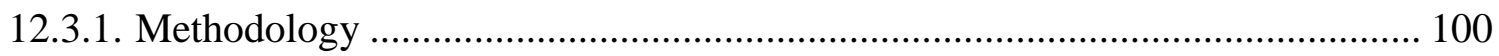

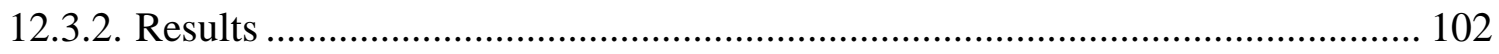

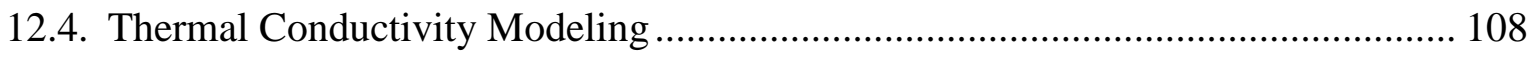

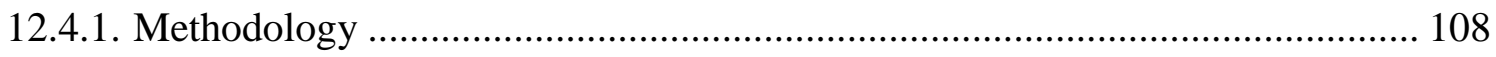

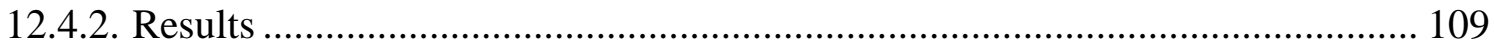

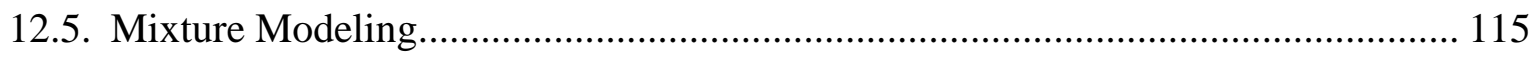

12.5.1. Pseudo-Pure Fluid Model ............................................................................. 116

12.5.2. Surrogate Mixture Models............................................................................... 120

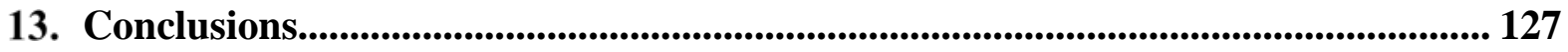

14. Acknowledgements ............................................................................................................ 129

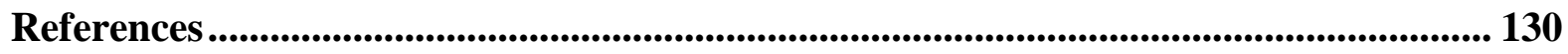

Appendix A: Thermal Conductivity Data Tables................................................................ 137

Appendix B: Viscosity Data Tables........................................................................................ 173

Appendix C: REFPROP Fluid Files...................................................................................... 185

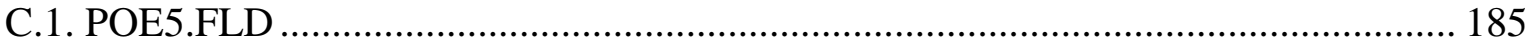

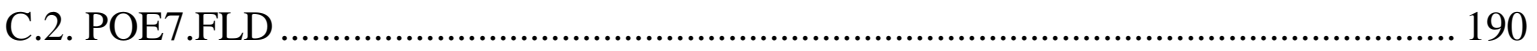

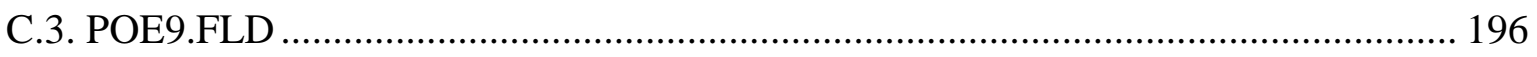

C.4. MILPRF23699.FLD …………………………………………………………. 201 


\section{List of Tables}

Table 1: GC-FID Analysis of Pentaerythritol Tetraester Base Oils. .............................................. 3

Table 2: Major Impurities in POE5 Sample Identified by the Acyl Moieties.................................. 4

Table 3: Major Impurities in POE7 Sample Identified by the Acyl Moieties. ................................. 4

Table 4: Major Impurities in POE9 Sample Identified by the Acyl Moieties................................. 4

Table 5: Thermal Decomposition Kinetic Data for POE5................................................................ 8

Table 6: Thermal Decomposition Kinetic Data for POE7 ............................................................... 11

Table 7: Thermal Decomposition Kinetic Data for POE9............................................................ 11

Table 8: Thermal Decomposition Kinetic Data for the MIL-PRF-23699 Qualified Lubricant.... 14

Table 9: Arrhenius Parameters for the Pure Base Oils and the Qualified Lubricant..................... 14

Table 10: The Vapor Pressures $\left(p_{\text {sat }}\right)$ and Combined Expanded $(k=2)$ Uncertainties $\left(U_{c}\right)$ for Measurements on POE5 and the Linear Alkane $n$-Octacosane $\left(\mathrm{C}_{28} \mathrm{H}_{58}\right.$, Measured as a Control Sample). ${ }^{\mathrm{a}}$ 21

Table 11: Measured Isobaric Heat Capacities for POE5 ............................................................... 30

Table 12: Measured Isobaric Heat Capacities for POE7 . ............................................................... 32

Table 13: Measured Isobaric Heat Capacities for POE9. ………................................................... 33

Table 14: Measured Isobaric Heat Capacities for MIL-PRF-23699.............................................. 34

Table 15: Measured Temperature T, Pressure $p$, Speed of Sound $w$, and Relative Combined, Expanded $(k=2)$ State Point Uncertainty in the Speed of Sound $U_{\mathrm{c}}$ for POE5; Average Values for the Four Sets of Three Replicate Measurements at Each $(T, p)$ State Point are Given. The Different Isochores Are Separated by Blank Lines.

Table 16: Measured Temperature T, Pressure $p$, Speed of Sound $w$, and Relative Combined, Expanded $(k=2)$ State Point Uncertainty in the Speed of Sound $U_{\mathrm{c}}$ for POE7; Average Values for the Four Sets of Three Replicate Measurements at Each $(T, p)$ State Point are Given. The Different Isochores Are Separated by Blank Lines.

Table 17: Measured Temperature T, Pressure $p$, Speed of Sound $w$, and Relative Combined, Expanded $(k=2)$ State Point Uncertainty in the Speed of Sound $U_{\mathrm{c}}$ for POE9; Average Values for the Four Sets of Three Replicate Measurements at Each $(T, p)$ State Point are Given. The Different Isochores Are Separated by Blank Lines. 
Table 18: Measured Temperature T, Pressure $p$, Speed of Sound $w$, and Relative Combined, Expanded $(k=2)$ State Point Uncertainty in the Speed of Sound $U_{\mathrm{c}}$ for MIL-PRF-23699; Average Values for the Four Sets of Three Replicate Measurements at Each $(T, p)$ State Point are Given. The Different Isochores Are Separated by Blank Lines...................... 46

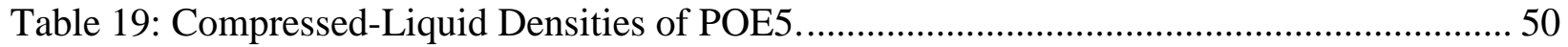

Table 20: Compressed-Liquid Densities of POE7 .................................................................... 51

Table 21: Compressed-Liquid Densities of POE9................................................................. 52

Table 22: Compressed-Liquid Densities of MIL-PRF-23699............................................. 53

Table 23: Measured Densities for Four Lubricant Samples at Ambient Pressure ( $83 \mathrm{kPa}) \ldots . . .60$

Table 24: Measured Speeds of Sound for Four Lubricants at Ambient Pressure ( $83 \mathrm{kPa}) . . . . .61$

Table 25: Calculated Adiabatic Compressibilities for Four Lubricants at Ambient Pressure

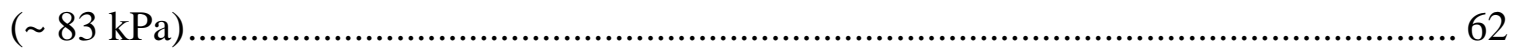

Table 26: Molar Mass and Critical Point Parameters. ............................................................... 83

Table 27: Coefficients of the Ideal-Gas Heat Capacity Equation............................................ 84

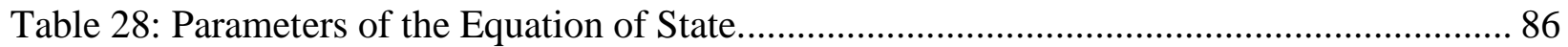

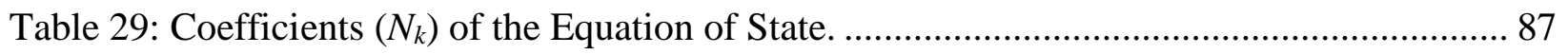

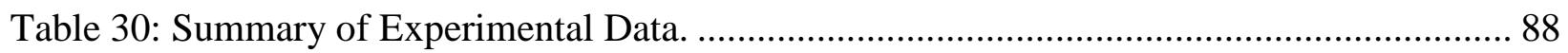

Table 31: Calculated Values of Properties for Algorithm Verification................................... 100

Table 32: Coefficients for the Thermal Conductivity Equation for POE5............................. 109

Table 33: Coefficients for the Thermal Conductivity Equation for POE7 ............................. 111

Table 34: Coefficients for the Thermal Conductivity Equation for POE9............................. 113

Table 35: Coefficients for the Thermal Conductivity Equation for MIL-PRF-23699............... 118

Table A1: Thermal Conductivity Data for POE5 at Temperatures from $300 \mathrm{~K}$ to $500 \mathrm{~K} . . . . . . . . .137$

Table A2: Thermal Conductivity Data for POE7 at Temperatures from $300 \mathrm{~K}$ to $500 \mathrm{~K} . . . . . . . . .145$

Table A3: Thermal Conductivity Data for POE9 at Temperatures from $300 \mathrm{~K}$ to $500 \mathrm{~K} . . . . . . . . .155$

Table A4: Thermal Conductivity Data for MIL-PRF-23699 Lubricant at Temperatures from

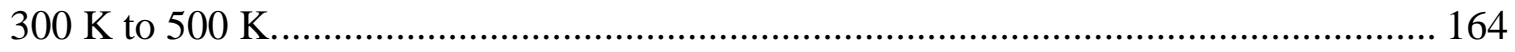

Table B1: Viscosity of POE5 at Temperatures from $275 \mathrm{~K}$ to $430 \mathrm{~K}$ with Pressures to $137 \mathrm{MPa}$. 173

Table B2: Viscosity of POE7 at Temperatures from $280 \mathrm{~K}$ to $450 \mathrm{~K}$ with Pressures to $137 \mathrm{MPa}$. 176 
Table B3: Viscosity of POE9 at Temperatures from $290 \mathrm{~K}$ to $450 \mathrm{~K}$ with Pressures to

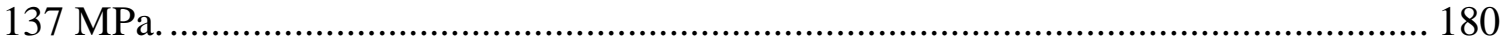
Table B4: Viscosity of Generic MIL-PRF-23699 Oil at Temperatures from $290 \mathrm{~K}$ to $450 \mathrm{~K}$ with

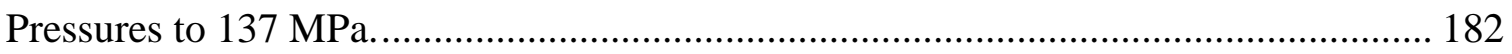




\section{List of Figures}

Figure 1: Chemical structure and nomenclature of the pentaerythritol tetraester base oils........... 2

Figure 2: High-pressure cell and thermostat block used for thermal decomposition studies of the

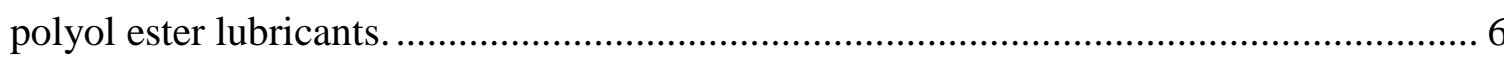

Figure 3: Data used to determine the decomposition rate constants $\left(k^{\prime}\right)$ for POE5. Both experimental data (circles) and their corresponding linear fits (solid and dashed lines) are

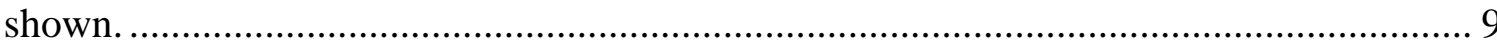

Figure 4: Data used to determine the decomposition rate constants $\left(k^{\prime}\right)$ for POE7. Both experimental data (circles) and their corresponding linear fits (solid and dashed lines) are shown. 10

Figure 5: Data used to determine the decomposition rate constants $\left(k^{\prime}\right)$ for POE9. Both experimental data (circles) and their corresponding linear fits (solid and dashed lines) are shown.

Figure 6: Data used to determine the decomposition rate constants ( $\left.k^{\prime}\right)$ for the MIL-PRF-23699 qualified lubricant. Both experimental data (circles) and their corresponding linear fits (solid and dashed lines) are shown.

Figure 7: Gas saturation apparatus with 18 saturator-adsorber pairs linked in series. An alternating pattern of long and short saturators was used (three long, three short, three long, etc.). The arrows show the direction of flow for the carrier gas.

Figure 8: Union between the saturator and adsorber. The PTFE sleeve insulates the carrier gas and vapor solute from the bulkhead union.

Figure 9: Differential scanning calorimeter.

Figure 10: Tools for sample preparation. From left to right: press used to seal sample pans, sample pans and lids, and microbalance used to determine sample mass.

Figure 11: Isobaric specific heat capacity measurements plotted as a function of temperature.

Only a subset of data points reported are plotted for clarity

Figure 12: Dual-path, pulse-echo, speed of sound instrument showing the thermostat (which contains the measuring cell) and the fluid manifold and pressure transducer; a vacuum system for evacuating the measuring cell is to the left of the thermostat.

Figure 13: Instrument rack for the pulse-echo speed of sound instrument 
Figure 14: Schematic diagram of the measuring cell inside the pressure vessel.

Figure 15: Measurements of the speed of sound for the POE lubricants; the lines connect the

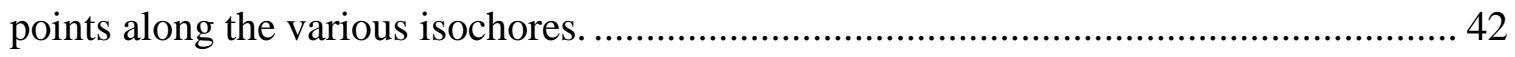

Figure 16: Schematic of the compressed-liquid density apparatus........................................ 48

Figure 17: Compressed-liquid density data as a function of pressure measured along isotherms at

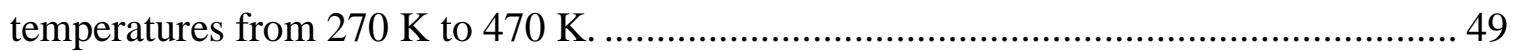

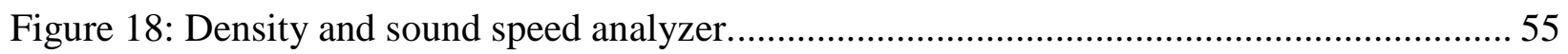

Figure 19: Vacuum system for degassing samples........................................................... 55

Figure 20: Ambient-pressure density measurements plotted as a function of temperature.......... 57

Figure 21: Ambient-pressure speed of sound measurements plotted as a function of

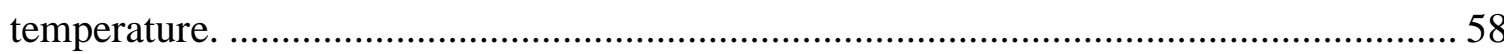

Figure 22: Calculated ambient-pressure adiabatic compressibilities plotted as a function of temperature. 59

Figure 23: (left) Transient hot-wire measurement electronics including: control computer, Wheatstone bridge containing hot-wires, power supplies, multimeters, multiplexer, and lock-in amplifier (for AC hot-wire experiments). (right) Temperature and pressure control system including: DC powered furnace, pressure manifold, piston pump, pressure transducer, and platinum resistance thermometers. 63

Figure 24: Transient hot-wire cell components including aluminum isothermal block, $5 \mathrm{~mL}$ microreactor, wire pressure feedthrough, and alumina hot-wire support and electrical insulation components.

Figure 25: Thermal conductivity data for POE5 as a function of pressure measured along isotherms at temperatures from $300 \mathrm{~K}$ to $500 \mathrm{~K}$.

Figure 26: Thermal conductivity data for POE7 as a function of pressure measured along isotherms at temperatures from $300 \mathrm{~K}$ to $500 \mathrm{~K}$

Figure 27: Thermal conductivity data for POE9 as a function of pressure measured along isotherms at temperatures from $300 \mathrm{~K}$ to $500 \mathrm{~K}$. 68

Figure 28: Thermal conductivity data for MIL-PRF-23699 lubricant as a function of pressure measured along isotherms at temperatures from $300 \mathrm{~K}$ to $500 \mathrm{~K}$

Figure 29: View of the oscillating-piston viscometer (above the bench drawers) with the vacuum system, sample cylinder, circulator, and syringe pump from left to right. 71 
Figure 30: Front panel of the custom LabVIEW virtual instrument developed in this project to automate the oscillating-piston viscometer with its associated circulator and syringe pump. Shown are the cell temperature (red) and the viscosity (blue) in the top plot, and the cell pressure (thin red) in the bottom plot for measurements of POE5 along the $320 \mathrm{~K}$ isotherm

Figure 31: Viscosity data for POE5 as a function of pressure measured along isotherms at temperatures from $275 \mathrm{~K}$ to $430 \mathrm{~K}$.

Figure 32: Viscosity data for POE7 as a function of pressure measured along isotherms at temperatures from $280 \mathrm{~K}$ to $450 \mathrm{~K}$. 75

Figure 33: Viscosity data for POE9 as a function of pressure measured along isotherms at temperatures from $290 \mathrm{~K}$ to $450 \mathrm{~K}$. 75

Figure 34: Viscosity data for MIL-PRF-23699 as a function of pressure measured along isotherms at temperatures from $290 \mathrm{~K}$ to $450 \mathrm{~K}$. 76

Figure 35: Comparisons of densities calculated with the equation of state to experimental data for POE5 as a function of pressure. 89

Figure 36: Comparisons of densities calculated with the equation of state to experimental data for POE7 as a function of pressure. 90

Figure 37: Comparisons of densities calculated with the equation of state to experimental data for POE9 as a function of pressure. 90

Figure 38: Comparisons of densities calculated with the equation of state to experimental data for MIL-PRF-23699 as a function of pressure. 91

Figure 39: Comparisons of speeds of sound calculated with the equation of state to experimental data for POE5 as a function of temperature. 91

Figure 40: Comparisons of speeds of sound calculated with the equation of state to experimental data for POE7 as a function of temperature. 92

Figure 41: Comparisons of speeds of sound calculated with the equation of state to experimental data for POE9 as a function of temperature. 92

Figure 42: Comparisons of speeds of sound calculated with the equation of state to experimental data for MIL-PRF-23699 as a function of temperature. 93 
Figure 43: Comparisons of isobaric heat capacities calculated with the equations of state for POE5, POE7, POE9, and MIL-PRF-23699 to experimental data as a function of temperature.

Figure 44: Phase Identification Parameter (PIP) versus temperature diagram showing isobars and saturation curves from 0 to $3 \mathrm{MPa}$ in steps of $0.1 \mathrm{MPa}$ for POE5. 95

Figure 45: Isothermal behavior of the POE7 equation of state at extreme conditions of temperature (up to $10^{12} \mathrm{~K}$ ) and pressure (up to $10^{10} \mathrm{MPa}$ ). 95

Figure 46: Temperature versus density diagram showing the saturation boundaries, the rectilinear diameter (the average of the saturation densities), and isobars up to $2 \mathrm{MPa}$ for POE9.

Figure 47: Calculations of $(Z-1) / \rho$ along isotherms versus density for POE5; isotherms are shown from temperatures of $400 \mathrm{~K}$ to $1200 \mathrm{~K}$ in steps of $25 \mathrm{~K}$. 96

Figure 48: Residual isochoric heat capacity versus temperature diagram for POE5; lines of constant density up to $2 \mathrm{~mol} \cdot \mathrm{dm}^{-3}$ are shown.

Figure 49: Calculated values of the second virial coefficient $B\left(\mathrm{dm}^{3} \cdot \mathrm{mol}^{-1}\right)$, third virial coefficient $C\left(\mathrm{dm}^{3} \cdot \mathrm{mol}^{-1}\right)^{2}$, and fourth virial coefficient $D\left(\mathrm{dm}^{3} \cdot \mathrm{mol}^{-1}\right)^{3}$ for POE7. 98

Figure 50: Characteristic (ideal) curves of the equation of state for POE9 as a function of temperature and pressure. 98

Figure 51: Percentage deviations in viscosity for POE5 as a function of temperature. 103

Figure 52: Percentage deviations in viscosity for POE5 as a function of pressure. 103

Figure 53: Percentage deviations in viscosity for POE5 as a function of density. 104

Figure 54: Percentage deviations in viscosity for POE7 as a function of temperature. 105

Figure 55: Percentage deviations in viscosity for POE7 as a function of pressure. 105

Figure 56: Percentage deviations in viscosity for POE7 as a function of density. 106

Figure 57: Percentage deviations in viscosity for POE9 as a function of temperature. 107

Figure 58: Percentage deviations in viscosity for POE9 as a function of pressure. 107

Figure 59: Percentage deviations in viscosity for POE9 as a function of density. 108

Figure 60: Percentage deviations in thermal conductivity for POE5 as a function of temperature. 110

Figure 61: Percentage deviations in thermal conductivity for POE5 as a function of pressure. 
Figure 62: Percentage deviations in thermal conductivity for POE5 as a function of density.

Figure 63: Percentage deviations in thermal conductivity for POE7 as a function of temperature.

Figure 64: Percentage deviations in thermal conductivity for POE7 as a function of pressure.

Figure 65: Percentage deviations in thermal conductivity for POE7 as a function of density.

Figure 66: Percentage deviations in thermal conductivity for POE9 as a function of temperature.

Figure 67: Percentage deviations in thermal conductivity for POE9 as a function of pressure.

Figure 68: Percentage deviations in thermal conductivity for POE9 as a function of density.

Figure 69: Percentage deviations in viscosity for MIL-PRF-23699 as a function of temperature.

Figure 70: Percentage deviations in viscosity for MIL-PRF-23699 as a function of pressure.

Figure 71: Percentage deviations in viscosity for MIL-PRF-23699 as a function of density.

Figure 72: Percentage deviations in thermal conductivity for MIL-PRF-23699 as a function of temperature.

Figure 73: Percentage deviations in thermal conductivity for MIL-PRF-23699 as a function of pressure.

Figure 74: Percentage deviations in thermal conductivity for MIL-PRF-23699 as a function of density.

Figure 75: Density at atmospheric pressure as a function of temperature for POE5, POE7, POE9, and MIL-PRF-23699.

Figure 76: Speed of sound at atmospheric pressure as a function of temperature for POE5, POE7, POE9, and MIL-PRF-23699. 
Figure 77: Heat capacity at atmospheric pressure as a function of temperature for POE5, POE7,

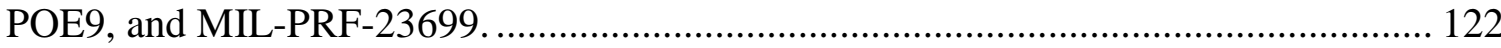

Figure 78: Thermal conductivity at atmospheric pressure as a function of temperature for POE5, POE7, POE9, and MIL-PRF-23699.

Figure 79: Viscosity at atmospheric pressure as a function of temperature for POE5, POE7, POE9, and MIL-PRF-23699.

Figure 80: Property deviations of Surrogate 1 model as a function of temperature.

Figure 81: Property deviations of Surrogate 2 model as a function of temperature; the inset shows the large deviations in viscosity at low temperatures.

Figure 82: Property deviations of Pseudo-pure fluid model as a function of temperature........ 125

Figure 83: Property deviations of Surrogate 1 model as a function of pressure........................ 125

Figure 84: Property deviations of Surrogate 2 model as a function of pressure; the inset shows

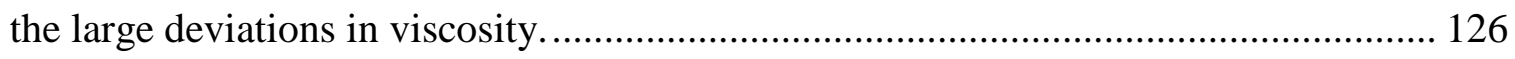

Figure 85: Property deviations of Pseudo-pure fluid model as a function of pressure.............. 126 


\section{Accomplishments and New Findings}

We performed an extensive experimental and modeling program on the thermophysical properties of three pure polyol ester fluids, and one fully qualified lubricant that meets military specification MIL-PRF-23699. The three pure fluids were pentaerythritol tetrapentanoate (POE5), pentaerythritol tetraheptanoate (POE7), and pentaerythritol tetranonanoate (POE9). Purity and chemical analysis indicated the MIL-PRF-23699 oil was a complex mixture of pentaerythritol tetraesters. The measured rates of thermal decomposition of the base fluids were comparable within the limits of uncertainty, and MIL-PRF-23699 was more thermally stable than the base fluids.

Thermodynamic property measurements (density, isobaric heat capacity, speed of sound, and vapor pressure) were carried out over a combined temperature range of (263 to 470) K, with pressures up to $63 \mathrm{MPa}$. These data represent the most comprehensive data set for fluids of this type.

The measured thermodynamic data were used to develop equations of state (EOS) based on Helmholtz energy for the pure fluids POE5, POE7, and POE9, and for the fully qualified lubricant MIL-PRF-23699, which was formulated as a pseudo-pure fluid. The EOS represent the entire fluid surface (liquid, vapor, and supercritical states) despite fluid critical temperatures approximately double the range of the experimental data. The experimental densities were fitted within $0.2 \%$, speed of sound within $0.1 \%$, and heat capacity to within $0.3 \%$. Development of these EOS required new fitting techniques and was possible only because of the accurate and wide-ranging data set.

Transport property measurements (viscosity and thermal conductivity) were measured over a combined temperature range of (275 to 500 ) $\mathrm{K}$, with pressures up to $138 \mathrm{MPa}$. These data were used to develop viscosity and thermal conductivity correlations for the four lubricants with deviations comparable to the experimental uncertainties.

Two surrogate mixture models were also developed using the base fluids POE5, POE7, and POE9, however we recommend the use of the pseudo-pure fluid model for the properties of the fully qualified lubricant. Finally, files compatible with the REFPROP computer program (version 10) are provided for the three pure fluids and the fully qualified lubricant. These will allow the modeling of these lubricants, including as a component in mixtures. 


\section{Purity Analysis of Polyol Ester Base Oils and MIL-PRF-23699 Oil}

\subsection{Summary}

The purity and chemical analysis of the fluids pentaerythritol tetrapentanoate (POE5), pentaerythritol tetraheptanoate (POE7), pentaerythritol tetranonanoate (POE9), and MIL-PRF23699 were determined by gas chromatography with flame ionization detection (GC-FID) and gas chromatography with mass spectrometry (GC-MS). The base oils POE5, POE7, and POE9 are approximately $96.7 \%, 97.3 \%$ and $93.0 \%$ of the title compound, respectively. Generic MIL-PRF23699 oil is a complex mixture of pentaerythritol tetraesters (details of the analysis are proprietary and are administratively restricted).

\subsection{GC-FID and GC-MS Analysis}

The pure fluid base oils, with the structure shown in Figure 1, are pentaerythritol-based with the esters pentanoate (POE5), heptanoate (POE7), and nonanoate (POE9). The qualified lubricant is a blend of pentaerythritol polyol esters in the $C_{5}$ to $C_{10}$ range (normal and branched alkane), and includes additives to prevent hydrolytic degradation.[1]<smiles>[R]C(=O)OCC(COC([R])=O)(COC([R])=O)COC([R])=O</smiles>

pentaerythritol tetrapentanoate (POE5): $\mathrm{R}=n-\mathrm{C}_{4} \mathrm{H}_{9}$ pentaerythritol tetraheptanoate (POE7): $\mathrm{R}=n-\mathrm{C}_{6} \mathrm{H}_{13}$ pentaerythritol tetranonanoate (POE9): $\mathrm{R}=n-\mathrm{C}_{8} \mathrm{H}_{17}$

Figure 1: Chemical structure and nomenclature of the pentaerythritol tetraester base oils.

Purity analysis by GC-FID was performed by temperature-programmed chromatographic separation of the diluted sample on a capillary column with a nonpolar stationary phase ((5\% phenyl)-methylpolysiloxane, $320 \mu \mathrm{m}$ i.d., $0.25 \mu \mathrm{m}$ thick film). Each sample was diluted to approximately 8 \% (by mass) in decane prior to chromatographic analysis. For the pure fluids (POE5, POE7, and POE9), $1 \mu \mathrm{L}$ of the diluted sample was injected by an autosampler into a split/splitless injector with split ratio of 50:1. The injector temperature was held at $573 \mathrm{~K}$, and the initial head pressure was $66.9 \mathrm{kPa}$. Flow of the carrier gas through the column was held constant 
at $1.6 \mathrm{~mL} \cdot \mathrm{min}^{-1}$. The temperature program for separation was: $393 \mathrm{~K}$ (for $0.5 \mathrm{~min}$ ) to $598 \mathrm{~K}$ (at $25 \mathrm{~K} \cdot \mathrm{min}^{-1}$ ), held $20 \mathrm{~min}$ for POE5 and POE7, and $28 \mathrm{~min}$ for POE9. The carrier gas for all GCFID experiments was research grade nitrogen. Reported purities and expanded uncertainties $(k=2)$ are based on three replicate injections of a minimum of 5 samples and an estimate for the complete separation and integration of components.

The GC-MS analysis was also performed by temperature-programmed chromatographic separation of the diluted sample on a capillary column with a nonpolar stationary phase ((5\% phenyl)-methylpolysiloxane, $250 \mu \mathrm{m}$ i.d., $0.25 \mu \mathrm{m}$ thick film). Approximately $1 \mu \mathrm{L}$ of the samples used for GC-FID analysis, described above, were injected manually. Chromatographic conditions for the pure fluids were as follows: $573 \mathrm{~K}$ inlet temperature, 70:1 injector split ratio, $135.1 \mathrm{kPa}$ initial column head pressure, and $1.5 \mathrm{~mL} \cdot \mathrm{min}^{-1}$ column flow. The oven temperature program was: $423 \mathrm{~K}$ (held for $0.5 \mathrm{~min}$ ), to $573 \mathrm{~K}$ (at a rate of $288 \mathrm{~K} \cdot \mathrm{min}^{-1}$, held $7 \mathrm{~min}$ ) to $593 \mathrm{~K}$ (at a rate of $283 \mathrm{~K} \cdot \mathrm{min}^{-1}$ ), held $10 \mathrm{~min}$ for POE5 and POE7, and $20 \mathrm{~min}$ for POE9. The carrier gas for these separations was ultra-high purity helium.

The percent purity of each base fluid is shown in Table 1 . The purity is based upon the uncalibrated area percent of the total FID response of the compound of interest, relative to the impurities. Identifications of the major impurities in the single-component base fluids are included in Table 2, Table 3, and Table 4. The impurities are listed in order of observed chromatographic retention time, relative to the specified fluid, which is an indicator of the size of the species (larger species elute later). The acyl moieties were determined by mass spectral fragmentation patterns.[2] The sizes of the ester groups present on the polyol are indicated; however, the exact structures (linear or branched chains, and number of ester groups) have not been determined. The most significant impurities are polyol tetra-esters with different acyl chain lengths (either larger, smaller, or branched); however, in each sample a small portion is a pentaerythritol (PE) triester, where one of the arms on the polyol is replaced by an unesterified hydroxyl group.

Table 1: GC-FID Analysis of Pentaerythritol Tetraester Base Oils.

\begin{tabular}{c|c|c|c|c}
\hline Fluid & Formula & CAS No. & Molar Mass & Purity (area \%) \\
\hline \hline POE5 & $\mathrm{C}_{25} \mathrm{H}_{44} \mathrm{O}_{8}$ & $15834-04-5$ & 472.6 & $96.7 \pm 0.2$ \\
POE7 & $\mathrm{C}_{33} \mathrm{H}_{60} \mathrm{O}_{8}$ & $25811-35-2$ & 584.8 & $97.3 \pm 0.8$ \\
POE9 & $\mathrm{C}_{41} \mathrm{H}_{76} \mathrm{O}_{8}$ & $14450-05-6$ & 697.0 & $93.0 \pm 1.0$ \\
\hline
\end{tabular}

${ }^{a}$ Area percent is uncalibrated GC-FID response 
Table 2: Major Impurities in POE5 Sample Identified by the Acyl Moieties.

\begin{tabular}{l|c}
\hline \multicolumn{1}{c|}{ Component } & Area \% $^{\mathbf{a}}$ \\
\hline \hline PE tripentanoate: $\left(\mathrm{C}_{4} \mathrm{H}_{9} \mathrm{C}(\mathrm{O}) \mathrm{OCH}_{2}\right)_{3} \mathrm{C}-\mathrm{CH}_{2} \mathrm{OH}$ & 0.4 \\
PE with $\mathrm{C}_{4}$ and $\mathrm{C}_{5}$ esters & 0.2 \\
PE with mixed linear/branched $\mathrm{C}_{5}$ esters & 0.6 \\
PE tetrapentanoate (POE5) & $96.7 \pm 0.2$ \\
PE with $\mathrm{C}_{5}$ and $\mathrm{C}_{7}$ esters & 1.5 \\
PE with $\mathrm{C}_{5}$ and $\mathrm{C}_{9}$ esters & 0.2 \\
PE with $\mathrm{C}_{5}$ esters (structure unknown) & 0.4 \\
\hline${ }^{\mathrm{a}}$ Area percent is uncalibrated GC-FID response
\end{tabular}

${ }^{a}$ Area percent is uncalibrated GC-FID response

Table 3: Major Impurities in POE7 Sample Identified by the Acyl Moieties.

\begin{tabular}{l|c}
\hline \multicolumn{1}{c|}{ Component } & Area \% $^{\mathbf{a}}$ \\
\hline \hline PE triheptanoate: $\left(\mathrm{C}_{6} \mathrm{H}_{13} \mathrm{C}(\mathrm{O}) \mathrm{OCH}_{2}\right)_{3} \mathrm{C}-\mathrm{CH}_{2} \mathrm{OH}$ & 0.1 \\
PE with $\mathrm{C}_{5}$ and $\mathrm{C}_{7}$ esters & 0.2 \\
PE with $\mathrm{C}_{6}$ and $\mathrm{C}_{7}$ esters & 1.8 \\
PE tetraheptanoate (POE7) & $97.3 \pm 0.8$ \\
PE with $\mathrm{C}_{7}$ and $\mathrm{C}_{8}$ esters & 0.2 \\
PE with $\mathrm{C}_{7}$ esters (structure unknown) & 0.3 \\
\hline
\end{tabular}

Table 4: Major Impurities in POE9 Sample Identified by the Acyl Moieties.

\begin{tabular}{l|c}
\hline \multicolumn{1}{c|}{ Component } & Area \% $^{\mathbf{a}}$ \\
\hline \hline PE trinonanoate: $\left(\mathrm{C}_{8} \mathrm{H}_{17} \mathrm{C}(\mathrm{O}) \mathrm{OCH}_{2}\right)_{3} \mathrm{C}-\mathrm{CH}_{2} \mathrm{OH}$ & 0.2 \\
PE with $\mathrm{C}_{6}$ and $\mathrm{C}_{9}$ esters & 0.2 \\
PE with $\mathrm{C}_{7}$ and $\mathrm{C}_{9}$ esters & 0.2 \\
PE with $\mathrm{C}_{8}$ and $\mathrm{C}_{9}$ esters & 0.3 \\
PE tetranonanoate (POE9) & $93.0 \pm 1.0$ \\
PE with other mixed tetra esters & 0.3 \\
PE with C9 and $\mathrm{C}_{10}$ esters & 4.9 \\
PE with C9 and $\mathrm{C}_{10}$ esters (larger species) & 0.5 \\
PE with larger mixed tetra esters & 0.3 \\
\hline
\end{tabular}

${ }^{a}$ Area percent is uncalibrated GC-FID response 


\section{Thermal Decomposition Kinetics of Polyol Ester Lubricants}

\subsection{Summary}

The global thermal decomposition kinetics of the fluids pentaerythritol tetrapentanoate (POE5), pentaerythritol tetraheptanoate (POE7), pentaerythritol tetranonanoate (POE9), and generic MILPRF-23699 were performed in stainless steel ampule reactors at approximately 20.7 MPa and the extent of decomposition determined by gas chromatography with flame ionization detection. The decomposition rates varied from $1.6( \pm 0.7) \times 10^{-8} \mathrm{~s}^{-1}$ at $496 \mathrm{~K}$ to $2.3( \pm 0.7) \times 10^{-4} \mathrm{~s}^{-1}$ at $676 \mathrm{~K}$ (Tables $5-7$ ), with expanded uncertainties given in parentheses. In general, the measured rates of decomposition of the base fluids POE5, POE7, and POE9 (which contained no additives) were comparable within the limits of uncertainty for each fluid measured; in other words, to the resolution of the experimental technique, no fluid was more thermally stable than another. As expected, the qualified lubricant, generic MIL-PRF-23699 (with additives) was more thermally stable than the base fluids. The rates of decomposition for generic MIL-PRF-23699 varied from $1.2( \pm 2.7) \times 10^{-8} \mathrm{~s}^{-1}$ at $497 \mathrm{~K}$ to $1.3( \pm 0.3) \times 10^{-4} \mathrm{~s}^{-1}$ at $676 \mathrm{~K}$ (Table 8). In general, the addition of anti-wear agents and antioxidants extended the lifetime of the base oils at the conditions tested by approximately a factor of 1.7. Finally, new (unpassivated) stainless steel reactors and alumina surfaces catalyzed the thermal decomposition of the base oils; the decomposition rate of generic MIL-PRF-23699 was impacted less by these factors, due to the inclusion of additives to prevent decomposition by surface reactions.

\subsection{Thermal Decomposition Kinetics}

The global thermal decomposition kinetics of three polyol ester lubricant base oils and a fully qualified (generic MIL-PRF-23699) formulation were performed in stainless steel ampule reactors to provide a guide for the subsequent property measurements and in-service constraints. The extent of decomposition, as a function of time, was determined by gas chromatography with flame ionization detection (GC-FID). With this information, pseudo-first-order rate constants and Arrhenius parameters were derived. Other techniques used for chemical characterization include gas chromatography with mass spectrometry (GC-MS), nuclear magnetic resonance spectroscopy, and Karl Fischer titration. Presented in this section is a brief description of the measurement procedure and a compilation of the kinetic measurements. The reader is referred to the paper by Urness et al.[3] for more details.

\subsubsection{Experimental Details}

Tubular stainless steel (316L) ampule reactors with an internal volume of approximately $190 \mu \mathrm{L}$ were used to test the fluids of interest. The reactors were sealed with a high-pressure valve and 
were heated in tight-fitting slots in a thermostated block, as shown in Figure 2. The reactor cell and thermostated block for temperature control are described in more detail elsewhere.[3, 4]

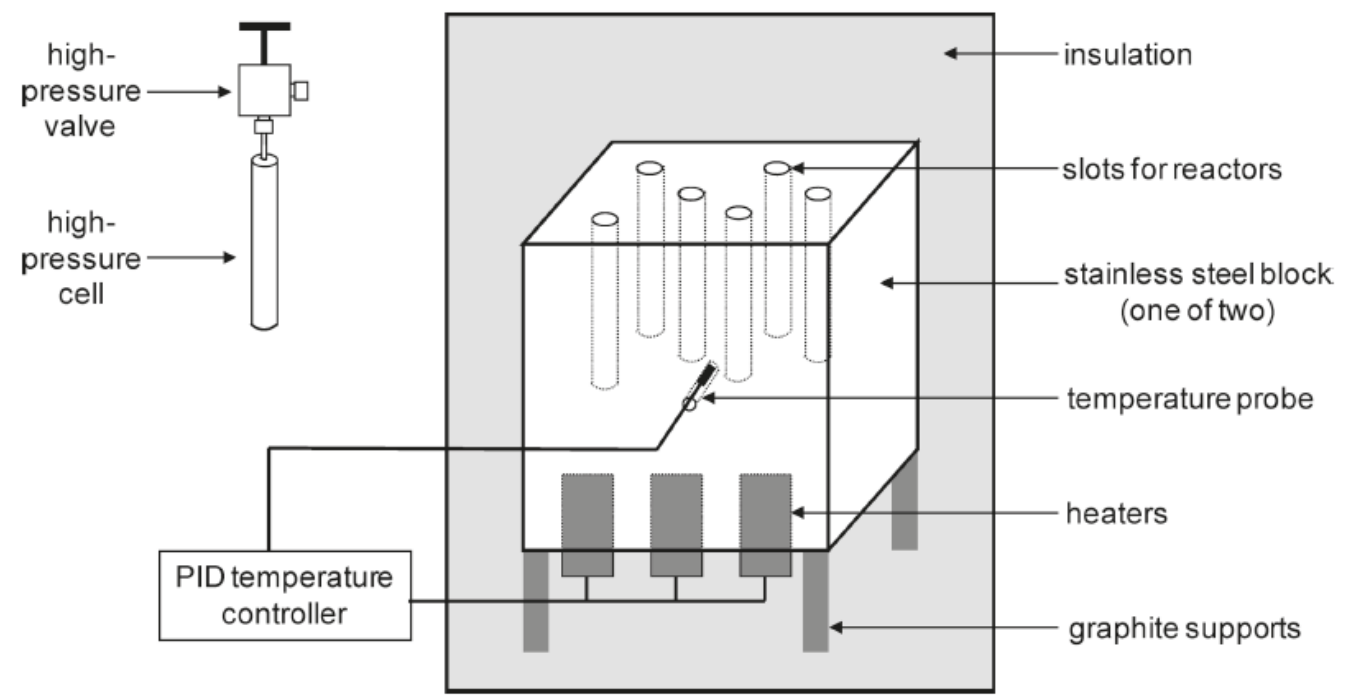

Figure 2: High-pressure cell and thermostat block used for thermal decomposition studies of the polyol ester lubricants.

The cells were loaded with the appropriate amount of fluid to achieve an initial pressure of approximately 20.7 MPa at a given temperature. The fluid mass was estimated with preliminary equations of state developed for POE5 or POE9. The appropriate mass loading of POE7 was the average of the predicted amount of POE5 and POE9. The cells were filled with approximately $150 \mathrm{mg}$ to $180 \mathrm{mg}$ of fluid, depending on the desired reaction temperature, nearly filling the entire cell volume. Prior to heating, the cell was degassed with a single freeze-pump-thaw cycle in liquid nitrogen. The samples were then heated in the temperature range of $498 \mathrm{~K}$ to $673 \mathrm{~K}$ with reaction times ranging from $10 \mathrm{~min}$ to $35 \mathrm{~min}$ at $673 \mathrm{~K}$ and up to $300 \mathrm{~h}$ to $700 \mathrm{~h}$ (12 days to 30 days) at $498 \mathrm{~K}$. The reactions were quenched by immersing the cell in a bath of room-temperature water.

\subsubsection{Data Analysis}

Due to the complexity involved during thermal decomposition, it is necessary to make assumptions to simplify the analysis and determine rate expressions to describe the thermal stability of these fluids. We assume that the reactant of interest, A, thermally decomposes to a set of products and that we can model the overall decomposition with a single, global pseudo-first-order rate constant, $k^{\prime}$. Since the product suite contains both hydrocarbons and oxygenated species, quantitative analysis by flame ionization detection (FID) of the product suite is complicated because the detector has a variable response to the amount of oxygen present. To avoid calibrating the FID 
response for all possible product species, we monitor the disappearance of the reactant(s), $[\mathrm{A}]_{t}$, relative to the products formed, and compare this to an unheated reference sample, $[\mathrm{A}]_{0}$, to measure the 'unreacted fraction':

$$
\ln \frac{[\mathrm{A}]_{t}}{[\mathrm{~A}]_{0}}=\ln (\text { unreacted fraction })=-k^{\prime} t
$$

In this equation, $k^{\prime}$ is the rate constant derived from the slope of the line in a plot of ln(unreacted fraction) vs. reaction time at a particular temperature.

We also assume that analysis of the remaining liquid fraction of the decomposed fluid is representative of the decomposition process. Based on our observations, the extent of decomposition that ensures this is a valid assumption is at most $40 \%$. Additional decomposition beyond this point results in a significant vapor phase and serves as an indication that the initial set of products is undergoing thermal decomposition. Analysis of samples with more than $40 \%$ of reactant decomposed will also not fall on a straight line with the less-decomposed samples, which serves as an indication of where the pseudo first-order approximation begins to break down.

Finally, the rate constants are measured over a range of temperatures to develop an Arrhenius expression that describes the fluid's thermal stability based on the activation energy, $E_{\mathrm{a}}\left(\mathrm{in} \mathrm{J} \cdot \mathrm{mol}^{-1}\right.$ ) and the pre-exponential factor, $A$ (units of $\mathrm{s}^{-1}$ ):

$$
k^{\prime}(T)=A \exp \left(-\frac{E_{\mathrm{a}}}{R T}\right)
$$

where $R$ is the universal gas constant $\left(8.314 \mathrm{~J} \cdot \mathrm{mol}^{-1} \cdot \mathrm{K}^{-1}\right)$ and $T$ is the reaction temperature.

The age of the ampule cell was also shown to be important for test repeatability, indicating that surface reactions can influence the decomposition rates of the polyol esters. This is contrary to previous decomposition studies using the ampule reactors to heat unoxygenated hydrocarbon fuels.[4, 5] Those experiments showed no correlation between cell age and measured decomposition rate. However, heating the pure polyol ester base oils in new, unpassivated cells resulted in decomposition products formed at a faster rate than when an older cell was used under the same conditions. The cells were passivated by several cycles of heating the reactors loaded with the polyol esters followed by cleaning to build up a sufficient surface oxide layer. A more detailed discussion of catalytic decomposition of these polyol esters is presented in Urness et al.[3]. 


\subsection{Results}

Linear regressions are used to determine the individual rate constants $\left(k^{\prime}\right)$ for the tested fluids. The figures give visual representations of how well the linear fits approximate the data. The data for the base oil POE5 tested at $496 \mathrm{~K}$ to $675 \mathrm{~K}$ is shown in Figure 3 and Table 5. Results for POE7 tested at $595 \mathrm{~K}$ to $675 \mathrm{~K}$ are included in Figure 4 and Table 6. Results for POE9 also tested at $595 \mathrm{~K}$ to $675 \mathrm{~K}$ are summarized in Figure 5 and Table 7. The data for the qualified lubricant at $497 \mathrm{~K}$ to $675 \mathrm{~K}$ are shown in Figure 6 and Table 8. The derived Arrhenius parameters for the four tested fluids are summarized in Table 9. A detailed discussion of the measured reaction rates, Arrhenius fits, and the formation of reaction products are included in Urness et al.[3].

Table 5: Thermal Decomposition Kinetic Data for POE5.

\begin{tabular}{c|c|c|c|c|c}
\hline $\boldsymbol{T}$ & $+/-$ & $\mathbf{1 0 0 0 / T}$ & $\boldsymbol{k}^{\prime}$ & $\mathbf{l n} \boldsymbol{k}^{\prime}$ & $\begin{array}{c}\text { fit uncert. (95 \%) } \\
/ \mathbf{K}\end{array}$ \\
\hline $\mathbf{K}$ & & $\mathbf{s}^{-\mathbf{1}}$ & & $6.92 \times 10^{-9}$ \\
\hline \hline 495.7 & 1.0 & 2.018 & $1.602 \times 10^{-8}$ & -17.949 & $2.12 \times 10^{-8}$ \\
520.3 & 1.0 & 1.922 & $3.736 \times 10^{-8}$ & -17.103 & $6.74 \times 10^{-7}$ \\
596.3 & 2.0 & 1.677 & $2.417 \times 10^{-6}$ & -12.933 & $9.09 \times 10^{-7}$ \\
599.7 & 3.2 & 1.668 & $3.015 \times 10^{-6}$ & -12.712 & $2.09 \times 10^{-6}$ \\
599.4 & 1.8 & 1.668 & $3.079 \times 10^{-6}$ & -12.691 & $3.28 \times 10^{-6}$ \\
621.1 & 2.4 & 1.610 & $1.047 \times 10^{-5}$ & -11.467 & $3.33 \times 10^{-6}$ \\
624.6 & 3.4 & 1.601 & $1.157 \times 10^{-5}$ & -11.367 & $1.94 \times 10^{-6}$ \\
624.3 & 2.0 & 1.602 & $9.991 \times 10^{-6}$ & -11.514 & $6.52 \times 10^{-6}$ \\
648.7 & 1.6 & 1.542 & $4.205 \times 10^{-5}$ & -10.077 & $1.04 \times 10^{-5}$ \\
649.7 & 2.6 & 1.539 & $5.259 \times 10^{-5}$ & -9.853 & $8.43 \times 10^{-6}$ \\
649.4 & 1.8 & 1.540 & $5.522 \times 10^{-5}$ & -9.804 & $3.70 \times 10^{-5}$ \\
670.7 & 2.0 & 1.491 & $1.818 \times 10^{-4}$ & -8.613 & $1.02 \times 10^{-4}$ \\
675.4 & 2.6 & 1.481 & $2.053 \times 10^{-4}$ & -8.491 & $6.91 \times 10^{-5}$ \\
675.6 & 2.0 & 1.480 & $2.340 \times 10^{-4}$ & -8.360 & \\
\hline
\end{tabular}



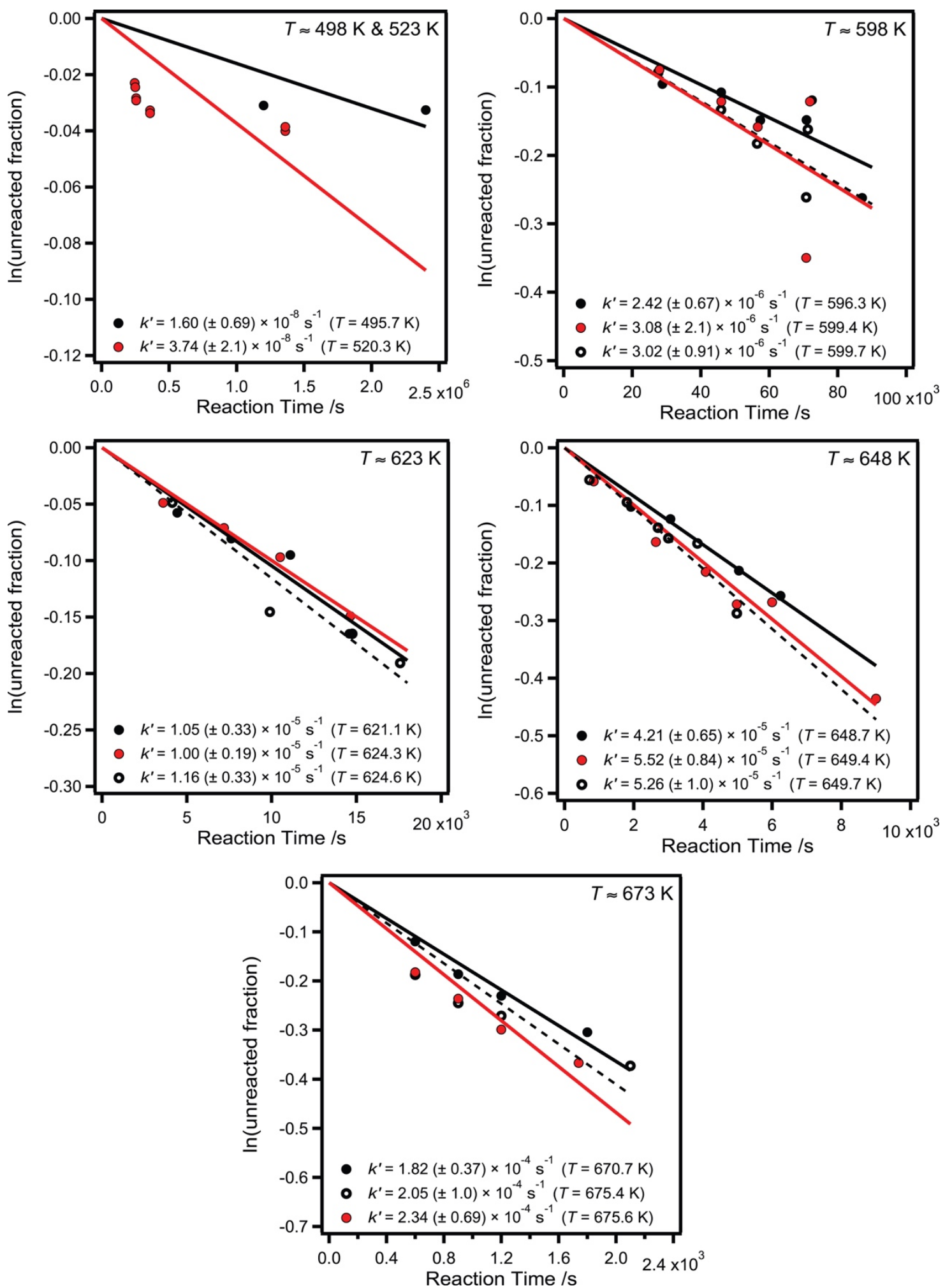

Figure 3: Data used to determine the decomposition rate constants $\left(k^{\prime}\right)$ for POE5. Both experimental data (circles) and their corresponding linear fits (solid and dashed lines) are shown. 

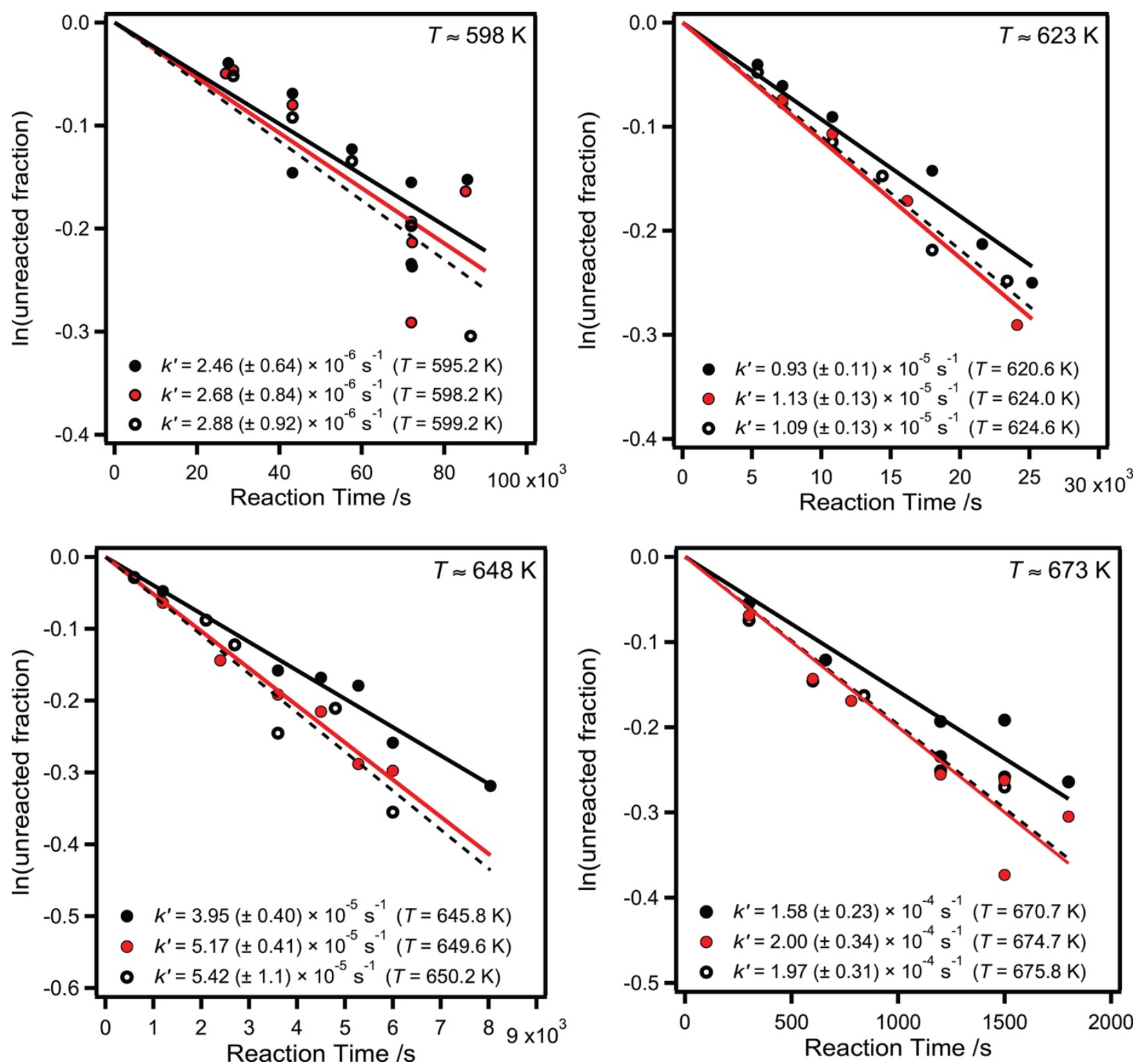

Figure 4: Data used to determine the decomposition rate constants $\left(k^{\prime}\right)$ for POE7. Both experimental data (circles) and their corresponding linear fits (solid and dashed lines) are shown. 
Table 6: Thermal Decomposition Kinetic Data for POE7.

\begin{tabular}{|c|c|c|c|c|c|}
\hline $\begin{array}{c}\mathbf{T} \\
/ \mathbf{K}\end{array}$ & $\begin{array}{l}+/- \\
/ \mathbf{K}\end{array}$ & $1000 / T$ & $\begin{array}{c}k^{\prime} \\
/ \mathbf{s}^{-1}\end{array}$ & $\ln k^{\prime}$ & $\begin{array}{c}\text { fit uncert. }(95 \%) \\
\qquad / \mathrm{s}^{-1}\end{array}$ \\
\hline 595.2 & 1.7 & 1.680 & $2.462 \times 10^{-6}$ & -12.915 & $6.41 \times 10^{-7}$ \\
\hline 599.2 & 3.5 & 1.669 & $2.879 \times 10^{-6}$ & -12.758 & $9.16 \times 10^{-7}$ \\
\hline 598.2 & 1.7 & 1.672 & $2.679 \times 10^{-6}$ & -12.830 & $8.42 \times 10^{-7}$ \\
\hline 620.6 & 2.2 & 1.611 & $9.298 \times 10^{-6}$ & -11.586 & $1.09 \times 10^{-6}$ \\
\hline 624.6 & 3.5 & 1.601 & $1.091 \times 10^{-5}$ & -11.426 & $1.26 \times 10^{-6}$ \\
\hline 624.0 & 2.2 & 1.603 & $1.131 \times 10^{-5}$ & -11.390 & $1.34 \times 10^{-6}$ \\
\hline 645.8 & 2.1 & 1.549 & $3.949 \times 10^{-5}$ & -10.140 & $3.98 \times 10^{-6}$ \\
\hline 650.2 & 3.9 & 1.538 & $5.422 \times 10^{-5}$ & -9.822 & $1.14 \times 10^{-5}$ \\
\hline 649.6 & 2.1 & 1.539 & $5.167 \times 10^{-5}$ & -9.871 & $4.07 \times 10^{-6}$ \\
\hline 670.7 & 2.4 & 1.491 & $1.579 \times 10^{-4}$ & -8.754 & $2.28 \times 10^{-5}$ \\
\hline 675.8 & 4.5 & 1.480 & $1.967 \times 10^{-4}$ & -8.534 & $3.13 \times 10^{-5}$ \\
\hline 674.7 & 2.3 & 1.482 & $2.000 \times 10^{-4}$ & -8.517 & $3.42 \times 10^{-5}$ \\
\hline
\end{tabular}

Table 7: Thermal Decomposition Kinetic Data for POE9.

\begin{tabular}{|c|c|c|c|c|c|}
\hline $\begin{array}{c}\mathbf{T} \\
/ \mathbf{K}\end{array}$ & $\begin{array}{l}+/- \\
/ \mathbf{K}\end{array}$ & $1000 / T$ & $\begin{array}{c}k^{\prime} \\
/ \mathbf{s}^{-1}\end{array}$ & $\ln k^{\prime}$ & $\begin{array}{c}\text { fit uncert. }(95 \%) \\
\qquad / \mathrm{s}^{-1}\end{array}$ \\
\hline 595.2 & 1.7 & 1.680 & $1.910 \times 10^{-6}$ & -13.168 & $6.16 \times 10^{-7}$ \\
\hline 599.2 & 3.5 & 1.669 & $2.364 \times 10^{-6}$ & -12.955 & $1.49 \times 10^{-6}$ \\
\hline 598.2 & 1.7 & 1.672 & $2.155 \times 10^{-6}$ & -13.048 & $1.37 \times 10^{-6}$ \\
\hline 620.6 & 2.2 & 1.611 & $1.002 \times 10^{-5}$ & -11.511 & $1.87 \times 10^{-6}$ \\
\hline 624.6 & 3.5 & 1.601 & $1.157 \times 10^{-5}$ & -11.367 & $3.30 \times 10^{-6}$ \\
\hline 624.0 & 2.2 & 1.603 & $1.207 \times 10^{-5}$ & -11.325 & $2.28 \times 10^{-6}$ \\
\hline 645.8 & 2.1 & 1.549 & $4.337 \times 10^{-5}$ & -10.046 & $2.90 \times 10^{-6}$ \\
\hline 650.2 & 3.9 & 1.538 & $5.175 \times 10^{-5}$ & -9.869 & $9.00 \times 10^{-6}$ \\
\hline 649.6 & 2.1 & 1.539 & $5.254 \times 10^{-5}$ & -9.854 & $2.64 \times 10^{-6}$ \\
\hline 670.7 & 2.4 & 1.491 & $1.921 \times 10^{-4}$ & -8.558 & $2.74 \times 10^{-5}$ \\
\hline 675.8 & 4.5 & 1.480 & $2.547 \times 10^{-4}$ & -8.276 & $4.45 \times 10^{-5}$ \\
\hline 674.7 & 2.3 & 1.482 & $2.449 \times 10^{-4}$ & -8.315 & $3.35 \times 10^{-5}$ \\
\hline
\end{tabular}



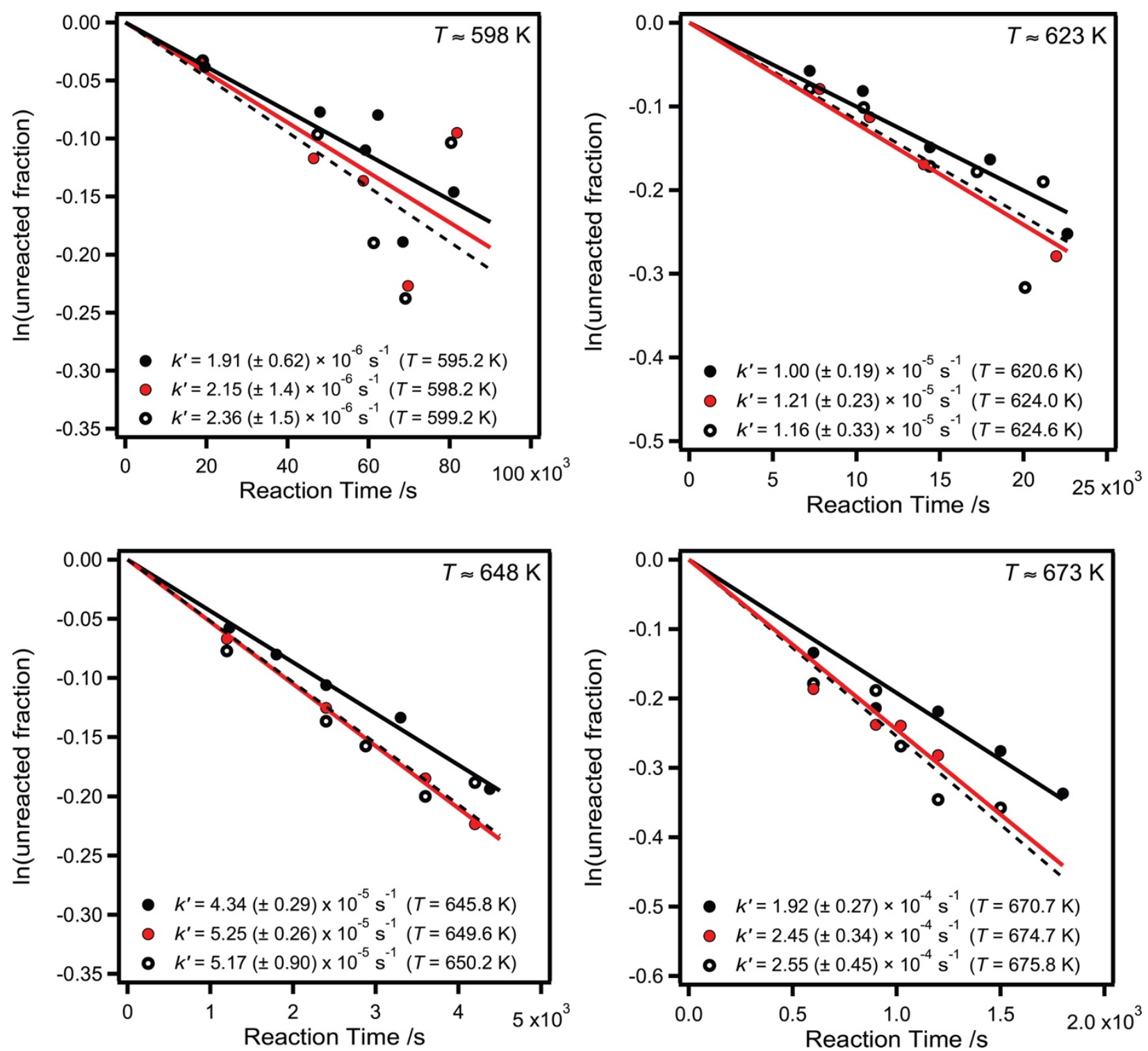

Figure 5: Data used to determine the decomposition rate constants $\left(k^{\prime}\right)$ for POE9. Both experimental data (circles) and their corresponding linear fits (solid and dashed lines) are shown. 

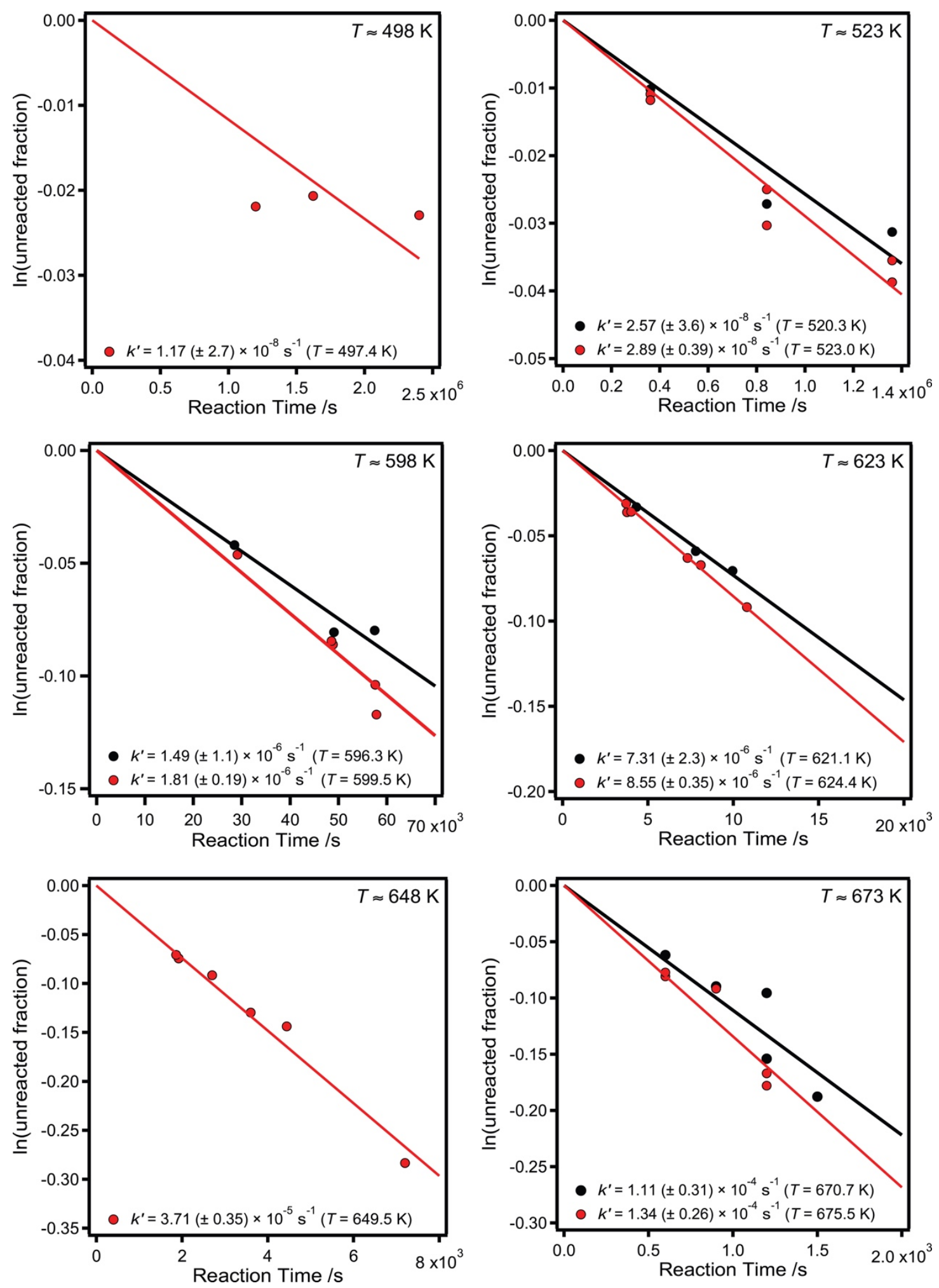

Figure 6: Data used to determine the decomposition rate constants $\left(k^{\prime}\right)$ for the MIL-PRF-23699 qualified lubricant. Both experimental data (circles) and their corresponding linear fits (solid and dashed lines) are shown. 
Table 8: Thermal Decomposition Kinetic Data for the MIL-PRF-23699 Qualified Lubricant.

\begin{tabular}{c|c|c|c|c|c}
\hline $\mathbf{T}$ & $+/-$ & $\mathbf{1 0 0 0 / T}$ & $\begin{array}{c}\boldsymbol{k}^{\prime} \\
/ \mathbf{s}^{-1}\end{array}$ & $\mathbf{I n} \boldsymbol{k}^{\prime}$ & $\begin{array}{c}\text { fit uncert. (95 \%) } \\
/ \mathbf{s}^{-1}\end{array}$ \\
\hline \hline 497.4 & 1.8 & 2.011 & $1.168 \times 10^{-8}$ & -18.266 & $2.74 \times 10^{-8}$ \\
520.3 & 1.0 & 1.922 & $2.568 \times 10^{-8}$ & -17.477 & $3.65 \times 10^{-8}$ \\
523.0 & 1.6 & 1.912 & $2.894 \times 10^{-8}$ & -17.358 & $3.90 \times 10^{-9}$ \\
596.3 & 2.0 & 1.677 & $1.493 \times 10^{-6}$ & -13.415 & $1.06 \times 10^{-6}$ \\
599.5 & 2.6 & 1.668 & $1.807 \times 10^{-6}$ & -13.224 & $1.90 \times 10^{-7}$ \\
621.1 & 2.4 & 1.610 & $7.313 \times 10^{-6}$ & -11.826 & $2.27 \times 10^{-6}$ \\
624.4 & 2.8 & 1.602 & $8.546 \times 10^{-6}$ & -11.670 & $3.53 \times 10^{-7}$ \\
649.5 & 2.2 & 1.540 & $3.707 \times 10^{-5}$ & -10.203 & $3.53 \times 10^{-6}$ \\
670.7 & 2.0 & 1.491 & $1.110 \times 10^{-4}$ & -9.106 & $3.12 \times 10^{-5}$ \\
675.5 & 2.4 & 1.480 & $1.342 \times 10^{-4}$ & -8.916 & $2.60 \times 10^{-5}$ \\
\hline
\end{tabular}

Table 9: Arrhenius Parameters for the Pure Base Oils and the Qualified Lubricant.

\begin{tabular}{c|c|c|c}
\hline fluid & $\boldsymbol{T} / \mathbf{K}$ & $\boldsymbol{A} / \mathbf{s}^{-\mathbf{1}}$ & $\boldsymbol{E}_{\mathbf{a}} / \mathbf{k J} \cdot \mathbf{~ m o l}^{-\mathbf{1}}$ \\
\hline \hline POE5 & $600-675$ & $2.28 \times 10^{8}$ & $158 \pm 10$ \\
POE7 & $600-675$ & $5.64 \times 10^{10}$ & $187 \pm 6$ \\
POE9 & $500-675$ & $1.41 \times 10^{12}$ & $204 \pm 5$ \\
MIL-PRF-23699 & $500-675$ & $2.62 \times 10^{8}$ & $161 \pm 7$ \\
\hline
\end{tabular}




\section{Vapor Pressure}

\subsection{Summary}

The vapor pressures ( $\left.p_{\text {sat }}\right)$ of POE5 and $n$-octacosane $\left(\mathrm{C}_{28} \mathrm{H}_{58}\right.$, a control compound) were measured by the gas saturation method at temperatures of $353.05 \mathrm{~K}$ and $362.93 \mathrm{~K}$. For POE5, the measured $p_{\text {sat }}$ was $(2.1 \pm 0.5) \mathrm{mPa}$ at $353.05 \mathrm{~K}$ and $(6.7 \pm 1.8) \mathrm{mPa}$ at $362.93 \mathrm{~K}$, with reported expanded uncertainties corresponding to a $95 \%$ confidence interval. Temperature gradients within the measurement apparatus were the principal source of uncertainty for these measurements. These values are in good agreement with the one prior report of $p_{\text {sat }}$ measurements on POE5.[6] Measurements on lubricant base fluids larger than POE5 were not attempted because their vapor pressures are expected to be below the measurable range of our apparatus.

\subsection{Vapor Pressure of POE5}

The vapor pressures ( $\left.p_{\text {sat }}\right)$ of POE5 and $n$-octacosane $\left(\mathrm{C}_{28} \mathrm{H}_{58}\right.$, a control compound) were measured by the gas saturation method [7-15] at temperatures of $353.05 \mathrm{~K}$ and $362.93 \mathrm{~K}$. The basis of the gas saturation method is the saturation of an inert carrier gas with vapor from a condensed phase. From measurements of the amount of vapor solute and the amount of carrier gas, one can calculate

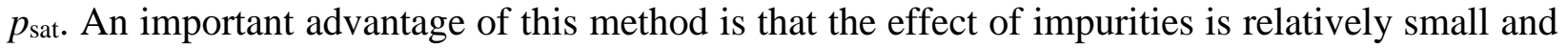
predictable, which means that samples of limited purity can be measured.

\subsubsection{Apparatus Description}

The gas saturation apparatus used for this work was developed at NIST and has been described in detail,[16, 17] so only a brief description is presented here. This type of apparatus (called a concatenated gas saturation apparatus) is an advancement on the classical technique in that it allows for various strategies that ensure good data quality. The principal components of the apparatus are a carrier-gas supply system, a series of saturator-adsorber pairs, and a temperaturecontrolled chamber (Figure 7). The carrier gas-supply system includes a gas cylinder filled with carrier gas, a pressure regulator, an ultra-low-flow mass flow controller, and a Bourdon-tube

pressure gauge (located just upstream from the first saturator-adsorber pair). For this work, 18 saturator-adsorber pairs were installed in series. The saturators, which are PTFE tubes filled with glass beads, were located inside a temperature-controlled forced-air chamber. The adsorbers, which are stainless steel tubes filled with porous polymer adsorbent, were located on a manifold above the chamber at room temperature (approximately $294 \mathrm{~K}$ ). The last adsorber vented to the lab at ambient pressure. 

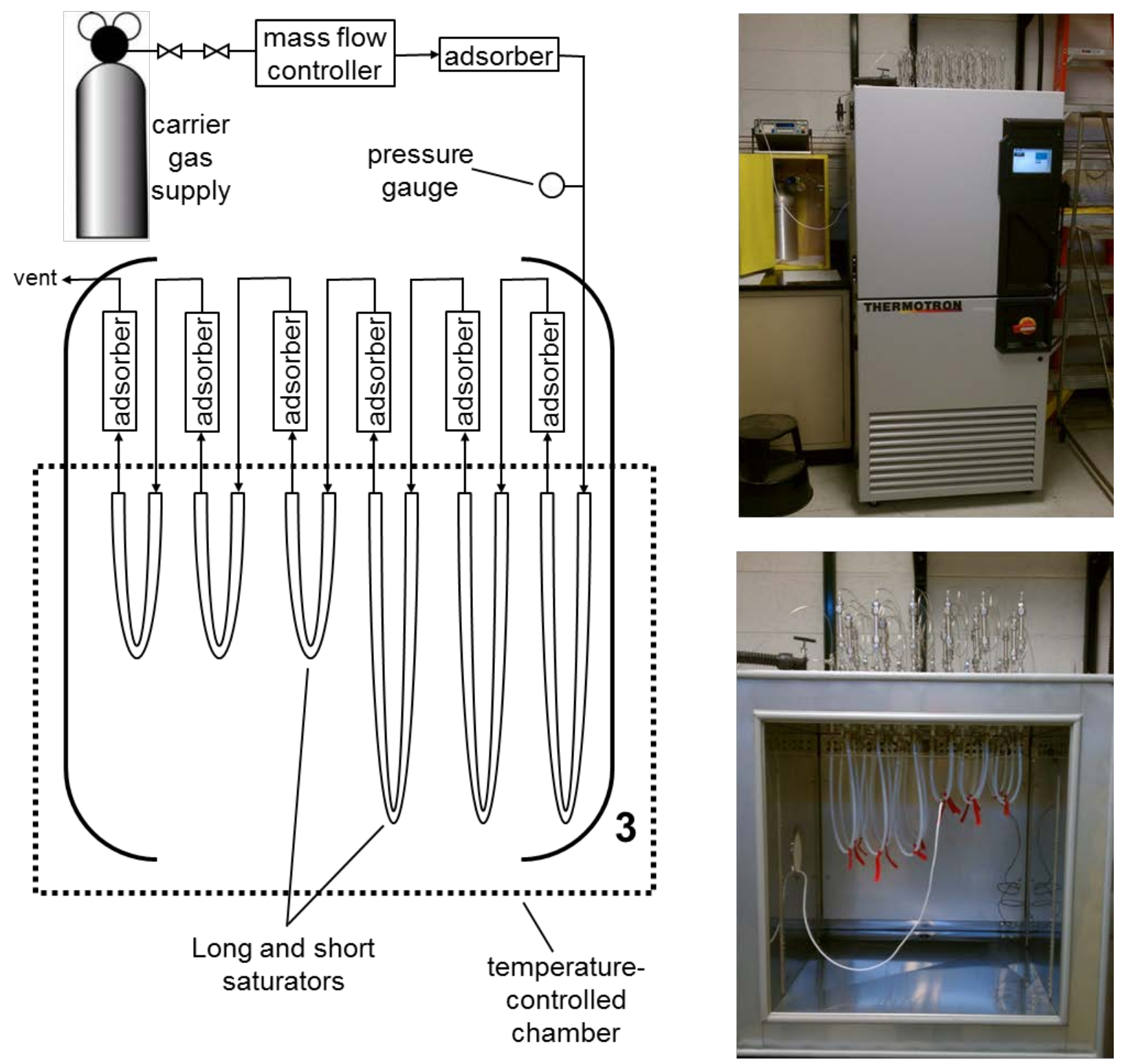

Figure 7: Gas saturation apparatus with 18 saturator-adsorber pairs linked in series. An alternating pattern of long and short saturators was used (three long, three short, three long, etc.). The arrows show the direction of flow for the carrier gas.

\subsubsection{Measurements}

The use of alternating long $(61 \mathrm{~cm})$ and short $(33 \mathrm{~cm})$ saturators for each sample compound provides a built-in test for flowrate effects on the measurement of $p_{\text {sat. }}[11,17,18]$ For these measurements, saturators \#2 (long), \#5 (short), \#8 (long), \#11 (short), \#14 (long) and \#17 (short) were coated with POE5. Saturators \#3 (long), \#6 (short), \#9 (long), \#12 (short), \#15 (long) and \#18 (short) were coated with $n$-octacosane. The other six saturators were left uncoated. The coating procedure consisted of the following steps. First, a $5 \%$ (mass/mass) solution of each sample 
compound in hexane was made. The glass beads in each saturator were wetted with $\sim 1.5 \mathrm{~mL}$ of one of these solutions, and the excess was poured out. Then the solvent was removed by a gentle flow of nitrogen through the adsorber at room temperature for $0.5 \mathrm{~h}$, after which the coated saturators were immediately installed in the temperature-controlled chamber of the apparatus. For the measurements reported herein, no significant difference was observed for the measurements with long or short saturators, which confirms the absence of flowrate effects.

At the beginning of each $p_{\text {sat }}$ measurement, the apparatus was flushed with $\geq 310 \mathrm{~cm}^{3}$ of UHPgrade nitrogen carrier gas at room temperature (the internal void volume of the gas saturation apparatus is approximately $120 \mathrm{~cm}^{3}$ ). The intent of this procedure was to remove the small amount of oxygen that is inevitably introduced into the gas saturation apparatus when the adsorbers are removed and reinstalled.[16] Some sample vapor is carried into the adsorbers during the flush but, because of the relatively small flush volume and low flush temperature, this type of measurement error was insignificant. After the flush, the oven temperature was raised to the measurement temperature. Total flow periods ranged from 14 days to 33 days. At the end of that time period, the flow of carrier gas was stopped and the adsorbers were removed for analysis of the trapped vapor. When an adsorber was removed, the lines were immediately capped in order to limit the infiltration of air into the gas saturation apparatus. The flowrate of carrier gas was $3.0 \mathrm{~cm}^{3} \cdot \mathrm{min}^{-1}$.

\subsubsection{Instrument Modifications}

The $p_{\text {sat }}$ measurements on POE5 and $n$-octacosane were at a higher temperature than any previous[16, 17] work in this gas saturation apparatus. At these temperatures, an apparatus modification was necessary in order to obtain accurate measurements of $p_{\text {sat. }}$ Specifically, a tightfitting PTFE sleeve was placed around the end of each adsorber inlet tube (Figure 8). These sleeves had an outer diameter that matched the inner diameter of the saturator tubing. This arrangement insulates the flow of gas from any temperature gradients created by the stainless-steel bulkhead union, which completely penetrates the insulated ceiling of the temperature-controlled chamber. The measured value of $p_{\text {sat }}$ for POE5 at $362.93 \mathrm{~K}$ increased by about $50 \%$ upon installation of the PTFE sleeves (i.e., the measured $p_{\text {sat }}$ was $4.4 \pm 1.1 \mathrm{mPa}$ before installing the PTFE sleeves and $6.7 \pm 1.8 \mathrm{mPa}$ afterwards). This is a good illustration of the importance of eliminating cold spots at the saturator-adsorber interface. 


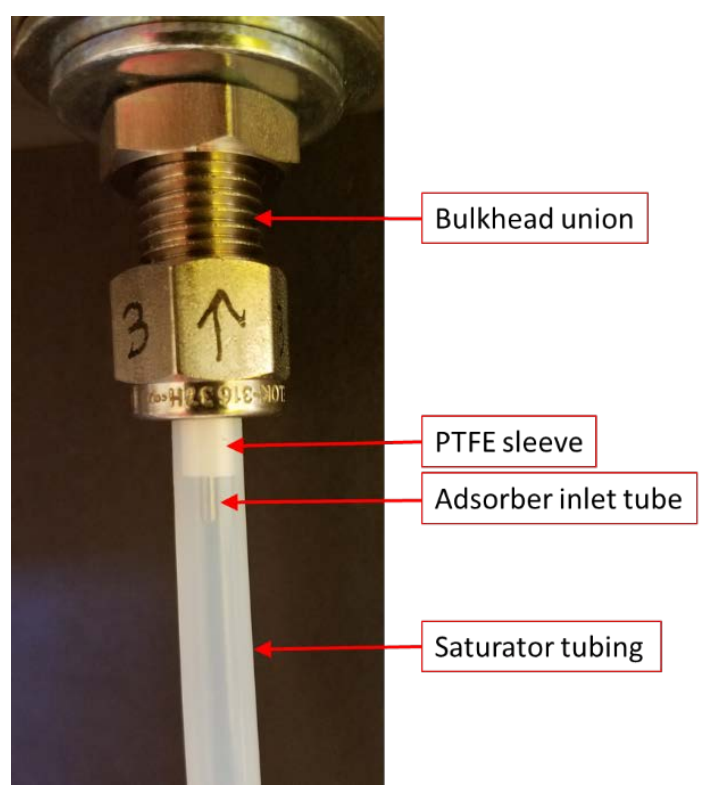

Figure 8: Union between the saturator and adsorber. The PTFE sleeve insulates the carrier gas and vapor solute from the bulkhead union.

\subsubsection{Temperature Measurements}

The temperature in the temperature-controlled chamber was measured with an ITS-90 calibrated platinum resistance thermometer (PRT). During the course of the $p_{\text {sat }}$ measurements, the calibration of the PRT was checked with both a water triple-point cell ( $T=273.16 \mathrm{~K})$ and a gallium fixed-point cell $(T=302.9146 \mathrm{~K})$. The temperatures measured by the PRT deviated from the two fixed points by $<0.01 \mathrm{~K}$. At each temperature set-point, the PRT was used to measure the temperature at the bottom/middle of each saturator loop and near the exit end of each saturator. Temperature fluctuations in a specific location in the temperature-controlled chamber were relatively small — the range of measured temperatures typically varied by less than $0.05 \mathrm{~K}$. A larger source of uncertainty comes from temperature gradients in the chamber, which increase in magnitude with temperature settings farther from ambient. For example, the average temperature difference between the middle (loop) and the exit end of a saturator was $0.80 \mathrm{~K}$ at a set-point of $353.05 \mathrm{~K}$, and it was $1.02 \mathrm{~K}$ at a set-point of $362.93 \mathrm{~K}$. These temperature gradients are the largest source of uncertainty in the $p_{\text {sat }}$ measurements.

\subsubsection{Mass of Carrier Gas}

The mass of carrier gas was determined by the mass flow controller, which has a relative standard uncertainty of $3.2 \%$ for the flow rate used for these measurements. The accuracy of the mass flow controller has been checked repeatedly by mass measurements.[16, 17] 


\subsubsection{Analysis of Vapor}

At the end of the flow period, the vapor collected in each adsorber was prepared for analysis by GC-FID.[17] This was done by elution of each adsorber with hexane into two GC autosampler vials, which already contained $\sim 0.3 \mathrm{~g}$ of a $\sim 0.1$ mass $\% n$-eicosane solution (in $n$-octane). Then the two eluent fractions, with $n$-eicosane as an internal standard, were analyzed by GC-FID. The relative sensitivity of the FID to $n$-eicosane, POE5, and $n$-octacosane was determined by analyzing known mixtures.

\subsection{Calculation of Vapor Pressure}

Vapor pressures were calculated from the raw data by a form of the ideal gas law that contains the Poynting correction,

$$
p_{\text {sat }}=(m \cdot R \cdot T) /(V \cdot M \cdot \Phi)
$$

where $p_{\text {sat }}$ is the vapor pressure of the sample, $m$ is the recovered mass of sample vapor, $R$ is the gas constant, $T$ is the temperature of the saturator, $V$ is the volume of carrier gas at the temperature and pressure of the saturator, $M$ is the molar mass of sample compound, and $\Phi$ is the Poynting correction. Assuming that the condensed phase is incompressible, and knowing that $p_{\text {sat }}$ is negligible compared to the experimental pressure $(p)$, then $\Phi=\exp (p \cdot v / R \cdot T)$, where $v$ is the molar volume[19] of the liquid sample. This is a small correction, which lowers all values of apparent $p_{\text {sat }}$ by $\sim 1 \%$. The volume of carrier gas, $V$, was determined from a high-accuracy equation of state for nitrogen[20] as implemented in REFPROP.[21] For these measurements, the effect of the sample vapor on the total vapor volume could be ignored because its mole fraction was very small compared to that of the carrier gas. The pressure in the saturators was taken to be ambient, which is $83.2 \mathrm{kPa}$ (with a standard deviation of $0.6 \mathrm{kPa}$ ) on the NIST site in Boulder, Colorado.[17] The values of $p_{\text {sat }}$ that were calculated from Eq. 3 were then corrected by dividing by the mole fraction purity of the sample (as determined by GC-FID). The POE5 had a mole fraction purity of 0.967.[3] The $n$-octacosane had a mole fraction purity of 0.995, so this correction is less important for the control compound. These corrected values of $p_{\text {sat }}$ are reported in Table 10.

\subsection{Uncertainty Calculations}

Temperature control is the principal source of uncertainty for these measurements. Considering both temperature gradients and temperature fluctuations, we estimate that the standard uncertainty in temperature is $0.80 \mathrm{~K}$ at a setting of $353.05 \mathrm{~K}$ and $1.02 \mathrm{~K}$ at a setting of $362.93 \mathrm{~K}$. These uncertainties in the measurement temperature result in standard uncertainties $(u)$ of $0.104 \cdot p_{\text {sat }}$ and 
0.125 $p_{\text {sat }}$ for $n$-octacosane at $T=353 \mathrm{~K}$ and $363 \mathrm{~K}$, respectively; for POE5 the uncertainties are $0.096 \cdot p_{\text {sat }}$ and $0.114 \cdot p_{\text {sat }}$ at $T=353 \mathrm{~K}$ and $363 \mathrm{~K}$, respectively. Other significant sources of measurement uncertainty included the following. Uncertainty in the determination of the mass of recovered vapor solute by GC-FID resulted in a standard uncertainty of $u=0.03 \cdot p_{\text {sat }}$. Uncertainty in the determination of the mass of $\mathrm{N}_{2}$ carrier gas by the mass flow controller resulted in a standard uncertainty of $u=0.032 \cdot p_{\text {sat. }}$ Uncertainty in the pressure in the saturators (caused by barometric variability and pressure drop across the apparatus) resulted in a standard uncertainty of $u=$ $0.024 \cdot p_{\text {sat. }}$ Impurities in the $n$-octacosane caused a standard uncertainty of $u=0.005 \cdot p_{\text {sat }}$ (based on the initial purity of the $n$-octacosane). Impurities in the POE5 caused a standard uncertainty of $u=$ $0.033 \cdot p_{\text {sat }}$ (based on the initial purity of the POE5). Finally, nonideality of the vapor phase mixture, which is caused by the interaction of the solute vapor with the $\mathrm{N}_{2}$ carrier gas, caused an estimated standard uncertainty of $u=0.02 \cdot p_{\text {sat. }}[3,17]$ The quadrature sum of the standard uncertainties associated with the measurement of $p_{\text {sat }}$ results in combined expanded $(k=2)$ uncertainties $\left(U_{\mathrm{c}}\right)$ of $0.234 \cdot p_{\text {sat }}$ and $0.272 \cdot p_{\text {sat }}$ for $n$-octacosane at $T=353 \mathrm{~K}$ and $363 \mathrm{~K}$, respectively, and $0.230 \cdot p_{\text {sat }}$ and $0.261 \cdot p_{\text {sat }}$ for POE5 at $T=353 \mathrm{~K}$ and $363 \mathrm{~K}$, respectively.

An important advantage of the design of the gas saturation apparatus used for this work is that a control sample can be measured simultaneously with the other sample(s). We have typically employed linear alkanes as the control sample for a variety of reasons.[3, 16, 22, 23] $n$-Octacosane was selected for this work because its $p_{\text {sat }}$ curve is very similar to that of POE5 in the temperature range studied; therefore, any systematic errors would have about the same magnitude effect on both. The results of the $p_{\text {sat }}$ measurements on $n$-octacosane are shown in Table 10.

Surprisingly, after the first two replicate measurements at $362.93 \mathrm{~K}$, the measured value of $p_{\text {sat }}$ drifted lower. At the same time, new peaks started to appear in the chromatograms of vapor trapped by the adsorbers. This is typical behavior for a compound that is decomposing during the $p_{\text {sat }}$ measurement,[16] but such decomposition was not expected for $n$-octacosane. Our hypothesis for why the $n$-octacosane started to decompose at these relatively low temperatures is that it contained low levels of olefinic impurities (as evidenced by multiple minor peaks in the ${ }^{1} \mathrm{H}$ NMR spectrum from $4.5-6.0 \mathrm{ppm}$ ), and olefins are known to initiate autoxidation chemistry under even mild measurement conditions.[16] The observation that the decomposition starts after an induction period is also consistent with autoxidation. In any case, the two initial measurements on $n$ octacosane at $362.93 \mathrm{~K}$ both indicated a $p_{\text {sat }}$ of $5.0 \pm 1.4 \mathrm{mPa}$. Within the uncertainty of the measurements, this is in agreement with the reference value from the correlation of Lemmon and 
Goodwin, which is $6.09 \pm 0.30 \mathrm{mPa}$.[24] This is evidence that, apart from sample decomposition, the measurement method was working properly.

\subsection{Results}

The results of $p_{\text {sat }}$ measurements on POE5 are shown in Table 10. Multiple replicates were performed at $362.93 \mathrm{~K}$ with the aim of checking for significant sample decomposition. Unlike $n$ octacosane, the $p_{\text {sat }}$ measurements on POE5 showed excellent repeatability and did not drift lower over time. This is evidence that there was no significant decomposition of POE5 during the measurements.[16] Additionally, chromatograms of vapor trapped by the adsorbers remained clear of decomposition product peaks. For these reasons, we believe that all of the measurements on POE5 are valid. The average value for the replicate measurements of $p_{\text {sat }}$ at $362.93 \mathrm{~K}$ was $6.7 \mathrm{mPa}$ with an expanded uncertainty of $1.8 \mathrm{mPa}$.

Table 10: The Vapor Pressures $\left(p_{\text {sat }}\right)$ and Combined Expanded $(k=2)$ Uncertainties $\left(U_{c}\right)$ for Measurements on POE5 and the Linear Alkane $n$-Octacosane $\left(\mathrm{C}_{28} \mathrm{H}_{58}\right.$, Measured as a Control Sample). ${ }^{a}$

\begin{tabular}{l|ccc}
\hline \multicolumn{1}{c|}{ Date } & $\boldsymbol{T} / \mathbf{K}$ & $\boldsymbol{p}_{\text {sat }} \mathbf{b} / \mathbf{m P a}$ & $\boldsymbol{U}_{\mathbf{c}} / \mathbf{m P a}$ \\
\hline \hline \multicolumn{4}{c}{ POE5 } \\
\hline 22-Jul-2016 & 362.93 & 6.7 & 1.8 \\
25-Aug-2016 & 362.93 & 6.8 & 1.8 \\
8-Sep-2016 & 362.93 & 6.8 & 1.8 \\
26-Sep-2016 & 353.05 & 2.1 & 0.5 \\
19-Oct-2016 & 362.93 & 6.6 & 1.7 \\
\hline \multicolumn{5}{c}{$\boldsymbol{n - O c t a c o s a n e}$} \\
22-Jul-2016 & 362.93 & 5.0 & 1.4 \\
25-Aug-2016 & 362.93 & 5.0 & 1.4 \\
8-Sep-2016 & 362.93 & 4.2 & $\mathrm{c}$ \\
26-Sep-2016 & 353.05 & 1.2 & $\mathrm{c}$ \\
19-Oct-2016 & 362.93 & 3.0 & $\mathrm{c}$ \\
\hline
\end{tabular}

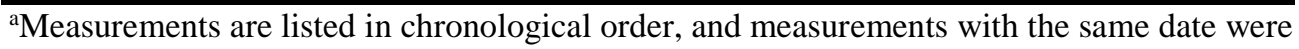
done simultaneously.

${ }^{\mathrm{b}}$ The values of $p_{\text {sat }}$ have been corrected for the mole fraction purity of each sample, as described in the text.

'Significant decomposition of $n$-octacosane was observed for this measurement.

There is only one prior report of $p_{\text {sat }}$ measurements on POE5 in the literature. By use of a gas saturation method that was designed for the measurement of relatively low pressures, Razzouk et 
al.[6] collected $p_{\text {sat }}$ data for POE5 in the temperature range $334-413 \mathrm{~K}$. They also reported a correlation of their data with the Antoine equation. They estimated a measurement uncertainty of $0.05 \cdot p_{\text {sat }}$, although the only sources of uncertainty that were considered were the GC-FID analysis, the gas flow rate, and the $p_{\text {sat }}$ of $n$-tetracosane (which was used to calibrate the measurement). In any case, by use of the reported Antoine coefficients for POE5,[6] one can calculate a $p_{\text {sat }}$ of $2.62 \pm 0.13 \mathrm{mPa}$ at $353.05 \mathrm{~K}$ and $7.99 \pm 0.40 \mathrm{mPa}$ at $362.93 \mathrm{~K}$. These values are within the combined uncertainties of the two measurements. The fact that the two data sets agree lends credence to both.

Measurements on lubricant base fluids larger than POE5 were not attempted because their vapor pressures were expected to be below the measurable range of our apparatus. For example, the $p_{\text {sat }}$ curve for POE7 is expected to be about two orders of magnitude lower than for POE5.[6] In principle, it is possible to conduct measurements in our apparatus at higher temperatures and with longer flow periods; however, the large measurement uncertainties at high temperatures and the potential for sample decomposition limit the value and practicality of such measurements. 


\section{Isobaric Specific Heat Capacity}

\subsection{Summary}

Measured isobaric specific heat capacities and associated expanded uncertainties are reported over the combined temperature range 208 to $503 \mathrm{~K}$; the specific temperature range varied for each fluid and was selected to avoid potential phase transitions. Heat capacities range from an overall low of $1.704 \mathrm{~J} \cdot \mathrm{g}^{-1} \cdot \mathrm{K}^{-1}$ to an overall high of $2.343 \mathrm{~J} \cdot \mathrm{g}^{-1} \cdot \mathrm{K}^{-1}$, with a relative ordering among the four fluids of POE5 < MIL-PRF-23699 < POE7 < POE9 and differences that range from $6.5 \%$ (at $313 \mathrm{~K}$ ) to $6.8 \%$ (at $408 \mathrm{~K})$. These results are also presented in Fortin.[25]

\subsection{Experimental Methods}

A commercial differential scanning calorimeter (DSC) (see Figure 9) was used to measure the isobaric specific heat capacity of the four lubricant samples. As the name implies, DSC is a differential technique in which the difference in heat flow between a sample and an inert reference is measured as a function of time and temperature while both are subjected to a controlled environment. More specifically, modulated differential scanning calorimetry (MDSC) was used for the measurements reported herein. The difference between conventional DSC and MDSC lies in the heating profile applied; in DSC, a simple linear profile is utilized, while with our MDSC, a sinusoidal modulation is overlaid on the conventional linear heating ramp. The net effect is equivalent to performing two experiments simultaneously, one experiment at the average linear heating rate and a second at an instantaneous sinusoidal heating rate. In addition, while it is only possible to measure total heat flow with conventional DSC, with MDSC it is possible to separate total heat flow into its two components: one that is a function of the sample's heat capacity and rate of temperature change (the heat capacity or "reversing" component) and one that is a function of the absolute temperature and time (the kinetic or "nonreversing" component). The combined net effect of these two advantages is a simpler, more precise measurement of heat capacity with MDSC compared to conventional DSC. Additional details regarding MDSC theory can be found in Boerio-Goates and Callanan,[26] Reading et al.,[27] Wunderlich et al.,[28] and Höhne et al.,[29] among other references. In this work, measurement runs were performed over a given temperature range using an underlying heating rate of $3 \mathrm{~K} \cdot \mathrm{min}^{-1}$ and modulated conditions of $\pm 1 \mathrm{~K}$ every $120 \mathrm{~s}$. The specific temperature range varied by sample in an effort to avoid phase changes observed during initial exploratory measurement scans. Measurements were made from 233 to $443 \mathrm{~K}$ for POE5, from 263 to $428 \mathrm{~K}$ for POE7, from 283 to $423 \mathrm{~K}$ for POE9, and from 208 to $503 \mathrm{~K}$ for MIL-PRF-23699. For all measurements, the measuring cell was continuously purged with a $50 \mathrm{~mL} \cdot \mathrm{min}^{-1}$ flow of dry nitrogen. 


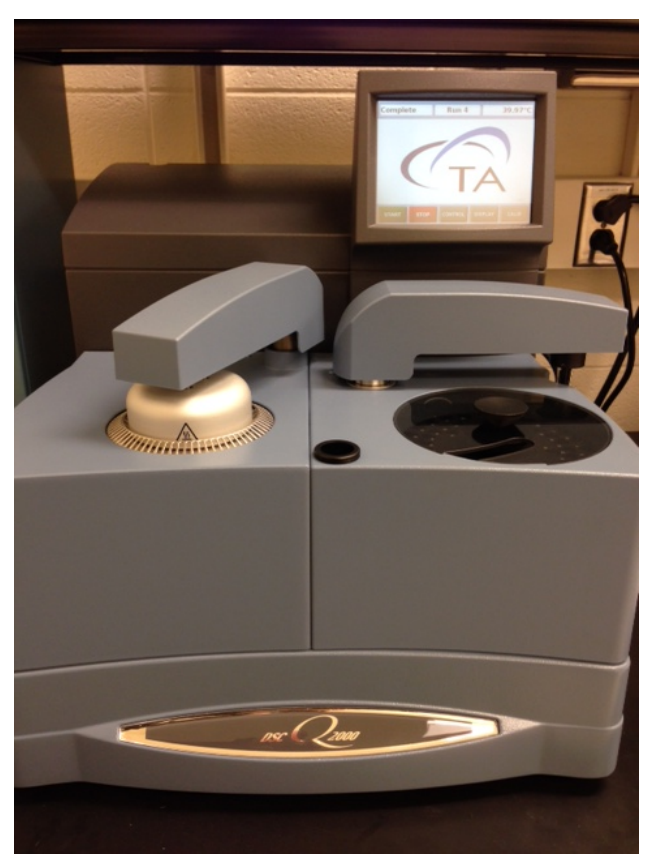

Figure 9: Differential scanning calorimeter.

\subsubsection{Instrument Calibration}

Determination of accurate heat capacities requires calibration of the instrument baseline, temperature, heat flow, and heat capacity. The instrument baseline was calibrated via two separate measurement runs; in the first the cell was empty and in the second sapphire disks were placed directly on the cell's sample and reference platforms. From these two runs, cell asymmetries were quantified, and the instrument's baseline was adjusted.

Temperature and heat flow were calibrated via measurements of materials with known transition temperatures and heats. A list of some typical calibration substances and recommended transition temperatures can be found in Della Gatta et al.[30] In this work, a combination of adamantane, indium, and tin were used. Indium SRM 2232 and tin SRM 2220 were obtained from the National Institute of Standards and Technology (NIST) and corresponding reference melting temperatures and heats of fusion were obtained from their respective certificates.[31, 32] Adamantane was not available as a certified reference material. The adamantane sample used was obtained from SigmaAldrich $^{\dagger}$ with a stated purity of $>99 \%$. Our own analysis by gas chromatography/mass spectrometry indicated a purity of $>99.95 \%$. The adamantane was used without further purification, and reference values for the temperature and heat of transition for the first order solidsolid transition were taken from Westrum.[33] The applied temperature calibration utilized

\footnotetext{
${ }^{\dagger}$ In order to describe materials and experimental procedures adequately, it is occasionally necessary to identify commercial products by manufacturers' names or labels. In no instance does such identification imply endorsement by the National Institute of Standards and Technology, nor does it imply that the particular product or equipment is necessarily the best available for the purpose.
} 
measurement results for all three calibrants for the heating rate of interest $\left(3 \mathrm{~K} \cdot \mathrm{min}^{-1}\right)$. For the heat flow calibration, we chose to use a single average calibration coefficient calculated using only results from indium and tin measurements.

Heat capacity was calibrated by measuring a material whose heat capacity is known. In this work, sapphire $\left(\alpha-\mathrm{Al}_{2} \mathrm{O}_{3}\right)$ was measured under the same experimental conditions as were used for the lubricant samples. The resulting calibration coefficient $\left(K_{c p}\right)$ is determined by dividing literature values[30, 34] by measured heat capacities. More specifically, $K_{c p}$ is calculated and applied as a function of temperature; to achieve this, the calibration coefficient is applied to the data during post-processing.

\subsubsection{Sample Preparation}

All calibration and lubricant samples were encapsulated in hermetically sealed aluminum pans (see Figure 10). Additionally, an empty hermetically sealed pan served as the reference. Five separate pans were prepared for each measured lubricant sample. Sample masses ranged from $8.344 \mathrm{mg}$ to 19.505 mg; multiple masses were measured to check for evidence of mass dependence, which could potentially indicate a significant contribution from the vapor phase. All mass measurements employed a microbalance (see Figure 10) and a double-substitution weighing scheme.[35] There are two main advantages to this process. First, the balance is calibrated over the same limited range as the sample weighings instead of assuming a linear response over the entire balance range, as is the case with most internal balance calibrations. And second, air buoyancy effects are reduced to small differences in volumes of the tare and sample pans and the relatively small effect of standard masses. Air buoyancy is explicitly accounted for via the inclusion of the ambient air density, which is determined from ambient temperature, pressure, and relative humidity measurements and the equation of Picard et al.[36]

Pans were first weighed empty to determine pan weight. They were then filled with sample, sealed closed with a press (see Figure 10), and reweighed to determine sample weight. The sealed pan was then allowed to sit for at least a day, and then it was reweighed to check for any mass loss, which would indicate that the pan was not properly sealed during encapsulation. Finally, pans were reweighed upon completion of the heat capacity measurements to again check for mass loss. A change in mass of $>0.3 \%$ would require data be discarded. No samples had to be discarded as a result of sample loss in this work. 


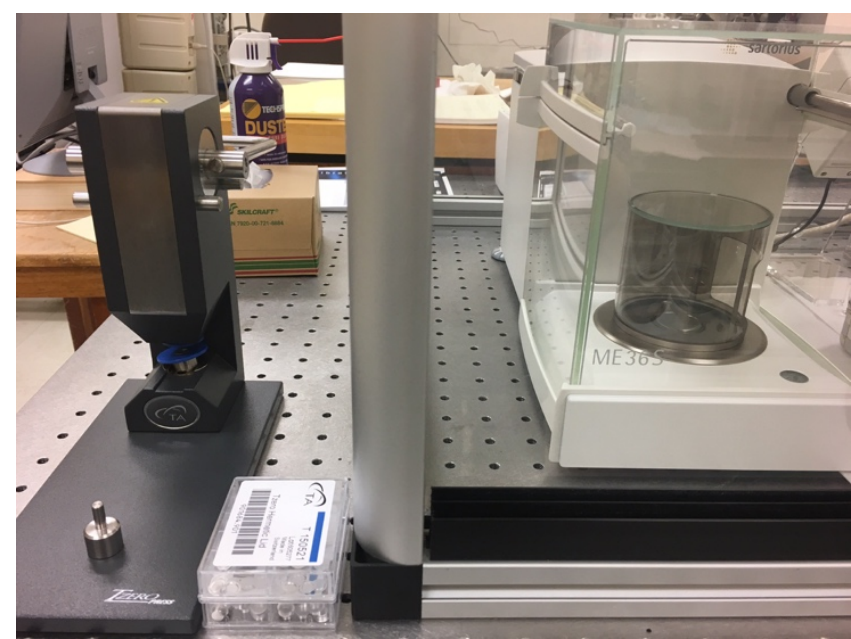

Figure 10: Tools for sample preparation. From left to right: press used to seal sample pans, sample pans and lids, and microbalance used to determine sample mass.

\subsubsection{Measurement Procedure}

Following the example of and building upon lessons learned during previous work,[37] heat capacity measurements for the four lubricant samples employed the following procedure, executed over two distinct measurement periods. First, the instrument baseline, temperature, and heat flow were calibrated as previously described. Then, prior to the start of sample measurements, the instrument was allowed to warm up and its performance was verified by performing repeat baseline runs, followed by a single indium verification measurement. These checks were repeated approximately every 24 hours if the instrument was set to run multiple samples over several days. For each of the five sample pans prepared for each fluid, two sets of two replicate runs were performed for a total of four replicates with the pan being removed in between. This provided valuable information about the instrument's repeatability and reproducibility, including contributions from pan placement upon the sample platform. Every run of a sample pan was bracketed by a single sapphire calibration run. Since a significant degree of variability was observed among the calibration runs over the course of each measurement period, and the variability appeared to be random in nature, it was determined that the application of an overall average $\bar{K}_{c p}(T)$ would be more representative of the instrument's behavior. A separate average $\bar{K}_{c p}(T)$ was determined and applied for each measurement period; for the first, an average of 80 separate sapphire calibrations was used, while for the second, 20 separate sapphire calibrations went into the average used. Finally, the fifth pan for each sample fluid was measured a second time using a quasi-isothermal method. For this method, rather than ramping the temperature at a constant rate of $3 \mathrm{~K} \cdot \mathrm{min}^{-1}$, the temperature was changed in $10 \mathrm{~K}$ steps and the sample allowed to 
sit at each temperature for a total of 15 minutes to allow for thermal equilibration within the sample. The information obtained from these runs was used in the subsequent uncertainty analysis.

\subsection{Results}

\subsubsection{Measurement Results}

Overall averaged heat capacity results $\left(\overline{\bar{c}}_{p}\right)$ are plotted as a function of temperature in Figure 11 and are reported in Tables 11 - 14 (Section 5.4) with POE5 in Table 11, POE7 in Table 12, POE9 in Table 13, and MIL-PRF-23699 in Table 14. For clarity, the plotted data in Figure 11 have been limited to a point every $5 \mathrm{~K}$. Additionally, the tabulated data (Tables $11-14$ ) represent a subset of the collected measurement data. Although measurement scans were performed over larger temperature ranges than what is shown in the tabulated data, only data from the stable portion of the heat flow curves are presented. Furthermore, although data were collected at $>1$ point per second, the data were further reduced to a point every $1 \mathrm{~K}$ to make the amount of data presented in the tables more reasonable.

As was previously discussed, for each fluid, five samples were prepared and each of those samples was measured four times for a total of twenty measurement runs per fluid. For a given fluid, replicate measurement results were first averaged for each of the five measured pans/masses and then those averages $\left(\bar{c}_{p}\right)$ were compared to check for any evidence of mass dependence. After the first measurement period, some of the fluids showed an apparent mass dependence among the first four pans/masses, while others did not. When present, the observed mass dependence could not be attributed to a contribution from the vapor phase. In an effort to better understand the initial observations, additional measurements were made during a second measurement period using two new sample pans/masses for each fluid, one pan with small masses ( $<6 \mathrm{mg}$ ) and one pan with larger masses (closer to previously measured masses, $12-19 \mathrm{mg}$ ). Results for the small masses were determined to be unreliable and have not been included here. Results for the larger masses were consistently higher than earlier measurements and eliminated any previously observed mass dependence. In the absence of an observed mass dependence, results were further averaged to give a single overall average $\left(\overline{\bar{c}}_{p}\right)$ as a function of temperature for each measured fluid.

Figure 11 shows that POE9 exhibits the highest heat capacities, ranging from $1.940 \mathrm{~J} \cdot \mathrm{g}^{-1} \cdot \mathrm{K}^{-1}$ at $313.13 \mathrm{~K}$ to $2.234 \mathrm{~J} \cdot \mathrm{g}^{-1} \cdot \mathrm{K}^{-1}$ at $412.14 \mathrm{~K}$. POE 5 exhibits the lowest heat capacities, ranging from $1.704 \mathrm{~J} \cdot \mathrm{g}^{-1} \cdot \mathrm{K}^{-1}$ at $263.14 \mathrm{~K}$ to $2.143 \mathrm{~J} \cdot \mathrm{g}^{-1} \cdot \mathrm{K}^{-1}$ at $432.15 \mathrm{~K}$. The difference in heat capacity between these two fluids is $6.5 \%$ at $313 \mathrm{~K}$ and $6.8 \%$ at $408 \mathrm{~K}$. MIL-PRF-23699, the fully qualified lubricant, has heat capacities that are between those of POE7 and POE5. Because the slope of 
MIL-PRF-23699's observed temperature dependence is different from the two lubricant base oils that bracket it, it has heat capacities that are closest to POE5 at lower temperatures (e.g., MILPRF-23699 is $0.8 \%$ higher at $273 \mathrm{~K}$ ) and closest to POE7 at higher temperatures (e.g., MIL-PRF23699 is $0.9 \%$ lower at $417 \mathrm{~K}$ ).

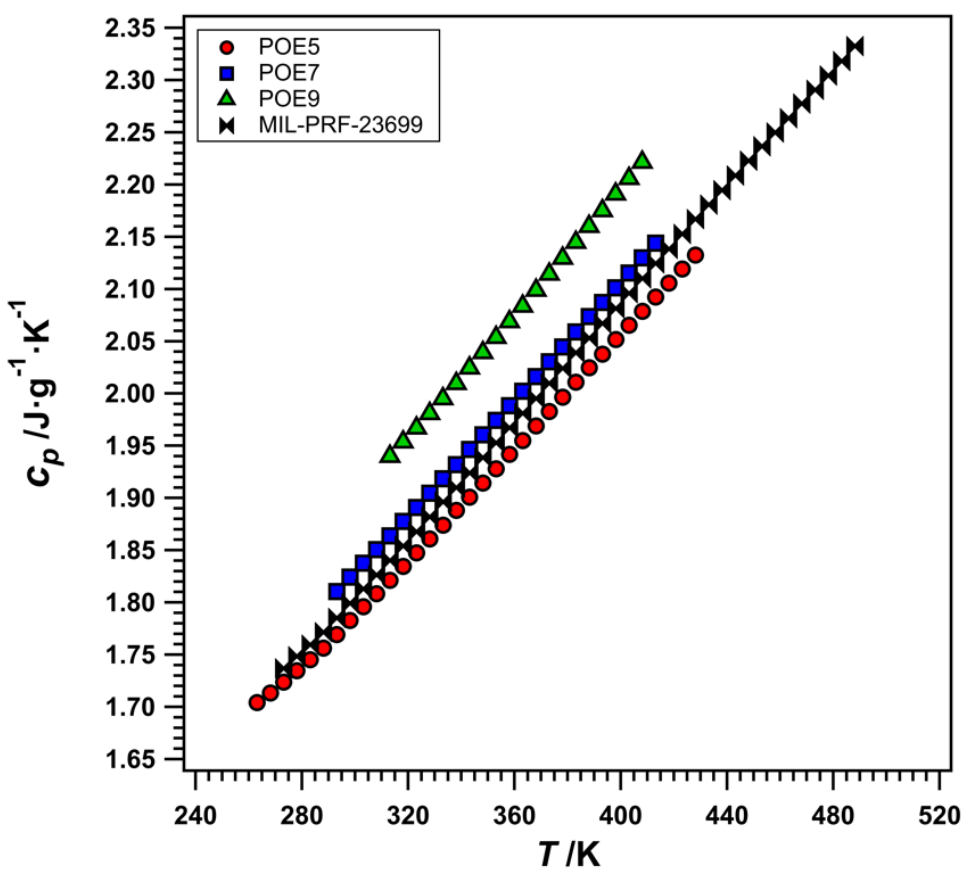

Figure 11: Isobaric specific heat capacity measurements plotted as a function of temperature. Only a subset of data points reported are plotted for clarity.

\subsubsection{Uncertainty Estimates}

Also included Tables 11 - 14 (Section 5.4) are the associated absolute expanded uncertainty estimates $\left(U\left(\overline{\bar{c}}_{p}\right)\right)$. The expanded uncertainty is calculated using the expression

$$
U\left(\overline{\bar{c}}_{p}\right)=t_{95}\left(d f_{c p}\right) \cdot u\left(\overline{\bar{c}}_{p}\right)
$$

where the coverage factor, $t_{95}\left(d f_{c p}\right)$, is taken from the $t$-distribution for $d f_{c p}$ degrees of freedom and a $95 \%$ level of confidence, and $\boldsymbol{u}\left(\overline{\bar{c}}_{p}\right)$ is the combined standard uncertainty for the averaged isobaric specific heat capacity measurements.[38]

The combined standard uncertainty for a single replicate measurement of a single sample mass can be expressed as 


$$
u\left(c_{p}\right)=\sqrt{\left(\frac{\partial c_{p}}{\partial K_{c p}}\right)^{2} u^{2}\left(K_{c p}\right)+\left(\frac{\partial c_{p}}{\partial m}\right)^{2} u^{2}(m)+\left(\frac{\partial c_{p}}{\partial c_{p m}}\right)^{2} u^{2}\left(c_{p m}\right)}
$$

where the first term is the contribution from the uncertainty associated with the heat capacity calibration $\left(K_{c p}\right)$, the second term is the contribution from the uncertainty in the sample mass $(m)$, and the last term is the uncertainty associated with the measured heat capacity signal produced by the instrument $\left(c_{p m}\right)$. Each of the three standard uncertainty terms $\left(u\left(K_{c p}\right), u(m)\right.$, and $\left.u\left(c_{p m}\right)\right)$ include both random (type A)[38] and systematic (type B)[38] sources of error. For example, $u\left(K_{c p}\right)$ incorporates the standard deviation for the averaged calibration coefficient $\left(\bar{K}_{c p}\right)$, as well as contributions from systematic error sources such as temperature alignment and uncertainties associated with the reference values used to calculate $K_{c p}$. Statistical methods are employed to combine single run standard uncertainties $\left(u\left(c_{p}\right)\right)$ to calculate the combined standard uncertainty for the average of replicate runs of a single sample mass $\left(\boldsymbol{u}\left(\bar{c}_{p}\right)\right)$, and to combine standard uncertainties for individual masses $\left(\boldsymbol{u}\left(\bar{c}_{p}\right)\right)$ to calculate the overall combined standard uncertainty for a given fluid $\left(\boldsymbol{u}\left(\overline{\bar{c}}_{p}\right)\right)$. The statistical methods used for this last calculation depend on the specific circumstances. In this work, the observed between-mass variances were often significantly larger than the observed within-mass variances. Under these circumstances, it is preferable to determine the variance of the overall average of the group means,[38] which is calculated using the expression

$$
u\left(\overline{\bar{c}}_{p}\right)=\frac{s_{\overline{\bar{c}} p}}{n_{\overline{\bar{c}} p}}
$$

where $\boldsymbol{s}_{\overline{\bar{c}} \boldsymbol{p}}$ is the standard deviation for the overall average specific heat capacity $\left(\overline{\bar{c}}_{p}\right)$ and $\boldsymbol{n}_{\overline{\bar{c}} \boldsymbol{p}}$ is the number of averaged masses ( $\boldsymbol{n}_{\overline{\bar{c}} \boldsymbol{p}}=5$ in this work). The degrees of freedom to use when determining the appropriate coverage factor for calculating expanded uncertainties (Eq. 4) are equal to $\boldsymbol{n}_{\overline{\bar{c}} \boldsymbol{p}}-1$. Corresponding coverage factors $\left(\boldsymbol{t}_{95}\left(d f_{c p}\right)\right)$ have not been included in Tables $11-$ 14 since, in this work, all coverage factors are equivalent $\left(t_{95}=2.776\right)$. Overall, expanded uncertainties range between $0.036 \mathrm{~J} \cdot \mathrm{g}^{-1} \cdot \mathrm{K}^{-1}$ and $0.065 \mathrm{~J} \cdot \mathrm{g}^{-1} \cdot \mathrm{K}^{-1}(1.61 \%$ to $2.93 \%)$. 


\subsection{Data Tables}

Table 11: Measured Isobaric Heat Capacities for POE5.

\begin{tabular}{|c|c|c|c|c|c|c|c|c|}
\hline $\begin{array}{c}\mathbf{T} \\
/ \mathrm{K} \\
\end{array}$ & $\begin{array}{c}\overline{\overline{\boldsymbol{c}}}_{\boldsymbol{p}} \\
/ \mathrm{J} \cdot \mathrm{g}^{-1} \cdot \mathbf{K}^{-1} \\
\end{array}$ & $\begin{array}{c}U\left(\overline{\bar{c}}_{p}\right) \\
/ \mathbf{J} \cdot \mathbf{g}^{-1} \cdot \mathbf{K}^{-1} \\
\end{array}$ & $\begin{array}{c}\mathbf{T} \\
/ \mathbf{K} \\
\end{array}$ & $\begin{array}{c}\overline{\bar{c}}_{p} \\
/ \mathbf{J} \cdot \mathbf{g}^{-1} \cdot \mathbf{K}^{-1} \\
\end{array}$ & $\begin{array}{c}U\left(\overline{\bar{c}}_{p}\right) \\
/ \mathbf{J} \cdot \mathbf{g}^{-1} \cdot \mathbf{K}^{-1}\end{array}$ & $\begin{array}{c}\mathbf{T} \\
/ \mathbf{K} \\
\end{array}$ & $\begin{array}{c}\overline{\overline{\boldsymbol{c}}}_{\boldsymbol{p}} \\
/ \mathrm{J} \cdot \mathrm{g}^{-1} \cdot \mathrm{K}^{-1} \\
\end{array}$ & $\begin{array}{c}\boldsymbol{U}\left(\overline{\overline{\boldsymbol{c}}}_{\boldsymbol{p}}\right) \\
/ \mathbf{J} \cdot \mathbf{g}^{-1} \cdot \mathbf{K}^{-1}\end{array}$ \\
\hline 263.14 & 1.704 & 0.040 & 293.14 & 1.769 & 0.039 & 323.14 & 1.848 & 0.037 \\
\hline 264.15 & 1.706 & 0.040 & 294.15 & 1.772 & 0.039 & 324.15 & 1.850 & 0.037 \\
\hline 265.15 & 1.708 & 0.040 & 295.14 & 1.775 & 0.039 & 325.14 & 1.853 & 0.037 \\
\hline 266.14 & 1.710 & 0.040 & 296.15 & 1.777 & 0.039 & 326.15 & 1.855 & 0.037 \\
\hline 267.14 & 1.712 & 0.040 & 297.14 & 1.780 & 0.038 & 327.15 & 1.858 & 0.037 \\
\hline 268.15 & 1.713 & 0.040 & 298.14 & 1.783 & 0.038 & 328.15 & 1.861 & 0.037 \\
\hline 269.14 & 1.715 & 0.040 & 299.15 & 1.785 & 0.038 & 329.14 & 1.863 & 0.037 \\
\hline 270.14 & 1.717 & 0.040 & 300.14 & 1.788 & 0.038 & 330.15 & 1.866 & 0.037 \\
\hline 271.15 & 1.719 & 0.040 & 301.15 & 1.791 & 0.038 & 331.15 & 1.869 & 0.037 \\
\hline 272.14 & 1.721 & 0.040 & 302.14 & 1.793 & 0.038 & 332.14 & 1.871 & 0.037 \\
\hline 273.14 & 1.724 & 0.040 & 303.15 & 1.796 & 0.038 & 333.14 & 1.874 & 0.037 \\
\hline 274.14 & 1.726 & 0.040 & 304.15 & 1.799 & 0.038 & 334.15 & 1.877 & 0.037 \\
\hline 275.15 & 1.728 & 0.039 & 305.14 & 1.801 & 0.038 & 335.14 & 1.880 & 0.037 \\
\hline 276.14 & 1.730 & 0.039 & 306.15 & 1.803 & 0.038 & 336.14 & 1.883 & 0.037 \\
\hline 277.14 & 1.733 & 0.039 & 307.15 & 1.806 & 0.038 & 337.14 & 1.886 & 0.037 \\
\hline 278.15 & 1.735 & 0.039 & 308.14 & 1.808 & 0.038 & 338.14 & 1.888 & 0.037 \\
\hline 279.15 & 1.737 & 0.039 & 309.14 & 1.811 & 0.038 & 339.15 & 1.891 & 0.037 \\
\hline 280.15 & 1.739 & 0.039 & 310.15 & 1.814 & 0.038 & 340.14 & 1.894 & 0.037 \\
\hline 281.14 & 1.741 & 0.039 & 311.15 & 1.816 & 0.038 & 341.14 & 1.896 & 0.037 \\
\hline 282.15 & 1.743 & 0.039 & 312.14 & 1.819 & 0.038 & 342.15 & 1.898 & 0.038 \\
\hline 283.15 & 1.745 & 0.038 & 313.15 & 1.821 & 0.037 & 343.14 & 1.901 & 0.037 \\
\hline 284.15 & 1.747 & 0.038 & 314.14 & 1.824 & 0.037 & 344.15 & 1.903 & 0.037 \\
\hline 285.15 & 1.750 & 0.038 & 315.15 & 1.827 & 0.037 & 345.15 & 1.906 & 0.037 \\
\hline 286.14 & 1.752 & 0.038 & 316.14 & 1.829 & 0.037 & 346.15 & 1.909 & 0.037 \\
\hline 287.14 & 1.754 & 0.038 & 317.15 & 1.832 & 0.037 & 347.14 & 1.911 & 0.037 \\
\hline 288.15 & 1.756 & 0.038 & 318.15 & 1.834 & 0.037 & 348.14 & 1.914 & 0.037 \\
\hline 289.15 & 1.759 & 0.039 & 319.14 & 1.837 & 0.037 & 349.15 & 1.917 & 0.037 \\
\hline 290.14 & 1.761 & 0.039 & 320.15 & 1.840 & 0.037 & 350.14 & 1.920 & 0.037 \\
\hline 291.14 & 1.764 & 0.039 & 321.15 & 1.842 & 0.037 & 351.14 & 1.922 & 0.037 \\
\hline 292.14 & 1.767 & 0.039 & 322.14 & 1.845 & 0.037 & 352.14 & 1.925 & 0.037 \\
\hline
\end{tabular}


Table 11, continued.

\begin{tabular}{|c|c|c|c|c|c|c|c|c|}
\hline $\begin{array}{c}T \\
/ \mathbf{K}\end{array}$ & $\begin{array}{c}\overline{\overline{\boldsymbol{c}}}_{\boldsymbol{p}} \\
/ \mathrm{J} \cdot \mathrm{g}^{-1} \cdot \mathbf{K}^{-1}\end{array}$ & $\begin{array}{c}U\left(\overline{\overline{\boldsymbol{c}}}_{\boldsymbol{p}}\right) \\
/ \mathbf{J} \cdot \mathrm{g}^{-1} \cdot \mathbf{K}^{-1}\end{array}$ & $\begin{array}{c}T \\
/ \mathbf{K}\end{array}$ & $\begin{array}{c}\overline{\overline{\boldsymbol{c}}}_{\boldsymbol{p}} \\
/ \mathrm{J} \cdot \mathrm{g}^{-1} \cdot \mathbf{K}^{-1}\end{array}$ & $\begin{array}{c}\boldsymbol{U}\left(\overline{\overline{\boldsymbol{c}}}_{\boldsymbol{p}}\right) \\
/ \mathbf{J} \cdot \mathbf{g}^{-1} \cdot \mathbf{K}^{-1}\end{array}$ & $\begin{array}{c}T \\
/ \mathbf{K}\end{array}$ & $\begin{array}{c}\overline{\overline{\boldsymbol{c}}}_{\boldsymbol{p}} \\
/ \mathrm{J} \cdot \mathbf{g}^{-1} \cdot \mathbf{K}^{-1}\end{array}$ & $\begin{array}{c}\boldsymbol{U}\left(\overline{\overline{\boldsymbol{c}}}_{\boldsymbol{p}}\right) \\
/ \mathbf{J} \cdot \mathbf{g}^{-1} \cdot \mathbf{K}^{-1}\end{array}$ \\
\hline 353.14 & 1.928 & 0.037 & 380.14 & 2.002 & 0.038 & 407.14 & 2.076 & 0.039 \\
\hline 354.15 & 1.931 & 0.037 & 381.15 & 2.005 & 0.038 & 408.14 & 2.079 & 0.039 \\
\hline 355.14 & 1.933 & 0.037 & 382.14 & 2.008 & 0.038 & 409.14 & 2.081 & 0.039 \\
\hline 356.15 & 1.936 & 0.037 & 383.14 & 2.011 & 0.038 & 410.15 & 2.084 & 0.039 \\
\hline 357.15 & 1.939 & 0.037 & 384.14 & 2.014 & 0.038 & 411.15 & 2.087 & 0.039 \\
\hline 358.14 & 1.942 & 0.037 & 385.15 & 2.016 & 0.038 & 412.14 & 2.090 & 0.039 \\
\hline 359.15 & 1.944 & 0.037 & 386.14 & 2.019 & 0.038 & 413.15 & 2.092 & 0.039 \\
\hline 360.15 & 1.947 & 0.037 & 387.14 & 2.022 & 0.038 & 414.15 & 2.095 & 0.039 \\
\hline 361.14 & 1.950 & 0.037 & 388.15 & 2.025 & 0.038 & 415.15 & 2.098 & 0.039 \\
\hline 362.15 & 1.952 & 0.037 & 389.15 & 2.027 & 0.038 & 416.14 & 2.101 & 0.039 \\
\hline 363.15 & 1.955 & 0.037 & 390.14 & 2.030 & 0.038 & 417.15 & 2.103 & 0.040 \\
\hline 364.14 & 1.958 & 0.037 & 391.15 & 2.033 & 0.038 & 418.15 & 2.106 & 0.040 \\
\hline 365.14 & 1.961 & 0.037 & 392.15 & 2.035 & 0.038 & 419.15 & 2.108 & 0.040 \\
\hline 366.15 & 1.963 & 0.037 & 393.15 & 2.038 & 0.039 & 420.14 & 2.111 & 0.040 \\
\hline 367.14 & 1.966 & 0.037 & 394.15 & 2.040 & 0.039 & 421.15 & 2.114 & 0.040 \\
\hline 368.14 & 1.969 & 0.037 & 395.15 & 2.043 & 0.039 & 422.15 & 2.116 & 0.040 \\
\hline 369.14 & 1.972 & 0.037 & 396.14 & 2.046 & 0.039 & 423.15 & 2.119 & 0.040 \\
\hline 370.14 & 1.975 & 0.037 & 397.14 & 2.049 & 0.039 & 424.14 & 2.122 & 0.040 \\
\hline 371.15 & 1.977 & 0.037 & 398.14 & 2.052 & 0.039 & 425.15 & 2.125 & 0.040 \\
\hline 372.14 & 1.980 & 0.037 & 399.14 & 2.055 & 0.039 & 426.15 & 2.127 & 0.041 \\
\hline 373.14 & 1.983 & 0.037 & 400.15 & 2.057 & 0.039 & 427.14 & 2.130 & 0.041 \\
\hline 374.15 & 1.986 & 0.037 & 401.14 & 2.060 & 0.039 & 428.15 & 2.133 & 0.041 \\
\hline 375.14 & 1.988 & 0.038 & 402.14 & 2.063 & 0.039 & 429.15 & 2.135 & 0.041 \\
\hline 376.14 & 1.991 & 0.038 & 403.15 & 2.065 & 0.039 & 430.14 & 2.138 & 0.041 \\
\hline 377.14 & 1.994 & 0.038 & 404.15 & 2.068 & 0.039 & 431.15 & 2.140 & 0.041 \\
\hline 378.15 & 1.997 & 0.038 & 405.14 & 2.071 & 0.039 & 432.15 & 2.143 & 0.041 \\
\hline 379.15 & 1.999 & 0.038 & 406.15 & 2.073 & 0.039 & & & \\
\hline
\end{tabular}


Table 12: Measured Isobaric Heat Capacities for POE7.

\begin{tabular}{|c|c|c|c|c|c|c|c|c|c|c|c|}
\hline $\begin{array}{c}T \\
/ \mathrm{K}\end{array}$ & $\begin{array}{c}\overline{\bar{c}}_{\boldsymbol{p}} \\
/ \mathrm{J} \cdot \mathbf{g}^{-1} \cdot \mathbf{K}^{-1}\end{array}$ & $\begin{array}{c}\boldsymbol{U}\left(\overline{\overline{\boldsymbol{c}}}_{\boldsymbol{p}}\right) \\
/ \mathbf{J} \cdot \mathbf{g}^{-1} \cdot \mathbf{K}^{-1}\end{array}$ & $\begin{array}{c}T \\
/ \mathbf{K}\end{array}$ & $\begin{array}{c}\overline{\overline{\boldsymbol{c}}}_{\boldsymbol{p}} \\
/ \mathrm{J} \cdot \mathrm{g}^{-1} \cdot \mathbf{K}^{-1}\end{array}$ & $\begin{array}{c}\boldsymbol{U}\left(\overline{\overline{\boldsymbol{c}}}_{\boldsymbol{p}}\right) \\
/ \mathbf{J} \cdot \mathbf{g}^{-1} \cdot \mathbf{K}^{-1}\end{array}$ & $\begin{array}{c}T \\
/ \mathbf{K}\end{array}$ & $\begin{array}{c}\overline{\overline{\boldsymbol{c}}}_{\boldsymbol{p}} \\
/ \mathrm{J} \cdot \mathbf{g}^{-1} \cdot \mathbf{K}^{-1}\end{array}$ & $\begin{array}{c}\boldsymbol{U}\left(\overline{\overline{\boldsymbol{c}}}_{\boldsymbol{p}}\right) \\
/ \mathbf{J} \cdot \mathbf{g}^{-1} \cdot \mathbf{K}^{-1}\end{array}$ & $\begin{array}{c}T \\
/ \mathbf{K}\end{array}$ & $\begin{array}{c}\overline{\overline{\boldsymbol{c}}}_{\boldsymbol{p}} \\
/ \mathbf{J} \cdot \mathbf{g}^{-1} \cdot \mathbf{K}^{-1}\end{array}$ & $\begin{array}{c}\boldsymbol{U}\left(\overline{\overline{\boldsymbol{c}}}_{\boldsymbol{p}}\right) \\
/ \mathbf{J} \cdot \mathbf{g}^{-1} \cdot \mathbf{K}^{-1}\end{array}$ \\
\hline 293.14 & 1.811 & 0.053 & 325.14 & 1.896 & 0.050 & 357.14 & 1.985 & 0.048 & 389.14 & 2.076 & 0.048 \\
\hline 294.14 & 1.813 & 0.053 & 326.14 & 1.899 & 0.050 & 358.14 & 1.988 & 0.048 & 390.14 & 2.079 & 0.048 \\
\hline 295.14 & 1.816 & 0.053 & 327.14 & 1.902 & 0.050 & 359.14 & 1.991 & 0.048 & 391.14 & 2.082 & 0.048 \\
\hline 296.14 & 1.819 & 0.053 & 328.14 & 1.905 & 0.050 & 360.14 & 1.994 & 0.048 & 392.14 & 2.085 & 0.048 \\
\hline 297.14 & 1.822 & 0.053 & 329.14 & 1.908 & 0.050 & 361.14 & 1.997 & 0.048 & 393.14 & 2.087 & 0.048 \\
\hline 298.14 & 1.824 & 0.053 & 330.14 & 1.910 & 0.050 & 362.14 & 1.999 & 0.048 & 394.14 & 2.090 & 0.048 \\
\hline 299.14 & 1.827 & 0.053 & 331.14 & 1.913 & 0.050 & 363.14 & 2.002 & 0.048 & 395.14 & 2.093 & 0.048 \\
\hline 300.14 & 1.830 & 0.053 & 332.14 & 1.916 & 0.050 & 364.14 & 2.005 & 0.048 & 396.14 & 2.095 & 0.048 \\
\hline 301.14 & 1.832 & 0.053 & 333.14 & 1.918 & 0.050 & 365.14 & 2.008 & 0.048 & 397.14 & 2.098 & 0.048 \\
\hline 302.14 & 1.835 & 0.053 & 334.13 & 1.921 & 0.050 & 366.14 & 2.011 & 0.048 & 398.14 & 2.101 & 0.048 \\
\hline 303.14 & 1.838 & 0.052 & 335.14 & 1.924 & 0.050 & 367.14 & 2.013 & 0.048 & 399.14 & 2.104 & 0.048 \\
\hline 304.14 & 1.840 & 0.052 & 336.14 & 1.927 & 0.050 & 368.14 & 2.016 & 0.048 & 400.14 & 2.107 & 0.048 \\
\hline 305.14 & 1.843 & 0.052 & 337.14 & 1.929 & 0.049 & 369.14 & 2.019 & 0.048 & 401.14 & 2.110 & 0.048 \\
\hline 306.14 & 1.845 & 0.052 & 338.14 & 1.932 & 0.049 & 370.14 & 2.022 & 0.048 & 402.14 & 2.113 & 0.048 \\
\hline 307.14 & 1.848 & 0.052 & 339.14 & 1.935 & 0.049 & 371.14 & 2.025 & 0.048 & 403.14 & 2.115 & 0.048 \\
\hline 308.14 & 1.851 & 0.052 & 340.14 & 1.938 & 0.049 & 372.14 & 2.027 & 0.048 & 404.14 & 2.118 & 0.048 \\
\hline 309.14 & 1.853 & 0.052 & 341.13 & 1.940 & 0.048 & 373.14 & 2.030 & 0.048 & 405.14 & 2.121 & 0.048 \\
\hline 310.14 & 1.856 & 0.052 & 342.14 & 1.943 & 0.048 & 374.14 & 2.033 & 0.048 & 406.14 & 2.124 & 0.048 \\
\hline 311.14 & 1.858 & 0.052 & 343.14 & 1.946 & 0.048 & 375.14 & 2.036 & 0.048 & 407.14 & 2.127 & 0.048 \\
\hline 312.14 & 1.861 & 0.051 & 344.14 & 1.949 & 0.048 & 376.14 & 2.039 & 0.048 & 408.14 & 2.130 & 0.048 \\
\hline 313.14 & 1.864 & 0.051 & 345.13 & 1.952 & 0.048 & 377.14 & 2.042 & 0.048 & 409.14 & 2.133 & 0.048 \\
\hline 314.14 & 1.867 & 0.051 & 346.14 & 1.955 & 0.049 & 378.14 & 2.045 & 0.048 & 410.14 & 2.135 & 0.048 \\
\hline 315.14 & 1.869 & 0.051 & 347.14 & 1.958 & 0.049 & 379.14 & 2.048 & 0.048 & 411.14 & 2.138 & 0.048 \\
\hline 316.14 & 1.872 & 0.051 & 348.14 & 1.960 & 0.048 & 380.14 & 2.050 & 0.048 & 412.13 & 2.141 & 0.049 \\
\hline 317.14 & 1.875 & 0.051 & 349.14 & 1.963 & 0.049 & 381.14 & 2.053 & 0.048 & 413.14 & 2.144 & 0.049 \\
\hline 318.14 & 1.878 & 0.050 & 350.14 & 1.966 & 0.049 & 382.14 & 2.056 & 0.048 & 414.13 & 2.147 & 0.049 \\
\hline 319.14 & 1.880 & 0.050 & 351.14 & 1.969 & 0.049 & 383.14 & 2.059 & 0.048 & 415.14 & 2.150 & 0.049 \\
\hline 320.14 & 1.883 & 0.050 & 352.14 & 1.972 & 0.049 & 384.14 & 2.062 & 0.048 & 416.14 & 2.152 & 0.049 \\
\hline 321.14 & 1.886 & 0.050 & 353.14 & 1.974 & 0.049 & 385.14 & 2.065 & 0.048 & 417.14 & 2.155 & 0.049 \\
\hline 322.13 & 1.888 & 0.050 & 354.14 & 1.977 & 0.049 & 386.14 & 2.068 & 0.048 & & & \\
\hline 323.14 & 1.891 & 0.050 & 355.14 & 1.980 & 0.048 & 387.14 & 2.071 & 0.048 & & & \\
\hline 324.14 & 1.894 & 0.050 & 356.14 & 1.983 & 0.048 & 388.14 & 2.074 & 0.048 & & & \\
\hline
\end{tabular}


Table 13: Measured Isobaric Heat Capacities for POE9.

\begin{tabular}{|c|c|c|c|c|c|c|c|c|}
\hline $\begin{array}{c}T \\
/ \mathbf{K}\end{array}$ & $\begin{array}{c}\overline{\overline{\boldsymbol{c}}}_{\boldsymbol{p}} \\
/ \mathrm{J} \cdot \mathrm{g}^{-1} \cdot \mathbf{K}^{-1}\end{array}$ & $\begin{array}{c}\boldsymbol{U}\left(\overline{\overline{\boldsymbol{c}}}_{\boldsymbol{p}}\right) \\
/ \mathbf{J} \cdot \mathbf{g}^{-1} \cdot \mathbf{K}^{-1}\end{array}$ & $\begin{array}{c}T \\
/ \mathbf{K}\end{array}$ & $\begin{array}{c}\overline{\overline{\boldsymbol{c}}}_{\boldsymbol{p}} \\
/ \mathbf{J} \cdot \mathbf{g}^{-1} \cdot \mathbf{K}^{-1}\end{array}$ & $\begin{array}{c}\boldsymbol{U}\left(\overline{\overline{\boldsymbol{c}}}_{\boldsymbol{p}}\right) \\
/ \mathbf{J} \cdot \mathbf{g}^{-1} \cdot \mathbf{K}^{-1}\end{array}$ & $\begin{array}{c}T \\
/ \mathbf{K}\end{array}$ & $\begin{array}{c}\overline{\overline{\boldsymbol{c}}}_{\boldsymbol{p}} \\
/ \mathbf{J} \cdot \mathbf{g}^{-1} \cdot \mathbf{K}^{-1}\end{array}$ & $\begin{array}{r}\boldsymbol{U}\left(\overline{\overline{\boldsymbol{c}}}_{\boldsymbol{p}}\right) \\
/ \mathbf{J} \cdot \mathbf{g}^{-1} \cdot \mathbf{K}^{-1}\end{array}$ \\
\hline 313.13 & 1.940 & 0.037 & 347.13 & 2.036 & 0.038 & 381.13 & 2.139 & 0.037 \\
\hline 314.13 & 1.943 & 0.037 & 348.13 & 2.039 & 0.037 & 382.13 & 2.142 & 0.037 \\
\hline 315.14 & 1.945 & 0.037 & 349.13 & 2.042 & 0.037 & 383.13 & 2.145 & 0.036 \\
\hline 316.13 & 1.948 & 0.037 & 350.14 & 2.045 & 0.037 & 384.13 & 2.148 & 0.036 \\
\hline 317.14 & 1.951 & 0.038 & 351.13 & 2.048 & 0.037 & 385.13 & 2.151 & 0.036 \\
\hline 318.13 & 1.954 & 0.037 & 352.13 & 2.051 & 0.037 & 386.13 & 2.154 & 0.036 \\
\hline 319.13 & 1.956 & 0.037 & 353.14 & 2.054 & 0.037 & 387.13 & 2.157 & 0.036 \\
\hline 320.14 & 1.959 & 0.037 & 354.13 & 2.057 & 0.037 & 388.13 & 2.160 & 0.036 \\
\hline 321.13 & 1.962 & 0.037 & 355.14 & 2.060 & 0.037 & 389.13 & 2.163 & 0.037 \\
\hline 322.13 & 1.964 & 0.037 & 356.13 & 2.063 & 0.037 & 390.13 & 2.166 & 0.037 \\
\hline 323.14 & 1.967 & 0.037 & 357.13 & 2.066 & 0.037 & 391.13 & 2.169 & 0.037 \\
\hline 324.13 & 1.970 & 0.038 & 358.13 & 2.069 & 0.037 & 392.13 & 2.172 & 0.037 \\
\hline 325.13 & 1.972 & 0.038 & 359.13 & 2.072 & 0.037 & 393.13 & 2.175 & 0.037 \\
\hline 326.13 & 1.975 & 0.038 & 360.13 & 2.075 & 0.037 & 394.13 & 2.178 & 0.037 \\
\hline 327.13 & 1.978 & 0.038 & 361.13 & 2.078 & 0.037 & 395.14 & 2.181 & 0.037 \\
\hline 328.14 & 1.981 & 0.037 & 362.13 & 2.081 & 0.037 & 396.13 & 2.185 & 0.037 \\
\hline 329.14 & 1.984 & 0.038 & 363.13 & 2.084 & 0.037 & 397.13 & 2.188 & 0.037 \\
\hline 330.13 & 1.986 & 0.037 & 364.13 & 2.087 & 0.037 & 398.13 & 2.191 & 0.037 \\
\hline 331.13 & 1.989 & 0.037 & 365.13 & 2.090 & 0.037 & 399.13 & 2.194 & 0.037 \\
\hline 332.13 & 1.992 & 0.037 & 366.13 & 2.093 & 0.037 & 400.13 & 2.197 & 0.037 \\
\hline 333.13 & 1.995 & 0.037 & 367.13 & 2.096 & 0.037 & 401.13 & 2.200 & 0.037 \\
\hline 334.13 & 1.998 & 0.037 & 368.13 & 2.099 & 0.037 & 402.14 & 2.203 & 0.036 \\
\hline 335.13 & 2.001 & 0.037 & 369.13 & 2.102 & 0.037 & 403.13 & 2.206 & 0.036 \\
\hline 336.14 & 2.004 & 0.037 & 370.13 & 2.105 & 0.037 & 404.14 & 2.209 & 0.036 \\
\hline 337.13 & 2.007 & 0.038 & 371.13 & 2.108 & 0.037 & 405.13 & 2.212 & 0.036 \\
\hline 338.13 & 2.010 & 0.038 & 372.13 & 2.111 & 0.037 & 406.13 & 2.215 & 0.036 \\
\hline 339.13 & 2.013 & 0.038 & 373.13 & 2.114 & 0.037 & 407.13 & 2.218 & 0.036 \\
\hline 340.13 & 2.016 & 0.038 & 374.14 & 2.117 & 0.038 & 408.13 & 2.221 & 0.036 \\
\hline 341.14 & 2.018 & 0.038 & 375.14 & 2.120 & 0.038 & 409.13 & 2.225 & 0.036 \\
\hline 342.14 & 2.021 & 0.038 & 376.13 & 2.123 & 0.037 & 410.14 & 2.228 & 0.036 \\
\hline 343.13 & 2.024 & 0.038 & 377.13 & 2.126 & 0.037 & 411.13 & 2.230 & 0.036 \\
\hline 344.13 & 2.027 & 0.038 & 378.13 & 2.129 & 0.037 & 412.14 & 2.234 & 0.036 \\
\hline 345.14 & 2.030 & 0.038 & 379.13 & 2.132 & 0.037 & & & \\
\hline 346.13 & 2.033 & 0.038 & 380.14 & 2.135 & 0.037 & & & \\
\hline
\end{tabular}


Table 14: Measured Isobaric Heat Capacities for MIL-PRF-23699.

\begin{tabular}{|c|c|c|c|c|c|c|c|c|}
\hline $\begin{array}{l}T \\
/ \mathrm{K}\end{array}$ & $\begin{array}{c}\overline{\overline{\boldsymbol{c}}}_{\boldsymbol{p}} \\
/ \mathrm{J} \cdot \mathrm{g}^{-1} \cdot \mathbf{K}^{-1}\end{array}$ & $\begin{array}{c}\boldsymbol{U}\left(\overline{\overline{\boldsymbol{c}}}_{\boldsymbol{p}}\right) \\
/ \mathbf{J} \cdot \mathbf{g}^{-1} \cdot \mathbf{K}^{-1}\end{array}$ & $\begin{array}{c}T \\
/ \mathrm{K}\end{array}$ & $\begin{array}{c}\overline{\bar{c}}_{p} \\
/ \mathbf{J} \cdot \mathbf{g}^{-1} \cdot K^{-1}\end{array}$ & $\begin{array}{c}\boldsymbol{U}\left(\overline{\overline{\boldsymbol{c}}}_{\boldsymbol{p}}\right) \\
/ \mathbf{J} \cdot \mathbf{g}^{-1} \cdot \mathbf{K}^{-1}\end{array}$ & $\begin{array}{c}T \\
/ \mathbf{K}\end{array}$ & $\begin{array}{c}\overline{\overline{\boldsymbol{c}}}_{\boldsymbol{p}} \\
/ \mathrm{J} \cdot \mathrm{g}^{-1} \cdot \mathbf{K}^{-1}\end{array}$ & $\begin{array}{c}\boldsymbol{U}\left(\overline{\overline{\boldsymbol{c}}}_{\boldsymbol{p}}\right) \\
/ \mathbf{J} \cdot \mathbf{g}^{-1} \cdot \mathbf{K}^{-1}\end{array}$ \\
\hline 273.15 & 1.737 & 0.048 & 312.15 & 1.837 & 0.048 & 351.15 & 1.947 & 0.050 \\
\hline 274.15 & 1.739 & 0.048 & 313.15 & 1.840 & 0.048 & 352.15 & 1.950 & 0.050 \\
\hline 275.15 & 1.742 & 0.048 & 314.15 & 1.843 & 0.048 & 353.15 & 1.953 & 0.050 \\
\hline 276.15 & 1.744 & 0.047 & 315.15 & 1.846 & 0.048 & 354.15 & 1.956 & 0.050 \\
\hline 277.16 & 1.746 & 0.047 & 316.15 & 1.849 & 0.048 & 355.15 & 1.959 & 0.050 \\
\hline 278.15 & 1.749 & 0.047 & 317.15 & 1.851 & 0.048 & 356.15 & 1.961 & 0.050 \\
\hline 279.15 & 1.751 & 0.047 & 318.15 & 1.854 & 0.048 & 357.15 & 1.964 & 0.050 \\
\hline 280.15 & 1.753 & 0.047 & 319.15 & 1.857 & 0.048 & 358.15 & 1.967 & 0.050 \\
\hline 281.15 & 1.755 & 0.047 & 320.15 & 1.860 & 0.048 & 359.15 & 1.970 & 0.050 \\
\hline 282.15 & 1.757 & 0.047 & 321.15 & 1.862 & 0.048 & 360.15 & 1.973 & 0.050 \\
\hline 283.15 & 1.760 & 0.047 & 322.15 & 1.865 & 0.048 & 361.15 & 1.976 & 0.050 \\
\hline 284.15 & 1.762 & 0.047 & 323.15 & 1.868 & 0.048 & 362.15 & 1.978 & 0.050 \\
\hline 285.15 & 1.764 & 0.047 & 324.15 & 1.870 & 0.048 & 363.15 & 1.981 & 0.050 \\
\hline 286.15 & 1.767 & 0.047 & 325.15 & 1.873 & 0.048 & 364.15 & 1.984 & 0.050 \\
\hline 287.15 & 1.769 & 0.047 & 326.15 & 1.876 & 0.048 & 365.15 & 1.987 & 0.050 \\
\hline 288.15 & 1.771 & 0.047 & 327.15 & 1.879 & 0.048 & 366.15 & 1.990 & 0.050 \\
\hline 289.15 & 1.774 & 0.048 & 328.15 & 1.882 & 0.048 & 367.15 & 1.992 & 0.050 \\
\hline 290.15 & 1.777 & 0.048 & 329.15 & 1.885 & 0.048 & 368.15 & 1.995 & 0.050 \\
\hline 291.15 & 1.779 & 0.048 & 330.15 & 1.888 & 0.048 & 369.15 & 1.998 & 0.050 \\
\hline 292.15 & 1.782 & 0.048 & 331.15 & 1.891 & 0.048 & 370.15 & 2.001 & 0.050 \\
\hline 293.15 & 1.785 & 0.048 & 332.15 & 1.893 & 0.048 & 371.15 & 2.004 & 0.050 \\
\hline 294.15 & 1.788 & 0.048 & 333.15 & 1.896 & 0.048 & 372.15 & 2.007 & 0.050 \\
\hline 295.15 & 1.791 & 0.048 & 334.15 & 1.899 & 0.048 & 373.15 & 2.010 & 0.050 \\
\hline 296.15 & 1.794 & 0.048 & 335.15 & 1.902 & 0.048 & 374.15 & 2.013 & 0.050 \\
\hline 297.15 & 1.797 & 0.048 & 336.15 & 1.904 & 0.048 & 375.15 & 2.015 & 0.050 \\
\hline 298.15 & 1.799 & 0.048 & 337.15 & 1.907 & 0.048 & 376.15 & 2.018 & 0.050 \\
\hline 299.15 & 1.802 & 0.048 & 338.15 & 1.910 & 0.048 & 377.15 & 2.021 & 0.050 \\
\hline 300.15 & 1.805 & 0.048 & 339.15 & 1.913 & 0.049 & 378.15 & 2.024 & 0.051 \\
\hline 301.15 & 1.808 & 0.048 & 340.15 & 1.916 & 0.049 & 379.15 & 2.027 & 0.051 \\
\hline 302.15 & 1.810 & 0.048 & 341.15 & 1.918 & 0.049 & 380.15 & 2.030 & 0.051 \\
\hline 303.15 & 1.813 & 0.048 & 342.15 & 1.921 & 0.049 & 381.15 & 2.033 & 0.051 \\
\hline 304.15 & 1.816 & 0.048 & 343.15 & 1.924 & 0.049 & 382.15 & 2.036 & 0.051 \\
\hline 305.15 & 1.818 & 0.048 & 344.15 & 1.927 & 0.049 & 383.15 & 2.039 & 0.051 \\
\hline 306.15 & 1.821 & 0.048 & 345.15 & 1.930 & 0.049 & 384.15 & 2.042 & 0.051 \\
\hline 307.15 & 1.824 & 0.048 & 346.15 & 1.933 & 0.049 & 385.15 & 2.045 & 0.051 \\
\hline 308.15 & 1.826 & 0.048 & 347.15 & 1.936 & 0.049 & 386.15 & 2.048 & 0.052 \\
\hline 309.15 & 1.829 & 0.048 & 348.15 & 1.939 & 0.049 & 387.15 & 2.050 & 0.052 \\
\hline 310.15 & 1.832 & 0.048 & 349.15 & 1.941 & 0.050 & 388.15 & 2.053 & 0.052 \\
\hline 311.15 & 1.835 & 0.048 & 350.15 & 1.944 & 0.050 & 389.15 & 2.056 & 0.052 \\
\hline
\end{tabular}


Table 14, continued.

\begin{tabular}{|c|c|c|c|c|c|c|c|c|}
\hline $\begin{array}{c}T \\
/ \mathrm{K} \\
\end{array}$ & $\begin{array}{c}\overline{\overline{\boldsymbol{c}}}_{\boldsymbol{p}} \\
/ \mathrm{J} \cdot \mathrm{g}^{-1} \cdot \mathbf{K}^{-1} \\
\end{array}$ & $\begin{array}{c}\boldsymbol{U}\left(\overline{\overline{\boldsymbol{c}}}_{\boldsymbol{p}}\right) \\
/ \mathbf{J} \cdot \mathbf{g}^{-1} \cdot \mathbf{K}^{-1}\end{array}$ & $\begin{array}{c}T \\
/ \mathrm{K} \\
\end{array}$ & $\begin{array}{c}\overline{\overline{\boldsymbol{c}}}_{\boldsymbol{p}} \\
/ \mathrm{J} \cdot \mathrm{g}^{-1} \cdot \mathbf{K}^{-1} \\
\end{array}$ & $\begin{array}{c}\boldsymbol{U}\left(\overline{\overline{\boldsymbol{c}}}_{\boldsymbol{p}}\right) \\
/ \mathbf{J} \cdot \mathbf{g}^{-1} \cdot \mathbf{K}^{-1}\end{array}$ & $\begin{array}{c}T \\
/ \mathbf{K} \\
\end{array}$ & $\begin{array}{c}\overline{\overline{\boldsymbol{c}}}_{\boldsymbol{p}} \\
/ \mathrm{J} \cdot \mathbf{g}^{-1} \cdot \mathbf{K}^{-1}\end{array}$ & $\begin{array}{c}\boldsymbol{U}\left(\overline{\overline{\boldsymbol{c}}}_{\boldsymbol{p}}\right) \\
/ \mathbf{J} \cdot \mathbf{g}^{-1} \cdot \mathbf{K}^{-1}\end{array}$ \\
\hline 390.15 & 2.059 & 0.052 & 425.15 & 2.158 & 0.056 & 460.14 & 2.255 & 0.061 \\
\hline 391.15 & 2.062 & 0.052 & 426.15 & 2.161 & 0.056 & 461.15 & 2.258 & 0.061 \\
\hline 392.15 & 2.064 & 0.052 & 427.15 & 2.164 & 0.057 & 462.15 & 2.261 & 0.061 \\
\hline 393.15 & 2.067 & 0.052 & 428.15 & 2.167 & 0.057 & 463.15 & 2.264 & 0.061 \\
\hline 394.15 & 2.070 & 0.052 & 429.15 & 2.170 & 0.057 & 464.15 & 2.266 & 0.062 \\
\hline 395.15 & 2.073 & 0.053 & 430.15 & 2.172 & 0.057 & 465.15 & 2.269 & 0.062 \\
\hline 396.15 & 2.076 & 0.053 & 431.15 & 2.175 & 0.057 & 466.15 & 2.272 & 0.062 \\
\hline 397.15 & 2.079 & 0.053 & 432.15 & 2.178 & 0.057 & 467.15 & 2.275 & 0.062 \\
\hline 398.15 & 2.082 & 0.053 & 433.15 & 2.181 & 0.058 & 468.15 & 2.278 & 0.062 \\
\hline 399.15 & 2.085 & 0.053 & 434.15 & 2.184 & 0.058 & 469.15 & 2.280 & 0.062 \\
\hline 400.15 & 2.087 & 0.054 & 435.15 & 2.186 & 0.058 & 470.15 & 2.283 & 0.062 \\
\hline 401.16 & 2.090 & 0.054 & 436.15 & 2.189 & 0.058 & 471.15 & 2.286 & 0.062 \\
\hline 402.16 & 2.093 & 0.054 & 437.15 & 2.192 & 0.058 & 472.15 & 2.288 & 0.062 \\
\hline 403.15 & 2.096 & 0.054 & 438.15 & 2.195 & 0.058 & 473.15 & 2.291 & 0.062 \\
\hline 404.15 & 2.099 & 0.054 & 439.15 & 2.197 & 0.058 & 474.15 & 2.294 & 0.062 \\
\hline 405.15 & 2.102 & 0.054 & 440.15 & 2.200 & 0.058 & 475.15 & 2.296 & 0.062 \\
\hline 406.15 & 2.105 & 0.054 & 441.15 & 2.203 & 0.058 & 476.15 & 2.299 & 0.062 \\
\hline 407.15 & 2.107 & 0.054 & 442.15 & 2.206 & 0.059 & 477.15 & 2.302 & 0.062 \\
\hline 408.15 & 2.110 & 0.054 & 443.15 & 2.209 & 0.059 & 478.15 & 2.304 & 0.063 \\
\hline 409.15 & 2.113 & 0.054 & 444.15 & 2.212 & 0.059 & 479.15 & 2.307 & 0.063 \\
\hline 410.15 & 2.116 & 0.055 & 445.15 & 2.214 & 0.059 & 480.15 & 2.310 & 0.063 \\
\hline 411.15 & 2.119 & 0.055 & 446.15 & 2.217 & 0.059 & 481.15 & 2.313 & 0.064 \\
\hline 412.15 & 2.122 & 0.055 & 447.15 & 2.220 & 0.059 & 482.15 & 2.315 & 0.064 \\
\hline 413.15 & 2.125 & 0.055 & 448.15 & 2.223 & 0.059 & 483.15 & 2.318 & 0.063 \\
\hline 414.15 & 2.127 & 0.055 & 449.15 & 2.226 & 0.059 & 484.15 & 2.321 & 0.063 \\
\hline 415.15 & 2.130 & 0.055 & 450.15 & 2.228 & 0.059 & 485.15 & 2.324 & 0.063 \\
\hline 416.16 & 2.133 & 0.055 & 451.15 & 2.231 & 0.060 & 486.15 & 2.327 & 0.063 \\
\hline 417.15 & 2.136 & 0.056 & 452.15 & 2.234 & 0.060 & 487.15 & 2.330 & 0.063 \\
\hline 418.15 & 2.138 & 0.056 & 453.15 & 2.237 & 0.060 & 488.15 & 2.333 & 0.064 \\
\hline 419.15 & 2.141 & 0.056 & 454.15 & 2.239 & 0.060 & 489.15 & 2.335 & 0.064 \\
\hline 420.15 & 2.144 & 0.056 & 455.15 & 2.242 & 0.060 & 490.15 & 2.338 & 0.064 \\
\hline 421.15 & 2.147 & 0.056 & 456.15 & 2.245 & 0.061 & 491.15 & 2.341 & 0.065 \\
\hline 422.15 & 2.150 & 0.056 & 457.15 & 2.247 & 0.061 & 492.15 & 2.343 & 0.064 \\
\hline 423.15 & 2.153 & 0.056 & 458.15 & 2.250 & 0.061 & & & \\
\hline 424.15 & 2.156 & 0.056 & 459.15 & 2.253 & 0.061 & & & \\
\hline
\end{tabular}




\section{Speed of Sound}

\subsection{Summary}

The speed of sound of the POE lubricants was measured over wide ranges of temperature and pressure in a dual-path, pulse-echo-type instrument. These measurements, together with the density and heat capacity measurements, provided the data necessary for fitting equations of state (EOS) for each of the POEs. The reason for the measurement of speed of sound may not be obvious. The speed of sound is closely related to heat capacity, which is important for any type of thermal calculation. Speed of sound data gives information on the change of heat capacity with pressure, but $c_{p}$ at a reference pressure is required; thus, the combination of $c_{p}$ data from the DSC measurements and the speed of sound over a wide range of pressure is very valuable for EOS fitting.

\subsection{Principle of the Measurement}

The speed of sound of the POE lubricants was measured over wide ranges of temperature and pressure in a dual-path, pulse-echo-type instrument. In this technique, a piezoelectric transducer is located within a sample volume of the test fluid. It is excited with a sinusoidal burst, near the crystal resonance frequency, from an arbitrary function generator, thus emitting ultrasonic pulses from each face of the crystal, which travel through the fluid sample, reflect off planar surfaces at each end of the sample volume, and return to the transducer, which also serves as the detector. The difference in the arrival times of the echo signals gives the speed of sound by

$$
w=\frac{2\left(L_{2}-L_{1}\right)}{\Delta t},
$$

where $w$ is the speed of sound, $L_{1}$ and $L_{2}$ are the path lengths, and $\Delta t$ is the time difference. The differential nature of this technique cancels end effects and improves the accuracy.

\subsection{Experimental Method}

\subsubsection{Instrument Description}

A quartz crystal with a diameter of $24 \mathrm{~mm}$, thickness of $0.36 \mathrm{~mm}$, and resonant frequency of 8.000 MHz served as the ultrasonic transducer. The quartz crystal is "X-cut," which means that its thickness expands and contracts when a voltage is applied to electrodes on opposite faces of the crystal. It was excited with a 10-cycle sinusoidal burst from an arbitrary function generator. The fluid path lengths on the opposite faces of the crystal were $30 \mathrm{~mm}$ and $12 \mathrm{~mm}$ (ratio of 2.5:1); these separations of the crystal and the reflectors were provided by tubular spacers fabricated of a 
machinable ceramic. A high-speed switch connected the crystal to the function generator during the input sinusoidal burst and then, after a delay of $6 \mu \mathrm{s}$, switched the crystal to the input of a threestage amplifier ( $5 \times$ per stage for a total of $125 \times$ ), which then fed into a digital storage oscilloscope. The echo signals were recorded for off-line analysis.

The measuring cell holding the crystal and fluid sample was contained in a pressure vessel rated to $93 \mathrm{MPa}$. This, in turn, was held in a thermostated oil bath operating from $228 \mathrm{~K}$ to $423 \mathrm{~K}$. A photo of the oil bath and associated fluid-handling manifold is shown as Figure 12. A photo of the instruments (which are located in the adjacent room) is shown as Figure 13. A schematic of the measuring cell is shown in Figure 14.

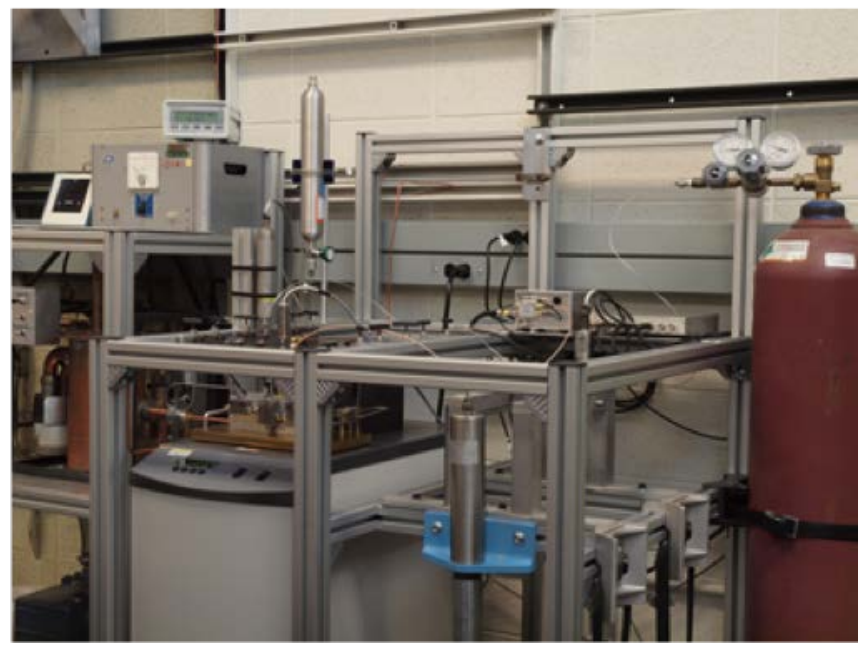

Figure 12: Dual-path, pulse-echo, speed of sound instrument showing the thermostat (which contains the measuring cell) and the fluid manifold and pressure transducer; a vacuum system for evacuating the measuring cell is to the left of the thermostat.

The temperature of the oil bath was measured with a long-stem 25-ohm standard platinum resistance thermometer (SPRT); the temperature-sensing portion of the SPRT was located immediately adjacent to the pressure vessel, as indicated in Figure 14. The resistance of the SPRT was ratioed to a standard resistor (which was held in a thermostated enclosure) with an AC resistance bridge. The SPRT, standard resistor, and resistance bridge were calibrated as a system over the range of $234.316 \mathrm{~K}$ to $429.749 \mathrm{~K}$ with fixed-point cells (mercury triple point, water triple point, and indium freezing point). The standard uncertainty in the SPRT/resistor/bridge system was estimated as $3 \mathrm{mK}$. The short-term (minute-to-minute) variations in the oil-bath temperature were $2 \mathrm{mK}$ or less. No long-term (hour-to-hour) variation was observed. The temperature gradients in the oil bath were less than $2.5 \mathrm{mK}$ over the region of the pressure vessel. The combined standard 
uncertainty in the temperature measurement, including the effects of the SPRT, standard resistor, resistance bridge, calibration standards, stability of the oil bath, and temperature gradients in the oil bath was $4 \mathrm{mK}$.

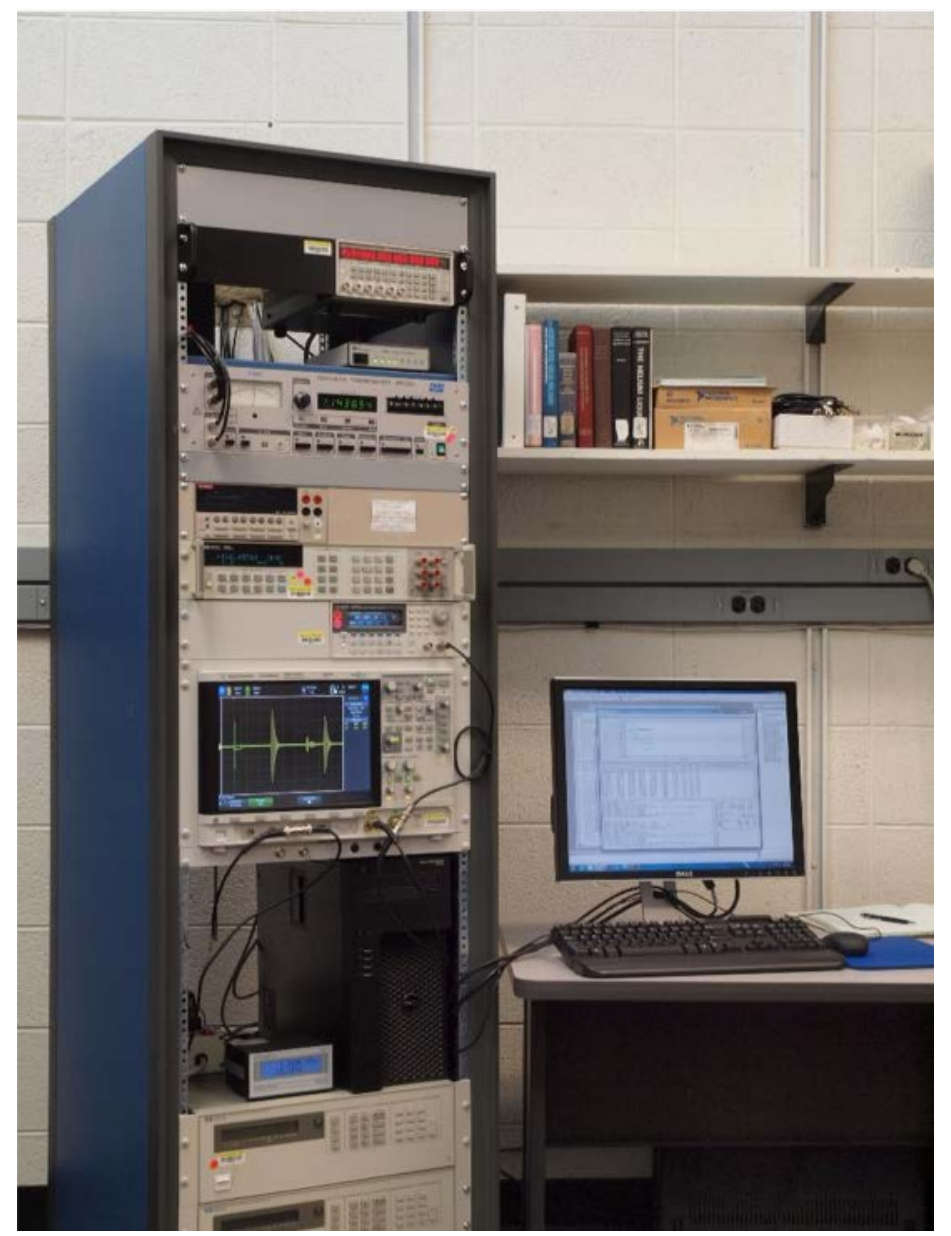

Figure 13: Instrument rack for the pulse-echo speed of sound instrument.

The pressure was measured with a vibrating-quartz-crystal pressure transducer with a maximum pressure of $138 \mathrm{MPa}$. The transducer was held at room temperature. The transducer was calibrated by the manufacturer with piston gages; this calibration included a temperature-compensation term. The zero of the transducer was checked regularly (while the system was evacuated between samples) and readings were corrected for any drift in the zero. The standard uncertainty in pressure was $0.007 \mathrm{MPa}$. 


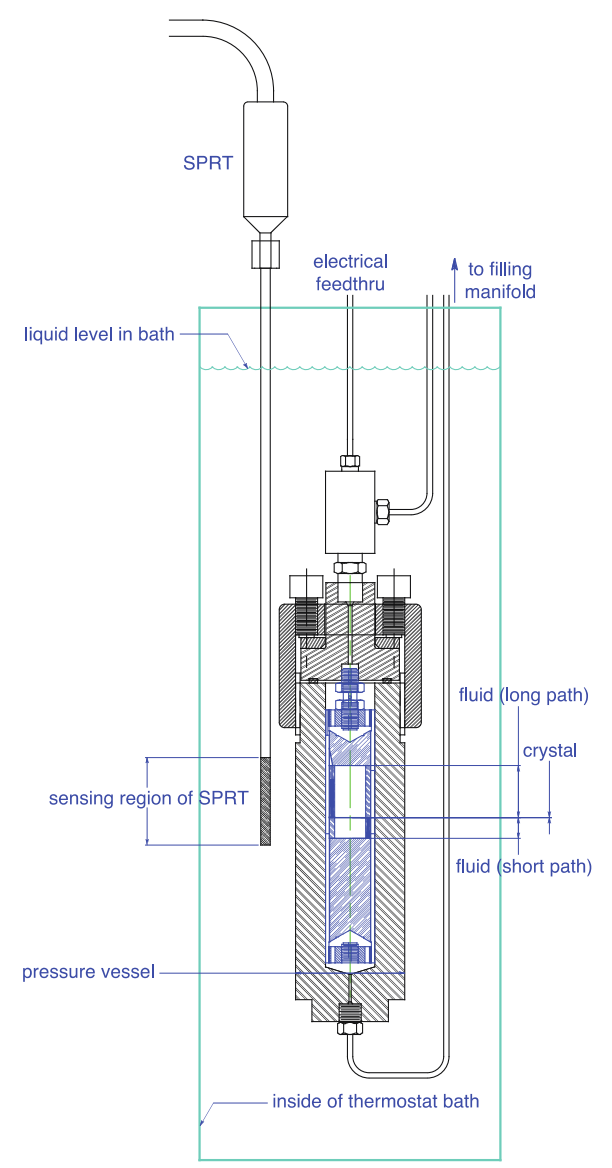

Figure 14: Schematic diagram of the measuring cell inside the pressure vessel.

\subsubsection{Measurement Sequence}

The fluid sample was loaded into a manual piston-type pump and degassed by pulling a vacuum on the head space of the pump. The sample was then pushed into the measuring cell (which had been evacuated), the pump was valved off, the oil bath was set to the first set-point temperature, and the experimental run was begun.

The entire experiment was controlled by a PC running a custom control program written in Visual Basic 6. At each $(T, p)$ state point multiple echo signals were recorded and analyzed. The pressure of the fluid sample, as well as the temperature of the oil bath, were scanned every $30 \mathrm{~s}$. The approach to equilibrium conditions was determined by monitoring three quantities: (1) the difference of the average temperature computed over the previous eight scans compared to the setpoint temperature; (2) the standard deviation of the previous eight temperature scans; (3) the rate of change of pressure with time, computed with a linear fit of the previous eight pressure readings. 
When all three of these were within preset tolerances a "converged" flag was set in the control program, and measurements commenced following an additional equilibration time of 20 minutes.

A single measurement set comprised recording three echo signals and the four temperature and pressure readings made at the start and end of the set and between the recording of the echoes. Four such sets, spaced 10 minutes apart, were recorded before moving to the next $(T, p)$ state point. These raw data were analyzed with a separate program to generate the $(T, p, w)$ data points.

When measurements at the first temperature were completed, the temperature was increased by an increment of $10 \mathrm{~K}$; since the cell was completely filled with liquid, the increase in temperature also increased the pressure. Measurements continued along this pseudo isochore (line of constant density) until either the desired maximum temperature or maximum pressure was reached. The bath was then cooled to a temperature $20 \mathrm{~K}$ above the initial temperature of the isochore, and the total quantity of sample in the measuring cell was reduced by opening the valve to the pump and backing out the pump piston to achieve a starting pressure for the next isochore of less than $1 \mathrm{MPa}$. The next isochore then commenced. This process was repeated to cover the liquid surface of the POE, within the operating limits of the pulse-echo instrument.

\subsection{Calibration of Path-Length Difference and Measurement Uncertainties}

The difference of the path lengths in the measuring cell, i.e., $\left(L_{2}-L_{1}\right)$ in Eq. 7, was calibrated as a function of temperature and pressure with measurements on propane over the temperature range of $260 \mathrm{~K}$ to $420 \mathrm{~K}$, with pressures to $56 \mathrm{MPa}$.

The propane speed of sound, as calculated with the equation of state (EOS) of Lemmon et al.[39], was taken as the known quantity in the calibration. The EOS, in turn, represents the high-accuracy propane measurements of Meier and Kabelac[40] with an average absolute deviation of $0.012 \%$. The RMS deviation of the calibration equation from the measurements was $0.010 \%$. Combining, in quadrature, the deviation of the EOS from the data of Meier and Kabelac[40] with the deviation of the path-length calibration equation from the present measurements yields an estimated combined standard uncertainty of $0.016 \%$ in our speed of sound measurements. This represents a conservative estimate of the uncertainty since it is likely that a portion of the difference between the path-length calibration and the present data is due to systematic deviations between the EOS and the data of Meier and Kabelac.[40] 
When reporting the uncertainties in experimental data it is customary to combine the effects of the state-point uncertainty (i.e., the effects of the uncertainties in temperature, pressure, and composition) with those in the uncertainty of the primary measurand (i.e., the speed of sound):

$$
U_{c}(w)=2 \times 100 \times\left\{u^{2}(w)+\left[\frac{\partial w}{\partial T}\right]^{2} u^{2}(T)+\left[\frac{\partial w}{\partial p}\right]^{2} u^{2}(p)\right\}^{\frac{1}{2}} / w
$$

where the $u(x)$ are the standard $(k=1)$ uncertainties in the different measurands (temperature, pressure, and speed of sound), the derivatives of the speed of sound with temperature and pressure are computed with an equation of state. The coverage factor of 2 corresponds to a $95 \%$ confidence interval, and the factor of 100 converts the relative deviation to a percentage deviation. The $U_{c}(w)$ is the relative, combined, expanded $(k=2)$ uncertainty in the speed of sound; it averaged $0.035 \%$ for the present measurements.

As discussed in Section 2, the purity of the POE base-stocks ranged from $93 \%$ to $97 \%$, and the effect of the impurities on the speed of sound could not be determined. Thus, the present measurements and their uncertainties must be considered as representative of the particular batches of POE base-stocks actually measured, rather than the corresponding pure fluids. Similarly, since the MIL-PRF-23699 lubricant is a complex mixture, those measurements apply only to that particular blend.

\subsection{Results}

The measurements carried out on the POE base-stocks and the general MIL-PRF-23699 lubricant are depicted in Figure 15. The measured data, as well as the relative, combined, expanded $(k=2)$ uncertainty in the speed of sound for each point, are reported in Table 15 through Table 18. As indicated above, the data in these tables are the averages of four sets of three replicates each.

The minimum temperature for the measurements was dictated by the fluid itself; these fluids are quite viscous at low temperatures, and this resulted in large damping of the ultrasonic signal. Thus, there was a minimum temperature (which varied from $283 \mathrm{~K}$ to $298 \mathrm{~K}$ for the different fluids) below which measurements were not possible. The maximum temperature was the upper limit of the temperature bath, $423 \mathrm{~K}$. 

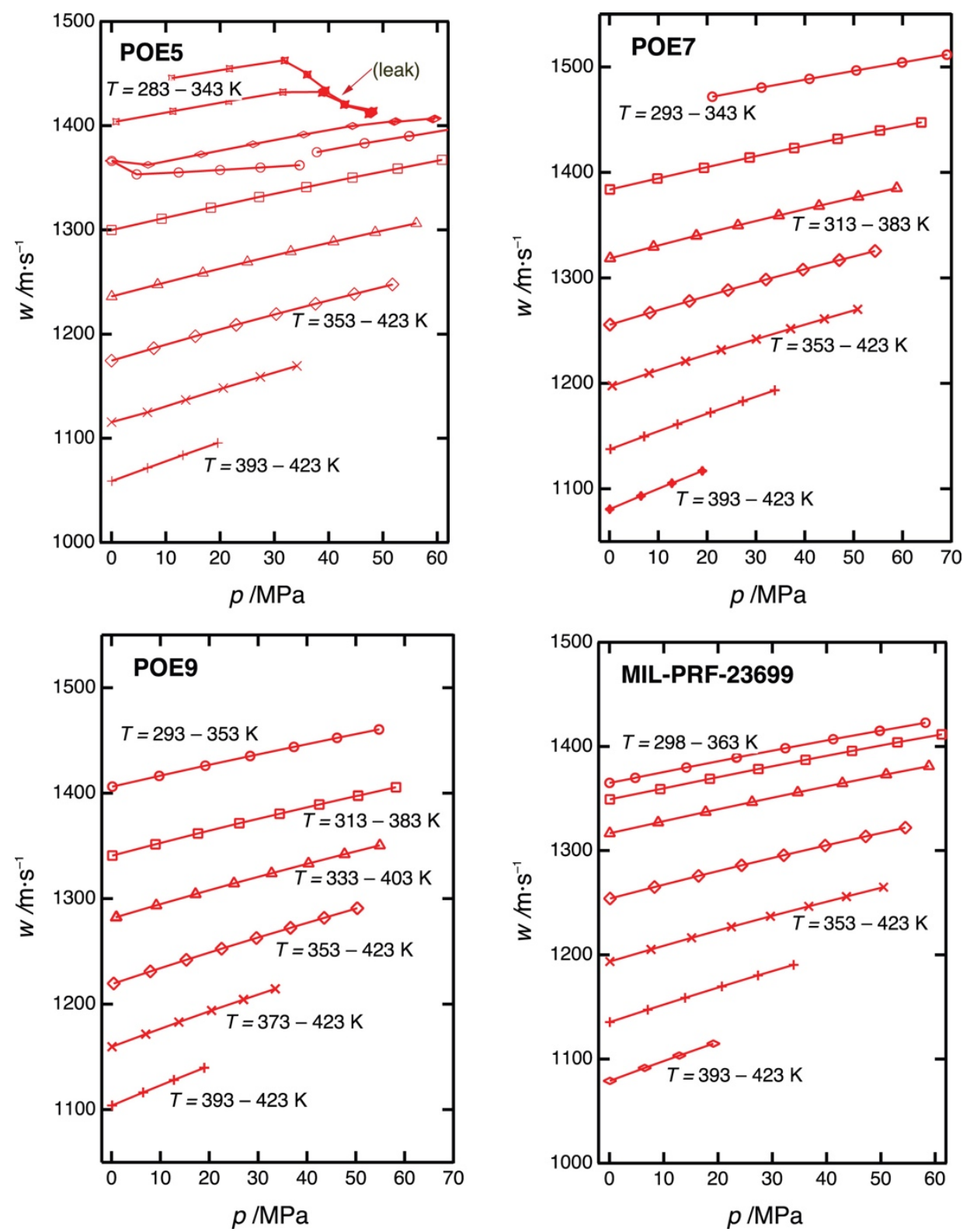

Figure 15: Measurements of the speed of sound for the POE lubricants; the lines connect the points along the various isochores. 


\subsection{Data Tables}

Table 15: Measured Temperature T, Pressure $p$, Speed of Sound $w$, and Relative Combined, Expanded $(k=2)$ State Point Uncertainty in the Speed of Sound $U_{c}$ for POE5; Average Values for the Four Sets of Three Replicate Measurements at Each $(T, p)$ State Point are Given. The Different Isochores Are Separated by Blank Lines.

\begin{tabular}{|c|c|c|c|c|c|c|c|}
\hline$T / K$ & P /MPa & $w / \mathbf{m} \cdot \mathbf{s}^{-1}$ & $U_{\mathrm{c}} / \%$ & $T / \mathbf{K}$ & P /MPa & $w / \mathbf{m} \cdot \mathbf{s}^{-\mathbf{1}}$ & $U_{\mathrm{c}} / \%$ \\
\hline 293.132 & 0.0181 & 1365.476 & 0.0336 & 373.195 & -0.0012 & 1114.547 & 0.0363 \\
\hline 303.139 & 4.6281 & 1352.481 & 0.0336 & 383.204 & 6.6170 & 1123.916 & 0.0356 \\
\hline 313.156 & 12.3615 & 1354.345 & 0.0334 & 393.214 & 13.6633 & 1135.933 & 0.0350 \\
\hline 323.159 & 19.9948 & 1356.712 & 0.0332 & 403.227 & 20.5941 & 1147.366 & 0.0346 \\
\hline 333.162 & 27.4316 & 1359.075 & 0.0331 & 413.244 & 27.4179 & 1158.290 & 0.0342 \\
\hline 343.150 & 34.6454 & 1361.348 & 0.0329 & 423.268 & 34.1569 & 1168.884 & 0.0339 \\
\hline 343.149 & 37.8186 & 1373.896 & 0.0328 & 393.212 & 0.0598 & 1058.043 & 0.0373 \\
\hline 353.181 & 46.6117 & 1382.536 & 0.0327 & 403.225 & 6.6308 & 1070.679 & 0.0364 \\
\hline 363.190 & 54.8395 & 1389.436 & 0.0326 & 413.243 & 13.1395 & 1083.017 & 0.0357 \\
\hline 373.203 & 62.9956 & 1396.435 & 0.0324 & 423.268 & 19.5660 & 1094.871 & 0.0352 \\
\hline 313.151 & 0.0116 & 1298.964 & 0.0341 & 293.132 & 0.0220 & 1365.564 & 0.0336 \\
\hline 323.158 & 9.2331 & 1309.912 & 0.0337 & 303.139 & 6.7167 & 1361.617 & 0.0335 \\
\hline 333.160 & 18.2892 & 1320.514 & 0.0334 & 313.155 & 16.5015 & 1371.845 & 0.0332 \\
\hline 343.150 & 27.2037 & 1330.844 & 0.0331 & 323.159 & 26.0576 & 1381.541 & 0.0329 \\
\hline 353.178 & 35.8680 & 1340.310 & 0.0330 & & & & \\
\hline 363.190 & 44.3935 & 1349.543 & 0.0328 & 333.161 & 35.3991 & 1390.771 & 0.0328 \\
\hline 373.203 & 52.7121 & 1358.259 & 0.0327 & 343.150 & 44.4116 & 1399.142 & 0.0326 \\
\hline \multirow[t]{2}{*}{383.211} & 60.8812 & 1366.682 & 0.0325 & 353.179 & 52.2295 & 1403.441 & 0.0329 \\
\hline & & & & 363.189 & 59.3763 & 1405.913 & 0.0329 \\
\hline 333.161 & 0.0235 & 1235.110 & 0.0347 & & & & \\
\hline 343.147 & 8.4892 & 1246.653 & 0.0342 & 283.156 & 0.8291 & 1403.250 & 0.0334 \\
\hline 353.177 & 16.8423 & 1257.782 & 0.0338 & 293.136 & 11.3041 & 1413.168 & 0.0332 \\
\hline 363.189 & 25.0229 & 1268.384 & 0.0335 & 303.143 & 21.5828 & 1422.759 & 0.0329 \\
\hline 373.203 & 33.0341 & 1278.414 & 0.0333 & 313.159 & 31.5667 & 1431.637 & 0.0327 \\
\hline 383.211 & 40.8868 & 1287.980 & 0.0331 & 323.162 & 38.8582 & 1431.089 & 0.0330 \\
\hline 393.219 & 48.5825 & 1297.095 & 0.0329 & 333.164 & 42.9600 & 1419.635 & 0.0330 \\
\hline 403.230 & 56.1271 & 1305.814 & 0.0328 & 343.151 & 47.7591 & 1411.782 & 0.0329 \\
\hline 353.174 & -0.0041 & 1173.564 & 0.0354 & 283.156 & 11.0513 & 1445.017 & 0.0330 \\
\hline 363.187 & 7.7667 & 1185.506 & 0.0348 & 293.138 & 21.7276 & 1454.206 & 0.0328 \\
\hline 373.200 & 15.4369 & 1197.063 & 0.0344 & 303.142 & 31.9038 & 1462.040 & 0.0329 \\
\hline 383.209 & 22.9599 & 1208.040 & 0.0340 & 313.160 & 36.0455 & 1448.525 & 0.0329 \\
\hline 393.218 & 30.3350 & 1218.414 & 0.0337 & 323.163 & 39.3803 & 1433.108 & 0.0328 \\
\hline 403.230 & 37.5838 & 1228.346 & 0.0335 & 333.165 & 43.0477 & 1419.990 & 0.0328 \\
\hline 413.246 & 44.7169 & 1237.920 & 0.0333 & 343.152 & 48.1996 & 1413.426 & 0.0329 \\
\hline \multirow[t]{2}{*}{423.268} & 51.7545 & 1247.224 & 0.0331 & 343.152 & 47.7037 & 1411.590 & 0.0327 \\
\hline & & & & 343.153 & 47.3341 & 1410.221 & 0.0327 \\
\hline
\end{tabular}


Table 16: Measured Temperature T, Pressure $p$, Speed of Sound $w$, and Relative Combined, Expanded $(k=2)$ State Point Uncertainty in the Speed of Sound $U_{\mathrm{c}}$ for POE7; Average Values for the Four Sets of Three Replicate Measurements at Each $(T, p)$ State Point are Given. The Different Isochores Are Separated by Blank Lines.

\begin{tabular}{|c|c|c|c|c|c|c|c|}
\hline$T / \mathbf{K}$ & P /MPa & $w / \mathbf{m} \cdot \mathbf{s}^{-1}$ & $U_{\mathrm{c}} / \%$ & $T / \mathbf{K}$ & P /MPa & $w / \mathbf{m} \cdot \mathbf{s}^{-1}$ & $U_{c} / \%$ \\
\hline 283.155 & 10.7533 & 1461.887 & 0.0329 & 353.171 & 0.5680 & 1196.837 & 0.0352 \\
\hline 293.135 & 21.0581 & 1471.087 & 0.0327 & 363.184 & 8.1298 & 1208.707 & 0.0347 \\
\hline 303.142 & 31.1244 & 1479.814 & 0.0326 & 373.199 & 15.5794 & 1220.118 & 0.0342 \\
\hline 313.158 & 40.9369 & 1488.077 & 0.0324 & 383.207 & 22.8906 & 1230.966 & 0.0339 \\
\hline 323.161 & 50.5395 & 1496.098 & 0.0323 & 393.217 & 30.0552 & 1241.250 & 0.0336 \\
\hline 333.163 & 59.9084 & 1503.747 & 0.0322 & 403.229 & 37.0907 & 1251.098 & 0.0334 \\
\hline \multirow[t]{2}{*}{343.151} & 69.0824 & 1511.182 & 0.0322 & 413.244 & 43.9979 & 1260.560 & 0.0332 \\
\hline & & & & 423.267 & 50.8041 & 1269.768 & 0.0330 \\
\hline 293.133 & 0.1014 & 1383.068 & 0.0336 & & & & \\
\hline 303.139 & 9.8016 & 1393.456 & 0.0332 & 373.193 & 0.2097 & 1136.740 & 0.0361 \\
\hline 313.158 & 19.3432 & 1403.608 & 0.0330 & 383.203 & 7.1101 & 1148.769 & 0.0354 \\
\hline 323.161 & 28.7051 & 1413.383 & 0.0328 & 393.212 & 13.9457 & 1160.488 & 0.0349 \\
\hline 333.163 & 37.8083 & 1422.467 & 0.0327 & 403.226 & 20.6757 & 1171.670 & 0.0344 \\
\hline 343.150 & 46.6989 & 1431.130 & 0.0325 & 413.243 & 27.3110 & 1182.451 & 0.0341 \\
\hline 353.180 & 55.3789 & 1439.184 & 0.0324 & 423.267 & 33.8513 & 1192.864 & 0.0338 \\
\hline \multirow[t]{2}{*}{363.190} & 63.8466 & 1446.854 & 0.0323 & & & & \\
\hline & & & & 393.212 & 0.0595 & 1079.740 & 0.0371 \\
\hline 313.152 & 0.1386 & 1317.694 & 0.0340 & 403.225 & 6.4499 & 1092.408 & 0.0363 \\
\hline 323.158 & 9.0420 & 1328.609 & 0.0336 & 413.242 & 12.7709 & 1104.602 & 0.0356 \\
\hline 333.160 & 17.7932 & 1339.162 & 0.0334 & 423.265 & 19.0114 & 1116.304 & 0.0351 \\
\hline 343.149 & 26.3088 & 1349.000 & 0.0331 & & & & \\
\hline 353.179 & 34.6727 & 1358.365 & 0.0329 & & & & \\
\hline 363.190 & 42.9057 & 1367.550 & 0.0328 & & & & \\
\hline 373.203 & 50.9466 & 1376.285 & 0.0326 & & & & \\
\hline 383.211 & 58.8016 & 1384.559 & 0.0325 & & & & \\
\hline 333.158 & 0.1071 & 1254.690 & 0.0346 & & & & \\
\hline 343.145 & 8.2742 & 1266.043 & 0.0341 & & & & \\
\hline 353.176 & 16.3619 & 1277.106 & 0.0338 & & & & \\
\hline 363.188 & 24.2793 & 1287.619 & 0.0335 & & & & \\
\hline 373.202 & 32.0324 & 1297.604 & 0.0332 & & & & \\
\hline 383.210 & 39.6262 & 1307.147 & 0.0331 & & & & \\
\hline 393.218 & 47.0703 & 1316.239 & 0.0329 & & & & \\
\hline 403.228 & 54.3619 & 1324.926 & 0.0328 & & & & \\
\hline
\end{tabular}


Table 17: Measured Temperature T, Pressure $p$, Speed of Sound $w$, and Relative Combined, Expanded $(k=2)$ State Point Uncertainty in the Speed of Sound $U_{\mathrm{c}}$ for POE9; Average Values for the Four Sets of Three Replicate Measurements at Each $(T, p)$ State Point are Given. The Different Isochores Are Separated by Blank Lines.

\begin{tabular}{cccc|cccc}
\hline $\boldsymbol{T} / \mathbf{K}$ & $\boldsymbol{P} / \mathbf{M P a}$ & $\boldsymbol{w} / \mathbf{m} \cdot \mathbf{s}^{-\mathbf{1}}$ & $\boldsymbol{U}_{\mathbf{c}} / \mathbf{\%}$ & $\boldsymbol{T} / \mathbf{K}$ & $\boldsymbol{P} / \mathbf{M P a}$ & $\boldsymbol{w} / \mathbf{m} \cdot \mathbf{s}^{-\mathbf{1}}$ & $\boldsymbol{U}_{\mathbf{c}} / \mathbf{\%}$ \\
\hline \hline 293.133 & 0.1710 & 1405.453 & 0.0335 & 353.170 & 0.4437 & 1218.551 & 0.0351 \\
303.138 & 9.7687 & 1415.509 & 0.0332 & 363.183 & 7.9422 & 1230.084 & 0.0346 \\
313.155 & 19.1881 & 1425.255 & 0.0330 & 373.197 & 15.3050 & 1241.121 & 0.0343 \\
323.159 & 28.3704 & 1434.416 & 0.0328 & 383.206 & 22.5374 & 1251.709 & 0.0338 \\
333.161 & 37.3299 & 1443.121 & 0.0326 & 393.213 & 29.6497 & 1261.885 & 0.0336 \\
343.149 & 46.1568 & 1451.746 & 0.0325 & 403.227 & 36.6317 & 1271.643 & 0.0333 \\
353.178 & 54.7552 & 1459.767 & 0.0324 & 413.243 & 43.5317 & 1281.214 & 0.0331 \\
& & & & 423.267 & 50.3031 & 1290.412 & 0.0330 \\
313.151 & 0.1498 & 1339.892 & 0.0340 & & & & \\
323.156 & 9.0089 & 1350.639 & 0.0337 & 373.193 & 0.0919 & 1158.622 & 0.0359 \\
333.160 & 17.6835 & 1360.935 & 0.0333 & 383.204 & 6.9885 & 1170.615 & 0.0353 \\
343.149 & 26.1628 & 1370.704 & 0.0331 & 393.213 & 13.7831 & 1182.159 & 0.0348 \\
353.178 & 34.4059 & 1379.683 & 0.0329 & 403.225 & 20.4689 & 1193.196 & 0.0343 \\
363.189 & 42.5199 & 1388.502 & 0.0327 & 413.241 & 27.0109 & 1203.621 & 0.0340 \\
373.202 & 50.4624 & 1396.960 & 0.0326 & 423.266 & 33.5078 & 1213.914 & 0.0337 \\
383.209 & 58.2317 & 1405.082 & 0.0325 & & & & \\
& & & & 393.209 & 0.1092 & 1103.001 & 0.0368 \\
333.158 & 1.0101 & 1281.574 & 0.0344 & 403.223 & 6.4763 & 1115.404 & 0.0361 \\
343.144 & 9.1619 & 1292.665 & 0.0340 & 413.240 & 12.7660 & 1127.381 & 0.0354 \\
353.175 & 17.1956 & 1303.384 & 0.0336 & 423.265 & 18.9870 & 1138.977 & 0.0349 \\
363.188 & 25.0554 & 1313.568 & 0.0334 & & & & \\
373.201 & 32.7592 & 1323.282 & 0.0332 & & & & \\
383.209 & 40.2988 & 1332.563 & 0.0330 & & & & \\
393.217 & 47.6836 & 1341.455 & 0.0328 & & & & \\
403.228 & 54.9122 & 1349.960 & 0.0327 & & & & \\
& & & & & & & \\
\hline
\end{tabular}


Table 18: Measured Temperature T, Pressure $p$, Speed of Sound $w$, and Relative Combined, Expanded $(k=2)$ State Point Uncertainty in the Speed of Sound $U_{c}$ for MIL-PRF-23699; Average Values for the Four Sets of Three Replicate Measurements at Each $(T, p)$ State Point are Given. The Different Isochores Are Separated by Blank Lines.

\begin{tabular}{|c|c|c|c|c|c|c|c|}
\hline$T / \mathbf{K}$ & $P / \mathbf{M P a}$ & $w / \mathbf{m} \cdot \mathbf{s}^{-\mathbf{1}}$ & $U_{\mathrm{c}} / \%$ & $T / K$ & $P / \mathbf{M P a}$ & $w / \mathbf{m} \cdot \mathbf{s}^{-1}$ & $U_{\mathrm{c}} / \%$ \\
\hline 298.164 & 0.0204 & 1364.191 & 0.0337 & 353.172 & 0.0875 & 1192.607 & 0.0353 \\
\hline 303.141 & 4.7472 & 1369.078 & 0.0336 & 363.185 & 7.6660 & 1204.184 & 0.0347 \\
\hline 313.157 & 14.1680 & 1378.989 & 0.0333 & 373.199 & 15.1458 & 1215.418 & 0.0343 \\
\hline 323.162 & 23.4335 & 1388.665 & 0.0331 & 383.208 & 22.4756 & 1226.067 & 0.0339 \\
\hline 333.164 & 32.3985 & 1397.558 & 0.0329 & 393.217 & 29.6654 & 1236.215 & 0.0336 \\
\hline 343.152 & 41.2161 & 1406.251 & 0.0327 & 403.229 & 36.7231 & 1245.936 & 0.0334 \\
\hline 353.181 & 49.8133 & 1414.365 & 0.0325 & 413.245 & 43.6624 & 1255.313 & 0.0332 \\
\hline 363.190 & 58.2269 & 1422.186 & 0.0324 & 423.267 & 50.5006 & 1264.450 & 0.0330 \\
\hline 303.137 & 0.0914 & 1348.400 & 0.0338 & 373.193 & 0.0908 & 1134.449 & 0.0361 \\
\hline 313.155 & 9.3595 & 1358.215 & 0.0335 & 383.202 & 7.0672 & 1146.398 & 0.0355 \\
\hline 323.159 & 18.4941 & 1368.108 & 0.0332 & 393.214 & 13.9420 & 1157.927 & 0.0349 \\
\hline 333.162 & 27.4283 & 1377.554 & 0.0330 & 403.226 & 20.7130 & 1168.982 & 0.0345 \\
\hline 343.150 & 36.1299 & 1386.451 & 0.0328 & 413.243 & 27.3789 & 1179.592 & 0.0341 \\
\hline 353.179 & 44.6903 & 1395.024 & 0.0327 & 423.268 & 33.9478 & 1189.828 & 0.0338 \\
\hline 363.191 & 53.0624 & 1403.284 & 0.0325 & & & & \\
\hline \multirow[t]{2}{*}{373.203} & 61.2328 & 1411.107 & 0.0324 & 393.213 & 0.0816 & 1078.164 & 0.0371 \\
\hline & & & & 403.226 & 6.5066 & 1090.583 & 0.0362 \\
\hline 313.151 & 0.0590 & 1315.825 & 0.0341 & 413.243 & 12.8606 & 1102.565 & 0.0356 \\
\hline 323.158 & 8.9728 & 1326.154 & 0.0337 & 423.267 & 19.1386 & 1114.092 & 0.0351 \\
\hline 333.161 & 17.7144 & 1336.232 & 0.0334 & & & & \\
\hline 343.149 & 26.2779 & 1345.893 & 0.0331 & & & & \\
\hline 353.179 & 34.6986 & 1355.166 & 0.0329 & & & & \\
\hline 363.190 & 42.9250 & 1363.991 & 0.0328 & & & & \\
\hline 373.204 & 50.9851 & 1372.464 & 0.0326 & & & & \\
\hline 383.211 & 58.8677 & 1380.595 & 0.0325 & & & & \\
\hline 333.158 & 0.4482 & 1254.829 & 0.0346 & & & & \\
\hline 333.158 & 0.1071 & 1253.110 & 0.0346 & & & & \\
\hline 343.148 & 8.3103 & 1264.178 & 0.0341 & & & & \\
\hline 353.177 & 16.4139 & 1274.888 & 0.0338 & & & & \\
\hline 363.189 & 24.3485 & 1285.088 & 0.0335 & & & & \\
\hline 373.203 & 32.1269 & 1294.812 & 0.0333 & & & & \\
\hline 383.210 & 39.7524 & 1304.125 & 0.0331 & & & & \\
\hline 393.219 & 47.2227 & 1313.050 & 0.0329 & & & & \\
\hline 403.229 & 54.5226 & 1321.542 & 0.0328 & & & & \\
\hline
\end{tabular}




\section{Compressed-Liquid Density}

\subsection{Summary}

The compressed-liquid densities of the fluids pentaerythritol tetrapentanoate (POE5), pentaerythritol tetraheptanoate (POE7), pentaerythritol tetranonanoate (POE9), and a fully qualified lubricant (MIL-PRF-23699) have been measured within a temperature range of $270 \mathrm{~K}$ to $470 \mathrm{~K}$ at pressures from $0.5 \mathrm{MPa}$ to $50 \mathrm{MPa}$ with a vibrating-tube densimeter. The high freezing points of all but the POE5 sample resulted in the pressure range of the measurements at lower isotherms (270 K and $290 \mathrm{~K}$ ) being reduced to avoid solidifying the sample. The compressedliquid densities of the lubricants studied ranged from $829 \mathrm{~kg} \cdot \mathrm{m}^{-3}$ to $1063 \mathrm{~kg} \cdot \mathrm{m}^{-3}$. The densities of the four fluids had a fair amount of overlap $\left(\sim 100 \mathrm{~kg} \cdot \mathrm{m}^{-3}\right)$ with the densities of POE5 > MILPRF-23699 > POE7 > POE9 for similar state points. These results are also presented in Outcalt.[41]

\subsection{Experimental Methods}

The apparatus used to measure compressed-liquid densities over the temperature range of $270 \mathrm{~K}$ to $470 \mathrm{~K}$ and to pressures of $50 \mathrm{MPa}$ is described in Outcalt and McLinden.[42] A schematic of the apparatus is illustrated in Figure 16. The heart of the apparatus is a commercial vibrating-tube densimeter; however, several physical and procedural improvements have been implemented beyond that of the commercial instrument operated in a stand-alone mode. The densimeter is housed in a specially designed two-stage thermostat for improved temperature control. The uncertainty in the temperature is $0.03 \mathrm{~K}$ with stability of $5 \mathrm{mK}$ during an isothermal measurement. Pressures are measured with an oscillating quartz crystal pressure transducer with an uncertainty of $5 \mathrm{kPa}$.

The densimeter apparatus has been designed, and software has been written so that the operation and data acquisition are fully automated. Electronically actuated pneumatic valves and a programmable syringe pump are used to move from one pressure to the next and/or flush fresh sample through the system. Operation of the densimeter in this manner allows for measurements to be made 24 hours a day.

Upon receipt, samples were transferred to $300 \mathrm{~mL}$ stainless steel cylinders. Closed cylinders were connected to a vacuum system and then submerged in liquid nitrogen to freeze the contained sample. Once the sample was frozen, the vapor space was evacuated. Once evacuated, the container was sealed, and the sample was heated to facilitate driving air that may have been 
entrained in the liquid, into the vapor space. This procedure was repeated three times for each sample.

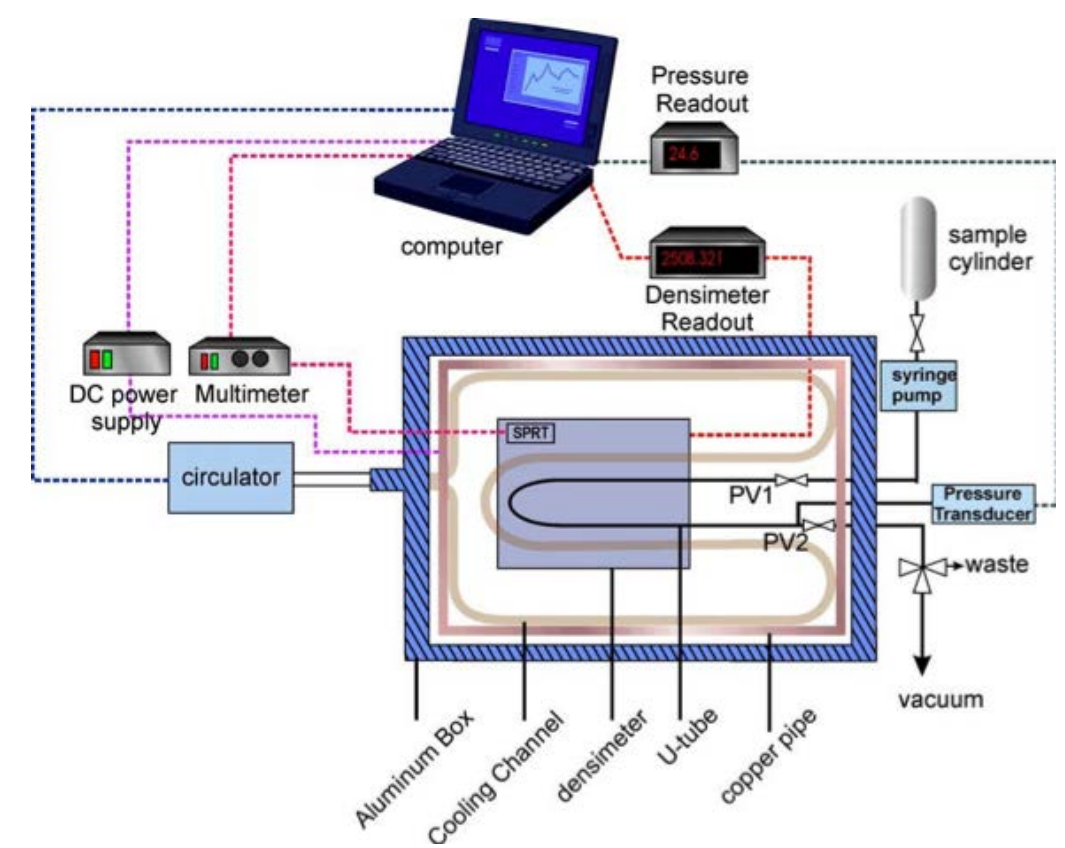

Figure 16: Schematic of the compressed-liquid density apparatus.

\subsection{Determination of Uncertainty}

In previous work, toluene (SRM 211d) and $99.999 \%$ propane had been used as calibration fluids. Due to the higher density ranges of the fluids studied in this work, toluene (SRM 211d) and HPLCgrade water were used as calibration fluids. The toluene and water samples underwent the same degassing procedure described above. Toluene was measured at 142 state points from $270 \mathrm{~K}$ to $470 \mathrm{~K}$ at pressures from $0.5 \mathrm{MPa}$ to $50 \mathrm{MPa}$, and water was measured at 104 points from $290 \mathrm{~K}$ to $470 \mathrm{~K}$ at pressures from $2 \mathrm{MPa}$ to $45 \mathrm{MPa}$. Additionally, the period of oscillation of the U-tube was measured under vacuum from $270 \mathrm{~K}$ to $470 \mathrm{~K}$ in $20 \mathrm{~K}$ increments. The NIST database REFPROP[21, 43, 44] was used to predict the densities of the water and toluene. The calibration data were combined and correlated with the equation of May et al.[45] The correlation represents the toluene and water data to average absolute deviations (AADs) of $0.13 \mathrm{~kg} \cdot \mathrm{m}^{-3}$ and $0.18 \mathrm{~kg} \cdot \mathrm{m}^{-3}$, respectively.

The uncertainty in densities predicted by the equation of state for water increases from less than $0.003 \%$ at temperatures below $423 \mathrm{~K}$ to $0.05 \%$ at temperatures above that in the temperature and pressure range of this work. The overall combined expanded uncertainty ( $k=2,95 \%$ confidence level) in densities presented here was calculated with the root sum of squares method (as described 
in the Guide to the Expression of Uncertainty in Measurement[38]) to be $0.71 \mathrm{~kg} \cdot \mathrm{m}^{-3}$ to $1.24 \mathrm{~kg} \cdot \mathrm{m}^{-3}$. The range of the uncertainty is a result of the range of uncertainty in the equation of state of water. The uncertainty encompasses the uncertainties in the temperature measurement, pressure measurement, equations of state for water and toluene, and the repeatability of the measurements of the lubricant samples.

\subsection{Results}

The compressed-liquid densities of the fluids POE5, POE7, POE9, and MIL-PRF-23699 were measured over the temperature range of $270 \mathrm{~K}$ to $470 \mathrm{~K}$ at pressures from $0.5 \mathrm{MPa}$ to $50 \mathrm{MPa}$. The high freezing points of all but the POE5 sample resulted in the pressure range of the measurements at lower isotherms (270 K and $290 \mathrm{~K}$ ) being reduced to avoid solidifying the samples. Additionally, there were no measurements of the POE9 sample at $270 \mathrm{~K}$ for the same reason. Measurement results are depicted in Figure 17 and reported in Table 19 to Table 22. The compressed-liquid densities of the lubricants studied range from $829 \mathrm{~kg} \cdot \mathrm{m}^{-3}$ to $1063 \mathrm{~kg} \cdot \mathrm{m}^{-3}$, with a relative order between the four fluids of POE9 < POE7 < MIL-PRF-23699 < POE5.
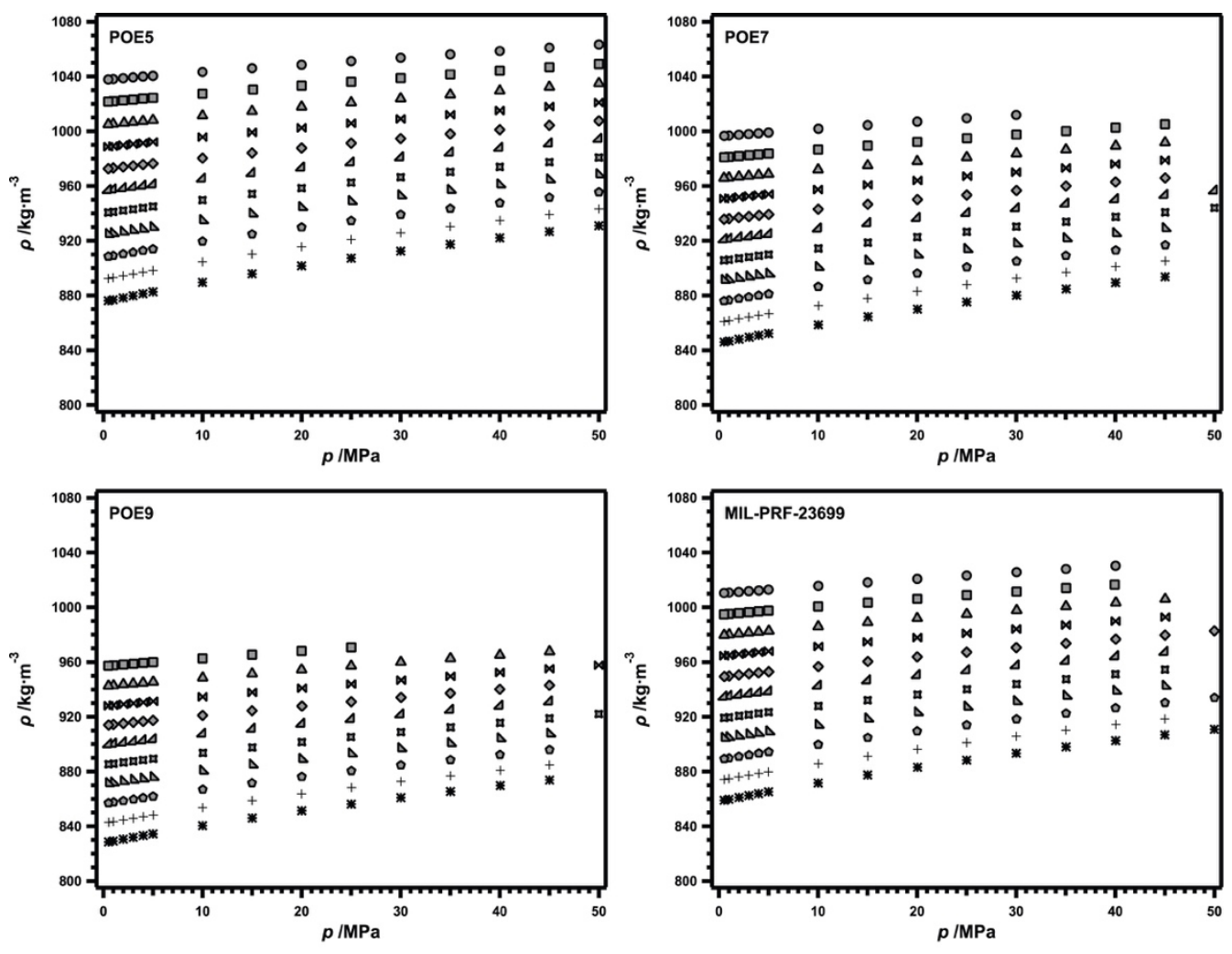

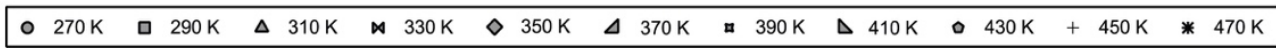

Figure 17: Compressed-liquid density data as a function of pressure measured along isotherms at temperatures from $270 \mathrm{~K}$ to $470 \mathrm{~K}$. 


\subsection{Data Tables}

Table 19: Compressed-Liquid Densities of POE5.

\begin{tabular}{|c|c|c|c|c|c|c|c|c|c|c|c|}
\hline \multicolumn{2}{|c|}{$270 \mathrm{~K}$} & \multicolumn{2}{|c|}{$290 \mathrm{~K}$} & \multicolumn{2}{|c|}{$310 \mathrm{~K}$} & \multicolumn{2}{|c|}{$330 \mathrm{~K}$} & \multicolumn{2}{|c|}{$350 \mathrm{~K}$} & \multicolumn{2}{|c|}{$370 \mathrm{~K}$} \\
\hline$\underset{/ \mathrm{MPa}}{\boldsymbol{p}}$ & $\begin{array}{c}\rho \\
/ \mathbf{k g} \cdot \mathbf{m}^{-3}\end{array}$ & $\begin{array}{c}p \\
/ \mathbf{M P a}\end{array}$ & $\begin{array}{c}\rho \\
/ \mathbf{k g} \cdot \mathbf{m}^{-3}\end{array}$ & $\underset{\text { /MPa }}{p}$ & $\begin{array}{c}\rho \\
/ \mathbf{k g} \cdot \mathbf{m}^{-3}\end{array}$ & $\underset{\text { /MPa }}{p}$ & $\begin{array}{c}\rho \\
/ \mathbf{k g} \cdot \mathbf{m}^{-3}\end{array}$ & $\begin{array}{c}\boldsymbol{p} \\
/ \mathbf{M P a}\end{array}$ & $\begin{array}{c}\rho \\
/ \mathbf{k g} \cdot \mathbf{m}^{-3}\end{array}$ & $\begin{array}{c}p \\
/ \mathrm{MPa}\end{array}$ & $\begin{array}{c}\rho \\
/ \mathbf{k g} \cdot \mathbf{m}^{-3}\end{array}$ \\
\hline 50.01 & $\begin{array}{l}1063.3 \\
\end{array}$ & 49.99 & $\begin{array}{l}1049.2 \\
\end{array}$ & 50.02 & 1035.0 & 50.02 & 1020.9 & 50.01 & 1007.5 & 50.0 & 994.0 \\
\hline 45.02 & 1061.0 & 45.04 & 1046.7 & 45.04 & 1032.3 & 45.03 & 1018.0 & 45.03 & 1004.3 & 45.0 & 990.7 \\
\hline 40.01 & 1058.6 & 40.04 & 1044.2 & 40.01 & 1029.6 & 40.02 & 1015.1 & 40.00 & 1001.1 & 40.0 & 987.4 \\
\hline 35.00 & 1056.2 & 35.02 & 1041.5 & 35.01 & 1026.7 & 35.01 & 1012.1 & 35.03 & 998.0 & 35.0 & 984.0 \\
\hline 30.01 & 1053.7 & 30.01 & 1038.8 & 30.01 & 1023.8 & 30.01 & 1009.0 & 30.02 & 994.6 & 30.0 & 980.5 \\
\hline 25.02 & 1051.2 & 25.02 & 1036.1 & 25.01 & 1020.9 & 25.01 & 1005.8 & 25.02 & 991.2 & 25.0 & 976.8 \\
\hline 20.02 & 1048.6 & 20.03 & 1033.3 & 20.01 & 1017.8 & 20.01 & 1002.5 & 20.04 & 987.7 & 19.9 & 973.0 \\
\hline 15.02 & 1046.0 & 15.05 & 1030.4 & 15.02 & 1014.7 & 15.02 & 999.2 & 15.02 & 984.1 & 15.0 & 969.0 \\
\hline 10.05 & 1043.3 & 10.04 & 1027.4 & 10.00 & 1011.4 & 10.03 & 995.7 & 10.04 & 980.3 & 10.0 & 965.0 \\
\hline 5.01 & 1040.4 & 5.03 & 1024.4 & 5.05 & 1008.1 & 5.04 & 992.1 & 5.04 & 976.4 & 5.02 & 960.7 \\
\hline 4.04 & 1039.9 & 4.00 & 1023.8 & 4.01 & 1007.4 & 4.02 & 991.3 & 4.01 & 975.6 & 4.01 & 959.8 \\
\hline 3.01 & 1039.3 & 3.04 & 1023.1 & 3.02 & 1006.7 & 3.03 & 990.6 & 3.02 & 974.7 & 3.05 & 958.9 \\
\hline 2.01 & 1038.7 & 2.03 & 1022.5 & 2.05 & 1006.0 & 2.01 & 989.8 & 2.00 & 973.9 & 2.02 & 958.0 \\
\hline 1.01 & 1038.1 & 1.02 & 1021.9 & 1.02 & 1005.3 & 1.01 & 989.1 & 1.02 & 973.1 & 1.01 & 957.0 \\
\hline 0.53 & 1037.8 & 0.51 & 1021.6 & 0.53 & 1005.0 & 0.50 & 988.7 & 0.52 & 972.6 & 0.51 & 956.6 \\
\hline \multicolumn{2}{|c|}{$390 \mathrm{~K}$} & \multicolumn{2}{|c|}{$410 \mathrm{~K}$} & \multicolumn{2}{|c|}{$430 \mathrm{~K}$} & \multicolumn{2}{|c|}{$450 \mathrm{~K}$} & \multicolumn{2}{|c|}{$470 \mathrm{~K}$} & & \\
\hline$\underset{/ \mathrm{MPa}}{\boldsymbol{p}}$ & $\begin{array}{c}\rho \\
/ \mathbf{k g} \cdot \mathbf{m}^{-3}\end{array}$ & $\begin{array}{c}p \\
/ \mathbf{M P a}\end{array}$ & $\underset{/ \mathbf{k g} \cdot \mathbf{m}^{-3}}{\rho}$ & $\underset{/ \mathrm{MPa}}{\boldsymbol{p}}$ & $\begin{array}{c}\rho \\
/ \mathbf{k g} \cdot \mathbf{m}^{-3}\end{array}$ & $\underset{\text { /MPa }}{p}$ & $\begin{array}{c}\rho \\
/ \mathbf{k g} \cdot \mathbf{m}^{-3}\end{array}$ & $\begin{array}{c}p \\
/ \mathbf{M P a}\end{array}$ & $\underset{/ \mathbf{k g} \cdot \mathbf{m}^{-3}}{\rho}$ & & \\
\hline 49.99 & 980.8 & 50.01 & 968.1 & 50.00 & 955.5 & 50.05 & 943.2 & 50.03 & 931.0 & & \\
\hline 45.02 & 977.4 & 45.02 & 964.4 & 45.01 & 951.6 & 45.01 & 939.1 & 45.01 & 926.6 & & \\
\hline 40.03 & 973.9 & 40.04 & 960.7 & 40.04 & 947.6 & 40.01 & 934.8 & 40.04 & 922.1 & & \\
\hline 35.02 & 970.2 & 35.01 & 956.7 & 35.03 & 943.5 & 35.01 & 930.3 & 35.02 & 917.4 & & \\
\hline 30.04 & 966.4 & 30.03 & 952.7 & 30.01 & 939.1 & 30.03 & 925.7 & 30.01 & 912.4 & & \\
\hline 25.01 & 962.5 & 25.05 & 948.5 & 25.02 & 934.6 & 25.02 & 920.8 & 25.01 & 907.2 & & \\
\hline 20.01 & 958.4 & 20.01 & 944.1 & 20.03 & 929.8 & 20.00 & 915.6 & 20.01 & 901.6 & & \\
\hline 15.03 & 954.2 & 15.01 & 939.4 & 15.03 & 924.8 & 15.01 & 910.2 & 15.02 & 895.8 & & \\
\hline 10.05 & 949.7 & 10.01 & 934.6 & 10.01 & 919.5 & 10.02 & 904.5 & 10.01 & 889.5 & & \\
\hline 5.02 & 945.0 & 5.03 & 929.5 & 5.04 & 913.9 & 5.04 & 898.3 & 5.02 & 882.7 & & \\
\hline 4.02 & 944.0 & 4.01 & 928.3 & 4.03 & 912.8 & 4.01 & 897.0 & 4.01 & 881.3 & & \\
\hline 3.02 & 943.0 & 3.01 & 927.3 & 3.01 & 911.5 & 3.01 & 895.7 & 3.01 & 879.8 & & \\
\hline 2.03 & 942.1 & 2.03 & 926.2 & 2.01 & 910.3 & 2.01 & 894.4 & 2.02 & 878.3 & & \\
\hline 1.03 & 941.1 & 1.04 & 925.1 & 1.03 & 909.1 & 1.03 & 893.0 & 1.03 & 876.8 & & \\
\hline 0.54 & 940.6 & 0.51 & 924.5 & 0.52 & 908.5 & 0.53 & 892.4 & 0.54 & 876.1 & & \\
\hline
\end{tabular}


Table 20: Compressed-Liquid Densities of POE7.

\begin{tabular}{|c|c|c|c|c|c|c|c|c|c|c|c|}
\hline \multicolumn{2}{|c|}{$270 \mathrm{~K}$} & \multicolumn{2}{|c|}{$290 \mathrm{~K}$} & \multicolumn{2}{|c|}{$310 \mathrm{~K}$} & \multicolumn{2}{|c|}{$330 \mathrm{~K}$} & \multicolumn{2}{|c|}{$350 \mathrm{~K}$} & \multicolumn{2}{|c|}{$370 \mathrm{~K}$} \\
\hline $\begin{array}{c}p \\
/ \mathbf{M P a} \\
\end{array}$ & $\begin{array}{c}\rho \\
/ \mathrm{kg} \cdot \mathbf{m}^{-3}\end{array}$ & $\begin{array}{c}p \\
/ \mathrm{MPa} \\
\end{array}$ & $\begin{array}{c}\rho \\
/ \mathrm{kg} \cdot \mathbf{m}^{-3}\end{array}$ & $\begin{array}{c}p \\
/ \mathbf{M P a}\end{array}$ & $\begin{array}{c}\rho \\
/ \mathbf{k g} \cdot \mathbf{m}^{-3}\end{array}$ & $\begin{array}{c}\boldsymbol{p} \\
/ \mathbf{M P a}\end{array}$ & $\begin{array}{c}\rho \\
/ \mathrm{kg} \cdot \mathbf{m}^{-3}\end{array}$ & $\begin{array}{c}p \\
/ \mathbf{M P a}\end{array}$ & $\begin{array}{c}\rho \\
/ \mathbf{k g} \cdot \mathbf{m}^{-3}\end{array}$ & $\begin{array}{c}p \\
/ \mathbf{M P a}\end{array}$ & $\begin{array}{c}\rho \\
/ \mathbf{k g} \cdot \mathbf{m}^{-3}\end{array}$ \\
\hline & & 50.01 & 1007.5 & 50.03 & 994.4 & 50.03 & 981.5 & 50.02 & 968.8 & 50.0 & 956.2 \\
\hline & & 45.01 & 1005.1 & 45.02 & 991.8 & 45.01 & 978.8 & 45.02 & 965.9 & 45.0 & 953.1 \\
\hline & & 40.01 & 1002.6 & 40.03 & 989.2 & 39.99 & 976.0 & 40.02 & 962.9 & 40.0 & 949.9 \\
\hline & & 35.03 & 1000.1 & 35.02 & 986.5 & 35.01 & 973.1 & 35.02 & 959.9 & 35.0 & 946.7 \\
\hline 29.99 & 1011.9 & 30.02 & 997.5 & 30.02 & 983.7 & 29.99 & 970.1 & 30.03 & 956.7 & 30.0 & 943.3 \\
\hline 25.01 & 1009.5 & 25.03 & 994.9 & 25.01 & 980.9 & 25.06 & 967.2 & 25.01 & 953.4 & 25.0 & 939.8 \\
\hline 20.01 & 1007.0 & 20.01 & 992.2 & 20.01 & 978.0 & 20.04 & 964.0 & 20.03 & 950.1 & 20.0 & 936.2 \\
\hline 15.01 & 1004.4 & 15.01 & 989.4 & 15.01 & 975.0 & 15.02 & 960.8 & 15.02 & 946.6 & 15.0 & 932.5 \\
\hline 10.04 & 1001.8 & 10.01 & 986.6 & 10.01 & 971.9 & 9.99 & 957.4 & 10.02 & 943.0 & 10.0 & 928.5 \\
\hline 5.01 & 999.0 & 5.02 & 983.7 & 5.01 & 968.7 & 5.02 & 954.0 & 5.03 & 939.2 & 5.03 & 924.4 \\
\hline 4.00 & 998.5 & 4.01 & 983.1 & 4.02 & 968.1 & 4.00 & 953.3 & 4.06 & 938.5 & 4.02 & 923.6 \\
\hline 3.01 & 997.9 & 3.06 & 982.5 & 3.01 & 967.4 & 3.02 & 952.6 & 3.03 & 937.7 & 3.04 & 922.7 \\
\hline 2.02 & 997.4 & 2.02 & 981.9 & 2.03 & 966.8 & 2.03 & 951.9 & 2.01 & 936.9 & 2.03 & 921.9 \\
\hline 1.02 & 996.8 & 1.02 & 981.2 & 1.01 & 966.1 & 1.04 & 951.1 & 1.02 & 936.1 & 1.03 & 921.0 \\
\hline 0.54 & 996.6 & 0.55 & 980.9 & 0.51 & 965.8 & 0.53 & 950.8 & 0.52 & 935.7 & 0.53 & 920.6 \\
\hline \multicolumn{2}{|c|}{$390 \mathrm{~K}$} & \multicolumn{2}{|c|}{$410 \mathrm{~K}$} & \multicolumn{2}{|c|}{$430 \mathrm{~K}$} & \multicolumn{2}{|c|}{$450 \mathrm{~K}$} & \multicolumn{2}{|c|}{$470 \mathrm{~K}$} & & \\
\hline $\begin{array}{c}p \\
/ \mathbf{M P a} \\
\end{array}$ & $\begin{array}{c}\rho \\
/ \mathbf{k g} \cdot \mathbf{m}^{-3} \\
\end{array}$ & $\begin{array}{c}p \\
/ \mathrm{MPa} \\
\end{array}$ & $\begin{array}{c}\rho \\
/ \mathbf{k g} \cdot \mathbf{m}^{-3} \\
\end{array}$ & $\begin{array}{c}p \\
/ \mathrm{MPa} \\
\end{array}$ & $\begin{array}{c}\rho \\
/ \mathbf{k g} \cdot \mathbf{m}^{-3}\end{array}$ & $\begin{array}{c}p \\
/ \mathbf{M P a}\end{array}$ & $\begin{array}{c}\rho \\
/ \mathrm{kg} \cdot \mathbf{m}^{-3}\end{array}$ & $\begin{array}{c}p \\
/ \mathrm{MPa}\end{array}$ & $\begin{array}{c}\rho \\
/ \mathbf{k g} \cdot \mathbf{m}^{-3}\end{array}$ & & \\
\hline 50.00 & 944.0 & 50.03 & 932.2 & 50.00 & 920.5 & 50.04 & 909.1 & 50.02 & 897.7 & & \\
\hline 45.03 & 940.7 & 45.02 & 928.7 & 45.03 & 916.8 & 45.03 & 905.2 & 45.02 & 893.6 & & \\
\hline 40.03 & 937.4 & 40.01 & 925.1 & 40.03 & 913.0 & 40.03 & 901.1 & 40.02 & 889.3 & & \\
\hline 35.03 & 933.9 & 35.02 & 921.4 & 35.01 & 909.1 & 35.03 & 896.9 & 35.04 & 884.8 & & \\
\hline 30.02 & 930.3 & 30.03 & 917.6 & 30.03 & 905.0 & 30.04 & 892.5 & 30.04 & 880.1 & & \\
\hline 25.01 & 926.5 & 25.01 & 913.5 & 25.01 & 900.7 & 25.02 & 887.9 & 25.01 & 875.2 & & \\
\hline 20.01 & 922.6 & 20.03 & 909.4 & 20.01 & 896.2 & 20.01 & 883.1 & 20.03 & 870.0 & & \\
\hline 15.03 & 918.6 & 15.02 & 905.0 & 15.02 & 891.4 & 14.99 & 877.9 & 15.05 & 864.5 & & \\
\hline 10.03 & 914.4 & 10.02 & 900.4 & 10.02 & 886.4 & 10.04 & 872.5 & 10.03 & 858.6 & & \\
\hline 5.02 & 909.9 & 5.04 & 895.5 & 5.03 & 881.1 & 5.05 & 866.7 & 5.02 & 852.2 & & \\
\hline 4.04 & 909.0 & 4.01 & 894.5 & 4.02 & 880.0 & 4.01 & 865.5 & 4.02 & 850.9 & & \\
\hline 3.03 & 908.1 & 3.03 & 893.5 & 3.01 & 878.9 & 3.02 & 864.3 & 3.05 & 849.5 & & \\
\hline 2.03 & 907.1 & 2.02 & 892.4 & 2.02 & 877.8 & 2.01 & 863.0 & 2.04 & 848.1 & & \\
\hline 1.01 & 906.1 & 1.01 & 891.4 & 1.01 & 876.6 & 1.03 & 861.7 & 1.05 & 846.7 & & \\
\hline 0.52 & 905.7 & 0.53 & 890.9 & 0.52 & 876.0 & 0.53 & 861.1 & 0.52 & 846.0 & & \\
\hline
\end{tabular}


Table 21: Compressed-Liquid Densities of POE9.

\begin{tabular}{|c|c|c|c|c|c|c|c|c|c|c|c|}
\hline \multicolumn{2}{|c|}{$290 \mathrm{~K}$} & \multicolumn{2}{|c|}{$310 \mathrm{~K}$} & \multicolumn{2}{|c|}{$330 \mathrm{~K}$} & \multicolumn{2}{|c|}{$350 \mathrm{~K}$} & \multicolumn{2}{|c|}{$370 \mathrm{~K}$} & \multicolumn{2}{|c|}{$390 \mathrm{~K}$} \\
\hline $\begin{array}{c}p \\
/ \mathbf{M P a} \\
\end{array}$ & $\begin{array}{c}\rho \\
/ \mathbf{k g} \cdot \mathbf{m}^{-3} \\
\end{array}$ & $\begin{array}{c}p \\
/ \mathbf{M P a} \\
\end{array}$ & $\begin{array}{c}\rho \\
/ \mathbf{k g} \cdot \mathbf{m}^{-3}\end{array}$ & $\begin{array}{c}\boldsymbol{p} \\
/ \mathbf{M P a}\end{array}$ & $\begin{array}{c}\rho \\
/ \mathbf{k g} \cdot \mathbf{m}^{-3}\end{array}$ & $\begin{array}{c}p \\
/ \mathbf{M P a}\end{array}$ & $\begin{array}{c}\rho \\
/ \mathbf{k g} \cdot \mathbf{m}^{-3}\end{array}$ & $\begin{array}{c}p \\
/ \mathbf{M P a}\end{array}$ & $\begin{array}{c}\rho \\
/ \mathbf{k g} \cdot \mathbf{m}^{-3}\end{array}$ & $\begin{array}{c}\boldsymbol{p} \\
/ \mathrm{MPa}\end{array}$ & $\begin{array}{c}\rho \\
/ \mathbf{k g} \cdot \mathbf{m}^{-3}\end{array}$ \\
\hline & & 50.02 & 970.2 & 49.99 & 957.7 & 50.08 & 945.8 & 50.01 & 933.7 & 49.99 & 9922.0 \\
\hline & & 45.01 & 967.7 & 45.01 & 955.1 & 45.00 & 943.0 & 45.02 & 930.8 & 45.01 & 918.8 \\
\hline & & 40.01 & 965.1 & 40.00 & 952.4 & 40.00 & 940.1 & 40.01 & 927.7 & 40.02 & 915.6 \\
\hline & & 35.01 & 962.6 & 35.02 & 949.6 & 35.01 & 937.2 & 35.03 & 924.6 & 35.03 & 912.3 \\
\hline & & 30.02 & 959.9 & 30.04 & 946.8 & 30.03 & 934.1 & 30.02 & 921.3 & 30.03 & 908.8 \\
\hline 25.01 & 970.7 & 25.00 & 957.2 & 25.02 & 943.9 & 25.01 & 931.0 & 25.01 & 918.0 & 24.99 & 905.2 \\
\hline 20.00 & 968.1 & 20.00 & 954.4 & 20.05 & 940.9 & 20.04 & 927.8 & 20.02 & 914.5 & 20.03 & 901.5 \\
\hline 15.00 & 965.4 & 15.02 & 951.5 & 15.02 & 937.8 & 15.01 & 924.4 & 15.03 & 910.9 & 15.02 & 897.6 \\
\hline 10.01 & 962.7 & 10.02 & 948.5 & 10.01 & 934.6 & 10.01 & 921.0 & 10.03 & 907.2 & 10.05 & 893.6 \\
\hline 5.01 & 959.9 & 5.03 & 945.5 & 5.03 & 931.3 & 5.01 & 917.4 & 5.03 & 903.2 & 5.03 & 889.3 \\
\hline 4.04 & 959.3 & 4.01 & 944.8 & 4.03 & 930.6 & 4.02 & 916.6 & 4.00 & 902.4 & 4.01 & 888.4 \\
\hline 3.05 & 958.7 & 3.01 & 944.2 & 3.04 & 929.9 & 3.02 & 915.9 & 3.03 & 901.6 & 3.03 & 887.5 \\
\hline 2.04 & 958.2 & 2.02 & 943.6 & 2.04 & 929.2 & 2.02 & 915.1 & 2.02 & 900.8 & 2.04 & 886.6 \\
\hline 1.02 & 957.6 & 1.03 & 942.9 & 1.02 & 928.5 & 1.02 & 914.4 & 1.04 & 899.9 & 1.03 & 885.7 \\
\hline 0.51 & 957.3 & 0.51 & 942.6 & 0.52 & 928.1 & 0.53 & 914.0 & 0.53 & 899.5 & 0.53 & 885.3 \\
\hline \multicolumn{2}{|c|}{$410 \mathrm{~K}$} & \multicolumn{2}{|c|}{$430 \mathrm{~K}$} & \multicolumn{2}{|c|}{$450 \mathrm{~K}$} & \multicolumn{2}{|c|}{$470 \mathrm{~K}$} & & & & \\
\hline$\underset{/ \mathrm{MPa}}{\boldsymbol{p}}$ & $\underset{/ \mathbf{k g} \cdot \mathbf{m}^{-3}}{\rho}$ & $\underset{\text { /MPa }}{p}$ & $\begin{array}{c}\rho \\
/ \mathbf{k g} \cdot \mathbf{m}^{-3}\end{array}$ & $\underset{\text { /MPa }}{p}$ & $\begin{array}{c}\rho \\
/ \mathbf{k g} \cdot \mathbf{m}^{-3}\end{array}$ & $\underset{/ \mathrm{MPa}}{p}$ & $\begin{array}{c}\rho \\
/ \mathbf{k g} \cdot \mathbf{m}^{-3}\end{array}$ & & & & \\
\hline 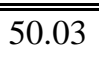 & 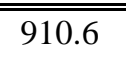 & 50.02 & $\begin{array}{l}899.4 \\
\end{array}$ & 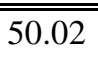 & 888.5 & 50.01 & $\begin{array}{l}877.7 \\
\end{array}$ & & & & \\
\hline 45.02 & 907.3 & 45.02 & 895.9 & 45.02 & 884.8 & 45.02 & 873.8 & & & & \\
\hline 40.01 & 903.8 & 40.02 & 892.3 & 40.04 & 880.9 & 40.04 & 869.7 & & & & \\
\hline 35.02 & 900.3 & 35.02 & 888.5 & 35.01 & 876.9 & 35.02 & 865.4 & & & & \\
\hline 30.00 & 896.6 & 30.01 & 884.6 & 30.02 & 872.7 & 30.01 & 860.9 & & & & \\
\hline 25.01 & 892.8 & 25.02 & 880.5 & 25.04 & 868.3 & 25.02 & 856.2 & & & & \\
\hline 20.02 & 888.8 & 20.02 & 876.2 & 20.00 & 863.7 & 20.03 & 851.3 & & & & \\
\hline 15.01 & 884.6 & 15.00 & 871.6 & 15.02 & 858.8 & 15.02 & 846.0 & & & & \\
\hline 10.00 & 880.2 & 10.03 & 866.9 & 10.02 & 853.6 & 10.01 & 840.4 & & & & \\
\hline 5.02 & 875.5 & 5.01 & 861.8 & 5.04 & 848.2 & 5.00 & 834.4 & & & & \\
\hline 4.02 & 874.5 & 4.01 & 860.8 & 4.04 & 847.0 & 4.03 & 833.2 & & & & \\
\hline 3.03 & 873.6 & 3.04 & 859.7 & 3.01 & 845.8 & 3.01 & 831.9 & & & & \\
\hline 2.01 & 872.6 & 2.02 & 858.6 & 2.02 & 844.6 & 2.01 & 830.6 & & & & \\
\hline 1.02 & 871.6 & 1.04 & 857.6 & 1.02 & 843.4 & 1.01 & 829.2 & & & & \\
\hline 0.52 & 871.1 & 0.51 & 857.0 & 0.54 & 842.9 & 0.53 & 828.6 & & & & \\
\hline
\end{tabular}


Table 22: Compressed-Liquid Densities of MIL-PRF-23699.

\begin{tabular}{|c|c|c|c|c|c|c|c|c|c|c|c|}
\hline \multicolumn{2}{|c|}{$270 \mathrm{~K}$} & \multicolumn{2}{|c|}{$290 \mathrm{~K}$} & \multicolumn{2}{|c|}{$310 \mathrm{~K}$} & \multicolumn{2}{|c|}{$330 \mathrm{~K}$} & \multicolumn{2}{|c|}{$350 \mathrm{~K}$} & \multicolumn{2}{|c|}{$370 \mathrm{~K}$} \\
\hline $\begin{array}{c}p \\
/ \mathbf{M P a}\end{array}$ & $\begin{array}{c}\rho \\
/ \mathbf{k g} \cdot \mathbf{m}^{-3}\end{array}$ & $\begin{array}{c}p \\
/ \mathbf{M P a}\end{array}$ & $\begin{array}{c}\rho \\
/ \mathbf{k g} \cdot \mathbf{m}^{-3}\end{array}$ & $\begin{array}{c}p \\
/ \mathbf{M P a}\end{array}$ & $\begin{array}{c}\rho \\
/ \mathbf{k g} \cdot \mathbf{m}^{-3}\end{array}$ & $\begin{array}{c}p \\
/ \mathbf{M P a}\end{array}$ & $\begin{array}{c}\rho \\
/ \mathbf{k g} \cdot \mathbf{m}^{-3}\end{array}$ & $\begin{array}{c}p \\
/ \mathbf{M P a}\end{array}$ & $\begin{array}{c}\rho \\
/ \mathbf{k g} \cdot \mathbf{m}^{-3}\end{array}$ & $\begin{array}{c}p \\
/ \mathrm{MPa}\end{array}$ & $\begin{array}{c}\rho \\
/ \mathbf{k g} \cdot \mathbf{m}^{-3}\end{array}$ \\
\hline & & & & 50.01 & 1008.6 & 50.00 & 995.5 & 49.99 & 982.7 & 50.04 & 970.1 \\
\hline & & & & 45.03 & 1006.0 & 45.04 & 992.8 & 45.01 & 979.7 & 45.01 & 967.0 \\
\hline 40.02 & 1030.3 & 39.94 & 1016.6 & 40.04 & 1003.3 & 40.01 & 989.9 & 40.04 & 976.7 & 40.03 & 963.8 \\
\hline 35.01 & 1028.0 & 35.02 & 1014.1 & 35.02 & 1000.6 & 35.02 & 987.0 & 35.02 & 973.6 & 35.03 & 960.5 \\
\hline 30.03 & 1025.6 & 30.04 & 1011.5 & 30.04 & 997.8 & 30.01 & 984.1 & 30.00 & 970.5 & 30.01 & 957.1 \\
\hline 25.01 & 1023.2 & 25.01 & 1008.9 & 25.01 & 995.0 & 25.01 & 981.0 & 25.01 & 967.2 & 25.01 & 953.6 \\
\hline 20.01 & 1020.7 & 20.01 & 1006.2 & 20.01 & 992.0 & 20.02 & 977.9 & 20.01 & 963.8 & 20.02 & 950.0 \\
\hline 15.01 & 1018.2 & 15.03 & 1003.4 & 15.02 & 989.0 & 15.01 & 974.7 & 15.03 & 960.4 & 15.01 & 946.2 \\
\hline 10.03 & 1015.6 & 10.01 & 1000.6 & 10.02 & 985.9 & 10.04 & 971.4 & 10.03 & 956.7 & 10.02 & 942.3 \\
\hline 5.01 & 1012.9 & 5.00 & 997.6 & 5.03 & 982.7 & 5.01 & 967.9 & 5.03 & 952.9 & 5.02 & 938.2 \\
\hline 4.04 & 1012.3 & 4.01 & 997.0 & 4.02 & 982.0 & 4.02 & 967.2 & 4.01 & 952.1 & 4.00 & 937.3 \\
\hline 3.01 & 1011.8 & 3.02 & 996.4 & 3.01 & 981.4 & 3.01 & 966.4 & 3.03 & 951.4 & 3.02 & 936.5 \\
\hline 2.01 & 1011.2 & 2.03 & 995.8 & 2.01 & 980.7 & 2.02 & 965.7 & 2.02 & 950.6 & 2.01 & 935.6 \\
\hline 1.03 & 1010.7 & 1.03 & 995.2 & 1.04 & 980.1 & 1.03 & 965.0 & 1.03 & 949.8 & 1.02 & 934.7 \\
\hline 0.52 & 1010.4 & 0.52 & 994.9 & 0.53 & 979.7 & 0.53 & 964.6 & 0.55 & 949.4 & 0.52 & 934.3 \\
\hline \multicolumn{2}{|c|}{$390 \mathrm{~K}$} & \multicolumn{2}{|c|}{$410 \mathrm{~K}$} & \multicolumn{2}{|c|}{$430 \mathrm{~K}$} & \multicolumn{2}{|c|}{$450 \mathrm{~K}$} & \multicolumn{2}{|c|}{$470 \mathrm{~K}$} & & \\
\hline $\begin{array}{c}\boldsymbol{p} \\
/ \mathbf{M P a}\end{array}$ & $\begin{array}{c}\rho \\
/ \mathbf{k g} \cdot \mathbf{m}^{-3}\end{array}$ & $\begin{array}{c}\boldsymbol{p} \\
/ \mathrm{MPa}\end{array}$ & $\begin{array}{c}\rho \\
/ \mathbf{k g} \cdot \mathbf{m}^{-3}\end{array}$ & $\begin{array}{c}p \\
/ \mathbf{M P a}\end{array}$ & $\begin{array}{c}\rho \\
/ \mathbf{k g} \cdot \mathbf{m}^{-3}\end{array}$ & $\begin{array}{c}\boldsymbol{p} \\
/ \mathbf{M P a}\end{array}$ & $\begin{array}{c}\rho \\
/ \mathrm{kg} \cdot \mathrm{m}^{-3}\end{array}$ & $\begin{array}{c}p \\
/ \mathbf{M P a}\end{array}$ & $\begin{array}{c}\rho \\
/ \mathbf{k g} \cdot \mathbf{m}^{-3}\end{array}$ & & \\
\hline 50.02 & 957.7 & 50.01 & 945.7 & 50.00 & 933.9 & 50.02 & 922.4 & 49.99 & 910.9 & & \\
\hline 45.01 & 954.5 & 45.02 & 942.2 & 45.01 & 930.3 & 44.99 & 918.4 & 44.99 & 906.8 & & \\
\hline 40.01 & 951.1 & 40.03 & 938.6 & 40.02 & 926.4 & 40.06 & 914.4 & 40.03 & 902.5 & & \\
\hline 35.03 & 947.5 & 35.02 & 934.9 & 35.03 & 922.5 & 35.01 & 910.2 & 35.02 & 898.0 & & \\
\hline 30.03 & 943.9 & 30.01 & 931.0 & 30.03 & 918.3 & 30.01 & 905.7 & 30.01 & 893.3 & & \\
\hline 25.03 & 940.1 & 25.02 & 927.0 & 25.00 & 914.0 & 25.00 & 901.1 & 25.00 & 888.3 & & \\
\hline 20.03 & 936.2 & 20.02 & 922.8 & 20.00 & 909.5 & 20.03 & 896.3 & 20.04 & 883.1 & & \\
\hline 15.01 & 932.1 & 15.01 & 918.3 & 15.02 & 904.7 & 15.02 & 891.1 & 15.01 & 877.5 & & \\
\hline 10.03 & 927.9 & 10.01 & 913.7 & 10.02 & 899.7 & 10.03 & 885.7 & 10.01 & 871.6 & & \\
\hline 5.02 & 923.4 & 5.03 & 908.9 & 5.02 & 894.4 & 5.00 & 879.8 & 5.01 & 865.2 & & \\
\hline 4.01 & 922.5 & 4.00 & 907.8 & 4.04 & 893.3 & 4.03 & 878.6 & 4.01 & 863.8 & & \\
\hline 3.01 & 921.5 & 3.04 & 906.8 & 3.03 & 892.1 & 3.01 & 877.3 & 3.03 & 862.5 & & \\
\hline 2.04 & 920.6 & 2.01 & 905.8 & 2.02 & 891.0 & 2.01 & 876.1 & 2.04 & 861.1 & & \\
\hline 1.03 & 919.6 & 1.01 & 904.7 & 1.02 & 889.8 & 1.02 & 874.8 & 1.03 & 859.7 & & \\
\hline 0.53 & 919.2 & 0.53 & 904.2 & 0.53 & 889.3 & 0.53 & 874.2 & 0.54 & 859.0 & & \\
\hline
\end{tabular}




\section{Ambient-Pressure Density and Speed of Sound}

\subsection{Summary}

Measured densities and speeds of sound, calculated adiabatic compressibilities, and all associated expanded uncertainties are reported over the combined temperature range $278 \mathrm{~K}$ to $343 \mathrm{~K}$ and at ambient pressure ( $83 \mathrm{kPa}$ ). Densities range from an overall low of $918.32 \mathrm{~kg} \cdot \mathrm{m}^{-3}$ to an overall high of $1030.29 \mathrm{~kg} \cdot \mathrm{m}^{-3}$, with a relative order between the four fluids of POE9 < POE7 < MILPRF-23699 < POE5 and differences that range from $6.5 \%$ (at $343.15 \mathrm{~K}$ ) to $6.7 \%$ (at $283.15 \mathrm{~K}$ ). Sound speeds range from an overall low of $1205.2 \mathrm{~m} \cdot \mathrm{s}^{-1}$ to an overall high of $1438.9 \mathrm{~m} \cdot \mathrm{s}^{-1}$, with a relative order of POE5 < MIL-PRF-23699 < POE7 < POE9 and differences that range from $3.4 \%$ (at $343.15 \mathrm{~K}$ ) to $2.8 \%$ (at $283.15 \mathrm{~K}$ ). Adiabatic compressibilities range from an overall low of 482.9 $\mathrm{TPa}^{-1}$ to an overall high of $709.4 \mathrm{TPa}^{-1}$, with POE7 exhibiting the highest overall values and POE5 and MIL-PRF-23699 exhibiting the lowest values, depending on temperature. Overall differences in adiabatic compressibilities range from $1.2 \%$ at $343.15 \mathrm{~K}$ to $1.6 \%$ at $283.15 \mathrm{~K}$. These results are also presented in Fortin.[25]

\subsection{Experimental Methods}

A commercial density $(\rho)$ and sound speed ( $w$ ) analyzer (see Figure 18) was used to simultaneously measure these two properties over the combined temperature range $278 \mathrm{~K}$ to $343 \mathrm{~K}$ and at ambient pressure ( $83 \mathrm{kPa}$ in Boulder, $\mathrm{CO}$ ). The instrument contains two measurement cells in series: a sound speed cell that measures the propagation time of $\sim 3 \mathrm{MHz}$ sound waves through a liquidfilled cavity and a density cell that utilizes a vibrating-tube densimeter constructed of borosilicate glass. Both cells are housed in a thermostated copper block whose temperature is controlled between $278 \mathrm{~K}$ and $343 \mathrm{~K}$ with a combination of thermoelectric Peltier elements and an integrated Pt-100 resistance thermometer. Measurements of air and water performed at $293 \mathrm{~K}, 313 \mathrm{~K}$, and $333 \mathrm{~K}$ are required to adjust the apparatus constants in the instrument's working equations for both sound speed and density; this is referred to as an adjustment procedure. Additionally, calibration measurements are performed to verify the instrument's performance before and after sample measurements. These involve measuring water and toluene standard reference material (SRM) 211d every $5 \mathrm{~K}$ from $343 \mathrm{~K}$ to $278 \mathrm{~K}$. Additional details about the density and sound speed analyzer can be found in Laesecke et al.,[46] Fortin,[47] and Fortin et al.[48] 


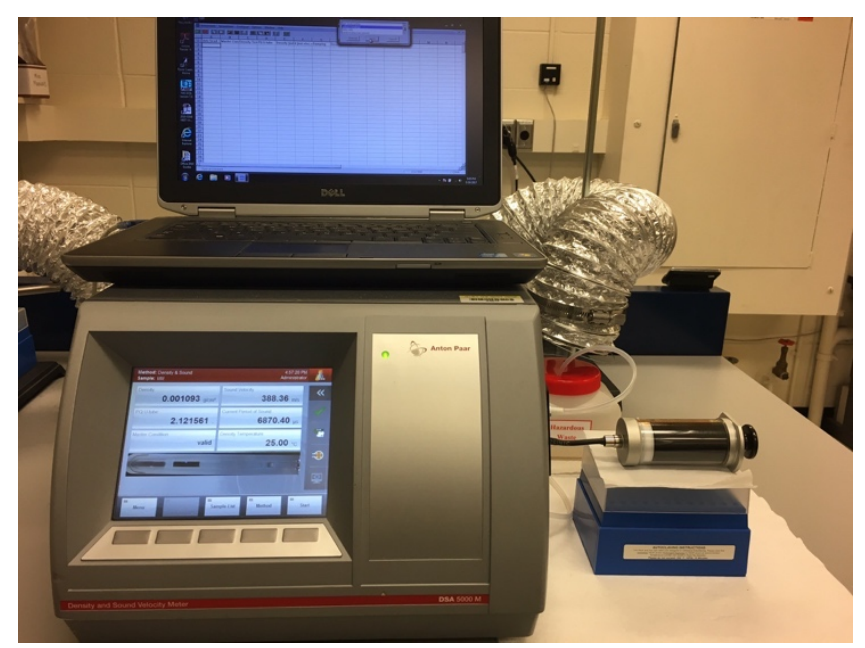

Figure 18: Density and sound speed analyzer.

Prior to measurements, each sample was degassed via repeat "freeze-pump-thaw" cycles. The sample was transferred to a glass bulb and the bulb was then attached to a vacuum system (see Figure 19). The first part of the cycle (the "freeze") is achieved by submerging the bulb in a Dewar of liquid nitrogen for several minutes. The vapor space over the still frozen sample is then evacuated by opening the bulb to the vacuum line (the "pump”). Once evacuated, the bulb is heated to facilitate driving any remaining dissolved impurities into the vapor space (the "thaw"). This cycle was repeated a minimum of five times to ensure complete removal of dissolved gases.

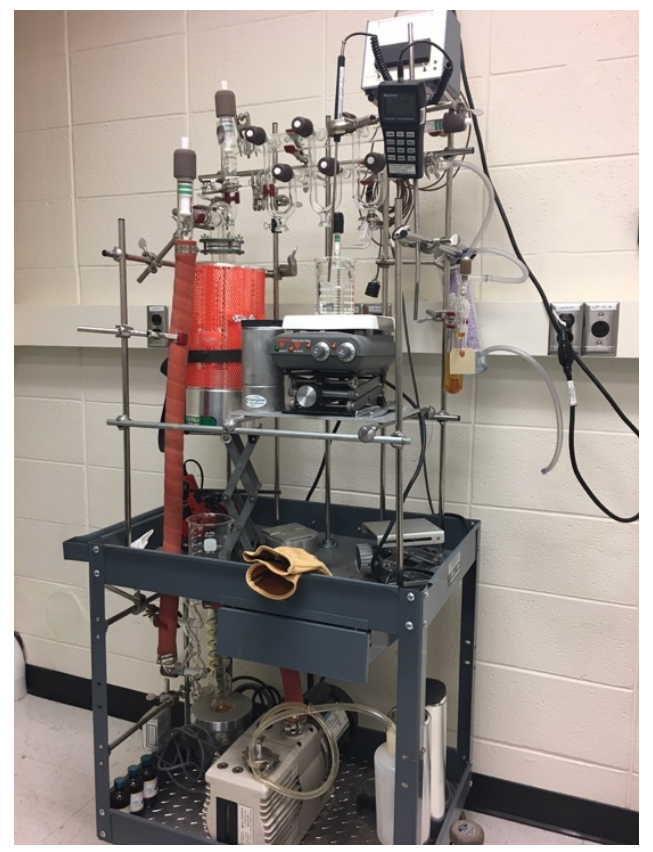

Figure 19: Vacuum system for degassing samples. 
Freshly degassed sample was then drawn up into a gas-tight $50 \mathrm{~mL}$ glass syringe, which remained attached to the instrument inlet for the duration of the measurements of a given sample (see Figure 18). Approximately $3 \mathrm{~mL}$ of sample was injected into the instrument (enough to fill both cells) and measurements were made via programmed scans between the maximum and minimum temperature in $5 \mathrm{~K}$ decrements. For all samples, the maximum temperature was $343 \mathrm{~K}$, and for all but POE9, the minimum temperature was $278 \mathrm{~K}$. POE9 could only be measured to $283 \mathrm{~K}$; below that, the sample solidified. Five to seven measurement scans were performed for each of the four samples with a fresh aliquot of sample fluid injected into the instrument prior to the start of each scan. In between samples, the measurement cells were thoroughly cleaned and dried. Additionally, since these samples proved difficult to clean out of the instrument, the first run of each new sample was discarded during analysis to minimize any chances of cross-contamination between samples.

\subsection{Results and Uncertainty Analysis}

Measurement results are presented in Table 23 and Table 24 for density $(\bar{\rho})$ and speed of sound $(\bar{w})$, respectively. The tabulated values represent the average of three to six replicate measurement scans, depending on the sample. Also included in the tables are the associated absolute and relative expanded uncertainty estimates $(U(\bar{\rho})$ and $U(\bar{w}))$. Using density as an example, the expanded uncertainty is calculated using the expression

$$
U(\bar{\rho})=t_{95}\left(d f_{\rho}\right) \cdot u(\bar{\rho})
$$

where the coverage factor, $t_{95}\left(d f_{\rho}\right)$, is taken from the $t$-distribution for $d f_{\rho}$ degrees of freedom and a $95 \%$ level of confidence, and $u(\bar{\rho})$ is the combined standard uncertainty for the averaged density measurements.[38] The combined standard uncertainty for density and speed of sound include contributions for the standard deviation of replicate temperature scans, instrument resolution, instrument calibration, as well as the uncertainty in measured temperature and pressure. Additional details concerning the uncertainty analysis calculations can be found in Fortin et al.[48] For the density data (Table 23), reported absolute expanded uncertainties (1.65 to $1.66 \mathrm{~kg} \cdot \mathrm{m}^{-3}$ ) correspond to relative expanded uncertainties of $0.12 \%$ to $0.18 \%$. For the sound speed data (Table 24), reported absolute expanded uncertainties $\left(1.7 \mathrm{~m} \cdot \mathrm{s}^{-1}\right)$ correspond to relative expanded uncertainties of $0.1 \%$ to $0.2 \%$.

To facilitate comparisons among the four samples, the averaged densities shown in Table 23 are plotted as a function of temperature in Figure 20. POE5 exhibits the highest densities, ranging from $977.88 \mathrm{~kg} \cdot \mathrm{m}^{-3}$ at $343.15 \mathrm{~K}$ to $1030.29 \mathrm{~kg} \cdot \mathrm{m}^{-3}$ at $278.15 \mathrm{~K}$. POE9 exhibits the lowest densities, 
ranging from $918.32 \mathrm{~kg} \cdot \mathrm{m}^{-3}$ at $343.15 \mathrm{~K}$ to $961.49 \mathrm{~kg} \cdot \mathrm{m}^{-3}$ at $283.15 \mathrm{~K}$. The difference in density between these two fluids is $6.5 \%$ at $343.15 \mathrm{~K}$ and $6.7 \%$ at $283 \mathrm{~K}$. MIL-PRF-23699, the fully qualified lubricant, exhibits densities most similar to POE7. MIL-PRF-23699 has densities ranging from 954.42 to $1003.53 \mathrm{~kg} \cdot \mathrm{m}^{-3}$, while POE7 has densities ranging from 940.20 to $989.09 \mathrm{~kg} \cdot \mathrm{m}^{-3}$, a difference of approximately $1.5 \%$.

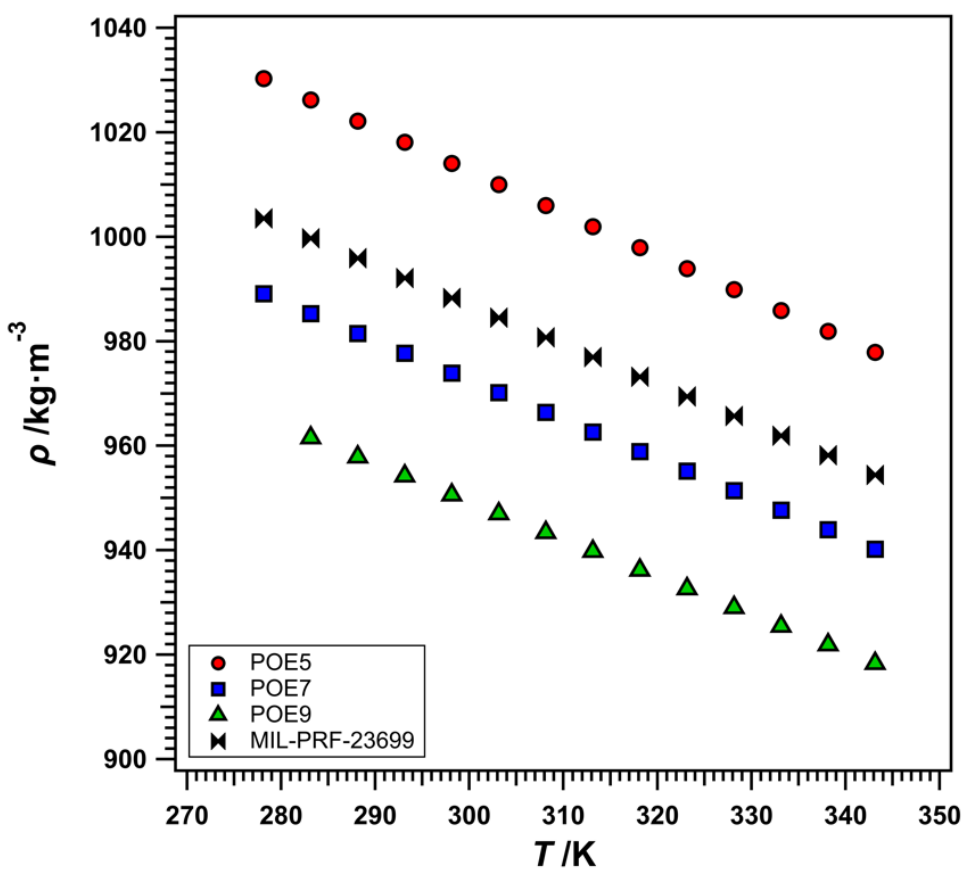

Figure 20: Ambient-pressure density measurements plotted as a function of temperature.

The averaged speeds of sound shown in Table 24 are plotted as a function of temperature in Figure 21. The trend in sound speeds is the opposite of that observed for density. POE9 exhibits the highest sound speeds, ranging from $1246.4 \mathrm{~m} \cdot \mathrm{s}^{-1}$ at $343.15 \mathrm{~K}$ to $1438.9 \mathrm{~m} \cdot \mathrm{s}^{-1}$ at $283.15 \mathrm{~K}$, while POE5 exhibits the lowest, ranging from 1205.2 to $1400.3 \mathrm{~m} \cdot \mathrm{s}^{-1}$ over the same temperature range. The corresponding difference in sound speeds for these two fluids ranges from $3.4 \%$ at $343.15 \mathrm{~K}$ to $2.8 \%$ at $283.15 \mathrm{~K}$. Similar to density, MIL-PRF-23699 exhibits sound speeds that are most similar to POE 7 with differences that range from $0.1 \%$ to $0.2 \%$ at $343.15 \mathrm{~K}$ and $278.15 \mathrm{~K}$, respectively. 


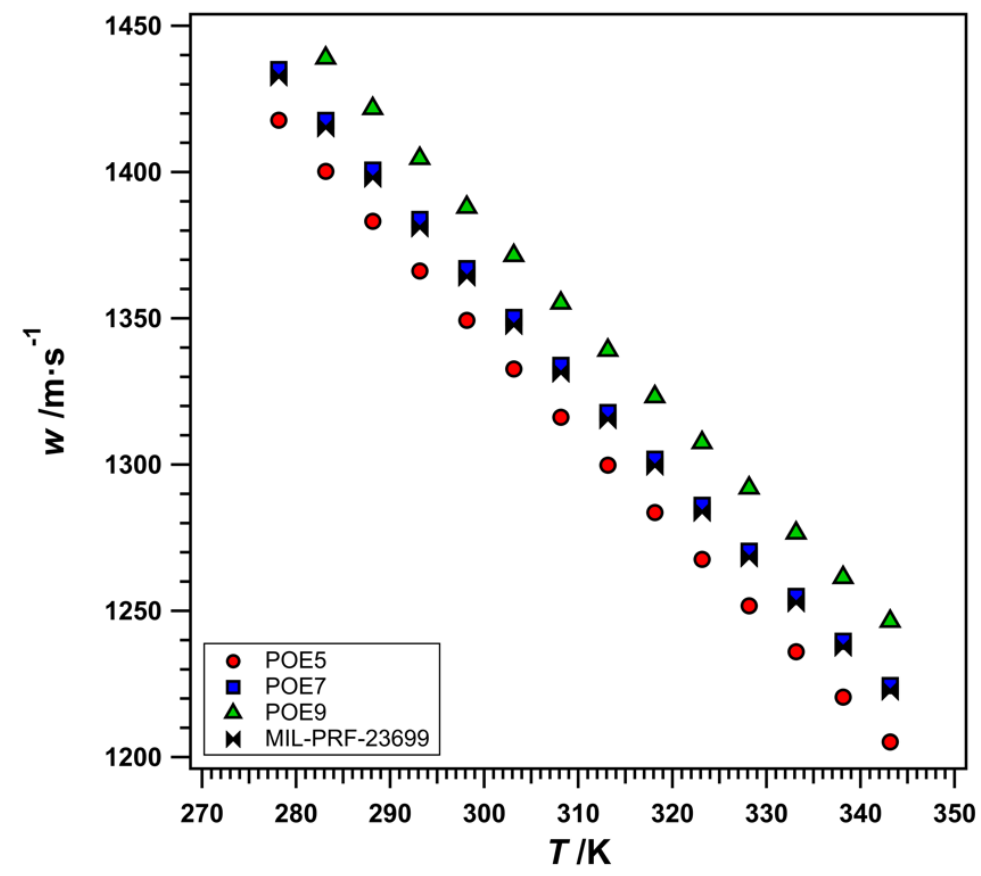

Figure 21: Ambient-pressure speed of sound measurements plotted as a function of temperature.

In addition to densities and speeds of sound, averaged adiabatic compressibilities $\left(\bar{\kappa}_{S}\right)$ were calculated from measured quantities via the relation

$$
\kappa_{s}=\frac{1}{\rho \cdot w^{2}},
$$

where $\rho$ is density, $w$ is speed of sound, and the subscript $s$ indicates "at constant entropy." Results for all four fluids are reported in Table 25 and shown plotted as a function of temperature in Figure 22. Also included in Table 25 are corresponding absolute and relative expanded uncertainties $\left(U\left(\bar{\kappa}_{s}\right)\right)$. Since adiabatic compressibility is a calculated quantity, its uncertainty is derived from the propagation of uncertainties for the two measured quantities. Reported absolute expanded uncertainties, ranging from 1.0 to $1.6 \mathrm{TPa}^{-1}$, correspond to relative expanded uncertainties of $0.2 \%$.

The observed trend in adiabatic compressibility (Figure 22) is less straightforward than that observed for either density or speed of sound. Here, POE7 exhibits the highest values, ranging from $709.4 \mathrm{TPa}^{-1}$ at $343.15 \mathrm{~K}$ to $491.0 \mathrm{TPa}^{-1}$ at $278.15 \mathrm{~K}$. However, the fluid with the lowest values changes with temperature. At $343.15 \mathrm{~K}$, MIL-PRF-23699 exhibits the lowest adiabatic compressibility $\left(700.8 \mathrm{TPa}^{-1}\right)$, while at $278.15 \mathrm{~K}$, POE5 is the lowest $\left(482.9 \mathrm{TPa}^{-1}\right)$. Additionally, 
the overall differences are significantly smaller than what was observed for the other properties, ranging from $1.2 \%$ at $343.15 \mathrm{~K}$ to $1.6 \%$ at $283.15 \mathrm{~K}$. Finally, at $343.15 \mathrm{~K}$, the adiabatic compressibility of MIL-PRF-23699 is essentially equivalent to that of POE9, differing by just $0.03 \%$, while at the low end of the temperature range MIL-PRF-23699 is most similar to POE5, with a difference of $0.5 \%$ at $278.15 \mathrm{~K}$.

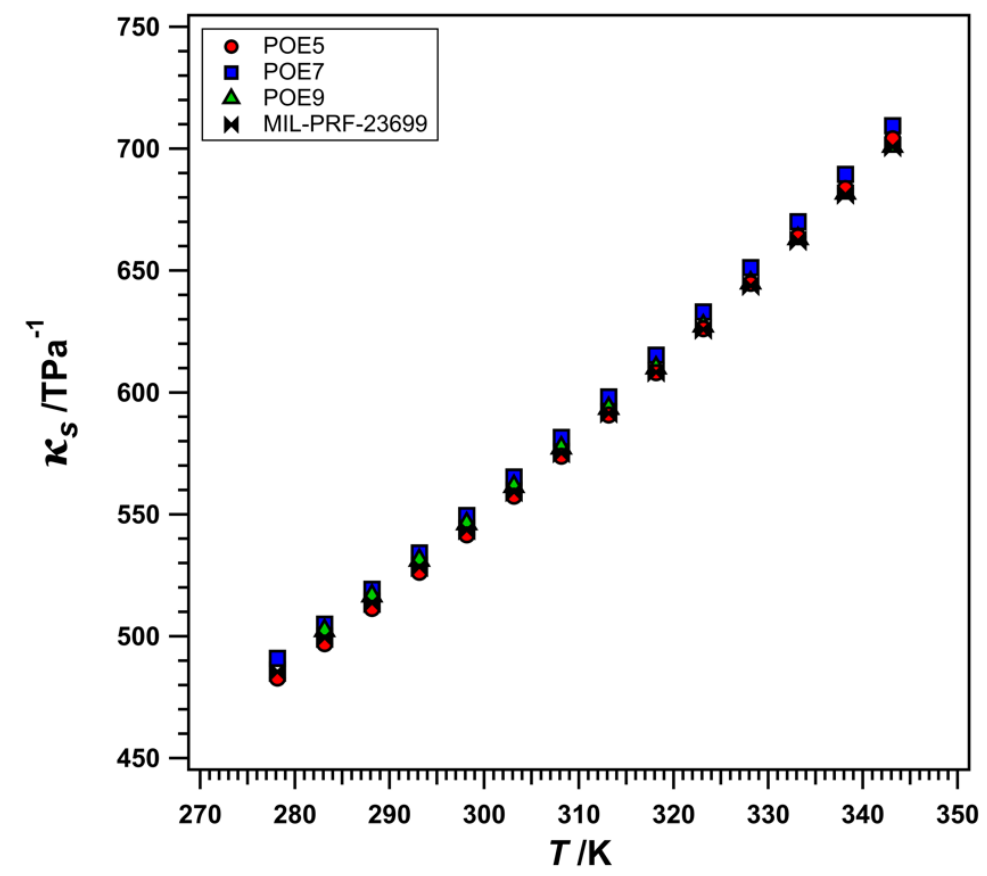

Figure 22: Calculated ambient-pressure adiabatic compressibilities plotted as a function of temperature. 
8.4. Data Tables

Table 23: Measured Densities for Four Lubricant Samples at Ambient Pressure ( 83 kPa).

\begin{tabular}{|c|c|c|c|c|c|c|c|c|}
\hline & \multicolumn{4}{|c|}{ POE5 } & \multicolumn{4}{|c|}{ POE7 } \\
\hline$T$ & $\bar{\rho}$ & $t_{95}$ & $\boldsymbol{U}(\overline{\boldsymbol{\rho}})$ & $\boldsymbol{U}(\overline{\boldsymbol{\rho}})$ & $\overline{\boldsymbol{\rho}}$ & $t_{95}$ & $\boldsymbol{U}(\overline{\boldsymbol{\rho}})$ & $\boldsymbol{U}(\overline{\boldsymbol{\rho}})$ \\
\hline$/ \mathbf{K}$ & $/ \mathbf{k g} \cdot \mathbf{m}^{-3}$ & & $/ \mathbf{k g} \cdot \mathbf{m}^{-3}$ & $1 \%$ & $/ \mathbf{k g} \cdot \mathbf{m}^{-3}$ & & $/ \mathrm{kg} \cdot \mathrm{m}^{-3}$ & $1 \%$ \\
\hline 343.15 & 977.88 & 2.041 & 1.66 & 0.17 & 940.20 & 2.042 & 1.65 & 0.14 \\
\hline 338.15 & 981.88 & 2.041 & 1.66 & 0.17 & 943.92 & 2.042 & 1.65 & 0.14 \\
\hline 333.15 & 985.88 & 2.041 & 1.66 & 0.17 & 947.66 & 2.042 & 1.65 & 0.13 \\
\hline 328.15 & 989.89 & 2.041 & 1.66 & 0.17 & 951.39 & 2.042 & 1.65 & 0.13 \\
\hline 323.15 & 993.90 & 2.041 & 1.66 & 0.17 & 955.13 & 2.042 & 1.65 & 0.13 \\
\hline 318.15 & 997.92 & 2.041 & 1.66 & 0.17 & 958.87 & 2.042 & 1.65 & 0.13 \\
\hline 313.15 & 1001.94 & 2.041 & 1.66 & 0.17 & 962.62 & 2.042 & 1.65 & 0.13 \\
\hline 308.15 & 1005.97 & 2.041 & 1.66 & 0.16 & 966.38 & 2.042 & 1.65 & 0.13 \\
\hline 303.15 & 1010.00 & 2.041 & 1.66 & 0.16 & 970.14 & 2.042 & 1.65 & 0.13 \\
\hline 298.15 & 1014.04 & 2.041 & 1.65 & 0.16 & 973.91 & 2.042 & 1.65 & 0.12 \\
\hline 293.15 & 1018.08 & 2.041 & 1.65 & 0.16 & 977.69 & 2.041 & 1.65 & 0.12 \\
\hline 288.15 & 1022.14 & 2.041 & 1.65 & 0.16 & 981.48 & 2.041 & 1.65 & 0.12 \\
\hline 283.15 & 1026.21 & 2.041 & 1.65 & 0.16 & 985.27 & 2.041 & 1.65 & 0.12 \\
\hline \multirow[t]{2}{*}{278.15} & 1030.29 & 2.041 & 1.65 & 0.16 & 989.09 & 2.041 & 1.65 & 0.12 \\
\hline & \multicolumn{4}{|c|}{ POE9 } & \multicolumn{4}{|c|}{ MIL-PRF-23699 } \\
\hline$T$ & $\bar{\rho}$ & $t_{95}$ & $\boldsymbol{U}(\overline{\boldsymbol{\rho}})$ & $\boldsymbol{U}(\overline{\boldsymbol{\rho}})$ & $\overline{\boldsymbol{\rho}}$ & $t_{95}$ & $\boldsymbol{U}(\overline{\boldsymbol{\rho}})$ & $\boldsymbol{U}(\overline{\boldsymbol{\rho}})$ \\
\hline$/ \mathbf{K}$ & $/ \mathbf{k g} \cdot \mathbf{m}^{-3}$ & & $/ \mathbf{k g} \cdot \mathbf{m}^{-3}$ & $/ \%$ & $/ \mathbf{k g} \cdot \mathbf{m}^{-3}$ & & $/ \mathbf{k g} \cdot \mathbf{m}^{-3}$ & $1 \%$ \\
\hline 343.15 & 918.32 & 2.042 & 1.65 & 0.18 & 954.42 & 2.042 & 1.65 & 0.17 \\
\hline 338.15 & 921.88 & 2.042 & 1.65 & 0.18 & 958.17 & 2.042 & 1.65 & 0.17 \\
\hline 333.15 & 925.45 & 2.042 & 1.65 & 0.18 & 961.92 & 2.042 & 1.65 & 0.17 \\
\hline 328.15 & 929.02 & 2.042 & 1.65 & 0.18 & 965.68 & 2.042 & 1.65 & 0.17 \\
\hline 323.15 & 932.60 & 2.042 & 1.65 & 0.18 & 969.44 & 2.042 & 1.65 & 0.17 \\
\hline 318.15 & 936.18 & 2.042 & 1.65 & 0.18 & 973.20 & 2.042 & 1.65 & 0.17 \\
\hline 313.15 & 939.77 & 2.042 & 1.65 & 0.18 & 976.97 & 2.042 & 1.65 & 0.17 \\
\hline 308.15 & 943.37 & 2.042 & 1.65 & 0.18 & 980.74 & 2.042 & 1.65 & 0.17 \\
\hline 303.15 & 946.97 & 2.042 & 1.65 & 0.17 & 984.52 & 2.042 & 1.65 & 0.17 \\
\hline 298.15 & 950.58 & 2.042 & 1.65 & 0.17 & 988.30 & 2.042 & 1.65 & 0.17 \\
\hline 293.15 & 954.20 & 2.042 & 1.65 & 0.17 & 992.09 & 2.042 & 1.65 & 0.17 \\
\hline 288.15 & 957.85 & 2.042 & 1.65 & 0.17 & 995.89 & 2.042 & 1.65 & 0.17 \\
\hline 283.15 & 961.49 & 2.042 & 1.65 & 0.17 & 999.71 & 2.042 & 1.65 & 0.17 \\
\hline 278.15 & & & & & 1003.53 & 2.042 & 1.65 & 0.16 \\
\hline
\end{tabular}


Table 24: Measured Speeds of Sound for Four Lubricants at Ambient Pressure ( 83 kPa).

\begin{tabular}{|c|c|c|c|c|c|c|c|c|}
\hline & \multicolumn{4}{|c|}{ POE5 } & \multicolumn{4}{|c|}{ POE7 } \\
\hline$T$ & $\overline{\bar{w}}$ & $t_{95}$ & $\boldsymbol{U}(\overline{\boldsymbol{w}})$ & $\boldsymbol{U}(\overline{\boldsymbol{w}})$ & $\overline{\boldsymbol{w}}$ & $t_{95}$ & $\boldsymbol{U}(\overline{\boldsymbol{w}})$ & $\boldsymbol{U}(\overline{\boldsymbol{w}})$ \\
\hline$/ \mathbf{K}$ & $/ \mathbf{m} \cdot \mathbf{s}^{-1}$ & & $/ \mathbf{m} \cdot \mathbf{s}^{-1}$ & $/ \%$ & $/ \mathbf{m} \cdot \mathbf{s}^{-1}$ & & $/ \mathbf{m} \cdot \mathbf{s}^{-1}$ & $/ \%$ \\
\hline 343.15 & 1205.2 & 2.034 & 1.7 & 0.1 & 1224.5 & 2.034 & 1.7 & 0.2 \\
\hline 338.15 & 1220.5 & 2.034 & 1.7 & 0.1 & 1239.6 & 2.034 & 1.7 & 0.2 \\
\hline 333.15 & 1236.1 & 2.034 & 1.7 & 0.1 & 1254.9 & 2.034 & 1.7 & 0.2 \\
\hline 328.15 & 1251.8 & 2.034 & 1.7 & 0.1 & 1270.4 & 2.034 & 1.7 & 0.2 \\
\hline 323.15 & 1267.6 & 2.034 & 1.7 & 0.1 & 1286.1 & 2.033 & 1.7 & 0.2 \\
\hline 318.15 & 1283.6 & 2.034 & 1.7 & 0.1 & 1301.9 & 2.033 & 1.7 & 0.2 \\
\hline 313.15 & 1299.8 & 2.033 & 1.7 & 0.1 & 1317.8 & 2.033 & 1.7 & 0.2 \\
\hline 308.15 & 1316.2 & 2.033 & 1.7 & 0.1 & 1333.9 & 2.033 & 1.7 & 0.2 \\
\hline 303.15 & 1332.7 & 2.033 & 1.7 & 0.1 & 1350.3 & 2.033 & 1.7 & 0.2 \\
\hline 298.15 & 1349.3 & 2.033 & 1.7 & 0.1 & 1367.0 & 2.032 & 1.7 & 0.2 \\
\hline 293.15 & 1366.2 & 2.033 & 1.7 & 0.1 & 1383.8 & 2.031 & 1.7 & 0.2 \\
\hline 288.15 & 1383.2 & 2.033 & 1.7 & 0.1 & 1400.7 & 2.029 & 1.7 & 0.2 \\
\hline 283.15 & 1400.3 & 2.033 & 1.7 & 0.1 & 1417.7 & 2.029 & 1.7 & 0.2 \\
\hline \multirow[t]{2}{*}{278.15} & 1417.7 & 2.033 & 1.7 & 0.1 & 1435.0 & 2.028 & 1.7 & 0.2 \\
\hline & \multicolumn{4}{|c|}{ POE9 } & \multicolumn{4}{|c|}{ MIL-PRF-23699 } \\
\hline$T$ & $\overline{\boldsymbol{w}}$ & $t_{95}$ & $\boldsymbol{U}(\overline{\boldsymbol{w}})$ & $\boldsymbol{U}(\overline{\boldsymbol{w}})$ & $\overline{\boldsymbol{w}}$ & $t_{95}$ & $\boldsymbol{U}(\overline{\boldsymbol{w}})$ & $\boldsymbol{U}(\overline{\boldsymbol{w}})$ \\
\hline$/ \mathbf{K}$ & $/ \mathbf{m} \cdot \mathbf{s}^{-1}$ & & $/ \mathbf{m} \cdot \mathbf{s}^{-1}$ & $/ \%$ & $/ \mathbf{m} \cdot \mathbf{s}^{-1}$ & & $/ \mathbf{m} \cdot \mathbf{s}^{-1}$ & $/ \%$ \\
\hline 343.15 & 1246.4 & 2.034 & 1.7 & 0.1 & 1222.8 & 2.033 & 1.7 & 0.1 \\
\hline 338.15 & 1261.4 & 2.034 & 1.7 & 0.1 & 1237.7 & 2.033 & 1.7 & 0.1 \\
\hline 333.15 & 1276.6 & 2.034 & 1.7 & 0.1 & 1252.9 & 2.032 & 1.7 & 0.1 \\
\hline 328.15 & 1292.0 & 2.034 & 1.7 & 0.1 & 1268.3 & 2.032 & 1.7 & 0.1 \\
\hline 323.15 & 1307.5 & 2.033 & 1.7 & 0.1 & 1283.9 & 2.031 & 1.7 & 0.1 \\
\hline 318.15 & 1323.2 & 2.033 & 1.7 & 0.1 & 1299.6 & 2.031 & 1.7 & 0.1 \\
\hline 313.15 & 1339.1 & 2.033 & 1.7 & 0.1 & 1315.5 & 2.031 & 1.7 & 0.1 \\
\hline 308.15 & 1355.2 & 2.033 & 1.7 & 0.1 & 1331.5 & 2.030 & 1.7 & 0.1 \\
\hline 303.15 & 1371.5 & 2.033 & 1.7 & 0.1 & 1347.7 & 2.029 & 1.7 & 0.1 \\
\hline 298.15 & 1387.9 & 2.032 & 1.7 & 0.1 & 1364.3 & 2.030 & 1.7 & 0.1 \\
\hline 293.15 & 1404.7 & 2.032 & 1.7 & 0.1 & 1381.1 & 2.030 & 1.7 & 0.1 \\
\hline 288.15 & 1421.7 & 2.032 & 1.7 & 0.1 & 1398.1 & 2.031 & 1.7 & 0.1 \\
\hline 283.15 & 1438.9 & 2.031 & 1.7 & 0.1 & 1415.3 & 2.031 & 1.7 & 0.1 \\
\hline 278.15 & & & & & 1432.7 & 2.031 & 1.7 & 0.1 \\
\hline
\end{tabular}


Table 25: Calculated Adiabatic Compressibilities for Four Lubricants at Ambient Pressure ( $83 \mathrm{kPa})$

\begin{tabular}{|c|c|c|c|c|c|c|}
\hline & \multicolumn{3}{|c|}{ POE5 } & \multicolumn{3}{|c|}{ POE7 } \\
\hline $\begin{array}{c}T \\
/ K\end{array}$ & $\begin{array}{c}\overline{\boldsymbol{\kappa}}_{\boldsymbol{s}} \\
/ \mathrm{TPa}^{-1}\end{array}$ & $\begin{array}{c}\boldsymbol{U}\left(\overline{\boldsymbol{\kappa}}_{\boldsymbol{s}}\right) \\
/ \mathrm{TPa}^{-1}\end{array}$ & $\begin{array}{c}\boldsymbol{U}\left(\overline{\boldsymbol{\kappa}}_{\boldsymbol{s}}\right) \\
/ \%\end{array}$ & $\begin{array}{c}\overline{\boldsymbol{\kappa}}_{\boldsymbol{s}} \\
/ \mathrm{TPa}^{-1}\end{array}$ & $\begin{array}{c}\boldsymbol{U}\left(\overline{\boldsymbol{\kappa}}_{\boldsymbol{s}}\right) \\
/ \mathbf{T P a}^{-1}\end{array}$ & $\begin{array}{c}\boldsymbol{U}\left(\overline{\boldsymbol{\kappa}}_{\boldsymbol{s}}\right) \\
/ \%\end{array}$ \\
\hline 343.15 & 704.1 & 1.5 & 0.2 & 709.4 & 1.6 & 0.2 \\
\hline 338.15 & 683.7 & 1.5 & 0.2 & 689.5 & 1.5 & 0.2 \\
\hline 333.15 & 663.9 & 1.4 & 0.2 & 670.1 & 1.5 & 0.2 \\
\hline 328.15 & 644.7 & 1.4 & 0.2 & 651.2 & 1.4 & 0.2 \\
\hline 323.15 & 626.1 & 1.3 & 0.2 & 633.0 & 1.4 & 0.2 \\
\hline 318.15 & 608.2 & 1.3 & 0.2 & 615.3 & 1.3 & 0.2 \\
\hline 313.15 & 590.7 & 1.2 & 0.2 & 598.2 & 1.3 & 0.2 \\
\hline 308.15 & 573.8 & 1.2 & 0.2 & 581.5 & 1.2 & 0.2 \\
\hline 303.15 & 557.5 & 1.2 & 0.2 & 565.3 & 1.2 & 0.2 \\
\hline 298.15 & 541.6 & 1.1 & 0.2 & 549.5 & 1.2 & 0.2 \\
\hline 293.15 & 526.3 & 1.1 & 0.2 & 534.2 & 1.1 & 0.2 \\
\hline 288.15 & 511.4 & 1.0 & 0.2 & 519.3 & 1.1 & 0.2 \\
\hline 283.15 & 497.0 & 1.0 & 0.2 & 505.0 & 1.0 & 0.2 \\
\hline \multirow[t]{2}{*}{278.15} & 482.9 & 1.0 & 0.2 & 491.0 & 1.0 & 0.2 \\
\hline & \multicolumn{3}{|c|}{ POE9 } & \multicolumn{3}{|c|}{ MIL-PRF-23699 } \\
\hline$T$ & $\overline{\boldsymbol{\kappa}}_{\boldsymbol{s}}$ & $\boldsymbol{U}\left(\overline{\boldsymbol{\kappa}}_{\boldsymbol{s}}\right)$ & $\boldsymbol{U}\left(\overline{\boldsymbol{\kappa}}_{\boldsymbol{s}}\right)$ & $\overline{\boldsymbol{\kappa}}_{\boldsymbol{s}}$ & $\boldsymbol{U}\left(\overline{\boldsymbol{\kappa}}_{\boldsymbol{s}}\right)$ & $\boldsymbol{U}\left(\overline{\boldsymbol{\kappa}}_{\boldsymbol{s}}\right)$ \\
\hline$/ \mathbf{K}$ & $/ \mathbf{T P a}^{-1}$ & $/ \mathrm{TPa}^{-1}$ & $1 \%$ & $/ \mathbf{T P a}^{-1}$ & $/ \mathbf{T P a}^{-1}$ & $1 \%$ \\
\hline 343.15 & 701.0 & 1.6 & 0.2 & 700.8 & 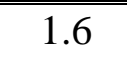 & 0.2 \\
\hline 338.15 & 681.8 & 1.5 & 0.2 & 681.2 & 1.5 & 0.2 \\
\hline 333.15 & 663.1 & 1.5 & 0.2 & 662.2 & 1.4 & 0.2 \\
\hline 328.15 & 644.9 & 1.4 & 0.2 & 643.8 & 1.4 & 0.2 \\
\hline 323.15 & 627.2 & 1.4 & 0.2 & 625.8 & 1.4 & 0.2 \\
\hline 318.15 & 610.1 & 1.3 & 0.2 & 608.4 & 1.3 & 0.2 \\
\hline 313.15 & 593.4 & 1.3 & 0.2 & 591.5 & 1.3 & 0.2 \\
\hline 308.15 & 577.2 & 1.2 & 0.2 & 575.2 & 1.2 & 0.2 \\
\hline 303.15 & 561.4 & 1.2 & 0.2 & 559.2 & 1.2 & 0.2 \\
\hline 298.15 & 546.1 & 1.2 & 0.2 & 543.6 & 1.1 & 0.2 \\
\hline 293.15 & 531.1 & 1.1 & 0.2 & 528.5 & 1.1 & 0.2 \\
\hline 288.15 & 516.5 & 1.1 & 0.2 & 513.7 & 1.1 & 0.2 \\
\hline 283.15 & 502.3 & 1.0 & 0.2 & 499.4 & 1.0 & 0.2 \\
\hline 278.15 & & & & 485.5 & 1.0 & 0.2 \\
\hline
\end{tabular}




\section{Thermal Conductivity}

\subsection{Summary}

The thermal conductivity of the liquid phase of three POE base oils and the fully qualified lubricant was measured over the temperature range of $300 \mathrm{~K}$ to $500 \mathrm{~K}$ at pressures up to $69 \mathrm{MPa}$ in a transient hot-wire apparatus. These measurements provided the data necessary for development of models for the thermal conductivity of POE lubricants.

\subsection{Instrument Description}

The thermal conductivity was measured with a high-temperature apparatus that allows isothermal control of the temperature of the hot-wire cell in a specially designed furnace. The experiment control electronics are shown in Figure 23 (left). The temperature control furnace, valve manifold, and piston pump are shown in Figure 23 (right). In the present measurements, the fluid was contained in the cylindrical bore of a microreactor, shown in Figure 24, with an inner diameter of $8 \mathrm{~mm}$, which is concentric with the hot wire of $12.7 \mu \mathrm{m}$ diameter that is $11.429 \mathrm{~cm}$ long and located along the central axis of the bore.
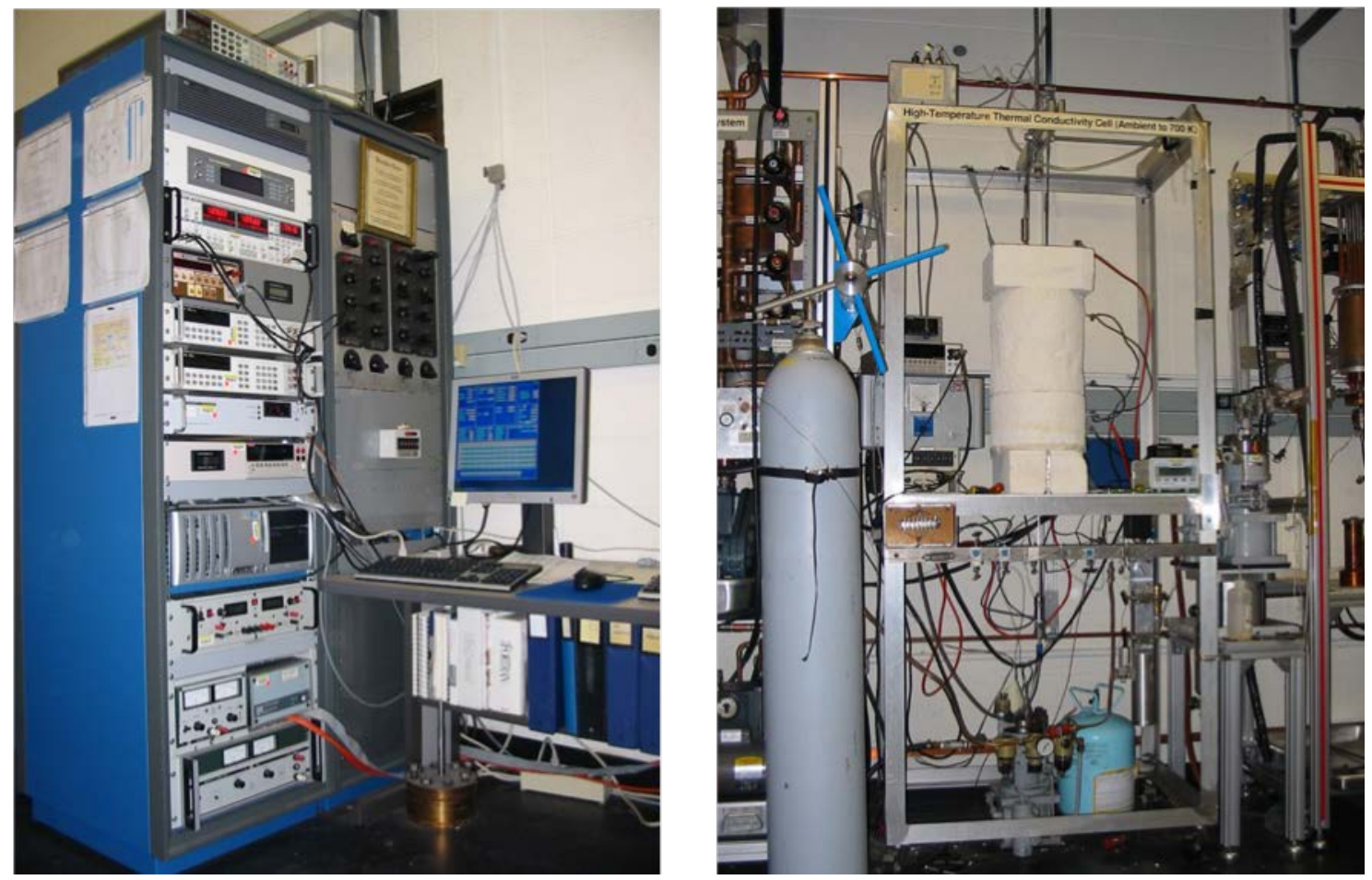

Figure 23: (left) Transient hot-wire measurement electronics including: control computer, Wheatstone bridge containing hot-wires, power supplies, multimeters, multiplexer, and lock-in amplifier (for AC hot-wire experiments). (right) Temperature and pressure control system including: DC powered furnace, pressure manifold, piston pump, pressure transducer, and platinum resistance thermometers. 
The hot wire has two lead wires at each end to allow separate current and sense leads for four-wire resistance measurements. The lead wires pass through a four-wire high-pressure seal with compressed Teflon sealant. The microreactor with a volume of $5 \mathrm{~mL}$ is located within an aluminum isothermal cylinder, also shown in Figure 24, with thermowells for platinum resistance sensors for control of temperature and measurement of temperature gradients, and for an SPRT to measure the initial cell temperature, $T_{\mathrm{i}}$, with an expanded uncertainty of $U\left(T_{\mathrm{i}}\right)=0.005 \mathrm{~K}$. The measurement cell/isothermal cylinder is in turn located within a cylindrical isothermal enclosure of aluminum with a wall thickness of $13 \mathrm{~mm}$, which is placed in the furnace that is controlled by the system computer at the desired temperature. An air gap between the cell/isothermal cylinder and the isothermal enclosure further reduces temperature gradients in the measurement cell. Pressures, $p$, are determined with a pressure transducer with an expanded uncertainty of $U(p)=7 \mathrm{kPa}$. All reported uncertainties are for a coverage factor of $k=2$, an approximately $95 \%$ confidence interval.

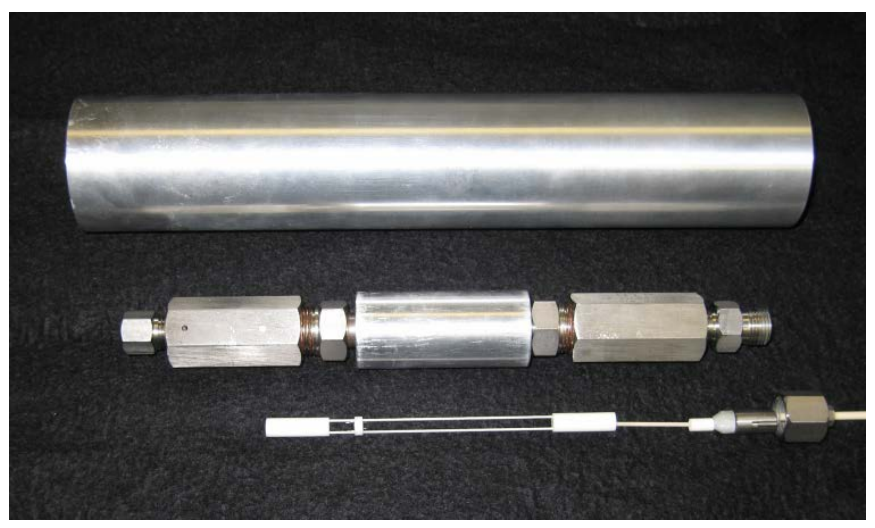

Figure 24: Transient hot-wire cell components including aluminum isothermal block, $5 \mathrm{~mL}$ microreactor, wire pressure feedthrough, and alumina hot-wire support and electrical insulation components.

\subsection{Measurement Principle}

Hot wires with a small diameter and large length-to-diameter ratio are used to approximate a transient line source as closely as possible as described many years ago by Healy et al.[49] Deviations from the transient line-source model are treated as corrections to the experimental temperature rise. In this approach, the ideal temperature rise, $\Delta T_{\text {id, }}$ is given by

$$
\Delta T_{\text {id }}=\frac{q}{4 \pi \lambda}\left[\ln (t)+\ln \left(\frac{4 a}{r_{0}{ }^{2} C}\right)\right]=\Delta T_{\mathrm{w}}+\sum_{i=1}^{10} \delta T_{i},
$$


where $q$ is the power applied per unit length, $\lambda$ is the thermal conductivity of the fluid, $t$ is the elapsed time from the onset of heating, $a=\lambda /\left(\rho c_{p}\right)$ is the thermal diffusivity of the fluid, $\rho$ is the density of the fluid, $c_{p}$ is the isobaric specific heat capacity of the fluid, $r_{0}$ is the radius of the hot wire, $C=1.781 \ldots$ is the exponential of Euler's constant. The ideal temperature rise is equivalent to the measured temperature rise of the wire, $\Delta T_{\mathrm{w}}$, summed with the corrections, $\delta T_{i}$, that account for deviations from ideal line-source conduction.[49] The form of Eq. 11 is that of a line, where the thermal conductivity can be found from the slope and the thermal diffusivity can be found from the intercept. A line is fit to $\Delta T_{\text {id }}$ versus $\ln (t)$ data over a time interval, typically from $0.1 \mathrm{~s}$ to $1.0 \mathrm{~s}$, with the thermal conductivity obtained from the slope of this fitted line. The thermal diffusivity from the intercept of this fitted line typically has an order of magnitude higher uncertainty and was not considered in this work. Assuming the thermal conductivity increases linearly with temperature for a small temperature rise, the experimental temperature, $T$, associated with the thermal conductivity is the average wire temperature over the time interval that was fit.

The largest correction for both gas and liquid measurements accounts for the finite dimensions and heat capacity of the hot wire. This correction for finite wire dimensions is most significant at short experiment times. The single-wire cell requires correction for finite diameter and length (axial conduction) during data analysis based on the two-dimensional transient analytical solution of Woodfield et al.[50] The end time for the fit interval was selected so the transient temperature gradient in the fluid did not penetrate to the cavity wall and fluid convection was not significant. An experiment duration of $1 \mathrm{~s}$ was considered optimal for all measurements.

Heat transfer by thermal radiation between media at two different temperatures $T_{1}$ and $T_{2}$ is proportional to $\left(T_{1}{ }^{4}-T_{2}{ }^{4}\right)$. When $T_{1}$ and $T_{2}$ are nearly the same (small temperature rise) this is approximately $T^{3}\left(T_{1}-T_{2}\right)$ so in the present hot-wire experiments the thermal radiation correction increases in proportion to $T^{3}$ and remains relatively small at temperatures up to $500 \mathrm{~K}$. The transparent fluid correction[49] was applied for the present measurements in both the gas and liquid phases. This correction is best for gas measurements, but even for higher density liquid measurements, where there is more absorption and emission of thermal radiation, this correction is considered reasonable at these temperatures.

\subsection{Measurement Process}

Measurements were started with a clean and evacuated hot-wire cell and pressure system. Pressure was controlled with a variable-volume piston pump. The pump was nearly filled with new sample 
such that the vapor headspace was at the top of the pump at the pump outlet. The pump was then connected to the pressure system and the entire system, including the pump, was evacuated to degas the sample in the pump. The vacuum was disconnected, and the degassed sample was then injected into the measurement system, which was at the desired temperature. Measurements were made along isotherms, starting at the lowest pressure and increasing pressure to $69 \mathrm{MPa}$ for a total of eight to nine pressures per isotherm. At each pressure, transient hot-wire measurements were made at five different power levels (yielding five different experimental temperatures and temperature rises) with two experiments of one second duration per power level. Thus, ten measurements were made at each initial temperature and pressure.

Each isotherm required 1 day to complete. Seven isotherms at temperatures of 300, 333, 367, 400, 433, 467, and $500 \mathrm{~K}$ were completed for each fluid. The liquid in the measurement cell was removed by pressurizing with carbon dioxide gas at the top of the measurement cell and withdrawing sample from the bottom of the cell through the pressure system manifold. The remaining liquid in the pump was also discharged through the pressure system manifold. The cell and pump were cleaned with multiple fills and discharges of liquid toluene. Between each fill and discharge of toluene solvent the system was filled with supercritical carbon dioxide that was discharged before the cell was evacuated. The pump was disassembled and cleaned between each sample. The final cleaning step was a fill of the entire pressure system, including the cleaned pump and hot-wire cell, with supercritical carbon dioxide. The discharge from this fill/soak was examined for residual liquid oil that might have been retained. Cleaning was continued until the carbon dioxide discharge contained no observable oil residue.

\subsubsection{Catalytic Alumina Support/ Insulator Problem}

Measurements were initially made with one of our typical single-wire cells with alumina components to support the hot wire and provide electrical insulation. The high-pressure wire feedthrough had a soapstone sealant. Measurements were made on the POE9 sample, with repeatability issues at temperatures above $400 \mathrm{~K}$. The problems became more pronounced with the POE7 sample. Chemical analysis of sample removed from the hot-wire apparatus after these issues were observed indicated decomposition. Subsequent testing of POE samples over ground alumina indicated catalytic activity, supporting our observations of decomposition producing changes in measured thermal conductivity. Macor machinable ceramic was then tested with no catalytic activity indicated. The cell used in all the measurements reported here used Macor, glass capillary, and Teflon for wire support and electrical insulation (i.e., the tests with POE9 and POE7 were repeated). There was no indication of decomposition during the measurements reported here. 


\subsection{Results and Uncertainties}

The measured thermal conductivity is shown as a function of pressure for each of the isotherms in Figure 25 through Figure 28. The measured thermal conductivity data are given in Appendix A in Tables A1 through A4. The measured thermal conductivity data are reported at nominal isotherm temperatures, $T_{\mathrm{i}}$, of 300, 333, 367, 400, 433, 467, and $500 \mathrm{~K}$ with pressures, $p$, up to $69 \mathrm{MPa}$. Since five different applied power levels were each measured twice, there are up to ten measurements reported for each fluid and each initial temperature and pressure.

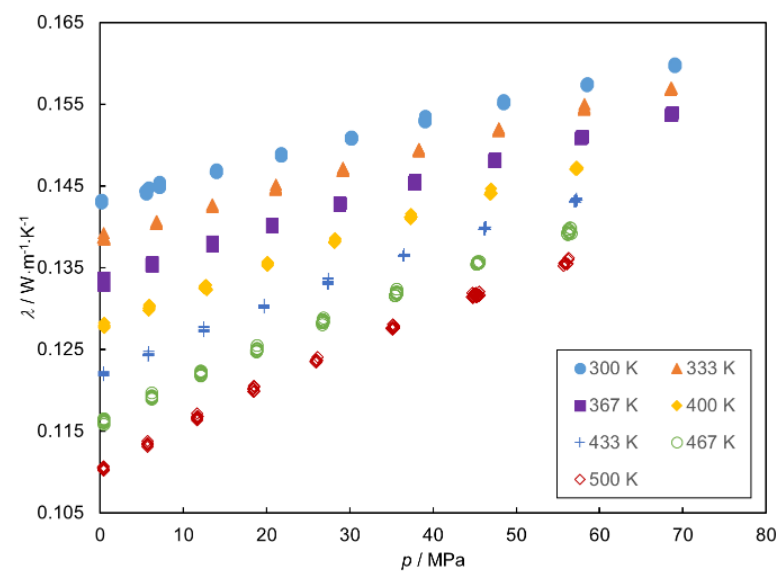

Figure 25: Thermal conductivity data for POE5 as a function of pressure measured along isotherms at temperatures from $300 \mathrm{~K}$ to $500 \mathrm{~K}$.

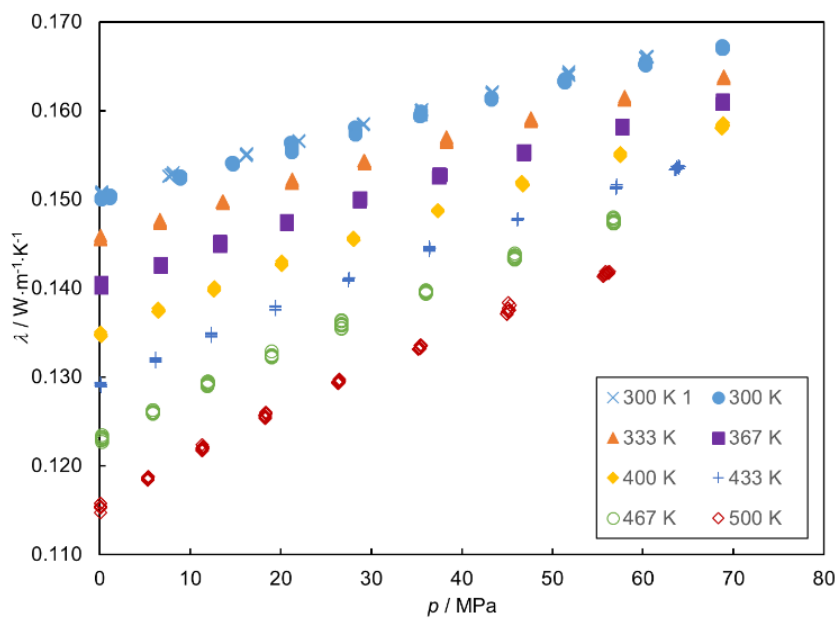

Figure 26: Thermal conductivity data for POE7 as a function of pressure measured along isotherms at temperatures from $300 \mathrm{~K}$ to $500 \mathrm{~K}$. 


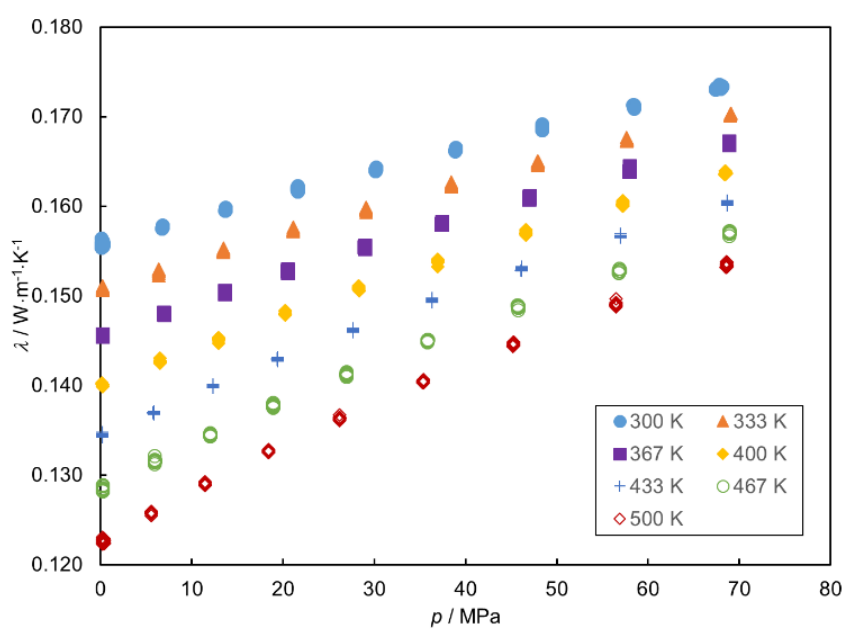

Figure 27: Thermal conductivity data for POE9 as a function of pressure measured along isotherms at temperatures from $300 \mathrm{~K}$ to $500 \mathrm{~K}$.

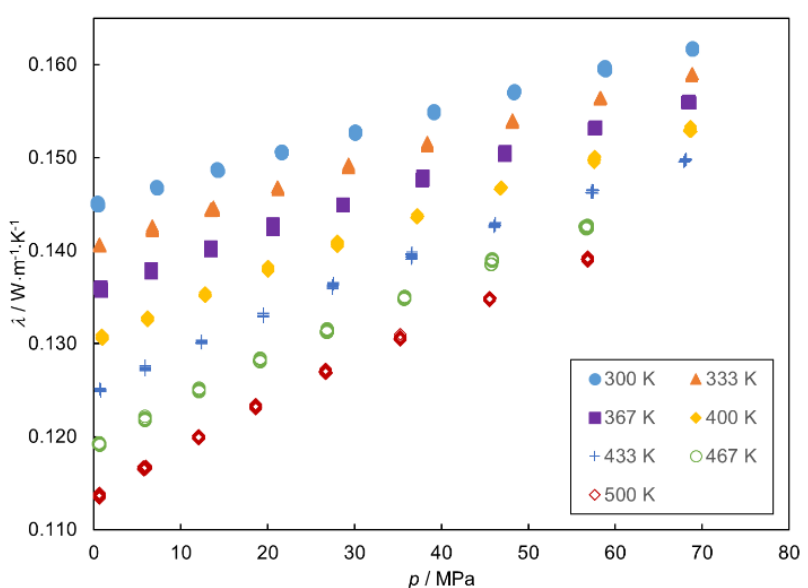

Figure 28: Thermal conductivity data for MIL-PRF-23699 lubricant as a function of pressure measured along isotherms at temperatures from $300 \mathrm{~K}$ to $500 \mathrm{~K}$.

Measurements were removed from analysis when deviation plots of the temperature rise as a function of $\ln (t)$ indicated convection occurred during a measurement. The measured thermal conductivity, $\lambda$, is associated with the experimental temperature, $T$, and $p$. The experiment temperature rise is reflected in the difference between $T$ and $T_{\mathrm{i}}$. The expanded uncertainty for $T$ is comparable to that of $T_{\mathrm{i}}$, and $U(T)=0.005 \mathrm{~K}$. The expanded relative uncertainty for the reported thermal conductivity data is $U(\lambda)=0.5 \%$. A parameter STAT was also calculated; it is the expanded relative uncertainty in the slope of the line fit to $\Delta T_{\text {id }}$ data as a function of $\ln (t)$ in Eq. 11 to get the experimental thermal conductivity. This parameter was 0.002 or less for all of the measurements reported here, which is much less than the expanded uncertainty. All expanded uncertainties are for a coverage factor of $k=2$, an approximately $95 \%$ confidence interval. 
The measurement sequence for the POE samples reported here was POE7, POE5, MIL-PRF23699, and POE9. POE7 was measured first since it was still in the pump after the alumina catalysis problem was identified and it was decided that the new hot-wire cell without alumina was required for these measurements. The first assembly of the new cell failed at the highest pressure of the initial $300 \mathrm{~K}$ isotherm when the lead wires slipped in the Teflon wire sealant. Mechanical support from an additional Teflon tube between the cell and the sealant was added in the second assembly that eliminated this problem. Thus, there are two independent measurements of the POE7 sample reported at $300 \mathrm{~K}$ from each assembly. Agreement between these two independent assemblies is within the expanded uncertainty of $U(\lambda)=0.5 \%$. The data from both assemblies are shown in Figure 26 (indicated as “300 K” and “300 K 1" in the figure legend).

At any given temperature, the thermal conductivity of the POE samples generally increases in the order of POE5, MIL-PRF-23699, POE7, and POE9. The corrections in Eq. 11 require estimates for the thermophysical properties of the measured liquid. These property estimates were based on preliminary values, but this adds only slightly to $U(\lambda)$ since the corrections are small for liquid samples. 


\section{Viscosity}

\subsection{Summary}

Viscosities of the four lubricants were measured with a commercial oscillating-piston viscometer that was modified at NIST with regard to thermal insulation, calibration, sample charging and pressurization, as well as automation and data acquisition. The instrument uses a variant of the falling body technique except that the motion of the sensing body is not unidirectional and driven by gravity but alternating and driven by electromagnetic induction from two magnetic coils. Measurements on the four lubricants were carried out over temperature ranges of $275 \mathrm{~K}$ to $430 \mathrm{~K}$ for POE5, $280 \mathrm{~K}$ to $450 \mathrm{~K}$ for POE7, $290 \mathrm{~K}$ to $450 \mathrm{~K}$ for POE9, and $290 \mathrm{~K}$ to $450 \mathrm{~K}$ for MILPRF-23699; in all cases the maximum pressure was $137 \mathrm{MPa}$. Measurements on squalane over a similar temperature and pressure range were required for calibration purposes. These results are also presented in Laesecke et al.[51]

\subsection{Experimental}

The experimental setup is shown in Figure 29. The tubular cell containing the sample and a viscosity-sensing cylinder is rated to $137 \mathrm{MPa}$. The cell can be thermostated between $270 \mathrm{~K}$ and $450 \mathrm{~K}$ with polydimethylsiloxane heat transfer liquid and a small laboratory circulator. For the samples in this project, three sensing cylinders were used in the viscosity ranges $1 \mathrm{mPa} \cdot \mathrm{s}$ to $20 \mathrm{mPa} \cdot \mathrm{s}, 2.5 \mathrm{mPa} \cdot \mathrm{s}$ to $50 \mathrm{mPa} \cdot \mathrm{s}$, and $5 \mathrm{mPa} \cdot \mathrm{s}$ to $100 \mathrm{mPa} \cdot \mathrm{s}$. Since these sensors had not been used at NIST previously, they had to be calibrated. Squalane $\left(\mathrm{C}_{30} \mathrm{H}_{64}\right)$ was chosen as reference liquid because its viscosity had been characterized recently in an international effort. The calibration required viscosity measurements of squalane with all three sensors in addition to the lubricant measurements. Mylona et al.[52] published two reference correlations for the viscosity of squalane, one formulated in terms of temperature and density and one in terms of temperature and pressure. Schmidt et al.[53] published a third reference correlation in terms of temperature and pressure and an additional viscosity data set that was measured at Imperial College (IC), London (UK) with a vibrating wire viscometer in the temperature range from $338.2 \mathrm{~K}$ to $473 \mathrm{~K}$ with pressures up to $200 \mathrm{MPa}$. This data set is the only one that overlaps with the temperature and pressure range of the viscometer used at NIST; it is therefore essential for the calibration of the three sensors. Unfortunately, it was found that none of the three reference correlations represents the IC data set within its estimated uncertainty of $2 \%$. The combined range of deviations extends from $-7.8 \%$ to $+7.4 \%$. If these correlations were used for calibrations, significant systematic errors would be propagated through other measurements. To avoid this, a new correlation of the 
IC data set had to be developed in this project which does represent these data within their quoted uncertainty of $2 \%$.

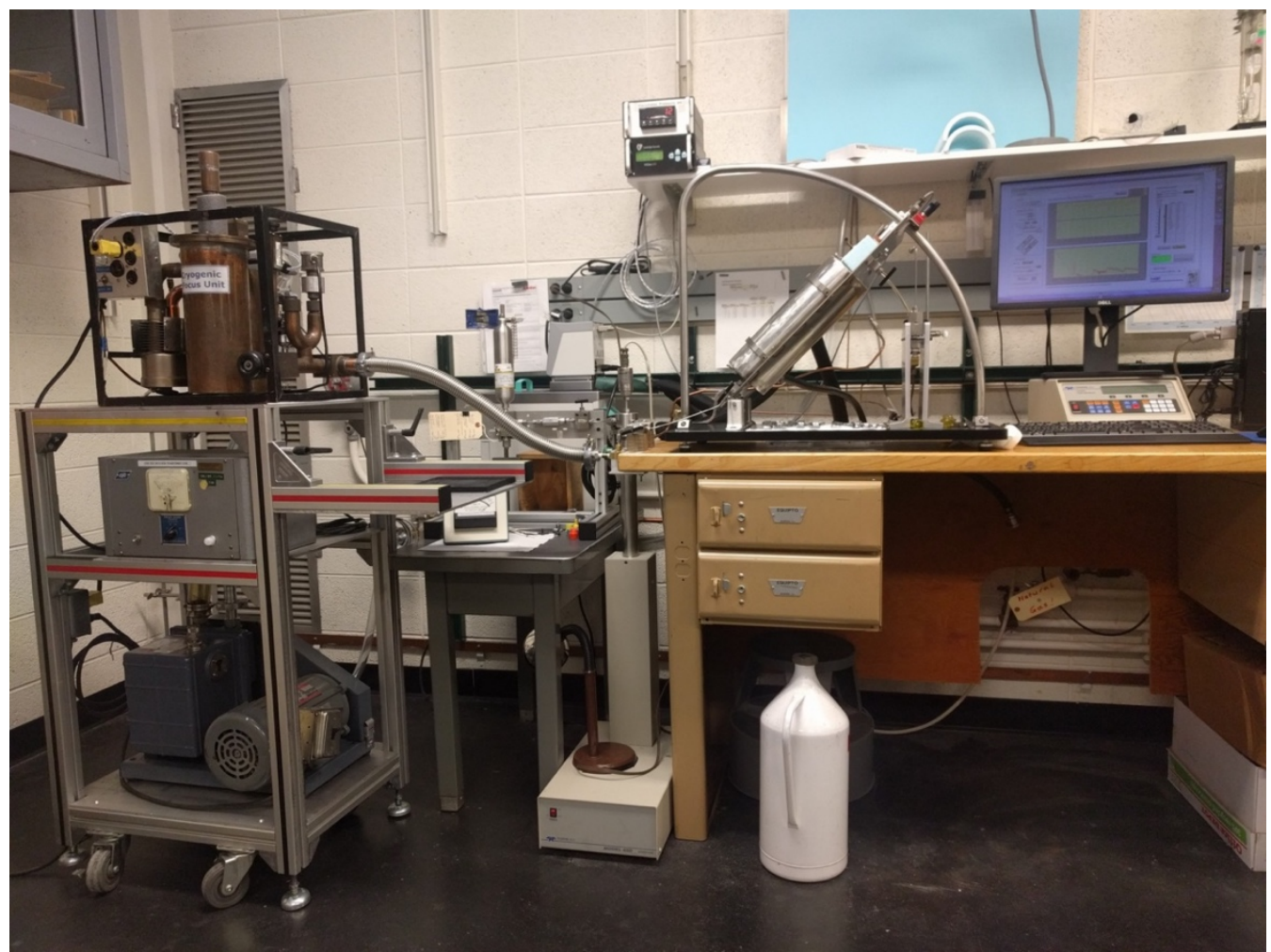

Figure 29: View of the oscillating-piston viscometer (above the bench drawers) with the vacuum system, sample cylinder, circulator, and syringe pump from left to right.

The temperature of the sample liquid in the viscometer was measured with a $100 \Omega$ platinum resistance temperature detector (RTD) that was calibrated and mounted on the outside of the pressure vessel by the manufacturer. The RTD is in a sealed space and cannot be removed from the pressure vessel to check its calibration. The manufacturer-quoted uncertainty of the temperature measurement is $0.1 \mathrm{~K}$. This uncertainty component contributes at most an uncertainty of $0.47 \%$ to the viscosity measurements of POE9 at $290 \mathrm{~K}$ and $4.8 \mathrm{MPa}$ where the maximum gradient $(\partial \eta / \partial T)_{p}$ occurs. The pressure in the viscometer was generated with a high-pressure syringe pump rated to $137 \mathrm{MPa}$ with a maximum sample volume of $65 \mathrm{~mL}$. Pressure was measured with a transducer rated to $207 \mathrm{MPa}$ with a full-scale uncertainty of $0.05 \%$, or $0.104 \mathrm{MPa}$. Prior to this project, the transducer calibration was checked with a primary pressure balance at $55.2 \mathrm{MPa}$, 41.4 MPa, 27.6 MPa, and 13.8 MPa, and the highest deviation was 0.024 MPa at the lowest calibration pressure. This uncertainty component contributes negligibly to the present measurements because the gradients $(\partial \eta / \partial p)_{T}$ are rather small for compressed liquids. 
The sample liquids were loaded into stainless steel cylinders and degassed by repeated freezing with liquid nitrogen-vacuum pumping-and thawing cycles. The sample cylinder was then mounted on the inlet of the viscometer manifold and the manifold was evacuated. The piston of the syringe pump was cycled during the vacuum pumping to remove residues on the walls of the syringe pump cylinder. With the syringe pump piston at the top position, the vacuum system was valved off and the sample cylinder valve opened. Sample liquid was drawn into the syringe pump cylinder until its volume of $65 \mathrm{~mL}$ was filled. After closing the sample cylinder valve, the valve between the syringe pump and the high-pressure cell was opened and sample was pumped into the viscometer manifold until it began to drain from the outlet of the manifold. Then, pumping was stopped, the outlet valve closed, and the measurement of an isotherm commenced with the customized LabVIEW software by programming the circulator to the desired temperature, setting the appropriate equilibration time, as well as the desired pressures and the measurement duration, at each pressure.

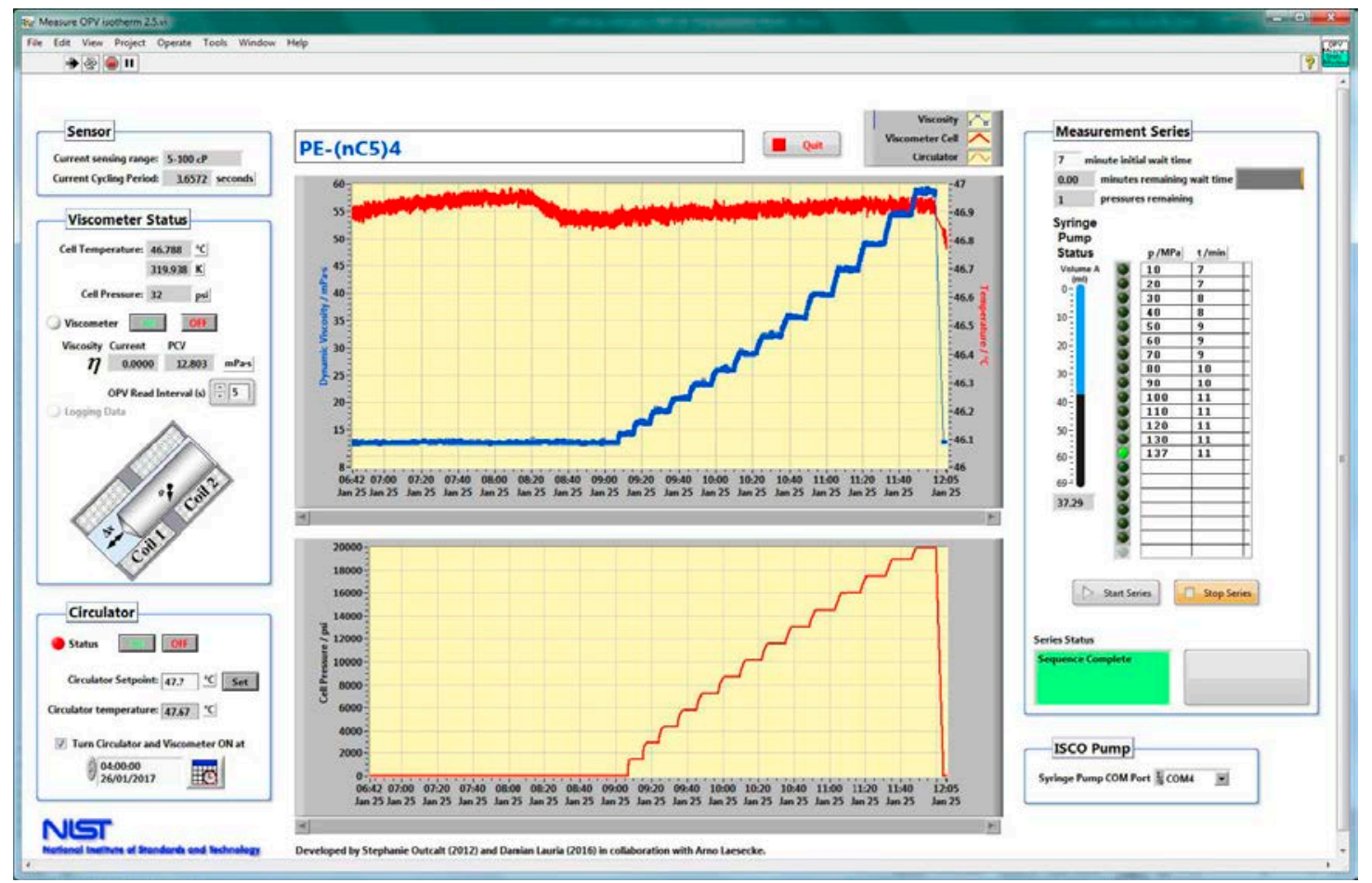

Figure 30: Front panel of the custom LabVIEW virtual instrument developed in this project to automate the oscillating-piston viscometer with its associated circulator and syringe pump. Shown are the cell temperature (red) and the viscosity (blue) in the top plot, and the cell pressure (thin red) in the bottom plot for measurements of POE5 along the $320 \mathrm{~K}$ isotherm. 
In the beginning of the project, viscosity data acquisition and scheduling were performed with software from the viscometer manufacturer. This was supplemented by a custom-developed LabVIEW utility that was adapted from the automated vibrating-tube densimeter (described in Section 7) to control and schedule the pressures to be generated by the high-pressure syringe pump (see Figure 30). This automation code was completed in November 2016 and accelerated the viscometer throughput by a factor of three. Due to its agility, it provides much more transparency of the internal viscometer state to the user. For instance, the presence of sample liquid or solvent in the viscometer cell is now indicated when the RTD registers a corresponding temperature change.

The viscosity measurements were carried out in the sequence POE9, squalane, POE7, squalane, POE5, MIL-PRF-23699, squalane with extensive rinsing of the viscometer between each sample. The solvent was a mixture of hexane isomers. During the rinsing, the high-pressure cell was thermostated to $308 \mathrm{~K}$. The volume of rinsing solvent was at least $300 \mathrm{~mL}$ which provided for 4.6 fillings of the cylinder of the high-pressure syringe pump. The internal volume of the high-pressure cell was approximately $8 \mathrm{~mL}$ and that of the remaining parts of the viscometer manifold approximately $5 \mathrm{~mL}$. Thus, the solvent volume was sufficient to rinse the viscometer volume several times over.

The MIL-PRF-23699 sample was measured last because prior measurements in the other instruments revealed that it was difficult to remove. During the viscosity measurements, it was observed that the MIL-PRF-23699 sample diffused through PTFE tape seals on the top and the bottom of the sample cylinder as these showed the brown color of the sample liquid after a few days. In addition, it was observed after the measurements of the MIL-PRF-23699 sample, that the hexane solvent was not miscible with that sample. Therefore, the viscometer was rinsed in addition with dodecane and then again with hexane. Despite this more extensive rinsing with two solvents, the following repeat squalane calibration measurements at $360 \mathrm{~K}$ and $370 \mathrm{~K}$ using the $5 \mathrm{mPa} \cdot \mathrm{s}$ to $100 \mathrm{mPa} \cdot \mathrm{s}$ sensing piston were invalid. Therefore, it was not possible to report calibrated viscosity data from measurements with this piston at these two temperatures. When results are reported at these two temperatures, they were obtained with one of the other two pistons.

\subsection{Results}

A total of 715 viscosity data points were measured with all three sensing pistons for the calibration liquid squalane from $277 \mathrm{~K}$ to $450 \mathrm{~K}$ with pressures to $137 \mathrm{MPa}$. Calibration functions in terms of pressure were obtained at each measured temperature by referencing the measured viscosities to 
those calculated from the VFT correlation of Mylona et al.[52] at temperatures below $340 \mathrm{~K}$, and the correlation of the IC data set that was developed in this work at temperatures above $340 \mathrm{~K}$. The VFT correlation of Mylona et al.[52] represents the experimental data of other laboratories within $\pm 3 \%$ and the correlation of the IC data set represents these data within their quoted uncertainty of $2 \%$. The calibration functions were subsequently applied in the analysis of the measured lubricant viscosities.

The viscosity measurement results for the lubricants are plotted as a function of pressure in Figures 31 through 34 and are reported in Tables B1 through B4 in Appendix B. The tabulated data are presented in the order they were measured with respect to temperature. A total of 269 data points are reported for POE5 in Table B1. The lowest temperature of these data is $275 \mathrm{~K}$. A total of 286 data points are reported for POE7 in Table B2. The lowest temperature of these data is $280 \mathrm{~K}$. A total of 161 data points are reported in Table B3 for POE9. Due to solidification of the sample, the lowest temperature of these measurements was limited to $290 \mathrm{~K}$. A total of 214 data points are reported for the MIL-PRF-23699 sample in Table B4. The lowest temperature of these data is 290 K. POE9 has the highest viscosity because it is the largest and most complex molecule with strong interlocking possibilities of the nonanoate side chains. As the length of these side chains decreases from POE9 to POE7 and POE5, so decreases the viscosity of the compounds. The viscosity of the MIL-PRF-23699 sample is close to that of POE9.

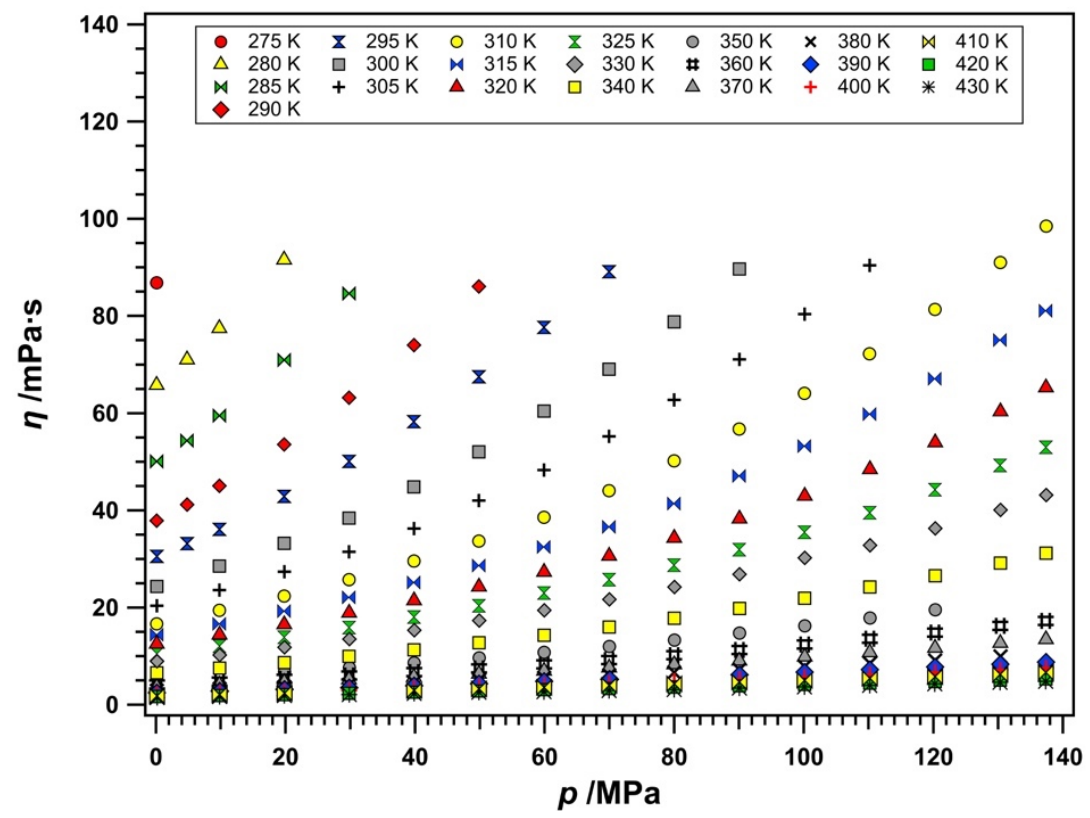

Figure 31: Viscosity data for POE5 as a function of pressure measured along isotherms at temperatures from $275 \mathrm{~K}$ to $430 \mathrm{~K}$. 


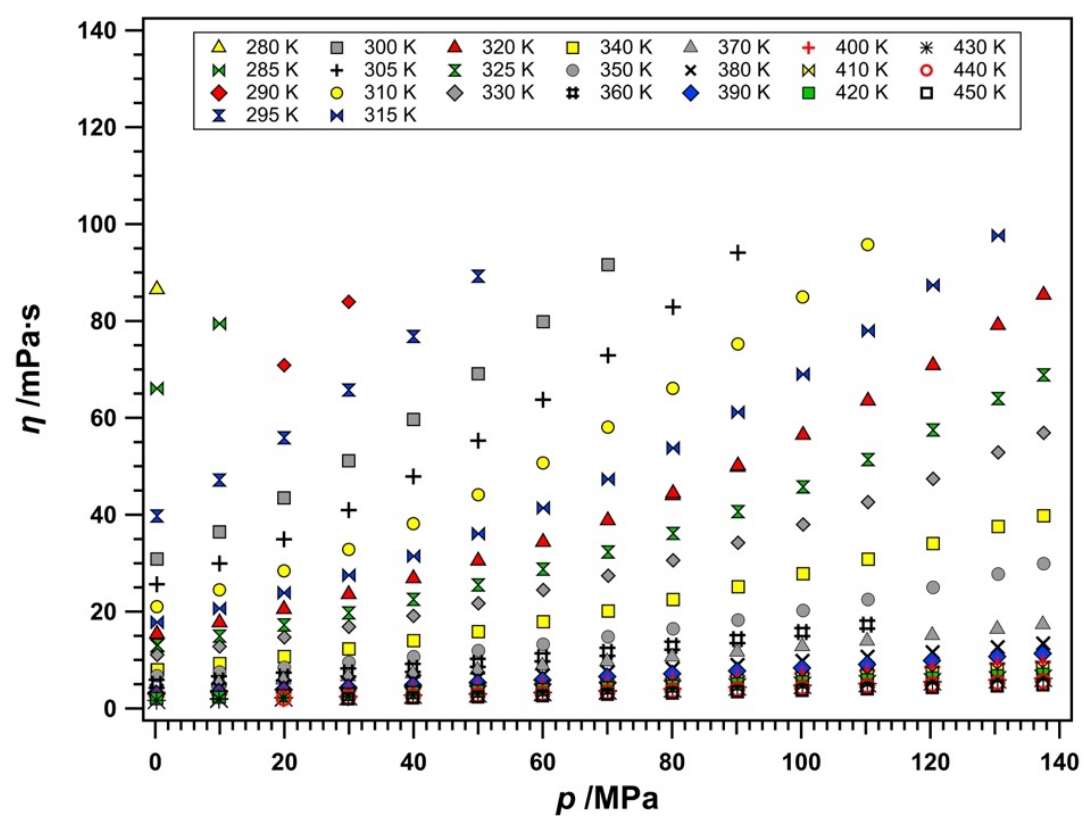

Figure 32: Viscosity data for POE7 as a function of pressure measured along isotherms at temperatures from $280 \mathrm{~K}$ to $450 \mathrm{~K}$.

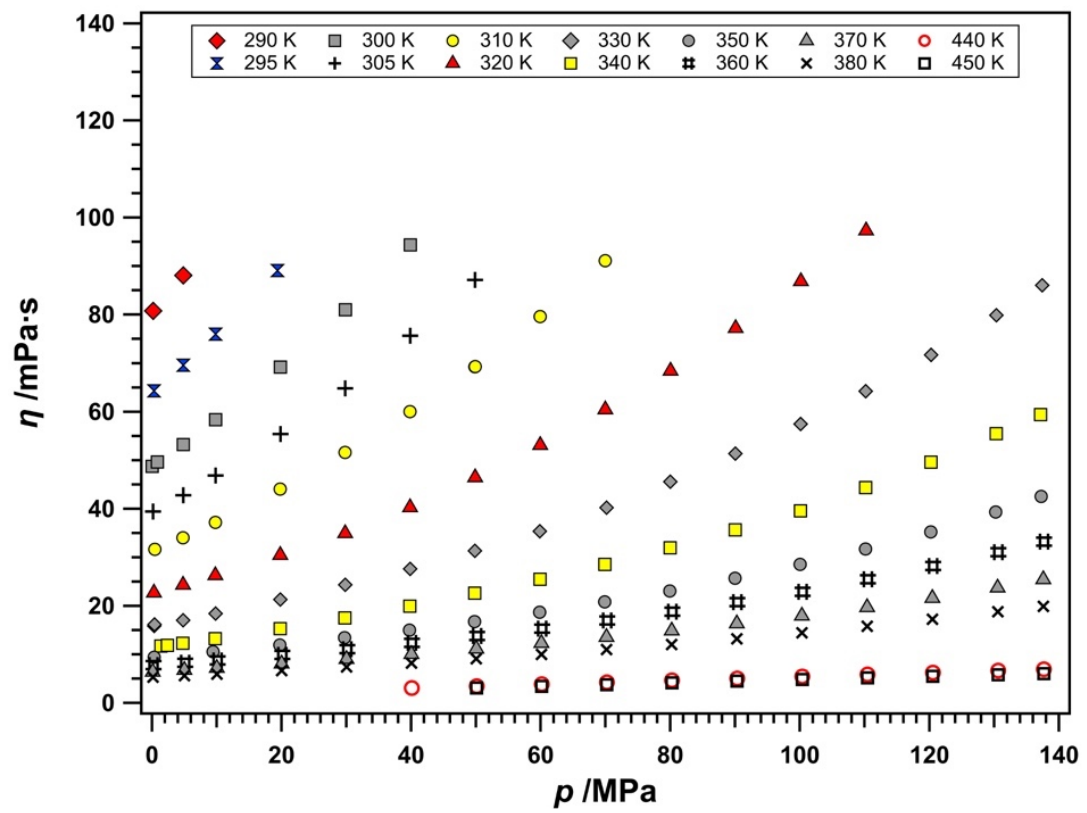

Figure 33: Viscosity data for POE9 as a function of pressure measured along isotherms at temperatures from $290 \mathrm{~K}$ to $450 \mathrm{~K}$. 


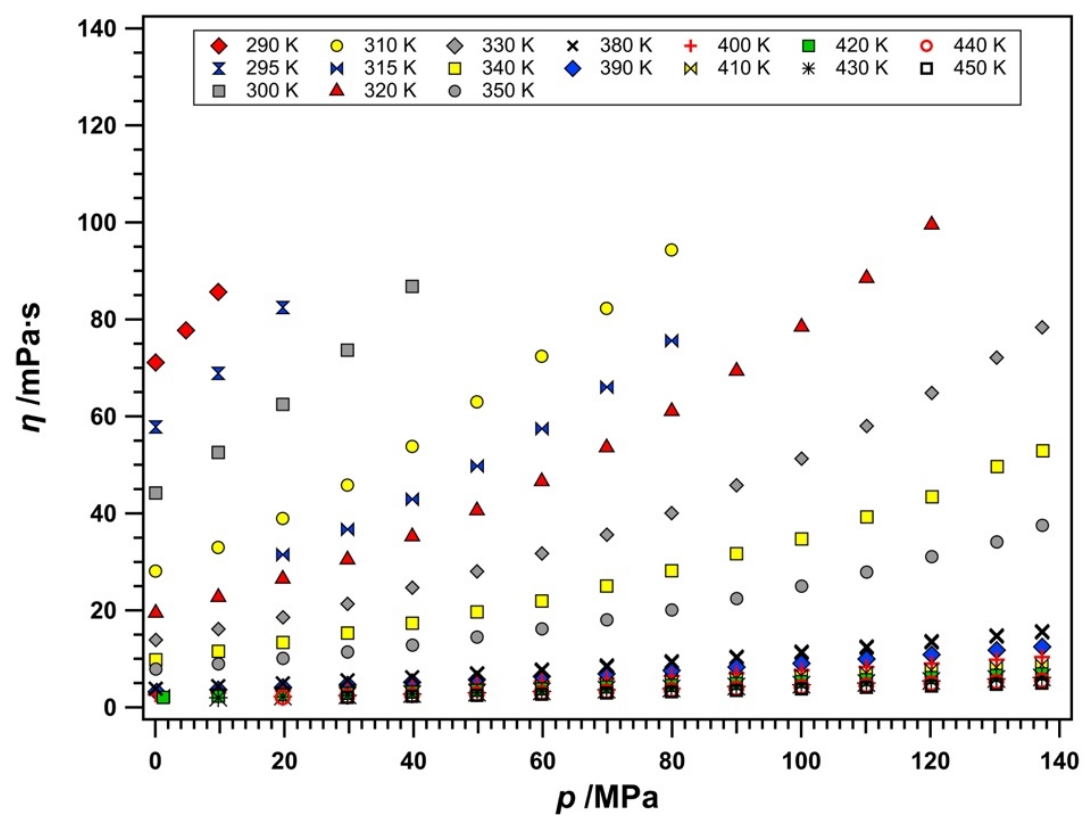

Figure 34: Viscosity data for MIL-PRF-23699 as a function of pressure measured along isotherms at temperatures from $290 \mathrm{~K}$ to $450 \mathrm{~K}$.

While the calibration with squalane ensures the mutual consistency of the measurements with the three sensing pistons within $\pm 3 \%$ below $340 \mathrm{~K}$ and $2 \%$ above $340 \mathrm{~K}$, it is difficult to assess the total uncertainty of the viscosity measurements with this instrument rigorously. This would require an in-depth analysis of the fluid mechanics and secondary flows around the alternating sensing pistons as was done for falling body viscometers by Schaschke et al.[54] Next, it would be necessary to derive the working equation that relates the period of the piston alternation to the viscosity of the sample taking into account the effect of thermal expansion and of sample compression on the viscometer tube and the sensing pistons. Absent such analysis, and based on our experience with falling body viscometers, $[55,56]$ the expanded uncertainty of the present measurements is estimated conservatively from $5 \%$ at atmospheric pressure to $10 \%$ at the maximum pressure of $137 \mathrm{MPa}$. 


\section{Advanced Distillation Curve of MIL-PRF-23699}

\subsection{Summary}

The advanced distillation curve methodology was applied at atmospheric pressure, in a noble gas environment, to a sample of MIL-PRF-23699 to establish an initial boiling temperature. Due to decomposition of the heated fluid, the distillation was carried out to only $10 \%$ distillate volume fraction. In this fraction, some of the additives were observed, in addition to decomposition products such as carboxylic acids; no polyol ester base oils were observed. No initial boiling temperature is defined under the conditions tested, because the fluid was not thermally stable.

\subsection{Advanced Distillation Curve Applied to MIL-PRF-23699}

The Advanced Distillation Curve[57, 58] was applied at atmospheric pressure to a sample of a qualified aviation turbine engine lubricant (MIL-PRF-23699). Since the boiling range of this fluid was predicted to be very high (boiling point reported by the manufacturer was $672 \mathrm{~K}$ at $3.98 \mathrm{kPa}$ ), the main objective was to establish an initial boiling temperature.

\subsubsection{Experimental Method}

A round bottom flask was filled with $200 \mathrm{~mL}$ of the fluid by volumetric pipette. Due to the viscous nature of the fluid, a small amount of sample was lost to the walls of the pipette. To reduce oxidative reactions, argon was bubbled through the fluid for approximately 10 minutes prior to heating. A flow of argon was also maintained on top of the fluid throughout heating to keep the environment free of oxygen; the flow was low enough to prevent cooling and displacement of the heated fluid. Two temperatures were recorded throughout the measurement, one in the fluid (kettle temperature, $T_{\mathrm{k}}$ ) and one in the vapor phase (head temperature, $T_{\mathrm{h}}$ ).

\subsection{Results}

The liquid mixture was heated to and maintained at $573 \mathrm{~K}$ for 30 minutes; no change in the fluid was observed. Further heating of the fluid in the kettle $\left(T_{\mathrm{k}}\right)$ to $620 \mathrm{~K}$ caused the colorless/faintly yellow fluid to become red $\left(T_{\mathrm{h}}=328 \mathrm{~K}\right)$. The fluid continued to darken with increased heating, becoming an opaque brown fluid. The first bubble in the fluid was observed at a kettle temperature $\left(T_{\mathrm{k}}\right)$ of $622 \mathrm{~K}\left(T_{\mathrm{h}}=333 \mathrm{~K}\right)$. A sustained boil was observed at $T_{\mathrm{k}}=639 \mathrm{~K}$ and $T_{\mathrm{h}}=361 \mathrm{~K}$ and the first drop of fluid was collected at $T_{\mathrm{k}}=649 \mathrm{~K}$ and $T_{\mathrm{h}}=399 \mathrm{~K}$. The fluid temperature was maintained between $643 \mathrm{~K}$ and $653 \mathrm{~K}$ for approximately 30 minutes; the head temperature $\left(T_{\mathrm{h}}\right)$ remained consistent between $400 \mathrm{~K}$ and $401 \mathrm{~K}$. The $10 \%$ volume fraction was collected at $T_{\mathrm{k}}=656 \mathrm{~K}$ and $T_{\mathrm{h}}=419 \mathrm{~K}$. 
Samples of the first drop, $10 \%$ volume fraction, and the remaining kettle fractions were diluted in hexane and analyzed by GC-MS. The first drop contained predominately species associated with the thermal decomposition of the fluid. Species included carboxylic acids, in addition to methyl esters of the acids, the corresponding aldehyde, alkanes, and larger species. The composition of the $10 \%$ fraction was similar to the composition of the 1 st drop. As expected, the $10 \%$ fraction contained a larger portion of species with higher boiling points than the first drop fraction. The remaining fluid in the kettle, although a very dark brown color, still contained primarily unreacted polyol esters, antioxidants, and anti-wear agents. Additional details of the analysis are proprietary and are administratively restricted. 


\section{Modeling}

\subsection{Summary}

Equations of state were developed for the pure fluids POE5, POE7, and POE9, and for the fully qualified lubricant (MIL-PRF-23699, formulated as a pseudo-pure fluid). The equations are formulated in terms of Helmholtz energy with independent variables of temperature and density. Equations expressed in terms of the Helmholtz energy have the advantage that properties are simple derivatives of this fundamental property, including those that cannot be measured, such as entropy. The experimental data measured as part of this project were used in a regression procedure to determine the coefficients of the equations of state. All densities measured in this work, including those measured in the atmospheric-pressure densimeter and those measured in the highpressure densimeter, were fitted to within $0.2 \%$, speed of sound to within $\sim 0.1 \%$, and the isobaric heat capacity to within $\sim 0.3 \%$. All equations extrapolate in a physically reasonable manner.

Viscosity models were developed for the pure fluids POE5, POE7, and POE9, and for the fully qualified lubricant (formulated as a pseudo-pure fluid). These correlations represent the viscosity of the liquid phase at pressures up to $140 \mathrm{MPa}$ to within 5 to $10 \%$, which is the estimated uncertainty of the experimental data. Similarly, correlations for the thermal conductivity of the pure fluids POE5, POE7, and POE9, and for the fully qualified lubricant (formulated as a pseudopure fluid) were developed that represent the thermal conductivity of the liquid phase at pressures up to $70 \mathrm{MPa}$ to within $0.4 \%$ to $0.7 \%$ depending on the fluid. The estimated uncertainty of the experimental data is $0.5 \%$. Two surrogate mixture models were developed using the base fluids POE5, POE7, and POE9. One focused on viscosity, and the other on thermodynamic properties. Neither were capable of representing the experimental data to within or near the experimental uncertainty, and we recommend the use of the pseudo-pure fluid model for the properties of the fully qualified lubricant.

\subsection{Equations of State}

Equations of state are tools to calculate the thermodynamic properties of pure fluids and mixtures, and have often been expressed as a function of pressure with independent variables of temperature and density. Modern-day equations of state now use the Helmholtz energy (also with independent variables of temperature and density) to enable the calculation of all thermodynamic properties. Equations expressed in terms of the Helmholtz energy have the advantage that properties are simple derivatives of this fundamental property, including those that cannot be measured, such as entropy. Equations expressed in terms of pressure require integration to calculate caloric properties (such as enthalpies and sound speeds) and are thus no longer used for high-accuracy applications. 
Through density $(\rho)$ derivatives of the Helmholtz energy, one can obtain properties such as pressure $(p)$, and its isothermal derivatives, such as $\mathrm{d} p / \mathrm{d} \rho$. At zero density, these properties are generally zero or simple values of temperature ( $T$ ) (for example, $\mathrm{d} p / \mathrm{d} \rho=R T$ at $p=0$, where $R$ is the gas constant). Non-ideal gas properties $(p>0)$ require a "real-gas" contribution (i.e., departure from ideal-gas behavior), which accounts for the interaction between molecules.

Unlike pressure and its derivatives, caloric properties require derivatives with respect to temperature, and this adds an additional complication. The calculation of caloric properties includes, in addition to the real-gas contribution, an ideal-gas contribution at zero pressure, which is represented by an equation that describes the isobaric heat capacity at zero pressure. This property can be obtained in a number of ways, through either experimental methods, or, for simple molecules, through simulation or group-contribution methods. When no such data or methods are available, the development of equations of state can become substantially more difficult, but is possible with current techniques as explained further below.

One of the most important properties in the development of equations of state is the critical temperature because of its use as a reducing parameter. This value is generally known for most fluids, especially for those where the critical temperature is less than $\sim 450 \mathrm{~K}$. At higher temperatures, fluids typically decompose below the critical temperature, but measurements that require the fluid to be at high temperatures for a small amount of time can obtain close estimates of the critical point. For the oils studied in this work, the critical temperatures are well beyond the decomposition limit, and no direct measurement is possible. Other methods are available that can be used for the estimation of the critical point properties, but the uncertainties in the calculated values are often much larger than that required to develop an equation of state. This work is one of the first to attempt to fit an equation of state where the critical temperature was only known within $~ 100 \mathrm{~K}$.

The critical density is also used as a reducing parameter in an equation of state. Because of the high uncertainty in the measurement of the density at the critical point, its value is almost always fitted simultaneously with the other parameters, and then compared to measured values to ensure it lies within the uncertainties from other sources. If the critical temperature were known, then an estimated value of the critical density could be obtained simply by the ratio of $T / T_{\mathrm{c}}$. For example, at a reduced value of $0.5 \times T_{c}$, the critical density would be about one-third the value of the densities in the liquid phase. At about $0.3 \times T_{\mathrm{c}}$, the critical density is about one-fourth the value of the liquid- 
phase densities. Thus, for example, for POE5 an estimated value of the critical density could be obtained as 0.25 to 0.33 of the liquid-phase values. From the final equation of state for POE5, the value of the liquid-phase density at $300 \mathrm{~K}\left(0.34 \times T_{\mathrm{c}}\right)$ showed it to be around $0.36 \times \rho_{\mathrm{c}}$.

The unknown value of the critical density was bound to the even more elusive value of the critical temperature. No previous work had ever demonstrated if the critical temperature could be determined for fluids where the temperatures of the measured properties are only a fraction of the critical temperature. This was initially thought to not be possible because only densities, heat capacities, and speeds of sound were being fitted. Through months of fitting, the sensitivity of the critical temperature in a high-accuracy equation of state became apparent, where "high-accuracy" in this work refers to the limited region where the measurements are available. Fitting each of the oils and the mixture showed that the critical temperature could only be varied within about 10 to 20 degrees while still maintaining low uncertainties in the measured data.

Recently, one of the key factors in the development of equations of state has become the rectilinear diameter. This value is the average of the liquid and vapor densities at saturation. Through significant practical experience, this property has been key to obtaining correct vapor-phase values when no measurements are available in that phase. The rectilinear diameter, in effect, mirrors the properties of the liquid phase onto those in the vapor phase and helps ensure the saturation dome and corresponding vapor properties are at least representative of the true fluid properties. Other constraints placed on the equation of state during its development contribute to the correct behavior in this phase, as explained in Section 12.2.3. These constraints involve properties, such as the phase identification parameter (PIP) and Gruneisen parameter, that are generally unfamiliar to all except those that fit equations.

The importance of correct vapor-phase properties comes into play when developing the ideal-gas heat capacity equation discussed earlier, and that is needed to obtain the ideal-gas part of the equation of state for caloric properties such as heat capacities, speeds of sound, enthalpies, and entropies. The ideal-gas heat capacities are related to the liquid-phase heat capacities by the contribution from the heat capacity in the vapor phase and from the second derivative of the vapor pressure curve with respect to temperature. Neither the vapor-phase properties nor vapor pressures at ambient conditions or higher are known; but with the techniques discussed above, the vaporphase properties can be at least qualitatively represented by the current state-of-the-art fitting in equations of state, and the vapor pressure can be extrapolated to higher temperatures to some degree based on the values available at very low pressures. 
The ability to make an equation with so many unknown properties that are generally required in fitting, which can then represent those properties with some degree of accuracy in regions far outside that of the available measurements, is made possible through very high accuracy data for density, speed of sound, and heat capacity in the liquid phase, along with the vapor pressure data at very low pressure. The key, however, to this success stems from not only the types and temperature ranges of the data, but more importantly from the high-pressure range of the data. If data had only been measured at saturation, the determination of the critical point would not have been possible. The high-pressure data enabled the ability to control the slope and curvature of the Helmholtz energy equation in the liquid phase; however, with the extensive temperature-pressure grid of data measured in this work, the derivatives of this fundamental thermodynamic property are fixed, and an entire surface can be extended far beyond the limits of the data. Because the surface is fixed due to the range and accuracy of the data, when done carefully, the surface can be extended to the critical point, and then into the vapor phase with state-of-the-art fitting techniques.

\subsubsection{Functional Form of the Equation of State}

Equations of state based on the Helmholtz energy are commonly expressed in a dimensionless form with dimensionless independent variables of density $\delta=\rho / \rho_{\mathrm{c}}$ and reciprocal temperature $\tau$ $=T_{\mathrm{C}} / T$. The critical point properties are given in Table 26. The form of this equation is

$$
\frac{a(\rho, T)}{R T}=\alpha(\delta, \tau)=\alpha^{0}(\delta, \tau)+\alpha^{\mathrm{r}}(\delta, \tau)
$$

The Helmholtz energy of the ideal gas is given by

$$
a^{0}=h^{0}-R T-T s^{0} .
$$

The ideal-gas enthalpy and entropy are given by

$$
\begin{gathered}
h^{0}=h_{0}^{0}+\int_{T_{0}}^{T} c_{p}^{0} \mathrm{~d} T \text { and } \\
S^{0}=S_{0}^{0}+\int_{T_{0}}^{T} \frac{C_{p}^{0}}{T} \mathrm{~d} T-R \ln \left(\frac{\rho T}{\rho_{0} T_{0}}\right),
\end{gathered}
$$


where $c_{p}{ }^{0}$ is the ideal-gas heat capacity. The subscript 0 for density and temperature indicate ideal gas properties, where $\rho_{0}$ is the ideal-gas density at $T_{0}$ and $p_{0}\left[\rho_{0}=p_{0} /\left(T_{0} R\right)\right]$, and $T_{0}$ and $p_{0}$ are arbitrary reference states. Combining these equations results in the following equations for the dimensional and dimensionless Helmholtz energies of the ideal gas,

$$
\begin{gathered}
a^{0}=h_{0}^{0}+\int_{T_{0}}^{T} c_{p}^{0} \mathrm{~d} T-R T-T\left[s_{0}^{0}+\int_{T_{0}}^{T} \frac{c_{p}^{0}}{T} \mathrm{~d} T-R \ln \left(\frac{\rho T}{\rho_{0} T_{0}}\right)\right] \text { and } \\
\alpha^{0}=\frac{h_{0}^{0} \tau}{R T_{\mathrm{c}}}-\frac{s_{0}^{0}}{R}-1+\ln \frac{\delta \tau_{0}}{\delta_{0} \tau}-\frac{\tau}{R} \int_{\tau_{0}}^{\tau} \frac{c_{p}^{0}}{\tau^{2}} \mathrm{~d} \tau+\frac{1}{R} \int_{\tau_{0}}^{\tau} \frac{c_{p}^{0}}{\tau} \mathrm{d} \tau,
\end{gathered}
$$

where $\delta_{0}=\rho_{0} / \rho_{\mathrm{c}}$ and $\tau_{0}=T_{\mathrm{c}} / T_{0}$.

\begin{tabular}{|c|c|c|c|c|}
\hline Substance & $\begin{array}{c}M \\
/ \mathrm{g} \cdot \mathrm{mol}^{-1}\end{array}$ & $\begin{array}{l}T_{\mathbf{c}} \\
/ \mathbf{K}\end{array}$ & $\begin{array}{c}p_{\mathbf{c}} \\
/ \mathbf{M P a}\end{array}$ & $\begin{array}{c}\rho_{c} \\
/ \mathbf{m o l}^{\prime} \mathbf{d m}^{-3}\end{array}$ \\
\hline POE5 & 472.612 & 890.0 & 1.27 & 0.556 \\
\hline POE7 & 584.835 & 940.0 & 1.03 & 0.412 \\
\hline POE9 & 697.051 & 970.0 & 0.885 & 0.316 \\
\hline MIL-PRF-23699 & 557.6 & 930.0 & 1.08 & 0.439 \\
\hline
\end{tabular}

Table 26: Molar Mass and Critical Point Parameters.

Models for the calculation of real fluid properties of fluids are abundant, requiring any number of terms depending on the complexity of the method. Fitting methods are often used to determine the coefficients of a particular model, being fitted to experimental data for the fluid and constrained by various criteria explained in some detail here and in more detail in other work, such as that of Span and Wagner[59], Lemmon and Jacobsen[60], and Lemmon et al.[39]

The equation used to describe the ideal-gas heat capacities used for the oils is given by

$$
\frac{c_{p}^{0}}{R}=v_{0}+\sum_{i=1}^{2} v_{i}\left(\frac{u_{i}}{T}\right)^{2} \frac{\exp \left(u_{i} / T\right)}{\left[\exp \left(u_{i} / T\right)-1\right]^{2}},
$$

where the parameters are given in Table 27. This is the Planck-Einstein functional form; it was used so that the shape of the ideal-gas heat capacity versus temperature would be similar to that derived from statistical mechanical models. 
Table 27: Coefficients of the Ideal-Gas Heat Capacity Equation.

\begin{tabular}{l|r|r|r|r}
\hline & POE5 & POE7 & POE9 & MIL-PRF-23699 \\
\hline \hline$v_{0}$ & 30.0 & 55.0 & 75.0 & 50.0 \\
$v_{1}$ & 850.0 & 1100.0 & 1000.0 & 1000.0 \\
$v_{2}$ & 2000.0 & 3000.0 & 2700.0 & 2000.0 \\
$u_{1}$ & 69.0 & 109.0 & 93.0 & 78.0 \\
$u_{2}$ & 98.0 & 240.5 & 360.0 & 105.0 \\
\hline
\end{tabular}

The real-gas contribution comes from the equation of state given further below. The parameters in this equation were fitted by minimizing the deviations between the experimental data measured in this work and the calculations from the equation. The fitting program is described by Lemmon and Jacobsen [60] and Lemmon et al.[39] In effect, each data point that is fitted is given a weight that is multiplied by the square of the deviation between the experimental point and the calculated value. The sum of these squared values multiplied by a weighting value, assigned by the one doing the fit, is then calculated. Additionally, a number of constraints are applied to ensure that the equation extrapolates well to very low temperatures and to high temperatures and pressures as explained in Section 12.2.3. This is necessary to use the developed EOS in mixture modeling where the equations used in the mixture often require calculations from the pure-fluid equation of state that are beyond the limits of the experimental data (in particular, below the triple point of the pure fluid). This is the modern practice in fitting equations of state and is done in all high-accuracy equations as described in multiple documents available in the literature.

The final functional form was determined through months of fitting POE5, POE7, and POE9 separately, but with each receiving about two weeks of fitting at a time. The approach used to develop the final equations of state was to develop a single equation that used the same exponents on density, temperature, and the parameters for the Gaussian terms (explained below), and to allow only the coefficients of the equation for each oil to change. This required several weeks of fitting one of the three pure fluids, and then the functional form consisting of all coefficients, exponents, parameters, and so on would be transferred to a different oil and the process would continue. Each transfer generally resulted in failure of the fit for the next oil in reproducing the experimental data based on fitting new coefficients only. In these situations, the exponents and other parameters were again fitted, and the process continued. After many iterations, a set of parameters began to emerge that would transfer easily from one fluid to the next.

The final set of parameters was developed during the fitting of POE7. After substituting these parameters into the fit for POE5, a new set of coefficients was fitted within an extremely short 
amount of time (compared to the work that was required up to this point), and this set of parameters was deemed adequate for these two fluids.

However, when applied to POE9, the situation was a bit different. The sample that was used to measure the properties of POE9 had nearly double the amount of impurities than in the samples delivered for POE5 and POE7. This resulted in twice the uncertainty in the molar mass of the substance. Densities and heat capacities were measured on a mass basis, and thus the density needs to be divided by the molar mass to obtain molar densities, and the heat capacities must be multiplied by the molar mass to obtain the molar properties required in fitting. The thermodynamic equation used to calculate the speed of sound from the Helmholtz energy is a function of the square root of the molar mass. Because of the lack of the true molar mass, our current program used to fit equations of state required modification to fit mass density and heat capacity while allowing the molar mass to change.

Ironically, due to this complication and the unknown values of the critical temperature and critical density, an optimum set of values for these three properties and the coefficients could not produce an equation that both represented the liquid-phase measurements and the vapor pressures. The substance delivered to NIST and labeled as POE9 is really a mixture of this substance and a number of other substances (as indicated in Table 4), and fitting this mixture to a pure fluid equation of state is yet another cause of the inability to fit it with the same uncertainties in vapor pressure as those for POE5 and POE7. The final equation was developed with much lower weights on the vapor pressures to obtain deviations in the other properties that were still generally within the uncertainties in those measurements. The average deviations in the measurements are larger (especially for vapor pressure) than is the case for those for POE5 and POE7. The deviations are discussed in the next section.

The functional form for the final equations of state for all three oils and the pseudo-pure fluid model for MIL-PRF-23699 is given below:

$$
\begin{aligned}
\alpha^{\mathrm{r}}(\delta, \tau) & =\sum N_{k} \delta^{d_{k}} \tau^{t_{k}}+\sum N_{k} \delta^{d_{k}} \tau^{t_{k}} \exp \left(-\delta^{l_{k}}\right) \\
& +\sum N_{k} \delta^{d_{k}} \tau^{t_{k}} \exp \left(-\eta_{k}\left(\delta-\varepsilon_{k}\right)^{2}-\beta_{k}\left(\tau-\gamma_{k}\right)^{2}\right)
\end{aligned}
$$


The first summation is a simple polynomial expression in terms of temperature and density, the second summation is composed of polynomial-exponential terms, and the third summation contains Gaussian bell-shaped terms (see Span and Wagner[59]). The exponents on temperature and density and the parameters inside the Gaussian terms are identical for all fluids and are given in Table 28. The coefficients $N_{k}$ for each fluid are given in Table 29.

\section{Table 28: Parameters of the Equation of State.}

\begin{tabular}{l|l|l|l|l|l|l|l}
\hline $\boldsymbol{k}$ & $\boldsymbol{t}_{\boldsymbol{k}}$ & $\boldsymbol{d}_{\boldsymbol{k}}$ & $\boldsymbol{l}_{\boldsymbol{k}}$ & $\boldsymbol{\eta}_{\boldsymbol{k}}$ & $\boldsymbol{\beta}_{\boldsymbol{k}}$ & $\boldsymbol{\gamma}_{\boldsymbol{k}}$ & $\boldsymbol{\varepsilon}_{\boldsymbol{k}}$ \\
\hline \hline 1 & 1.0 & 5 & & & & & \\
2 & 0.08 & 1 & & & & & \\
3 & 1.2 & 1 & & & & & \\
4 & 1.162 & 2 & & & & & \\
5 & 0.54 & 3 & & & & & \\
6 & 1.08 & 2 & 1 & & & & \\
7 & 1.56 & 3 & 1 & & & & \\
8 & 1.78 & 3 & 1 & & & & \\
9 & 1.42 & 4 & 1 & & & & \\
10 & 1.62 & 5 & 1 & & & & \\
11 & 2.0 & 1 & & 1.0 & 0.36 & 1.57 & 1.7 \\
12 & 1.0 & 2 & & 1.31 & 0.35 & 1.45 & 0.55 \\
13 & 1.0 & 4 & & 0.46 & 0.55 & 1.63 & 0.95 \\
14 & 1.0 & 3 & & 20.0 & 1000.0 & 1.09 & 0.91 \\
\hline
\end{tabular}


Table 29: Coefficients $\left(N_{k}\right)$ of the Equation of State.

\begin{tabular}{l|c|c|c|c}
\hline $\boldsymbol{k}$ & POE5 & POE7 & POE9 & MIL-PRF-23699 \\
\hline \hline 1 & 0.0106971 & 0.00996664 & 0.00811 & 0.00990044 \\
2 & 1.510321 & 1.801441 & 1.446665 & 1.633544 \\
3 & -2.596894 & -2.872737 & -2.303871 & -2.6606 \\
4 & -2.311273 & -2.347331 & -2.320483 & -2.328001 \\
5 & 0.3332414 & 0.3403607 & 0.336244 & 0.3281252 \\
6 & 2.500028 & 2.550799 & 2.383751 & 2.4664585 \\
7 & 7.527764 & 7.625433 & 8.210469 & 7.657825 \\
8 & -5.405529 & -5.44993 & -6.200227 & -5.569829 \\
9 & 0.94954 & 0.8612612 & 0.813201 & 0.914727 \\
10 & 0.3303842 & 0.3734221 & 0.377317 & 0.3668741 \\
11 & -0.0234605 & -0.00711446 & 0.0023328 & -0.007457 \\
12 & 0.0156448 & 0.02289254 & 0.0217847 & 0.028916 \\
13 & -0.0092188 & -0.00893703 & -0.009014 & -0.0103879 \\
14 & -0.5415831 & -0.75359521 & -1.54251 & -0.645008 \\
\hline
\end{tabular}

\subsubsection{Experimental Data and Comparisons to the Equation of State}

The accuracy of the equation of state can be determined by statistical comparisons of calculated property values to experimental data. These statistics are based on the percent deviation in any property, $X$, defined as

$$
\Delta X=100\left(\frac{X_{\text {data }}-X_{\text {calc }}}{X_{\text {data }}}\right)
$$

With this definition, the average absolute deviation is defined as

$$
\mathrm{AAD}=\frac{1}{n} \sum_{i=1}^{n}\left|\Delta X_{i}\right|
$$

where $n$ is the number of data points. Table 30 lists the AAD values between the experimental data and those calculated from the equation of state. Comparisons given in the following paragraphs use the average absolute deviations given by Eq. 21 unless otherwise stated (such as the maximum value). Discussions of maximum errors or of systematic offsets use the absolute values of the deviations, unless otherwise noted. In the plots, data points with excessive deviations are shown at the outer limits to indicate where these outliers were measured. 
Table 30: Summary of Experimental Data.

\begin{tabular}{|c|c|c|c|c|c|}
\hline Apparatus & $\begin{array}{l}\text { No. of } \\
\text { points }\end{array}$ & $\begin{array}{c}\mathbf{T} \\
/ \mathbf{K} \\
\end{array}$ & $\begin{array}{c}p \\
/ \mathrm{MPa} \\
\end{array}$ & $\begin{array}{c}\rho \\
/ \mathbf{m o l}^{-} \mathbf{d m}^{-3} \\
\end{array}$ & $\begin{array}{c}\text { AAD } \\
/ \% \\
\end{array}$ \\
\hline \multicolumn{6}{|c|}{ POE5 } \\
\hline \multicolumn{6}{|l|}{ Density } \\
\hline High-pressure densimeter & 165 & $270-470$ & $0.5-50$ & $1.85-2.25$ & 0.06 \\
\hline Atmospheric-pressure densimeter & 14 & $278-343$ & 0.083 & $2.07-2.18$ & 0.048 \\
\hline \multicolumn{6}{|l|}{ Speed of Sound } \\
\hline Atmospheric-pressure instrument & 14 & $278-343$ & 0.083 & & 0.027 \\
\hline High-pressure pulse-echo & 68 & $283-423$ & $0.001-63$ & & 0.052 \\
\hline \multicolumn{6}{|l|}{ Heat Capacity } \\
\hline DSC & 170 & $263-432$ & 0.083 & & 0.097 \\
\hline \multicolumn{6}{|l|}{ Vapor pressure } \\
\hline Gas saturation \& literature & 10 & $334-413$ & & & 11.7 \\
\hline \multicolumn{6}{|c|}{ POE7 } \\
\hline \multicolumn{6}{|l|}{ Density } \\
\hline High-pressure densimeter & 161 & $270-470$ & $0.511-50$ & $1.45-1.73$ & 0.063 \\
\hline Atmospheric-pressure densimeter & 14 & $278-343$ & 0.083 & $1.61-1.69$ & 0.053 \\
\hline \multicolumn{6}{|l|}{ Speed of Sound } \\
\hline Atmospheric-pressure instrument & 14 & 278-343 & 0.083 & & 0.077 \\
\hline High-pressure pulse-echo & 49 & $283-423$ & $0.059-69.1$ & & 0.081 \\
\hline \multicolumn{6}{|l|}{ Heat Capacity } \\
\hline DSC & 125 & 293-417 & 0.083 & & 0.088 \\
\hline \multicolumn{6}{|l|}{ Vapor pressure } \\
\hline Literature & 4 & $374-433$ & & & 4.4 \\
\hline \multicolumn{6}{|c|}{ POE9 } \\
\hline \multicolumn{6}{|l|}{ Density } \\
\hline High-pressure densimeter & 145 & $290-470$ & $0.506-50.1$ & $1.19-1.39$ & 0.051 \\
\hline Atmospheric-pressure densimeter & 13 & 283-343 & 0.083 & $1.32-1.38$ & 0.073 \\
\hline \multicolumn{6}{|l|}{ Speed of Sound } \\
\hline Atmospheric-pressure instrument & 13 & 283-343 & 0.083 & & 0.053 \\
\hline High-pressure pulse-echo & 41 & $293-423$ & $0.092-58.2$ & & 0.052 \\
\hline \multicolumn{6}{|l|}{ Heat Capacity } \\
\hline DSC & 100 & $313-412$ & 0.083 & & 0.267 \\
\hline \multicolumn{6}{|l|}{ Vapor pressure } \\
\hline Literature & 5 & $395-476$ & & & \\
\hline
\end{tabular}


Table 30, continued.

\begin{tabular}{|c|c|c|c|c|c|}
\hline Apparatus & $\begin{array}{l}\text { No. of } \\
\text { points }\end{array}$ & $\begin{array}{c}T \\
/ \mathbf{K} \\
\end{array}$ & $\begin{array}{c}p \\
/ \mathrm{MPa} \\
\end{array}$ & $\begin{array}{c}\rho \\
/ \mathbf{m o l}^{\prime} \cdot \mathbf{d m}^{-3} \\
\end{array}$ & $\begin{array}{c}\text { AAD } \\
/ \% \\
\end{array}$ \\
\hline \multicolumn{6}{|c|}{ MIL-PRF-23699 } \\
\hline \multicolumn{6}{|l|}{ Density } \\
\hline High-pressure densimeter & 161 & $270-470$ & $0.518-50$ & $1.54-1.85$ & 0.074 \\
\hline Atmospheric-pressure densimeter & 14 & 278-343 & 0.083 & $1.71-1.8$ & 0.068 \\
\hline \multicolumn{6}{|l|}{ Speed of Sound } \\
\hline Atmospheric-pressure instrument & 14 & 278-343 & 0.083 & & 0.04 \\
\hline High-pressure pulse-echo & 51 & 298-423 & $0.02-61.2$ & & 0.053 \\
\hline \multicolumn{6}{|l|}{ Heat Capacity } \\
\hline DSC & 220 & 273-492 & 0.083 & & 0.171 \\
\hline
\end{tabular}

All densities measured in this work, including those measured in the atmospheric-pressure densimeter and those measured in the high-pressure densimeter, were fitted to within $0.2 \%$ as shown in Figures 35 - 38. The average absolute deviations for each set of measurements and for each of the four fluids is between $0.05 \%$ and $0.07 \%$. The bias in the deviations between the equations of state and the density measurements for POE5 and POE7 are mostly negligible, whereas those for POE9 are $-0.03 \%$ and $-0.07 \%$ for the two data sets. This bias is caused by the higher impurities in the sample and the difficulty of fitting the equation as explained in section 12.2.2.

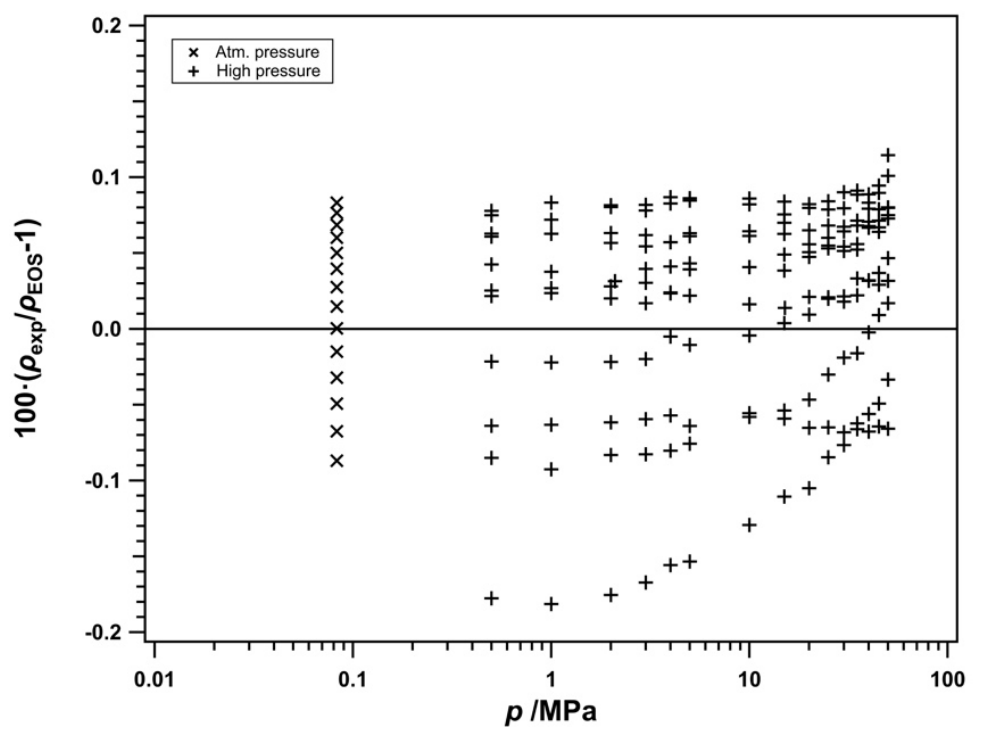

Figure 35: Comparisons of densities calculated with the equation of state to experimental data for POE5 as a function of pressure. 


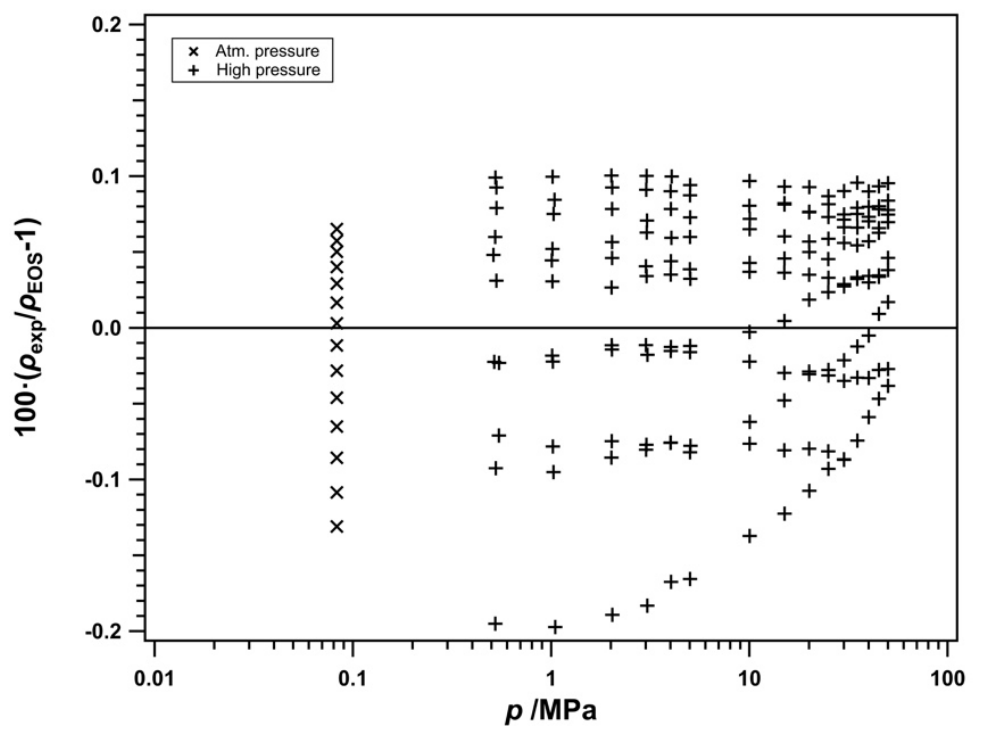

Figure 36: Comparisons of densities calculated with the equation of state to experimental data for POE7 as a function of pressure.

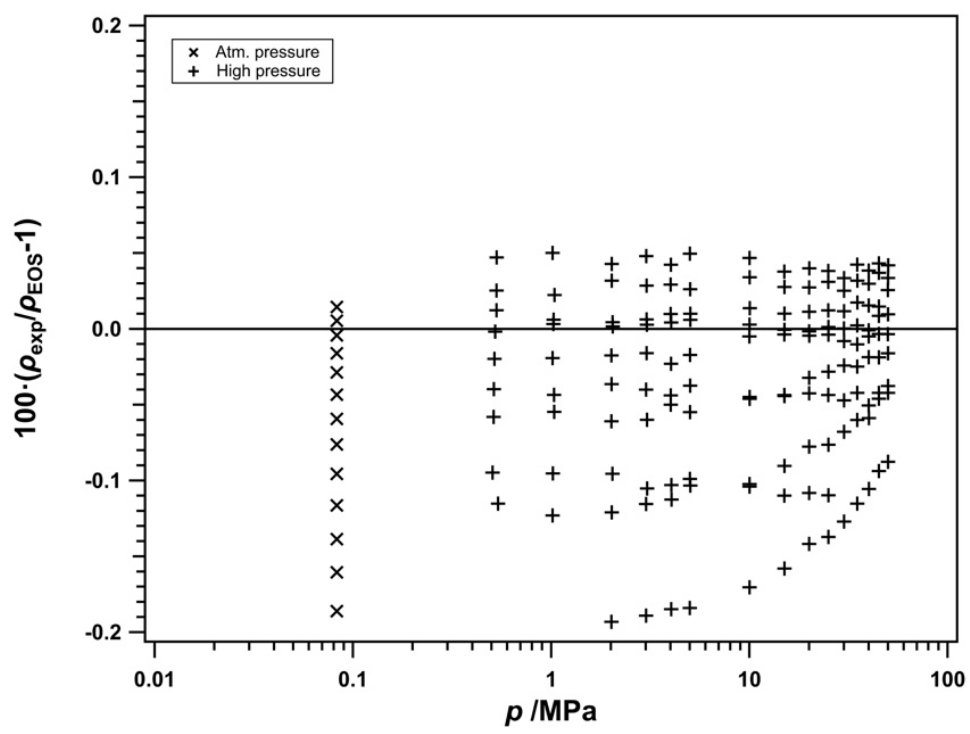

Figure 37: Comparisons of densities calculated with the equation of state to experimental data for POE9 as a function of pressure. 


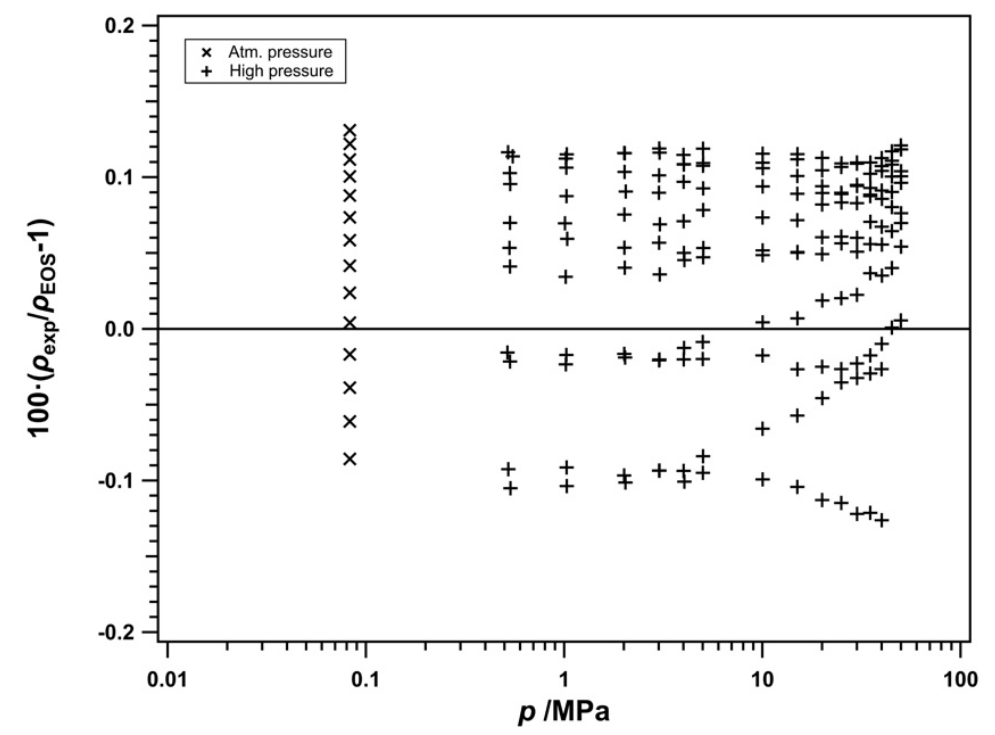

Figure 38: Comparisons of densities calculated with the equation of state to experimental data for MIL-PRF-23699 as a function of pressure.

Deviations between the equation of state and the measurements for the speed of sound given in this work are nearly identical for all four fluids. As shown in Figures 39 to 42, deviations generally range from $+0.1 \%$ to $-0.4 \%$. Most of the data lie between $\pm 0.1 \%$, except 3 to 6 points for each fluid. The average absolute deviations range between $0.05 \%$ for POE5, POE9, and MIL-PRF23699 to $0.08 \%$ for POE7. The equations of state represent both sets of measurements (those taken at atmospheric pressure and those taken at high pressure) with nearly equal distributions (excluding the few outliers below $-0.1 \%$ ).

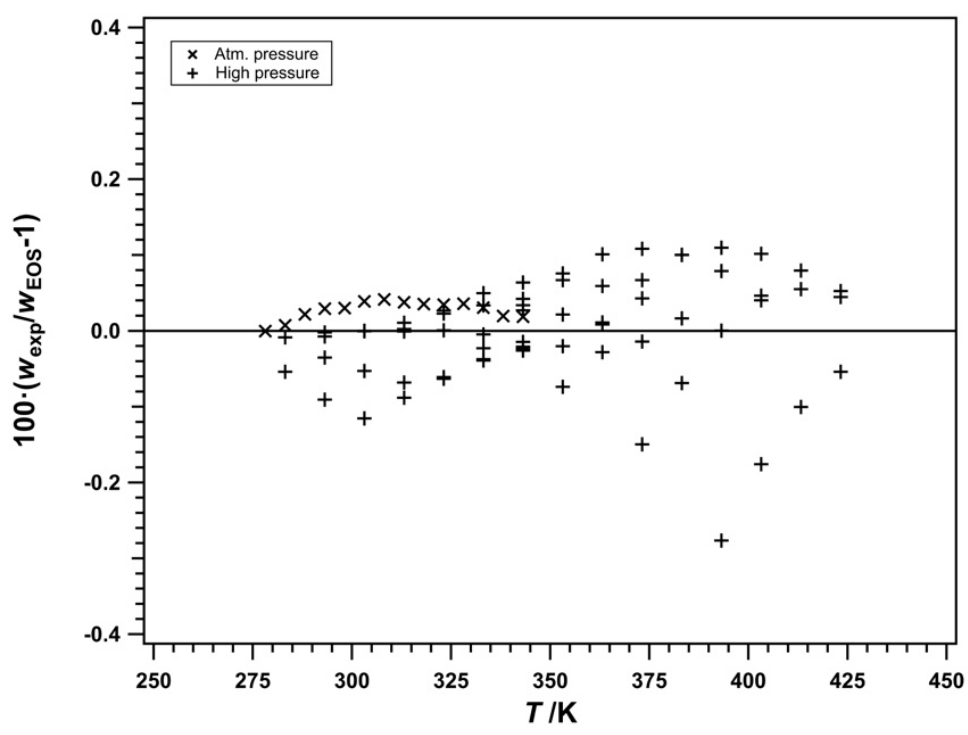

Figure 39: Comparisons of speeds of sound calculated with the equation of state to experimental data for POE5 as a function of temperature. 


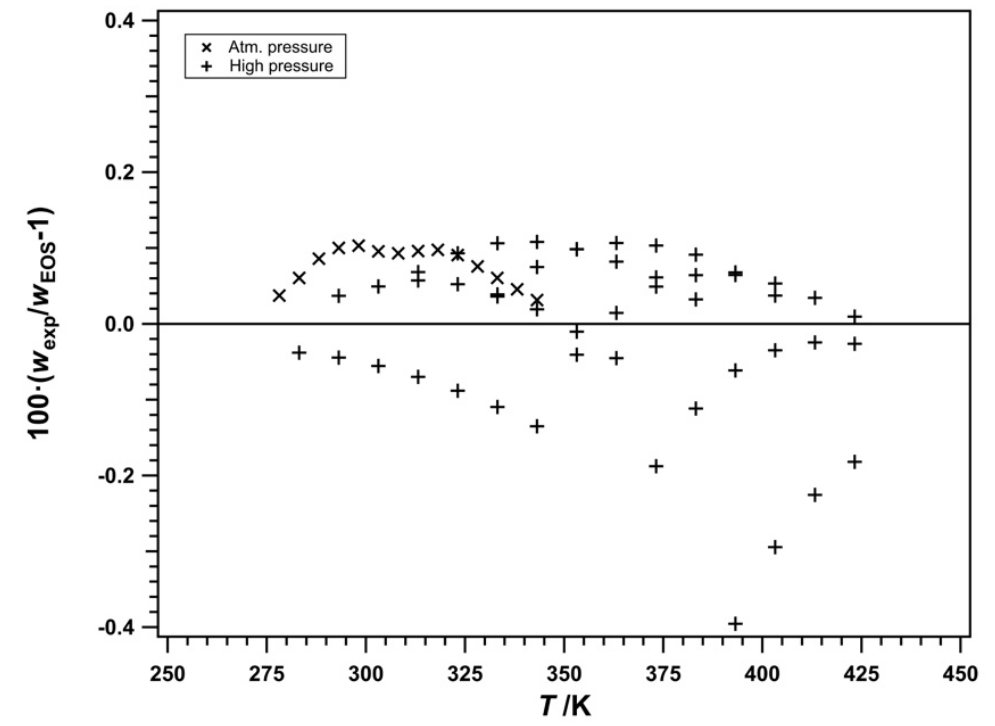

Figure 40: Comparisons of speeds of sound calculated with the equation of state to experimental data for POE7 as a function of temperature.

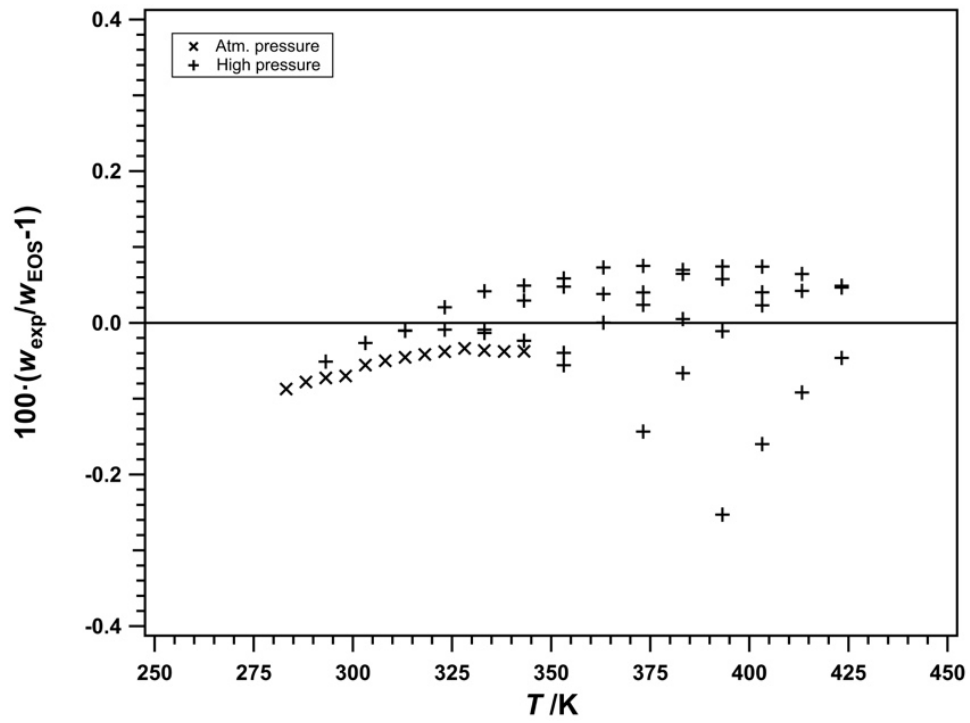

Figure 41: Comparisons of speeds of sound calculated with the equation of state to experimental data for POE9 as a function of temperature. 


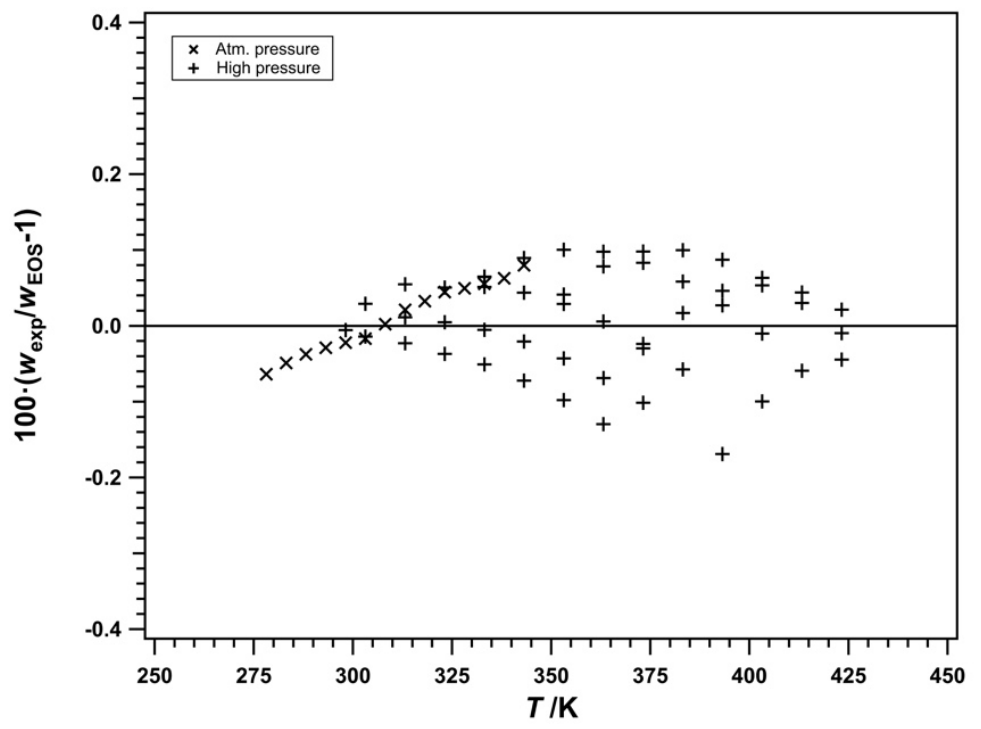

Figure 42: Comparisons of speeds of sound calculated with the equation of state to experimental data for MIL-PRF-23699 as a function of temperature.

The equations of state represent the isobaric heat capacity data within $0.3 \%$ for POE5, POE7, and MIL-PRF-23699 as shown in Figure 43. The average absolute deviations are less than $0.1 \%$ for POE5 and POE7, and $0.17 \%$ for MIL-PRF-23699. For POE9, the largest deviation is $-0.85 \%$ and the AAD is $0.27 \%$. As with the other properties, POE9 shows larger errors as explained elsewhere.

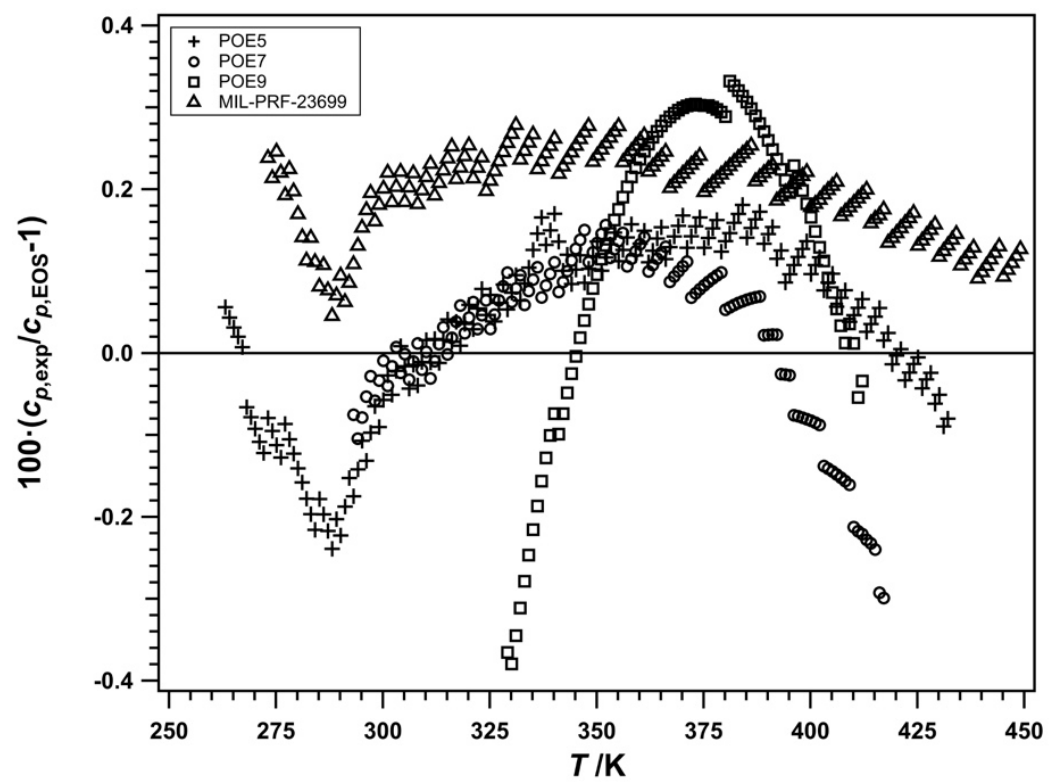

Figure 43: Comparisons of isobaric heat capacities calculated with the equations of state for POE5, POE7, POE9, and MIL-PRF-23699 to experimental data as a function of temperature. 
The vapor pressures were by far the most difficult property to accurately model while keeping all other data within their uncertainties. However, without these data it would not have been possible to obtain the critical temperatures with the "low" uncertainty that we believe we have achieved. The values at the critical point (temperature and density) were extremely sensitive to the vapor pressures, and fitting these at the same time as all other measurements to a functional form that was required to maintain smooth behavior to lower extremes than previously done with any other fluid presented many new challenges. The use of one functional form where only the coefficients changed between the four fluids but the temperature exponents and Gaussian bell-shaped parameters were identical for each fluid contributed in both positive and negative ways. The vapor pressures for POE5 and POE7 benefitted from the same functional form. The equations for these fluids represent the vapor pressures generally within $3 \%$, except one point with a deviation of $10 \%$ that could not be fitted without damaging the representation of all other points. The situation with POE9 is quite different due to the much higher impurities. The rigid functional form could not be forced to fit these data to within the same deviations and comparing with data on a percent deviation basis gives a false impression, especially since the lowest pressure is less than $1 \mathrm{mPa}$ (a factor of one billionth of the critical pressure). Five points are available for POE9.[6] At $476 \mathrm{~K}$, the vapor pressure measurement is $0.17 \mathrm{~Pa}$, and the calculated value is $0.94 \mathrm{~Pa}$. At $395 \mathrm{~K}$, the measured value is $0.05 \mathrm{mPa}$ and the calculated value is $0.39 \mathrm{mPa}$. These calculated values are still within the uncertainty of the measured values.

\subsubsection{Extrapolation Behavior}

The extrapolation behavior of equations of state has become an extremely important part of fitting equations of state over the last 15 years. Each of the approximately 50 publications on equations of state in that time frame show this behavior to demonstrate that the equation has been fitted correctly. The current criteria for each equation to be accepted as final includes some of the following:

1) All properties must extrapolate to near $0 \mathrm{~K}$ without extreme changes, and must be very smooth and follow the correct path to around 50 to $100 \mathrm{~K}$. Most equations start to deviate from the desired path below these temperatures, but each new generation of equations continues to improve at lower temperatures as better starting functional forms become available. An example for POE5 is shown in Figure 44 for the Phase Identification Parameter, or PIP. 


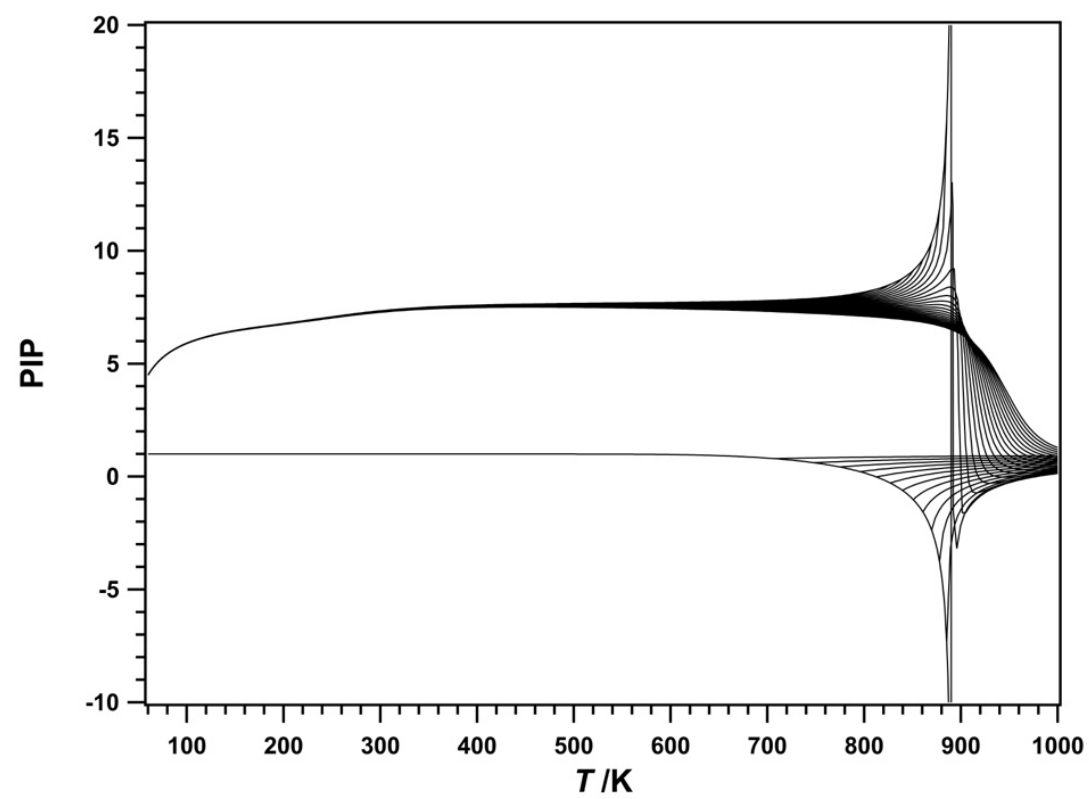

Figure 44: Phase Identification Parameter (PIP) versus temperature diagram showing isobars and saturation curves from 0 to $3 \mathrm{MPa}$ in steps of $0.1 \mathrm{MPa}$ for POE5.

2) All properties must extrapolate to infinite temperature, pressure, and density. This is verified continuously while fitting with the use of pressure-density plots showing isotherms over a large range of temperatures. The isotherms should converge at extremely high pressures, but not cross. Plots of the correct behavior are shown in the R-125 publication of Lemmon and Jacobsen[60]. This is demonstrated in Figure 45 for POE7.

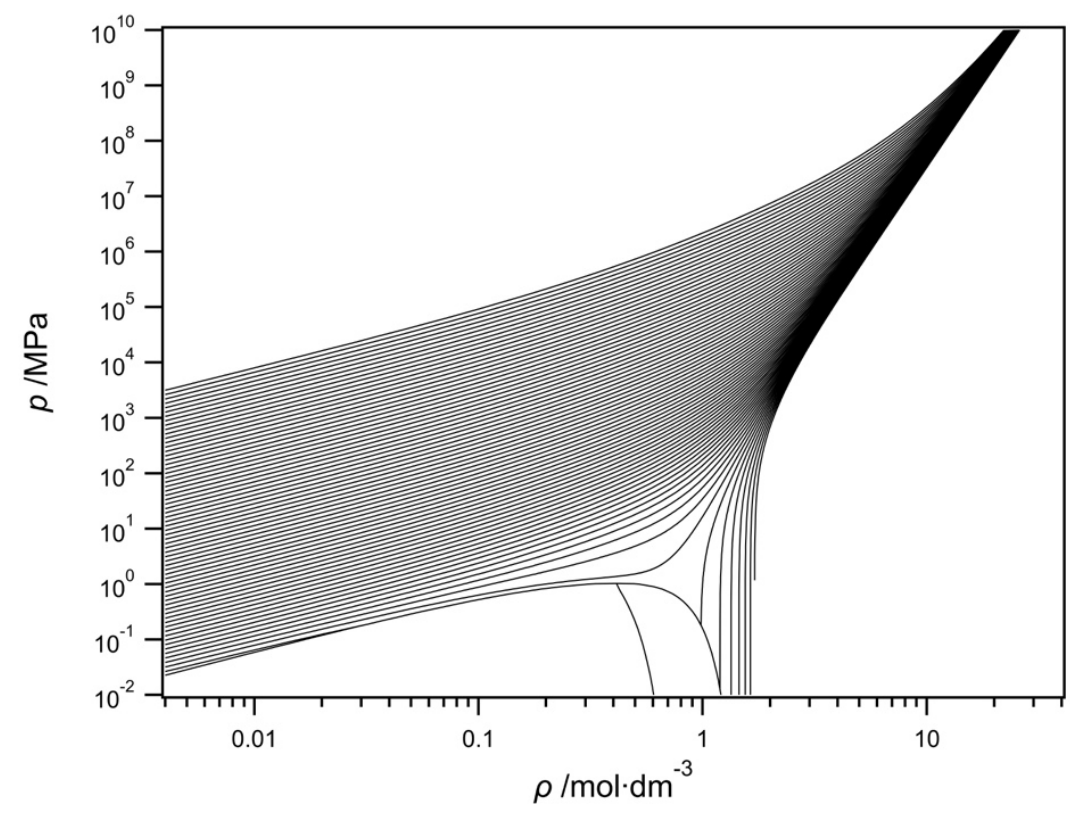

Figure 45: Isothermal behavior of the POE7 equation of state at extreme conditions of temperature (up to $10^{12} \mathrm{~K}$ ) and pressure (up to $10^{10} \mathrm{MPa}$ ). 
3) The rectilinear diameter (the average of the saturated liquid and vapor densities) must be linear in the critical region down to about one-half of the critical temperature. Below that, the line bends slightly to allow the liquid-phase density to increase at low temperatures. Figure 46 shows this for POE9.

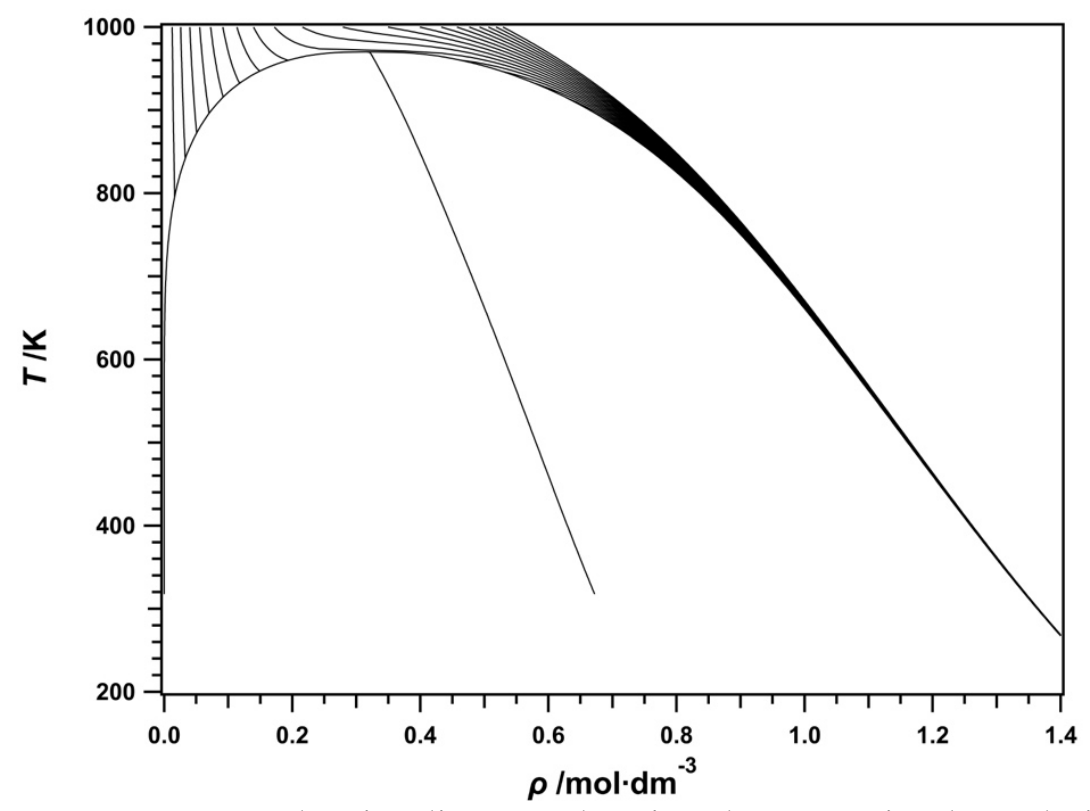

Figure 46: Temperature versus density diagram showing the saturation boundaries, the rectilinear diameter (the average of the saturation densities), and isobars up to 2 MPa for POE9.

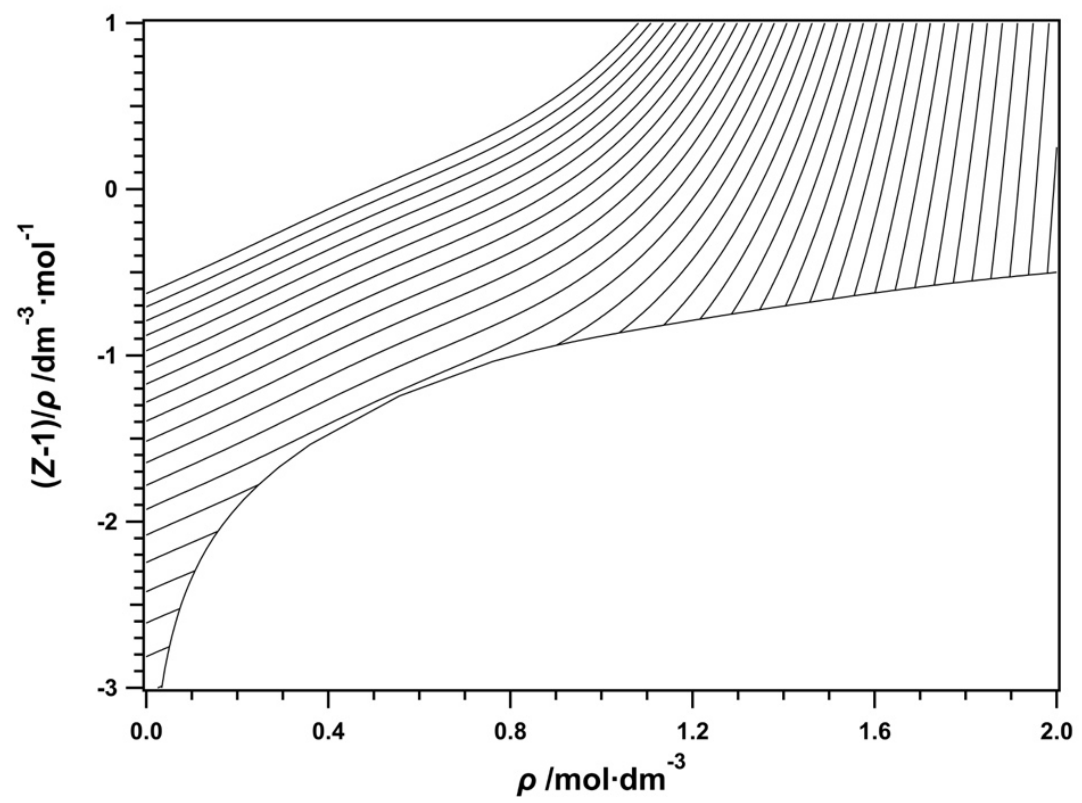

Figure 47: Calculations of $(Z-1) / \rho$ along isotherms versus density for POE5; isotherms are shown from temperatures of $400 \mathrm{~K}$ to $1200 \mathrm{~K}$ in steps of $25 \mathrm{~K}$. 
4) The slopes of isotherms in the vapor phase for the property $(Z-1) / \rho$, where $Z$ is the compressibility factor and $\rho$ is the density, should have little or no curvature, except for highly associating fluids such as water. Figure 47 shows this for POE5. This is extremely important for fluids that have no data in the vapor phase, as is case for the oils fitted in this work.

5) The first partial derivative of pressure with respect to density at constant temperature for the critical isotherm absolutely must never be negative, and the second derivative must be negative in the vapor phase and positive in the liquid phase, with the transition exactly at the critical point. If these criteria are not met, the equation will generally have two critical points (the critical point is the point where these two derivatives are both equal to zero).

6) The derivatives of the residual isochoric heat capacity $\left(c_{v}\right)$ with respect to saturation temperature for both the liquid and vapor phases should be positive near the critical point. In the vapor phase, this should be true everywhere except for highly associating fluids. During fitting, the 3rd and 4th derivatives are also forced to be positive very close to the critical point to ensure proper critical region behavior.

7) For most fluids, the slope of isochores in both vapor, liquid, and critical states with respect to temperature should be negative (it is unclear if this criterion is also correct for associating fluids). Figure 48 shows this and the $c_{v}$ criteria above for POE5.

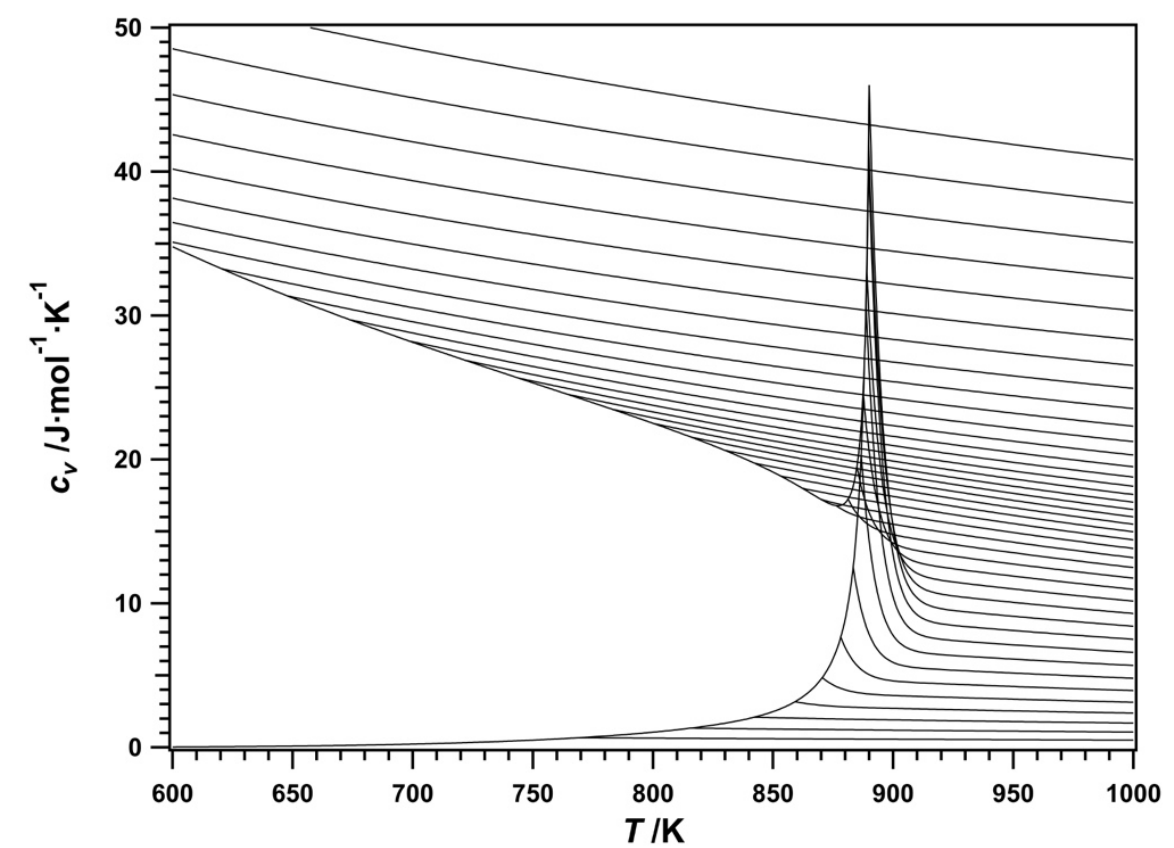

Figure 48: Residual isochoric heat capacity versus temperature diagram for POE5; lines of constant density up to $2 \mathrm{~mol} \cdot \mathrm{dm}^{-3}$ are shown. 
8) The second, third, and fourth virial coefficients must conform to the same behavior as manifested in calculations from molecular simulations. The behavior of these virial coefficients from the equation of state for POE7 is shown in Figure 49; the curves shown are typical of those from statistical thermodynamic models.

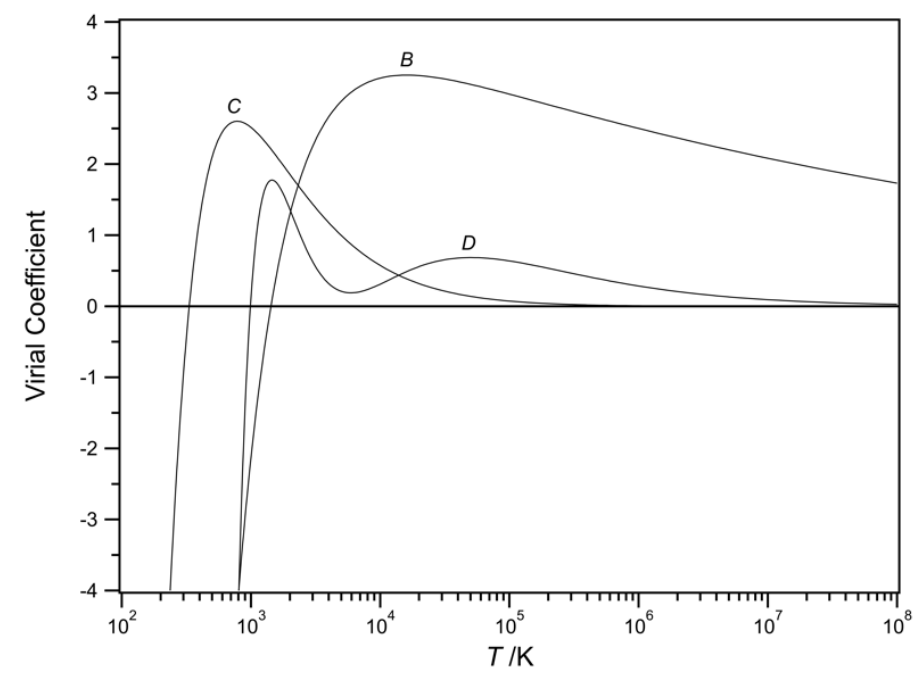

Figure 49: Calculated values of the second virial coefficient $B\left(\mathrm{dm}^{3} \cdot \mathrm{mol}^{-1}\right)$, third virial coefficient $C\left(\mathrm{dm}^{3} \cdot \mathrm{mol}^{-1}\right)^{2}$, and fourth virial coefficient $D\left(\mathrm{dm}^{3} \cdot \mathrm{mol}^{-1}\right)^{3}$ for POE7.

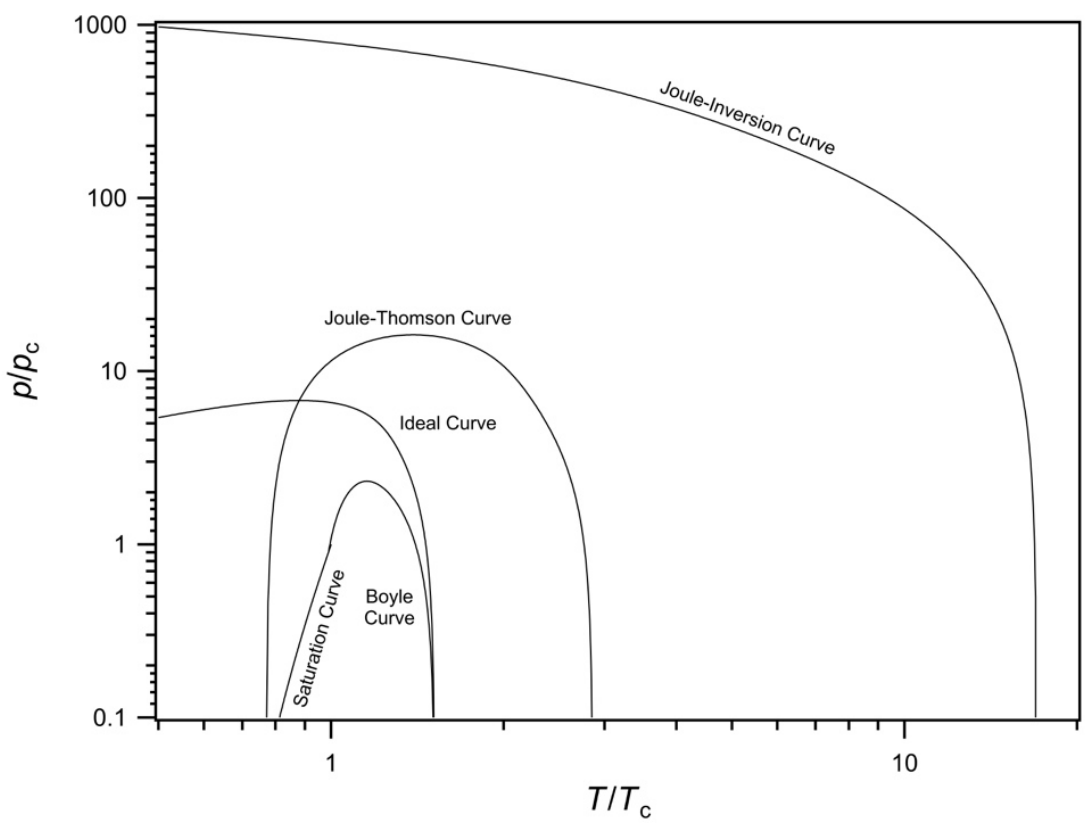

Figure 50: Characteristic (ideal) curves of the equation of state for POE9 as a function of temperature and pressure. 
9) The ideal curves, as described in other publications on equations of state, must be smooth and appear similar to that shown in the R-125 publication.[60] The curves shown in Figure 50 for POE9 do not fully meet these criteria but are sufficiently correct for the application of the oils fitted in this work.

There are numerous other criteria that must be fulfilled before an equation can be considered "finished", but these are not documented here. The nine above are the most important, and the equations here meet all of the other criteria.

\subsubsection{Estimated Uncertainties of Calculated Properties}

The expanded uncertainty of calculated values in the liquid phase for the oils that were measured in this work is $0.2 \%$ in density and speed of sound between $260 \mathrm{~K}$ and $450 \mathrm{~K}$ with pressures to $70 \mathrm{MPa}$. For speed of sound, the uncertainty may increase to $0.3 \%$ above $380 \mathrm{~K}$. Outside of these ranges, the uncertainties will slowly increase as a function of the distance from the upper or lower bounds given above. The amount of the increase is unknown but will be gradual (based on experience in fitting other equations where the extrapolation behavior of the equation is correct). For these fluids, the values could be as small as $0.4 \%$ at $220 \mathrm{~K}$ (assuming the oil is still fluid) or, for density, at temperatures up to $600 \mathrm{~K}$. The uncertainties in the critical region and vapor phase are unknown, but not expected to exceed $1 \%$ for density at temperatures up to $600 \mathrm{~K}$ in the vapor phase. The uncertainty in vapor-phase speed of sound is unknown due to the lack of information pertaining to the ideal-gas isobaric heat capacity.

The uncertainties in heat capacities in the liquid phase are $0.5 \%$ from $250 \mathrm{~K}$ to $450 \mathrm{~K}$, except for POE9 where the uncertainty increases to $1 \%$ or more below $330 \mathrm{~K}$. The uncertainties for vapor pressures are difficult to quantify due to the extremely low vapor pressures below $500 \mathrm{~K}$. An approximate value would be one order of magnitude; for example, at a temperature where the calculated value of the vapor pressure is $1 \mathrm{~Pa}$, the true value could be between $0.1 \mathrm{~Pa}$ to $10 \mathrm{~Pa}$. For vapor pressures above $0.1 \mathrm{MPa}$, the same may be true, but it is estimated that the uncertainties would be less than $50 \%$. For example, the equation for POE 5 calculates a temperature at $0.1 \mathrm{MPa}$ of $707 \mathrm{~K}$, but the true value may lie between $671 \mathrm{~K}$ (where the vapor pressure is $0.05 \mathrm{MPa}$ ) and $730 \mathrm{~K}$ (where the vapor pressure is $0.15 \mathrm{MPa}$ ).

\subsubsection{Calculated Values}

As an aid in code implementation, calculated values of properties from the equation of state are given in Table 31. The number of digits displayed does not indicate the accuracy in the values but are given for validation of computer code. 
Table 31: Calculated Values of Properties for Algorithm Verification.

\begin{tabular}{|c|c|c|c|c|c|c|}
\hline & $\begin{array}{c}T \\
/ \mathbf{K}\end{array}$ & $\begin{array}{c}\rho \\
/ \mathbf{m o l}^{-} \mathbf{d m}^{-3}\end{array}$ & $\begin{array}{c}p \\
/ \mathbf{M P a}\end{array}$ & $\begin{array}{c}c_{v} \\
/ \mathbf{J} \cdot \mathbf{m o l}^{-1} \cdot \mathbf{K}^{-1}\end{array}$ & $\begin{array}{c}c_{p} \\
/ \mathbf{J} \cdot \mathbf{m o l}^{-1} \cdot \mathbf{K}^{-1}\end{array}$ & $\begin{array}{c}w \\
/ \mathbf{m} \cdot \mathbf{s}^{-1}\end{array}$ \\
\hline \multirow[t]{4}{*}{ POE5 } & 300.0 & 2.24 & 86.7515 & 710.515 & 845.862 & 1658.37 \\
\hline & 890.0 & 0.555 & 1.26998 & 1362.9 & 52007400.0 & 32.6999 \\
\hline & 800.0 & 0.079 & 0.422597 & 1260.95 & 1283.6 & 93.9009 \\
\hline & 500.0 & 0.0 & 0.0 & 942.314 & 950.628 & 94.2014 \\
\hline \multirow[t]{4}{*}{ POE7 } & 300.0 & 1.74 & 87.1105 & 906.79 & 1073.18 & 1683.26 \\
\hline & 940.0 & 0.41 & 1.03 & 2229.74 & 8161290.0 & 25.6542 \\
\hline & 800.0 & 0.027 & 0.160361 & 1919.11 & 1933.27 & 94.9921 \\
\hline & 500.0 & 0.0 & 0.0 & 1243.0 & 1251.32 & 84.5927 \\
\hline \multirow[t]{4}{*}{ POE9 } & 300.0 & 1.43 & 95.8767 & 1151.98 & 1352.93 & 1733.14 \\
\hline & 970.0 & 0.32 & 0.884998 & 3064.23 & 9291810.0 & 18.8048 \\
\hline & 800.0 & 0.017 & 0.104378 & 2546.39 & 2558.4 & 90.0762 \\
\hline & 500.0 & 0.0 & 0.0 & 1572.95 & 1581.26 & 77.4309 \\
\hline \multirow[t]{4}{*}{ MIL-PRF-23699 } & 300.0 & 1.8 & 28.5227 & 841.412 & 1003.51 & 1478.92 \\
\hline & 930.0 & 0.44 & 1.08 & 1652.07 & 36093600.0 & 28.9772 \\
\hline & 800.0 & 0.036 & 0.209763 & 1509.9 & 1525.29 & 95.3025 \\
\hline & 500.0 & 0.0 & 0.0 & 1142.46 & 1150.77 & 86.6593 \\
\hline
\end{tabular}

\subsection{Viscosity Modeling}

\subsubsection{Methodology}

There are a wide variety of models that can be used to represent the viscosity of a pure fluid. In this project, the primary concern was to represent the liquid-phase viscosity over a wide range of pressures including up to relatively high pressures of $140 \mathrm{MPa}$. Since the final goal is to provide a model that can be incorporated into REFPROP[21], the model must also be able to represent the viscosity of the gas and supercritical phases, even though experimental data is not available in these regions. Thus we have chosen a hybrid approach[61] that represents the viscosity as a sum of terms;

\section{Error! Bookmark not defined.

$$
\eta\left(\rho_{\mathrm{r}}, T_{\mathrm{r}}\right)=\eta_{0}\left(T_{\mathrm{r}}\right)+\Delta \eta\left(\rho_{\mathrm{r}}, T_{\mathrm{r}}\right)+
$$$$
\Delta \eta_{\mathrm{c}}\left(\rho_{\mathrm{r}}, T_{\mathrm{r}}\right) \text {, }
$$

where $\rho_{\mathrm{r}}$ and $T_{\mathrm{r}}$ are reduced density and temperature given by $\rho_{\mathrm{r}}=\rho / \rho_{\mathrm{c}}$ and $T_{\mathrm{r}}=T / T_{\mathrm{c}}$, the subscript c denotes critical values, and the first term, $\eta_{0}(T)$, is the contribution to the viscosity in the dilutegas limit. The critical enhancement term, $\Delta \eta_{\mathrm{c}}(\rho, T)$, arises from the long-range density fluctuations that occur in a fluid near its critical point, which contribute to divergence of the viscosity at the critical point. Finally, the term $\Delta \eta(\rho, T)$, the residual term, represents the contribution of all other 
effects to the viscosity of the fluid at elevated densities including many-body collisions, molecularvelocity correlations, and collisional transfer. In this work, we will use the estimation method of Chung et al.[62] for the zero-density term, we will neglect the critical enhancement term, and use an empirical model for the residual contribution. The critical enhancement for viscosity contributes only very near to the critical point, and we will not discuss it further here.

As mentioned above, the dilute-gas viscosity is calculated with the model of Chung et al.[62]:

$$
\eta_{0}(T)=4.0785 \sqrt{M T} \frac{F_{c}}{V_{c}^{2 / 3} \Omega_{\eta}^{*}},
$$

Where $\Omega_{\eta}^{*}$ is the viscosity collision integral, $F_{c}$ is the higher order correction factor (neglecting the contribution from the dipole moment and association effects), given as

$$
\begin{aligned}
& \Omega_{\eta}^{*}=1.16145 T^{*-0.14874}+0.52487 \exp \left(-0.77320 T^{*}\right)+2.16178 \exp \left(-2.43787 T^{*}\right) \\
& -6.435 \times 10^{-4} T^{* 0.14874} \sin \left(18.0323 T^{*-0.76830}-7.27371\right)
\end{aligned}
$$

and

$$
F_{c}=1-0.2756 \omega,
$$

where $T^{*}=T / \varepsilon$, is the reduced temperature and $\varepsilon(\mathrm{K})$ is the energy scaling parameter. This parameter was obtained with the method of Chung et al.[62] according to

$$
\varepsilon / k=T_{\mathrm{c}} / 1.2593,
$$

where $V_{\mathrm{c}}$ is the critical volume in $\mathrm{cm}^{3} \cdot \mathrm{mol}^{-1}, T_{\mathrm{c}}$ is in $\mathrm{K}$, and $M$ is the molar mass of the fluid in $\mathrm{g} \cdot \mathrm{mol}^{-1}, \omega$ is the dimensionless acentric factor, and the viscosity is in $\mu \mathrm{Pa} \cdot \mathrm{s}$.

The residual viscosity term, $\Delta \eta\left(\rho_{\mathrm{r}}, T_{\mathrm{r}}\right)$, represents the contribution of all other effects to the viscosity of the fluid at elevated densities including many-body collisions, molecular-velocity correlations, and collisional transfer. Because there is little theoretical guidance concerning this term, we develop an empirical model based on our experimentally obtained data. The procedure adopted during this analysis uses symbolic regression software[63] to fit the experimental data to the residual viscosity. Symbolic regression is a type of genetic programming that allows the 
exploration of arbitrary functional forms to regress data. The functional form is obtained by use of a set of operators, parameters, and variables as building blocks. We first adopt a form suggested by the hard sphere model of Assael et al.[64] and pull out a factor $\left(\rho_{\mathrm{r}}{ }^{2 / 3} T_{\mathrm{r}}{ }^{1 / 2}\right)$, such that $\Delta \eta\left(\rho_{\mathrm{r}}, T_{\mathrm{r}}\right)=\left(\rho_{\mathrm{r}}^{2 / 3} T_{\mathrm{r}}^{1 / 2}\right) F\left(\rho_{\mathrm{r}}, T_{\mathrm{r}}\right)$. We have used this approach successfully for a variety of fluids, including alkanes[65] and refrigerants[66], and most recently, ammonia[67]. Since we are interested in the viscosity of highly viscous fluids at high pressures, we adopt a scaled variable $\Gamma=\rho_{\mathrm{r}}^{\gamma} / T_{\mathrm{r}}$, also referred to as thermodynamic scaling, suggested by the works of Bair and Casalini.[68] Scaling in this variable has been suggested as a way to obtain good extrapolation to high pressures. The power $\gamma$ is determined by plotting the residual viscosity $\left(\eta-\eta_{0}\right) /\left(\rho_{\mathrm{r}}{ }^{2 / 3} T_{\mathrm{r}}{ }^{1 / 2}\right)$ vs $\rho_{\mathrm{r}}^{\gamma} / T_{\mathrm{r}}$ and finding the value of $\gamma$ that causes the data to collapse into a single line. Symbolic regression is then used to find the function $\Delta \eta\left(\rho_{\mathrm{r}}, T_{\mathrm{r}}\right)=\left(\rho_{\mathrm{r}}^{2 / 3} T_{\mathrm{r}}^{1 / 2}\right) F\left(\rho_{\mathrm{r}}, T_{\mathrm{r}}\right)$. In the present work, we restricted the operators to the set $(+,-, \times, /)$ and the operands (constant, $T_{\mathrm{r}}, \rho_{\mathrm{r}}$ and $\rho_{\mathrm{r}}^{\gamma} / T_{\mathrm{r}}$ ), with $\gamma$ fixed as described previously. All densities are obtained from the equations of state described earlier in this report. The functional form for each fluid will be determined by the symbolic regression algorithm based on the available data and will not be the same for each fluid.

\subsubsection{Results}

\subsubsection{POE5}

The equation of state for POE5 discussed in Section 12.2 has the following values for critical temperature and density, that we adopt here as well: $T_{\mathrm{C}}=890.0 \mathrm{~K}, \rho_{\mathrm{c}}=0.556 \mathrm{~mol} \cdot \mathrm{L}^{-1}$. The molar mass is $472.612 \mathrm{~g} \cdot \mathrm{mol}^{-1}$ and the acentric factor is 0.89 . Using Eqs. 25 - 26 leads to $\varepsilon / k=706.7418 \mathrm{~K}$ and $F_{c}=0.75472$. The density scaling factor was found to be $\gamma=3.58$. The equation for residual viscosity obtained from symbolic regression was

$$
\Delta \eta\left(\rho_{\mathrm{r}}, T_{\mathrm{r}}\right)=\left(a_{1} \Gamma+a_{2} \Gamma^{2}+a_{3} \Gamma^{6}+a_{4} \Gamma^{13}\right) \sqrt{T_{\mathrm{r}}} \rho_{\mathrm{r}}^{2 / 3}
$$

where $\Gamma=\rho_{\mathrm{r}}^{3.58} / T_{\mathrm{r}}, \quad a_{1}=1.4307113, \quad a_{2}=0.019090959, \quad a_{3}=4.0904180 \times 10^{-12}$ and $a_{4}=1.3888478 \times 10^{-30}$. Eqs. $22-26$ combined with Eq. 27 are then used to calculate the viscosity. Comparisons with our experimental data in Section 10 resulted in an average absolute deviation of $1.8 \%$, a bias of $0.13 \%$, and an uncertainty at the $95 \%$ level of $4.7 \%$. This is consistent with the experimental uncertainty estimated to be $5 \%$ at the lowest pressures rising to $10 \%$ at the highest pressure nearing $140 \mathrm{MPa}$. Deviation plots as a function of temperature, pressure and density are shown in Figures 51 - 53. As shown in Figure 52, we did not observe any trends in the deviations as a function of pressure. 


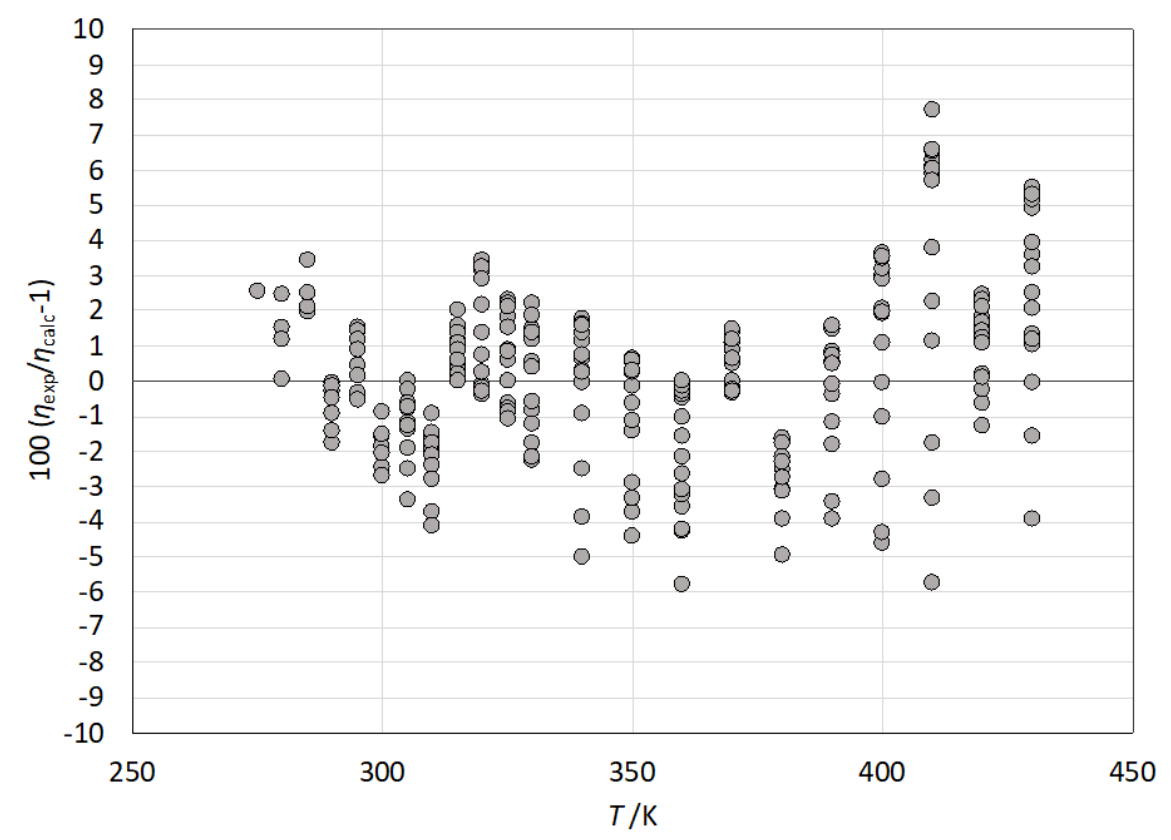

Figure 51: Percentage deviations in viscosity for POE5 as a function of temperature.

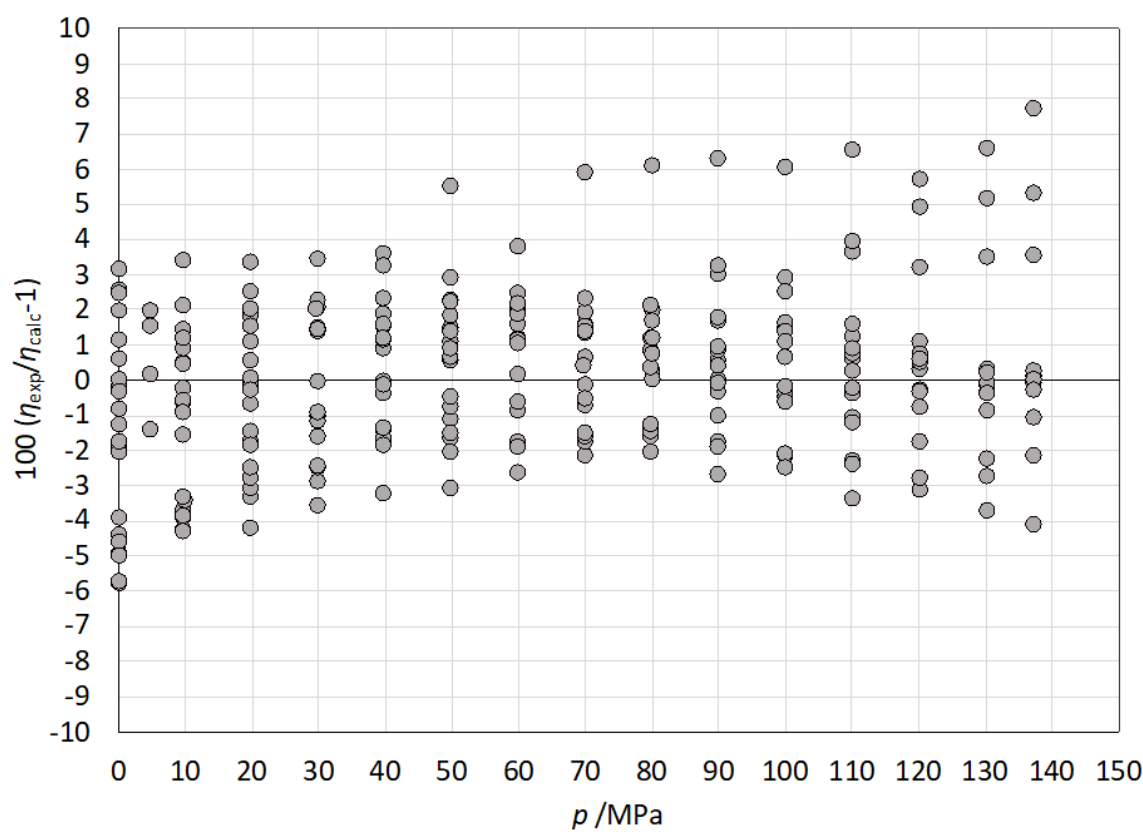

Figure 52: Percentage deviations in viscosity for POE5 as a function of pressure. 


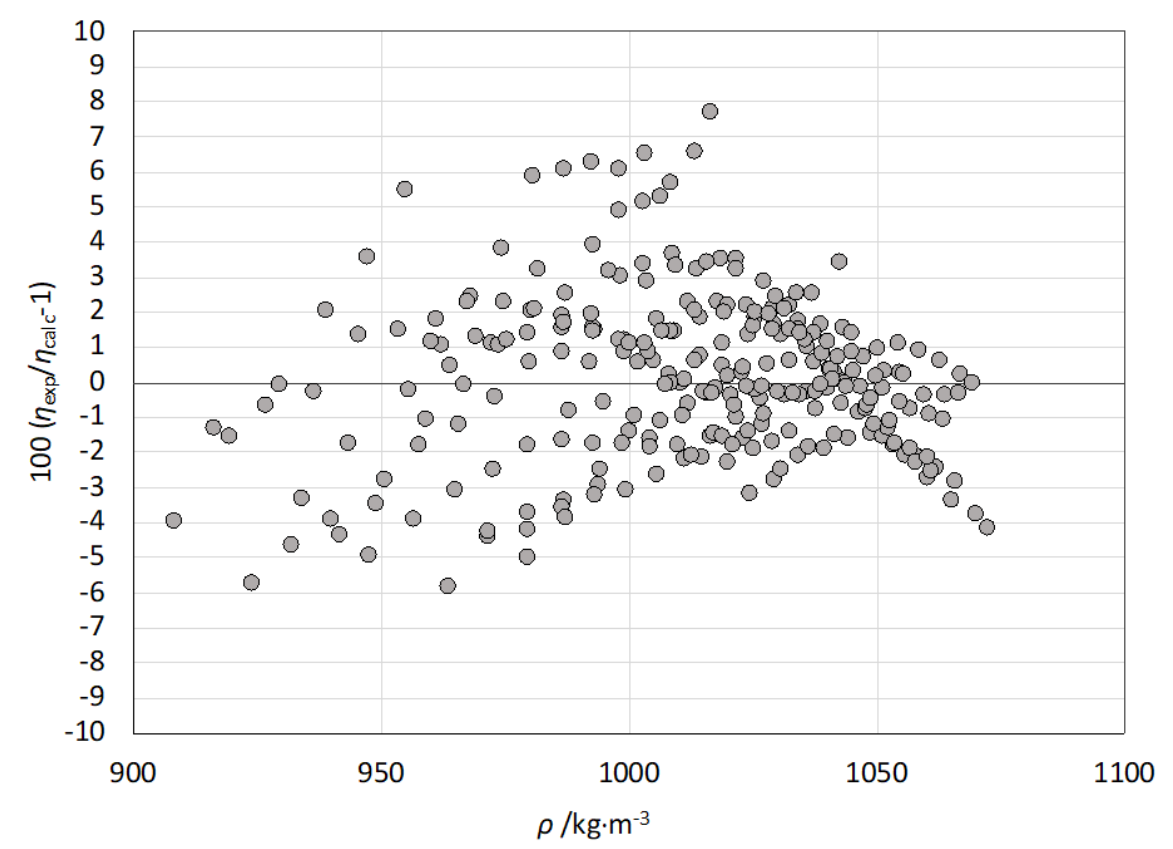

Figure 53: Percentage deviations in viscosity for POE5 as a function of density.

\subsubsection{POE7}

The equation of state for POE7 discussed in section 12.2 has the following values for critical temperature and density, that we adopt here as well: $T_{\mathrm{C}}=940.0 \mathrm{~K}, \rho_{\mathrm{c}}=0.412 \mathrm{~mol} \cdot \mathrm{L}^{-1}$. The molar mass is $584.835 \mathrm{~g} \cdot \mathrm{mol}^{-1}$ and the acentric factor is 1.06. Using Eqs. $25-26$ leads to $\varepsilon / k=746.4464 \mathrm{~K}$ and $F_{c}=0.70786$. The density scaling factor was found to be $\gamma=3.43$. The equation for residual viscosity obtained from symbolic regression was

$$
\Delta \eta\left(\rho_{\mathrm{r}}, T_{\mathrm{r}}\right)=\left(a_{1} \Gamma^{5}+a_{2} \Gamma^{13}+a_{3} T_{\mathrm{r}}^{2} \Gamma^{2}\right) \sqrt{T_{\mathrm{r}}} \rho_{\mathrm{r}}^{2 / 3}
$$

where $\Gamma=\rho_{\mathrm{r}}^{3.43} / T_{\mathrm{r}}, a_{1}=1.8292961 \times 10^{-9}, a_{2}=2.2667078 \times 10^{-30}$, and $a_{3}=0.10216630$. Eqs. $22-26$ combined with Eq. 28 are then used to calculate the viscosity. Comparisons with our experimental data in Section 10 resulted in an average absolute deviation of $1.8 \%$, a bias of $0.0 \%$, and an uncertainty at the $95 \%$ level of $4.5 \%$. This is consistent with the estimated experimental uncertainty estimated to be $5 \%$ at the lowest pressures rising to $10 \%$ at the highest pressure nearing $140 \mathrm{MPa}$. Deviation plots as a function of temperature, pressure and density are shown in Figures 54 - 56. We did not observe any systematic trends in the deviations. 


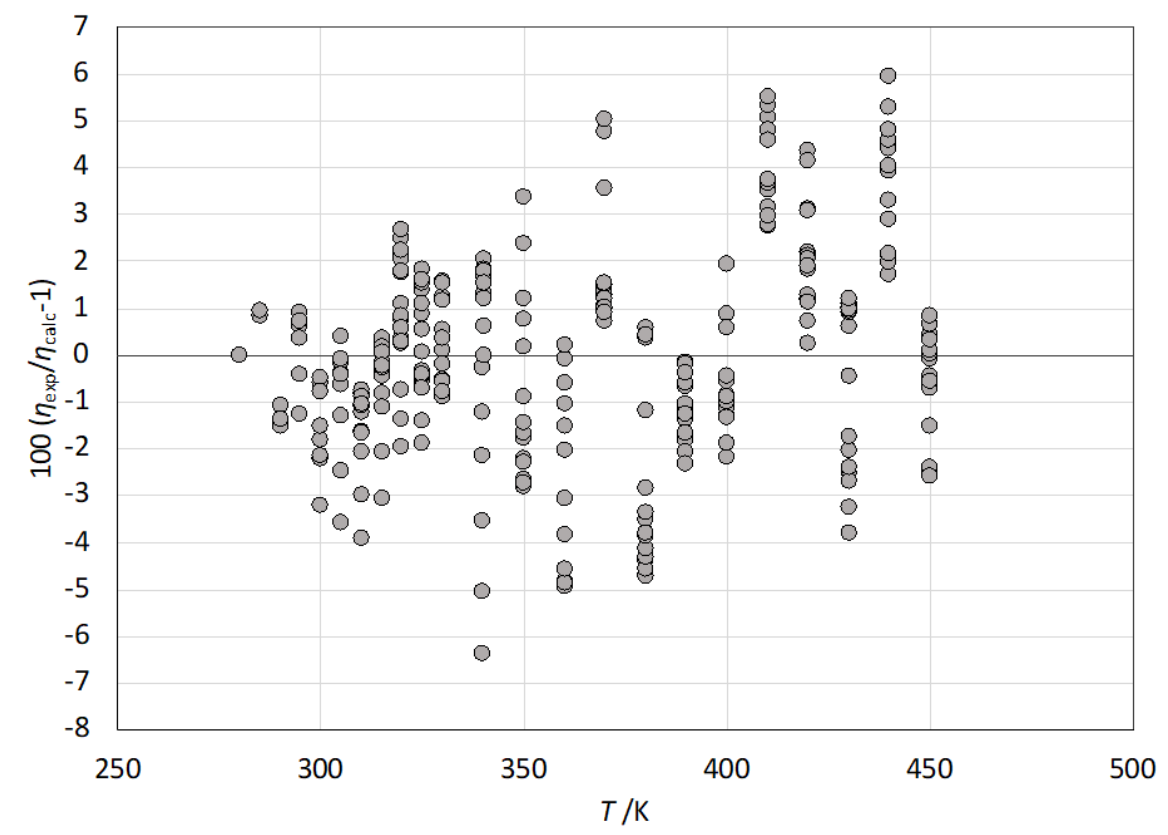

Figure 54: Percentage deviations in viscosity for POE7 as a function of temperature.

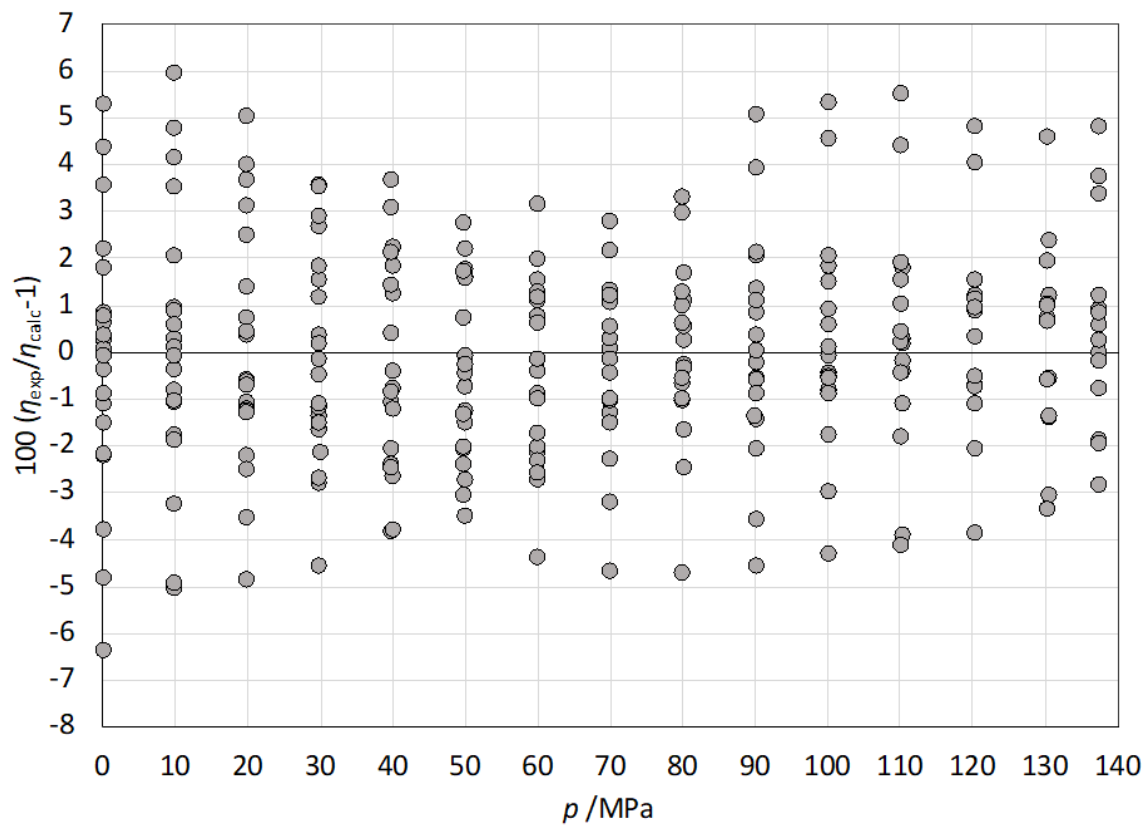

Figure 55: Percentage deviations in viscosity for POE7 as a function of pressure. 


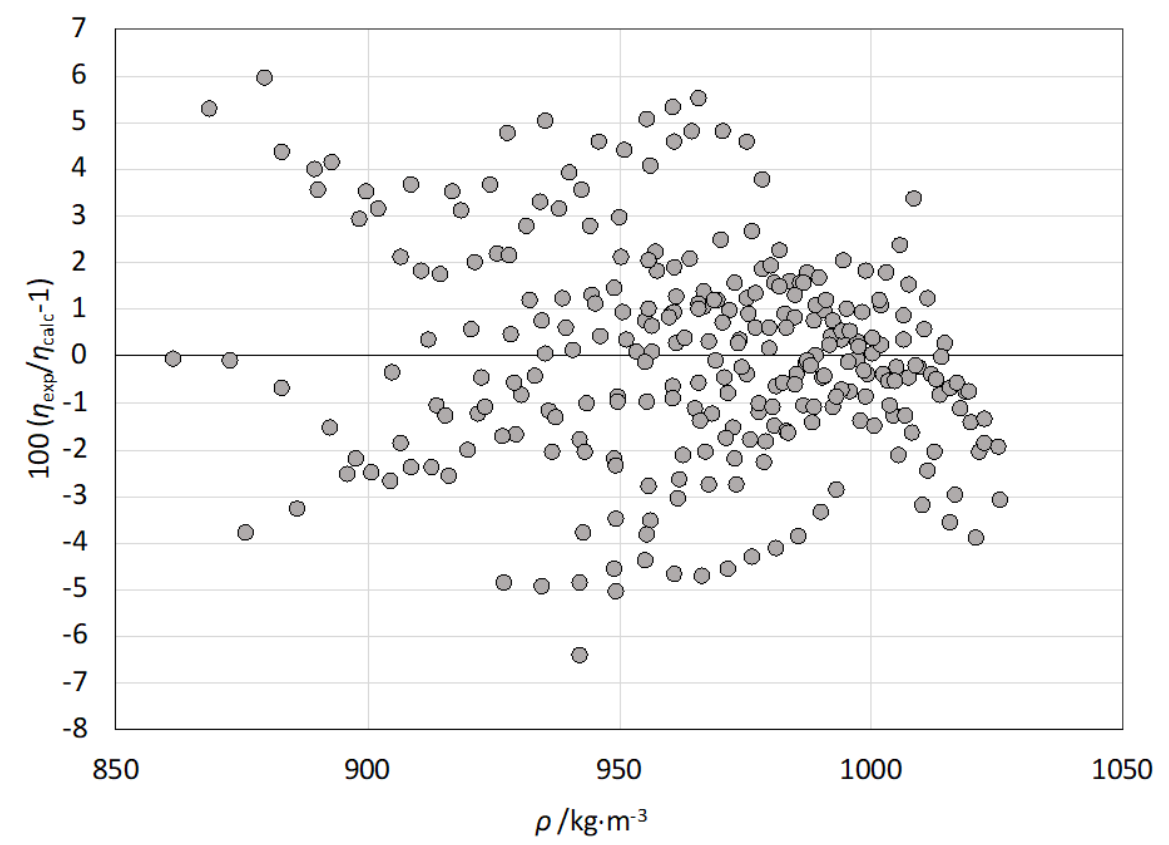

Figure 56: Percentage deviations in viscosity for POE7 as a function of density.

\subsubsection{POE9}

The equation of state for POE9 discussed in Section 12.2 has the following values for critical temperature and density, that we adopt here as well: $T_{\mathrm{C}}=970.0 \mathrm{~K}$ and $\rho_{\mathrm{c}}=0.316 \mathrm{~mol} \cdot \mathrm{L}^{-1}$. The molar mass is $697.051 \mathrm{~g} \cdot \mathrm{mol}^{-1}$ and the acentric factor is 0.998 . Using Eqs. $25-26$ leads to $\varepsilon / k=770.2692 \mathrm{~K}$, and $F_{c}=0.72495$. The density scaling factor was found to be $\gamma=3.36$. The equation for residual viscosity obtained from symbolic regression was

$$
\Delta \eta\left(\rho_{\mathrm{r}}, T_{\mathrm{r}}\right)=\left(a_{1} \Gamma+a_{2} \Gamma^{2}+a_{3} \Gamma^{3}+a_{4} \Gamma^{11}\right) \sqrt{T_{\mathrm{r}}} \rho_{\mathrm{r}}^{2 / 3}
$$

where $\Gamma=\rho_{\mathrm{r}}^{3.36} / T_{\mathrm{r}}, \quad a_{1}=4.9977918, \quad a_{2}=-0.049733553, a_{3}=0.00025851171$, and $a_{4}=1.6282415 \times 10^{-25}$. Eqs. $22-26$ combined with Eq. 29 are then used to calculate the viscosity. Comparisons with our experimental data in Section 10 resulted in an average absolute deviation of $2.5 \%$, a bias of $-0.8 \%$, and an uncertainty at the $95 \%$ level of $6.8 \%$. This is slightly larger than the estimated experimental uncertainty estimated to be $5 \%$ at the lowest pressures rising to $10 \%$ at the highest pressure nearing $140 \mathrm{MPa}$. Deviation plots as a function of temperature, pressure and density are shown in Figures 57 - 59. We did not observe any trends in the deviations. 


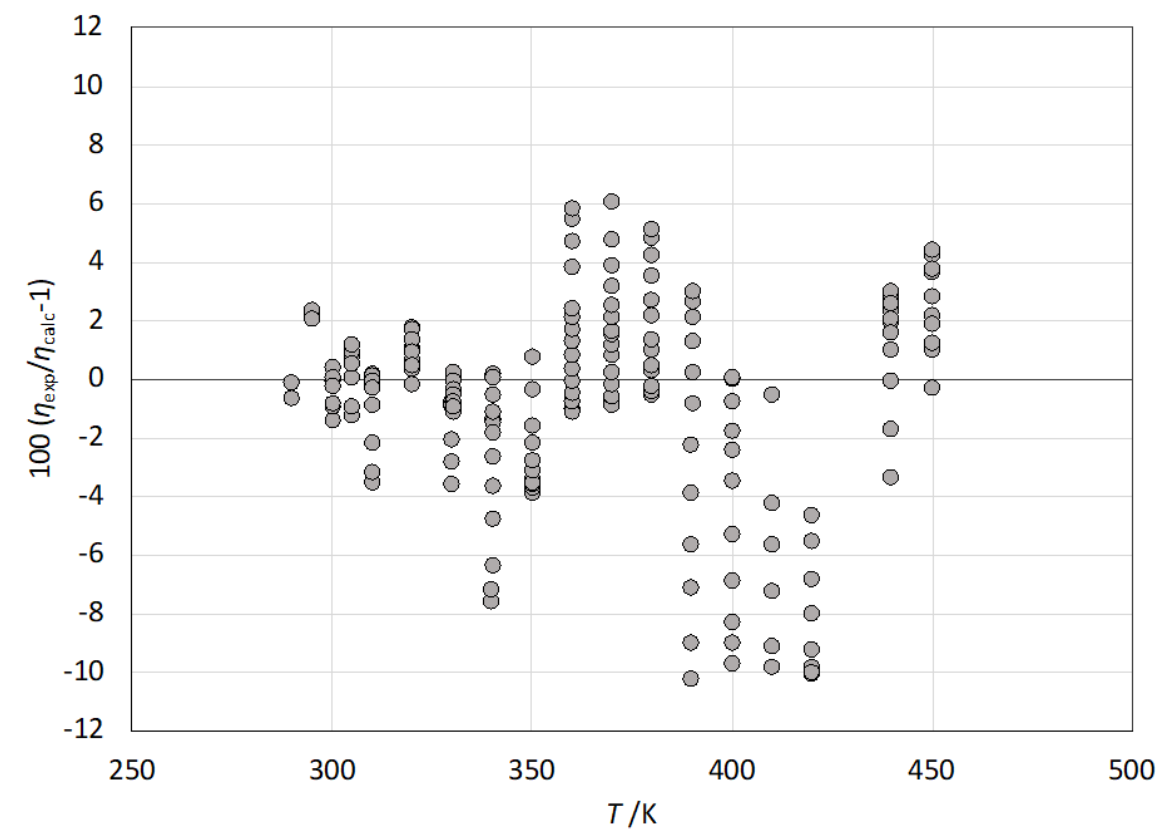

Figure 57: Percentage deviations in viscosity for POE9 as a function of temperature.

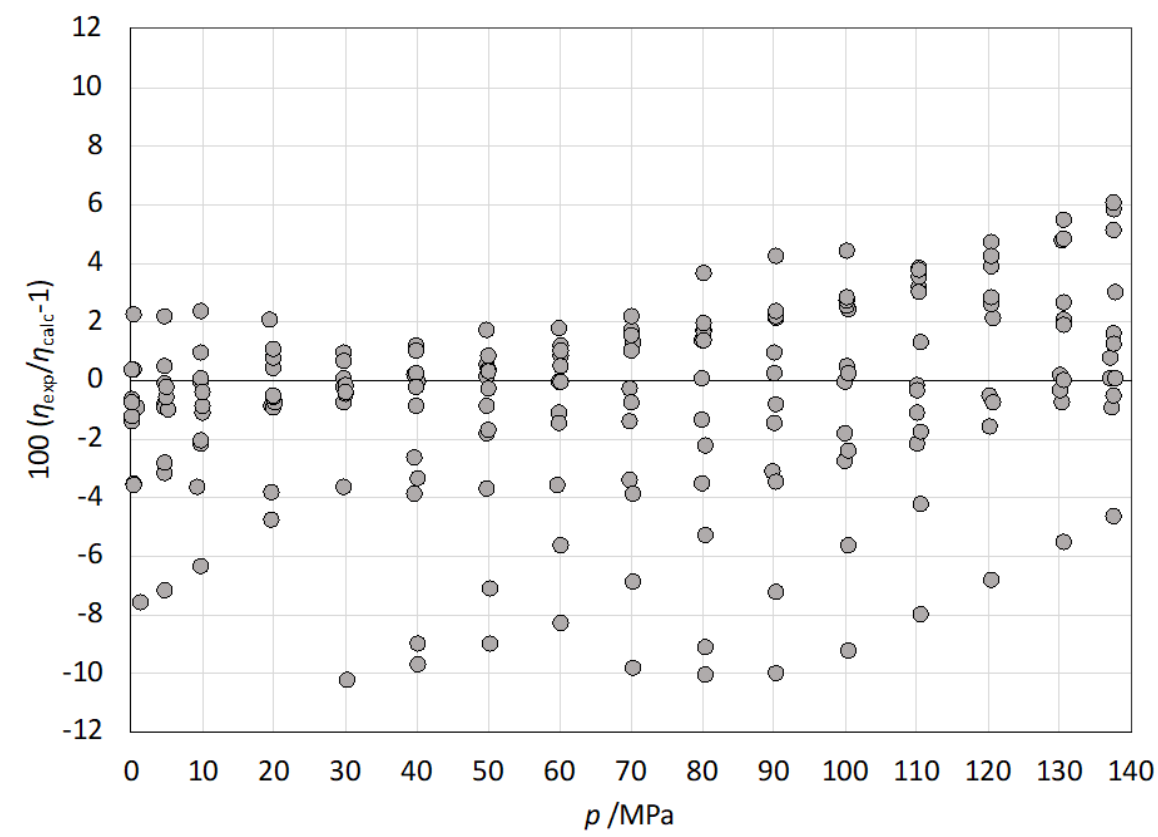

Figure 58: Percentage deviations in viscosity for POE9 as a function of pressure. 


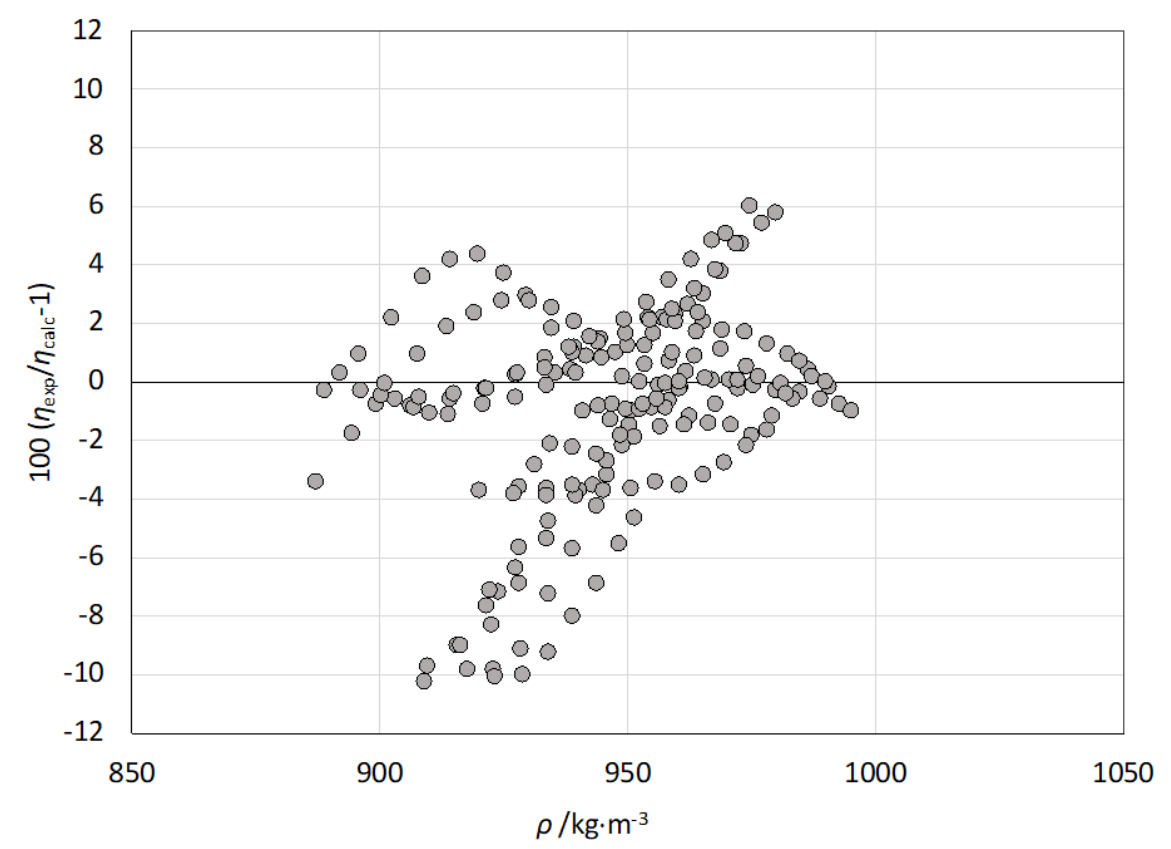

Figure 59: Percentage deviations in viscosity for POE9 as a function of density.

\subsection{Thermal Conductivity Modeling}

\subsubsection{Methodology}

Typically, similar to how viscosity is treated, the thermal conductivity is expressed in terms of a dilute gas, residual, and critical enhancement terms:

$$
\lambda\left(T_{\mathrm{r}}, \rho_{\mathrm{r}}\right)=\lambda^{0}\left(T_{\mathrm{r}}\right)+\Delta \lambda^{\mathrm{res}}\left(T_{\mathrm{r}}, \rho_{\mathrm{r}}\right)+\lambda^{\mathrm{crit}}\left(T_{\mathrm{r}}, \rho_{\mathrm{r}}\right)
$$

where the dilute gas contribution $\lambda_{0}$ is a function of $T$ only. In this work we do not have any experimental data in the gas phase, so we use Chapman-Enskog theory as described in Huber[61] to generate estimates of zero-density thermal conductivity values, which are then fit to a simple polynomial form:

$$
\lambda^{0}\left(T_{\mathrm{r}}\right)=\sum_{k=0}^{n} \alpha_{k} T_{\mathrm{r}}^{k}
$$

The residual contribution $\lambda^{\text {res }}$ is written as a polynomial in terms of temperature and density,

$$
\Delta \lambda^{\mathrm{res}}(T, \rho)=\sum_{l=1}^{3}\left(\beta_{1, l}+\beta_{2, l}\left(T / T_{\mathrm{c}}\right)\right)\left(\rho / \rho_{\mathrm{c}}\right)^{l}
$$


and the coefficients are found by fitting experimental data. The critical enhancement contribution is estimated using the generalized method described in Perkins et al.[69] that requires only $T_{\mathrm{c}}, \rho_{\mathrm{c}}$, $p_{c}$, the acentric factor $\omega$ and the molar mass of the component.

\subsubsection{Results}

\subsubsection{POE5}

We fit the liquid-phase thermal conductivity data described in Section 9, incorporating estimated vapor-phase predictions, and obtained the following coefficients for Eq. 31 and Eq. 32. All densities were determined from the EOS described in Section 12.2. The resulting coefficients are given in Table 32. Comparisons with our experimental data (Section 9) resulted in an average absolute deviation of $0.12 \%$, a bias of $0.0 \%$, and an uncertainty at the $95 \%$ level of $0.3 \%$. This is well within the estimated experimental uncertainty estimated to be $0.5 \%$ for the entire range of the experiments that covered $301 \mathrm{~K}$ to $502 \mathrm{~K}$ at pressures up to $69 \mathrm{MPa}$. Deviation plots as a function of temperature, pressure and density are shown in Figures $60-62$.

Table 32: Coefficients for the Thermal Conductivity Equation for POE5.

\begin{tabular}{l|c}
\hline \multicolumn{1}{c|}{ Coefficients } & \\
\hline \hline$\alpha_{0}$ & 1.847472 \\
$\alpha_{1}$ & -18.00409 \\
$\alpha_{2}$ & 108.2592 \\
$\alpha_{3}$ & -80.25605 \\
$\alpha_{4}$ & 26.80722 \\
$\alpha_{5}$ & -3.439569 \\
$\beta_{1,1}$ & 0.00804625 \\
$\beta_{1,2}$ & 0.00500470 \\
$\beta_{1,3}$ & -0.000211256 \\
$\beta_{2,1}$ & 0.0561869 \\
$\beta_{2,2}$ & -0.0542289 \\
$\beta_{2,3}$ & 0.0126351 \\
\hline
\end{tabular}




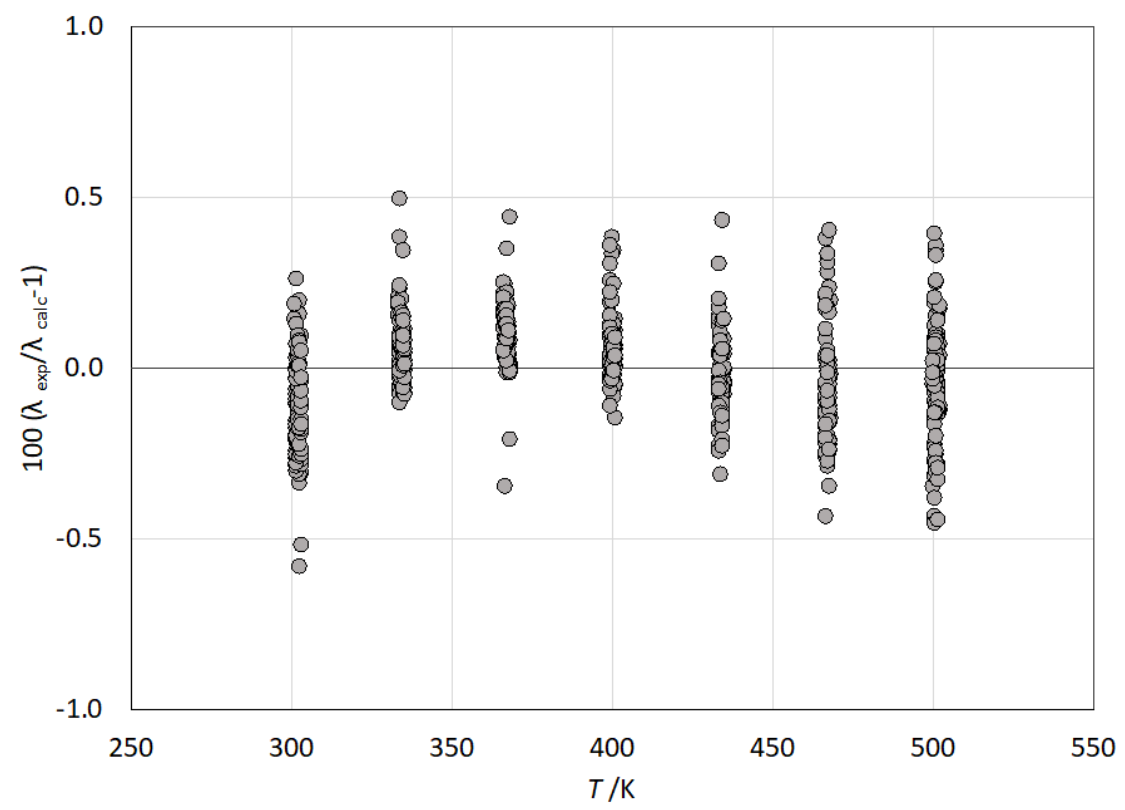

Figure 60: Percentage deviations in thermal conductivity for POE5 as a function of temperature.

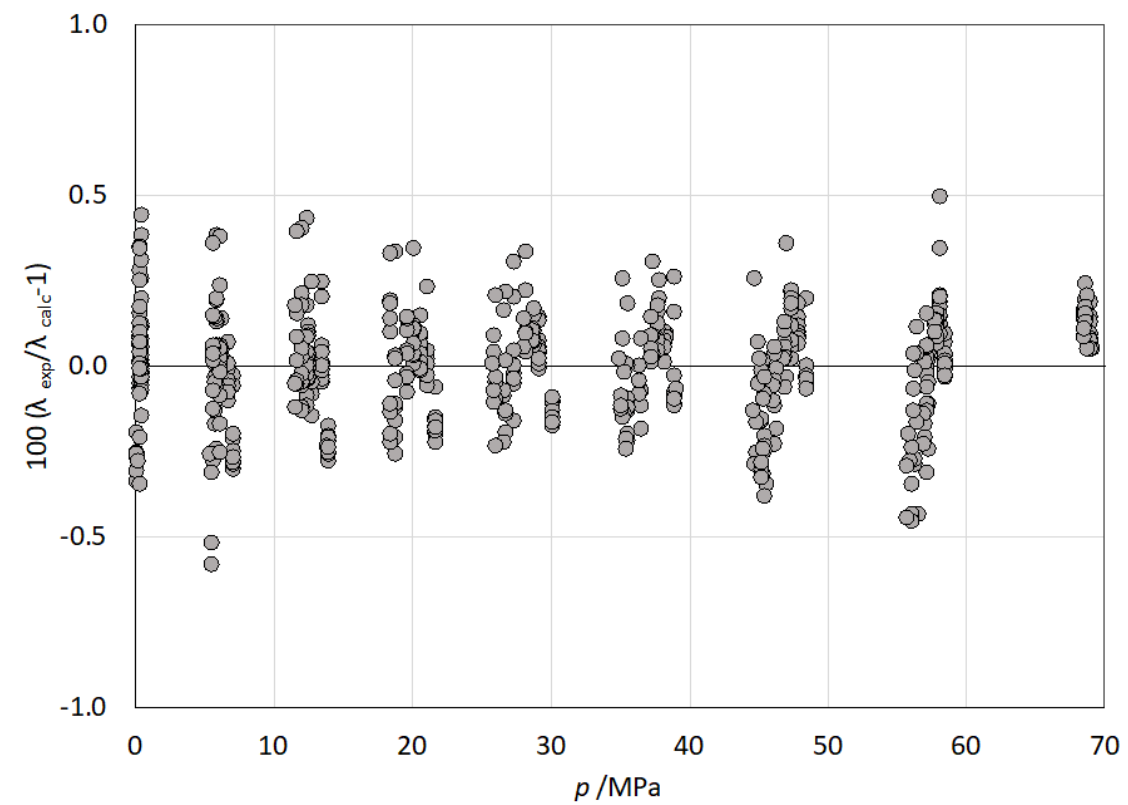

Figure 61: Percentage deviations in thermal conductivity for POE5 as a function of pressure. 


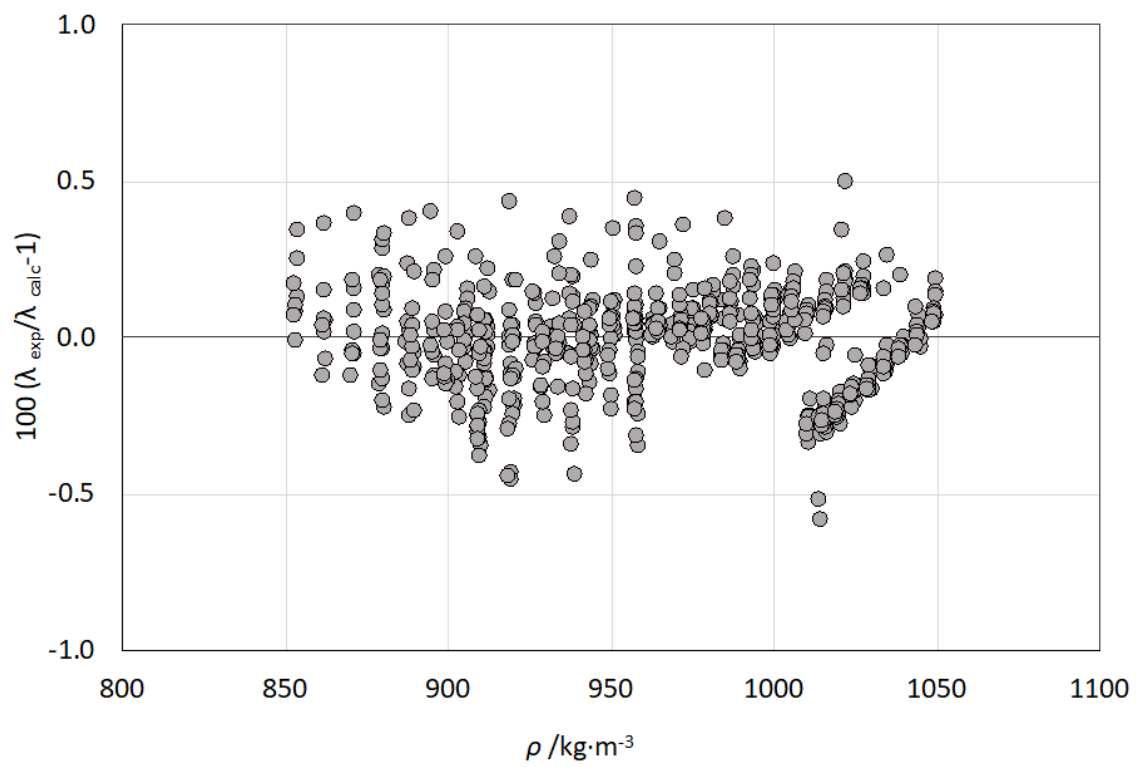

Figure 62: Percentage deviations in thermal conductivity for POE5 as a function of density.

\subsubsection{POE7}

We fit the liquid-phase thermal conductivity data described in Section 9, incorporating estimated vapor-phase predictions, and obtained the following coefficients for Eq. 31 and Eq. 32. All densities were determined from the EOS described in Section 12.2. The resulting coefficients are given in Table 33.

Table 33: Coefficients for the Thermal Conductivity Equation for POE7.

\begin{tabular}{l|c}
\hline \multicolumn{1}{c|}{ Coefficients } & \\
\hline \hline$\alpha_{0}$ & 5.0156798 \\
$\alpha_{1}$ & -32.028343 \\
$\alpha_{2}$ & 112.07684 \\
$\alpha_{3}$ & -52.063516 \\
$\alpha_{4}$ & 7.7818950 \\
$\alpha_{5}$ & 0.16886652 \\
$\beta_{1,1}$ & 0.0314251 \\
$\beta_{1,2}$ & -0.00484656 \\
$\beta_{1,3}$ & 0.000729462 \\
$\beta_{2,1}$ & 0.0124139 \\
$\beta_{2,2}$ & -0.0359579 \\
$\beta_{2,3}$ & 0.0105320 \\
\hline
\end{tabular}


Comparisons with our experimental data (Section 9) resulted in an average absolute deviation of $0.24 \%$, a bias of $0.0 \%$, and an uncertainty at the $95 \%$ level of $0.7 \%$. This is slightly above the estimated experimental uncertainty estimated to be $0.5 \%$ for the entire range of the experiments that covered $300 \mathrm{~K}$ to $504 \mathrm{~K}$ at pressures up to $69 \mathrm{MPa}$. Deviation plots as a function of temperature, pressure and density are shown in Figures 63 - 65.

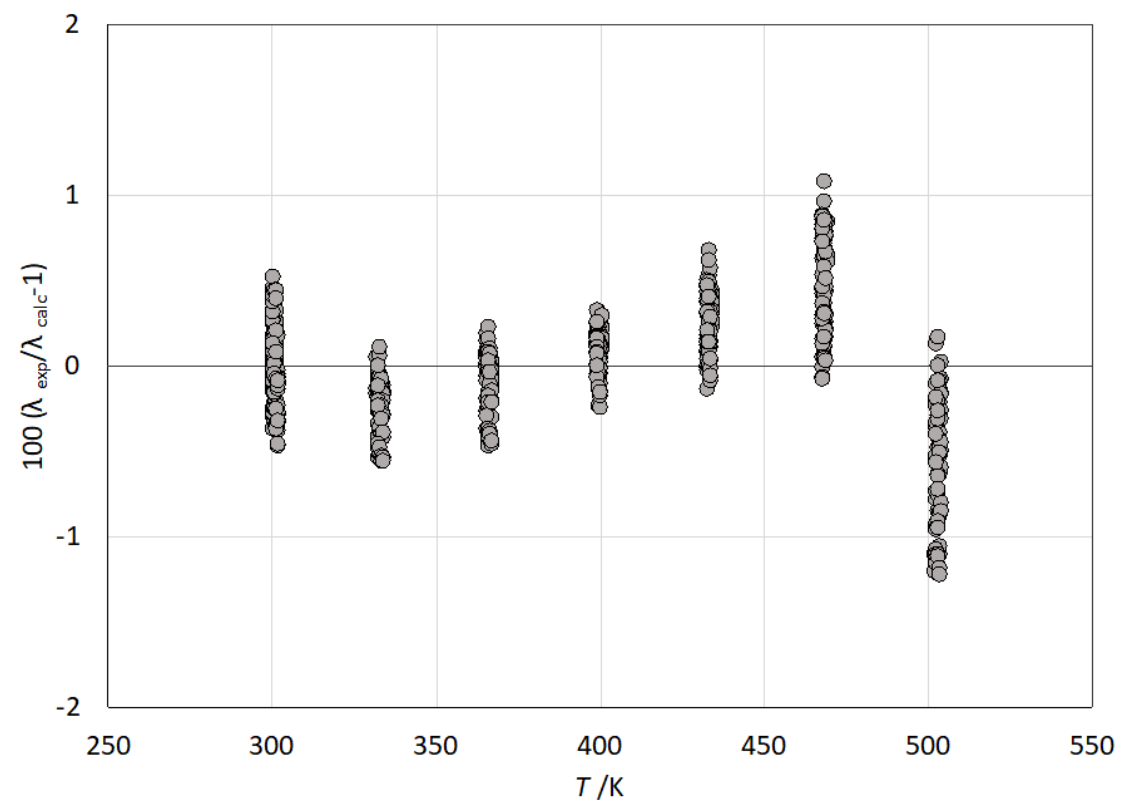

Figure 63: Percentage deviations in thermal conductivity for POE7 as a function of temperature.

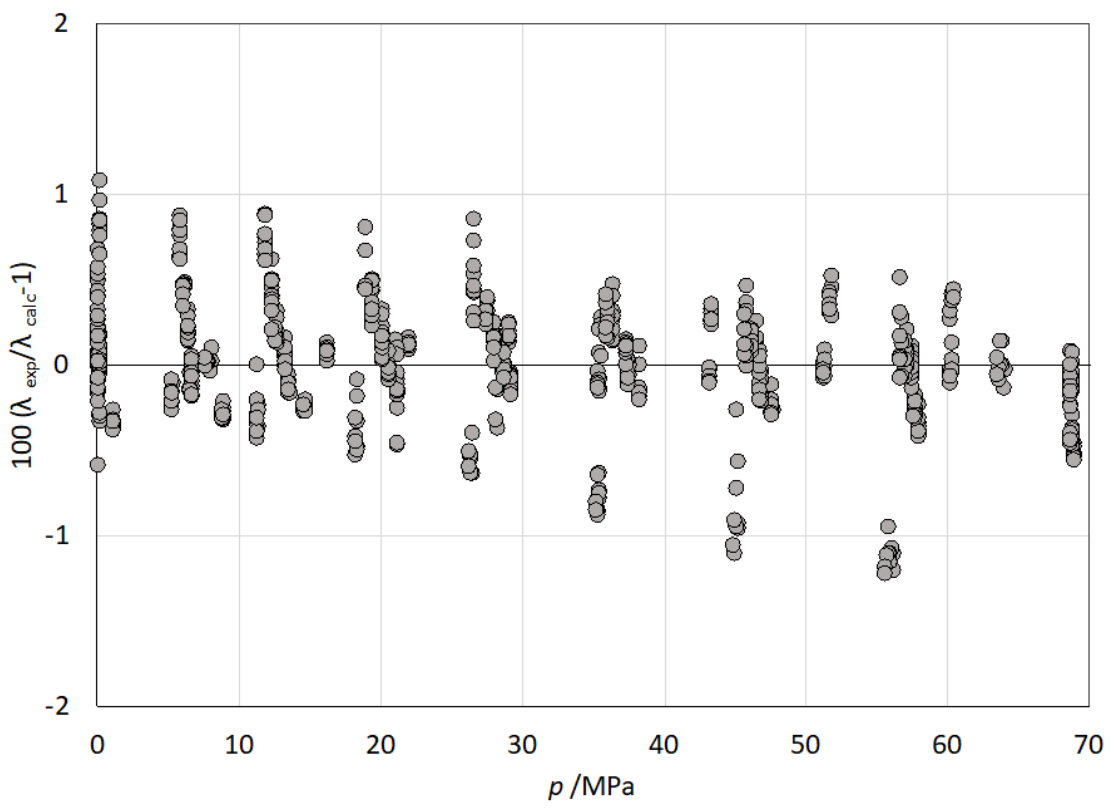

Figure 64: Percentage deviations in thermal conductivity for POE7 as a function of pressure. 


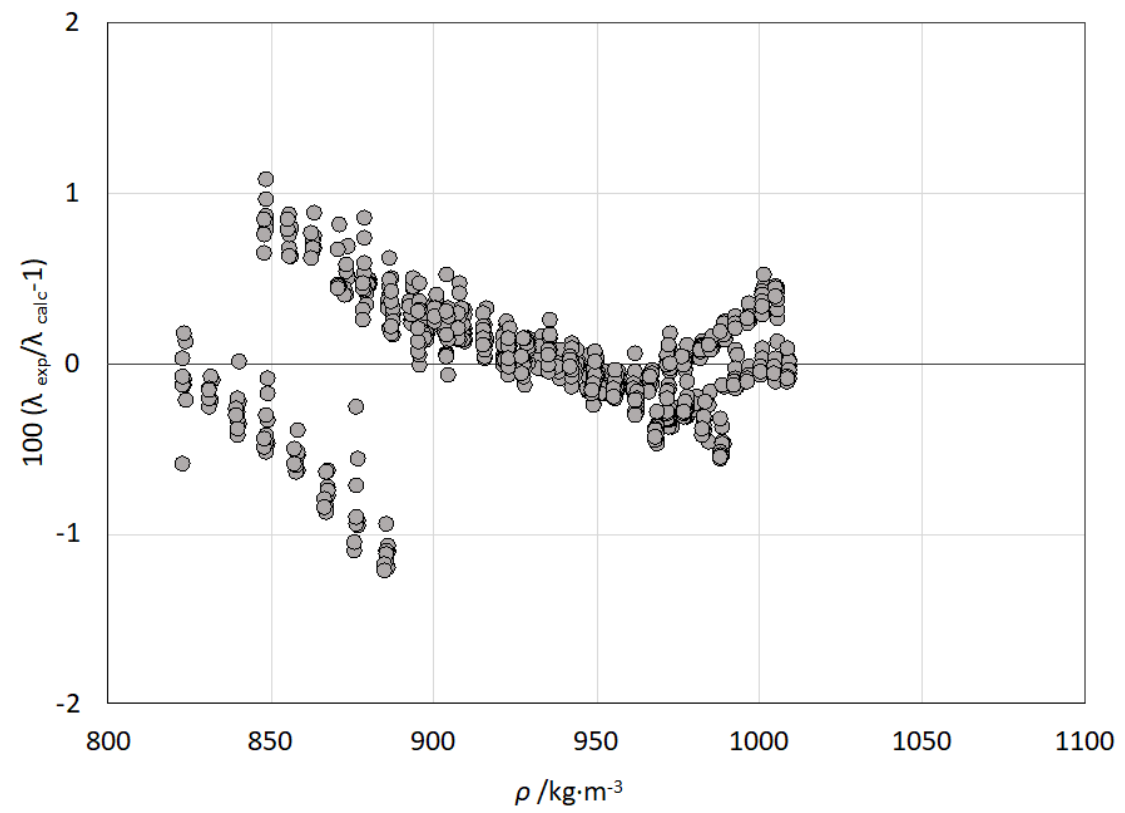

Figure 65: Percentage deviations in thermal conductivity for POE7 as a function of density.

\subsubsection{POE9}

We fit the liquid-phase thermal conductivity data described in Section 9, incorporating estimated vapor-phase predictions, and obtained the following coefficients for Eq. 31 and Eq. 32. All densities were determined from the EOS described in Section 12.2. The resulting coefficients are given in Table 34.

Table 34: Coefficients for the Thermal Conductivity Equation for POE9.

\begin{tabular}{l|c}
\hline \multicolumn{1}{c|}{ Coefficients } & \\
\hline \hline$\alpha_{0}$ & -2.617097 \\
$\alpha_{1}$ & 43.187405 \\
$\alpha_{2}$ & -160.22117 \\
$\alpha_{3}$ & 404.97948 \\
$\alpha_{4}$ & -339.90316 \\
$\alpha_{5}$ & 99.120658 \\
$\beta_{1,1}$ & 0.0375194 \\
$\beta_{1,2}$ & -0.00967900 \\
$\beta_{1,3}$ & 0.00151768 \\
$\beta_{2,1}$ & 0.00348471 \\
$\beta_{2,2}$ & -0.0229526 \\
$\beta_{2,3}$ & 0.00707870 \\
\hline
\end{tabular}


Comparisons with our experimental data (Section 9) resulted in an average absolute deviation of $0.13 \%$, a bias of $0.0 \%$, and an uncertainty at the $95 \%$ level of $0.4 \%$. This is within the estimated experimental uncertainty estimated to be $0.5 \%$ for the entire range of the experiments that covered $301 \mathrm{~K}$ to $500 \mathrm{~K}$ at pressures up to $69 \mathrm{MPa}$. Deviation plots as a function of temperature, pressure and density are shown in Figures 66 - 68.

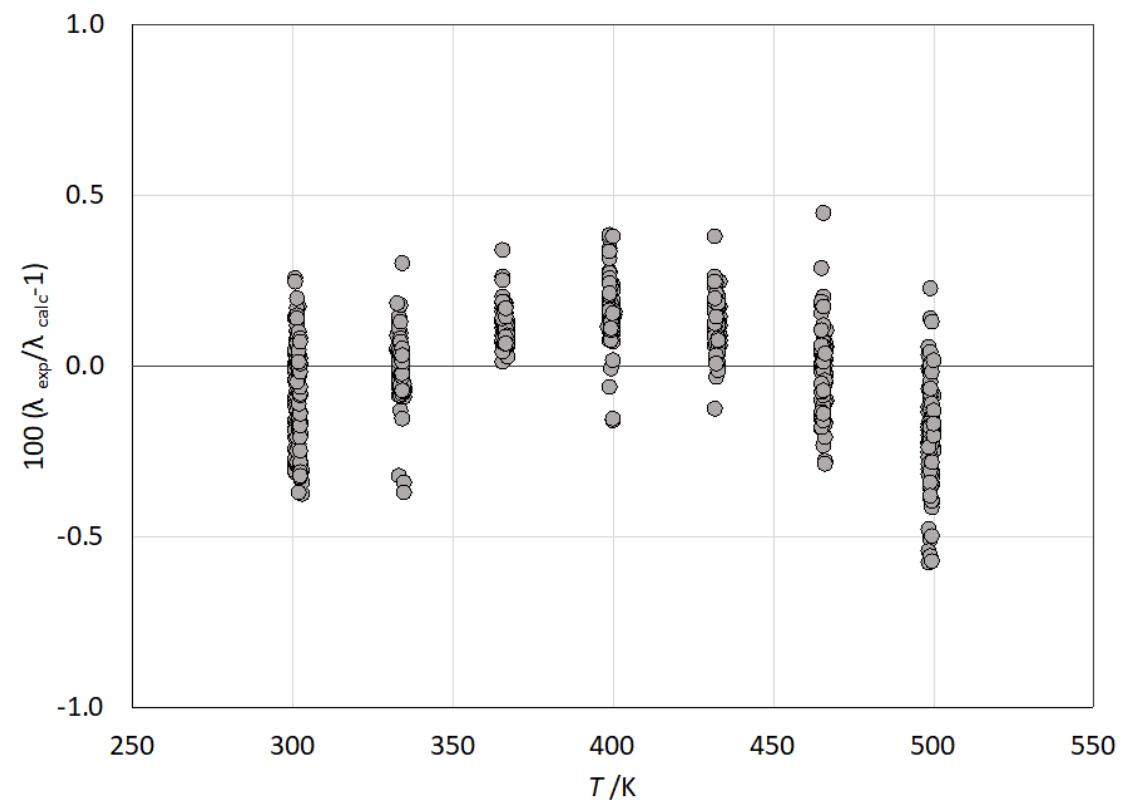

Figure 66: Percentage deviations in thermal conductivity for POE9 as a function of temperature.

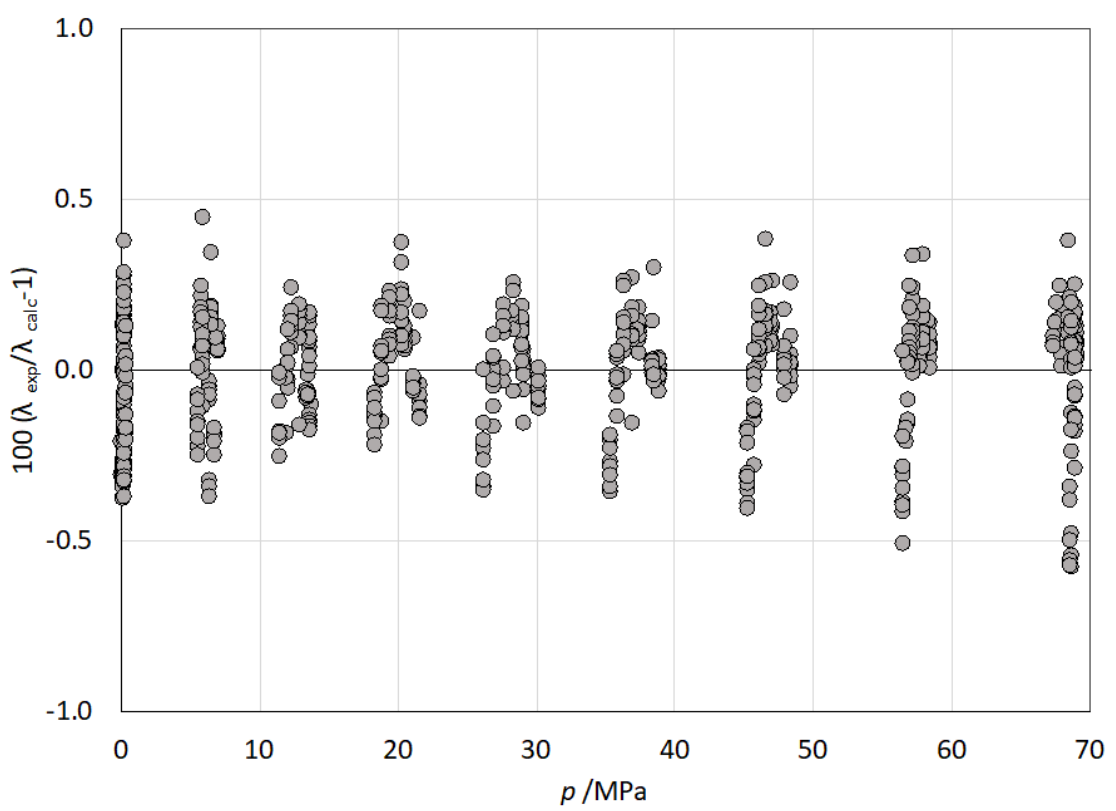

Figure 67: Percentage deviations in thermal conductivity for POE9 as a function of pressure. 


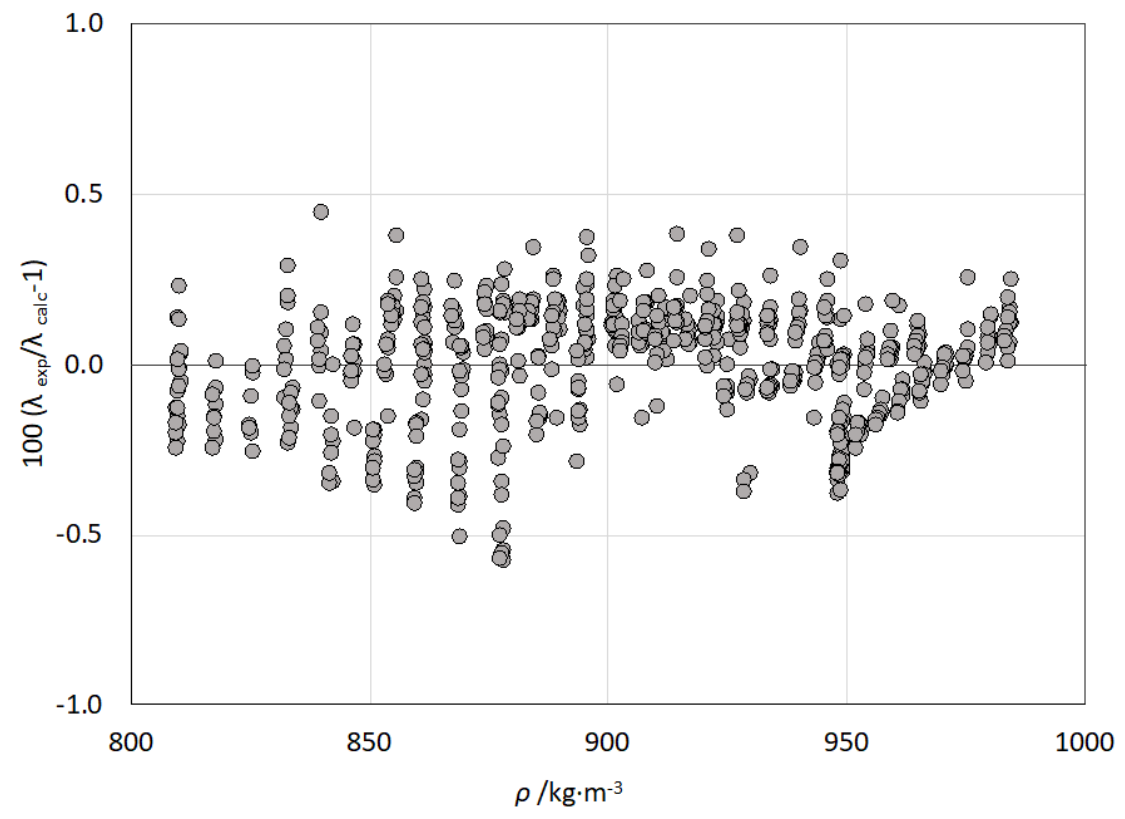

Figure 68: Percentage deviations in thermal conductivity for POE9 as a function of density.

\subsection{Mixture Modeling}

We have taken two different approaches to modeling the fully qualified lubricant, which is a mixture of numerous components. The first method treats the mixture as a pseudo-pure fluid, and an equation of state, viscosity and thermal conductivity correlations are developed by fitting experimental mixture data. This approach has been used successfully to model mixtures such as air[70], as well as several azeotropic and near-azeotropic refrigerant mixtures such as R404A, R407C, R410A and R507A.[71] Advantages of this approach are that the data can be fit well, as it is treated exactly like a pure fluid. A disadvantage of the pseudo-pure fluid model is that one cannot model the volatility with a distillation curve with this type of model, because a pure fluid distills at a single temperature while a mixture will distill over a range of temperatures beginning with the most volatile component and ending with the least volatile component. An alternative approach, that we also take here, is to approximate the actual lubricant mixture with a surrogate mixture model[72-74]. In this approach, the lubricant is modeled as a defined mixture of components whose composition is determined by finding a best match with the experimental data. The components are selected such that they are chemically similar to the components in the mixture but are not necessarily the actual components in the mixture. For the lubricant mixture considered here, the three pure POE fluids will be combined in such a way as to represent the properties of the actual lubricant. 


\subsubsection{Pseudo-Pure Fluid Model}

\subsubsection{MIL-PRF-23699 Viscosity Model}

The equation of state for MIL-PRF-23699 discussed in Section 12.2 has the following values for critical temperature and density, that we adopt here as well: $T_{\mathrm{C}}=930.0 \mathrm{~K}$ and $\rho_{\mathrm{c}}=0.439 \mathrm{~mol} \cdot \mathrm{L}^{-1}$. The molar mass is $557.6 \mathrm{~g} \cdot \mathrm{mol}^{-1}$ and the acentric factor is 0.97. Using Eqs. $25-26$ leads to $\varepsilon / k=738.5055 \mathrm{~K}$, and $F_{c}=0.73267$. The density scaling factor was found to be $\gamma=3.58$. The equation for residual viscosity obtained from symbolic regression was:

$$
\Delta \eta\left(\rho_{\mathrm{r}}, T_{\mathrm{r}}\right)=\left(a_{1} \Gamma+a_{2} \Gamma^{4}+a_{3} \Gamma^{5}+a_{4} \Gamma^{11}+a_{5} T_{\mathrm{r}}+a_{6} T_{\mathrm{r}}^{2}\right) \sqrt{T_{\mathrm{r}}} \rho_{\mathrm{r}}^{2 / 3}
$$

where $\Gamma=\rho_{\mathrm{r}}^{3.35} / T_{\mathrm{r}}, \quad a_{1}=3.0384588, \quad a_{2}=9.5157314 \times 10^{-07}, \quad a_{3}=-3.5296759 \times 10^{-24}$, $a_{4}=-3.5296759 \times 10^{-24}$, and $a_{5}=0.014280851$, and $a_{6}=0.014280851$. Eqs. $22-26$ combined with Eq. 33 are then used to calculate the viscosity. Comparisons with our experimental data (Section 10) resulted in an average absolute deviation of $1.9 \%$, a bias of $-0.1 \%$, and an uncertainty at the $95 \%$ level of $5.0 \%$. The temperature range covered is $290 \mathrm{~K}$ to $450 \mathrm{~K}$ at pressures up to 137.4 MPa. This is consistent with the estimated experimental uncertainty, which was estimated to be $5 \%$ at the lowest pressures rising to $10 \%$ at the highest pressure nearing $140 \mathrm{MPa}$. Deviation plots as a function of temperature, pressure and density are shown in Figures 69 - 71. As shown in Figure 70, we observe only slight systematic trends in the deviations as a function of pressure.

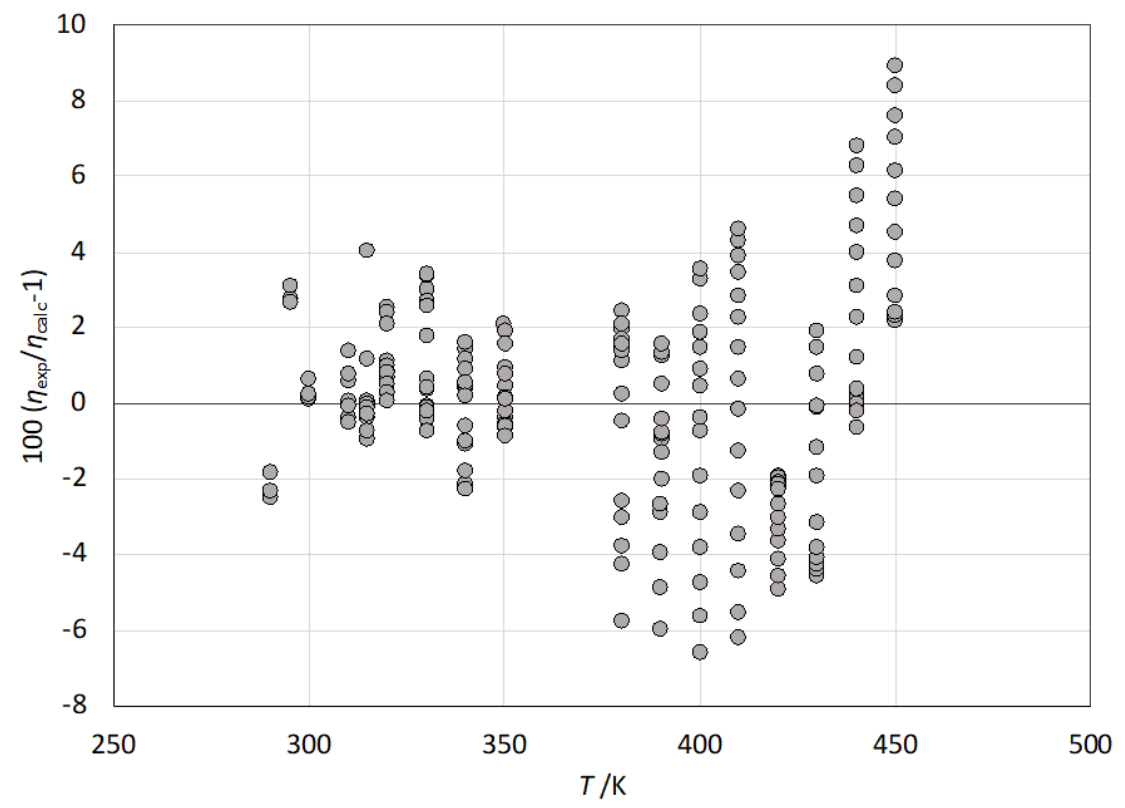

Figure 69: Percentage deviations in viscosity for MIL-PRF-23699 as a function of temperature. 


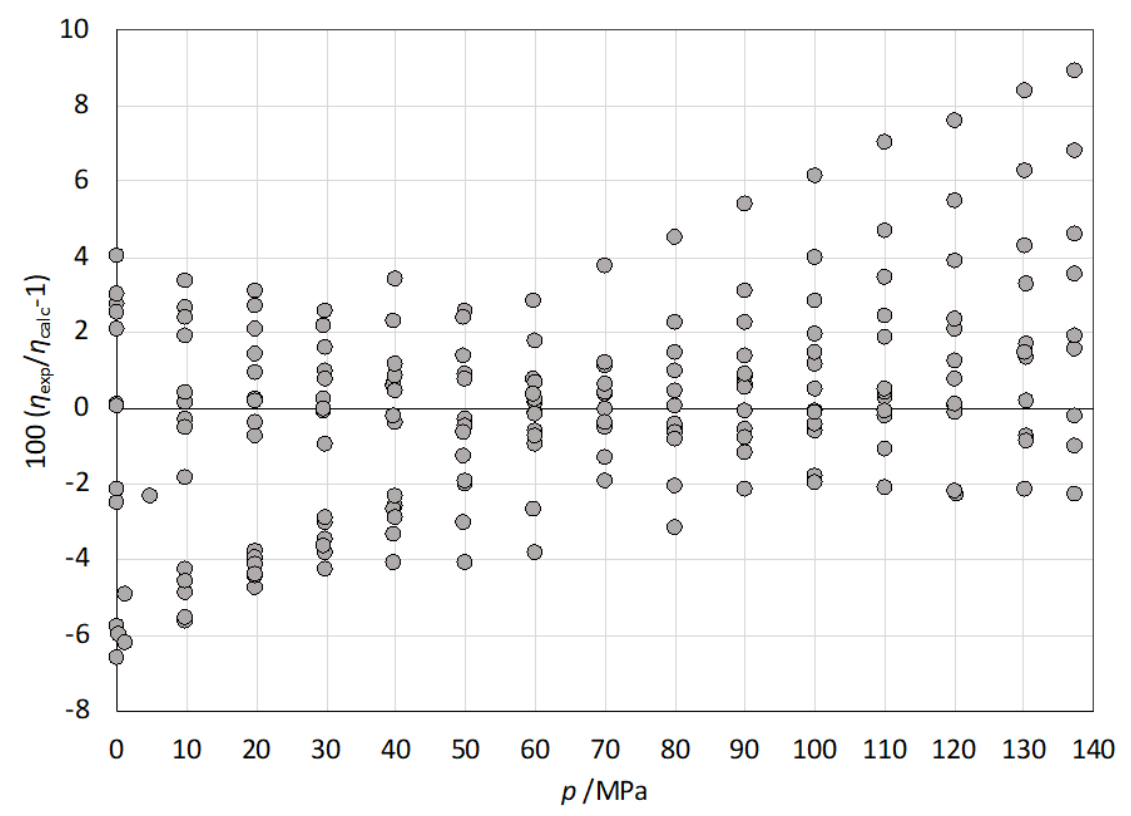

Figure 70: Percentage deviations in viscosity for MIL-PRF-23699 as a function of pressure.

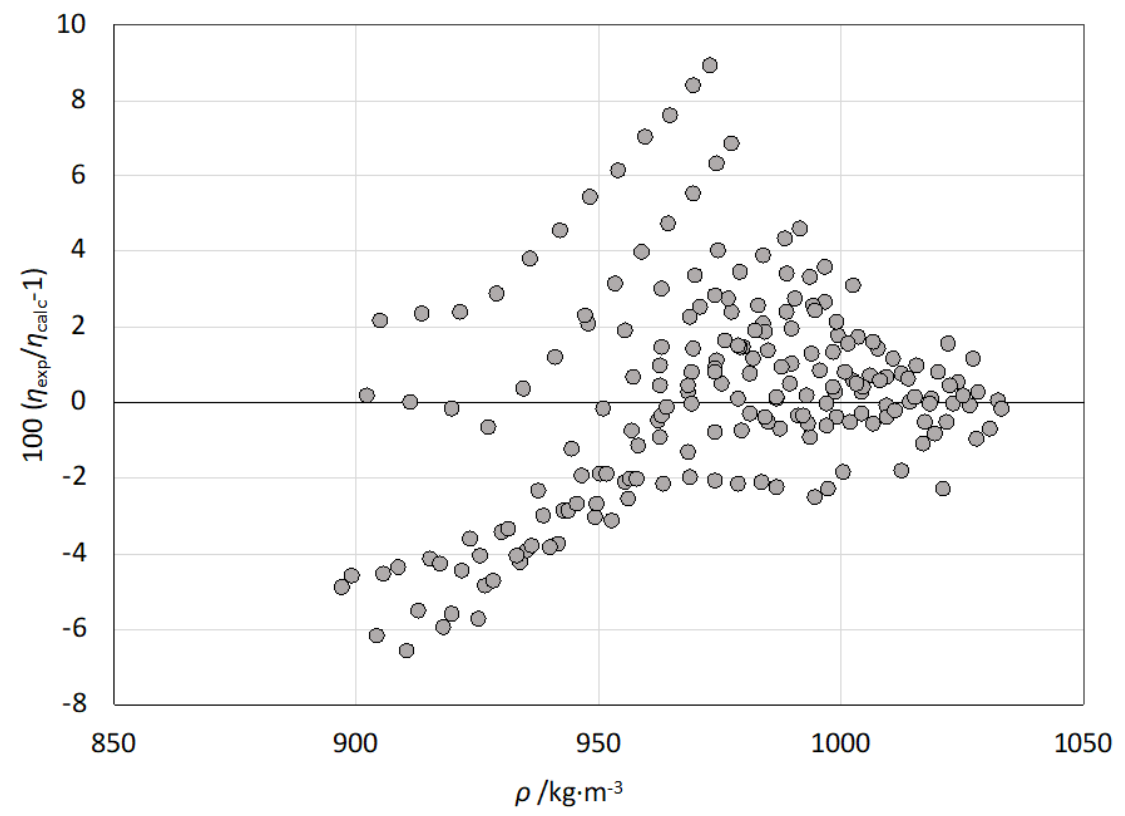

Figure 71: Percentage deviations in viscosity for MIL-PRF-23699 as a function of density.

\subsubsection{MIL-PRF-23699 Thermal Conductivity Model}

We fit the liquid-phase thermal conductivity data described in Section 9, incorporating estimated vapor-phase predictions, and obtained the following coefficients for Eq. 31 and Eq. 32. All densities were determined from the EOS described in Section 12.2. The resulting coefficients are given in Table 35. Comparisons with our experimental data (Section 9) resulted in an average 
absolute deviation of $0.19 \%$, a bias of $0.0 \%$, and an uncertainty at the $95 \%$ level of $0.5 \%$. This is consistent with the estimated experimental uncertainty, which was estimated to be $0.5 \%$ for the entire range of the experiments that covered $301 \mathrm{~K}$ to $501 \mathrm{~K}$ at pressures up to $69 \mathrm{MPa}$. Deviation plots as a function of temperature, pressure and density are shown in Figures $72-74$.

Table 35: Coefficients for the Thermal Conductivity Equation for MIL-PRF-23699.

\begin{tabular}{l|c}
\hline \multicolumn{1}{c|}{ Coefficients } & \\
\hline \hline$\alpha_{0}$ & 2.029847 \\
$\alpha_{1}$ & -17.66899 \\
$\alpha_{2}$ & 106.7431 \\
$\alpha_{3}$ & -83.01913 \\
$\alpha_{4}$ & 29.21753 \\
$\alpha_{5}$ & -3.957349 \\
$\beta_{1,1}$ & -0.0717307 \\
$\beta_{1,2}$ & 0.0411848 \\
$\beta_{1,3}$ & -0.00433472 \\
$\beta_{2,1}$ & 0.1242990 \\
$\beta_{2,2}$ & -0.0759461 \\
$\beta_{2,3}$ & 0.0133060 \\
\hline
\end{tabular}

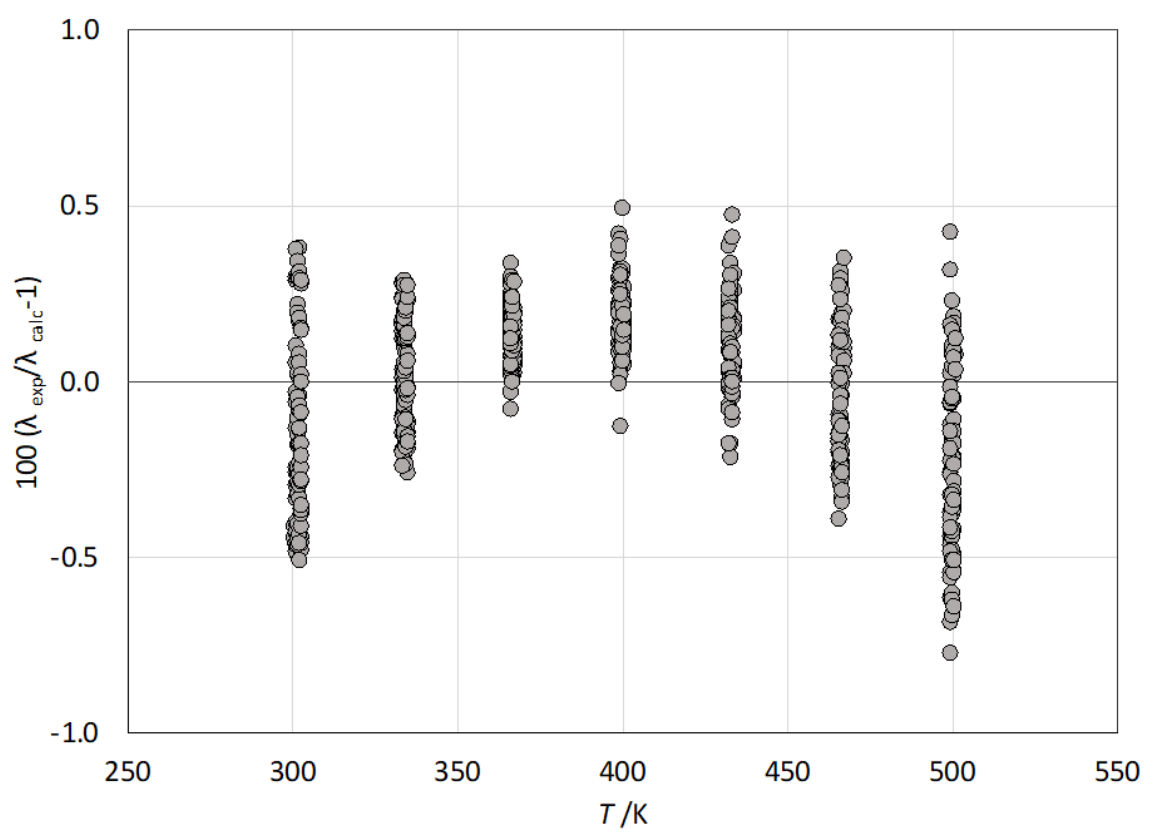

Figure 72: Percentage deviations in thermal conductivity for MIL-PRF-23699 as a function of temperature. 


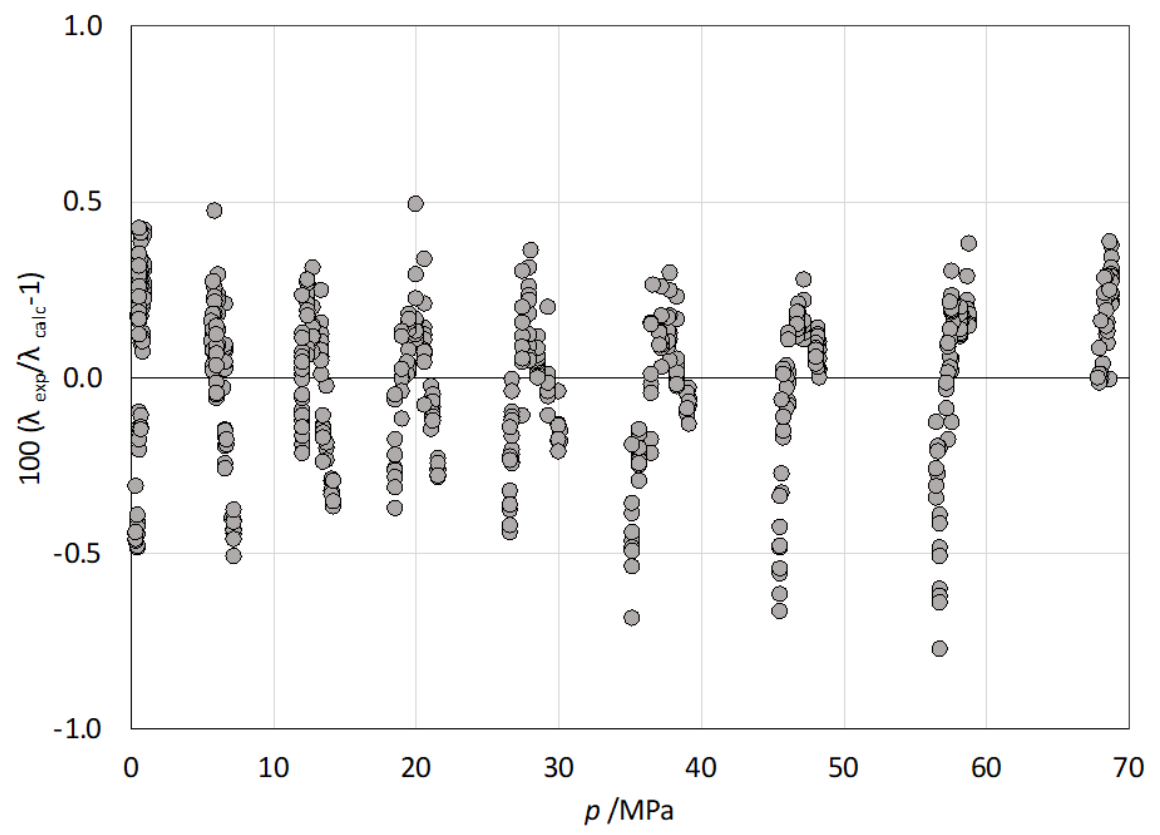

Figure 73: Percentage deviations in thermal conductivity for MIL-PRF-23699 as a function of pressure.

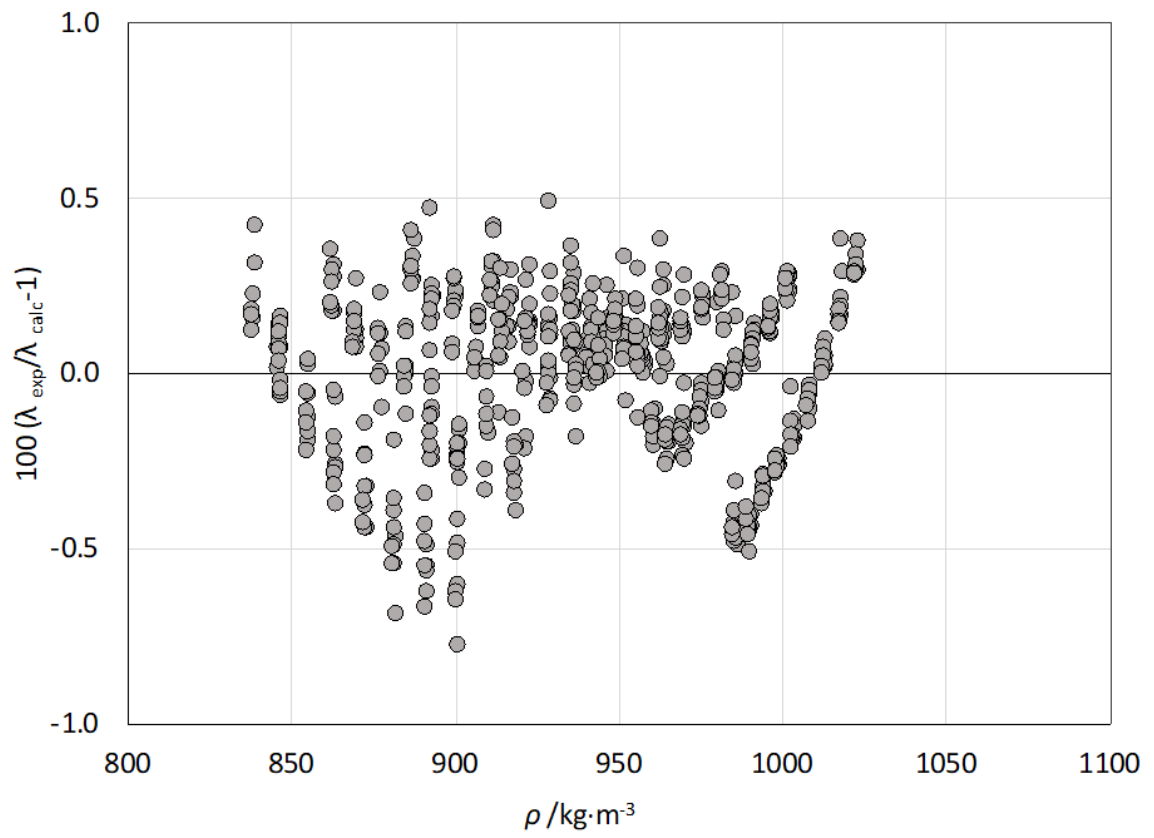

Figure 74: Percentage deviations in thermal conductivity for MIL-PRF-23699 as a function of density. 


\subsubsection{Surrogate Mixture Models}

In order to model a complex fluid mixture, such as jet fuels, diesel fuels or rocket propellants, an approach that has been used successfully is to model the complex fluid as a surrogate mixture[7274]. The general philosophy is to match desired properties (such as density, viscosity, thermal conductivity, sound speed, heat capacity) of the real mixture by finding compositions of a limited slate of well-characterized fluids that can be mixed together as a surrogate for the real mixture. The exact fluids that are in the actual mixture do not have to be present, but fluids that represent the chemical nature of the constituents should be present. In this project, we have three wellcharacterized fluids (POE5, POE7, and POE9) that are all polyol esters of the approximate weights of those in the actual mixture and the goal is to combine them in such a way as to represent the properties of the lubricant mixture MIL-PRF-23699.

It is instructive to observe trends among the POE fluids for the properties of interest at standard atmospheric pressure. Figures 75 - 79 show the properties density, sound speed, heat capacity, thermal conductivity, and viscosity at atmospheric pressure as a function of temperature for the three pure POEs, and for the actual lubricant mixture. The curves were generated with the EOS and transport models discussed in earlier sections that represent the experimental data to within, or close to within, the experimental uncertainty. For density, heat capacity, and thermal conductivity there is a clear pattern that the properties of the lubricant mixture fall in between those of POE5 and POE7. For the speed of sound, the lubricant mixture is very close to pure POE7. However, for the viscosity, the properties of the mixture fall in between those of POE7 and POE9, being closest to POE9 at lower temperatures and then becoming closer to POE7 as the temperature approaches $450 \mathrm{~K}$. This presents a significant problem to modeling the lubricant mixture as a surrogate composed of POE5, POE7, and POE9. It just is not possible to combine these three fluids in such a way to capture all properties with a surrogate model approach. That said, we will present here two surrogate model choices. One that focuses more on the representation of the viscosity, and a second that focuses on the equilibrium properties and thermal conductivity. The performance of either of these two models is not as accurate as the pseudo-pure fluid approach discussed earlier but serves as an alternative methodology if a true mixture is required. 


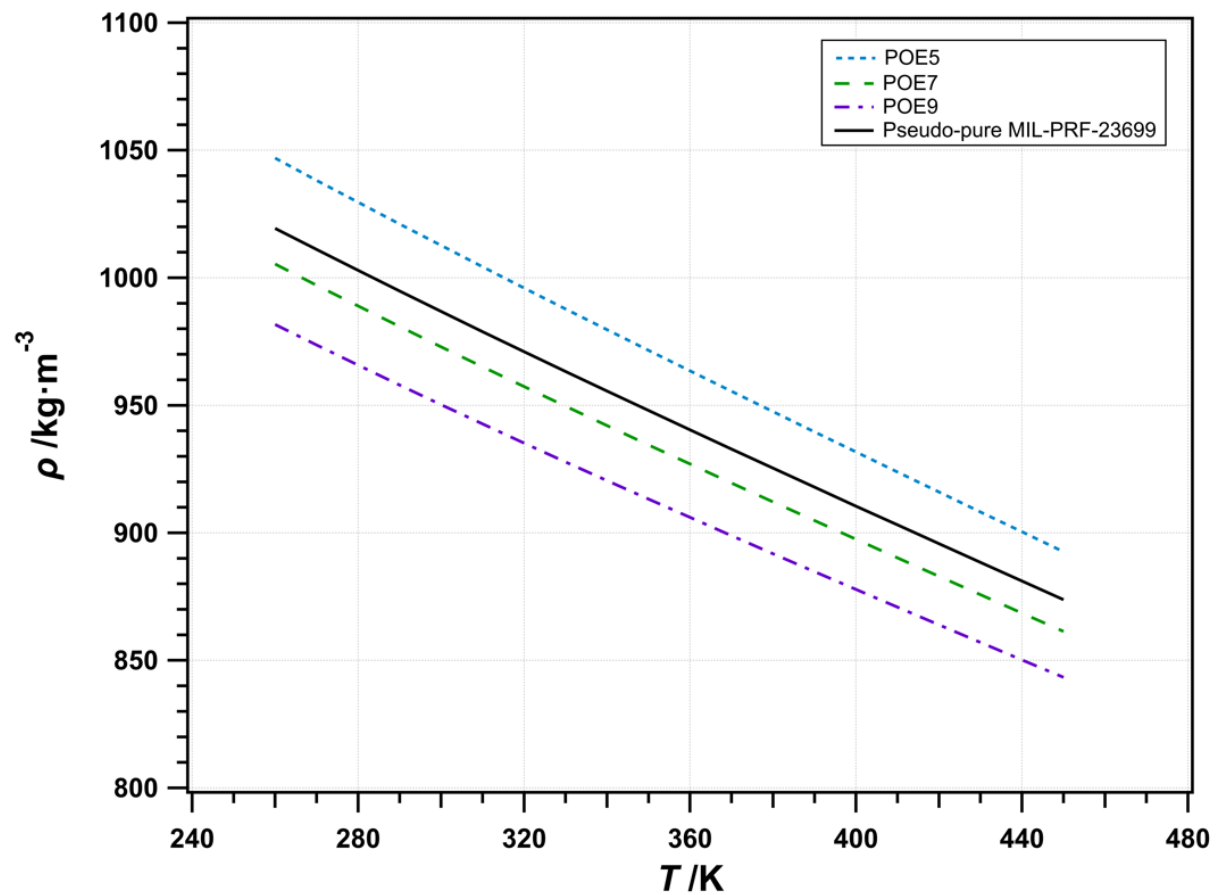

Figure 75: Density at atmospheric pressure as a function of temperature for POE5, POE7, POE9, and MIL-PRF-23699.

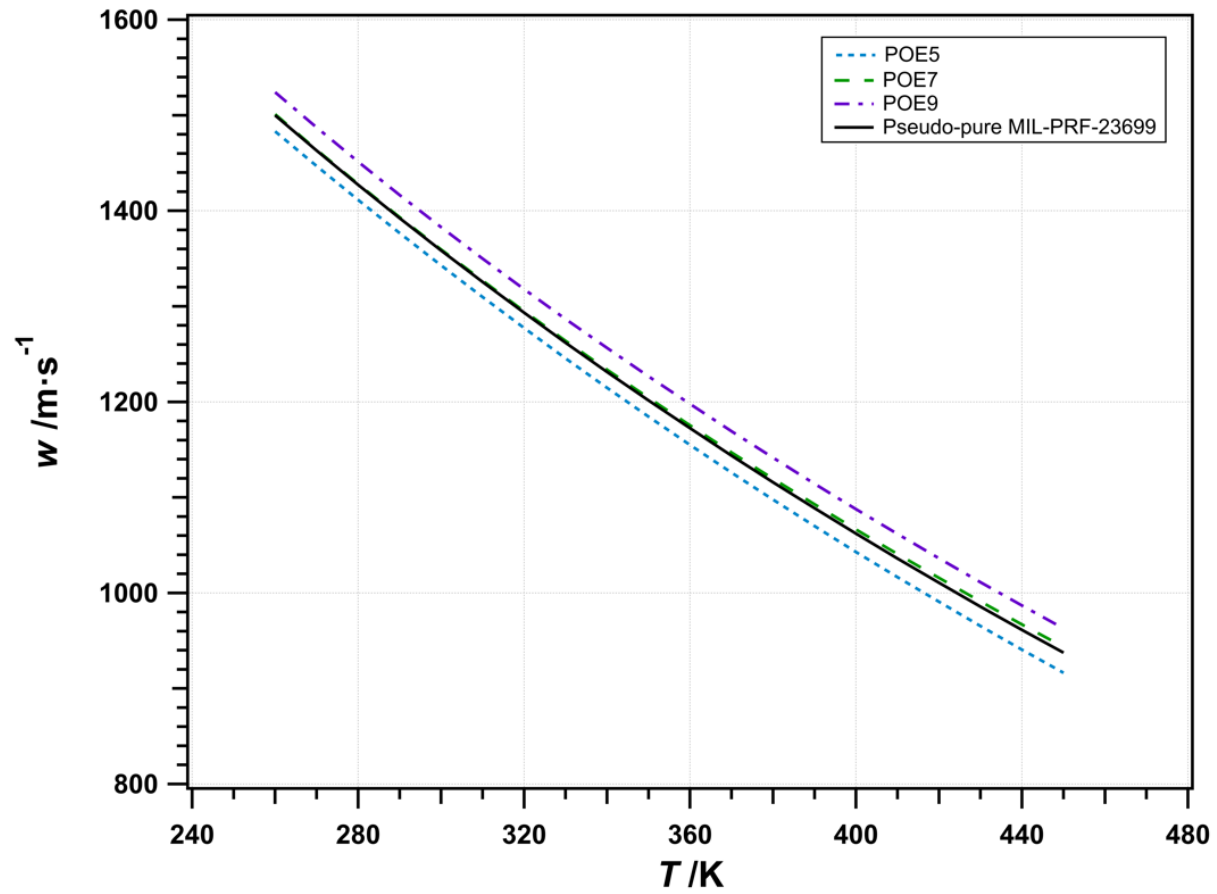

Figure 76: Speed of sound at atmospheric pressure as a function of temperature for POE5, POE7, POE9, and MIL-PRF-23699. 


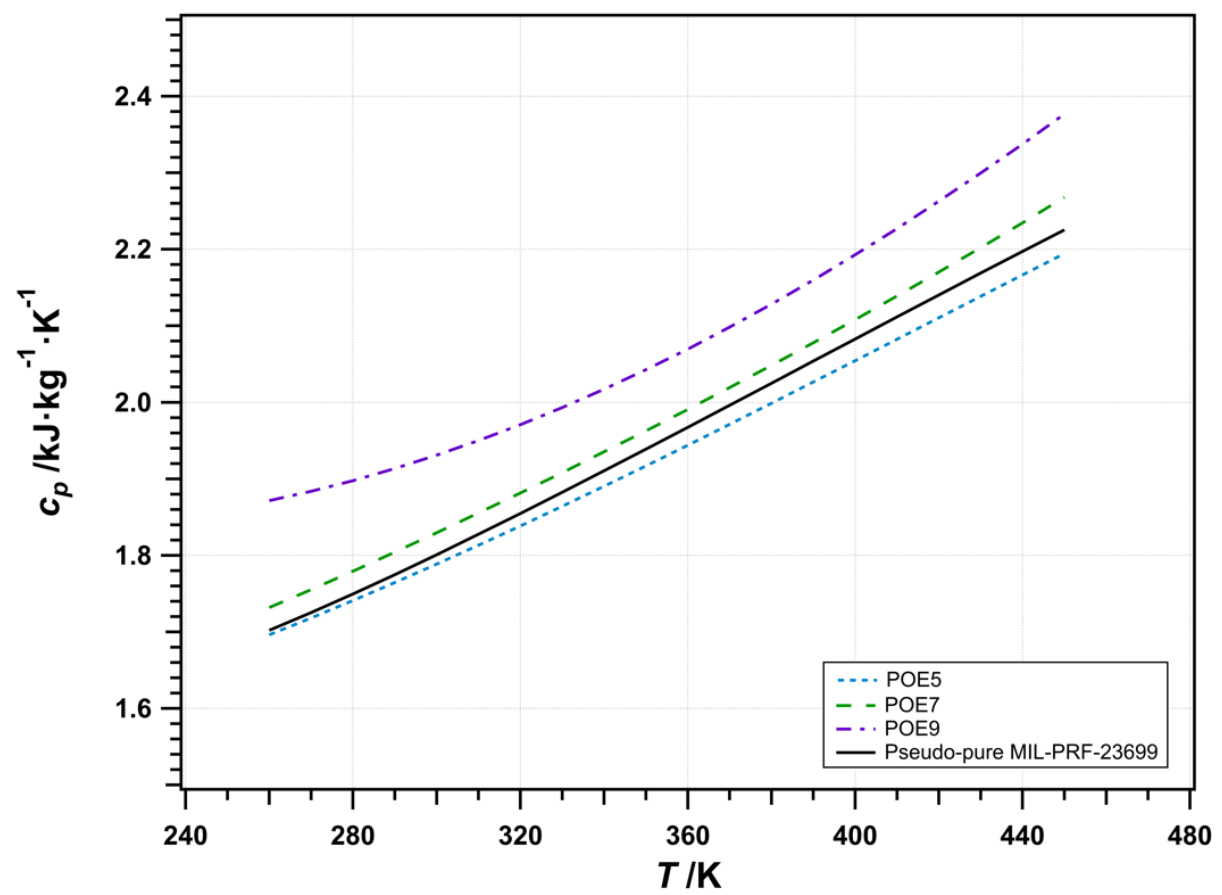

Figure 77: Heat capacity at atmospheric pressure as a function of temperature for POE5, POE7, POE9, and MIL-PRF-23699.

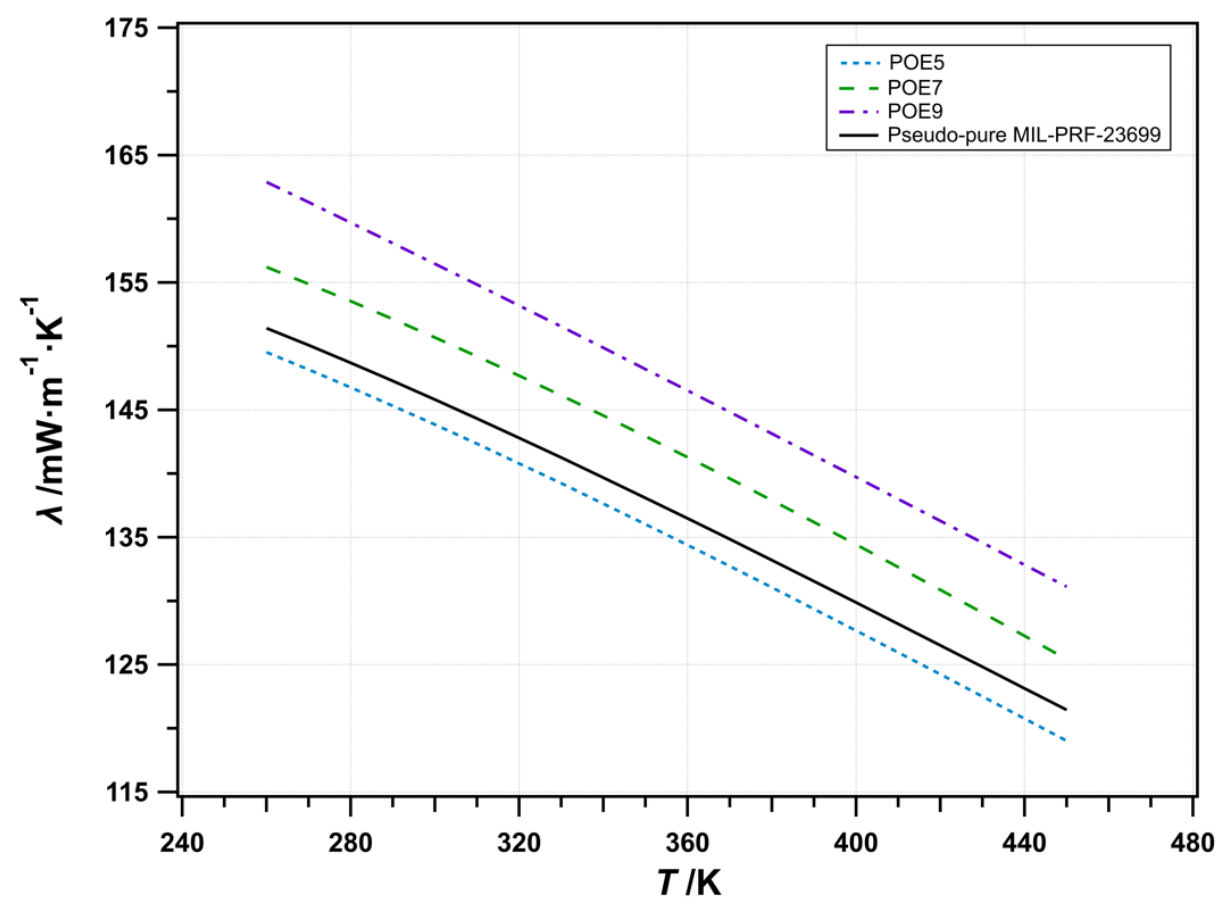

Figure 78: Thermal conductivity at atmospheric pressure as a function of temperature for POE5, POE7, POE9, and MIL-PRF-23699. 


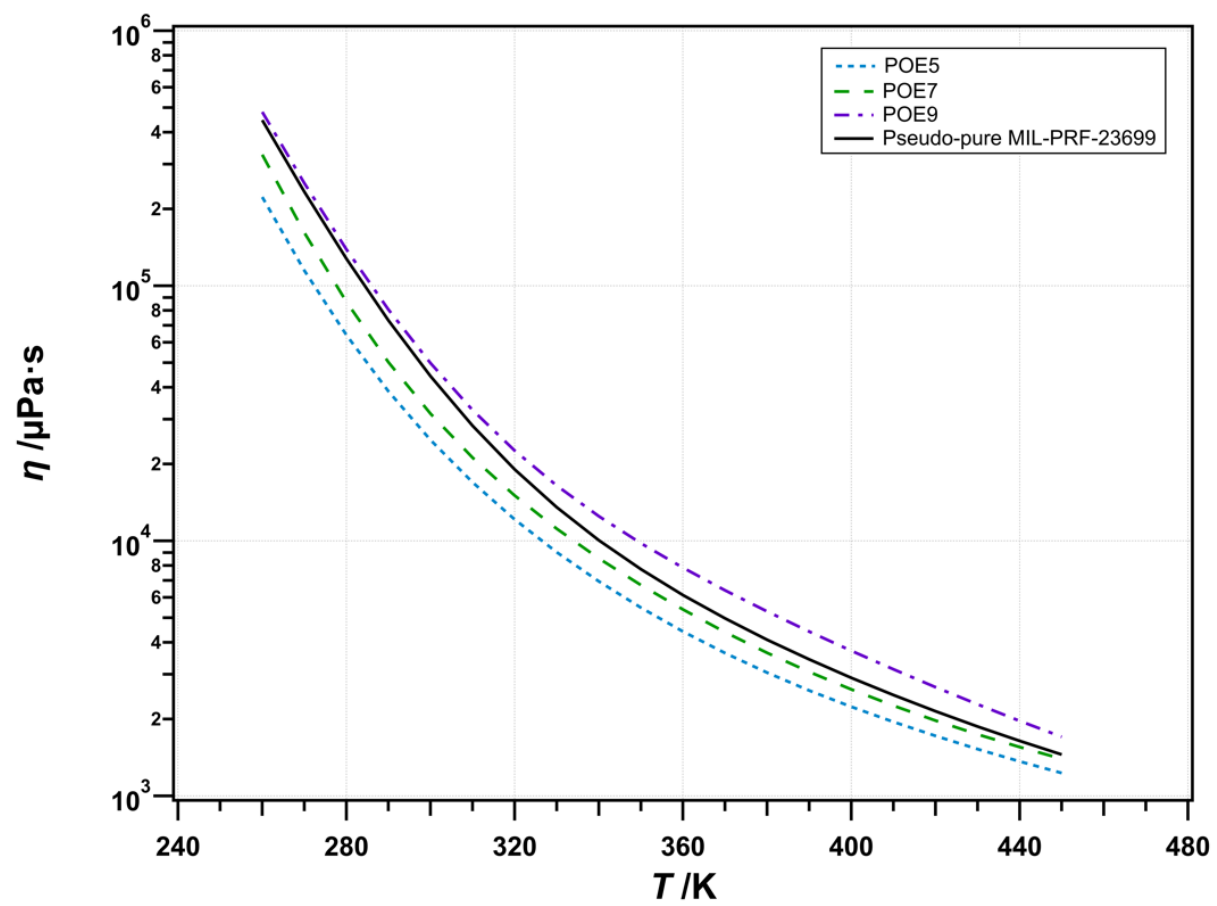

Figure 79: Viscosity at atmospheric pressure as a function of temperature for POE5, POE7, POE9, and MIL-PRF-23699.

The first surrogate model was designed to put more weight on matching the viscosity, resulting in very poor fits for the other properties. The composition of this surrogate, Surrogate 1, is 10 mole \% POE5 and 90 mole \% POE9. A second surrogate model was developed that weighted the equilibrium properties (density, sound speed, heat capacity), and thermal conductivity much more heavily than viscosity. The composition of this surrogate, Surrogate 2, is 54 mole \% POE5 and 46 mole \% POE7. The third option, discussed earlier, is to treat the mixture as a pseudo-pure fluid. Deviation plots for the properties as a function of temperature and with pressure for all three models are shown in Figures 80 - 85. The deviations were computed with the experimental data obtained in this work for the lubricant MIL-PRF-23699. The viscosity deviations show that even with an emphasis on fitting viscosity (Surrogate 1, Figures 80 and 83), the results are not within the experimental uncertainty. This can be compared with Figures 82 and 85, which show the deviations with experimental data for the pseudo-pure model. The pseudo-pure fluid model for MIL-PRF-23699 is obviously superior to either of the surrogate models. This is due in part to the fact that the surrogate model lacks any components lighter than POE9 that have a viscosity greater than, or at least close to, that of POE9. A possible solution is found in the work of Pensado et al.[75] who previously observed that the viscosity increases with increasing branching in POEs. 


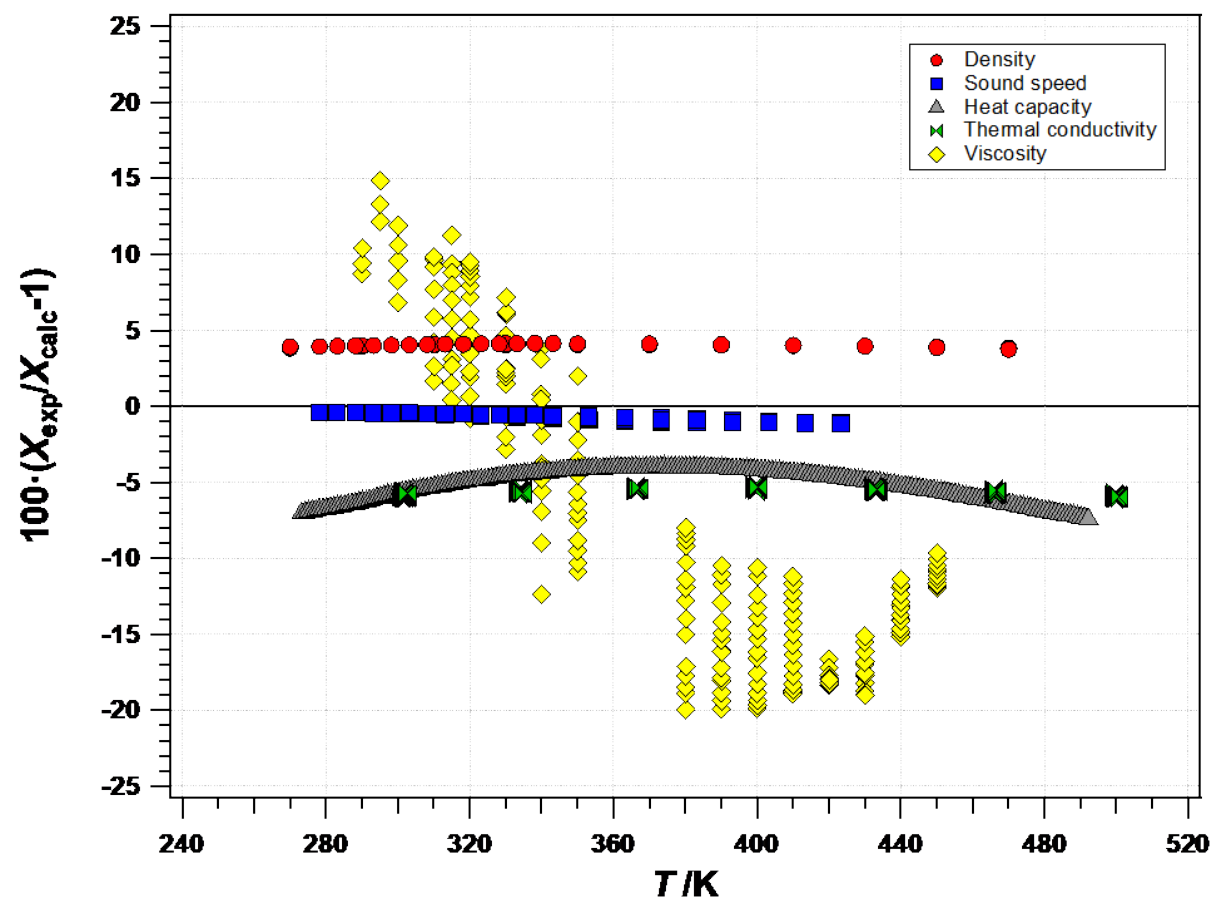

Figure 80: Property deviations of Surrogate 1 model as a function of temperature.

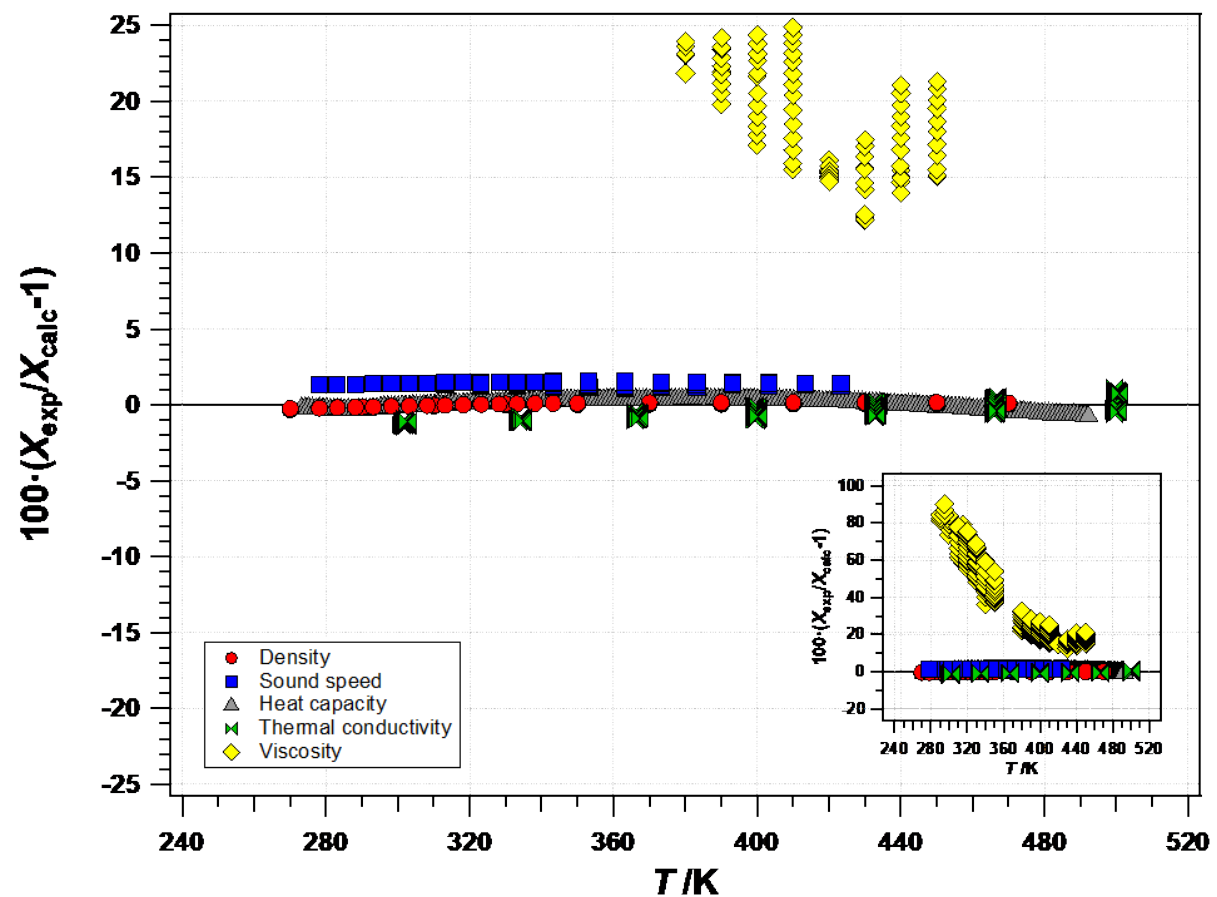

Figure 81: Property deviations of Surrogate 2 model as a function of temperature; the inset shows the large deviations in viscosity at low temperatures. 


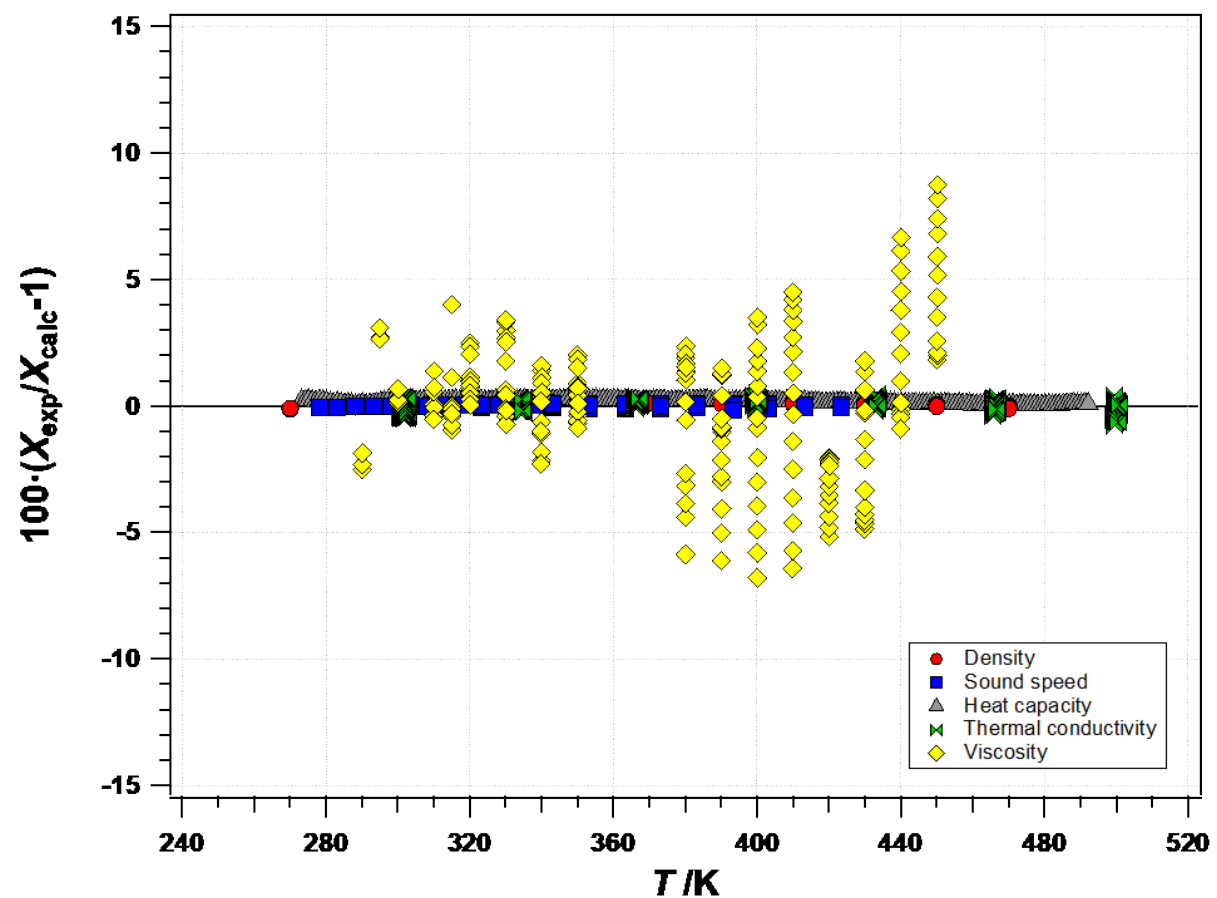

Figure 82: Property deviations of Pseudo-pure fluid model as a function of temperature.

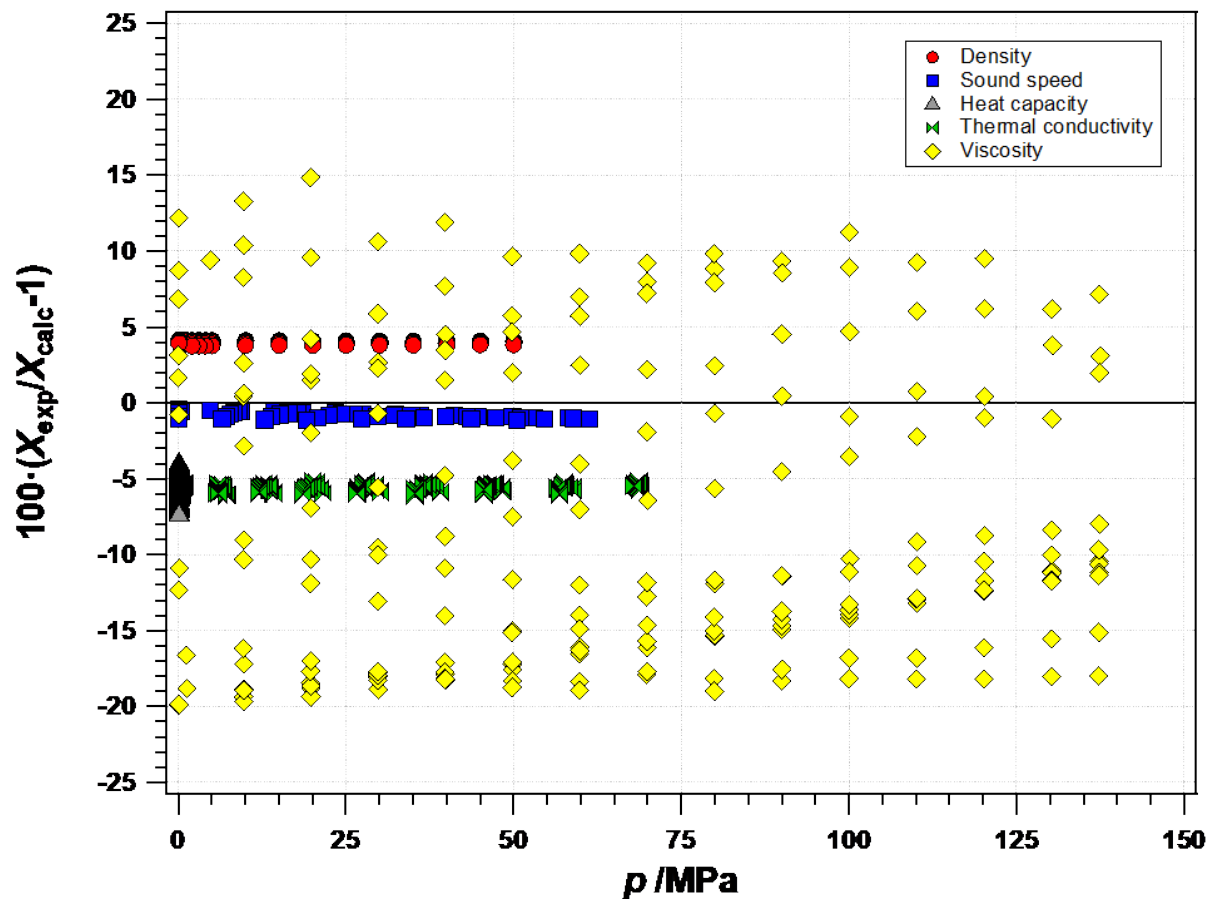

Figure 83: Property deviations of Surrogate 1 model as a function of pressure. 


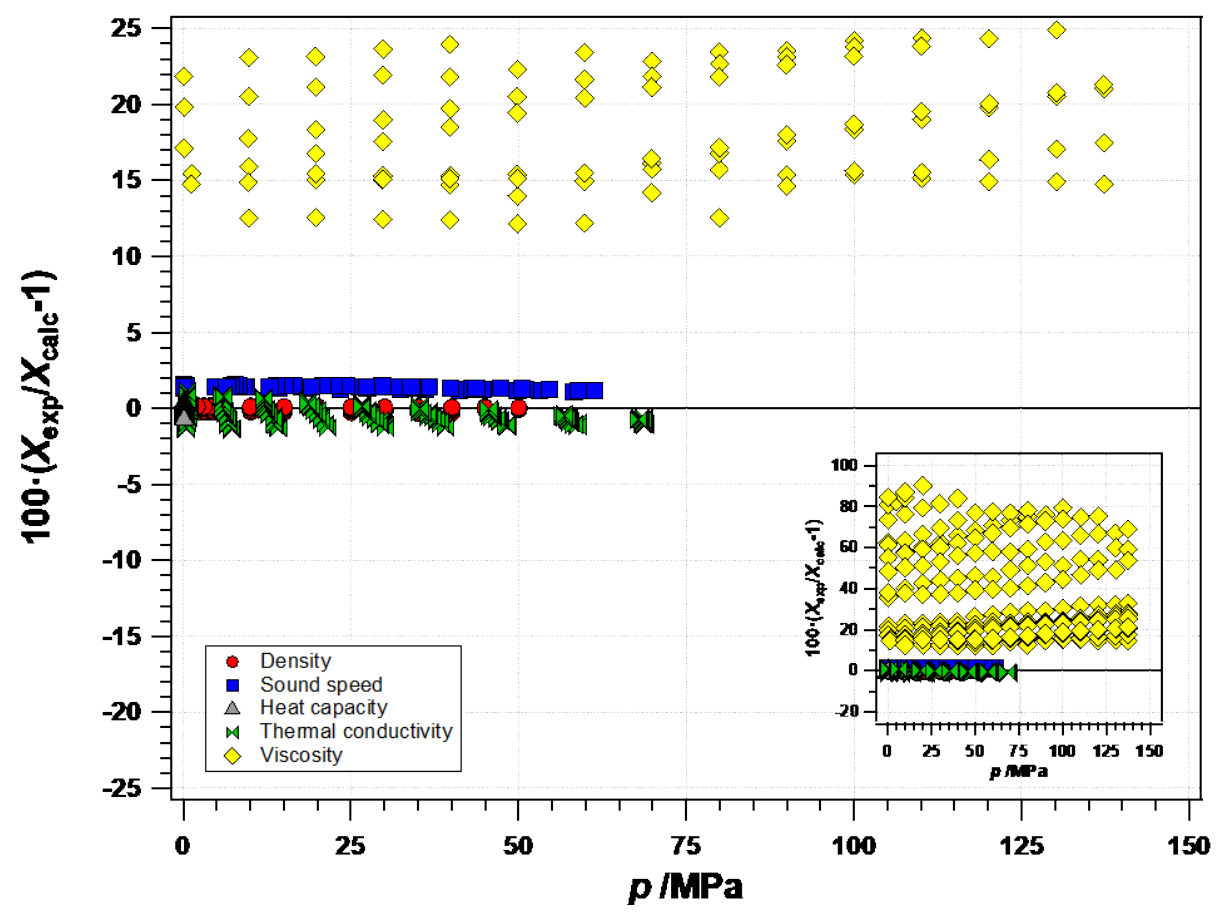

Figure 84: Property deviations of Surrogate 2 model as a function of pressure; the inset shows the large deviations in viscosity.

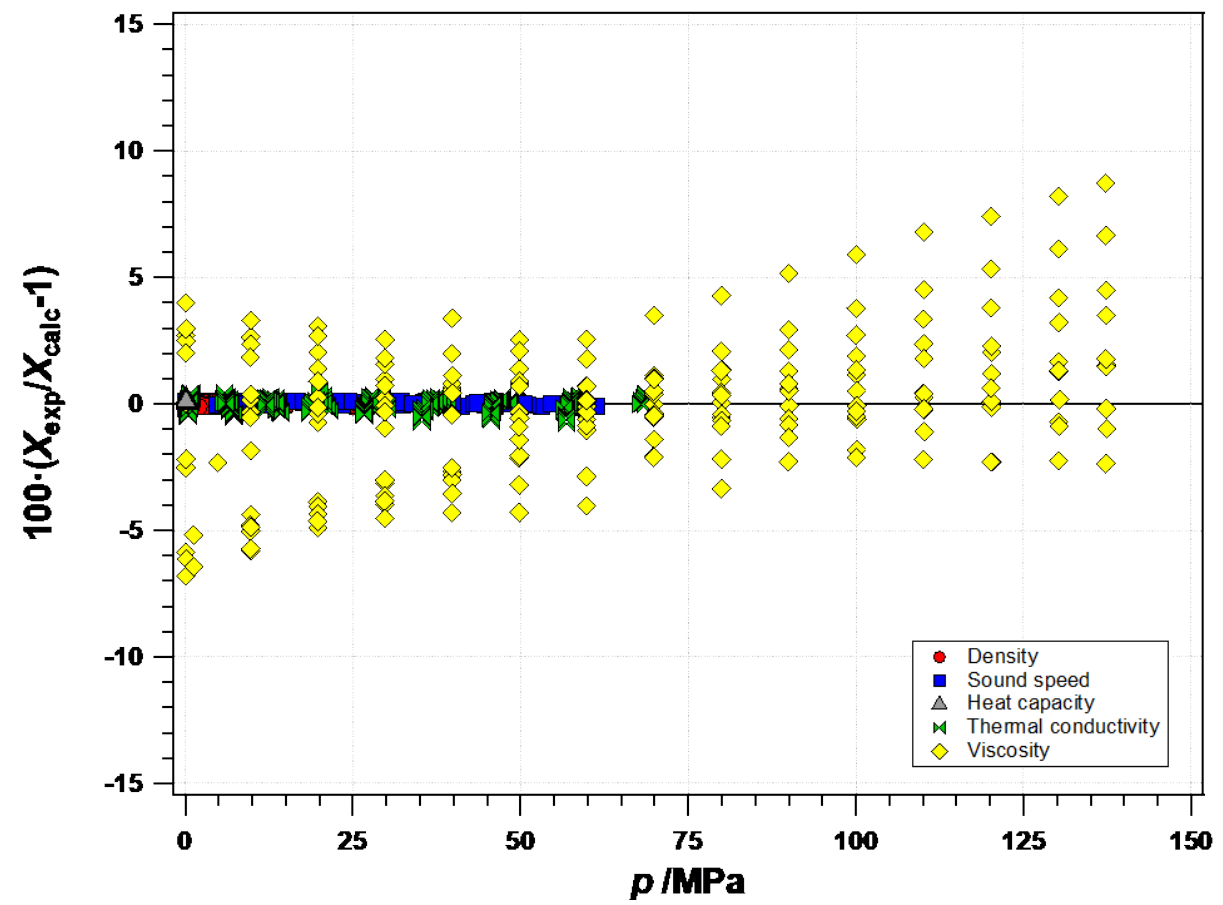

Figure 85: Property deviations of Pseudo-pure fluid model as a function of pressure. 


\section{Conclusions}

An extensive experimental and modeling program was completed that is summarized by the following points:

1. We performed purity and chemical analysis of the fluids pentaerythritol tetrapentanoate (POE5), pentaerythritol tetraheptanoate (POE7), pentaerythritol tetranonanoate (POE9), and MIL-PRF-23699 by gas chromatography with flame ionization detection (GC-FID) and gas chromatography with mass spectrometry (GC-MS).

2. The global thermal decomposition kinetics of the fluids POE5, POE7, POE9, and MILPRF-23699 were performed in stainless steel ampule reactors at pressures of approximately $20 \mathrm{MPa}$ with the extent of decomposition determined by GC-FID.

3. We completed thermodynamic (density, heat capacity, speed of sound) and transport (viscosity and thermal conductivity) property measurements of three pure polyol ester lubricants fluids (POE5, POE7, and POE9) and MIL-PRF-23699. Vapor pressure was measured for POE5; however, measurements on lubricant base fluids larger than POE5 were not attempted because their vapor pressures were below the measurable range of our apparatus.

4. The advanced distillation curve methodology was applied at atmospheric pressure, in an argon gas environment, to a sample of MIL-PRF-23699 to establish an initial boiling temperature. Due to decomposition of the heated fluid, the distillation was only carried out to $10 \%$ distillate volume fraction. No initial boiling temperature could be defined under the conditions tested, because the fluid was not thermally stable.

5. Equations of state and transport property models were constructed for the three pure base fluids, and for the fully qualified lubricant (treated as a pseudo-pure fluid). Two surrogate mixture models were developed for MIL-PRF-23699 based on the three pure POEs, one with an emphasis on thermodynamic properties and thermal conductivity, and one with an emphasis on viscosity. Both surrogate models failed to represent the properties to within their experimental uncertainty, and we recommend use of the pseudo-pure-fluid models.

6. Files that are compatible with the REFPROP computer program (version 10) are provided for the three pure fluids and the fully qualified lubricant (see Appendix C). 
In conclusion, we provide models for the thermophysical properties of three pure POE base fluids (POE5, POE7, POE9) and a fully qualified lubricant that meets MIL-PRF-23699 that are primarily based upon the comprehensive experimental work described in this document. The models are implemented in files that are compatible with the NIST computer program REFPROP, version 10. The thermophysical properties of the properties measured are predominantly represented by the models to within their experimental uncertainty. 


\section{Acknowledgements}

The authors thank James McDonnell and Dawn Schmidt, both of the Naval Air Systems Command (NAVAIR), for funding (Contract No. N0042115IP00008) and for supplying the four polyol ester lubricant samples. K.N.U. gratefully acknowledges a National Academy of Sciences/National Research Council postdoctoral fellowship. Contribution of the National Institute of Standards and Technology; not subject to U.S. Copyright. 


\section{References}

1. Cottington, R. L.; Ravner, H. Interactions of Neopentyl Polyol Ester-Tricresyl PhosphateIron Systems at $500^{\circ} \mathrm{F}$. ASLE Trans. 1969, 12, 280-286.

2. Zeman, A.; Bartl, P.; Schaaff, A. Mass-Spectrometric Fragmentation of Neopentylpolyol Esters. 1. Pentaerythritol Tetra Esters of Fatty-Acids. Org. Mass Spectrom. 1978, 13, 248-253.

3. Urness, K. N.; Gough, R. V.; Widegren, J. A.; Bruno, T. J. Thermal Decomposition Kinetics of Polyol Ester Lubricants. Energy Fuels 2016, 30, 10161-10170.

4. Widegren, J. A.; Bruno, T. J. Thermal Decomposition Kinetics of the Aviation Turbine Fuel Jet A. Ind. Eng. Chem. Res. 2008, 47, 4342-43448.

5. Widegren, J. A.; Bruno, T. J. Thermal Decomposition Kinetics of Propylcyclohexane. Ind. Eng. Chem. Res. 2009, 48, 654-659.

6. Razzouk, A.; Mokbel, I.; García, J.; Fernandez, J.; Msakni, N.; Jose, J. Vapor Pressure Measurements in the Range $10^{-5} \mathrm{~Pa}$ to $1 \mathrm{~Pa}$ of Four Pentaerythritol Esters - Density and Vapor-Liquid Equilibria Modeling of Ester Lubricants. Fluid Phase Equilibr. 2007, 260, 248-261.

7. Bruno, T. J.; Mayrath, J. E. Concatenated Gas Saturation Vapor Pressure Apparatus. Rev. Sci. Instrum. 1997, 68, 2864-2870.

8. Carruth, G. F.; Kobayashi, R. Vapor Pressure of Normal Paraffins Ethane through nDecane from Their Triple Points to About 10 mm Hg. J. Chem. Eng. Data 1973, 18, 115126.

9. Delle Site, A. The Vapor Pressure of Environmentally Significant Organic Chemicals: A Review of Methods and Data at Ambient Temperature. J. Phys. Chem. Ref. Data 1997, 26, 157-193.

10. Gerry, H. T.; Gillespie, L. J. The Calculation of Normal Vapor Pressures from the Data of the Gas Current Method, Particularly in the Case of Iodine. Phys. Rev. 1932, 40, 269280.

11. Kvande, H.; Wahlbeck, P. G. Theory for Determination of Vapour Pressures by the Transpiration Method. Acta Chem. Scand. A 1976, 30, 297-302.

12. Macknick, A. B.; Prausnitz, J. M. Vapor-Pressures of High-Molecular-Weight Hydrocarbons. J. Chem. Eng. Data 1979, 24, 175-178. 
13. Mokbel, I.; Razzouk, A.; Hajjaji, A.; Msakni, N.; Jose, J. A Gas Saturation Apparatus for Very Low Vapor or Sublimation Pressure Measurements $\left(10^{-3} \mathrm{~Pa}\right)$ : Vapor-Liquid Equilibria of $n$-Alkanes (n-C $\left.10, n-C_{24}, n-C_{28}\right)$. J. Chem. Eng. Data 2007, 52, 1720-1725.

14. Razzouk, A.; Abou Naccoul, R.; Mokbel, I.; Saab, J.; Jose, J. Vapor and Sublimation Pressures of Three Normal Alkanes: C-20, C-24, and C-28. J. Chem. Eng. Data 2009, 54, 1214-1219.

15. Verevkin, S. P.; Emel'yaneriko, V. N. Transpiration Method: Vapor Pressures and Enthalpies of Vaporization of Some Low-Boiling Esters. Fluid Phase Equilibr. 2008, 266, 64-75.

16. Widegren, J. A.; Beall, C. E.; Tolbert, A. E.; Lovestead, T. M.; Bruno, T. J. The Use of Antioxidants to Improve Vapor Pressure Measurements on Compounds with Oxidative Instability: Methyl Oleate with tert-Butylhydroquinone. J. Chem. Eng. Data 2017, 62, 539-546.

17. Widegren, J. A.; Harvey, A. H.; McLinden, M. O.; Bruno, T. J. Vapor Pressure Measurements by the Gas Saturation Method: The Influence of the Carrier Gas. J. Chem. Eng. Data 2015, 60, 1173-1180.

18. Merten, U. Diffusion Effects in the Transpiration Method of Vapor Pressure Measurement. J. Phys. Chem. 1959, 63, 443-445.

19. Frenkel, M.; Chirico, R. D.; Diky, V.; Kroenlein, K.; Muzny, C. D.; Kazakov, A. F.; Magee, J. W.; Abdulagatov, I. M. Thermodata Engine (TDE), Version 8.0; National Institute of Standards and Technology: Gaithersburg, MD, 2013.

20. Span, R.; Lemmon, E. W.; Jacobsen, R. T.; Wagner, W.; Yokozeki, A. A Reference Equation of State for the Thermodynamic Properties of Nitrogen for Temperatures from 63.151 to $1000 \mathrm{~K}$ and Pressures to $2200 \mathrm{MPa}$. J. Phys. Chem. Ref. Data 2000, 29, 13611433.

21. Lemmon, E. W.; Bell, I. H.; Huber, M. L.; McLinden, M. O. Reference Fluid Thermodynamic and Transport Properties (REFPROP), Version 10.0; National Institute of Standards and Technology: Gaithersburg, MD, 2018.

22. Yang, S.; Schultz, A. J.; Kofke, D. A.; Harvey, A. H. Interpreting Gas-Saturation Vapor Pressure Measurements Using Virial Coefficients Derived from Molecular Models. J. Chem. Eng. Data 2014, 59, 3183-3192.

23. Widegren, J. A.; Bruno, T. J. Gas Saturation Vapor Pressure Measurements of Mononitrotoluene Isomers from (283.15 to 313.15) K. J. Chem. Eng. Data 2010, 55, 159164. 
24. Lemmon, E. W.; Goodwin, A. R. H. Critical Properties and Vapor Pressure Equation for Alkanes $\mathrm{C}_{n} \mathrm{H}_{2 n+2}$ : Normal Alkanes with $n \leq 36$ and Isomers for $n=4$ through $n=9$. $J$. Phys. Chem. Ref. Data 2000, 29, 1-39.

25. Fortin, T. J. Density, Speed of Sound, and Heat Capacity Measurements of Polyol Ester Lubricants. J. Chem. Eng. Data 2018, 63, 4325-4338.

26. Boerio-Goates, J.; Callanan, J. E. Differential Thermal Methods. In Physical Methods of Chemistry, Volume VI: Determination of Thermodynamic Properties; Rossiter, B. W., Baetzold, R. C., Eds.; John Wiley \& Sons: New York, NY, 1992; pp 621-717.

27. Reading, M.; Luget, A.; Wilson, R. Modulated Differential Scanning Calorimetry. Thermochim. Acta 1994, 238, 295-307.

28. Wunderlich, B.; Jin, Y.; Boller, A. Mathematical Description of Differential Scanning Calorimetry Based on Periodic Temperature Modulation. Thermochim. Acta 1994, 238, 277-293.

29. Höhne, G. W. H.; Hemminger, W.; Flammersheim, H. J. Differential Scanning Calorimetry: An Introduction for Practitioners; Springer-Verlag: Berlin, Germany, 1996.

30. Della Gatta, G.; Richardson, M. J.; Sarge, S. M.; Stølen, S. Standards, Calibration, and Guidelines in Microcalorimetry Part 2. Calibration Standards for Differential Scanning Calorimetry. Pure Appl. Chem. 2006, 78, 1455-1476.

31. Rosasco, G. J.; Whetstone, J. R.; Watters, R. L. Certificate of Analysis - Standard Reference Material 2232; National Institute of Standards and Technology: Gaithersburg, MD, 2003.

32. Rasberry, S. D. Certificate of Analysis - Standard Reference Material 2220; National Institute of Standards and Technology: Gaithersburg, MD, 1989.

33. Westrum, E. F. The Thermophysical Properties of Three Globular Molecules. J. Phys. Chem. Solids 1961, 18, 83-85.

34. Archer, D. G. Thermodynamic Properties of Synthetic Sapphire $\left(\alpha-\mathrm{Al}_{2} \mathrm{O}_{3}\right)$, Standard Reference Material 720 and the Effect of Temperature-Scale Differences on Thermodynamic Properties. J. Phys. Chem. Ref. Data 1993, 22, 1441-1453.

35. Harris, G. L.; Torres, J. A. Selected Laboratory and Measurement Practices and Procedures to Support Basic Mass Calibrations, NISTIR 6969; National Institute of Standards and Technology: Gaithersburg, MD, 2003.

36. Picard, A.; Davis, R. S.; Glaser, M.; Fujii, K. Revised Formula for the Density of Moist Air (CIPM-2007). Metrologia 2008, 45, 149-155. 
37. Schneider, N.; Fortin, T. J.; Span, R.; Gerber, M. Thermophysical Properties of the Marine Microalgae Nannochloropsis salina. Fuel Process. Technol. 2016, 152, 390-398.

38. Guide to the Expression of Uncertainty in Measurement; International Organization for Standardization: Geneva, Switzerland, 1995.

39. Lemmon, E. W.; McLinden, M. O.; Wagner, W. Thermodynamic Properties of Propane. III. A Reference Equation of State for Temperatures from the Melting Line to $650 \mathrm{~K}$ and Pressures up to 1000 MPa. J. Chem. Eng. Data 2009, 54, 3141-3180.

40. Meier, K.; Kabelac, S. Thermodynamic Properties of Propane. IV. Speed of Sound in the Liquid and Supercritical Regions. J. Chem. Eng. Data 2012, 57, 3391-3398.

41. Outcalt, S. L. Compressed-Liquid Density Measurements of Four Polyol Ester-Based Lubricants. Energy Fuels 2018, 32, 3775-3782.

42. Outcalt, S. L.; McLinden, M. O. Automated Densimeter for the Rapid Characterization of Industrial Fluids. Ind. Eng. Chem. Res. 2007, 46, 8264-8269.

43. Wagner, W.; Pruß, A. The IAPWS Formulation 1995 for the Thermodynamic Properties of Ordinary Water Substance for General and Scientific Use. J. Phys. Chem. Ref. Data 2002, 31, 387-535.

44. Lemmon, E. W.; Span, R. Short Fundamental Equations of State for 20 Industrial Fluids. J. Chem. Eng. Data 2006, 51, 785-850.

45. May, E. F.; Tay, W. J.; Nania, M.; Aleji, A.; Al-Ghafri, S.; Trusler, J. P. M. Physical Apparatus Parameters and Model for Vibrating Tube Densimeters at Pressures to 140 MPa and Temperatures to 473 K. Rev. Sci. Instrum. 2014, 85, 095111.

46. $\quad$ Laesecke, A.; Fortin, T. J.; Splett, J. D. Density, Speed of Sound, and Viscosity Measurements of Reference Materials for Biofuels. Energy Fuels 2012, 26, 1844-1861.

47. Fortin, T. J. Assessment of Variability in the Thermophysical Properties of Rocket Propellant RP-1. Energy Fuels 2012, 26, 4383-4394.

48. Fortin, T. J.; Laesecke, A.; Freund, M.; Outcalt, S. L. Advanced Calibration, Adjustment, and Operation of a Density and Sound Speed Analyzer. J. Chem. Thermodyn. 2013, 57, 276-285.

49. Healy, J.; DeGroot, J. J.; Kestin, J. The Theory of the Transient Hot-Wire Method for Measuring the Thermal Conductivity. Physica 1976, 82C, 392-408.

50. Woodfield, P. L.; Fukai, J.; Fujii, M.; Takata, Y.; Shinzato, K. A Two-Dimensional Analytical Solution for the Transient Short-Hot-Wire Method. Int. J. Thermophys. 2008, 29, 1278-1298. 
51. Laesecke, A.; Junker, C.; Lauria, D. S. Viscosity Measurements of Three Base Oils and One Fully Formulated Lubricant and New Viscosity Correlations for the Calibration Liquid Squalane. J. Res. NIST 2019, 124, 124002.

52. Mylona, S. K.; Assael, M. J.; Comuñas, M. J. P.; Parades, X.; Gaciño, F. M.; Fernández, J.; Bazile, J. P.; Boned, C.; Daridon, J. L.; Galliero, G.; Pauly, J.; Harris, K. R. Reference Correlations for the Density and Viscosity of Squalane from 273 to $473 \mathrm{~K}$ at Pressures to 200 MPa. J. Phys. Chem. Ref. Data 2014, 43, 013104.

53. Schmidt, K. A.; Pagnutti, D.; Curran, M. D.; Singh, A.; Trusler, J. P. M.; Maitland, G. C.; McBride-Wright, M. New Experimental Data and Reference Models for the Viscosity and Density of Squalane. J. Chem. Eng. Data 2015, 60, 137-150.

54. Schaschke, C. J.; Abid, S.; Fletcher, I.; Heslop, M. J. Evaluation of a Falling Sinker-Type Viscometer at High Pressure Using Edible Oil. J. Food. Eng. 2008, 87, 51-58.

55. $\quad$ Laesecke, A.; Bair, S. High Pressure Viscosity Measurements of 1,1,1,2Tetrafluoroethane. Int. J. Thermophys. 2011, 32, 925-941.

56. Bair, S.; Laesecke, A. Viscosity Measurements of R32 and R410a to 350 MPa. Int. J. Refrig. 2017, 83, 157-167.

57. Bruno, T. J.; Ott, L. S.; Smith, B. L.; Lovestead, T. M. Complex Fluid Analysis with the Advanced Distillation Curve Approach. Anal. Chem. 2010, 82, 777-783.

58. Smith, B. L.; Ott, L. S.; Bruno, T. J. Composition-Explicit Distillation Curves of Commercial Biodiesel Fuels: Comparison of Petroleum-Derived Fuels with B20 and B100. Ind. Eng. Chem. Res. 2008, 47, 5832-5840.

59. Span, R.; Wagner, W. Equations of State for Technical Applications. II. Results for Nonpolar Fluids. Int. J. Thermophys. 2003, 24, 41-109.

60. Lemmon, E. W.; Jacobsen, R. T. A New Functional Form and New Fitting Techniques for Equations of State with Application to Pentafluoroethane (HFC-125). J. Phys. Chem. Ref. Data 2005, 34, 69-108.

61. Huber, M. L. Models for the Viscosity, Thermal Conductivity, and Surface Tension of Selected Pure Fluids as Implemented in REFPROP V10.0, NISTIR 8209; National Institute of Standards and Technology: Gaithersburg, MD, 2018.

62. Chung, T.-H.; Ajlan, M.; Lee, L. L.; Starling, K. E. Generalized Multiparameter Correlation for Nonpolar and Polar Fluid Transport Properties. Ind. Eng. Chem. Res. 1988, 27, 671-679.

63. Eureqa Formulize, v.098.1; Nutonian Inc.: Cambridge, MA. 
64. Assael, M. J.; Dymond, J. H.; Papadaki, M.; Patterson, P. M. Correlation and Prediction of Dense Fluid Transport Coefficients. I. n-Alkanes. Int. J. Thermophys. 1992, 13, 269281.

65. Assael, M. J.; Papalas, T. B.; Huber, M. L. Reference Correlations for the Viscosity and Thermal Conductivity of n-Undecane. J. Phys. Chem. Ref. Data 2017, 46, 033103.

66. Tsolakidou, C. M.; Assael, M. J.; Huber, M. L.; Perkins, R. A. Correlations for the Viscosity and Thermal Conductivity of Ethyl Fluoride (R161). J. Phys. Chem. Ref. Data 2017, 46, 023103.

67. Monogenidou, S. A.; Assael, M. J.; Huber, M. L. Reference Correlation for the Viscosity of Ammonia from the Triple Point to $725 \mathrm{~K}$ and up to $50 \mathrm{MPa}$. J. Phys. Chem. Ref. Data 2018, 47, 023102.

68. Bair, S.; Casalini, R. A Scaling Parameter and Function for the Accurate Correlation of Viscosity with Temperature and Pressure across Eight Orders of Magnitude of Viscosity. J. Tribol. 2008, 130, 041802.

69. Perkins, R. A.; Sengers, J. V.; Abdulagatov, I. M.; Huber, M. L. Simplified Model for the Critical Thermal-Conductivity Enhancement in Molecular Fluids. Int. J. Thermophys. 2013, 34, 191-212.

70. Lemmon, E. W.; Jacobsen, R. T.; Penoncello, S. G.; Friend, D. G. Thermodynamic Properties of Air and Mixtures of Nitrogen, Argon, and Oxygen from 60 to $2000 \mathrm{~K}$ at Pressures to 2000 MPa. J. Phys. Chem. Ref. Data 2000, 29, 331-385.

71. Lemmon, E. W. Pseudo-Pure Fluid Equations of State for the Refrigerant Blends R-410a, R-404a, R-507a, and R-407c. Int. J. Refrig. 2003, 24, 991-1006.

72. Huber, M. L.; Lemmon, E. W.; Ott, L. S.; Bruno, T. J. Preliminary Surrogate Mixture Models for the Thermophysical Properties of Rocket Propellants RP-1 and RP-2. Energy Fuels 2009, 23, 3083-3088.

73. Huber, M. L.; Lemmon, E. W.; Bruno, T. J. Surrogate Mixture Models for the Thermophysical Properties of Aviation Fuel Jet-A. Energy Fuels 2010, 24, 3565-3571.

74. Mueller, C. J.; Cannella, W. J.; Bruno, T. J.; Bunting, B.; Dettman, H. D.; Franz, J. A.; Huber, M. L.; Natarajan, M.; Pitz, W. J.; Ratcliff, M. A.; Wright, K. Methodology for Formulating Diesel Surrogate Fuels with Accurate Compositional, Ignition-Quality, and Volatility Characteristics. Energy Fuels 2012, 26, 3284-3303.

75. Pensado, A. S.; Comuñas, M. J. P.; Lugo, L.; Fernández, J. High-Pressure Characterization of Dynamic Viscosity and Derived Properties for Squalane and Two 
Pentaerythritol Ester Lubricants: Pentaerythritol Tetra-2-ethylhexanoate and Pentaerythritol Tetranonanoate. Ind. Eng. Chem. Res. 2006, 45, 2394-2404. 


\section{Appendix A: Thermal Conductivity Data Tables}

Table A1: Thermal Conductivity Data for POE5 at Temperatures from $300 \mathrm{~K}$ to $500 \mathrm{~K}$.

\begin{tabular}{|c|c|c|c|c|c|c|c|}
\hline$T_{\mathrm{i}}^{\mathrm{a}} / \mathbf{K}$ & $p / \mathbf{M P a}$ & $T / K$ & $\lambda / \mathbf{W} \cdot \mathbf{m}^{-1} \mathbf{K}^{-1}$ & $T_{\mathrm{i}}^{\mathrm{a}} / \mathbf{K}$ & $p / \mathbf{M P a}$ & $T / K$ & $\lambda / \mathbf{W} \cdot \mathbf{m}^{-1} \mathbf{K}^{-1}$ \\
\hline 299.549 & 0.155 & 301.436 & 0.14327 & 299.590 & 21.709 & 301.791 & 0.14902 \\
\hline 299.554 & 0.161 & 301.836 & 0.14323 & 299.591 & 21.708 & 301.795 & 0.14888 \\
\hline 299.549 & 0.164 & 301.830 & 0.14331 & 299.593 & 21.701 & 302.214 & 0.14879 \\
\hline 299.555 & 0.167 & 302.275 & 0.14315 & 299.592 & 21.716 & 302.215 & 0.14884 \\
\hline 299.554 & 0.170 & 302.276 & 0.14317 & 299.592 & 21.721 & 302.676 & 0.14867 \\
\hline 299.552 & 0.173 & 302.752 & 0.14298 & 299.596 & 21.708 & 302.677 & 0.14876 \\
\hline 299.552 & 0.177 & 302.750 & 0.14309 & 299.590 & 21.724 & 303.171 & 0.14866 \\
\hline 299.559 & 0.180 & 303.275 & 0.14294 & 299.589 & 21.735 & 303.166 & 0.14867 \\
\hline 299.561 & 0.184 & 303.273 & 0.14298 & 299.586 & 30.144 & 301.377 & 0.15101 \\
\hline 299.583 & 5.854 & 301.456 & 0.14481 & 299.578 & 30.158 & 301.367 & 0.15093 \\
\hline 299.580 & 5.646 & 302.274 & 0.14459 & 299.580 & 30.181 & 301.742 & 0.15100 \\
\hline 299.580 & 5.588 & 302.748 & 0.14445 & 299.579 & 30.153 & 301.740 & 0.15098 \\
\hline 299.579 & 5.548 & 302.747 & 0.14405 & 299.579 & 30.152 & 302.160 & 0.15088 \\
\hline 299.577 & 5.495 & 303.255 & 0.14406 & 299.577 & 30.167 & 302.156 & 0.15086 \\
\hline 299.575 & 5.439 & 303.252 & 0.14442 & 299.585 & 30.160 & 302.618 & 0.15077 \\
\hline 299.579 & 7.141 & 301.449 & 0.14546 & 299.578 & 30.161 & 302.608 & 0.15089 \\
\hline 299.576 & 7.127 & 301.439 & 0.14505 & 299.578 & 30.146 & 303.099 & 0.15074 \\
\hline 299.576 & 7.135 & 301.832 & 0.14513 & 299.584 & 30.154 & 303.106 & 0.15072 \\
\hline 299.577 & 7.125 & 301.828 & 0.14502 & 299.596 & 39.004 & 301.378 & 0.15317 \\
\hline 299.585 & 7.122 & 302.269 & 0.14497 & 299.596 & 39.013 & 301.379 & 0.15309 \\
\hline 299.585 & 7.127 & 302.270 & 0.14530 & 299.598 & 39.016 & 301.753 & 0.15357 \\
\hline 299.586 & 7.143 & 302.744 & 0.14495 & 299.591 & 39.012 & 301.742 & 0.15303 \\
\hline 299.585 & 7.142 & 302.741 & 0.14502 & 299.596 & 39.014 & 302.161 & 0.15300 \\
\hline 299.585 & 7.130 & 303.251 & 0.14483 & 299.596 & 39.013 & 302.159 & 0.15296 \\
\hline 299.587 & 7.140 & 303.250 & 0.14485 & 299.595 & 39.018 & 302.605 & 0.15298 \\
\hline 299.588 & 13.947 & 301.439 & 0.14698 & 299.592 & 39.015 & 302.604 & 0.15332 \\
\hline 299.587 & 13.973 & 301.434 & 0.14683 & 299.593 & 39.012 & 303.087 & 0.15284 \\
\hline 299.591 & 13.976 & 301.819 & 0.14689 & 299.591 & 38.999 & 303.085 & 0.15287 \\
\hline 299.589 & 13.946 & 301.816 & 0.14682 & 299.581 & 48.515 & 301.325 & 0.15533 \\
\hline 299.587 & 13.960 & 302.248 & 0.14681 & 299.579 & 48.501 & 301.342 & 0.15533 \\
\hline 299.585 & 13.965 & 302.240 & 0.14682 & 299.580 & 48.488 & 301.706 & 0.15525 \\
\hline 299.581 & 13.946 & 302.704 & 0.14669 & 299.576 & 48.474 & 302.111 & 0.15516 \\
\hline 299.582 & 13.892 & 302.700 & 0.14671 & 299.583 & 48.475 & 302.117 & 0.15519 \\
\hline 299.581 & 13.938 & 303.211 & 0.14663 & 299.582 & 48.468 & 302.558 & 0.15515 \\
\hline 299.579 & 13.937 & 303.200 & 0.14665 & 299.581 & 48.456 & 302.551 & 0.15550 \\
\hline 299.593 & 21.709 & 301.414 & 0.14886 & 299.581 & 48.454 & 303.033 & 0.15508 \\
\hline 299.593 & 21.721 & 301.413 & 0.14893 & 299.583 & 48.462 & 303.036 & 0.15504 \\
\hline
\end{tabular}

${ }^{\text {a} I n i t i a l ~ c e l l ~ t e m p e r a t u r e . ~}$ 
Table A1, continued.

\begin{tabular}{|c|c|c|c|c|c|c|c|}
\hline$T_{\mathbf{i}^{\mathrm{a}}} / \mathbf{K}$ & $p / \mathbf{M P a}$ & $T / \mathbf{K}$ & $\lambda / \mathbf{W} \cdot \mathbf{m}^{-1} \mathbf{K}^{-1}$ & $T_{\mathbf{i}^{\mathrm{a}}} / \mathbf{K}$ & $p / \mathbf{M P a}$ & $T / \mathbf{K}$ & $\lambda / \mathbf{W} \cdot \mathbf{m}^{-1} \mathbf{K}^{-1}$ \\
\hline 299.596 & 58.504 & 301.330 & 0.15742 & 332.002 & 6.740 & 335.405 & 0.14031 \\
\hline 299.596 & 58.490 & 301.330 & 0.15746 & 332.013 & 13.507 & 333.719 & 0.14266 \\
\hline 299.594 & 58.493 & 301.689 & 0.15755 & 332.009 & 13.496 & 333.723 & 0.14271 \\
\hline 299.596 & 58.487 & 301.693 & 0.15749 & 332.006 & 13.500 & 334.074 & 0.14269 \\
\hline 299.591 & 58.473 & 302.089 & 0.15741 & 332.013 & 13.490 & 334.078 & 0.14256 \\
\hline 299.593 & 58.483 & 302.089 & 0.15746 & 332.010 & 13.488 & 334.473 & 0.14247 \\
\hline 299.589 & 58.463 & 302.520 & 0.15738 & 332.013 & 13.488 & 334.474 & 0.14250 \\
\hline 299.587 & 58.448 & 302.518 & 0.15736 & 332.012 & 13.499 & 334.910 & 0.14246 \\
\hline 299.587 & 58.460 & 302.989 & 0.15727 & 332.010 & 13.498 & 334.904 & 0.14243 \\
\hline 299.582 & 58.473 & 302.983 & 0.15746 & 332.014 & 13.493 & 335.377 & 0.14243 \\
\hline 299.574 & 69.033 & 301.297 & 0.15996 & 332.008 & 13.498 & 335.365 & 0.14241 \\
\hline 299.577 & 69.035 & 301.299 & 0.15989 & 332.016 & 21.097 & 333.717 & 0.14483 \\
\hline 299.578 & 69.039 & 301.653 & 0.15984 & 332.014 & 21.087 & 333.708 & 0.14513 \\
\hline 299.574 & 69.024 & 301.649 & 0.15974 & 332.011 & 21.089 & 334.061 & 0.14471 \\
\hline 299.576 & 69.035 & 302.046 & 0.15975 & 332.012 & 21.080 & 334.059 & 0.14476 \\
\hline 299.583 & 69.059 & 302.071 & 0.15968 & 332.012 & 21.090 & 334.455 & 0.14464 \\
\hline 299.581 & 69.041 & 302.503 & 0.15969 & 332.013 & 21.086 & 334.450 & 0.14465 \\
\hline 299.584 & 69.021 & 302.505 & 0.15968 & 332.014 & 21.088 & 334.876 & 0.14471 \\
\hline 299.586 & 68.999 & 302.953 & 0.15959 & 332.014 & 21.091 & 334.874 & 0.14456 \\
\hline 299.588 & 69.004 & 302.956 & 0.15959 & 332.011 & 21.079 & 335.333 & 0.14458 \\
\hline 331.980 & 0.383 & 333.737 & 0.13864 & 332.015 & 21.082 & 335.340 & 0.14453 \\
\hline 331.988 & 0.413 & 333.742 & 0.13867 & 332.020 & 29.158 & 333.689 & 0.14706 \\
\hline 331.981 & 0.439 & 334.109 & 0.13877 & 332.019 & 29.155 & 333.681 & 0.14720 \\
\hline 331.979 & 0.457 & 334.107 & 0.13923 & 332.023 & 29.156 & 334.039 & 0.14705 \\
\hline 331.981 & 0.473 & 334.513 & 0.13861 & 332.019 & 29.154 & 334.031 & 0.14693 \\
\hline 331.983 & 0.487 & 334.514 & 0.13858 & 332.023 & 29.144 & 334.420 & 0.14697 \\
\hline 331.987 & 0.498 & 334.960 & 0.13849 & 332.021 & 29.137 & 334.424 & 0.14709 \\
\hline 331.991 & 0.507 & 334.962 & 0.13852 & 332.023 & 29.142 & 334.844 & 0.14685 \\
\hline 331.987 & 0.516 & 335.433 & 0.13866 & 332.024 & 29.144 & 334.844 & 0.14689 \\
\hline 331.986 & 0.523 & 335.431 & 0.13841 & 332.020 & 29.131 & 335.293 & 0.14685 \\
\hline 331.995 & 6.770 & 333.731 & 0.14057 & 332.019 & 29.133 & 335.289 & 0.14681 \\
\hline 332.000 & 6.769 & 333.722 & 0.14067 & 332.023 & 38.323 & 333.661 & 0.14952 \\
\hline 331.997 & 6.769 & 334.080 & 0.14049 & 332.024 & 38.298 & 333.655 & 0.14951 \\
\hline 332.001 & 6.765 & 334.093 & 0.14063 & 332.018 & 38.285 & 333.992 & 0.14948 \\
\hline 331.996 & 6.760 & 334.483 & 0.14066 & 332.021 & 38.304 & 334.375 & 0.14942 \\
\hline 331.999 & 6.756 & 334.493 & 0.14050 & 332.025 & 38.279 & 334.377 & 0.14938 \\
\hline 331.998 & 6.731 & 334.928 & 0.14041 & 332.023 & 38.278 & 334.793 & 0.14934 \\
\hline 331.996 & 6.750 & 334.920 & 0.14039 & 332.024 & 38.265 & 335.233 & 0.14920 \\
\hline 331.997 & 6.745 & 335.396 & 0.14034 & 332.019 & 38.259 & 335.229 & 0.14926 \\
\hline
\end{tabular}

anitial cell temperature. 
Table A1, continued.

\begin{tabular}{|c|c|c|c|c|c|c|c|}
\hline$T_{\mathbf{i}^{\mathrm{a}}} / \mathbf{K}$ & $p / \mathbf{M P a}$ & $T / \mathbf{K}$ & $\lambda / \mathbf{W} \cdot \mathbf{m}^{-1} \mathbf{K}^{-1}$ & $T_{\mathbf{i}^{\mathrm{a}}}^{\mathrm{a}} / \mathbf{K}$ & $p / \mathbf{M P a}$ & $T / \mathbf{K}$ & $\lambda / \mathbf{W} \cdot \mathbf{m}^{-1} \mathbf{K}^{-1}$ \\
\hline 332.017 & 47.909 & 333.620 & 0.15206 & 364.893 & 6.232 & 366.861 & 0.13545 \\
\hline 332.016 & 47.896 & 333.962 & 0.15188 & 364.893 & 6.204 & 367.236 & 0.13534 \\
\hline 332.016 & 47.898 & 333.960 & 0.15196 & 364.891 & 6.211 & 367.233 & 0.13542 \\
\hline 332.019 & 47.881 & 334.335 & 0.15188 & 364.885 & 6.195 & 367.642 & 0.13533 \\
\hline 332.015 & 47.870 & 334.744 & 0.15180 & 364.894 & 6.188 & 367.659 & 0.13532 \\
\hline 332.017 & 47.862 & 334.742 & 0.15179 & 364.892 & 6.207 & 368.101 & 0.13521 \\
\hline 332.020 & 47.853 & 335.187 & 0.15172 & 364.907 & 13.465 & 366.514 & 0.13794 \\
\hline 332.014 & 47.845 & 335.175 & 0.15170 & 364.913 & 13.471 & 366.523 & 0.13816 \\
\hline 332.019 & 58.184 & 333.585 & 0.15455 & 364.910 & 13.467 & 366.858 & 0.13789 \\
\hline 332.016 & 58.174 & 333.578 & 0.15458 & 364.907 & 13.494 & 366.843 & 0.13818 \\
\hline 332.017 & 58.170 & 333.948 & 0.15449 & 364.912 & 13.493 & 367.224 & 0.13777 \\
\hline 332.018 & 58.149 & 333.946 & 0.15499 & 364.907 & 13.486 & 367.217 & 0.13777 \\
\hline 332.018 & 58.135 & 334.317 & 0.15449 & 364.906 & 13.474 & 367.624 & 0.13778 \\
\hline 332.016 & 58.149 & 334.312 & 0.15440 & 364.914 & 13.490 & 367.631 & 0.13779 \\
\hline 332.019 & 58.123 & 334.716 & 0.15432 & 364.911 & 13.468 & 368.062 & 0.13765 \\
\hline 332.018 & 58.118 & 334.718 & 0.15428 & 364.905 & 13.477 & 368.056 & 0.13766 \\
\hline 332.022 & 58.118 & 335.160 & 0.15462 & 364.903 & 20.645 & 366.483 & 0.14026 \\
\hline 332.014 & 58.112 & 335.143 & 0.15432 & 364.899 & 20.655 & 366.487 & 0.14032 \\
\hline 332.017 & 68.637 & 333.593 & 0.15693 & 364.903 & 20.652 & 366.820 & 0.14035 \\
\hline 332.013 & 68.602 & 333.584 & 0.15698 & 364.900 & 20.664 & 366.817 & 0.14023 \\
\hline 332.015 & 68.588 & 333.916 & 0.15686 & 364.899 & 20.637 & 367.189 & 0.14021 \\
\hline 332.012 & 68.585 & 333.908 & 0.15702 & 364.903 & 20.659 & 367.184 & 0.14022 \\
\hline 332.012 & 68.566 & 334.280 & 0.15684 & 364.903 & 20.641 & 367.581 & 0.14008 \\
\hline 332.014 & 68.552 & 334.278 & 0.15686 & 364.900 & 20.634 & 367.593 & 0.14005 \\
\hline 332.007 & 68.541 & 334.665 & 0.15680 & 364.900 & 20.648 & 368.011 & 0.13995 \\
\hline 332.008 & 68.535 & 334.667 & 0.15679 & 364.903 & 20.650 & 368.014 & 0.13996 \\
\hline 332.011 & 68.510 & 335.097 & 0.15676 & 364.906 & 28.803 & 366.472 & 0.14290 \\
\hline 332.013 & 68.504 & 335.097 & 0.15674 & 364.907 & 28.770 & 366.475 & 0.14291 \\
\hline 364.917 & 0.397 & 367.302 & 0.13376 & 364.907 & 28.793 & 366.796 & 0.14273 \\
\hline 364.917 & 0.403 & 367.722 & 0.13330 & 364.907 & 28.759 & 367.158 & 0.14268 \\
\hline 364.918 & 0.406 & 368.168 & 0.13287 & 364.904 & 28.778 & 367.158 & 0.14274 \\
\hline 364.919 & 0.409 & 368.176 & 0.13317 & 364.906 & 28.762 & 367.542 & 0.14269 \\
\hline 364.917 & 0.423 & 366.586 & 0.13296 & 364.906 & 28.771 & 367.549 & 0.14265 \\
\hline 364.919 & 0.431 & 367.735 & 0.13322 & 364.903 & 28.738 & 367.978 & 0.14262 \\
\hline 364.919 & 0.432 & 367.726 & 0.13325 & 364.901 & 28.740 & 367.966 & 0.14258 \\
\hline 364.922 & 0.434 & 368.185 & 0.13327 & 364.906 & 37.832 & 366.421 & 0.14563 \\
\hline 364.922 & 0.435 & 368.189 & 0.13375 & 364.908 & 37.815 & 366.419 & 0.14570 \\
\hline 364.889 & 6.265 & 366.511 & 0.13566 & 364.905 & 37.806 & 366.724 & 0.14552 \\
\hline 364.893 & 6.227 & 366.512 & 0.13552 & 364.906 & 37.785 & 366.731 & 0.14552 \\
\hline
\end{tabular}

anitial cell temperature. 
Table A1, continued.

\begin{tabular}{|c|c|c|c|c|c|c|c|}
\hline$T_{\mathbf{i}^{\mathrm{a}}} / \mathbf{K}$ & $p / \mathbf{M P a}$ & $T / \mathbf{K}$ & $\lambda / \mathbf{W} \cdot \mathbf{m}^{-1} \mathbf{K}^{-1}$ & $T_{\mathbf{i}^{\mathrm{a}}}^{\mathrm{a}} / \mathbf{K}$ & $p / \mathbf{M P a}$ & $T / \mathbf{K}$ & $\lambda / \mathbf{W} \cdot \mathbf{m}^{-1} \mathbf{K}^{-1}$ \\
\hline 364.909 & 37.769 & 367.094 & 0.14540 & 397.992 & 0.487 & 399.893 & 0.12781 \\
\hline 364.903 & 37.771 & 367.082 & 0.14536 & 397.995 & 0.489 & 400.256 & 0.12781 \\
\hline 364.908 & 37.753 & 367.482 & 0.14544 & 397.990 & 0.490 & 400.252 & 0.12795 \\
\hline 364.909 & 37.761 & 367.496 & 0.14537 & 397.993 & 0.492 & 400.668 & 0.12770 \\
\hline 364.907 & 37.760 & 367.910 & 0.14524 & 397.994 & 0.494 & 400.658 & 0.12776 \\
\hline 364.912 & 37.756 & 367.912 & 0.14520 & 397.993 & 0.495 & 401.102 & 0.12766 \\
\hline 364.908 & 47.403 & 366.411 & 0.14834 & 397.994 & 0.497 & 401.093 & 0.12765 \\
\hline 364.908 & 47.434 & 366.406 & 0.14828 & 397.975 & 5.940 & 399.513 & 0.13026 \\
\hline 364.901 & 47.454 & 366.720 & 0.14809 & 397.979 & 5.948 & 399.514 & 0.13035 \\
\hline 364.908 & 47.455 & 366.718 & 0.14808 & 397.978 & 5.922 & 399.827 & 0.13010 \\
\hline 364.907 & 47.437 & 367.063 & 0.14829 & 397.978 & 5.902 & 399.825 & 0.13029 \\
\hline 364.908 & 47.391 & 367.069 & 0.14797 & 397.980 & 5.892 & 400.196 & 0.13004 \\
\hline 364.905 & 47.409 & 367.443 & 0.14802 & 397.982 & 5.891 & 400.212 & 0.13046 \\
\hline 364.907 & 47.387 & 367.457 & 0.14819 & 397.979 & 5.878 & 400.612 & 0.12982 \\
\hline 364.906 & 47.405 & 367.853 & 0.14804 & 397.980 & 5.868 & 400.598 & 0.13007 \\
\hline 364.905 & 47.362 & 367.856 & 0.14812 & 397.981 & 5.867 & 401.047 & 0.12975 \\
\hline 364.918 & 57.906 & 366.409 & 0.15107 & 397.983 & 5.859 & 401.030 & 0.12975 \\
\hline 364.915 & 57.895 & 366.406 & 0.15102 & 398.003 & 12.783 & 400.605 & 0.13242 \\
\hline 364.916 & 57.868 & 366.726 & 0.15099 & 398.000 & 12.763 & 400.601 & 0.13286 \\
\hline 364.916 & 57.870 & 366.712 & 0.15090 & 398.003 & 12.767 & 401.029 & 0.13227 \\
\hline 364.920 & 57.850 & 367.059 & 0.15089 & 398.003 & 12.760 & 401.019 & 0.13240 \\
\hline 364.915 & 57.833 & 367.059 & 0.15093 & 398.014 & 20.158 & 399.523 & 0.13553 \\
\hline 364.916 & 57.805 & 367.431 & 0.15090 & 398.014 & 20.137 & 399.522 & 0.13537 \\
\hline 364.921 & 57.741 & 367.423 & 0.15088 & 398.010 & 20.119 & 399.828 & 0.13535 \\
\hline 364.916 & 57.755 & 367.837 & 0.15085 & 398.014 & 20.107 & 399.833 & 0.13546 \\
\hline 364.914 & 57.737 & 367.842 & 0.15079 & 398.022 & 20.111 & 400.191 & 0.13534 \\
\hline 364.918 & 68.713 & 366.375 & 0.15394 & 398.013 & 20.105 & 400.180 & 0.13530 \\
\hline 364.913 & 68.686 & 366.372 & 0.15369 & 398.012 & 20.112 & 400.566 & 0.13534 \\
\hline 364.913 & 68.683 & 366.684 & 0.15385 & 398.015 & 20.107 & 400.565 & 0.13567 \\
\hline 364.925 & 68.674 & 366.688 & 0.15381 & 398.014 & 20.087 & 400.970 & 0.13528 \\
\hline 364.920 & 68.656 & 367.024 & 0.15381 & 398.014 & 20.085 & 400.973 & 0.13522 \\
\hline 364.919 & 68.623 & 367.023 & 0.15377 & 398.020 & 28.213 & 399.474 & 0.13845 \\
\hline 364.915 & 68.598 & 367.389 & 0.15369 & 398.023 & 28.206 & 399.487 & 0.13828 \\
\hline 364.915 & 68.560 & 367.371 & 0.15362 & 398.021 & 28.203 & 399.797 & 0.13856 \\
\hline 364.913 & 68.541 & 367.774 & 0.15361 & 398.020 & 28.189 & 399.782 & 0.13816 \\
\hline 364.913 & 68.526 & 367.775 & 0.15361 & 398.017 & 28.173 & 400.131 & 0.13817 \\
\hline 397.990 & 0.479 & 399.557 & 0.12823 & 398.018 & 28.171 & 400.131 & 0.13810 \\
\hline 397.990 & 0.482 & 399.564 & 0.12788 & 398.019 & 28.140 & 400.511 & 0.13806 \\
\hline 397.992 & 0.484 & 399.883 & 0.12786 & 398.019 & 28.151 & 400.511 & 0.13818 \\
\hline
\end{tabular}

${ }^{\text {a} I n i t i a l ~ c e l l ~ t e m p e r a t u r e . ~}$ 
Table A1, continued.

\begin{tabular}{|c|c|c|c|c|c|c|c|}
\hline$T_{\mathbf{i}^{\mathrm{a}}} / \mathbf{K}$ & $p / \mathbf{M P a}$ & $T / \mathbf{K}$ & $\lambda / \mathbf{W} \cdot \mathbf{m}^{-1} \mathbf{K}^{-1}$ & $T_{\mathbf{i}^{\mathrm{a}}} / \mathbf{K}$ & $p / \mathbf{M P a}$ & $T / \mathbf{K}$ & $\lambda / \mathbf{W} \cdot \mathbf{m}^{-1} \mathbf{K}^{-1}$ \\
\hline 398.020 & 28.156 & 400.912 & 0.13801 & 398.042 & 12.566 & 400.629 & 0.13244 \\
\hline 398.020 & 28.151 & 400.913 & 0.13801 & 398.049 & 12.561 & 401.059 & 0.13250 \\
\hline 398.035 & 37.384 & 399.474 & 0.14118 & 398.050 & 12.557 & 401.055 & 0.13243 \\
\hline 398.036 & 37.376 & 399.483 & 0.14157 & 431.982 & 0.399 & 433.510 & 0.12223 \\
\hline 398.038 & 37.365 & 399.783 & 0.14115 & 431.983 & 0.401 & 433.512 & 0.12219 \\
\hline 398.038 & 37.354 & 399.777 & 0.14123 & 431.981 & 0.403 & 433.811 & 0.12200 \\
\hline 398.036 & 37.350 & 400.112 & 0.14118 & 431.986 & 0.405 & 433.818 & 0.12210 \\
\hline 398.041 & 37.320 & 400.112 & 0.14117 & 431.990 & 0.406 & 434.169 & 0.12183 \\
\hline 398.033 & 37.303 & 400.473 & 0.14101 & 431.986 & 0.407 & 434.168 & 0.12201 \\
\hline 398.038 & 37.294 & 400.477 & 0.14106 & 431.990 & 0.408 & 434.572 & 0.12196 \\
\hline 398.035 & 37.248 & 400.862 & 0.14097 & 431.986 & 0.409 & 434.550 & 0.12182 \\
\hline 398.032 & 37.231 & 400.859 & 0.14112 & 431.990 & 0.410 & 434.973 & 0.12184 \\
\hline 398.035 & 47.003 & 399.443 & 0.14409 & 431.994 & 0.411 & 434.984 & 0.12178 \\
\hline 398.034 & 46.981 & 399.446 & 0.14465 & 431.963 & 5.844 & 433.454 & 0.12471 \\
\hline 398.032 & 46.960 & 399.744 & 0.14400 & 431.964 & 5.837 & 433.445 & 0.12432 \\
\hline 398.037 & 46.946 & 399.751 & 0.14415 & 431.960 & 5.839 & 433.758 & 0.12449 \\
\hline 398.027 & 46.916 & 400.062 & 0.14414 & 431.962 & 5.835 & 433.763 & 0.12444 \\
\hline 398.032 & 46.918 & 400.078 & 0.14412 & 431.962 & 5.828 & 434.105 & 0.12446 \\
\hline 398.035 & 46.882 & 400.431 & 0.14414 & 431.963 & 5.811 & 434.105 & 0.12425 \\
\hline 398.034 & 46.858 & 400.419 & 0.14418 & 431.963 & 5.820 & 434.496 & 0.12429 \\
\hline 398.035 & 46.867 & 400.827 & 0.14398 & 431.963 & 5.801 & 434.475 & 0.12442 \\
\hline 398.035 & 46.839 & 400.824 & 0.14396 & 431.961 & 5.800 & 434.883 & 0.12435 \\
\hline 398.048 & 57.341 & 399.445 & 0.14724 & 431.961 & 5.793 & 434.897 & 0.12421 \\
\hline 398.046 & 57.298 & 399.438 & 0.14704 & 431.983 & 12.456 & 433.454 & 0.12761 \\
\hline 398.045 & 57.270 & 399.719 & 0.14721 & 431.983 & 12.462 & 433.461 & 0.12744 \\
\hline 398.046 & 57.223 & 399.717 & 0.14738 & 431.990 & 12.448 & 433.777 & 0.12738 \\
\hline 398.045 & 57.212 & 400.044 & 0.14715 & 431.985 & 12.444 & 434.099 & 0.12717 \\
\hline 398.044 & 57.187 & 400.061 & 0.14709 & 431.982 & 12.456 & 434.110 & 0.12713 \\
\hline 398.046 & 57.157 & 400.403 & 0.14713 & 431.984 & 12.450 & 434.484 & 0.12719 \\
\hline 398.044 & 57.131 & 400.406 & 0.14702 & 431.981 & 12.441 & 434.471 & 0.12777 \\
\hline 398.039 & 57.109 & 400.780 & 0.14709 & 431.982 & 12.439 & 434.881 & 0.12716 \\
\hline 398.043 & 57.071 & 400.786 & 0.14701 & 431.982 & 12.441 & 434.875 & 0.12727 \\
\hline 398.042 & 12.587 & 399.456 & 0.13279 & 431.996 & 19.709 & 433.427 & 0.13030 \\
\hline 398.041 & 12.577 & 399.567 & 0.13256 & 432.001 & 19.696 & 433.455 & 0.13039 \\
\hline 398.042 & 12.585 & 399.900 & 0.13257 & 432.000 & 19.695 & 433.747 & 0.13034 \\
\hline 398.044 & 12.584 & 399.888 & 0.13270 & 432.004 & 19.693 & 433.754 & 0.13043 \\
\hline 398.046 & 12.588 & 400.252 & 0.13247 & 432.006 & 19.694 & 434.085 & 0.13014 \\
\hline 398.041 & 12.565 & 400.252 & 0.13252 & 432.001 & 19.692 & 434.083 & 0.13021 \\
\hline 398.046 & 12.559 & 400.635 & 0.13249 & 432.008 & 19.671 & 434.446 & 0.13036 \\
\hline
\end{tabular}

anitial cell temperature. 
Table A1, continued.

\begin{tabular}{|c|c|c|c|c|c|c|c|}
\hline$T_{\mathbf{i}^{\mathrm{a}}} / \mathbf{K}$ & $p / \mathbf{M P a}$ & $T / \mathbf{K}$ & $\lambda / \mathbf{W} \cdot \mathbf{m}^{-1} \mathbf{K}^{-1}$ & $T_{\mathbf{i}^{\mathrm{a}}} / \mathbf{K}$ & $p / \mathbf{M P a}$ & $T / \mathbf{K}$ & $\lambda / \mathbf{W} \cdot \mathbf{m}^{-1} \mathbf{K}^{-1}$ \\
\hline 432.001 & 19.676 & 434.451 & 0.13014 & 465.227 & 0.393 & 466.727 & 0.11646 \\
\hline 432.002 & 19.677 & 434.839 & 0.13032 & 465.226 & 0.407 & 466.727 & 0.11637 \\
\hline 432.004 & 19.672 & 434.851 & 0.13002 & 465.220 & 0.415 & 467.017 & 0.11655 \\
\hline 432.012 & 27.422 & 433.415 & 0.13372 & 465.223 & 0.421 & 467.031 & 0.11624 \\
\hline 432.008 & 27.428 & 433.424 & 0.13358 & 465.229 & 0.426 & 467.384 & 0.11653 \\
\hline 432.011 & 27.417 & 433.704 & 0.13324 & 465.227 & 0.429 & 467.377 & 0.11613 \\
\hline 432.002 & 27.408 & 433.712 & 0.13333 & 465.224 & 0.432 & 467.743 & 0.11607 \\
\hline 432.008 & 27.396 & 434.031 & 0.13317 & 465.223 & 0.434 & 467.744 & 0.11610 \\
\hline 432.010 & 27.380 & 434.396 & 0.13296 & 465.225 & 0.436 & 468.151 & 0.11587 \\
\hline 432.002 & 27.371 & 434.377 & 0.13317 & 465.228 & 0.437 & 468.165 & 0.11627 \\
\hline 432.006 & 27.369 & 434.774 & 0.13304 & 465.214 & 6.186 & 466.650 & 0.11929 \\
\hline 432.004 & 27.342 & 434.755 & 0.13305 & 465.222 & 6.189 & 466.670 & 0.11971 \\
\hline 432.004 & 36.556 & 433.380 & 0.13640 & 465.218 & 6.182 & 466.958 & 0.11901 \\
\hline 431.999 & 36.538 & 433.662 & 0.13644 & 465.221 & 6.181 & 466.970 & 0.11891 \\
\hline 432.002 & 36.521 & 433.643 & 0.13650 & 465.223 & 6.174 & 467.302 & 0.11914 \\
\hline 432.000 & 36.507 & 433.986 & 0.13666 & 465.219 & 6.175 & 467.310 & 0.11921 \\
\hline 432.000 & 36.470 & 434.315 & 0.13639 & 465.220 & 6.168 & 467.671 & 0.11937 \\
\hline 431.997 & 36.456 & 434.316 & 0.13643 & 465.226 & 6.163 & 467.678 & 0.11898 \\
\hline 432.003 & 36.436 & 434.694 & 0.13644 & 465.227 & 6.168 & 468.076 & 0.11900 \\
\hline 432.001 & 36.426 & 434.708 & 0.13637 & 465.226 & 12.106 & 466.635 & 0.12198 \\
\hline 432.010 & 46.328 & 433.354 & 0.14000 & 465.224 & 12.120 & 466.627 & 0.12217 \\
\hline 432.010 & 46.275 & 433.634 & 0.13970 & 465.224 & 12.102 & 466.942 & 0.12201 \\
\hline 432.008 & 46.246 & 433.621 & 0.13964 & 465.223 & 12.093 & 466.923 & 0.12234 \\
\hline 432.003 & 46.218 & 433.936 & 0.13985 & 465.225 & 12.096 & 467.247 & 0.12225 \\
\hline 432.012 & 46.193 & 433.932 & 0.13995 & 465.222 & 12.087 & 467.250 & 0.12209 \\
\hline 432.006 & 46.165 & 434.275 & 0.13993 & 465.220 & 12.080 & 467.625 & 0.12180 \\
\hline 432.010 & 46.143 & 434.290 & 0.13968 & 465.227 & 12.069 & 467.611 & 0.12190 \\
\hline 432.010 & 46.120 & 434.640 & 0.13966 & 465.230 & 12.061 & 468.021 & 0.12186 \\
\hline 432.010 & 46.096 & 434.670 & 0.13970 & 465.226 & 12.060 & 468.005 & 0.12239 \\
\hline 432.010 & 57.289 & 433.326 & 0.14324 & 465.247 & 18.818 & 466.621 & 0.12487 \\
\hline 432.007 & 57.250 & 433.321 & 0.14351 & 465.247 & 18.817 & 466.912 & 0.12509 \\
\hline 432.008 & 57.209 & 433.590 & 0.14345 & 465.244 & 18.809 & 466.910 & 0.12499 \\
\hline 432.007 & 57.175 & 433.591 & 0.14337 & 465.247 & 18.806 & 467.232 & 0.12513 \\
\hline 432.009 & 57.134 & 433.900 & 0.14303 & 465.251 & 18.801 & 467.239 & 0.12551 \\
\hline 432.010 & 57.110 & 433.916 & 0.14328 & 465.250 & 18.797 & 467.597 & 0.12507 \\
\hline 432.012 & 57.071 & 434.235 & 0.14319 & 465.251 & 18.779 & 467.581 & 0.12477 \\
\hline 432.007 & 57.015 & 434.246 & 0.14311 & 465.251 & 18.782 & 467.978 & 0.12477 \\
\hline 432.006 & 56.979 & 434.596 & 0.14316 & 465.248 & 18.771 & 467.968 & 0.12483 \\
\hline 432.007 & 56.941 & 434.588 & 0.14302 & 465.257 & 26.803 & 466.608 & 0.12888 \\
\hline
\end{tabular}

anitial cell temperature. 
Table A1, continued.

\begin{tabular}{|c|c|c|c|c|c|c|c|}
\hline$T_{\mathbf{i}^{\mathrm{a}}} / \mathbf{K}$ & $p / \mathbf{M P a}$ & $T / \mathbf{K}$ & $\lambda / \mathbf{W} \cdot \mathbf{m}^{-1} \mathbf{K}^{-1}$ & $T_{\mathbf{i}^{\mathrm{a}}} / \mathbf{K}$ & $p / \mathbf{M P a}$ & $T / \mathbf{K}$ & $\lambda / \mathbf{W} \cdot \mathbf{m}^{-1} \mathbf{K}^{-1}$ \\
\hline 465.255 & 26.774 & 466.898 & 0.12837 & 499.180 & 0.397 & 501.311 & 0.11033 \\
\hline 465.260 & 26.757 & 466.902 & 0.12829 & 499.189 & 0.402 & 501.688 & 0.11029 \\
\hline 465.255 & 26.738 & 467.216 & 0.12832 & 499.196 & 0.404 & 501.685 & 0.11017 \\
\hline 465.258 & 26.722 & 467.210 & 0.12851 & 499.193 & 0.406 & 502.089 & 0.11030 \\
\hline 465.257 & 26.700 & 467.551 & 0.12864 & 499.201 & 0.407 & 502.093 & 0.11019 \\
\hline 465.260 & 26.694 & 467.566 & 0.12832 & 499.135 & 5.706 & 500.548 & 0.11353 \\
\hline 465.258 & 26.669 & 467.916 & 0.12828 & 499.131 & 5.707 & 500.529 & 0.11340 \\
\hline 465.261 & 26.654 & 467.939 & 0.12807 & 499.126 & 5.702 & 500.831 & 0.11335 \\
\hline 465.287 & 35.633 & 466.610 & 0.13202 & 499.124 & 5.701 & 501.175 & 0.11339 \\
\hline 465.279 & 35.618 & 466.601 & 0.13239 & 499.127 & 5.705 & 501.166 & 0.11378 \\
\hline 465.290 & 35.608 & 466.880 & 0.13194 & 499.128 & 5.705 & 501.541 & 0.11338 \\
\hline 465.286 & 35.594 & 466.895 & 0.13181 & 499.128 & 5.699 & 501.524 & 0.11348 \\
\hline 465.285 & 35.562 & 467.193 & 0.13179 & 499.125 & 5.696 & 501.920 & 0.11311 \\
\hline 465.287 & 35.545 & 467.198 & 0.13204 & 499.129 & 5.691 & 501.922 & 0.11328 \\
\hline 465.290 & 35.531 & 467.540 & 0.13184 & 499.173 & 11.686 & 500.573 & 0.11682 \\
\hline 465.289 & 35.499 & 467.519 & 0.13200 & 499.172 & 11.673 & 500.566 & 0.11717 \\
\hline 465.292 & 35.473 & 467.898 & 0.13165 & 499.164 & 11.667 & 500.842 & 0.11669 \\
\hline 465.287 & 35.403 & 467.903 & 0.13158 & 499.162 & 11.661 & 501.140 & 0.11680 \\
\hline 465.306 & 45.511 & 466.585 & 0.13578 & 499.155 & 11.654 & 501.168 & 0.11655 \\
\hline 465.305 & 45.464 & 466.579 & 0.13553 & 499.149 & 11.649 & 501.514 & 0.11651 \\
\hline 465.314 & 45.428 & 466.847 & 0.13555 & 499.156 & 11.639 & 501.501 & 0.11649 \\
\hline 465.307 & 45.357 & 467.146 & 0.13579 & 499.152 & 11.632 & 501.887 & 0.11670 \\
\hline 465.308 & 45.302 & 467.145 & 0.13562 & 499.151 & 11.623 & 501.904 & 0.11634 \\
\hline 465.307 & 45.269 & 467.466 & 0.13568 & 499.222 & 18.488 & 500.582 & 0.12039 \\
\hline 465.305 & 45.237 & 467.475 & 0.13546 & 499.238 & 18.483 & 500.608 & 0.11988 \\
\hline 465.309 & 45.168 & 467.832 & 0.13541 & 499.240 & 18.479 & 500.896 & 0.12051 \\
\hline 465.308 & 56.563 & 466.563 & 0.13921 & 499.245 & 18.455 & 501.222 & 0.11981 \\
\hline 465.301 & 56.500 & 466.537 & 0.13985 & 499.248 & 18.445 & 501.225 & 0.12017 \\
\hline 465.305 & 56.445 & 466.807 & 0.13991 & 499.254 & 18.435 & 501.579 & 0.11983 \\
\hline 465.311 & 56.398 & 466.812 & 0.13951 & 499.258 & 18.431 & 501.590 & 0.12016 \\
\hline 465.304 & 56.341 & 467.110 & 0.13929 & 499.265 & 18.426 & 501.967 & 0.12015 \\
\hline 465.302 & 56.284 & 467.110 & 0.13929 & 499.263 & 18.435 & 501.966 & 0.11981 \\
\hline 465.307 & 56.228 & 467.414 & 0.13967 & 499.183 & 26.100 & 500.458 & 0.12403 \\
\hline 465.304 & 56.184 & 467.421 & 0.13951 & 499.171 & 26.064 & 500.419 & 0.12364 \\
\hline 465.308 & 56.135 & 467.771 & 0.13907 & 499.159 & 26.042 & 500.731 & 0.12365 \\
\hline 465.309 & 56.067 & 467.761 & 0.13919 & 499.154 & 26.015 & 500.717 & 0.12341 \\
\hline 499.176 & 0.382 & 500.657 & 0.11049 & 499.143 & 25.999 & 501.043 & 0.12361 \\
\hline 499.178 & 0.388 & 500.965 & 0.11058 & 499.139 & 25.967 & 501.012 & 0.12369 \\
\hline 499.183 & 0.393 & 500.977 & 0.11068 & 499.140 & 25.941 & 501.353 & 0.12369 \\
\hline
\end{tabular}

anitial cell temperature. 
Table A1, continued.

\begin{tabular}{|c|c|c|c|}
\hline$T_{\mathrm{i}}^{\mathrm{a}} / \mathbf{K}$ & $p / \mathbf{M P a}$ & $T / \mathbf{K}$ & $\lambda / \mathbf{W} \cdot \mathbf{m}^{-1} \mathbf{K}^{-1}$ \\
\hline 499.134 & 25.908 & 501.314 & 0.12344 \\
\hline 499.134 & 25.895 & 501.704 & 0.12342 \\
\hline 499.133 & 25.864 & 501.701 & 0.12351 \\
\hline 499.194 & 35.320 & 500.490 & 0.12781 \\
\hline 499.186 & 35.289 & 500.740 & 0.12760 \\
\hline 499.189 & 35.246 & 500.755 & 0.12757 \\
\hline 499.182 & 35.230 & 501.024 & 0.12782 \\
\hline 499.178 & 35.178 & 501.025 & 0.12803 \\
\hline 499.176 & 35.142 & 501.370 & 0.12753 \\
\hline 499.174 & 35.108 & 501.325 & 0.12747 \\
\hline 499.179 & 35.085 & 501.711 & 0.12743 \\
\hline 499.176 & 34.992 & 501.693 & 0.12757 \\
\hline 499.011 & 45.161 & 500.153 & 0.13194 \\
\hline 498.996 & 45.094 & 500.115 & 0.13183 \\
\hline 498.989 & 45.049 & 500.374 & 0.13148 \\
\hline 498.984 & 44.991 & 500.362 & 0.13175 \\
\hline 498.978 & 44.938 & 500.656 & 0.13186 \\
\hline 498.973 & 44.874 & 500.643 & 0.13152 \\
\hline 498.972 & 44.825 & 500.958 & 0.13136 \\
\hline 498.969 & 44.755 & 500.922 & 0.13128 \\
\hline 498.969 & 44.723 & 501.357 & 0.13194 \\
\hline 498.968 & 44.664 & 501.325 & 0.13141 \\
\hline 498.975 & 45.581 & 500.210 & 0.13161 \\
\hline 498.977 & 45.522 & 500.193 & 0.13201 \\
\hline 498.980 & 45.500 & 500.500 & 0.13170 \\
\hline 498.981 & 45.457 & 500.482 & 0.13149 \\
\hline 498.982 & 45.404 & 500.775 & 0.13152 \\
\hline 498.980 & 45.350 & 500.770 & 0.13150 \\
\hline 498.983 & 45.317 & 501.107 & 0.13155 \\
\hline 498.986 & 45.268 & 501.100 & 0.13145 \\
\hline 498.979 & 45.231 & 501.452 & 0.13142 \\
\hline 498.982 & 45.184 & 501.441 & 0.13134 \\
\hline 499.024 & 56.282 & 500.256 & 0.13616 \\
\hline 499.026 & 56.211 & 500.507 & 0.13595 \\
\hline 499.027 & 56.133 & 500.503 & 0.13551 \\
\hline 499.029 & 56.059 & 500.796 & 0.13542 \\
\hline 499.032 & 55.910 & 501.078 & 0.13557 \\
\hline 499.040 & 55.840 & 501.103 & 0.13566 \\
\hline 499.033 & 55.753 & 501.419 & 0.13546 \\
\hline 499.031 & 55.675 & 501.423 & 0.13523 \\
\hline
\end{tabular}

anitial cell temperature. 
Table A2: Thermal Conductivity Data for POE7 at Temperatures from $300 \mathrm{~K}$ to $500 \mathrm{~K}$.

\begin{tabular}{|c|c|c|c|c|c|c|c|}
\hline$T_{\mathbf{i}}{ }^{\mathrm{a}} / \mathbf{K}$ & $p / \mathrm{MPa}$ & $T / K$ & $\lambda / \mathbf{W} \cdot \mathbf{m}^{-1} \mathbf{K}^{-1}$ & $T_{\mathbf{i}}^{\mathrm{a}} / \mathbf{K}$ & $p / \mathbf{M P a}$ & $T / K$ & $\lambda / \mathbf{W} \cdot \mathbf{m}^{-1} \mathbf{K}^{-1}$ \\
\hline \multicolumn{4}{|c|}{ Wire 1} & 299.327 & 16.201 & 301.365 & 0.15493 \\
\hline 299.103 & 0.220 & 300.046 & 0.15072 & 299.326 & 16.193 & 301.368 & 0.15495 \\
\hline 299.107 & 0.220 & 300.054 & 0.15079 & 299.335 & 21.992 & 300.222 & 0.15662 \\
\hline 299.113 & 0.220 & 300.311 & 0.15060 & 299.337 & 21.986 & 300.462 & 0.15662 \\
\hline 299.115 & 0.220 & 300.314 & 0.15065 & 299.339 & 21.998 & 300.465 & 0.15657 \\
\hline 299.119 & 0.221 & 300.604 & 0.15090 & 299.337 & 21.992 & 300.729 & 0.15664 \\
\hline 299.127 & 0.221 & 300.612 & 0.15063 & 299.339 & 21.993 & 301.025 & 0.15653 \\
\hline 299.130 & 0.221 & 300.919 & 0.15065 & 299.344 & 22.002 & 301.031 & 0.15656 \\
\hline 299.138 & 0.220 & 300.928 & 0.15057 & 299.346 & 21.992 & 301.355 & 0.15651 \\
\hline 299.141 & 0.221 & 301.273 & 0.15055 & 299.344 & 21.993 & 301.355 & 0.15651 \\
\hline 299.146 & 0.221 & 301.281 & 0.15058 & 299.350 & 29.127 & 300.211 & 0.15846 \\
\hline 299.257 & 0.212 & 300.185 & 0.15065 & 299.345 & 29.136 & 300.204 & 0.15852 \\
\hline 299.260 & 0.212 & 300.186 & 0.15079 & 299.346 & 29.132 & 300.440 & 0.15841 \\
\hline 299.264 & 0.212 & 300.443 & 0.15075 & 299.347 & 29.137 & 300.440 & 0.15859 \\
\hline 299.263 & 0.212 & 300.442 & 0.15076 & 299.340 & 29.120 & 300.696 & 0.15848 \\
\hline 299.265 & 0.212 & 300.724 & 0.15063 & 299.341 & 29.130 & 300.986 & 0.15840 \\
\hline 299.274 & 0.212 & 300.734 & 0.15069 & 299.342 & 29.124 & 300.986 & 0.15851 \\
\hline 299.276 & 0.212 & 301.038 & 0.15057 & 299.338 & 29.110 & 301.301 & 0.15837 \\
\hline 299.280 & 0.212 & 301.044 & 0.15063 & 299.343 & 35.513 & 300.718 & 0.15957 \\
\hline 299.276 & 0.211 & 301.376 & 0.15059 & 299.339 & 35.500 & 300.715 & 0.15983 \\
\hline 299.280 & 0.211 & 301.379 & 0.15050 & 299.337 & 35.480 & 300.997 & 0.15945 \\
\hline 299.299 & 8.128 & 300.198 & 0.15302 & 299.340 & 35.473 & 300.994 & 0.16001 \\
\hline 299.295 & 8.060 & 300.196 & 0.15288 & 299.341 & 35.504 & 301.315 & 0.15953 \\
\hline 299.294 & 8.000 & 300.457 & 0.15274 & 299.338 & 35.534 & 301.320 & 0.16000 \\
\hline 299.295 & 7.933 & 300.458 & 0.15271 & 299.334 & 35.523 & 300.449 & 0.15982 \\
\hline 299.298 & 7.885 & 300.738 & 0.15274 & 299.334 & 35.597 & 300.996 & 0.16014 \\
\hline 299.296 & 7.832 & 300.735 & 0.15273 & 299.333 & 35.552 & 301.312 & 0.16001 \\
\hline 299.299 & 7.691 & 301.035 & 0.15265 & 299.331 & 35.565 & 301.309 & 0.16004 \\
\hline 299.301 & 7.648 & 301.370 & 0.15256 & 299.339 & 43.397 & 300.177 & 0.16215 \\
\hline 299.298 & 7.594 & 301.368 & 0.15261 & 299.333 & 43.373 & 300.410 & 0.16209 \\
\hline 299.334 & 16.220 & 300.238 & 0.15518 & 299.339 & 43.366 & 300.434 & 0.16200 \\
\hline 299.336 & 16.218 & 300.485 & 0.15511 & 299.337 & 43.375 & 300.691 & 0.16214 \\
\hline 299.332 & 16.210 & 300.484 & 0.15510 & 299.333 & 43.310 & 300.966 & 0.16193 \\
\hline 299.327 & 16.219 & 300.750 & 0.15504 & 299.337 & 43.323 & 300.966 & 0.16197 \\
\hline 299.336 & 16.204 & 300.755 & 0.15505 & 299.335 & 43.320 & 301.283 & 0.16187 \\
\hline 299.332 & 16.192 & 301.042 & 0.15491 & 299.341 & 43.306 & 301.283 & 0.16192 \\
\hline
\end{tabular}

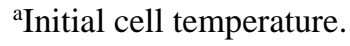


Table A2, continued.

\begin{tabular}{|c|c|c|c|c|c|c|c|}
\hline$T_{\mathbf{i}^{\mathrm{a}}} / \mathbf{K}$ & $p / \mathbf{M P a}$ & $T / K$ & $\lambda / \mathbf{W} \cdot \mathbf{m}^{-1} \mathbf{K}^{-1}$ & $T_{\mathbf{i}^{\mathrm{a}}} / \mathbf{K}$ & $p / \mathbf{M P a}$ & $T / \mathbf{K}$ & $\lambda / \mathbf{W} \cdot \mathbf{m}^{-1} \mathbf{K}^{-1}$ \\
\hline 299.345 & 51.809 & 300.219 & 0.16428 & 299.317 & 8.893 & 300.408 & 0.15264 \\
\hline 299.341 & 51.802 & 300.200 & 0.16439 & 299.312 & 8.875 & 300.686 & 0.15266 \\
\hline 299.340 & 51.789 & 300.429 & 0.16402 & 299.311 & 8.882 & 300.688 & 0.15250 \\
\hline 299.343 & 51.764 & 300.686 & 0.16417 & 299.313 & 8.897 & 301.017 & 0.15247 \\
\hline 299.342 & 51.788 & 300.687 & 0.16415 & 299.316 & 8.849 & 301.009 & 0.15247 \\
\hline 299.344 & 51.775 & 300.968 & 0.16405 & 299.311 & 8.866 & 301.365 & 0.15249 \\
\hline 299.346 & 51.799 & 300.973 & 0.16394 & 299.314 & 8.824 & 301.359 & 0.15241 \\
\hline 299.338 & 51.770 & 301.270 & 0.16397 & 299.307 & 8.860 & 301.754 & 0.15236 \\
\hline 299.352 & 60.273 & 300.161 & 0.16586 & 299.315 & 8.877 & 301.760 & 0.15239 \\
\hline 299.349 & 60.262 & 300.154 & 0.16594 & 299.307 & 14.596 & 300.336 & 0.15416 \\
\hline 299.347 & 60.303 & 300.389 & 0.16603 & 299.310 & 14.638 & 300.359 & 0.15413 \\
\hline 299.348 & 60.387 & 300.395 & 0.16611 & 299.311 & 14.539 & 300.632 & 0.15413 \\
\hline 299.349 & 60.416 & 300.650 & 0.16614 & 299.303 & 14.651 & 300.963 & 0.15409 \\
\hline 299.353 & 60.426 & 300.687 & 0.16614 & 299.306 & 14.651 & 300.973 & 0.15410 \\
\hline 299.347 & 60.443 & 300.959 & 0.16612 & 299.310 & 14.673 & 301.326 & 0.15413 \\
\hline 299.346 & 60.450 & 300.957 & 0.16613 & 299.307 & 14.661 & 301.713 & 0.15397 \\
\hline 299.351 & 60.459 & 301.270 & 0.16609 & 299.303 & 14.628 & 301.699 & 0.15402 \\
\hline 299.349 & 60.464 & 301.270 & 0.16602 & 299.318 & 21.057 & 300.364 & 0.15644 \\
\hline \multicolumn{4}{|c|}{ Wire 2} & 299.313 & 21.167 & 300.656 & 0.15593 \\
\hline 299.323 & 1.126 & 300.421 & 0.15035 & 299.315 & 21.150 & 300.970 & 0.15627 \\
\hline 299.321 & 1.127 & 300.419 & 0.15052 & 299.314 & 21.168 & 301.319 & 0.15627 \\
\hline 299.322 & 1.138 & 300.712 & 0.15039 & 299.315 & 21.154 & 301.316 & 0.15572 \\
\hline 299.322 & 1.120 & 301.041 & 0.15030 & 299.311 & 21.170 & 301.699 & 0.15534 \\
\hline 299.318 & 1.123 & 301.032 & 0.15032 & 299.315 & 21.170 & 301.703 & 0.15535 \\
\hline 299.319 & 1.052 & 301.378 & 0.15024 & 299.320 & 28.228 & 300.340 & 0.15777 \\
\hline 299.316 & 1.103 & 301.392 & 0.15023 & 299.320 & 28.195 & 301.302 & 0.15766 \\
\hline 299.320 & 1.113 & 301.793 & 0.15014 & 299.322 & 28.240 & 301.306 & 0.15729 \\
\hline 299.320 & 1.120 & 301.790 & 0.15021 & 299.319 & 28.150 & 301.669 & 0.15811 \\
\hline 299.303 & 0.157 & 300.396 & 0.15020 & 299.318 & 28.193 & 301.681 & 0.15732 \\
\hline 299.304 & 0.158 & 300.398 & 0.15022 & 299.323 & 35.419 & 300.365 & 0.15951 \\
\hline 299.305 & 0.158 & 300.694 & 0.15016 & 299.320 & 35.386 & 300.632 & 0.15964 \\
\hline 299.310 & 0.158 & 301.020 & 0.15009 & 299.318 & 35.421 & 300.636 & 0.15956 \\
\hline 299.307 & 0.159 & 301.021 & 0.15006 & 299.319 & 35.376 & 300.942 & 0.15945 \\
\hline 299.309 & 0.160 & 301.384 & 0.15001 & 299.321 & 35.423 & 300.950 & 0.15943 \\
\hline 299.305 & 0.160 & 301.379 & 0.15005 & 299.320 & 35.428 & 301.281 & 0.15996 \\
\hline 299.306 & 0.162 & 301.777 & 0.15001 & 299.317 & 35.398 & 301.280 & 0.15946 \\
\hline 299.313 & 8.897 & 300.406 & 0.15261 & 299.319 & 35.351 & 301.653 & 0.15939 \\
\hline
\end{tabular}

anitial cell temperature. 
Table A2, continued.

\begin{tabular}{|c|c|c|c|c|c|c|c|}
\hline$T_{\mathrm{i}^{\mathrm{a}}}^{\mathrm{a}} / \mathrm{K}$ & $p / \mathbf{M P a}$ & $T / K$ & $\lambda / \mathbf{W} \cdot \mathbf{m}^{-1} \mathbf{K}^{-1}$ & $T_{\mathrm{i}^{\mathrm{a}}}^{\mathrm{a}} / \mathbf{K}$ & $p / \mathbf{M P a}$ & $T / K$ & $\lambda / \mathbf{W} \cdot \mathbf{m}^{-1} \mathbf{K}^{-1}$ \\
\hline 299.320 & 35.396 & 301.662 & 0.15937 & 299.311 & 68.747 & 301.573 & 0.16694 \\
\hline 299.323 & 43.233 & 300.351 & 0.16152 & 299.314 & 68.766 & 301.571 & 0.16699 \\
\hline 299.321 & 43.232 & 300.627 & 0.16149 & 330.744 & 0.109 & 331.790 & 0.14593 \\
\hline 299.317 & 43.210 & 300.617 & 0.16144 & 330.746 & 0.109 & 331.793 & 0.14561 \\
\hline 299.322 & 43.232 & 300.934 & 0.16148 & 330.745 & 0.110 & 332.079 & 0.14561 \\
\hline 299.325 & 43.208 & 300.931 & 0.16139 & 330.746 & 0.110 & 332.073 & 0.14557 \\
\hline 299.322 & 43.230 & 301.268 & 0.16132 & 330.744 & 0.111 & 332.387 & 0.14553 \\
\hline 299.319 & 43.227 & 301.637 & 0.16129 & 330.744 & 0.111 & 332.729 & 0.14547 \\
\hline 299.322 & 43.204 & 301.636 & 0.16126 & 330.743 & 0.112 & 332.726 & 0.14559 \\
\hline 299.318 & 51.321 & 300.332 & 0.16357 & 330.745 & 0.112 & 333.110 & 0.14544 \\
\hline 299.316 & 51.286 & 300.324 & 0.16343 & 330.757 & 6.618 & 332.377 & 0.14761 \\
\hline 299.316 & 51.312 & 300.604 & 0.16341 & 330.757 & 6.627 & 332.382 & 0.14762 \\
\hline 299.318 & 51.298 & 300.603 & 0.16331 & 330.756 & 6.618 & 332.714 & 0.14771 \\
\hline 299.320 & 51.327 & 300.913 & 0.16335 & 330.758 & 6.635 & 332.718 & 0.14764 \\
\hline 299.320 & 51.333 & 300.912 & 0.16342 & 330.754 & 6.636 & 333.087 & 0.14754 \\
\hline 299.315 & 51.293 & 301.233 & 0.16321 & 330.755 & 6.648 & 333.092 & 0.14746 \\
\hline 299.317 & 51.315 & 301.240 & 0.16329 & 330.756 & 6.630 & 333.497 & 0.14740 \\
\hline 299.316 & 51.321 & 301.605 & 0.16319 & 330.756 & 6.625 & 333.494 & 0.14746 \\
\hline 299.318 & 51.315 & 301.609 & 0.16322 & 330.754 & 6.624 & 333.936 & 0.14738 \\
\hline 299.315 & 60.217 & 300.325 & 0.16528 & 330.759 & 6.631 & 333.942 & 0.14734 \\
\hline 299.319 & 60.290 & 300.341 & 0.16562 & 330.760 & 13.583 & 332.356 & 0.14982 \\
\hline 299.318 & 60.247 & 300.596 & 0.16534 & 330.755 & 13.573 & 332.351 & 0.14975 \\
\hline 299.316 & 60.287 & 300.605 & 0.16532 & 330.762 & 13.581 & 332.692 & 0.14982 \\
\hline 299.317 & 60.235 & 300.894 & 0.16526 & 330.761 & 13.569 & 332.686 & 0.14970 \\
\hline 299.316 & 60.286 & 300.907 & 0.16541 & 330.760 & 13.580 & 333.063 & 0.14962 \\
\hline 299.315 & 60.268 & 301.226 & 0.16524 & 330.762 & 13.569 & 333.056 & 0.14964 \\
\hline 299.313 & 60.243 & 301.227 & 0.16514 & 330.756 & 13.575 & 333.455 & 0.14964 \\
\hline 299.317 & 60.285 & 301.596 & 0.16526 & 330.756 & 13.581 & 333.452 & 0.14956 \\
\hline 299.317 & 60.213 & 301.590 & 0.16518 & 330.763 & 13.589 & 333.897 & 0.14949 \\
\hline 299.319 & 68.743 & 300.326 & 0.16708 & 330.760 & 13.582 & 333.893 & 0.14952 \\
\hline 299.317 & 68.738 & 300.315 & 0.16719 & 330.764 & 21.235 & 332.308 & 0.15207 \\
\hline 299.313 & 68.773 & 300.590 & 0.16725 & 330.762 & 21.236 & 332.306 & 0.15207 \\
\hline 299.312 & 68.781 & 300.583 & 0.16724 & 330.766 & 21.234 & 332.638 & 0.15209 \\
\hline 299.318 & 68.779 & 300.889 & 0.16710 & 330.766 & 21.237 & 332.641 & 0.15225 \\
\hline 299.319 & 68.770 & 300.885 & 0.16712 & 330.765 & 21.222 & 333.005 & 0.15189 \\
\hline 299.319 & 68.775 & 301.217 & 0.16704 & 330.764 & 21.210 & 332.999 & 0.15191 \\
\hline 299.316 & 68.712 & 301.199 & 0.16730 & 330.767 & 21.224 & 333.403 & 0.15180 \\
\hline
\end{tabular}

anitial cell temperature. 
Table A2, continued.

\begin{tabular}{|c|c|c|c|c|c|c|c|}
\hline$T_{\mathrm{i}^{\mathrm{a}}}^{\mathrm{a}} / \mathrm{K}$ & $p / \mathrm{MPa}$ & $T / K$ & $\lambda / \mathbf{W} \cdot \mathbf{m}^{-1} \mathbf{K}^{-1}$ & $T_{\mathrm{i}^{\mathrm{a}}}^{\mathrm{a}} / \mathbf{K}$ & $p / \mathbf{M P a}$ & $T / K$ & $\lambda / \mathbf{W} \cdot \mathbf{m}^{-1} \mathbf{K}^{-1}$ \\
\hline 330.764 & 21.219 & 333.393 & 0.15182 & 330.781 & 57.967 & 333.300 & 0.16136 \\
\hline 330.762 & 21.233 & 333.827 & 0.15182 & 330.778 & 57.959 & 333.289 & 0.16122 \\
\hline 330.760 & 21.223 & 333.825 & 0.15178 & 330.777 & 57.963 & 333.700 & 0.16115 \\
\hline 330.769 & 29.200 & 332.313 & 0.15438 & 330.782 & 57.951 & 333.703 & 0.16120 \\
\hline 330.768 & 29.201 & 332.310 & 0.15435 & 330.785 & 68.911 & 332.240 & 0.16384 \\
\hline 330.766 & 29.209 & 332.638 & 0.15432 & 330.781 & 68.913 & 332.542 & 0.16371 \\
\hline 330.769 & 29.211 & 332.639 & 0.15423 & 330.778 & 68.930 & 332.540 & 0.16384 \\
\hline 330.765 & 29.203 & 332.993 & 0.15418 & 330.781 & 68.915 & 332.891 & 0.16372 \\
\hline 330.767 & 29.214 & 332.998 & 0.15427 & 330.779 & 68.898 & 332.878 & 0.16378 \\
\hline 330.768 & 29.209 & 333.390 & 0.15415 & 330.779 & 68.904 & 333.252 & 0.16367 \\
\hline 330.766 & 29.204 & 333.386 & 0.15420 & 330.781 & 68.909 & 333.255 & 0.16361 \\
\hline 330.767 & 29.201 & 333.810 & 0.15403 & 330.780 & 68.910 & 333.657 & 0.16361 \\
\hline 330.766 & 29.200 & 333.811 & 0.15401 & 330.786 & 68.891 & 333.657 & 0.16359 \\
\hline 330.772 & 38.294 & 332.281 & 0.15693 & 364.066 & 0.126 & 365.625 & 0.14062 \\
\hline 330.775 & 38.290 & 332.621 & 0.15666 & 364.073 & 0.127 & 365.626 & 0.14056 \\
\hline 330.774 & 38.298 & 332.640 & 0.15668 & 364.073 & 0.127 & 365.934 & 0.14062 \\
\hline 330.775 & 38.286 & 332.992 & 0.15659 & 364.071 & 0.128 & 366.279 & 0.14023 \\
\hline 330.773 & 38.283 & 332.990 & 0.15701 & 364.073 & 0.128 & 366.289 & 0.14026 \\
\hline 330.770 & 38.291 & 333.372 & 0.15655 & 364.071 & 0.129 & 366.692 & 0.14029 \\
\hline 330.774 & 38.280 & 333.373 & 0.15651 & 364.071 & 0.129 & 366.685 & 0.14024 \\
\hline 330.773 & 38.280 & 333.794 & 0.15649 & 364.075 & 0.129 & 367.121 & 0.14015 \\
\hline 330.771 & 38.301 & 333.789 & 0.15644 & 364.071 & 0.130 & 367.115 & 0.14011 \\
\hline 330.777 & 47.667 & 332.290 & 0.15895 & 364.085 & 0.149 & 365.640 & 0.14049 \\
\hline 330.776 & 47.658 & 332.287 & 0.15919 & 364.087 & 0.151 & 365.648 & 0.14046 \\
\hline 330.775 & 47.660 & 332.599 & 0.15898 & 364.088 & 0.152 & 365.972 & 0.14029 \\
\hline 330.774 & 47.660 & 332.598 & 0.15902 & 364.087 & 0.153 & 366.002 & 0.14045 \\
\hline 330.779 & 47.659 & 332.959 & 0.15891 & 364.087 & 0.154 & 366.353 & 0.14023 \\
\hline 330.775 & 47.633 & 332.947 & 0.15887 & 364.091 & 0.156 & 366.364 & 0.14034 \\
\hline 330.778 & 47.633 & 333.336 & 0.15881 & 364.093 & 0.156 & 366.759 & 0.14022 \\
\hline 330.776 & 47.648 & 333.338 & 0.15889 & 364.092 & 0.156 & 367.182 & 0.14009 \\
\hline 330.774 & 47.638 & 333.743 & 0.15877 & 364.091 & 0.155 & 367.188 & 0.14012 \\
\hline 330.776 & 47.644 & 333.746 & 0.15876 & 364.119 & 6.728 & 365.669 & 0.14276 \\
\hline 330.783 & 57.967 & 332.266 & 0.16160 & 364.117 & 6.738 & 365.984 & 0.14279 \\
\hline 330.776 & 57.957 & 332.571 & 0.16128 & 364.118 & 6.733 & 365.978 & 0.14278 \\
\hline 330.778 & 57.966 & 332.574 & 0.16139 & 364.118 & 6.708 & 366.331 & 0.14264 \\
\hline 330.780 & 57.967 & 332.925 & 0.16129 & 364.121 & 6.707 & 366.344 & 0.14271 \\
\hline 330.780 & 57.956 & 332.917 & 0.16131 & 364.120 & 6.710 & 366.719 & 0.14260 \\
\hline
\end{tabular}

anitial cell temperature. 
Table A2, continued.

\begin{tabular}{|c|c|c|c|c|c|c|c|}
\hline$T_{\mathrm{i}^{\mathrm{a}}}^{\mathrm{a}} / \mathrm{K}$ & $p / \mathrm{MPa}$ & $T / K$ & $\lambda / \mathbf{W} \cdot \mathbf{m}^{-1} \mathbf{K}^{-1}$ & $T_{\mathrm{i}^{\mathrm{a}}}^{\mathrm{a}} / \mathbf{K}$ & $p / \mathbf{M P a}$ & $T / K$ & $\lambda / \mathbf{W} \cdot \mathbf{m}^{-1} \mathbf{K}^{-1}$ \\
\hline 364.120 & 6.711 & 366.713 & 0.14263 & 364.134 & 37.471 & 366.992 & 0.15246 \\
\hline 364.117 & 6.715 & 367.136 & 0.14246 & 364.130 & 37.418 & 366.991 & 0.15250 \\
\hline 364.120 & 6.715 & 367.159 & 0.14242 & 364.122 & 46.876 & 365.552 & 0.15544 \\
\hline 364.127 & 13.279 & 365.641 & 0.14514 & 364.116 & 46.873 & 365.557 & 0.15544 \\
\hline 364.125 & 13.286 & 365.641 & 0.14511 & 364.114 & 46.853 & 365.853 & 0.15523 \\
\hline 364.126 & 13.291 & 365.965 & 0.14523 & 364.113 & 46.846 & 365.858 & 0.15547 \\
\hline 364.125 & 13.294 & 365.954 & 0.14511 & 364.114 & 46.840 & 366.195 & 0.15516 \\
\hline 364.124 & 13.300 & 366.309 & 0.14494 & 364.114 & 46.830 & 366.185 & 0.15524 \\
\hline 364.126 & 13.307 & 366.316 & 0.14495 & 364.108 & 46.834 & 366.543 & 0.15529 \\
\hline 364.122 & 13.300 & 366.697 & 0.14486 & 364.111 & 46.823 & 366.545 & 0.15538 \\
\hline 364.128 & 13.314 & 366.700 & 0.14504 & 364.109 & 46.828 & 366.939 & 0.15517 \\
\hline 364.126 & 13.299 & 367.107 & 0.14486 & 364.110 & 46.829 & 366.936 & 0.15509 \\
\hline 364.124 & 13.295 & 367.102 & 0.14479 & 364.113 & 57.708 & 365.481 & 0.15832 \\
\hline 364.128 & 20.614 & 365.592 & 0.14738 & 364.108 & 57.707 & 365.480 & 0.15822 \\
\hline 364.128 & 20.634 & 365.601 & 0.14750 & 364.111 & 57.717 & 365.773 & 0.15822 \\
\hline 364.132 & 20.636 & 365.921 & 0.14759 & 364.108 & 57.723 & 365.774 & 0.15833 \\
\hline 364.127 & 20.637 & 365.933 & 0.14746 & 364.106 & 57.711 & 366.129 & 0.15811 \\
\hline 364.126 & 20.628 & 366.276 & 0.14734 & 364.106 & 57.712 & 366.140 & 0.15823 \\
\hline 364.129 & 20.620 & 366.649 & 0.14736 & 364.103 & 57.696 & 366.489 & 0.15806 \\
\hline 364.132 & 20.628 & 366.660 & 0.14729 & 364.103 & 57.709 & 366.490 & 0.15804 \\
\hline 364.128 & 20.618 & 367.064 & 0.14727 & 364.102 & 57.697 & 366.871 & 0.15815 \\
\hline 364.138 & 28.765 & 365.589 & 0.15014 & 364.105 & 57.683 & 366.880 & 0.15800 \\
\hline 364.130 & 28.727 & 365.588 & 0.15010 & 364.104 & 68.798 & 365.467 & 0.16104 \\
\hline 364.130 & 28.721 & 365.898 & 0.15005 & 364.105 & 68.801 & 365.468 & 0.16116 \\
\hline 364.136 & 28.706 & 365.901 & 0.15007 & 364.105 & 68.803 & 365.762 & 0.16087 \\
\hline 364.134 & 28.717 & 366.236 & 0.14997 & 364.101 & 68.782 & 365.760 & 0.16099 \\
\hline 364.132 & 28.677 & 366.231 & 0.14996 & 364.100 & 68.787 & 366.094 & 0.16090 \\
\hline 364.129 & 28.679 & 366.599 & 0.14996 & 364.102 & 68.781 & 366.097 & 0.16080 \\
\hline 364.136 & 28.691 & 366.630 & 0.15007 & 364.104 & 68.778 & 366.455 & 0.16091 \\
\hline 364.132 & 28.697 & 367.032 & 0.14987 & 364.103 & 68.768 & 366.450 & 0.16089 \\
\hline 364.129 & 28.705 & 367.022 & 0.14981 & 364.103 & 68.767 & 366.823 & 0.16075 \\
\hline 364.134 & 37.591 & 365.559 & 0.15275 & 364.099 & 68.753 & 366.820 & 0.16079 \\
\hline 364.135 & 37.544 & 365.875 & 0.15279 & 397.623 & 0.103 & 399.106 & 0.13503 \\
\hline 364.134 & 37.517 & 365.878 & 0.15282 & 397.623 & 0.103 & 399.101 & 0.13484 \\
\hline 364.136 & 37.487 & 366.220 & 0.15261 & 397.625 & 0.107 & 399.424 & 0.13472 \\
\hline 364.134 & 37.456 & 366.579 & 0.15253 & 397.622 & 0.107 & 399.419 & 0.13483 \\
\hline 364.130 & 37.462 & 366.583 & 0.15260 & 397.623 & 0.103 & 399.762 & 0.13469 \\
\hline
\end{tabular}

anitial cell temperature. 
Table A2, continued.

\begin{tabular}{|c|c|c|c|c|c|c|c|}
\hline$T_{\mathrm{i}^{\mathrm{a}}}^{\mathrm{a}} / \mathrm{K}$ & $p / \mathrm{MPa}$ & $T / K$ & $\lambda / \mathbf{W} \cdot \mathbf{m}^{-1} \mathbf{K}^{-1}$ & $T_{\mathrm{i}^{\mathrm{a}}}^{\mathrm{a}} / \mathbf{K}$ & $p / \mathbf{M P a}$ & $T / K$ & $\lambda / \mathbf{W} \cdot \mathbf{m}^{-1} \mathbf{K}^{-1}$ \\
\hline 397.628 & 0.114 & 399.775 & 0.13471 & 397.638 & 28.020 & 399.323 & 0.14564 \\
\hline 397.621 & 0.115 & 400.153 & 0.13477 & 397.637 & 28.027 & 399.322 & 0.14575 \\
\hline 397.626 & 0.126 & 400.159 & 0.13480 & 397.642 & 28.025 & 399.657 & 0.14560 \\
\hline 397.626 & 0.127 & 400.557 & 0.13457 & 397.642 & 28.040 & 399.658 & 0.14538 \\
\hline 397.624 & 0.132 & 400.558 & 0.13454 & 397.644 & 28.041 & 400.005 & 0.14551 \\
\hline 397.617 & 6.475 & 399.095 & 0.13771 & 397.643 & 28.036 & 400.012 & 0.14551 \\
\hline 397.617 & 6.485 & 399.099 & 0.13745 & 397.643 & 28.038 & 400.394 & 0.14548 \\
\hline 397.617 & 6.481 & 399.414 & 0.13749 & 397.644 & 28.059 & 400.398 & 0.14541 \\
\hline 397.626 & 6.486 & 399.413 & 0.13761 & 397.651 & 37.368 & 399.021 & 0.14865 \\
\hline 397.623 & 6.487 & 399.761 & 0.13740 & 397.650 & 37.365 & 399.304 & 0.14880 \\
\hline 397.625 & 6.482 & 399.757 & 0.13736 & 397.650 & 37.357 & 399.298 & 0.14872 \\
\hline 397.621 & 6.483 & 400.120 & 0.13735 & 397.648 & 37.349 & 399.616 & 0.14871 \\
\hline 397.629 & 6.465 & 400.120 & 0.13739 & 397.651 & 37.354 & 399.621 & 0.14879 \\
\hline 397.631 & 6.464 & 400.531 & 0.13734 & 397.654 & 37.339 & 399.965 & 0.14872 \\
\hline 397.636 & 6.453 & 400.532 & 0.13733 & 397.647 & 37.348 & 400.340 & 0.14866 \\
\hline 397.646 & 12.656 & 399.070 & 0.14001 & 397.647 & 37.344 & 400.336 & 0.14865 \\
\hline 397.643 & 12.653 & 399.065 & 0.13991 & 397.646 & 46.595 & 398.957 & 0.15191 \\
\hline 397.637 & 12.651 & 399.371 & 0.14012 & 397.653 & 46.585 & 398.964 & 0.15193 \\
\hline 397.643 & 12.643 & 399.387 & 0.14006 & 397.645 & 46.581 & 399.236 & 0.15177 \\
\hline 397.638 & 12.639 & 399.710 & 0.13987 & 397.649 & 46.592 & 399.252 & 0.15204 \\
\hline 397.636 & 12.642 & 399.714 & 0.13983 & 397.646 & 46.676 & 399.570 & 0.15162 \\
\hline 397.633 & 12.637 & 400.067 & 0.13981 & 397.645 & 46.715 & 399.585 & 0.15176 \\
\hline 397.636 & 12.628 & 400.065 & 0.13981 & 397.645 & 46.737 & 399.931 & 0.15174 \\
\hline 397.631 & 12.629 & 400.462 & 0.13981 & 397.653 & 46.748 & 399.936 & 0.15155 \\
\hline 397.634 & 12.623 & 400.469 & 0.13969 & 397.652 & 46.758 & 400.308 & 0.15157 \\
\hline 397.638 & 20.130 & 399.054 & 0.14277 & 397.657 & 46.763 & 400.307 & 0.15167 \\
\hline 397.640 & 20.133 & 399.066 & 0.14303 & 397.714 & 57.482 & 399.041 & 0.15527 \\
\hline 397.640 & 20.131 & 399.359 & 0.14257 & 397.715 & 57.482 & 399.041 & 0.15522 \\
\hline 397.642 & 20.129 & 399.355 & 0.14259 & 397.711 & 57.484 & 399.318 & 0.15508 \\
\hline 397.638 & 20.119 & 399.688 & 0.14261 & 397.720 & 57.487 & 399.326 & 0.15485 \\
\hline 397.640 & 20.120 & 399.689 & 0.14280 & 397.720 & 57.489 & 399.631 & 0.15491 \\
\hline 397.642 & 20.119 & 400.055 & 0.14269 & 397.724 & 57.491 & 399.647 & 0.15496 \\
\hline 397.638 & 20.111 & 400.039 & 0.14261 & 397.725 & 57.485 & 399.976 & 0.15505 \\
\hline 397.641 & 20.115 & 400.441 & 0.14260 & 397.726 & 57.483 & 399.974 & 0.15502 \\
\hline 397.639 & 20.120 & 400.430 & 0.14279 & 397.725 & 57.487 & 400.332 & 0.15492 \\
\hline 397.643 & 28.026 & 399.046 & 0.14569 & 397.725 & 57.482 & 400.333 & 0.15492 \\
\hline 397.638 & 28.027 & 399.051 & 0.14566 & 397.738 & 68.861 & 398.967 & 0.15860 \\
\hline
\end{tabular}

anitial cell temperature. 
Table A2, continued.

\begin{tabular}{|c|c|c|c|c|c|c|c|}
\hline$T_{\mathrm{i}^{\mathrm{a}}}^{\mathrm{a}} / \mathrm{K}$ & $p / \mathrm{MPa}$ & $T / K$ & $\lambda / \mathbf{W} \cdot \mathbf{m}^{-1} \mathbf{K}^{-1}$ & $T_{\mathrm{i}^{\mathrm{a}}}^{\mathrm{a}} / \mathbf{K}$ & $p / \mathbf{M P a}$ & $T / K$ & $\lambda / \mathbf{W} \cdot \mathbf{m}^{-1} \mathbf{K}^{-1}$ \\
\hline 397.734 & 68.849 & 398.969 & 0.15841 & 431.347 & 12.322 & 433.654 & 0.13466 \\
\hline 397.737 & 68.847 & 399.234 & 0.15838 & 431.348 & 12.326 & 433.673 & 0.13464 \\
\hline 397.738 & 68.836 & 399.539 & 0.15836 & 431.344 & 12.324 & 434.048 & 0.13455 \\
\hline 397.739 & 68.831 & 399.546 & 0.15830 & 431.343 & 12.314 & 434.034 & 0.13449 \\
\hline 397.736 & 68.828 & 399.863 & 0.15823 & 431.356 & 19.427 & 432.729 & 0.13801 \\
\hline 397.743 & 68.819 & 399.877 & 0.15817 & 431.350 & 19.433 & 432.730 & 0.13792 \\
\hline 397.740 & 68.810 & 400.222 & 0.15814 & 431.354 & 19.424 & 433.009 & 0.13790 \\
\hline 397.745 & 68.735 & 399.048 & 0.15834 & 431.352 & 19.418 & 433.009 & 0.13795 \\
\hline 397.748 & 68.729 & 399.061 & 0.15845 & 431.351 & 19.414 & 433.325 & 0.13757 \\
\hline 397.749 & 68.721 & 399.325 & 0.15805 & 431.353 & 19.418 & 433.329 & 0.13763 \\
\hline 397.746 & 68.710 & 399.620 & 0.15818 & 431.345 & 19.419 & 433.664 & 0.13750 \\
\hline 397.741 & 68.705 & 399.616 & 0.15819 & 431.349 & 19.417 & 433.667 & 0.13756 \\
\hline 397.748 & 68.698 & 399.950 & 0.15796 & 431.349 & 19.413 & 434.030 & 0.13762 \\
\hline 397.748 & 68.686 & 400.304 & 0.15803 & 431.342 & 19.406 & 434.018 & 0.13757 \\
\hline 397.744 & 68.678 & 400.299 & 0.15806 & 431.348 & 27.520 & 432.695 & 0.14114 \\
\hline 431.317 & 0.101 & 432.783 & 0.12922 & 431.357 & 27.510 & 432.691 & 0.14117 \\
\hline 431.311 & 0.106 & 433.064 & 0.12940 & 431.355 & 27.504 & 432.971 & 0.14102 \\
\hline 431.318 & 0.111 & 433.083 & 0.12916 & 431.357 & 27.495 & 433.280 & 0.14088 \\
\hline 431.318 & 0.118 & 433.421 & 0.12915 & 431.356 & 27.489 & 433.289 & 0.14091 \\
\hline 431.311 & 0.119 & 433.402 & 0.12898 & 431.356 & 27.496 & 433.616 & 0.14092 \\
\hline 431.319 & 0.121 & 433.778 & 0.12914 & 431.360 & 27.492 & 433.984 & 0.14076 \\
\hline 431.316 & 0.129 & 433.779 & 0.12892 & 431.359 & 27.481 & 433.985 & 0.14080 \\
\hline 431.321 & 0.133 & 434.161 & 0.12890 & 431.353 & 36.447 & 432.643 & 0.14449 \\
\hline 431.318 & 0.138 & 434.165 & 0.12885 & 431.354 & 36.427 & 432.641 & 0.14450 \\
\hline 431.363 & 6.164 & 432.763 & 0.13212 & 431.351 & 36.432 & 432.909 & 0.14469 \\
\hline 431.369 & 6.166 & 432.777 & 0.13208 & 431.352 & 36.417 & 432.904 & 0.14420 \\
\hline 431.375 & 6.153 & 433.073 & 0.13204 & 431.357 & 36.423 & 433.230 & 0.14455 \\
\hline 431.372 & 6.160 & 433.392 & 0.13199 & 431.355 & 36.419 & 433.211 & 0.14436 \\
\hline 431.372 & 6.150 & 433.395 & 0.13198 & 431.356 & 36.406 & 433.557 & 0.14434 \\
\hline 431.374 & 6.149 & 433.744 & 0.13191 & 431.353 & 36.414 & 433.538 & 0.14413 \\
\hline 431.371 & 6.147 & 434.149 & 0.13177 & 431.355 & 36.405 & 433.899 & 0.14417 \\
\hline 431.369 & 6.141 & 434.159 & 0.13168 & 431.353 & 36.395 & 433.891 & 0.14417 \\
\hline 431.353 & 12.338 & 432.710 & 0.13495 & 431.367 & 46.217 & 432.579 & 0.14780 \\
\hline 431.339 & 12.342 & 432.999 & 0.13452 & 431.357 & 46.199 & 432.563 & 0.14773 \\
\hline 431.347 & 12.343 & 433.000 & 0.13485 & 431.364 & 46.192 & 432.841 & 0.14784 \\
\hline 431.342 & 12.339 & 433.312 & 0.13484 & 431.368 & 46.193 & 432.913 & 0.14781 \\
\hline 431.346 & 12.334 & 433.320 & 0.13501 & 431.365 & 46.181 & 433.207 & 0.14770 \\
\hline
\end{tabular}

anitial cell temperature. 
Table A2, continued.

\begin{tabular}{|c|c|c|c|c|c|c|c|}
\hline$T_{\mathrm{i}^{\mathrm{a}}}^{\mathrm{a}} / \mathrm{K}$ & $p / \mathbf{M P a}$ & $T / K$ & $\lambda / \mathbf{W} \cdot \mathbf{m}^{-1} \mathbf{K}^{-1}$ & $T_{\mathrm{i}^{\mathrm{a}}}^{\mathrm{a}} / \mathbf{K}$ & $p / \mathbf{M P a}$ & $T / K$ & $\lambda / \mathbf{W} \cdot \mathbf{m}^{-1} \mathbf{K}^{-1}$ \\
\hline 431.359 & 46.184 & 433.197 & 0.14782 & 466.396 & 5.814 & 468.345 & 0.12602 \\
\hline 431.368 & 46.178 & 433.537 & 0.14780 & 466.394 & 5.820 & 468.710 & 0.12589 \\
\hline 431.365 & 46.167 & 433.528 & 0.14778 & 466.397 & 5.833 & 468.722 & 0.12606 \\
\hline 431.367 & 46.156 & 433.866 & 0.14765 & 466.398 & 5.836 & 469.095 & 0.12603 \\
\hline 431.365 & 46.137 & 433.858 & 0.14760 & 466.399 & 5.829 & 469.079 & 0.12611 \\
\hline 431.378 & 57.128 & 432.639 & 0.15145 & 466.440 & 11.900 & 467.803 & 0.12953 \\
\hline 431.375 & 57.124 & 432.637 & 0.15168 & 466.439 & 11.893 & 467.799 & 0.12935 \\
\hline 431.374 & 57.107 & 432.897 & 0.15148 & 466.438 & 11.896 & 468.085 & 0.12921 \\
\hline 431.380 & 57.102 & 432.897 & 0.15133 & 466.446 & 11.886 & 468.072 & 0.12947 \\
\hline 431.382 & 57.085 & 433.192 & 0.15146 & 466.441 & 11.879 & 468.391 & 0.12921 \\
\hline 431.380 & 57.071 & 433.187 & 0.15144 & 466.440 & 11.875 & 468.375 & 0.12913 \\
\hline 431.381 & 57.058 & 433.506 & 0.15148 & 466.438 & 11.868 & 468.726 & 0.12909 \\
\hline 431.383 & 57.039 & 433.494 & 0.15115 & 466.434 & 11.862 & 469.078 & 0.12914 \\
\hline 431.389 & 57.037 & 433.854 & 0.15118 & 466.434 & 11.874 & 469.105 & 0.12896 \\
\hline 431.384 & 57.015 & 433.836 & 0.15126 & 466.413 & 18.950 & 467.720 & 0.13288 \\
\hline 431.377 & 64.069 & 432.600 & 0.15364 & 466.412 & 18.937 & 467.986 & 0.13236 \\
\hline 431.372 & 63.989 & 432.607 & 0.15345 & 466.413 & 18.950 & 468.008 & 0.13237 \\
\hline 431.369 & 63.916 & 432.870 & 0.15382 & 466.414 & 18.947 & 468.321 & 0.13232 \\
\hline 431.370 & 63.851 & 432.873 & 0.15359 & 466.416 & 18.938 & 468.298 & 0.13233 \\
\hline 431.367 & 63.796 & 433.155 & 0.15354 & 466.420 & 18.940 & 468.648 & 0.13226 \\
\hline 431.367 & 63.725 & 433.140 & 0.15373 & 466.422 & 18.932 & 469.010 & 0.13219 \\
\hline 431.370 & 63.668 & 433.455 & 0.15344 & 466.432 & 18.930 & 469.022 & 0.13249 \\
\hline 431.368 & 63.628 & 433.458 & 0.15332 & 466.452 & 26.665 & 467.720 & 0.13631 \\
\hline 431.372 & 63.573 & 433.784 & 0.15345 & 466.452 & 26.664 & 467.725 & 0.13592 \\
\hline 431.368 & 63.527 & 433.793 & 0.15329 & 466.451 & 26.651 & 467.982 & 0.13600 \\
\hline 466.376 & 0.203 & 467.798 & 0.12324 & 466.451 & 26.653 & 468.003 & 0.13586 \\
\hline 466.379 & 0.192 & 467.796 & 0.12320 & 466.445 & 26.649 & 468.278 & 0.13603 \\
\hline 466.375 & 0.196 & 468.083 & 0.12346 & 466.447 & 26.632 & 468.274 & 0.13639 \\
\hline 466.374 & 0.193 & 468.407 & 0.12303 & 466.443 & 26.648 & 468.618 & 0.13578 \\
\hline 466.371 & 0.199 & 468.400 & 0.12326 & 466.441 & 26.634 & 468.596 & 0.13582 \\
\hline 466.374 & 0.198 & 468.760 & 0.12293 & 466.441 & 26.633 & 468.968 & 0.13555 \\
\hline 466.377 & 0.189 & 469.137 & 0.12273 & 466.440 & 26.634 & 468.949 & 0.13549 \\
\hline 466.381 & 0.196 & 469.151 & 0.12297 & 466.452 & 36.020 & 467.693 & 0.13972 \\
\hline 466.398 & 5.823 & 467.765 & 0.12627 & 466.448 & 36.014 & 467.695 & 0.13956 \\
\hline 466.399 & 5.823 & 467.756 & 0.12606 & 466.456 & 36.011 & 467.960 & 0.13963 \\
\hline 466.403 & 5.812 & 468.039 & 0.12632 & 466.451 & 36.008 & 467.963 & 0.13970 \\
\hline 466.398 & 5.825 & 468.360 & 0.12597 & 466.454 & 36.007 & 468.263 & 0.13970 \\
\hline
\end{tabular}

anitial cell temperature. 
Table A2, continued.

\begin{tabular}{|c|c|c|c|c|c|c|c|}
\hline$T_{\mathrm{i}^{\mathrm{a}}}^{\mathrm{a}} / \mathrm{K}$ & $p / \mathrm{MPa}$ & $T / K$ & $\lambda / \mathbf{W} \cdot \mathbf{m}^{-1} \mathbf{K}^{-1}$ & $T_{\mathrm{i}^{\mathrm{a}}}^{\mathrm{a}} / \mathbf{K}$ & $p / \mathbf{M P a}$ & $T / K$ & $\lambda / \mathbf{W} \cdot \mathbf{m}^{-1} \mathbf{K}^{-1}$ \\
\hline 466.456 & 36.001 & 468.254 & 0.13959 & 500.984 & 5.326 & 502.946 & 0.11871 \\
\hline 466.457 & 35.985 & 468.566 & 0.13971 & 500.990 & 5.321 & 503.290 & 0.11865 \\
\hline 466.459 & 35.977 & 468.570 & 0.13978 & 500.982 & 5.302 & 503.665 & 0.11836 \\
\hline 466.453 & 35.977 & 468.898 & 0.13939 & 500.973 & 5.319 & 503.635 & 0.11844 \\
\hline 466.455 & 35.971 & 468.907 & 0.13945 & 500.971 & 5.314 & 504.045 & 0.11843 \\
\hline 466.461 & 45.826 & 467.658 & 0.14395 & 500.964 & 5.327 & 504.032 & 0.11843 \\
\hline 466.471 & 45.805 & 467.654 & 0.14326 & 500.895 & 11.398 & 502.460 & 0.12199 \\
\hline 466.464 & 45.804 & 467.921 & 0.14376 & 500.887 & 11.374 & 502.440 & 0.12214 \\
\hline 466.463 & 45.789 & 467.902 & 0.14330 & 500.885 & 11.334 & 502.743 & 0.12236 \\
\hline 466.470 & 45.780 & 468.200 & 0.14364 & 500.885 & 11.284 & 502.737 & 0.12197 \\
\hline 466.468 & 45.778 & 468.203 & 0.14335 & 500.879 & 11.324 & 503.135 & 0.12203 \\
\hline 466.467 & 45.770 & 468.513 & 0.14324 & 500.878 & 11.335 & 503.138 & 0.12187 \\
\hline 466.473 & 45.759 & 468.518 & 0.14333 & 500.884 & 11.338 & 503.512 & 0.12170 \\
\hline 466.470 & 45.745 & 468.835 & 0.14353 & 500.880 & 11.332 & 503.491 & 0.12175 \\
\hline 466.473 & 45.736 & 468.844 & 0.14340 & 500.879 & 11.362 & 503.882 & 0.12185 \\
\hline 466.463 & 56.777 & 467.607 & 0.14734 & 500.884 & 11.340 & 503.881 & 0.12178 \\
\hline 466.462 & 56.764 & 467.622 & 0.14784 & 500.884 & 18.368 & 502.465 & 0.12597 \\
\hline 466.463 & 56.750 & 467.853 & 0.14760 & 500.877 & 18.362 & 502.459 & 0.12578 \\
\hline 466.464 & 56.741 & 467.871 & 0.14729 & 500.874 & 18.344 & 502.735 & 0.12604 \\
\hline 466.466 & 56.721 & 468.132 & 0.14781 & 500.863 & 18.301 & 502.725 & 0.12553 \\
\hline 466.470 & 56.706 & 468.142 & 0.14743 & 500.858 & 18.254 & 503.027 & 0.12553 \\
\hline 466.466 & 56.698 & 468.427 & 0.14739 & 500.856 & 18.214 & 503.030 & 0.12565 \\
\hline 466.470 & 56.695 & 468.449 & 0.14757 & 500.857 & 18.275 & 503.407 & 0.12535 \\
\hline 466.467 & 56.675 & 468.765 & 0.14732 & 500.857 & 18.306 & 503.403 & 0.12543 \\
\hline 466.465 & 56.661 & 468.766 & 0.14803 & 500.853 & 18.307 & 503.771 & 0.12535 \\
\hline 501.023 & 0.092 & 502.705 & 0.11534 & 500.855 & 18.300 & 503.772 & 0.12541 \\
\hline 501.024 & 0.099 & 502.707 & 0.11574 & 500.902 & 26.437 & 502.434 & 0.12957 \\
\hline 501.021 & 0.096 & 503.026 & 0.11573 & 500.897 & 26.459 & 502.439 & 0.12977 \\
\hline 501.022 & 0.093 & 503.035 & 0.11542 & 500.896 & 26.463 & 502.721 & 0.12944 \\
\hline 501.017 & 0.097 & 503.377 & 0.11537 & 500.897 & 26.434 & 502.704 & 0.12956 \\
\hline 501.012 & 0.100 & 503.356 & 0.11532 & 500.895 & 26.413 & 503.043 & 0.12936 \\
\hline 501.005 & 0.103 & 503.721 & 0.11526 & 500.890 & 26.409 & 503.046 & 0.12935 \\
\hline 500.996 & 0.097 & 503.707 & 0.11472 & 500.889 & 26.361 & 503.360 & 0.12929 \\
\hline 501.000 & 0.092 & 504.134 & 0.11535 & 500.890 & 26.294 & 503.372 & 0.12930 \\
\hline 500.992 & 0.100 & 504.128 & 0.11524 & 500.890 & 26.297 & 503.734 & 0.12926 \\
\hline 500.985 & 5.344 & 502.608 & 0.11877 & 500.890 & 26.281 & 503.730 & 0.12936 \\
\hline 500.990 & 5.317 & 502.939 & 0.11855 & 500.939 & 35.510 & 502.432 & 0.13351 \\
\hline
\end{tabular}

anitial cell temperature. 
Table A2, continued.

\begin{tabular}{cccc}
\hline $\boldsymbol{T}_{\mathbf{i}}^{\mathbf{a}} / \mathbf{K}$ & $\boldsymbol{p} / \mathbf{M P a}$ & $\boldsymbol{T} / \mathbf{K}$ & $\boldsymbol{\lambda} / \mathbf{W} \cdot \mathbf{m}^{-1} \mathbf{K}^{-1}$ \\
\hline \hline 500.943 & 35.486 & 502.435 & 0.13357 \\
500.949 & 35.445 & 502.717 & 0.13348 \\
500.955 & 35.430 & 502.735 & 0.13363 \\
500.956 & 35.380 & 503.062 & 0.13328 \\
500.956 & 35.361 & 503.049 & 0.13355 \\
500.972 & 35.359 & 503.402 & 0.13320 \\
500.975 & 35.292 & 503.390 & 0.13315 \\
500.973 & 35.242 & 503.779 & 0.13319 \\
500.981 & 35.202 & 503.784 & 0.13310 \\
500.930 & 45.302 & 502.360 & 0.13811 \\
500.924 & 45.254 & 502.639 & 0.13754 \\
500.917 & 45.215 & 502.634 & 0.13749 \\
500.909 & 45.143 & 502.910 & 0.13775 \\
500.901 & 45.103 & 502.909 & 0.13837 \\
500.895 & 45.075 & 503.212 & 0.13738 \\
500.878 & 45.029 & 503.199 & 0.13741 \\
500.872 & 44.966 & 503.553 & 0.13707 \\
500.855 & 44.925 & 503.547 & 0.13712 \\
500.769 & 56.262 & 502.177 & 0.14186 \\
500.763 & 56.188 & 502.160 & 0.14169 \\
500.764 & 56.129 & 502.441 & 0.14182 \\
500.761 & 56.029 & 502.426 & 0.14173 \\
500.763 & 55.948 & 502.704 & 0.14160 \\
500.763 & 55.888 & 502.716 & 0.14187 \\
500.763 & 55.821 & 503.044 & 0.14159 \\
500.765 & 55.775 & 503.039 & 0.14155 \\
500.757 & 55.681 & 503.369 & 0.14139 \\
500.754 & 55.624 & 503.359 & 0.14130 \\
\hline
\end{tabular}

anitial cell temperature. 
Table A3: Thermal Conductivity Data for POE9 at Temperatures from $300 \mathrm{~K}$ to $500 \mathrm{~K}$.

\begin{tabular}{|c|c|c|c|c|c|c|c|}
\hline$T_{\mathrm{i}^{\mathrm{a}}}^{\mathrm{a}} / \mathrm{K}$ & $p / \mathrm{MPa}$ & $T / \mathbf{K}$ & $\lambda / \mathbf{W} \cdot \mathbf{m}^{-1} \mathbf{K}^{-1}$ & $T_{\mathbf{i}^{\mathrm{a}}}^{\mathrm{a}} / \mathbf{K}$ & $p / \mathbf{M P a}$ & $T / K$ & $\lambda / \mathbf{W} \cdot \mathbf{m}^{-1} \mathbf{K}^{-1}$ \\
\hline 299.474 & 0.117 & 301.250 & 0.15582 & 299.473 & 0.209 & 302.439 & 0.15566 \\
\hline 299.471 & 0.133 & 301.241 & 0.15579 & 299.473 & 0.230 & 302.921 & 0.15554 \\
\hline 299.472 & 0.150 & 301.610 & 0.15578 & 299.469 & 0.229 & 302.913 & 0.15553 \\
\hline 299.469 & 0.091 & 301.594 & 0.15576 & 299.465 & 0.213 & 301.220 & 0.15605 \\
\hline 299.469 & 0.125 & 302.014 & 0.15568 & 299.466 & 0.214 & 301.224 & 0.15612 \\
\hline 299.463 & 0.108 & 302.000 & 0.15574 & 299.464 & 0.214 & 301.589 & 0.15603 \\
\hline 299.467 & 0.122 & 302.409 & 0.15563 & 299.466 & 0.214 & 301.590 & 0.15598 \\
\hline 299.465 & 0.075 & 302.401 & 0.15561 & 299.465 & 0.214 & 302.000 & 0.15590 \\
\hline 299.462 & 0.124 & 302.885 & 0.15601 & 299.467 & 0.214 & 301.996 & 0.15590 \\
\hline 299.467 & 0.115 & 302.908 & 0.15549 & 299.465 & 0.214 & 302.440 & 0.15586 \\
\hline 299.465 & 0.060 & 301.218 & 0.15593 & 299.462 & 0.215 & 302.437 & 0.15580 \\
\hline 299.467 & 0.059 & 301.234 & 0.15578 & 299.465 & 0.215 & 302.918 & 0.15572 \\
\hline 299.469 & 0.108 & 301.602 & 0.15575 & 299.461 & 0.215 & 302.910 & 0.15571 \\
\hline 299.468 & 0.066 & 301.590 & 0.15641 & 299.487 & 6.767 & 301.225 & 0.15786 \\
\hline 299.472 & 0.089 & 302.009 & 0.15570 & 299.488 & 6.785 & 301.226 & 0.15785 \\
\hline 299.471 & 0.076 & 302.008 & 0.15570 & 299.487 & 6.786 & 301.587 & 0.15777 \\
\hline 299.472 & 0.071 & 302.452 & 0.15557 & 299.483 & 6.778 & 301.987 & 0.15774 \\
\hline 299.471 & 0.096 & 302.450 & 0.15561 & 299.485 & 6.754 & 301.987 & 0.15776 \\
\hline 299.473 & 0.091 & 302.934 & 0.15540 & 299.487 & 6.756 & 302.427 & 0.15765 \\
\hline 299.473 & 0.099 & 302.929 & 0.15546 & 299.487 & 6.754 & 302.428 & 0.15769 \\
\hline 299.473 & 0.257 & 301.232 & 0.15593 & 299.488 & 6.742 & 302.898 & 0.15749 \\
\hline 299.473 & 0.240 & 301.227 & 0.15593 & 299.485 & 6.743 & 302.898 & 0.15755 \\
\hline 299.472 & 0.256 & 301.600 & 0.15580 & 299.480 & 13.701 & 301.181 & 0.15989 \\
\hline 299.474 & 0.251 & 301.594 & 0.15584 & 299.485 & 13.686 & 301.543 & 0.15975 \\
\hline 299.475 & 0.256 & 302.007 & 0.15573 & 299.484 & 13.686 & 301.540 & 0.15978 \\
\hline 299.475 & 0.245 & 302.004 & 0.15574 & 299.480 & 13.687 & 301.935 & 0.15970 \\
\hline 299.474 & 0.211 & 302.440 & 0.15559 & 299.479 & 13.686 & 302.365 & 0.15962 \\
\hline 299.480 & 0.231 & 302.451 & 0.15568 & 299.484 & 13.678 & 302.371 & 0.15962 \\
\hline 299.477 & 0.222 & 302.924 & 0.15555 & 299.482 & 13.659 & 302.832 & 0.15953 \\
\hline 299.480 & 0.257 & 302.936 & 0.15556 & 299.480 & 13.625 & 302.833 & 0.15950 \\
\hline 299.475 & 0.207 & 301.233 & 0.15604 & 299.471 & 21.550 & 301.140 & 0.16205 \\
\hline 299.475 & 0.209 & 301.233 & 0.15587 & 299.470 & 21.578 & 301.146 & 0.16198 \\
\hline 299.478 & 0.225 & 301.604 & 0.15585 & 299.471 & 21.565 & 301.491 & 0.16193 \\
\hline 299.476 & 0.210 & 301.598 & 0.15580 & 299.472 & 21.568 & 301.496 & 0.16196 \\
\hline 299.474 & 0.214 & 302.003 & 0.15560 & 299.470 & 21.555 & 301.884 & 0.16190 \\
\hline 299.475 & 0.209 & 302.003 & 0.15582 & 299.467 & 21.558 & 301.882 & 0.16190 \\
\hline 299.472 & 0.220 & 302.443 & 0.15565 & 299.468 & 21.574 & 302.309 & 0.16225 \\
\hline
\end{tabular}

${ }^{\text {a} I n i t i a l ~ c e l l ~ t e m p e r a t u r e . ~}$ 
Table A3, continued.

\begin{tabular}{|c|c|c|c|c|c|c|c|}
\hline$T_{\mathrm{i}^{\mathrm{a}}}^{\mathrm{a}} / \mathrm{K}$ & $p / \mathbf{M P a}$ & $T / K$ & $\lambda / \mathbf{W} \cdot \mathbf{m}^{-1} \mathbf{K}^{-1}$ & $T_{\mathbf{i}^{\mathrm{a}}} / \mathbf{K}$ & $p / \mathbf{M P a}$ & $T / K$ & $\lambda / \mathbf{W} \cdot \mathbf{m}^{-1} \mathbf{K}^{-1}$ \\
\hline 299.469 & 21.577 & 302.311 & 0.16179 & 299.459 & 58.425 & 302.196 & 0.17099 \\
\hline 299.469 & 21.576 & 302.770 & 0.16168 & 299.455 & 58.418 & 302.186 & 0.17104 \\
\hline 299.466 & 21.573 & 302.768 & 0.16167 & 299.455 & 58.428 & 302.630 & 0.17089 \\
\hline 299.463 & 30.163 & 301.116 & 0.16429 & 299.455 & 68.112 & 301.374 & 0.17339 \\
\hline 299.460 & 30.160 & 301.115 & 0.16433 & 299.456 & 68.053 & 301.750 & 0.17342 \\
\hline 299.465 & 30.158 & 301.469 & 0.16422 & 299.458 & 68.034 & 301.750 & 0.17324 \\
\hline 299.460 & 30.158 & 301.851 & 0.16413 & 299.456 & 67.990 & 302.148 & 0.17316 \\
\hline 299.462 & 30.142 & 301.857 & 0.16414 & 299.458 & 67.925 & 302.146 & 0.17308 \\
\hline 299.461 & 30.139 & 302.277 & 0.16411 & 299.454 & 67.787 & 301.045 & 0.17357 \\
\hline 299.460 & 30.124 & 302.276 & 0.16398 & 299.453 & 67.730 & 301.380 & 0.17336 \\
\hline 299.458 & 30.144 & 302.733 & 0.16396 & 299.454 & 67.699 & 301.382 & 0.17330 \\
\hline 299.460 & 30.142 & 302.737 & 0.16397 & 299.457 & 67.624 & 301.742 & 0.17338 \\
\hline 299.458 & 38.919 & 301.113 & 0.16654 & 299.455 & 67.420 & 301.730 & 0.17323 \\
\hline 299.457 & 38.917 & 301.112 & 0.16647 & 299.456 & 67.354 & 302.134 & 0.17311 \\
\hline 299.455 & 38.909 & 301.451 & 0.16646 & 299.452 & 67.353 & 302.153 & 0.17310 \\
\hline 299.451 & 38.903 & 301.446 & 0.16643 & 299.452 & 67.355 & 302.591 & 0.17303 \\
\hline 299.453 & 38.906 & 301.834 & 0.16640 & 299.454 & 67.355 & 302.592 & 0.17301 \\
\hline 299.453 & 38.875 & 301.826 & 0.16643 & 331.658 & 0.196 & 333.293 & 0.15113 \\
\hline 299.454 & 38.868 & 302.245 & 0.16627 & 331.654 & 0.197 & 333.627 & 0.15084 \\
\hline 299.452 & 38.874 & 302.247 & 0.16631 & 331.655 & 0.198 & 333.633 & 0.15097 \\
\hline 299.452 & 38.863 & 302.694 & 0.16617 & 331.653 & 0.198 & 334.007 & 0.15081 \\
\hline 299.450 & 38.852 & 302.687 & 0.16624 & 331.657 & 0.199 & 334.013 & 0.15071 \\
\hline 299.453 & 48.362 & 301.073 & 0.16916 & 331.656 & 0.199 & 334.422 & 0.15071 \\
\hline 299.457 & 48.352 & 301.078 & 0.16881 & 331.655 & 0.199 & 334.415 & 0.15072 \\
\hline 299.455 & 48.365 & 301.418 & 0.16886 & 331.659 & 0.199 & 334.866 & 0.15066 \\
\hline 299.451 & 48.377 & 301.790 & 0.16857 & 331.659 & 0.200 & 334.868 & 0.15062 \\
\hline 299.456 & 48.369 & 301.795 & 0.16869 & 331.675 & 6.392 & 333.276 & 0.15252 \\
\hline 299.456 & 48.345 & 302.205 & 0.16861 & 331.675 & 6.392 & 333.282 & 0.15294 \\
\hline 299.453 & 48.369 & 302.202 & 0.16863 & 331.674 & 6.367 & 333.610 & 0.15290 \\
\hline 299.452 & 48.367 & 302.647 & 0.16859 & 331.675 & 6.372 & 333.993 & 0.15276 \\
\hline 299.450 & 48.349 & 302.641 & 0.16851 & 331.677 & 6.364 & 333.983 & 0.15280 \\
\hline 299.453 & 58.442 & 301.057 & 0.17130 & 331.674 & 6.349 & 334.395 & 0.15271 \\
\hline 299.456 & 58.341 & 301.049 & 0.17128 & 331.677 & 6.343 & 334.839 & 0.15223 \\
\hline 299.460 & 58.409 & 301.421 & 0.17111 & 331.674 & 6.372 & 334.837 & 0.15219 \\
\hline 299.453 & 58.435 & 301.411 & 0.17115 & 331.680 & 13.478 & 333.265 & 0.15519 \\
\hline 299.453 & 58.425 & 301.783 & 0.17110 & 331.675 & 13.473 & 333.261 & 0.15511 \\
\hline 299.454 & 58.428 & 301.785 & 0.17115 & 331.675 & 13.449 & 333.582 & 0.15535 \\
\hline
\end{tabular}

anitial cell temperature. 
Table A3, continued.

\begin{tabular}{|c|c|c|c|c|c|c|c|}
\hline$T_{\mathrm{i}^{\mathrm{a}}}^{\mathrm{a}} / \mathrm{K}$ & $p / \mathrm{MPa}$ & $T / K$ & $\lambda / \mathbf{W} \cdot \mathbf{m}^{-1} \mathbf{K}^{-1}$ & $T_{\mathrm{i}^{\mathrm{a}}}^{\mathrm{a}} / \mathbf{K}$ & $p / \mathbf{M P a}$ & $T / K$ & $\lambda / \mathbf{W} \cdot \mathbf{m}^{-1} \mathbf{K}^{-1}$ \\
\hline 331.678 & 13.462 & 333.594 & 0.15513 & 331.699 & 47.926 & 333.204 & 0.16494 \\
\hline 331.674 & 13.472 & 333.959 & 0.15500 & 331.699 & 47.905 & 333.512 & 0.16487 \\
\hline 331.677 & 13.414 & 333.943 & 0.15500 & 331.700 & 47.918 & 333.518 & 0.16495 \\
\hline 331.674 & 13.446 & 334.360 & 0.15490 & 331.698 & 47.904 & 333.866 & 0.16507 \\
\hline 331.677 & 13.458 & 334.377 & 0.15493 & 331.700 & 47.896 & 333.861 & 0.16481 \\
\hline 331.682 & 13.473 & 334.814 & 0.15485 & 331.699 & 47.910 & 334.245 & 0.16474 \\
\hline 331.677 & 13.471 & 334.809 & 0.15486 & 331.703 & 47.918 & 334.252 & 0.16477 \\
\hline 331.676 & 21.127 & 333.235 & 0.15745 & 331.701 & 47.887 & 334.651 & 0.16465 \\
\hline 331.679 & 21.107 & 333.225 & 0.15763 & 331.699 & 47.899 & 334.651 & 0.16457 \\
\hline 331.677 & 21.112 & 333.564 & 0.15735 & 331.704 & 57.689 & 333.157 & 0.16745 \\
\hline 331.677 & 21.103 & 333.573 & 0.15737 & 331.702 & 57.666 & 333.146 & 0.16767 \\
\hline 331.676 & 21.102 & 333.940 & 0.15734 & 331.702 & 57.673 & 333.503 & 0.16741 \\
\hline 331.681 & 21.094 & 333.941 & 0.15735 & 331.700 & 57.681 & 333.501 & 0.16735 \\
\hline 331.678 & 21.102 & 334.332 & 0.15725 & 331.706 & 57.676 & 333.853 & 0.16734 \\
\hline 331.674 & 21.106 & 334.336 & 0.15729 & 331.704 & 57.672 & 333.847 & 0.16745 \\
\hline 331.682 & 21.108 & 334.769 & 0.15716 & 331.705 & 57.671 & 334.225 & 0.16731 \\
\hline 331.678 & 21.108 & 334.761 & 0.15718 & 331.704 & 57.662 & 334.222 & 0.16732 \\
\hline 331.679 & 29.091 & 333.220 & 0.15987 & 331.703 & 57.629 & 334.619 & 0.16724 \\
\hline 331.680 & 29.104 & 333.225 & 0.15981 & 331.702 & 57.661 & 334.619 & 0.16722 \\
\hline 331.682 & 29.118 & 333.551 & 0.15978 & 331.702 & 69.046 & 333.130 & 0.17026 \\
\hline 331.683 & 29.112 & 333.548 & 0.15974 & 331.698 & 69.058 & 333.129 & 0.17032 \\
\hline 331.683 & 29.077 & 333.902 & 0.15958 & 331.698 & 69.048 & 333.432 & 0.17033 \\
\hline 331.683 & 29.108 & 334.298 & 0.15961 & 331.696 & 69.030 & 333.430 & 0.17022 \\
\hline 331.681 & 29.087 & 334.294 & 0.15963 & 331.700 & 69.036 & 333.771 & 0.17032 \\
\hline 331.683 & 29.075 & 334.717 & 0.15954 & 331.698 & 69.032 & 333.776 & 0.17024 \\
\hline 331.679 & 29.087 & 334.715 & 0.15932 & 331.695 & 69.011 & 334.136 & 0.17012 \\
\hline 331.695 & 38.437 & 333.210 & 0.16240 & 331.699 & 69.003 & 334.137 & 0.17018 \\
\hline 331.690 & 38.429 & 333.207 & 0.16258 & 331.695 & 69.000 & 334.532 & 0.17011 \\
\hline 331.688 & 38.432 & 333.517 & 0.16231 & 331.696 & 68.974 & 334.530 & 0.17006 \\
\hline 331.688 & 38.432 & 333.520 & 0.16230 & 364.342 & 0.232 & 365.896 & 0.14573 \\
\hline 331.691 & 38.418 & 333.875 & 0.16230 & 364.341 & 0.234 & 366.217 & 0.14576 \\
\hline 331.685 & 38.433 & 333.872 & 0.16228 & 364.338 & 0.235 & 366.205 & 0.14566 \\
\hline 331.694 & 38.447 & 334.268 & 0.16271 & 364.337 & 0.236 & 366.566 & 0.14554 \\
\hline 331.689 & 38.423 & 334.257 & 0.16223 & 364.337 & 0.237 & 366.557 & 0.14561 \\
\hline 331.696 & 38.441 & 334.686 & 0.16211 & 364.336 & 0.238 & 366.951 & 0.14567 \\
\hline 331.693 & 38.443 & 334.697 & 0.16214 & 364.342 & 0.239 & 366.960 & 0.14564 \\
\hline 331.705 & 47.934 & 333.206 & 0.16494 & 364.339 & 0.240 & 367.353 & 0.14551 \\
\hline
\end{tabular}

anitial cell temperature. 
Table A3, continued.

\begin{tabular}{|c|c|c|c|c|c|c|c|}
\hline$T_{\mathrm{i}^{\mathrm{a}}}^{\mathrm{a}} / \mathrm{K}$ & $p / \mathrm{MPa}$ & $T / K$ & $\lambda / \mathbf{W} \cdot \mathbf{m}^{-1} \mathbf{K}^{-1}$ & $T_{\mathrm{i}^{\mathrm{a}}}^{\mathrm{a}} / \mathbf{K}$ & $p / \mathbf{M P a}$ & $T / K$ & $\lambda / \mathbf{W} \cdot \mathbf{m}^{-1} \mathbf{K}^{-1}$ \\
\hline 364.337 & 0.241 & 367.375 & 0.14548 & 364.336 & 28.938 & 367.136 & 0.15522 \\
\hline 364.336 & 6.947 & 365.841 & 0.14815 & 364.330 & 37.437 & 365.715 & 0.15818 \\
\hline 364.334 & 6.946 & 366.139 & 0.14811 & 364.330 & 37.441 & 365.739 & 0.15827 \\
\hline 364.336 & 6.933 & 366.147 & 0.14806 & 364.335 & 37.441 & 366.065 & 0.15817 \\
\hline 364.331 & 6.945 & 366.493 & 0.14800 & 364.327 & 37.380 & 366.047 & 0.15809 \\
\hline 364.333 & 6.965 & 366.500 & 0.14807 & 364.335 & 37.405 & 366.393 & 0.15797 \\
\hline 364.333 & 6.948 & 366.871 & 0.14805 & 364.331 & 37.423 & 366.388 & 0.15807 \\
\hline 364.334 & 6.953 & 366.868 & 0.14796 & 364.331 & 37.368 & 366.743 & 0.15801 \\
\hline 364.334 & 6.963 & 367.292 & 0.14789 & 364.333 & 37.370 & 366.748 & 0.15797 \\
\hline 364.329 & 6.936 & 367.272 & 0.14793 & 364.327 & 37.363 & 367.130 & 0.15794 \\
\hline 364.337 & 13.582 & 365.803 & 0.15036 & 364.331 & 37.318 & 367.130 & 0.15796 \\
\hline 364.334 & 13.583 & 365.804 & 0.15054 & 364.331 & 47.040 & 365.696 & 0.16093 \\
\hline 364.334 & 13.595 & 366.109 & 0.15054 & 364.335 & 47.049 & 365.709 & 0.16102 \\
\hline 364.336 & 13.601 & 366.463 & 0.15035 & 364.333 & 47.011 & 365.990 & 0.16119 \\
\hline 364.335 & 13.600 & 366.455 & 0.15050 & 364.331 & 46.979 & 365.992 & 0.16103 \\
\hline 364.327 & 13.614 & 366.837 & 0.15032 & 364.335 & 46.995 & 366.324 & 0.16096 \\
\hline 364.335 & 13.583 & 366.825 & 0.15025 & 364.334 & 46.989 & 366.317 & 0.16091 \\
\hline 364.330 & 13.586 & 367.235 & 0.15026 & 364.336 & 46.953 & 366.672 & 0.16087 \\
\hline 364.336 & 20.492 & 365.776 & 0.15296 & 364.336 & 46.972 & 366.680 & 0.16091 \\
\hline 364.335 & 20.474 & 365.779 & 0.15275 & 364.339 & 46.949 & 367.059 & 0.16078 \\
\hline 364.337 & 20.500 & 366.090 & 0.15273 & 364.339 & 46.930 & 367.056 & 0.16076 \\
\hline 364.339 & 20.491 & 366.122 & 0.15277 & 364.342 & 57.979 & 365.726 & 0.16416 \\
\hline 364.337 & 20.490 & 366.453 & 0.15271 & 364.346 & 57.981 & 365.724 & 0.16446 \\
\hline 364.333 & 20.504 & 366.456 & 0.15265 & 364.345 & 57.969 & 366.019 & 0.16411 \\
\hline 364.336 & 20.501 & 366.829 & 0.15264 & 364.347 & 57.985 & 366.013 & 0.16418 \\
\hline 364.336 & 20.487 & 366.819 & 0.15268 & 364.339 & 57.989 & 366.327 & 0.16403 \\
\hline 364.340 & 20.503 & 367.238 & 0.15256 & 364.344 & 57.975 & 366.337 & 0.16414 \\
\hline 364.335 & 20.499 & 367.221 & 0.15265 & 364.342 & 57.960 & 366.677 & 0.16395 \\
\hline 364.337 & 28.939 & 365.737 & 0.15557 & 364.346 & 57.926 & 366.686 & 0.16396 \\
\hline 364.345 & 28.965 & 365.766 & 0.15547 & 364.346 & 57.943 & 367.058 & 0.16385 \\
\hline 364.337 & 28.956 & 366.046 & 0.15562 & 364.342 & 57.920 & 367.052 & 0.16389 \\
\hline 364.341 & 28.971 & 366.062 & 0.15555 & 364.353 & 68.874 & 365.676 & 0.16692 \\
\hline 364.335 & 28.956 & 366.378 & 0.15546 & 364.353 & 68.857 & 365.676 & 0.16727 \\
\hline 364.339 & 28.969 & 366.389 & 0.15547 & 364.354 & 68.864 & 365.965 & 0.16705 \\
\hline 364.337 & 28.927 & 366.739 & 0.15547 & 364.357 & 68.864 & 365.970 & 0.16713 \\
\hline 364.339 & 28.967 & 366.760 & 0.15542 & 364.355 & 68.852 & 366.281 & 0.16690 \\
\hline 364.331 & 28.975 & 367.145 & 0.15531 & 364.354 & 68.861 & 366.278 & 0.16706 \\
\hline
\end{tabular}

anitial cell temperature. 
Table A3, continued.

\begin{tabular}{|c|c|c|c|c|c|c|c|}
\hline$T_{\mathrm{i}^{\mathrm{a}}}^{\mathrm{a}} / \mathrm{K}$ & $p / \mathrm{MPa}$ & $T / K$ & $\lambda / \mathbf{W} \cdot \mathbf{m}^{-1} \mathbf{K}^{-1}$ & $T_{\mathrm{i}^{\mathrm{a}}}^{\mathrm{a}} / \mathbf{K}$ & $p / \mathbf{M P a}$ & $T / K$ & $\lambda / \mathbf{W} \cdot \mathbf{m}^{-1} \mathbf{K}^{-1}$ \\
\hline 364.353 & 68.832 & 366.617 & 0.16699 & 397.592 & 20.257 & 399.231 & 0.14799 \\
\hline 364.351 & 68.860 & 366.624 & 0.16689 & 397.590 & 20.258 & 399.548 & 0.14809 \\
\hline 364.353 & 68.858 & 366.990 & 0.16700 & 397.591 & 20.253 & 399.552 & 0.14789 \\
\hline 364.355 & 68.855 & 367.000 & 0.16683 & 397.591 & 20.246 & 399.886 & 0.14794 \\
\hline 397.579 & 0.186 & 399.052 & 0.14030 & 397.591 & 20.238 & 399.906 & 0.14793 \\
\hline 397.580 & 0.187 & 399.058 & 0.14016 & 397.592 & 20.237 & 400.268 & 0.14788 \\
\hline 397.582 & 0.188 & 399.370 & 0.14009 & 397.589 & 20.246 & 400.281 & 0.14796 \\
\hline 397.584 & 0.188 & 399.365 & 0.14007 & 397.595 & 28.293 & 398.963 & 0.15090 \\
\hline 397.581 & 0.189 & 399.696 & 0.14007 & 397.596 & 28.315 & 398.967 & 0.15110 \\
\hline 397.585 & 0.190 & 399.705 & 0.14005 & 397.599 & 28.326 & 399.261 & 0.15058 \\
\hline 397.586 & 0.190 & 400.078 & 0.13984 & 397.591 & 28.299 & 399.554 & 0.15084 \\
\hline 397.585 & 0.191 & 400.064 & 0.14007 & 397.598 & 28.310 & 399.571 & 0.15097 \\
\hline 397.586 & 0.191 & 400.478 & 0.13988 & 397.596 & 28.298 & 399.913 & 0.15081 \\
\hline 397.588 & 0.192 & 400.481 & 0.13989 & 397.597 & 28.295 & 400.274 & 0.15078 \\
\hline 397.576 & 6.488 & 399.018 & 0.14302 & 397.595 & 28.304 & 400.290 & 0.15071 \\
\hline 397.583 & 6.489 & 399.021 & 0.14280 & 397.606 & 36.962 & 398.913 & 0.15408 \\
\hline 397.582 & 6.491 & 399.327 & 0.14272 & 397.596 & 36.971 & 398.931 & 0.15395 \\
\hline 397.584 & 6.488 & 399.327 & 0.14267 & 397.594 & 36.969 & 399.196 & 0.15391 \\
\hline 397.581 & 6.493 & 399.657 & 0.14266 & 397.597 & 36.960 & 399.509 & 0.15373 \\
\hline 397.582 & 6.494 & 399.655 & 0.14269 & 397.596 & 36.971 & 399.522 & 0.15375 \\
\hline 397.581 & 6.485 & 400.018 & 0.14255 & 397.596 & 36.964 & 399.843 & 0.15383 \\
\hline 397.575 & 6.480 & 400.019 & 0.14255 & 397.593 & 36.967 & 399.855 & 0.15370 \\
\hline 397.576 & 6.490 & 400.400 & 0.14252 & 397.597 & 36.944 & 400.209 & 0.15326 \\
\hline 397.577 & 6.484 & 400.398 & 0.14249 & 397.591 & 36.970 & 400.208 & 0.15375 \\
\hline 397.583 & 12.905 & 398.967 & 0.14529 & 397.589 & 46.634 & 398.898 & 0.15742 \\
\hline 397.580 & 12.926 & 398.951 & 0.14522 & 397.594 & 46.639 & 398.904 & 0.15722 \\
\hline 397.583 & 12.911 & 399.256 & 0.14529 & 397.594 & 46.644 & 399.179 & 0.15706 \\
\hline 397.580 & 12.920 & 399.242 & 0.14529 & 397.591 & 46.628 & 399.171 & 0.15697 \\
\hline 397.582 & 12.917 & 399.577 & 0.14517 & 397.596 & 46.645 & 399.483 & 0.15699 \\
\hline 397.580 & 12.908 & 399.560 & 0.14517 & 397.585 & 46.631 & 399.472 & 0.15699 \\
\hline 397.586 & 12.917 & 399.939 & 0.14469 & 397.592 & 46.641 & 399.809 & 0.15697 \\
\hline 397.579 & 12.909 & 399.917 & 0.14517 & 397.592 & 46.635 & 399.817 & 0.15691 \\
\hline 397.578 & 12.918 & 400.308 & 0.14514 & 397.587 & 46.624 & 400.157 & 0.15678 \\
\hline 397.583 & 12.909 & 400.316 & 0.14500 & 397.592 & 46.635 & 400.166 & 0.15693 \\
\hline 397.588 & 20.259 & 398.934 & 0.14830 & 397.588 & 57.231 & 398.866 & 0.16037 \\
\hline 397.591 & 20.243 & 398.931 & 0.14793 & 397.594 & 57.231 & 398.867 & 0.16065 \\
\hline 397.586 & 20.240 & 399.207 & 0.14833 & 397.591 & 57.230 & 399.140 & 0.16041 \\
\hline
\end{tabular}

anitial cell temperature. 
Table A3, continued.

\begin{tabular}{|c|c|c|c|c|c|c|c|}
\hline$T_{\mathrm{i}^{\mathrm{a}}}^{\mathrm{a}} / \mathrm{K}$ & $p / \mathrm{MPa}$ & $T / K$ & $\lambda / \mathbf{W} \cdot \mathbf{m}^{-1} \mathbf{K}^{-1}$ & $T_{\mathrm{i}^{\mathrm{a}}}^{\mathrm{a}} / \mathbf{K}$ & $p / \mathbf{M P a}$ & $T / K$ & $\lambda / \mathbf{W} \cdot \mathbf{m}^{-1} \mathbf{K}^{-1}$ \\
\hline 397.588 & 57.218 & 399.128 & 0.16047 & 430.690 & 12.329 & 432.637 & 0.13985 \\
\hline 397.590 & 57.218 & 399.433 & 0.16004 & 430.690 & 12.321 & 432.629 & 0.13988 \\
\hline 397.593 & 57.199 & 399.423 & 0.16024 & 430.696 & 12.322 & 432.984 & 0.13973 \\
\hline 397.587 & 57.197 & 399.757 & 0.16013 & 430.694 & 12.330 & 433.344 & 0.13983 \\
\hline 397.587 & 57.197 & 399.751 & 0.16018 & 430.695 & 12.340 & 433.348 & 0.13979 \\
\hline 397.584 & 57.205 & 400.110 & 0.16015 & 430.694 & 19.391 & 431.982 & 0.14300 \\
\hline 397.584 & 57.199 & 400.109 & 0.16000 & 430.693 & 19.407 & 431.985 & 0.14310 \\
\hline 397.597 & 68.575 & 398.827 & 0.16367 & 430.693 & 19.402 & 432.248 & 0.14286 \\
\hline 397.593 & 68.546 & 399.118 & 0.16379 & 430.697 & 19.391 & 432.254 & 0.14287 \\
\hline 397.592 & 68.503 & 399.422 & 0.16359 & 430.692 & 19.402 & 432.557 & 0.14295 \\
\hline 397.589 & 68.480 & 399.414 & 0.16360 & 430.696 & 19.413 & 432.578 & 0.14299 \\
\hline 397.594 & 68.465 & 399.748 & 0.16357 & 430.697 & 19.412 & 432.907 & 0.14271 \\
\hline 397.588 & 68.421 & 399.733 & 0.16353 & 430.696 & 19.401 & 432.894 & 0.14289 \\
\hline 397.593 & 68.425 & 400.080 & 0.16394 & 430.692 & 19.421 & 433.273 & 0.14273 \\
\hline 397.597 & 68.418 & 400.098 & 0.16356 & 430.693 & 19.426 & 433.262 & 0.14271 \\
\hline 430.704 & 0.227 & 432.115 & 0.13477 & 430.697 & 27.649 & 431.993 & 0.14634 \\
\hline 430.706 & 0.228 & 432.409 & 0.13455 & 430.699 & 27.666 & 432.265 & 0.14598 \\
\hline 430.708 & 0.229 & 432.401 & 0.13442 & 430.697 & 27.654 & 432.264 & 0.14625 \\
\hline 430.708 & 0.230 & 432.724 & 0.13434 & 430.698 & 27.666 & 432.578 & 0.14615 \\
\hline 430.702 & 0.230 & 432.733 & 0.13443 & 430.701 & 27.663 & 432.576 & 0.14615 \\
\hline 430.708 & 0.231 & 433.084 & 0.13433 & 430.703 & 27.670 & 432.922 & 0.14595 \\
\hline 430.707 & 0.231 & 433.087 & 0.13430 & 430.700 & 27.651 & 433.243 & 0.14605 \\
\hline 430.708 & 0.232 & 433.464 & 0.13419 & 430.696 & 27.661 & 433.244 & 0.14609 \\
\hline 430.707 & 0.233 & 433.472 & 0.13422 & 430.703 & 36.356 & 431.949 & 0.14974 \\
\hline 430.694 & 5.816 & 432.054 & 0.13712 & 430.700 & 36.361 & 431.944 & 0.14951 \\
\hline 430.699 & 5.822 & 432.052 & 0.13698 & 430.701 & 36.360 & 432.234 & 0.14954 \\
\hline 430.703 & 5.799 & 432.342 & 0.13693 & 430.695 & 36.352 & 432.207 & 0.14968 \\
\hline 430.702 & 5.826 & 432.349 & 0.13715 & 430.699 & 36.357 & 432.527 & 0.14949 \\
\hline 430.700 & 5.818 & 432.666 & 0.13704 & 430.692 & 36.346 & 432.840 & 0.14931 \\
\hline 430.698 & 5.815 & 432.648 & 0.13699 & 430.691 & 36.360 & 432.841 & 0.14922 \\
\hline 430.701 & 5.819 & 433.009 & 0.13691 & 430.690 & 36.338 & 433.170 & 0.14930 \\
\hline 430.705 & 5.799 & 433.010 & 0.13696 & 430.684 & 46.136 & 431.957 & 0.15299 \\
\hline 430.699 & 5.812 & 433.379 & 0.13702 & 430.683 & 46.140 & 431.937 & 0.15323 \\
\hline 430.700 & 5.820 & 433.404 & 0.13676 & 430.684 & 46.121 & 432.190 & 0.15307 \\
\hline 430.693 & 12.302 & 432.019 & 0.13994 & 430.681 & 46.110 & 432.193 & 0.15310 \\
\hline 430.692 & 12.318 & 432.332 & 0.13996 & 430.682 & 46.109 & 432.485 & 0.15293 \\
\hline 430.687 & 12.342 & 432.317 & 0.14009 & 430.683 & 46.127 & 432.484 & 0.15281 \\
\hline
\end{tabular}

anitial cell temperature. 
Table A3, continued.

\begin{tabular}{|c|c|c|c|c|c|c|c|}
\hline$T_{\mathrm{i}^{\mathrm{a}}}^{\mathrm{a}} / \mathrm{K}$ & $p / \mathbf{M P a}$ & $T / K$ & $\lambda / \mathbf{W} \cdot \mathbf{m}^{-1} \mathbf{K}^{-1}$ & $T_{\mathrm{i}^{\mathrm{a}}}^{\mathrm{a}} / \mathbf{K}$ & $p / \mathbf{M P a}$ & $T / K$ & $\lambda / \mathbf{W} \cdot \mathbf{m}^{-1} \mathbf{K}^{-1}$ \\
\hline 430.687 & 46.107 & 432.796 & 0.15281 & 464.026 & 5.926 & 466.268 & 0.13143 \\
\hline 430.683 & 46.118 & 432.799 & 0.15292 & 464.020 & 5.940 & 466.635 & 0.13150 \\
\hline 430.681 & 46.120 & 433.138 & 0.15280 & 464.016 & 5.914 & 466.609 & 0.13144 \\
\hline 430.680 & 46.107 & 433.137 & 0.15280 & 464.021 & 11.994 & 465.310 & 0.13460 \\
\hline 430.686 & 56.970 & 431.886 & 0.15692 & 464.025 & 12.008 & 465.325 & 0.13434 \\
\hline 430.691 & 56.950 & 432.150 & 0.15668 & 464.020 & 12.017 & 465.589 & 0.13464 \\
\hline 430.695 & 56.962 & 432.152 & 0.15663 & 464.026 & 12.006 & 465.590 & 0.13452 \\
\hline 430.698 & 56.976 & 432.441 & 0.15658 & 464.021 & 12.018 & 465.895 & 0.13459 \\
\hline 430.693 & 56.980 & 432.749 & 0.15652 & 464.033 & 12.016 & 465.920 & 0.13467 \\
\hline 430.692 & 56.972 & 432.735 & 0.15653 & 464.029 & 12.017 & 466.232 & 0.13442 \\
\hline 430.690 & 56.955 & 433.060 & 0.15647 & 464.027 & 12.014 & 466.225 & 0.13449 \\
\hline 430.692 & 56.963 & 433.077 & 0.15670 & 464.026 & 12.019 & 466.587 & 0.13434 \\
\hline 430.688 & 68.654 & 431.842 & 0.16051 & 464.026 & 12.007 & 466.585 & 0.13437 \\
\hline 430.695 & 68.655 & 431.862 & 0.16062 & 464.051 & 18.864 & 465.313 & 0.13807 \\
\hline 430.686 & 68.657 & 432.096 & 0.16008 & 464.047 & 18.869 & 465.313 & 0.13788 \\
\hline 430.691 & 68.636 & 432.379 & 0.16030 & 464.046 & 18.855 & 465.560 & 0.13784 \\
\hline 430.690 & 68.647 & 432.372 & 0.16048 & 464.047 & 18.865 & 465.576 & 0.13801 \\
\hline 430.688 & 68.654 & 432.688 & 0.16024 & 464.049 & 18.869 & 465.873 & 0.13783 \\
\hline 430.683 & 68.647 & 432.992 & 0.16032 & 464.054 & 18.864 & 465.876 & 0.13752 \\
\hline 430.688 & 68.650 & 432.999 & 0.16031 & 464.052 & 18.870 & 466.207 & 0.13765 \\
\hline 464.034 & 0.238 & 465.413 & 0.12891 & 464.058 & 18.868 & 466.197 & 0.13777 \\
\hline 464.031 & 0.239 & 465.406 & 0.12877 & 464.054 & 18.858 & 466.549 & 0.13760 \\
\hline 464.034 & 0.240 & 465.689 & 0.12819 & 464.056 & 18.859 & 466.548 & 0.13763 \\
\hline 464.031 & 0.241 & 465.694 & 0.12875 & 464.058 & 26.904 & 465.295 & 0.14151 \\
\hline 464.038 & 0.240 & 466.011 & 0.12857 & 464.060 & 26.917 & 465.296 & 0.14137 \\
\hline 464.031 & 0.241 & 466.003 & 0.12846 & 464.057 & 26.915 & 465.538 & 0.14140 \\
\hline 464.033 & 0.243 & 466.354 & 0.12845 & 464.061 & 26.915 & 465.556 & 0.14138 \\
\hline 464.032 & 0.243 & 466.357 & 0.12836 & 464.059 & 26.912 & 465.844 & 0.14125 \\
\hline 464.031 & 0.243 & 466.726 & 0.12838 & 464.061 & 26.915 & 465.847 & 0.14123 \\
\hline 464.031 & 0.244 & 466.716 & 0.12819 & 464.062 & 26.906 & 466.159 & 0.14110 \\
\hline 464.025 & 5.933 & 465.329 & 0.13177 & 464.060 & 26.900 & 466.151 & 0.14131 \\
\hline 464.023 & 5.940 & 465.337 & 0.13162 & 464.058 & 26.876 & 466.483 & 0.14116 \\
\hline 464.019 & 5.938 & 465.618 & 0.13164 & 464.063 & 26.904 & 466.501 & 0.14097 \\
\hline 464.021 & 5.935 & 465.600 & 0.13212 & 464.052 & 35.833 & 465.269 & 0.14513 \\
\hline 464.020 & 5.935 & 465.935 & 0.13146 & 464.047 & 35.811 & 465.258 & 0.14505 \\
\hline 464.022 & 5.932 & 465.924 & 0.13149 & 464.046 & 35.817 & 465.513 & 0.14493 \\
\hline 464.026 & 5.926 & 466.253 & 0.13128 & 464.047 & 35.797 & 465.502 & 0.14510 \\
\hline
\end{tabular}

anitial cell temperature. 
Table A3, continued.

\begin{tabular}{|c|c|c|c|c|c|c|c|}
\hline$T_{\mathrm{i}^{\mathrm{a}}}^{\mathrm{a}} / \mathrm{K}$ & $p / \mathbf{M P a}$ & $T / K$ & $\lambda / \mathbf{W} \cdot \mathbf{m}^{-1} \mathbf{K}^{-1}$ & $T_{\mathbf{i}^{\mathrm{a}}} / \mathbf{K}$ & $p / \mathbf{M P a}$ & $T / K$ & $\lambda / \mathbf{W} \cdot \mathbf{m}^{-1} \mathbf{K}^{-1}$ \\
\hline 464.052 & 35.823 & 465.793 & 0.14481 & 497.514 & 0.246 & 499.438 & 0.12287 \\
\hline 464.048 & 35.825 & 466.117 & 0.14492 & 497.526 & 0.246 & 499.778 & 0.12236 \\
\hline 464.049 & 35.804 & 466.092 & 0.14504 & 497.514 & 0.247 & 499.784 & 0.12245 \\
\hline 464.047 & 35.812 & 466.431 & 0.14490 & 497.516 & 0.248 & 500.144 & 0.12228 \\
\hline 464.058 & 45.750 & 465.233 & 0.14893 & 497.519 & 0.248 & 500.143 & 0.12242 \\
\hline 464.053 & 45.733 & 465.220 & 0.14881 & 497.514 & 5.561 & 498.828 & 0.12584 \\
\hline 464.053 & 45.723 & 465.457 & 0.14871 & 497.514 & 5.569 & 498.846 & 0.12571 \\
\hline 464.052 & 45.690 & 465.451 & 0.14900 & 497.509 & 5.568 & 499.099 & 0.12586 \\
\hline 464.059 & 45.707 & 465.766 & 0.14888 & 497.510 & 5.564 & 499.102 & 0.12595 \\
\hline 464.048 & 45.744 & 465.746 & 0.14888 & 497.512 & 5.555 & 499.393 & 0.12564 \\
\hline 464.054 & 45.747 & 466.076 & 0.14869 & 497.517 & 5.567 & 499.415 & 0.12571 \\
\hline 464.045 & 45.749 & 466.058 & 0.14870 & 497.515 & 5.570 & 499.734 & 0.12565 \\
\hline 464.055 & 45.751 & 466.403 & 0.14842 & 497.518 & 5.569 & 500.096 & 0.12568 \\
\hline 464.053 & 45.747 & 466.384 & 0.14877 & 497.517 & 5.568 & 500.104 & 0.12548 \\
\hline 464.063 & 56.877 & 465.200 & 0.15283 & 497.508 & 11.426 & 498.766 & 0.12894 \\
\hline 464.073 & 56.860 & 465.464 & 0.15281 & 497.506 & 11.432 & 499.013 & 0.12921 \\
\hline 464.072 & 56.878 & 465.473 & 0.15282 & 497.499 & 11.431 & 499.009 & 0.12923 \\
\hline 464.067 & 56.842 & 465.718 & 0.15305 & 497.501 & 11.435 & 499.323 & 0.12907 \\
\hline 464.060 & 56.801 & 465.707 & 0.15302 & 497.500 & 11.420 & 499.615 & 0.12888 \\
\hline 464.073 & 56.784 & 466.021 & 0.15282 & 497.507 & 11.434 & 499.646 & 0.12889 \\
\hline 464.072 & 56.729 & 466.334 & 0.15258 & 497.501 & 11.436 & 499.974 & 0.12887 \\
\hline 464.067 & 56.730 & 466.331 & 0.15264 & 497.507 & 11.437 & 499.985 & 0.12886 \\
\hline 464.021 & 68.900 & 465.143 & 0.15699 & 497.510 & 18.395 & 498.723 & 0.13282 \\
\hline 464.019 & 68.902 & 465.148 & 0.15706 & 497.507 & 18.386 & 498.730 & 0.13273 \\
\hline 464.022 & 68.909 & 465.379 & 0.15713 & 497.510 & 18.385 & 498.976 & 0.13262 \\
\hline 464.022 & 68.872 & 465.381 & 0.15715 & 497.501 & 18.394 & 499.261 & 0.13267 \\
\hline 464.030 & 68.898 & 465.655 & 0.15724 & 497.500 & 18.393 & 499.262 & 0.13273 \\
\hline 464.028 & 68.883 & 465.664 & 0.15696 & 497.503 & 18.388 & 499.567 & 0.13250 \\
\hline 464.028 & 68.889 & 465.941 & 0.15708 & 497.504 & 18.383 & 499.904 & 0.13254 \\
\hline 464.033 & 68.867 & 465.947 & 0.15696 & 497.501 & 18.380 & 499.910 & 0.13259 \\
\hline 464.031 & 68.887 & 466.263 & 0.15722 & 497.524 & 26.223 & 498.725 & 0.13642 \\
\hline 464.039 & 68.883 & 466.272 & 0.15671 & 497.521 & 26.222 & 498.959 & 0.13670 \\
\hline 497.520 & 0.244 & 498.879 & 0.12278 & 497.530 & 26.222 & 498.977 & 0.13622 \\
\hline 497.519 & 0.244 & 498.852 & 0.12284 & 497.526 & 26.205 & 499.235 & 0.13629 \\
\hline 497.515 & 0.245 & 499.153 & 0.12303 & 497.528 & 26.225 & 499.550 & 0.13634 \\
\hline 497.526 & 0.246 & 499.148 & 0.12267 & 497.527 & 26.218 & 499.552 & 0.13641 \\
\hline 497.520 & 0.246 & 499.455 & 0.12260 & 497.532 & 26.205 & 499.894 & 0.13609 \\
\hline
\end{tabular}

anitial cell temperature. 
Table A3, continued.

\begin{tabular}{|c|c|c|c|c|c|c|c|}
\hline$T_{\mathrm{i}^{\mathrm{a}}}^{\mathrm{a}} / \mathrm{K}$ & $p / \mathrm{MPa}$ & $T / K$ & $\lambda / \mathbf{W} \cdot \mathbf{m}^{-1} \mathbf{K}^{-1}$ & $T_{\mathbf{i}^{\mathrm{a}}} / \mathbf{K}$ & $p / \mathbf{M P a}$ & $T / K$ & $\lambda / \mathbf{W} \cdot \mathbf{m}^{-1} \mathbf{K}^{-1}$ \\
\hline 497.533 & 26.201 & 499.889 & 0.13613 & 497.635 & 68.573 & 499.449 & 0.15320 \\
\hline 497.535 & 35.370 & 498.682 & 0.14063 & 497.630 & 68.568 & 499.739 & 0.15326 \\
\hline 497.531 & 35.367 & 498.680 & 0.14060 & 497.636 & 68.555 & 499.754 & 0.15315 \\
\hline 497.531 & 35.355 & 498.923 & 0.14048 & 497.767 & 0.390 & 499.140 & 0.12289 \\
\hline 497.533 & 35.349 & 499.205 & 0.14042 & 497.762 & 0.389 & 499.130 & 0.12278 \\
\hline 497.534 & 35.354 & 499.211 & 0.14033 & 497.758 & 0.389 & 499.396 & 0.12258 \\
\hline 497.534 & 35.354 & 499.512 & 0.14047 & 497.763 & 0.388 & 499.398 & 0.12271 \\
\hline 497.532 & 35.339 & 499.492 & 0.14052 & 497.754 & 0.388 & 499.702 & 0.12290 \\
\hline 497.541 & 35.355 & 499.833 & 0.14027 & 497.756 & 0.390 & 499.709 & 0.12272 \\
\hline 497.540 & 35.345 & 499.828 & 0.14032 & 497.754 & 0.390 & 500.034 & 0.12271 \\
\hline 497.556 & 45.239 & 498.665 & 0.14485 & 497.747 & 0.390 & 500.035 & 0.12253 \\
\hline 497.551 & 45.258 & 498.697 & 0.14467 & 497.750 & 0.391 & 500.392 & 0.12238 \\
\hline 497.548 & 45.231 & 498.900 & 0.14461 & 497.753 & 0.392 & 500.394 & 0.12242 \\
\hline 497.556 & 45.257 & 498.929 & 0.14482 & & & & \\
\hline 497.558 & 45.258 & 499.196 & 0.14454 & & & & \\
\hline 497.551 & 45.267 & 499.191 & 0.14475 & & & & \\
\hline 497.551 & 45.265 & 499.473 & 0.14445 & & & & \\
\hline 497.556 & 45.240 & 499.469 & 0.14453 & & & & \\
\hline 497.553 & 45.241 & 499.797 & 0.14453 & & & & \\
\hline 497.559 & 45.222 & 499.786 & 0.14438 & & & & \\
\hline 497.596 & 56.482 & 498.710 & 0.14967 & & & & \\
\hline 497.592 & 56.472 & 498.709 & 0.14915 & & & & \\
\hline 497.596 & 56.472 & 498.949 & 0.14927 & & & & \\
\hline 497.597 & 56.471 & 498.956 & 0.14910 & & & & \\
\hline 497.600 & 56.460 & 499.215 & 0.14877 & & & & \\
\hline 497.601 & 56.466 & 499.217 & 0.14895 & & & & \\
\hline 497.610 & 56.455 & 499.490 & 0.14888 & & & & \\
\hline 497.611 & 56.455 & 499.527 & 0.14891 & & & & \\
\hline 497.613 & 56.424 & 499.804 & 0.14904 & & & & \\
\hline 497.614 & 56.432 & 499.827 & 0.14894 & & & & \\
\hline 497.636 & 68.666 & 498.711 & 0.15327 & & & & \\
\hline 497.631 & 68.646 & 498.696 & 0.15341 & & & & \\
\hline 497.631 & 68.634 & 498.926 & 0.15329 & & & & \\
\hline 497.632 & 68.628 & 498.927 & 0.15376 & & & & \\
\hline 497.633 & 68.623 & 499.174 & 0.15383 & & & & \\
\hline 497.631 & 68.606 & 499.184 & 0.15357 & & & & \\
\hline 497.632 & 68.594 & 499.442 & 0.15348 & & & & \\
\hline
\end{tabular}

anitial cell temperature. 
Table A4: Thermal Conductivity Data for MIL-PRF-23699 Lubricant at Temperatures from $300 \mathrm{~K}$ to $500 \mathrm{~K}$.

\begin{tabular}{|c|c|c|c|c|c|c|c|}
\hline$T_{\mathbf{i}^{\mathrm{a}}}^{\mathrm{a}} / \mathbf{K}$ & $p / \mathrm{MPa}$ & $T / K$ & $\lambda / \mathbf{W} \cdot \mathbf{m}^{-1} \mathbf{K}^{-1}$ & $T_{\mathbf{i}^{\mathrm{a}}}^{\mathrm{a}} / \mathbf{K}$ & $p / \mathbf{M P a}$ & $T / K$ & $\lambda / \mathbf{W} \cdot \mathbf{m}^{-1} \mathbf{K}^{-1}$ \\
\hline 299.137 & 0.421 & 300.666 & 0.14515 & 299.705 & 14.275 & 302.821 & 0.14856 \\
\hline 299.158 & 0.427 & 300.702 & 0.14520 & 299.710 & 14.267 & 302.822 & 0.14855 \\
\hline 299.175 & 0.436 & 301.038 & 0.14504 & 299.743 & 21.603 & 301.208 & 0.15069 \\
\hline 299.208 & 0.447 & 301.486 & 0.14508 & 299.743 & 21.569 & 301.204 & 0.15070 \\
\hline 299.223 & 0.449 & 301.508 & 0.14506 & 299.747 & 21.579 & 301.554 & 0.15063 \\
\hline 299.242 & 0.451 & 301.968 & 0.14500 & 299.748 & 21.570 & 301.554 & 0.15066 \\
\hline 299.259 & 0.468 & 301.996 & 0.14505 & 299.746 & 21.580 & 301.933 & 0.15059 \\
\hline 299.273 & 0.481 & 302.492 & 0.14485 & 299.748 & 21.544 & 301.934 & 0.15063 \\
\hline 299.288 & 0.492 & 302.515 & 0.14490 & 299.748 & 21.561 & 302.351 & 0.15050 \\
\hline 299.475 & 0.348 & 300.997 & 0.14505 & 299.745 & 21.580 & 302.349 & 0.15056 \\
\hline 299.479 & 0.356 & 301.005 & 0.14506 & 299.747 & 21.587 & 302.808 & 0.15045 \\
\hline 299.483 & 0.367 & 301.371 & 0.14523 & 299.743 & 21.598 & 302.804 & 0.15046 \\
\hline 299.483 & 0.374 & 301.371 & 0.14504 & 299.765 & 30.059 & 301.200 & 0.15286 \\
\hline 299.493 & 0.381 & 301.773 & 0.14498 & 299.768 & 30.071 & 301.546 & 0.15274 \\
\hline 299.493 & 0.387 & 301.774 & 0.14495 & 299.768 & 30.088 & 301.548 & 0.15280 \\
\hline 299.496 & 0.393 & 302.221 & 0.14491 & 299.773 & 30.080 & 301.926 & 0.15270 \\
\hline 299.500 & 0.402 & 302.631 & 0.14483 & 299.769 & 30.073 & 301.919 & 0.15271 \\
\hline 299.507 & 0.409 & 302.638 & 0.14487 & 299.768 & 30.064 & 302.329 & 0.15287 \\
\hline 299.610 & 7.170 & 301.112 & 0.14692 & 299.772 & 30.068 & 302.336 & 0.15272 \\
\hline 299.613 & 7.182 & 301.111 & 0.14687 & 299.776 & 30.074 & 302.789 & 0.15261 \\
\hline 299.627 & 7.205 & 301.486 & 0.14687 & 299.777 & 30.071 & 302.788 & 0.15256 \\
\hline 299.620 & 7.235 & 301.485 & 0.14682 & 299.785 & 39.133 & 301.188 & 0.15505 \\
\hline 299.622 & 7.231 & 301.869 & 0.14667 & 299.786 & 39.128 & 301.194 & 0.15500 \\
\hline 299.625 & 7.235 & 301.873 & 0.14678 & 299.785 & 39.114 & 301.525 & 0.15490 \\
\hline 299.631 & 7.253 & 302.312 & 0.14673 & 299.782 & 39.116 & 301.534 & 0.15499 \\
\hline 299.632 & 7.263 & 302.294 & 0.14669 & 299.785 & 39.129 & 301.906 & 0.15481 \\
\hline 299.629 & 7.257 & 302.760 & 0.14669 & 299.783 & 39.120 & 301.902 & 0.15490 \\
\hline 299.640 & 7.250 & 302.773 & 0.14674 & 299.778 & 39.126 & 302.305 & 0.15485 \\
\hline 299.677 & 14.120 & 301.155 & 0.14882 & 299.777 & 39.124 & 302.306 & 0.15487 \\
\hline 299.680 & 14.127 & 301.159 & 0.14877 & 299.773 & 39.103 & 302.740 & 0.15479 \\
\hline 299.686 & 14.137 & 301.518 & 0.14879 & 299.785 & 48.381 & 301.183 & 0.15725 \\
\hline 299.689 & 14.147 & 301.524 & 0.14874 & 299.786 & 48.368 & 301.182 & 0.15717 \\
\hline 299.692 & 14.152 & 301.911 & 0.14867 & 299.784 & 48.384 & 301.516 & 0.15709 \\
\hline 299.697 & 14.190 & 301.920 & 0.14874 & 299.786 & 48.367 & 301.519 & 0.15709 \\
\hline 299.700 & 14.226 & 302.349 & 0.14860 & 299.789 & 48.333 & 301.877 & 0.15713 \\
\hline 299.700 & 14.239 & 302.351 & 0.14859 & 299.789 & 48.321 & 302.281 & 0.15705 \\
\hline
\end{tabular}

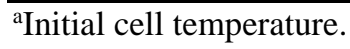


Table A4, continued.

\begin{tabular}{|c|c|c|c|c|c|c|c|}
\hline$T_{\mathbf{i}^{\mathrm{a}}} / \mathbf{K}$ & $p / \mathbf{M P a}$ & $T / K$ & $\lambda / \mathbf{W} \cdot \mathbf{m}^{-1} \mathbf{K}^{-1}$ & $T_{\mathbf{i}^{\mathrm{a}}} / \mathbf{K}$ & $p / \mathbf{M P a}$ & $T / K$ & $\lambda / \mathbf{W} \cdot \mathbf{m}^{-1} \mathbf{K}^{-1}$ \\
\hline 299.792 & 48.314 & 302.286 & 0.15705 & 332.028 & 6.690 & 334.104 & 0.14237 \\
\hline 299.792 & 48.290 & 302.719 & 0.15695 & 332.028 & 6.692 & 334.504 & 0.14237 \\
\hline 299.792 & 48.293 & 302.721 & 0.15692 & 332.030 & 6.696 & 334.938 & 0.14226 \\
\hline 299.791 & 58.660 & 301.151 & 0.15968 & 332.029 & 6.693 & 334.935 & 0.14228 \\
\hline 299.788 & 58.674 & 301.495 & 0.15954 & 332.033 & 13.746 & 333.401 & 0.14454 \\
\hline 299.790 & 58.680 & 301.499 & 0.15950 & 332.035 & 13.734 & 334.080 & 0.14469 \\
\hline 299.788 & 58.776 & 301.864 & 0.15945 & 332.033 & 13.736 & 334.470 & 0.14440 \\
\hline 299.792 & 58.812 & 301.868 & 0.15980 & 332.035 & 13.736 & 334.471 & 0.14433 \\
\hline 299.786 & 58.824 & 302.259 & 0.15942 & 332.029 & 21.153 & 333.351 & 0.14669 \\
\hline 299.785 & 58.825 & 302.258 & 0.15944 & 332.029 & 21.146 & 333.353 & 0.14687 \\
\hline 299.785 & 58.840 & 302.691 & 0.15936 & 332.030 & 21.173 & 333.679 & 0.14676 \\
\hline 299.789 & 58.852 & 302.694 & 0.15935 & 332.031 & 21.191 & 333.679 & 0.14681 \\
\hline 299.814 & 68.848 & 301.159 & 0.16172 & 332.033 & 21.193 & 334.028 & 0.14674 \\
\hline 299.824 & 68.847 & 301.171 & 0.16185 & 332.030 & 21.196 & 334.025 & 0.14666 \\
\hline 299.816 & 68.857 & 301.488 & 0.16170 & 332.028 & 21.195 & 334.406 & 0.14664 \\
\hline 299.826 & 68.857 & 301.516 & 0.16177 & 332.028 & 21.199 & 334.407 & 0.14661 \\
\hline 299.822 & 68.861 & 301.867 & 0.16168 & 332.027 & 21.209 & 334.828 & 0.14657 \\
\hline 299.824 & 68.860 & 301.868 & 0.16169 & 332.024 & 21.208 & 334.823 & 0.14656 \\
\hline 299.819 & 68.869 & 302.259 & 0.16163 & 332.033 & 29.304 & 333.366 & 0.14912 \\
\hline 299.827 & 68.871 & 302.267 & 0.16163 & 332.028 & 29.303 & 333.362 & 0.14895 \\
\hline 299.830 & 68.879 & 302.697 & 0.16158 & 332.024 & 29.305 & 333.676 & 0.14905 \\
\hline 299.828 & 68.879 & 302.694 & 0.16159 & 332.028 & 29.304 & 333.678 & 0.14900 \\
\hline 332.027 & 0.614 & 333.431 & 0.14074 & 332.026 & 29.297 & 334.020 & 0.14932 \\
\hline 332.025 & 0.629 & 333.761 & 0.14054 & 332.029 & 29.291 & 334.021 & 0.14897 \\
\hline 332.027 & 0.643 & 333.767 & 0.14064 & 332.025 & 29.272 & 334.399 & 0.14889 \\
\hline 332.030 & 0.655 & 334.131 & 0.14053 & 332.025 & 29.280 & 334.396 & 0.14889 \\
\hline 332.025 & 0.675 & 334.526 & 0.14053 & 332.027 & 29.275 & 334.813 & 0.14886 \\
\hline 332.027 & 0.684 & 334.528 & 0.14058 & 332.023 & 29.272 & 334.810 & 0.14890 \\
\hline 332.027 & 0.690 & 334.963 & 0.14046 & 332.020 & 38.358 & 333.337 & 0.15169 \\
\hline 332.026 & 0.696 & 334.966 & 0.14046 & 332.029 & 38.353 & 333.346 & 0.15148 \\
\hline 332.027 & 6.684 & 333.418 & 0.14247 & 332.026 & 38.353 & 333.655 & 0.15148 \\
\hline 332.030 & 6.687 & 333.752 & 0.14249 & 332.025 & 38.353 & 333.652 & 0.15140 \\
\hline 332.025 & 6.681 & 334.101 & 0.14268 & 332.022 & 38.346 & 333.990 & 0.15134 \\
\hline 332.031 & 6.689 & 334.506 & 0.14235 & 332.024 & 38.347 & 334.368 & 0.15134 \\
\hline 332.031 & 6.687 & 334.505 & 0.14224 & 332.028 & 38.343 & 334.372 & 0.15128 \\
\hline 332.030 & 6.690 & 334.933 & 0.14215 & 332.021 & 38.333 & 334.772 & 0.15124 \\
\hline
\end{tabular}

anitial cell temperature. 
Table A4, continued.

\begin{tabular}{|c|c|c|c|c|c|c|c|}
\hline$T_{\mathrm{i}^{\mathrm{a}}}^{\mathrm{a}} / \mathrm{K}$ & $p / \mathbf{M P a}$ & $T / K$ & $\lambda / \mathbf{W} \cdot \mathbf{m}^{-1} \mathbf{K}^{-1}$ & $T_{\mathbf{i}^{\mathrm{a}}} / \mathbf{K}$ & $p / \mathbf{M P a}$ & $T / K$ & $\lambda / \mathbf{W} \cdot \mathbf{m}^{-1} \mathbf{K}^{-1}$ \\
\hline 332.025 & 38.335 & 334.775 & 0.15162 & 331.987 & 13.458 & 334.845 & 0.14431 \\
\hline 332.036 & 48.175 & 333.323 & 0.15406 & 331.984 & 13.456 & 334.841 & 0.14429 \\
\hline 332.038 & 48.177 & 333.326 & 0.15404 & 364.655 & 0.724 & 365.981 & 0.13597 \\
\hline 332.034 & 48.166 & 333.628 & 0.15398 & 364.655 & 0.746 & 365.986 & 0.13613 \\
\hline 332.027 & 48.160 & 333.621 & 0.15399 & 364.654 & 0.764 & 366.291 & 0.13583 \\
\hline 332.029 & 48.158 & 333.954 & 0.15382 & 364.655 & 0.778 & 366.294 & 0.13596 \\
\hline 332.034 & 48.153 & 333.961 & 0.15392 & 364.650 & 0.790 & 366.629 & 0.13575 \\
\hline 332.035 & 48.149 & 334.331 & 0.15387 & 364.652 & 0.799 & 366.631 & 0.13580 \\
\hline 332.030 & 48.144 & 334.324 & 0.15380 & 364.648 & 0.806 & 367.005 & 0.13577 \\
\hline 332.036 & 48.134 & 334.737 & 0.15381 & 364.650 & 0.812 & 367.009 & 0.13587 \\
\hline 332.031 & 48.132 & 334.730 & 0.15378 & 364.651 & 0.818 & 367.417 & 0.13568 \\
\hline 332.035 & 58.292 & 333.303 & 0.15647 & 364.656 & 0.822 & 367.424 & 0.13567 \\
\hline 332.034 & 58.289 & 333.308 & 0.15649 & 364.654 & 6.595 & 365.972 & 0.13788 \\
\hline 332.036 & 58.281 & 333.610 & 0.15637 & 364.654 & 6.595 & 365.969 & 0.13792 \\
\hline 332.034 & 58.276 & 333.606 & 0.15644 & 364.656 & 6.578 & 366.264 & 0.13806 \\
\hline 332.033 & 58.267 & 333.933 & 0.15634 & 364.655 & 6.569 & 366.275 & 0.13772 \\
\hline 332.032 & 58.264 & 333.932 & 0.15643 & 364.654 & 6.593 & 366.612 & 0.13783 \\
\hline 332.031 & 58.257 & 334.298 & 0.15643 & 364.658 & 6.580 & 366.614 & 0.13776 \\
\hline 332.031 & 58.251 & 334.293 & 0.15631 & 364.657 & 6.582 & 366.985 & 0.13769 \\
\hline 332.032 & 58.245 & 334.690 & 0.15628 & 364.657 & 6.587 & 366.983 & 0.13777 \\
\hline 332.031 & 58.233 & 334.686 & 0.15629 & 364.659 & 6.587 & 367.386 & 0.13773 \\
\hline 332.028 & 68.853 & 333.261 & 0.15904 & 364.659 & 6.587 & 367.390 & 0.13766 \\
\hline 332.027 & 68.843 & 333.259 & 0.15897 & 364.660 & 13.411 & 365.937 & 0.14030 \\
\hline 332.030 & 68.835 & 333.560 & 0.15900 & 364.663 & 13.416 & 365.928 & 0.14031 \\
\hline 332.027 & 68.825 & 333.556 & 0.15893 & 364.665 & 13.425 & 366.240 & 0.14031 \\
\hline 332.024 & 68.821 & 333.879 & 0.15899 & 364.659 & 13.421 & 366.229 & 0.14044 \\
\hline 332.031 & 68.809 & 333.883 & 0.15896 & 364.660 & 13.424 & 366.569 & 0.14022 \\
\hline 332.023 & 68.801 & 334.238 & 0.15885 & 364.663 & 13.426 & 366.566 & 0.14006 \\
\hline 332.024 & 68.794 & 334.237 & 0.15883 & 364.662 & 13.424 & 366.935 & 0.14013 \\
\hline 332.023 & 68.791 & 334.647 & 0.15884 & 364.664 & 13.428 & 366.937 & 0.14008 \\
\hline 332.029 & 68.780 & 334.650 & 0.15889 & 364.663 & 13.427 & 367.333 & 0.14005 \\
\hline 331.982 & 13.452 & 333.409 & 0.14439 & 364.666 & 13.430 & 367.342 & 0.14001 \\
\hline 331.985 & 13.455 & 333.669 & 0.14450 & 364.677 & 20.571 & 365.941 & 0.14233 \\
\hline 331.985 & 13.458 & 333.670 & 0.14449 & 364.673 & 20.572 & 365.932 & 0.14264 \\
\hline 331.982 & 13.453 & 334.018 & 0.14445 & 364.675 & 20.570 & 366.237 & 0.14257 \\
\hline 331.984 & 13.453 & 334.021 & 0.14445 & 364.678 & 20.574 & 366.246 & 0.14288 \\
\hline 331.983 & 13.454 & 334.414 & 0.14444 & 364.679 & 20.570 & 366.565 & 0.14251 \\
\hline
\end{tabular}

anitial cell temperature. 
Table A4, continued.

\begin{tabular}{|c|c|c|c|c|c|c|c|}
\hline$T_{\mathrm{i}^{\mathrm{a}}}^{\mathrm{a}} / \mathrm{K}$ & $p / \mathrm{MPa}$ & $T / K$ & $\lambda / \mathbf{W} \cdot \mathbf{m}^{-1} \mathbf{K}^{-1}$ & $T_{\mathrm{i}^{\mathrm{a}}}^{\mathrm{a}} / \mathbf{K}$ & $p / \mathbf{M P a}$ & $T / K$ & $\lambda / \mathbf{W} \cdot \mathbf{m}^{-1} \mathbf{K}^{-1}$ \\
\hline 364.679 & 20.569 & 366.565 & 0.14245 & 364.722 & 57.651 & 366.467 & 0.15320 \\
\hline 364.682 & 20.570 & 366.932 & 0.14242 & 364.722 & 57.639 & 366.485 & 0.15320 \\
\hline 364.676 & 20.567 & 366.923 & 0.14241 & 364.722 & 57.626 & 366.825 & 0.15314 \\
\hline 364.678 & 20.569 & 367.324 & 0.14255 & 364.725 & 57.617 & 366.831 & 0.15322 \\
\hline 364.675 & 20.564 & 367.320 & 0.14231 & 364.721 & 57.589 & 367.191 & 0.15311 \\
\hline 364.696 & 28.628 & 365.942 & 0.14494 & 364.726 & 68.494 & 365.896 & 0.15592 \\
\hline 364.694 & 28.623 & 365.937 & 0.14496 & 364.721 & 68.477 & 365.891 & 0.15587 \\
\hline 364.695 & 28.619 & 366.236 & 0.14504 & 364.721 & 68.465 & 366.167 & 0.15602 \\
\hline 364.698 & 28.621 & 366.236 & 0.14493 & 364.726 & 68.428 & 366.474 & 0.15606 \\
\hline 364.698 & 28.616 & 366.561 & 0.14483 & 364.727 & 68.415 & 366.467 & 0.15596 \\
\hline 364.699 & 28.617 & 366.559 & 0.14490 & 364.724 & 68.400 & 366.800 & 0.15591 \\
\hline 364.698 & 28.623 & 366.918 & 0.14487 & 364.725 & 68.378 & 366.800 & 0.15594 \\
\hline 364.699 & 28.622 & 366.917 & 0.14488 & 364.724 & 68.349 & 367.165 & 0.15597 \\
\hline 364.697 & 28.617 & 367.296 & 0.14486 & 397.909 & 0.902 & 399.167 & 0.13062 \\
\hline 364.700 & 28.618 & 367.306 & 0.14486 & 397.904 & 0.923 & 399.163 & 0.13091 \\
\hline 364.701 & 37.824 & 365.934 & 0.14797 & 397.911 & 0.938 & 399.464 & 0.13074 \\
\hline 364.706 & 37.816 & 365.934 & 0.14803 & 397.907 & 0.951 & 399.469 & 0.13085 \\
\hline 364.704 & 37.809 & 366.227 & 0.14763 & 397.912 & 0.962 & 399.788 & 0.13062 \\
\hline 364.705 & 37.799 & 366.223 & 0.14775 & 397.912 & 0.972 & 399.793 & 0.13068 \\
\hline 364.709 & 37.796 & 366.547 & 0.14768 & 397.912 & 0.980 & 400.146 & 0.13064 \\
\hline 364.704 & 37.799 & 366.533 & 0.14778 & 397.902 & 0.987 & 400.143 & 0.13055 \\
\hline 364.702 & 37.782 & 366.890 & 0.14760 & 397.907 & 0.993 & 400.536 & 0.13052 \\
\hline 364.699 & 37.772 & 366.891 & 0.14766 & 397.906 & 0.998 & 400.530 & 0.13046 \\
\hline 364.700 & 37.775 & 367.273 & 0.14758 & 397.914 & 6.159 & 399.153 & 0.13280 \\
\hline 364.700 & 37.772 & 367.270 & 0.14768 & 397.913 & 6.158 & 399.149 & 0.13290 \\
\hline 364.710 & 47.275 & 365.903 & 0.15064 & 397.915 & 6.160 & 399.448 & 0.13278 \\
\hline 364.710 & 47.266 & 365.901 & 0.15040 & 397.917 & 6.159 & 399.446 & 0.13278 \\
\hline 364.709 & 47.264 & 366.190 & 0.15035 & 397.918 & 6.162 & 399.772 & 0.13260 \\
\hline 364.706 & 47.253 & 366.185 & 0.15038 & 397.914 & 6.159 & 399.759 & 0.13271 \\
\hline 364.709 & 47.247 & 366.502 & 0.15048 & 397.918 & 6.161 & 400.128 & 0.13248 \\
\hline 364.711 & 47.233 & 366.844 & 0.15032 & 397.921 & 6.162 & 400.516 & 0.13248 \\
\hline 364.713 & 47.226 & 366.849 & 0.15030 & 397.921 & 6.164 & 400.519 & 0.13254 \\
\hline 364.713 & 47.216 & 367.222 & 0.15028 & 397.930 & 12.782 & 399.136 & 0.13529 \\
\hline 364.715 & 47.205 & 367.226 & 0.15030 & 397.930 & 12.782 & 399.142 & 0.13528 \\
\hline 364.724 & 57.694 & 365.887 & 0.15330 & 397.932 & 12.781 & 399.425 & 0.13547 \\
\hline 364.722 & 57.684 & 365.885 & 0.15321 & 397.931 & 12.781 & 399.428 & 0.13532 \\
\hline 364.719 & 57.672 & 366.163 & 0.15329 & 397.936 & 12.784 & 399.744 & 0.13519 \\
\hline
\end{tabular}

anitial cell temperature. 
Table A4, continued.

\begin{tabular}{|c|c|c|c|c|c|c|c|}
\hline$T_{\mathrm{i}^{\mathrm{a}}}^{\mathrm{a}} / \mathrm{K}$ & $p / \mathrm{MPa}$ & $T / K$ & $\lambda / \mathbf{W} \cdot \mathbf{m}^{-1} \mathbf{K}^{-1}$ & $T_{\mathrm{i}^{\mathrm{a}}}^{\mathrm{a}} / \mathbf{K}$ & $p / \mathbf{M P a}$ & $T / K$ & $\lambda / \mathbf{W} \cdot \mathbf{m}^{-1} \mathbf{K}^{-1}$ \\
\hline 397.939 & 12.787 & 399.757 & 0.13510 & 397.957 & 46.827 & 399.300 & 0.14676 \\
\hline 397.938 & 12.788 & 400.104 & 0.13515 & 397.952 & 46.817 & 399.590 & 0.14674 \\
\hline 397.936 & 12.785 & 400.096 & 0.13510 & 397.954 & 46.809 & 399.602 & 0.14676 \\
\hline 397.939 & 12.786 & 400.471 & 0.13509 & 397.953 & 46.798 & 399.947 & 0.14662 \\
\hline 397.941 & 12.784 & 400.477 & 0.13505 & 397.954 & 46.788 & 399.955 & 0.14665 \\
\hline 397.953 & 20.069 & 399.145 & 0.13792 & 397.953 & 46.775 & 400.298 & 0.14660 \\
\hline 397.944 & 20.053 & 399.415 & 0.13813 & 397.956 & 46.767 & 400.308 & 0.14662 \\
\hline 397.950 & 20.051 & 399.421 & 0.13803 & 397.985 & 57.640 & 399.097 & 0.15001 \\
\hline 397.950 & 20.054 & 399.724 & 0.13790 & 397.986 & 57.620 & 399.363 & 0.14977 \\
\hline 397.951 & 20.053 & 399.732 & 0.13785 & 397.982 & 57.605 & 399.345 & 0.15014 \\
\hline 397.953 & 20.057 & 400.077 & 0.13832 & 397.988 & 57.589 & 399.648 & 0.14968 \\
\hline 397.951 & 20.055 & 400.072 & 0.13783 & 397.987 & 57.575 & 399.642 & 0.14946 \\
\hline 397.953 & 20.056 & 400.448 & 0.13782 & 397.993 & 57.557 & 399.972 & 0.14970 \\
\hline 397.946 & 20.049 & 400.439 & 0.13777 & 397.983 & 57.538 & 399.955 & 0.14993 \\
\hline 397.955 & 28.030 & 399.109 & 0.14105 & 397.989 & 57.524 & 400.310 & 0.14973 \\
\hline 397.956 & 28.027 & 399.114 & 0.14090 & 397.990 & 57.506 & 400.310 & 0.14976 \\
\hline 397.952 & 28.021 & 399.386 & 0.14094 & 398.006 & 68.707 & 399.104 & 0.15279 \\
\hline 397.953 & 28.018 & 399.382 & 0.14062 & 398.005 & 68.682 & 399.090 & 0.15338 \\
\hline 397.953 & 28.012 & 399.689 & 0.14071 & 398.005 & 68.658 & 399.356 & 0.15314 \\
\hline 397.957 & 28.007 & 399.691 & 0.14079 & 398.006 & 68.635 & 399.351 & 0.15300 \\
\hline 397.957 & 28.002 & 400.028 & 0.14059 & 398.005 & 68.609 & 399.626 & 0.15297 \\
\hline 397.954 & 27.999 & 400.028 & 0.14050 & 398.010 & 68.562 & 399.947 & 0.15283 \\
\hline 397.951 & 27.995 & 400.386 & 0.14068 & 398.005 & 68.533 & 399.943 & 0.15288 \\
\hline 397.954 & 27.990 & 400.394 & 0.14044 & 398.007 & 68.513 & 400.282 & 0.15286 \\
\hline 397.954 & 37.264 & 399.082 & 0.14370 & 398.002 & 68.493 & 400.282 & 0.15293 \\
\hline 397.954 & 37.255 & 399.097 & 0.14372 & 430.815 & 0.712 & 432.322 & 0.12521 \\
\hline 397.953 & 37.235 & 399.374 & 0.14367 & 430.811 & 0.722 & 432.631 & 0.12503 \\
\hline 397.957 & 37.227 & 399.381 & 0.14390 & 430.813 & 0.730 & 432.622 & 0.12511 \\
\hline 397.952 & 37.212 & 399.669 & 0.14353 & 430.816 & 0.736 & 432.979 & 0.12497 \\
\hline 397.948 & 37.203 & 399.662 & 0.14364 & 430.818 & 0.746 & 433.368 & 0.12509 \\
\hline 397.949 & 37.188 & 400.004 & 0.14364 & 430.814 & 0.750 & 433.354 & 0.12495 \\
\hline 397.953 & 37.190 & 400.005 & 0.14370 & 430.815 & 0.753 & 433.754 & 0.12489 \\
\hline 397.958 & 37.173 & 400.371 & 0.14358 & 430.816 & 0.756 & 433.764 & 0.12483 \\
\hline 397.950 & 37.165 & 400.366 & 0.14353 & 430.818 & 5.910 & 432.283 & 0.12747 \\
\hline 397.954 & 46.868 & 399.033 & 0.14687 & 430.819 & 5.905 & 432.586 & 0.12746 \\
\hline 397.952 & 46.853 & 399.029 & 0.14676 & 430.818 & 5.901 & 432.586 & 0.12735 \\
\hline 397.950 & 46.842 & 399.297 & 0.14680 & 430.811 & 5.902 & 432.916 & 0.12737 \\
\hline
\end{tabular}

anitial cell temperature. 
Table A4, continued.

\begin{tabular}{|c|c|c|c|c|c|c|c|}
\hline$T_{\mathrm{i}^{\mathrm{a}}}^{\mathrm{a}} / \mathrm{K}$ & $p / \mathrm{MPa}$ & $T / K$ & $\lambda / \mathbf{W} \cdot \mathbf{m}^{-1} \mathbf{K}^{-1}$ & $T_{\mathrm{i}^{\mathrm{a}}}^{\mathrm{a}} / \mathbf{K}$ & $p / \mathbf{M P a}$ & $T / K$ & $\lambda / \mathbf{W} \cdot \mathbf{m}^{-1} \mathbf{K}^{-1}$ \\
\hline 430.814 & 5.899 & 432.921 & 0.12735 & 430.873 & 36.574 & 432.512 & 0.13919 \\
\hline 430.808 & 5.901 & 433.291 & 0.12762 & 430.868 & 36.566 & 432.510 & 0.13966 \\
\hline 430.821 & 5.898 & 433.292 & 0.12710 & 430.866 & 36.548 & 432.822 & 0.13910 \\
\hline 430.814 & 5.897 & 433.688 & 0.12714 & 430.873 & 36.536 & 433.171 & 0.13929 \\
\hline 430.816 & 5.893 & 433.694 & 0.12719 & 430.866 & 36.527 & 433.160 & 0.13956 \\
\hline 430.839 & 12.372 & 432.283 & 0.13034 & 430.870 & 36.518 & 433.541 & 0.13932 \\
\hline 430.842 & 12.371 & 432.287 & 0.13036 & 430.886 & 46.204 & 432.200 & 0.14298 \\
\hline 430.839 & 12.369 & 432.585 & 0.13035 & 430.885 & 46.188 & 432.196 & 0.14270 \\
\hline 430.834 & 12.365 & 432.578 & 0.13036 & 430.885 & 46.172 & 432.483 & 0.14265 \\
\hline 430.840 & 12.366 & 432.916 & 0.13022 & 430.891 & 46.155 & 432.484 & 0.14273 \\
\hline 430.838 & 12.363 & 432.911 & 0.13020 & 430.894 & 46.133 & 432.787 & 0.14273 \\
\hline 430.834 & 12.363 & 433.273 & 0.13001 & 430.892 & 46.110 & 432.788 & 0.14286 \\
\hline 430.839 & 12.366 & 433.290 & 0.12998 & 430.896 & 46.082 & 433.124 & 0.14266 \\
\hline 430.838 & 12.361 & 433.668 & 0.13007 & 430.888 & 46.081 & 433.127 & 0.14271 \\
\hline 430.841 & 12.359 & 433.672 & 0.13007 & 430.894 & 46.069 & 433.486 & 0.14258 \\
\hline 430.839 & 19.491 & 432.240 & 0.13320 & 430.889 & 46.045 & 433.492 & 0.14248 \\
\hline 430.839 & 19.488 & 432.244 & 0.13326 & 430.899 & 57.479 & 432.189 & 0.14649 \\
\hline 430.839 & 19.487 & 432.537 & 0.13319 & 430.899 & 57.424 & 432.455 & 0.14615 \\
\hline 430.840 & 19.481 & 432.540 & 0.13319 & 430.892 & 57.399 & 432.455 & 0.14653 \\
\hline 430.841 & 19.475 & 432.867 & 0.13303 & 430.897 & 57.376 & 432.765 & 0.14639 \\
\hline 430.842 & 19.472 & 432.871 & 0.13294 & 430.898 & 57.357 & 432.764 & 0.14649 \\
\hline 430.838 & 19.471 & 433.228 & 0.13293 & 430.898 & 57.331 & 433.086 & 0.14646 \\
\hline 430.841 & 19.470 & 433.222 & 0.13292 & 430.896 & 57.306 & 433.085 & 0.14626 \\
\hline 430.837 & 19.464 & 433.602 & 0.13283 & 430.901 & 57.283 & 433.441 & 0.14614 \\
\hline 430.834 & 19.462 & 433.609 & 0.13288 & 430.896 & 57.256 & 433.435 & 0.14624 \\
\hline 430.852 & 27.523 & 432.231 & 0.13632 & 430.904 & 68.198 & 432.175 & 0.14979 \\
\hline 430.851 & 27.516 & 432.238 & 0.13642 & 430.900 & 68.132 & 432.429 & 0.14968 \\
\hline 430.848 & 27.513 & 432.521 & 0.13653 & 430.900 & 68.100 & 432.421 & 0.14992 \\
\hline 430.849 & 27.508 & 432.526 & 0.13617 & 430.900 & 68.069 & 432.717 & 0.14965 \\
\hline 430.851 & 27.496 & 432.845 & 0.13619 & 430.896 & 68.036 & 432.709 & 0.14975 \\
\hline 430.852 & 27.497 & 432.850 & 0.13628 & 430.905 & 67.973 & 433.040 & 0.14960 \\
\hline 430.860 & 27.487 & 433.188 & 0.13587 & 430.898 & 67.941 & 433.378 & 0.14953 \\
\hline 430.851 & 27.487 & 433.184 & 0.13609 & 430.898 & 67.908 & 433.375 & 0.14954 \\
\hline 430.854 & 27.482 & 433.562 & 0.13618 & 464.206 & 0.519 & 465.673 & 0.11924 \\
\hline 430.857 & 27.477 & 433.574 & 0.13604 & 464.210 & 0.539 & 465.678 & 0.11925 \\
\hline 430.865 & 36.593 & 432.225 & 0.13985 & 464.210 & 0.554 & 465.974 & 0.11937 \\
\hline 430.874 & 36.578 & 432.228 & 0.13945 & 464.210 & 0.569 & 465.987 & 0.11934 \\
\hline
\end{tabular}

anitial cell temperature. 
Table A4, continued.

\begin{tabular}{|c|c|c|c|c|c|c|c|}
\hline$T_{\mathrm{i}^{\mathrm{a}}}^{\mathrm{a}} / \mathrm{K}$ & $p / \mathrm{MPa}$ & $T / K$ & $\lambda / \mathbf{W} \cdot \mathbf{m}^{-1} \mathbf{K}^{-1}$ & $T_{\mathrm{i}^{\mathrm{a}}}^{\mathrm{a}} / \mathbf{K}$ & $p / \mathbf{M P a}$ & $T / K$ & $\lambda / \mathbf{W} \cdot \mathbf{m}^{-1} \mathbf{K}^{-1}$ \\
\hline 464.210 & 0.584 & 466.319 & 0.11931 & 464.249 & 26.784 & 465.861 & 0.13150 \\
\hline 464.214 & 0.593 & 466.318 & 0.11917 & 464.249 & 26.782 & 466.173 & 0.13130 \\
\hline 464.210 & 0.602 & 466.683 & 0.11914 & 464.254 & 26.786 & 466.168 & 0.13144 \\
\hline 464.209 & 0.609 & 466.681 & 0.11922 & 464.255 & 26.768 & 466.508 & 0.13122 \\
\hline 464.216 & 0.615 & 467.086 & 0.11927 & 464.247 & 26.769 & 466.489 & 0.13127 \\
\hline 464.213 & 0.623 & 467.089 & 0.11909 & 464.248 & 26.750 & 466.867 & 0.13127 \\
\hline 464.216 & 5.813 & 465.632 & 0.12200 & 464.247 & 26.754 & 466.863 & 0.13133 \\
\hline 464.218 & 5.817 & 465.622 & 0.12222 & 464.259 & 35.732 & 465.560 & 0.13509 \\
\hline 464.218 & 5.810 & 465.928 & 0.12192 & 464.262 & 35.735 & 465.562 & 0.13504 \\
\hline 464.220 & 5.814 & 465.939 & 0.12199 & 464.266 & 35.714 & 465.829 & 0.13507 \\
\hline 464.218 & 5.810 & 466.262 & 0.12199 & 464.274 & 35.703 & 465.836 & 0.13486 \\
\hline 464.221 & 5.807 & 466.258 & 0.12193 & 464.271 & 35.700 & 466.142 & 0.13494 \\
\hline 464.218 & 5.806 & 466.616 & 0.12194 & 464.275 & 35.707 & 466.148 & 0.13489 \\
\hline 464.225 & 5.807 & 466.619 & 0.12190 & 464.274 & 35.686 & 466.466 & 0.13488 \\
\hline 464.224 & 5.807 & 467.028 & 0.12177 & 464.280 & 35.682 & 466.479 & 0.13486 \\
\hline 464.223 & 5.808 & 467.022 & 0.12175 & 464.278 & 35.651 & 466.828 & 0.13480 \\
\hline 464.222 & 12.061 & 465.592 & 0.12490 & 464.272 & 35.634 & 466.828 & 0.13485 \\
\hline 464.225 & 12.039 & 465.596 & 0.12510 & 464.291 & 45.827 & 465.512 & 0.13891 \\
\hline 464.222 & 12.051 & 465.881 & 0.12498 & 464.292 & 45.816 & 465.527 & 0.13893 \\
\hline 464.216 & 12.051 & 465.886 & 0.12526 & 464.291 & 45.780 & 465.782 & 0.13894 \\
\hline 464.223 & 12.062 & 466.203 & 0.12507 & 464.288 & 45.774 & 465.766 & 0.13913 \\
\hline 464.221 & 12.052 & 466.191 & 0.12494 & 464.292 & 45.758 & 466.078 & 0.13907 \\
\hline 464.217 & 12.041 & 466.539 & 0.12503 & 464.290 & 45.745 & 466.076 & 0.13897 \\
\hline 464.214 & 12.044 & 466.551 & 0.12486 & 464.289 & 45.722 & 466.397 & 0.13856 \\
\hline 464.215 & 12.047 & 466.935 & 0.12489 & 464.289 & 45.684 & 466.390 & 0.13854 \\
\hline 464.214 & 12.049 & 466.922 & 0.12496 & 464.290 & 45.654 & 466.728 & 0.13858 \\
\hline 464.236 & 19.060 & 465.580 & 0.12832 & 464.307 & 56.764 & 465.561 & 0.14246 \\
\hline 464.238 & 19.082 & 465.585 & 0.12847 & 464.308 & 56.745 & 465.533 & 0.14273 \\
\hline 464.238 & 19.082 & 465.877 & 0.12841 & 464.305 & 56.684 & 465.789 & 0.14269 \\
\hline 464.242 & 19.067 & 466.193 & 0.12806 & 464.309 & 56.670 & 465.797 & 0.14258 \\
\hline 464.242 & 19.051 & 466.196 & 0.12821 & 464.305 & 56.628 & 466.079 & 0.14263 \\
\hline 464.246 & 19.068 & 466.541 & 0.12816 & 464.306 & 56.592 & 466.400 & 0.14239 \\
\hline 464.247 & 19.072 & 466.542 & 0.12812 & 464.296 & 56.588 & 466.392 & 0.14244 \\
\hline 464.245 & 19.031 & 466.900 & 0.12813 & 464.300 & 56.555 & 466.715 & 0.14247 \\
\hline 464.250 & 26.810 & 465.587 & 0.13166 & 464.302 & 56.503 & 466.716 & 0.14264 \\
\hline 464.246 & 26.809 & 465.578 & 0.13135 & 498.013 & 0.622 & 499.455 & 0.11381 \\
\hline 464.255 & 26.779 & 465.862 & 0.13157 & 498.015 & 0.628 & 499.443 & 0.11393 \\
\hline
\end{tabular}

anitial cell temperature. 
Table A4, continued.

\begin{tabular}{|c|c|c|c|c|c|c|c|}
\hline$T_{\mathrm{i}^{\mathrm{a}}}^{\mathrm{a}} / \mathrm{K}$ & $p / \mathrm{MPa}$ & $T / K$ & $\lambda / \mathbf{W} \cdot \mathbf{m}^{-1} \mathbf{K}^{-1}$ & $T_{\mathrm{i}^{\mathrm{a}}}^{\mathrm{a}} / \mathbf{K}$ & $p / \mathbf{M P a}$ & $T / K$ & $\lambda / \mathbf{W} \cdot \mathbf{m}^{-1} \mathbf{K}^{-1}$ \\
\hline 498.014 & 0.633 & 499.756 & 0.11366 & 498.020 & 26.693 & 499.597 & 0.12725 \\
\hline 498.015 & 0.636 & 500.083 & 0.11353 & 498.013 & 26.698 & 499.870 & 0.12684 \\
\hline 498.020 & 0.638 & 500.090 & 0.11361 & 498.012 & 26.699 & 499.875 & 0.12692 \\
\hline 498.021 & 0.641 & 500.464 & 0.11350 & 498.007 & 26.688 & 500.201 & 0.12706 \\
\hline 498.021 & 0.641 & 500.453 & 0.11348 & 498.005 & 26.671 & 500.189 & 0.12694 \\
\hline 498.024 & 0.646 & 500.852 & 0.11337 & 498.015 & 26.675 & 500.545 & 0.12685 \\
\hline 498.017 & 5.724 & 499.408 & 0.11676 & 498.018 & 26.649 & 500.544 & 0.12676 \\
\hline 498.004 & 5.737 & 499.684 & 0.11663 & 498.044 & 35.247 & 499.299 & 0.13039 \\
\hline 498.011 & 5.751 & 499.692 & 0.11666 & 498.050 & 35.239 & 499.309 & 0.13068 \\
\hline 498.003 & 5.726 & 499.994 & 0.11660 & 498.051 & 35.251 & 499.564 & 0.13076 \\
\hline 498.004 & 5.730 & 500.353 & 0.11647 & 498.053 & 35.238 & 499.564 & 0.13101 \\
\hline 498.004 & 5.732 & 500.340 & 0.11659 & 498.049 & 35.241 & 499.858 & 0.13059 \\
\hline 498.003 & 5.731 & 500.728 & 0.11646 & 498.048 & 35.218 & 499.839 & 0.13051 \\
\hline 497.997 & 5.740 & 500.709 & 0.11640 & 498.043 & 35.229 & 500.165 & 0.13072 \\
\hline 498.001 & 12.033 & 499.367 & 0.12006 & 498.048 & 35.234 & 500.170 & 0.13061 \\
\hline 497.996 & 12.031 & 499.366 & 0.11998 & 498.041 & 35.231 & 500.500 & 0.13044 \\
\hline 497.995 & 12.033 & 499.637 & 0.12012 & 498.040 & 35.251 & 500.508 & 0.13051 \\
\hline 497.998 & 12.041 & 499.650 & 0.11986 & 498.035 & 45.556 & 499.246 & 0.13474 \\
\hline 497.987 & 12.019 & 499.935 & 0.12009 & 498.028 & 45.559 & 499.231 & 0.13484 \\
\hline 497.988 & 12.005 & 499.938 & 0.11983 & 498.033 & 45.551 & 499.497 & 0.13479 \\
\hline 497.987 & 12.029 & 500.300 & 0.11974 & 498.029 & 45.562 & 499.501 & 0.13489 \\
\hline 497.978 & 12.035 & 500.269 & 0.11987 & 498.027 & 45.547 & 499.778 & 0.13462 \\
\hline 497.981 & 12.036 & 500.660 & 0.11978 & 498.030 & 45.572 & 500.090 & 0.13485 \\
\hline 497.980 & 12.040 & 500.643 & 0.11989 & 498.027 & 45.567 & 500.079 & 0.13492 \\
\hline 498.009 & 18.615 & 499.316 & 0.12349 & 498.027 & 45.523 & 500.398 & 0.13471 \\
\hline 498.005 & 18.597 & 499.301 & 0.12324 & 498.028 & 45.526 & 500.397 & 0.13499 \\
\hline 498.007 & 18.605 & 499.617 & 0.12319 & 498.044 & 56.816 & 499.231 & 0.13933 \\
\hline 498.007 & 18.617 & 499.601 & 0.12307 & 498.047 & 56.817 & 499.481 & 0.13921 \\
\hline 498.011 & 18.617 & 499.927 & 0.12327 & 498.045 & 56.816 & 499.487 & 0.13881 \\
\hline 498.007 & 18.596 & 499.905 & 0.12342 & 498.047 & 56.817 & 499.779 & 0.13902 \\
\hline 498.005 & 18.589 & 500.244 & 0.12316 & 498.045 & 56.794 & 500.063 & 0.13911 \\
\hline 498.004 & 18.601 & 500.241 & 0.12309 & 498.045 & 56.809 & 500.068 & 0.13896 \\
\hline 498.008 & 18.605 & 500.602 & 0.12317 & 498.047 & 56.787 & 500.374 & 0.13908 \\
\hline 498.010 & 18.596 & 500.603 & 0.12300 & 498.045 & 56.808 & 500.400 & 0.13890 \\
\hline 498.022 & 26.629 & 499.312 & 0.12703 & 498.054 & 5.995 & 499.472 & 0.11665 \\
\hline 498.018 & 26.629 & 499.303 & 0.12688 & 498.049 & 5.991 & 499.459 & 0.11670 \\
\hline 498.018 & 26.678 & 499.595 & 0.12714 & 498.049 & 5.993 & 499.753 & 0.11684 \\
\hline
\end{tabular}

anitial cell temperature. 
Table A4, continued.

\begin{tabular}{cccc}
\hline $\boldsymbol{T}_{\mathbf{i}}^{\mathbf{a}} / \mathbf{K}$ & $\boldsymbol{p} / \mathbf{M P a}$ & $\boldsymbol{T} / \mathbf{K}$ & $\boldsymbol{\lambda} / \mathbf{W} \cdot \mathbf{m}^{-1} \mathbf{K}^{-\mathbf{1}}$ \\
\hline \hline 498.048 & 5.995 & 499.736 & 0.11662 \\
498.054 & 5.991 & 500.068 & 0.11671 \\
498.053 & 5.990 & 500.083 & 0.11657 \\
498.053 & 5.988 & 500.431 & 0.11667 \\
498.049 & 5.992 & 500.427 & 0.11665 \\
498.054 & 5.993 & 500.795 & 0.11665 \\
498.049 & 5.994 & 500.795 & 0.11655 \\
\hline
\end{tabular}

anitial cell temperature. 


\section{Appendix B: Viscosity Data Tables}

Table B1: Viscosity of POE5 at Temperatures from $275 \mathrm{~K}$ to $430 \mathrm{~K}$ with Pressures to $137 \mathrm{MPa}$.

\begin{tabular}{|c|c|c|c|c|c|}
\hline $\begin{array}{c}T \\
/ \mathbf{K} \\
\end{array}$ & $\begin{array}{c}\boldsymbol{p} \\
/ \mathrm{MPa}\end{array}$ & $\begin{array}{c}\eta \\
/ \mathrm{mPa} \cdot \mathrm{s}\end{array}$ & $\begin{array}{c}T \\
/ \mathbf{K} \\
\end{array}$ & $\begin{array}{c}p \\
/ \mathrm{MPa}\end{array}$ & $\begin{array}{c}\eta \\
/ \mathrm{mPa} \cdot \mathrm{s} \\
\end{array}$ \\
\hline 350.03 & 19.828 & 6.740 & 380.02 & 9.816 & 3.278 \\
\hline 350.03 & 29.842 & 7.603 & 380.02 & 19.814 & 3.691 \\
\hline 350.04 & 39.859 & 8.632 & 380.02 & 29.814 & 4.124 \\
\hline 350.04 & 49.893 & 9.649 & 380.03 & 39.838 & 4.594 \\
\hline 350.04 & 59.928 & 10.78 & 380.03 & 49.874 & 5.067 \\
\hline 350.05 & 69.986 & 12.00 & 380.04 & 59.913 & 5.558 \\
\hline 350.05 & 80.043 & 13.32 & 380.03 & 69.958 & 6.088 \\
\hline 350.05 & 90.090 & 14.74 & 380.04 & 79.990 & 6.657 \\
\hline 350.06 & 100.160 & 16.24 & 380.04 & 90.044 & 7.242 \\
\hline 350.06 & 110.223 & 17.85 & 380.05 & 100.123 & 7.846 \\
\hline 350.06 & 120.297 & 19.55 & 380.05 & 110.186 & 8.507 \\
\hline 360.04 & 0.154 & 4.156 & 380.00 & 120.265 & 9.148 \\
\hline 360.05 & 9.822 & 4.756 & 379.97 & 130.367 & 9.950 \\
\hline 360.05 & 19.817 & 5.347 & 389.95 & 0.142 & 2.494 \\
\hline 360.06 & 29.820 & 6.020 & 389.97 & 9.812 & 2.835 \\
\hline 360.07 & 39.838 & 6.727 & 389.97 & 19.818 & 3.162 \\
\hline 360.07 & 49.865 & 7.475 & 389.98 & 29.821 & 3.532 \\
\hline 360.07 & 59.911 & 8.306 & 389.99 & 39.842 & 3.926 \\
\hline 360.07 & 69.946 & 9.205 & 390.00 & 49.876 & 4.353 \\
\hline 360.07 & 79.989 & 10.19 & 390.01 & 59.915 & 4.812 \\
\hline 360.07 & 90.042 & 11.26 & 390.00 & 69.967 & 5.239 \\
\hline 360.08 & 100.115 & 12.40 & 390.02 & 90.075 & 6.168 \\
\hline 360.08 & 110.191 & 13.59 & 390.02 & 100.138 & 6.726 \\
\hline 360.09 & 120.245 & 14.86 & 390.03 & 110.205 & 7.228 \\
\hline 360.08 & 130.316 & 16.23 & 390.03 & 120.273 & 7.786 \\
\hline 360.08 & 137.352 & 17.27 & 390.03 & 130.341 & 8.350 \\
\hline 369.98 & 0.131 & 3.629 & 390.04 & 137.389 & 8.797 \\
\hline 369.98 & 9.798 & 4.102 & 390.01 & 70.073 & 5.257 \\
\hline 369.99 & 19.800 & 4.623 & 390.00 & 19.872 & 3.171 \\
\hline 370.00 & 29.803 & 5.165 & 390.01 & 9.864 & 2.799 \\
\hline 370.00 & 39.827 & 5.702 & 399.93 & 0.151 & 2.136 \\
\hline 370.00 & 49.861 & 6.342 & 399.93 & 9.831 & 2.390 \\
\hline 370.01 & 59.906 & 6.967 & 399.92 & 19.832 & 2.699 \\
\hline 370.01 & 69.949 & 7.612 & 399.93 & 29.832 & 3.034 \\
\hline 370.02 & 79.999 & 8.290 & 399.93 & 39.851 & 3.370 \\
\hline 370.02 & 90.058 & 9.037 & 399.94 & 49.882 & 3.732 \\
\hline 370.03 & 100.140 & 9.862 & 399.95 & 59.932 & 4.113 \\
\hline 370.04 & 110.197 & 10.76 & 399.94 & 69.965 & 4.471 \\
\hline 370.04 & 120.266 & 11.70 & 399.94 & 80.016 & 4.857 \\
\hline 370.04 & 130.336 & 12.70 & 399.94 & 90.074 & 5.315 \\
\hline 370.04 & 137.395 & 13.46 & 399.95 & 100.134 & 5.738 \\
\hline 380.01 & 0.139 & 2.896 & 399.96 & 110.208 & 6.235 \\
\hline
\end{tabular}


Table B1, continued.

\begin{tabular}{|c|c|c|c|c|c|}
\hline $\begin{array}{c}T \\
/ \mathrm{K} \\
\end{array}$ & $\begin{array}{c}p \\
/ \mathrm{MPa} \\
\end{array}$ & $\begin{array}{c}\eta \\
/ \mathbf{m P a} \cdot \mathbf{s} \\
\end{array}$ & $\begin{array}{c}T \\
/ \mathrm{K} \\
\end{array}$ & $\begin{array}{c}p \\
/ \mathrm{MPa} \\
\end{array}$ & $\begin{array}{c}\eta \\
/ \mathrm{mPa} \cdot \mathrm{s} \\
\end{array}$ \\
\hline 399.96 & 120.267 & 6.687 & 410.01 & 120.267 & 5.802 \\
\hline 399.97 & 130.345 & 7.212 & 410.01 & 130.324 & 6.275 \\
\hline 399.97 & 137.390 & 7.585 & 410.03 & 137.369 & 6.653 \\
\hline 419.97 & 0.131 & 1.698 & 310.02 & 0.111 & 16.65 \\
\hline 419.96 & 9.807 & 1.903 & 310.02 & 9.781 & 19.43 \\
\hline 419.96 & 19.810 & 2.116 & 310.02 & 19.790 & 22.36 \\
\hline 419.96 & 29.807 & 2.367 & 310.00 & 29.803 & 25.75 \\
\hline 419.97 & 39.831 & 2.594 & 309.99 & 39.826 & 29.57 \\
\hline 419.96 & 49.859 & 2.838 & 310.02 & 49.838 & 33.69 \\
\hline 419.95 & 59.908 & 3.102 & 310.02 & 59.889 & 38.56 \\
\hline 419.95 & 69.950 & 3.354 & 310.03 & 69.928 & 44.05 \\
\hline 419.96 & 79.988 & 3.616 & 310.03 & 79.984 & 50.18 \\
\hline 419.96 & 90.057 & 3.881 & 310.04 & 90.024 & 56.75 \\
\hline 419.96 & 100.121 & 4.164 & 310.04 & 100.092 & 64.09 \\
\hline 419.98 & 110.197 & 4.459 & 310.04 & 110.149 & 72.22 \\
\hline 419.98 & 120.262 & 4.773 & 310.01 & 120.246 & 81.36 \\
\hline 419.98 & 130.326 & 5.062 & 309.97 & 130.335 & 91.00 \\
\hline 419.98 & 137.362 & 5.296 & 309.96 & 137.408 & 98.51 \\
\hline 430.04 & 0.172 & 1.467 & 300.03 & 0.120 & 24.33 \\
\hline 430.02 & 9.821 & 1.674 & 300.04 & 9.797 & 28.53 \\
\hline 430.03 & 19.810 & 1.880 & 300.04 & 19.791 & 33.22 \\
\hline 430.03 & 29.819 & 2.111 & 300.05 & 29.795 & 38.41 \\
\hline 430.04 & 39.839 & 2.341 & 300.04 & 39.803 & 44.84 \\
\hline 430.03 & 49.850 & 2.595 & 300.04 & 49.837 & 52.04 \\
\hline 430.04 & 59.890 & 2.694 & 300.03 & 59.890 & 60.44 \\
\hline 430.03 & 69.936 & 2.920 & 300.03 & 69.933 & 69.05 \\
\hline 430.02 & 79.987 & 3.143 & 300.03 & 79.980 & 78.80 \\
\hline 430.03 & 90.042 & 3.448 & 300.02 & 90.042 & 89.67 \\
\hline 430.04 & 100.109 & 3.674 & 305.03 & 0.117 & 20.39 \\
\hline 430.04 & 110.163 & 3.987 & 305.03 & 9.797 & 23.63 \\
\hline 430.05 & 120.225 & 4.304 & 305.02 & 19.798 & 27.36 \\
\hline 430.06 & 130.297 & 4.605 & 305.03 & 29.802 & 31.49 \\
\hline 430.07 & 137.350 & 4.823 & 305.03 & 39.827 & 36.28 \\
\hline 410.03 & 0.144 & 1.839 & 305.03 & 49.849 & 42.01 \\
\hline 410.03 & 9.822 & 2.101 & 305.03 & 59.901 & 48.29 \\
\hline 410.00 & 19.822 & 2.369 & 305.03 & 69.942 & 55.22 \\
\hline 410.00 & 39.849 & 2.950 & 305.03 & 79.982 & 62.73 \\
\hline 410.00 & 49.872 & 3.259 & 305.02 & 90.041 & 71.10 \\
\hline 410.00 & 59.914 & 3.601 & 305.02 & 100.117 & 80.39 \\
\hline 410.01 & 69.960 & 3.987 & 305.03 & 110.175 & 90.42 \\
\hline 410.01 & 80.008 & 4.326 & 340.05 & 0.129 & 6.58 \\
\hline 410.02 & 90.064 & 4.681 & 340.03 & 9.808 & 7.56 \\
\hline 410.02 & 100.132 & 5.036 & 340.02 & 19.809 & 8.70 \\
\hline 410.02 & 110.200 & 5.444 & 340.01 & 29.813 & 9.97 \\
\hline
\end{tabular}


Table B1, continued.

\begin{tabular}{|c|c|c|c|c|c|}
\hline $\begin{array}{c}T \\
/ \mathrm{K} \\
\end{array}$ & $\begin{array}{c}p \\
/ \mathrm{MPa} \\
\end{array}$ & $\begin{array}{c}\eta \\
/ \mathbf{m P a} \cdot \mathbf{s} \\
\end{array}$ & $\begin{array}{c}T \\
/ \mathrm{K} \\
\end{array}$ & $\begin{array}{c}p \\
/ \mathrm{MPa} \\
\end{array}$ & $\begin{array}{c}\eta \\
/ \mathrm{mPa} \cdot \mathrm{s} \\
\end{array}$ \\
\hline 340.01 & 39.830 & 11.31 & 315.08 & 29.762 & 22.10 \\
\hline 340.01 & 49.873 & 12.76 & 315.08 & 39.783 & 25.17 \\
\hline 340.01 & 59.921 & 14.32 & 315.07 & 49.805 & 28.64 \\
\hline 340.01 & 69.965 & 16.00 & 315.08 & 59.848 & 32.46 \\
\hline 340.02 & 80.018 & 17.83 & 315.08 & 69.898 & 36.60 \\
\hline 340.02 & 90.083 & 19.81 & 315.09 & 79.942 & 41.40 \\
\hline 340.02 & 100.138 & 21.92 & 315.09 & 89.995 & 47.08 \\
\hline 340.01 & 110.210 & 24.23 & 315.08 & 100.072 & 53.23 \\
\hline 340.01 & 120.288 & 26.55 & 315.09 & 110.138 & 59.82 \\
\hline 340.02 & 130.348 & 29.16 & 315.09 & 120.185 & 67.07 \\
\hline 340.01 & 137.399 & 31.21 & 315.08 & 130.257 & 75.04 \\
\hline 325.01 & 0.125 & 10.49 & 315.09 & 137.303 & 81.09 \\
\hline 324.97 & 9.798 & 12.05 & 320.02 & 0.135 & 12.53 \\
\hline 324.97 & 19.792 & 13.90 & 320.03 & 9.815 & 14.40 \\
\hline 324.96 & 29.794 & 15.90 & 320.03 & 19.819 & 16.52 \\
\hline 324.96 & 39.817 & 18.05 & 320.04 & 29.820 & 18.88 \\
\hline 324.96 & 49.841 & 20.39 & 320.05 & 39.838 & 21.45 \\
\hline 324.97 & 59.886 & 22.98 & 320.05 & 49.874 & 24.28 \\
\hline 324.95 & 69.930 & 25.74 & 320.05 & 59.913 & 27.31 \\
\hline 324.96 & 79.968 & 28.70 & 320.06 & 69.966 & 30.62 \\
\hline 324.96 & 90.030 & 31.91 & 320.06 & 80.017 & 34.29 \\
\hline 324.96 & 100.096 & 35.51 & 320.07 & 90.073 & 38.28 \\
\hline 324.96 & 110.176 & 39.52 & 320.07 & 100.143 & 42.97 \\
\hline 324.96 & 120.235 & 44.25 & 320.07 & 110.217 & 48.43 \\
\hline 324.96 & 130.297 & 49.25 & 320.07 & 120.293 & 53.94 \\
\hline 324.97 & 137.355 & 53.00 & 320.07 & 130.358 & 60.32 \\
\hline 330.00 & 0.158 & 8.965 & 320.07 & 137.402 & 65.25 \\
\hline 330.01 & 9.831 & 10.25 & 275.06 & 0.107 & 86.85 \\
\hline 330.01 & 19.834 & 11.81 & 279.97 & 0.116 & 65.78 \\
\hline 330.01 & 29.845 & 13.52 & 279.98 & 4.793 & 71.02 \\
\hline 330.02 & 39.868 & 15.35 & 279.98 & 9.781 & 77.48 \\
\hline 330.02 & 49.896 & 17.35 & 279.97 & 19.788 & 91.56 \\
\hline 330.02 & 59.935 & 19.45 & 285.06 & 0.094 & 50.09 \\
\hline 330.02 & 69.987 & 21.70 & 285.08 & 4.776 & 54.38 \\
\hline 330.03 & 80.034 & 24.24 & 285.07 & 9.777 & 59.49 \\
\hline 330.02 & 90.083 & 26.87 & 285.07 & 19.776 & 70.94 \\
\hline 330.03 & 100.146 & 30.25 & 285.07 & 29.795 & 84.65 \\
\hline 330.03 & 110.224 & 32.82 & 290.02 & 0.106 & 37.87 \\
\hline 330.04 & 120.301 & 36.30 & 290.02 & 4.794 & 41.20 \\
\hline 330.04 & 130.355 & 40.10 & 290.02 & 9.790 & 45.07 \\
\hline 330.05 & 137.405 & 43.16 & 290.01 & 19.794 & 53.59 \\
\hline 315.06 & 0.101 & 14.41 & 290.01 & 29.797 & 63.18 \\
\hline 315.07 & 9.768 & 16.64 & 290.01 & 39.813 & 74.00 \\
\hline 315.07 & 19.766 & 19.28 & 290.02 & 49.841 & 86.08 \\
\hline
\end{tabular}


Table B1, continued.

\begin{tabular}{ccc}
\hline $\boldsymbol{T}$ & $\boldsymbol{p}$ & $\boldsymbol{\eta}$ \\
$\boldsymbol{/ K}$ & $/ \mathbf{M P a}$ & $/ \mathbf{m P a} \cdot \mathbf{s}$ \\
\hline \hline 295.07 & 0.120 & 30.55 \\
295.08 & 4.802 & 33.17 \\
295.08 & 9.798 & 36.10 \\
295.07 & 19.794 & 42.88 \\
295.07 & 29.795 & 50.09 \\
295.07 & 39.808 & 58.26 \\
295.07 & 49.848 & 67.51 \\
295.07 & 59.886 & 77.67 \\
295.07 & 69.939 & 89.12 \\
\hline
\end{tabular}

Table B2: Viscosity of POE7 at Temperatures from $280 \mathrm{~K}$ to $450 \mathrm{~K}$ with Pressures to $137 \mathrm{MPa}$.

\begin{tabular}{ccc|ccc}
\hline $\boldsymbol{T}$ & $\boldsymbol{p}$ & $\boldsymbol{\eta}$ & $\boldsymbol{T}$ & $\boldsymbol{p}$ & $\boldsymbol{\eta}$ \\
$\boldsymbol{/ K}$ & $\boldsymbol{M} \mathbf{M P a}$ & $/ \mathbf{m P a} \mathbf{s}$ & $\boldsymbol{h}$ & $\mathbf{M P a}$ & $\mathbf{\text { mPa} \cdot \mathbf { s }}$ \\
\hline \hline 290.01 & 19.939 & 70.87 & 309.99 & 9.917 & 24.53 \\
290.01 & 29.938 & 83.96 & 309.99 & 19.921 & 28.43 \\
295.01 & 0.247 & 39.75 & 309.99 & 29.931 & 32.84 \\
295.03 & 9.927 & 47.19 & 309.99 & 39.947 & 38.17 \\
295.03 & 19.934 & 55.88 & 310.00 & 49.980 & 44.15 \\
295.02 & 29.944 & 65.78 & 309.99 & 60.019 & 50.72 \\
295.02 & 39.968 & 76.83 & 310.00 & 70.071 & 58.09 \\
295.03 & 49.996 & 89.27 & 310.00 & 80.111 & 66.12 \\
300.04 & 0.259 & 30.87 & 310.00 & 90.179 & 75.26 \\
300.04 & 9.918 & 36.47 & 310.01 & 100.222 & 84.96 \\
300.04 & 19.927 & 43.50 & 310.01 & 110.281 & 95.79 \\
300.02 & 29.934 & 51.17 & 280.04 & 0.266 & 86.54 \\
300.02 & 39.958 & 59.69 & 284.96 & 0.251 & 66.09 \\
300.02 & 49.996 & 69.11 & 284.96 & 9.935 & 79.44 \\
300.02 & 60.040 & 79.87 & 315.03 & 0.282 & 17.84 \\
300.01 & 70.097 & 91.65 & 315.03 & 9.952 & 20.65 \\
305.03 & 0.234 & 25.66 & 315.04 & 19.954 & 23.91 \\
305.03 & 9.929 & 29.94 & 315.03 & 29.964 & 27.54 \\
305.03 & 19.927 & 34.96 & 315.04 & 39.988 & 31.46 \\
305.03 & 29.935 & 40.98 & 315.03 & 50.017 & 36.08 \\
305.03 & 39.953 & 47.92 & 315.03 & 60.061 & 41.40 \\
305.03 & 49.983 & 55.31 & 315.03 & 70.118 & 47.36 \\
305.03 & 60.025 & 63.75 & 315.03 & 80.168 & 53.78 \\
305.03 & 70.082 & 72.91 & 315.03 & 90.228 & 61.17 \\
305.03 & 80.144 & 82.91 & 315.03 & 100.283 & 69.02 \\
305.03 & 90.202 & 94.13 & 315.03 & 110.358 & 78.01 \\
309.98 & 0.246 & 21.03 & 315.04 & 120.424 & 87.38 \\
\hline
\end{tabular}


Table B2, continued.

\begin{tabular}{|c|c|c|c|c|c|}
\hline $\begin{array}{c}T \\
/ \mathbf{K} \\
\end{array}$ & $\begin{array}{c}p \\
/ \mathrm{MPa} \\
\end{array}$ & $\begin{array}{c}\eta \\
/ \mathrm{mPa} \cdot \mathrm{s} \\
\end{array}$ & $\begin{array}{c}T \\
/ \mathbf{K} \\
\end{array}$ & $\begin{array}{c}p \\
/ \mathrm{MPa} \\
\end{array}$ & $\begin{array}{c}\eta \\
/ \mathrm{mPa} \cdot \mathrm{s} \\
\end{array}$ \\
\hline 315.04 & 130.486 & 97966 & ב330.03 & 100.280 & 37.99 \\
\hline 320.03 & 0.262 & 15.41 & 330.03 & 110.341 & 42.61 \\
\hline 320.03 & 9.935 & 17.74 & 330.03 & 120.413 & 47.42 \\
\hline 320.03 & 19.928 & 20.52 & 330.03 & 130.485 & 52.87 \\
\hline 320.03 & 29.941 & 23.60 & 330.03 & 137.529 & 56.91 \\
\hline 320.03 & 39.959 & 26.88 & 339.96 & 0.274 & 8.022 \\
\hline 320.03 & 49.982 & 30.51 & 339.98 & 9.961 & 9.284 \\
\hline 320.04 & 60.030 & 34.36 & 339.98 & 19.967 & 10.74 \\
\hline 320.04 & 70.070 & 38.84 & 339.99 & 29.978 & 12.33 \\
\hline 320.04 & 80.122 & 44.00 & 340.00 & 39.993 & 14.01 \\
\hline 320.05 & 90.176 & 49.83 & 340.01 & 50.023 & 15.88 \\
\hline 325.04 & 0.263 & 12.93 & 340.02 & 60.068 & 17.93 \\
\hline 325.05 & 9.942 & 14.97 & 340.02 & 70.113 & 20.15 \\
\hline 325.05 & 19.944 & 17.26 & 340.02 & 80.176 & 22.53 \\
\hline 325.05 & 29.954 & 19.75 & 340.02 & 90.233 & 25.14 \\
\hline 325.06 & 39.979 & 22.55 & 340.02 & 100.290 & 27.84 \\
\hline 325.06 & 50.001 & 25.53 & 340.03 & 110.362 & 30.85 \\
\hline 325.06 & 60.043 & 28.76 & 340.03 & 120.422 & 34.07 \\
\hline 325.06 & 70.099 & 32.33 & 340.04 & 130.497 & 37.57 \\
\hline 325.05 & 80.154 & 36.17 & 340.03 & 137.545 & 39.81 \\
\hline 325.04 & 90.219 & 40.66 & 340.00 & 0.341 & 8.026 \\
\hline 325.04 & 100.281 & 45.77 & 349.97 & 0.266 & 6.777 \\
\hline 325.05 & 110.355 & 51.41 & 350.00 & 9.959 & 7.521 \\
\hline 325.04 & 120.430 & 57.51 & 350.01 & 19.962 & 8.498 \\
\hline 325.03 & 130.502 & 63.99 & 350.02 & 29.965 & 9.524 \\
\hline 325.03 & 137.543 & 68.89 & 350.03 & 39.985 & 10.70 \\
\hline 325.00 & 0.331 & 13.14 & 350.04 & 50.022 & 11.94 \\
\hline 319.99 & 0.269 & 15.37 & 350.05 & 60.053 & 13.28 \\
\hline 320.01 & 80.160 & 44.45 & 350.05 & 70.103 & 14.81 \\
\hline 320.03 & 90.220 & 50.14 & 350.06 & 80.169 & 16.49 \\
\hline 320.03 & 100.281 & 56.49 & 350.05 & 90.225 & 18.26 \\
\hline 320.04 & 110.347 & 63.52 & 350.06 & 100.284 & 20.24 \\
\hline 320.04 & 120.417 & 70.83 & 350.07 & 110.348 & 22.51 \\
\hline 320.05 & 130.479 & 79.14 & 350.07 & 120.415 & 25.00 \\
\hline 320.04 & 137.533 & 85.39 & 350.08 & 130.499 & 27.76 \\
\hline 329.99 & 0.275 & 11.09 & 350.07 & 137.549 & 29.92 \\
\hline 330.00 & 9.955 & 12.83 & 360.02 & 0.178 & 5.120 \\
\hline 330.00 & 19.958 & 14.71 & 360.03 & 9.854 & 5.822 \\
\hline 330.01 & 29.961 & 16.88 & 360.04 & 19.860 & 6.601 \\
\hline 330.01 & 39.977 & 19.15 & 360.03 & 29.863 & 7.451 \\
\hline 330.01 & 50.017 & 21.72 & 360.04 & 39.875 & 8.398 \\
\hline 330.01 & 60.064 & 24.48 & 360.04 & 49.903 & 9.425 \\
\hline 330.01 & 70.110 & 27.44 & 360.05 & 59.942 & 10.56 \\
\hline 330.02 & 80.158 & 30.61 & 360.05 & 69.986 & 11.72 \\
\hline 330.03 & 90.216 & 34.24 & 360.05 & 80.037 & 12.98 \\
\hline
\end{tabular}


Table B2, continued.

\begin{tabular}{|c|c|c|c|c|c|}
\hline $\begin{array}{c}T \\
/ \mathrm{K} \\
\end{array}$ & $\begin{array}{c}p \\
/ \mathrm{MPa} \\
\end{array}$ & $\begin{array}{c}\eta \\
/ \mathbf{m P a} \cdot \mathbf{s} \\
\end{array}$ & $\begin{array}{c}T \\
/ \mathrm{K} \\
\end{array}$ & $\begin{array}{c}\boldsymbol{p} \\
/ \mathbf{M P a} \\
\end{array}$ & $\begin{array}{c}\eta \\
/ \mathrm{mPa} \cdot \mathrm{s} \\
\end{array}$ \\
\hline 360.06 & 90.100 & 14.33 & 390.01 & 19.861 & 3.907 \\
\hline 360.07 & 100.150 & 15.78 & 390.01 & 29.872 & 4.365 \\
\hline 360.08 & 110.220 & 17.33 & 390.02 & 39.885 & 4.843 \\
\hline 369.99 & 9.843 & 5.241 & 390.03 & 49.909 & 5.363 \\
\hline 370.00 & 19.836 & 5.948 & 390.03 & 59.950 & 5.894 \\
\hline 370.00 & 29.844 & 6.589 & 400.01 & 0.216 & 2.562 \\
\hline 370.00 & 39.864 & 7.207 & 399.98 & 9.885 & 2.930 \\
\hline 369.99 & 49.900 & 7.952 & 399.96 & 19.876 & 3.337 \\
\hline 369.99 & 59.931 & 8.839 & 399.97 & 29.879 & 3.749 \\
\hline 369.98 & 69.974 & 9.717 & 399.97 & 39.898 & 4.186 \\
\hline 369.98 & 80.019 & 10.66 & 399.99 & 49.921 & 4.607 \\
\hline 369.98 & 90.082 & 11.71 & 399.99 & 59.963 & 5.088 \\
\hline 369.99 & 100.151 & 12.81 & 400.00 & 70.002 & 5.575 \\
\hline 369.99 & 110.216 & 13.97 & 400.00 & 80.054 & 6.085 \\
\hline 370.00 & 120.291 & 15.14 & 400.01 & 90.106 & 6.624 \\
\hline 370.00 & 130.361 & 16.42 & 400.01 & 100.153 & 7.205 \\
\hline 370.00 & 137.397 & 17.36 & 400.02 & 110.215 & 7.800 \\
\hline 380.00 & 0.195 & 3.660 & 400.00 & 120.294 & 8.531 \\
\hline 380.01 & 9.874 & 4.175 & 399.99 & 130.353 & 9.280 \\
\hline 380.01 & 19.886 & 4.720 & 399.98 & 137.408 & 9.635 \\
\hline 380.02 & 29.888 & 5.213 & 399.96 & 0.188 & 2.591 \\
\hline 380.01 & 39.917 & 5.744 & 410.02 & 0.151 & 2.342 \\
\hline 380.02 & 49.946 & 6.295 & 410.02 & 9.821 & 2.669 \\
\hline 380.02 & 59.995 & 6.883 & 410.01 & 19.837 & 3.026 \\
\hline 380.03 & 70.033 & 7.539 & 410.02 & 29.846 & 3.388 \\
\hline 380.03 & 80.088 & 8.255 & 410.03 & 39.861 & 3.776 \\
\hline 380.04 & 90.142 & 9.025 & 410.03 & 49.885 & 4.138 \\
\hline 380.05 & 100.200 & 9.850 & 410.03 & 59.921 & 4.568 \\
\hline 380.05 & 110.268 & 10.72 & 410.04 & 69.971 & 4.980 \\
\hline 380.05 & 120.338 & 11.65 & 410.03 & 80.029 & 5.441 \\
\hline 380.06 & 130.406 & 12.67 & 410.04 & 90.091 & 6.030 \\
\hline 380.06 & 137.466 & 13.45 & 410.04 & 100.152 & 6.545 \\
\hline 380.02 & 40.035 & 5.667 & 410.03 & 110.208 & 7.079 \\
\hline 390.04 & 0.139 & 3.094 & 410.04 & 120.277 & 7.572 \\
\hline 390.04 & 69.958 & 6.609 & 410.05 & 130.341 & 8.120 \\
\hline 390.04 & 80.008 & 7.186 & 410.05 & 137.393 & 8.461 \\
\hline 390.04 & 90.064 & 7.773 & 420.01 & 0.201 & 2.064 \\
\hline 390.05 & 100.115 & 8.408 & 420.02 & 9.893 & 2.351 \\
\hline 390.05 & 110.189 & 9.109 & 420.03 & 19.891 & 2.635 \\
\hline 390.06 & 120.249 & 9.915 & 420.02 & 29.907 & 2.917 \\
\hline 390.06 & 130.331 & 10.75 & 420.02 & 39.921 & 3.284 \\
\hline 390.06 & 137.377 & 11.37 & 420.03 & 49.936 & 3.596 \\
\hline 390.01 & 0.176 & 3.057 & 420.03 & 59.971 & 3.912 \\
\hline 390.00 & 9.845 & 3.459 & 420.02 & 70.021 & 4.279 \\
\hline
\end{tabular}


Table B2, continued.

\begin{tabular}{|c|c|c|c|c|c|}
\hline $\begin{array}{c}T \\
/ \mathrm{K} \\
\end{array}$ & $\begin{array}{c}p \\
/ \mathrm{MPa} \\
\end{array}$ & $\begin{array}{c}\eta \\
/ \mathbf{m P a} \cdot \mathbf{s} \\
\end{array}$ & $\begin{array}{c}T \\
/ \mathrm{K} \\
\end{array}$ & $\begin{array}{c}\boldsymbol{p} \\
/ \mathbf{M P a} \\
\end{array}$ & $\begin{array}{c}\eta \\
/ \mathrm{mPa} \cdot \mathrm{s} \\
\end{array}$ \\
\hline 419.98 & 80.068 & 4.666 & 450.10 & 49.909 & 2.433 \\
\hline 419.97 & 90.122 & 5.105 & 450.11 & 59.946 & 2.663 \\
\hline 420.03 & 100.177 & 5.512 & 450.10 & 69.994 & 2.968 \\
\hline 420.07 & 110.236 & 5.931 & 450.11 & 80.042 & 3.218 \\
\hline 420.06 & 120.315 & 6.331 & 450.10 & 90.100 & 3.503 \\
\hline 420.07 & 130.382 & 6.764 & 450.11 & 100.146 & 3.778 \\
\hline 420.08 & 137.431 & 7.064 & 450.11 & 110.212 & 4.073 \\
\hline 430.00 & 9.841 & 2.039 & 450.11 & 120.281 & 4.361 \\
\hline 430.02 & 19.850 & 2.297 & 450.11 & 130.350 & 4.677 \\
\hline 430.01 & 29.861 & 2.504 & 450.12 & 137.400 & 4.904 \\
\hline 429.98 & 39.886 & 2.788 & & & \\
\hline 429.96 & 49.931 & 3.056 & & & \\
\hline 429.95 & 59.972 & 3.328 & & & \\
\hline 430.14 & 70.030 & 3.642 & & & \\
\hline 430.04 & 0.190 & 1.680 & & & \\
\hline 430.03 & 9.858 & 1.929 & & & \\
\hline 430.03 & 19.869 & 2.202 & & & \\
\hline 430.02 & 29.875 & 2.464 & & & \\
\hline 430.02 & 39.894 & 2.747 & & & \\
\hline 430.02 & 49.929 & 3.045 & & & \\
\hline 430.03 & 59.967 & 3.352 & & & \\
\hline 430.03 & 70.016 & 3.710 & & & \\
\hline 430.03 & 80.067 & 4.079 & & & \\
\hline 430.04 & 90.123 & 4.441 & & & \\
\hline 430.04 & 100.181 & 4.789 & & & \\
\hline 430.03 & 110.240 & 5.163 & & & \\
\hline 430.04 & 120.318 & 5.541 & & & \\
\hline 430.04 & 130.396 & 5.942 & & & \\
\hline 430.05 & 137.451 & 6.240 & & & \\
\hline 440.07 & 19.855 & 2.097 & & & \\
\hline 440.07 & 29.869 & 2.325 & & & \\
\hline 440.06 & 39.882 & 2.564 & & & \\
\hline 440.08 & 49.908 & 2.818 & & & \\
\hline 440.09 & 59.945 & 3.099 & & & \\
\hline 440.11 & 69.997 & 3.387 & & & \\
\hline 440.11 & 80.034 & 3.722 & & & \\
\hline 440.10 & 90.083 & 4.054 & & & \\
\hline 440.09 & 100.143 & 4.402 & & & \\
\hline 440.10 & 110.208 & 4.728 & & & \\
\hline 440.09 & 120.272 & 5.056 & & & \\
\hline 440.09 & 130.352 & 5.440 & & & \\
\hline 440.09 & 137.415 & 5.710 & & & \\
\hline 450.10 & 29.851 & 2.004 & & & \\
\hline 450.10 & 39.875 & 2.205 & & & \\
\hline
\end{tabular}


Table B3: Viscosity of POE9 at Temperatures from $290 \mathrm{~K}$ to $450 \mathrm{~K}$ with Pressures to 137 MPa.

\begin{tabular}{|c|c|c|c|c|c|}
\hline $\begin{array}{c}T \\
/ \mathrm{K}\end{array}$ & $\begin{array}{c}p \\
/ \mathbf{M P a}\end{array}$ & $\begin{array}{c}\eta \\
/ \mathbf{m P a} \cdot \mathrm{s}\end{array}$ & $\begin{array}{c}T \\
/ \mathbf{K}\end{array}$ & $\begin{array}{c}p \\
/ \mathbf{M P a}\end{array}$ & $\begin{array}{c}\eta \\
/ \mathbf{m P a} \cdot \mathrm{s}\end{array}$ \\
\hline 300.11 & 0.025 & 48.73 & 320.10 & 69.999 & 60.44 \\
\hline 300.11 & 0.839 & 49.65 & 320.10 & 80.053 & 68.41 \\
\hline 300.12 & 4.823 & 53.24 & 320.11 & 90.112 & 77.19 \\
\hline 300.12 & 9.810 & 58.37 & 320.10 & 100.180 & 86.85 \\
\hline 300.13 & 19.819 & 69.18 & 320.10 & 110.255 & 97.30 \\
\hline 300.12 & 29.860 & 81.03 & 330.11 & 0.344 & 15.87 \\
\hline 300.12 & 39.886 & 94.35 & 330.10 & 4.805 & 17.04 \\
\hline 289.89 & 0.181 & 80.78 & 330.10 & 9.814 & 18.40 \\
\hline 289.94 & 4.824 & 88.09 & 330.12 & 19.817 & 21.30 \\
\hline 295.07 & 0.317 & 64.31 & 330.14 & 29.836 & 24.34 \\
\hline 295.09 & 4.819 & 69.57 & 330.17 & 39.836 & 27.62 \\
\hline 295.10 & 9.819 & 75.97 & 330.19 & 49.849 & 31.33 \\
\hline 295.10 & 19.375 & 89.09 & 330.20 & 59.876 & 35.36 \\
\hline 305.03 & 0.150 & 39.43 & 330.20 & 70.179 & 40.23 \\
\hline 305.04 & 4.820 & 42.77 & 330.21 & 79.996 & 45.58 \\
\hline 305.06 & 9.813 & 46.85 & 330.22 & 90.052 & 51.35 \\
\hline 305.08 & 19.822 & 55.39 & 330.23 & 100.117 & 57.48 \\
\hline 305.10 & 29.825 & 64.80 & 330.23 & 110.184 & 64.23 \\
\hline 305.10 & 39.849 & 75.62 & 330.23 & 120.265 & 71.72 \\
\hline 305.09 & 49.881 & 87.16 & 330.24 & 130.340 & 79.87 \\
\hline 309.97 & 0.446 & 31.64 & 330.23 & 137.405 & 86.05 \\
\hline 310.00 & 4.788 & 34.03 & 330.21 & 0.396 & 16.16 \\
\hline 310.02 & 9.777 & 37.20 & 340.06 & 1.367 & 11.72 \\
\hline 310.03 & 19.788 & 44.05 & 340.08 & 4.748 & 12.31 \\
\hline 310.04 & 29.801 & 51.60 & 340.10 & 9.759 & 13.25 \\
\hline 310.05 & 39.811 & 60.01 & 340.10 & 19.772 & 15.30 \\
\hline 310.06 & 49.841 & 69.26 & 340.11 & 29.782 & 17.50 \\
\hline 310.06 & 49.888 & 69.29 & 340.12 & 39.806 & 19.94 \\
\hline 310.06 & 59.913 & 79.60 & 340.13 & 49.829 & 22.61 \\
\hline 310.06 & 69.950 & 91.10 & 340.13 & 59.878 & 25.48 \\
\hline 320.05 & 0.313 & 22.69 & 340.14 & 69.918 & 28.55 \\
\hline 320.07 & 4.806 & 24.29 & 340.14 & 79.978 & 31.96 \\
\hline 320.08 & 9.798 & 26.28 & 340.14 & 90.031 & 35.65 \\
\hline 320.08 & 19.810 & 30.44 & 340.15 & 100.102 & 39.59 \\
\hline 320.09 & 29.826 & 34.93 & 340.15 & 110.176 & 44.38 \\
\hline 320.09 & 39.856 & 40.28 & 340.16 & 120.240 & 49.61 \\
\hline 320.10 & 49.892 & 46.45 & 340.16 & 130.311 & 55.47 \\
\hline 320.10 & 59.935 & 53.08 & 340.16 & 137.153 & 59.40 \\
\hline
\end{tabular}


Table B3, continued.

\begin{tabular}{|c|c|c|c|c|c|}
\hline $\begin{array}{c}T \\
/ \mathbf{K}\end{array}$ & $\begin{array}{c}p \\
/ \mathbf{M P a}\end{array}$ & $\begin{array}{c}\eta \\
/ \mathrm{mPa} \cdot \mathrm{s}\end{array}$ & $\begin{array}{c}T \\
/ \mathrm{K}\end{array}$ & $\begin{array}{c}p \\
/ \mathbf{M P a}\end{array}$ & $\begin{array}{c}\eta \\
/ \mathrm{mPa} \cdot \mathrm{s}\end{array}$ \\
\hline 340.12 & 2.365 & 11.85 & 370.06 & 70.168 & "13.55 \\
\hline 350.16 & 9.421 & 10.56 & 370.07 & 80.227 & 14.87 \\
\hline 350.17 & 19.732 & 11.94 & 370.08 & 90.283 & 16.34 \\
\hline 350.18 & 29.736 & 13.43 & 370.08 & 100.332 & 17.92 \\
\hline 350.18 & 39.750 & 15.00 & 370.08 & 110.402 & 19.677 \\
\hline 350.19 & 49.781 & 16.77 & 370.09 & 120.476 & 21.591 \\
\hline 350.20 & 59.825 & 18.69 & 370.10 & 130.555 & 23.703 \\
\hline 350.20 & 69.874 & 20.82 & 370.10 & 137.602 & 25.454 \\
\hline 350.20 & 79.934 & 23.08 & 379.98 & 0.151 & 5.306 \\
\hline 350.20 & 89.999 & 25.68 & 379.99 & 5.018 & 5.61 \\
\hline 350.20 & 100.059 & 28.53 & 379.99 & 10.024 & 5.94 \\
\hline 350.20 & 110.139 & 31.73 & 380.00 & 20.014 & 6.65 \\
\hline 350.21 & 120.220 & 35.22 & 380.02 & 30.030 & 7.41 \\
\hline 350.20 & 130.234 & 39.32 & 380.01 & 40.047 & 8.21 \\
\hline 350.20 & 137.274 & 42.54 & 380.02 & 50.061 & 9.09 \\
\hline 350.18 & 0.333 & 9.40 & 380.03 & 60.116 & 9.99 \\
\hline 359.99 & 0.220 & 7.79 & 380.03 & 70.169 & 10.99 \\
\hline 360.02 & 5.114 & 8.26 & 380.03 & 80.220 & 12.04 \\
\hline 360.03 & 10.114 & 8.76 & 380.05 & 90.285 & 13.21 \\
\hline 360.04 & 20.125 & 9.88 & 380.05 & 100.335 & 14.44 \\
\hline 360.05 & 30.135 & 11.07 & 380.06 & 110.410 & 15.803 \\
\hline 360.06 & 40.142 & 12.37 & 380.07 & 120.483 & 17.242 \\
\hline 360.06 & 50.170 & 13.78 & 380.07 & 130.567 & 18.789 \\
\hline 360.08 & 60.216 & 15.32 & 380.08 & 137.599 & 19.899 \\
\hline 360.08 & 70.268 & 16.99 & 440.03 & 40.107 & 3.081 \\
\hline 360.09 & 80.317 & 18.80 & 440.03 & 50.129 & 3.454 \\
\hline 360.10 & 90.374 & 20.77 & 440.03 & 60.172 & 3.85 \\
\hline 360.10 & 100.429 & 22.89 & 440.02 & 70.227 & 4.24 \\
\hline 360.10 & 110.503 & 25.48 & 440.02 & 80.274 & 4.64 \\
\hline 360.11 & 120.584 & 28.184 & 440.01 & 90.335 & 5.04 \\
\hline 360.12 & 130.664 & 31.076 & 440.02 & 100.384 & 5.45 \\
\hline 360.12 & 137.703 & 33.22 & 440.02 & 110.468 & 5.86 \\
\hline 369.83 & 0.123 & 6.37 & 440.02 & 120.548 & 6.250 \\
\hline 369.89 & 5.015 & 6.77 & 440.02 & 130.625 & 6.644 \\
\hline 369.93 & 10.012 & 7.16 & 440.03 & 137.675 & 6.915 \\
\hline 369.97 & 20.023 & 8.05 & 450.12 & 50.112 & 3.060 \\
\hline 370.00 & 30.029 & 9.00 & 450.13 & 60.151 & 3.398 \\
\hline 370.02 & 40.055 & 10.02 & 450.12 & 70.204 & 3.751 \\
\hline 370.04 & 50.084 & 11.13 & 450.12 & 80.262 & 4.130 \\
\hline 370.04 & 60.120 & 12.30 & 450.11 & 90.320 & 4.49 \\
\hline
\end{tabular}


Table B3, continued.

\begin{tabular}{ccc}
\hline $\boldsymbol{T}$ & $\boldsymbol{p}$ & $\boldsymbol{\eta}$ \\
$\mathbf{K}$ & $/ \mathbf{M P a}$ & $/ \mathbf{m P a} \cdot \mathbf{s}$ \\
\hline \hline 450.11 & 100.380 & 4.84 \\
450.11 & 110.450 & 5.17 \\
450.11 & 120.533 & 5.48 \\
450.11 & 130.613 & 5.80 \\
450.12 & 137.677 & 6.03 \\
\hline
\end{tabular}

Table B4: Viscosity of Generic MIL-PRF-23699 Oil at Temperatures from $290 \mathrm{~K}$ to $450 \mathrm{~K}$ with Pressures to $137 \mathrm{MPa}$.

\begin{tabular}{ccc|ccc}
\hline $\boldsymbol{T}$ & $\boldsymbol{p}$ & $\boldsymbol{\eta}$ & $\boldsymbol{T}$ & $\boldsymbol{p}$ & $\boldsymbol{\eta}$ \\
$\mathbf{K}$ & $\mathbf{M P a}$ & $/ \mathbf{m P a} \cdot \mathbf{s}$ & $\mathbf{K}$ & $\mathbf{M P a}$ & $\mathbf{m P a} \mathbf{s}$ \\
\hline \hline 315.02 & 79.941 & 75.61 & 320.08 & 49.798 & 40.60 \\
315.02 & 69.898 & 66.05 & 320.09 & 59.836 & 46.59 \\
315.01 & 59.865 & 57.49 & 320.09 & 69.885 & 53.59 \\
315.00 & 49.827 & 49.78 & 320.09 & 79.958 & 61.08 \\
315.01 & 39.787 & 42.94 & 320.09 & 90.010 & 69.36 \\
315.01 & 29.759 & 36.72 & 320.09 & 100.067 & 78.43 \\
315.02 & 19.733 & 31.49 & 320.08 & 110.145 & 88.50 \\
300.01 & 0.051 & 44.20 & 320.09 & 120.214 & 99.51 \\
300.02 & 9.732 & 52.58 & 330.06 & 0.088 & 13.92 \\
300.03 & 19.737 & 62.48 & 330.07 & 9.767 & 16.15 \\
300.04 & 29.740 & 73.69 & 330.07 & 19.758 & 18.57 \\
300.04 & 39.759 & 86.81 & 330.08 & 29.760 & 21.36 \\
310.09 & 0.030 & 28.13 & 330.09 & 39.782 & 24.71 \\
310.08 & 9.717 & 33.00 & 330.08 & 49.811 & 28.05 \\
310.08 & 19.721 & 38.97 & 330.09 & 59.853 & 31.75 \\
310.07 & 29.725 & 45.85 & 330.09 & 69.897 & 35.62 \\
310.07 & 39.748 & 53.83 & 330.10 & 79.942 & 40.06 \\
310.08 & 49.776 & 63.00 & 330.10 & 89.996 & 45.79 \\
310.07 & 59.807 & 72.39 & 330.10 & 100.062 & 51.31 \\
310.08 & 69.868 & 82.28 & 330.11 & 110.129 & 58.02 \\
310.08 & 79.917 & 94.34 & 330.10 & 120.210 & 64.82 \\
290.04 & 0.064 & 71.13 & 330.11 & 130.265 & 72.13 \\
290.05 & 4.748 & 77.76 & 330.11 & 137.310 & 78.39 \\
290.04 & 9.747 & 85.65 & 339.96 & 0.087 & 9.846 \\
295.06 & 0.056 & 57.81 & 339.97 & 9.758 & 11.58 \\
295.07 & 9.736 & 68.88 & 339.98 & 19.763 & 13.41 \\
295.06 & 19.747 & 82.45 & 339.98 & 29.763 & 15.34 \\
320.09 & 0.075 & 19.48 & 339.98 & 39.775 & 17.38 \\
320.07 & 9.753 & 22.74 & 339.96 & 49.808 & 19.68 \\
320.07 & 19.742 & 26.48 & 339.96 & 59.843 & 21.94 \\
320.07 & 29.753 & 30.47 & 339.95 & 69.896 & 25.03 \\
320.07 & 39.776 & 35.22 & 339.96 & 79.938 & 28.20 \\
\hline & & & & & \\
\hline
\end{tabular}


Table B4, continued.

\begin{tabular}{|c|c|c|c|c|c|}
\hline $\begin{array}{c}T \\
/ \mathrm{K} \\
\end{array}$ & $\begin{array}{c}p \\
/ \mathrm{MPa} \\
\end{array}$ & $\begin{array}{c}\eta \\
/ \mathbf{m P a} \cdot \mathbf{s} \\
\end{array}$ & $\begin{array}{c}T \\
/ \mathrm{K} \\
\end{array}$ & $\begin{array}{c}p \\
/ \mathrm{MPa} \\
\end{array}$ & $\begin{array}{c}\eta \\
/ \mathrm{mPa} \cdot \mathrm{s} \\
\end{array}$ \\
\hline 339.96 & 89.993 & 31.72 & 390.10 & 79.923 & 7.635 \\
\hline 339.95 & 100.061 & 34.76 & 390.11 & 89.978 & 8.325 \\
\hline 339.89 & 110.161 & 39.28 & 390.11 & 100.046 & 9.092 \\
\hline 339.86 & 120.259 & 43.41 & 390.12 & 110.109 & 9.966 \\
\hline 339.83 & 130.343 & 49.68 & 390.13 & 120.174 & 10.89 \\
\hline 339.83 & 137.402 & 52.93 & 390.13 & 130.250 & 11.81 \\
\hline 349.99 & 0.076 & 7.893 & 390.14 & 137.313 & 12.51 \\
\hline 350.01 & 9.755 & 8.969 & 400.00 & 0.088 & 2.709 \\
\hline 350.02 & 19.762 & 10.11 & 400.01 & 9.758 & 3.079 \\
\hline 350.03 & 29.770 & 11.43 & 400.02 & 19.756 & 3.479 \\
\hline 350.03 & 39.799 & 12.86 & 400.02 & 29.765 & 3.904 \\
\hline 350.04 & 49.816 & 14.51 & 400.03 & 39.789 & 4.355 \\
\hline 350.05 & 59.857 & 16.19 & 400.04 & 49.804 & 4.836 \\
\hline 350.05 & 69.913 & 18.05 & 400.05 & 59.833 & 5.360 \\
\hline 350.05 & 79.959 & 20.12 & 400.05 & 69.890 & 5.875 \\
\hline 350.06 & 90.003 & 22.46 & 400.06 & 79.936 & 6.448 \\
\hline 350.06 & 100.071 & 25.01 & 400.07 & 89.981 & 7.034 \\
\hline 350.07 & 110.144 & 27.91 & 400.08 & 100.051 & 7.667 \\
\hline 350.07 & 120.213 & 31.09 & 400.08 & 110.114 & 8.331 \\
\hline 350.06 & 130.275 & 34.13 & 400.10 & 120.183 & 9.043 \\
\hline 350.06 & 137.337 & 37.55 & 400.10 & 130.249 & 9.846 \\
\hline 380.10 & 0.072 & 3.849 & 400.11 & 137.281 & 10.40 \\
\hline 380.10 & 9.752 & 4.404 & 409.88 & 1.271 & 2.367 \\
\hline 380.10 & 19.745 & 4.970 & 409.90 & 9.753 & 2.643 \\
\hline 380.11 & 29.752 & 5.585 & 409.92 & 19.744 & 2.990 \\
\hline 380.11 & 39.770 & 6.233 & 409.93 & 29.748 & 3.354 \\
\hline 380.11 & 49.804 & 7.043 & 409.94 & 39.769 & 3.743 \\
\hline 380.12 & 59.853 & 7.822 & 409.95 & 49.785 & 4.154 \\
\hline 380.13 & 69.902 & 8.673 & 409.96 & 59.826 & 4.591 \\
\hline 380.13 & 79.954 & 9.549 & 409.97 & 69.861 & 5.042 \\
\hline 380.14 & 90.003 & 10.45 & 409.98 & 79.900 & 5.518 \\
\hline 380.14 & 100.064 & 11.49 & 409.98 & 89.947 & 6.025 \\
\hline 380.15 & 110.128 & 12.59 & 409.99 & 100.016 & 6.548 \\
\hline 380.16 & 120.187 & 13.68 & 409.99 & 110.068 & 7.106 \\
\hline 380.17 & 130.255 & 14.84 & 409.99 & 120.141 & 7.686 \\
\hline 380.18 & 137.317 & 15.72 & 410.00 & 130.205 & 8.296 \\
\hline 390.03 & 0.204 & 3.223 & 410.01 & 137.266 & 8.746 \\
\hline 390.04 & 9.747 & 3.662 & 420.01 & 1.215 & 2.067 \\
\hline 390.05 & 19.752 & 4.145 & 420.02 & 9.730 & 2.302 \\
\hline 390.05 & 29.751 & 4.663 & 420.01 & 19.732 & 2.588 \\
\hline 390.07 & 39.760 & 5.171 & 420.02 & 29.736 & 2.887 \\
\hline 390.08 & 49.796 & 5.739 & 420.03 & 39.749 & 3.192 \\
\hline 390.09 & 59.837 & 6.374 & 420.03 & 49.775 & 3.512 \\
\hline 390.09 & 69.870 & 6.954 & 420.04 & 59.817 & 3.847 \\
\hline
\end{tabular}


Table B4, continued.

\begin{tabular}{|c|c|c|c|c|c|}
\hline $\begin{array}{c}T \\
/ \mathrm{K} \\
\end{array}$ & $\begin{array}{c}p \\
/ \mathrm{MPa} \\
\end{array}$ & $\begin{array}{c}\eta \\
/ \mathbf{m P a} \cdot \mathbf{s} \\
\end{array}$ & $\begin{array}{c}T \\
/ \mathrm{K} \\
\end{array}$ & $\begin{array}{c}\boldsymbol{p} \\
/ \mathbf{M P a} \\
\end{array}$ & $\begin{array}{c}\eta \\
/ \mathrm{mPa} \cdot \mathrm{s} \\
\end{array}$ \\
\hline 420.05 & 69.864 & 4.216 & 450.11 & 120.147 & 4.435 \\
\hline 420.05 & 79.904 & 4.564 & 450.13 & 130.209 & 4.763 \\
\hline 420.07 & 89.945 & 4.926 & 450.13 & 137.252 & 5.001 \\
\hline 420.07 & 100.016 & 5.321 & 380.06 & 0.048 & 3.856 \\
\hline 420.08 & 110.074 & 5.719 & 380.06 & 9.742 & 4.372 \\
\hline 420.08 & 120.137 & 6.136 & 380.07 & 19.740 & 4.950 \\
\hline 420.09 & 130.199 & 6.583 & 380.06 & 29.748 & 5.550 \\
\hline 420.09 & 137.243 & 6.897 & 380.08 & 39.764 & 6.205 \\
\hline 429.94 & 9.742 & 2.008 & 380.08 & 49.803 & 6.886 \\
\hline 429.93 & 19.738 & 2.253 & 380.08 & 59.835 & 7.594 \\
\hline 429.93 & 29.749 & 2.502 & 380.08 & 69.882 & 8.371 \\
\hline 429.93 & 39.765 & 2.763 & 380.08 & 79.927 & 9.268 \\
\hline 429.94 & 49.798 & 3.027 & 380.10 & 89.991 & 10.21 \\
\hline 429.95 & 59.824 & 3.309 & 380.10 & 100.055 & 11.23 \\
\hline 429.96 & 69.862 & 3.665 & 380.11 & 110.119 & 12.28 \\
\hline 429.96 & 79.913 & 3.917 & 380.11 & 120.181 & 13.40 \\
\hline 429.96 & 89.956 & 4.313 & 380.11 & 130.250 & 14.61 \\
\hline 429.97 & 100.021 & 4.691 & 380.12 & 137.307 & 15.49 \\
\hline 429.98 & 110.086 & 5.041 & & & \\
\hline 429.99 & 120.141 & 5.446 & & & \\
\hline 429.99 & 130.208 & 5.868 & & & \\
\hline 429.99 & 137.260 & 6.173 & & & \\
\hline 439.97 & 19.717 & 2.071 & & & \\
\hline 439.97 & 29.716 & 2.293 & & & \\
\hline 439.96 & 39.731 & 2.523 & & & \\
\hline 439.96 & 49.763 & 2.749 & & & \\
\hline 439.97 & 59.814 & 3.027 & & & \\
\hline 439.97 & 69.867 & 3.312 & & & \\
\hline 439.97 & 79.917 & 3.619 & & & \\
\hline 439.98 & 89.958 & 3.932 & & & \\
\hline 439.98 & 100.025 & 4.262 & & & \\
\hline 439.99 & 110.087 & 4.601 & & & \\
\hline 440.00 & 120.142 & 4.959 & & & \\
\hline 440.00 & 130.198 & 5.336 & & & \\
\hline 440.00 & 137.254 & 5.610 & & & \\
\hline 450.11 & 29.738 & 2.070 & & & \\
\hline 450.11 & 39.747 & 2.283 & & & \\
\hline 450.11 & 49.776 & 2.501 & & & \\
\hline 450.11 & 59.820 & 2.736 & & & \\
\hline 450.10 & 69.855 & 2.993 & & & \\
\hline 450.11 & 79.907 & 3.257 & & & \\
\hline 450.11 & 89.955 & 3.536 & & & \\
\hline 450.11 & 100.022 & 3.822 & & & \\
\hline 450.11 & 110.091 & 4.128 & & & \\
\hline
\end{tabular}




\title{
Appendix C: REFPROP Fluid Files
}

\section{C.1. POE5.FLD}

\author{
POE5 !Short name \\ 15834-04-5 !CAS number \\ pentaerythritol tetrapentanoate !Full name \\ C25H4408 !Chemical formula \\ POE5 \\ 472.612 \\ ! Synonym \\ 250. \\ ! Molar mass $[\mathrm{g} / \mathrm{mol}]$ \\ 707.618 \\ 890.0 \\ 1270.0 \\ $\odot .556$ \\ 0.89 \\ -1 . \\ NBP \\ !Triple point temperature [K] \\ !Normal boiling point [K] \\ !Critical temperature [K] \\ !Critical pressure [kPa] \\ !Critical density $[\mathrm{mol} / \mathrm{L}]$ \\ ! Acentric factor \\ !Dipole moment [Debye] \\ 10.0 \\ !Default reference state \\ !Version number \\ ! compiled by E.W. Lemmon, NIST Physical and Chemical Properties Division, Boulder, \\ Colorado \\ ! 12-01-17 EWL, Original version. \\ ! 08-09-18 EWL, Add final equation of state. \\ ! 08-29-18 MLH, Add viscosity correlation. \\ ! 09-06-18 MLH, Add thermal conductivity correlation.
}

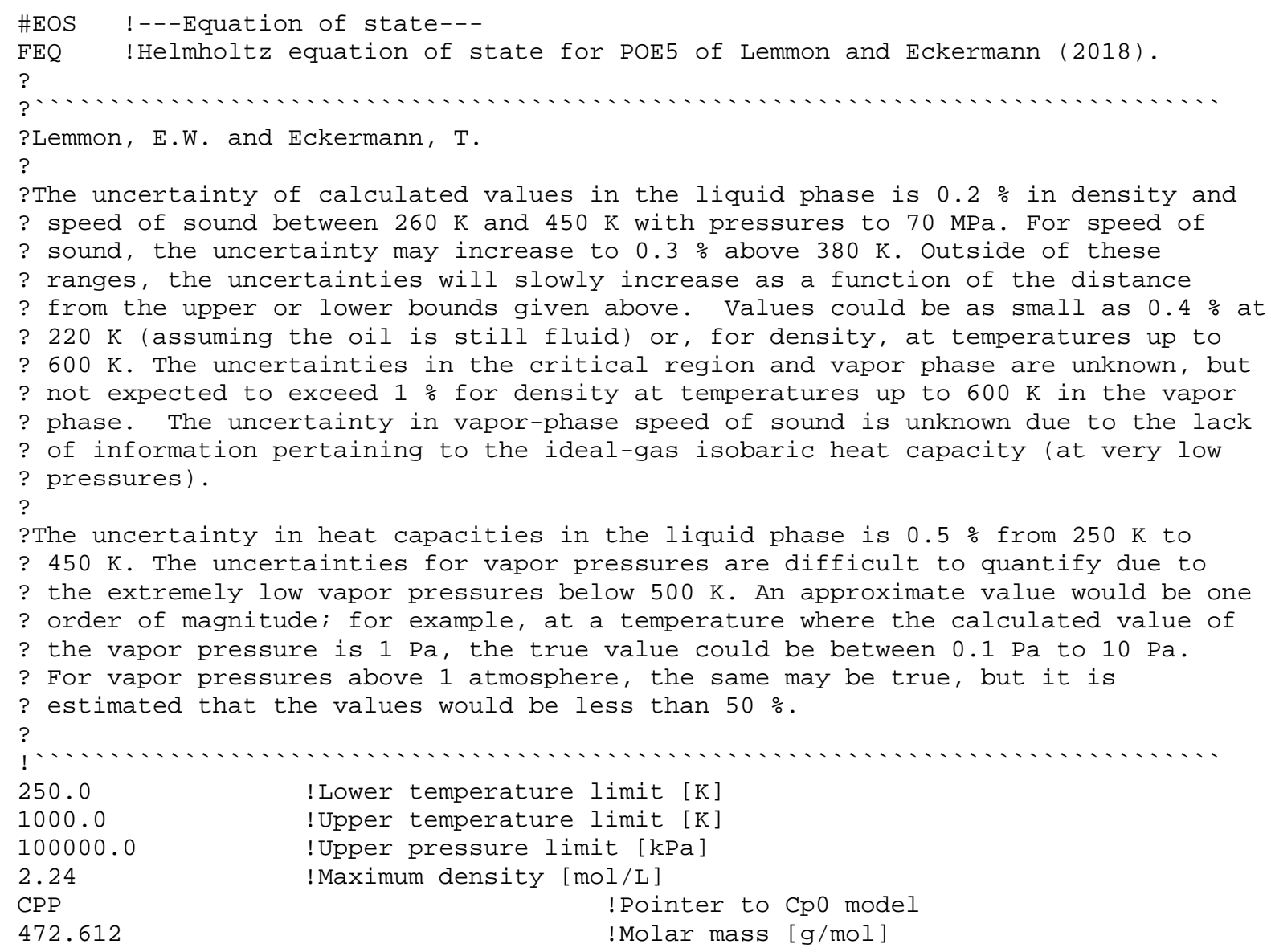


250.0

0.000000000000001

2.23

707.618

0.89

890.0

890.0

8.3144598

104

Gaussian terms, and Gao terms

$0.0106971 \quad 1.0 \quad 5.0$.

$1.510321 \quad 0.08 \quad 1.0$.

$\begin{array}{llll}-2.596894 & 1.2 & 1 . & 0 .\end{array}$

$\begin{array}{llll}-2.311273 & 1.162 & 2 . & 0 .\end{array}$

$\begin{array}{llll}0.3332414 & 0.54 & 3 . & 0 .\end{array}$

$\begin{array}{llll}2.500028 & 1.08 & 2 . & 1 .\end{array}$

$\begin{array}{llll}7.527764 & 1.56 & 3 . & 1 .\end{array}$

$\begin{array}{lll}-5.405529 & 1.78 & 3 . \\ 1 .\end{array}$

$\begin{array}{llll}0.94954 & 1.42 & 4 . & 1 .\end{array}$

$0.3303842 \quad 1.62 \quad 5.1$.

$-0.0234605$

$\odot .0156448$

$-0.0092188$

$-\odot .5415831$
2.0

1.0

1.0

1.0
!Triple point temperature [K]

!Pressure at triple point [kPa]

!Density at triple point [mol/L]

! Normal boiling point temperature [K]

!Acentric factor

$\begin{array}{ll}\odot .556 & \text { ! Tc }[\mathrm{K}], \mathrm{pc}[\mathrm{KPa}], \text { rhoc }[\mathrm{mol} / \mathrm{L}] \\ 0.556 & \text { !Reducing parameters }[\mathrm{K}, \mathrm{mol} / \mathrm{L}]\end{array}$

! Gas constant [ $\mathrm{J} / \mathrm{mol}-\mathrm{K}]$

$\odot \quad \odot \quad \odot \odot$ !\# terms and \# coefs/term for normal terms,

\#AUX !---Auxiliary function for cp®

CPP IIdeal gas heat capacity function for P0E5 of Lemmon and Eckermann (2018).

?

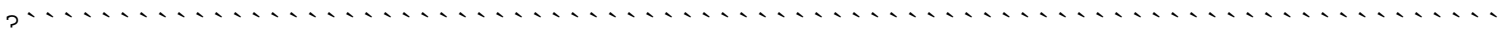

?Lemmon, E.W. and Eckermann, T.

?

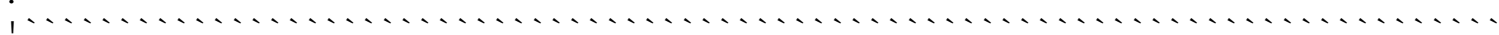

0 .

10000 .

$\odot$.

$\odot$.

$1.0 \quad 8.3144598$ !Reducing parameters for $\mathrm{T}, \mathrm{Cp} \odot$

$12 \odot \odot \odot \odot \odot \odot$ !Nterms: polynomial, exponential, cosh, sinh

$30.0 \quad 0.0$

$69.0 \quad 850.0$

$98.0 \quad 2000.0$

$\wedge \wedge \wedge \wedge \wedge \wedge \wedge \wedge \wedge \wedge \wedge \wedge \wedge \wedge \wedge \wedge \wedge \wedge \wedge \wedge \wedge \wedge \wedge \wedge \wedge \wedge \wedge \wedge \wedge \wedge \wedge \wedge \wedge \wedge \wedge \wedge \wedge \wedge \wedge \wedge \wedge \wedge \wedge \wedge \wedge \wedge \wedge \wedge \wedge \wedge \wedge \wedge \wedge \wedge \wedge \wedge \wedge \wedge \wedge \wedge \wedge \wedge \wedge \wedge \wedge \wedge \wedge \wedge \wedge \wedge \wedge \wedge \wedge \wedge \wedge \wedge \wedge \wedge \wedge \wedge$

\#ETA !---Viscosity---

VS7 !Pure fluid viscosity model for P0E5 of Huber (2018).

?

?

? Estimated uncertainty is $5 \%$ for the liquid over the temperature range $275 \mathrm{~K}$ to $430 \mathrm{~K}$ at pressures to $140 \mathrm{MPa}$.

? Larger uncertainties in the gas phase; data unavailable for comparisons.

$?$

250 .

1000 .

150000 .

3.

CIO

$\odot .9838$

706.7418

$1.0 \quad 1.0$

$1.0 \quad 0.5$

! Lower temperature limit [K]

!Upper temperature limit [K]

!Upper pressure limit [kPa]

!Maximum density [mol/L]

!Pointer to reduced effective collision cross-section model

!Lennard-Jones coefficient sigma [nm]

! Lennard-Jones coefficient epsilon/kappa [K]

!Reducing parameters for eta, T, rho

!Chapman-Enskog term 


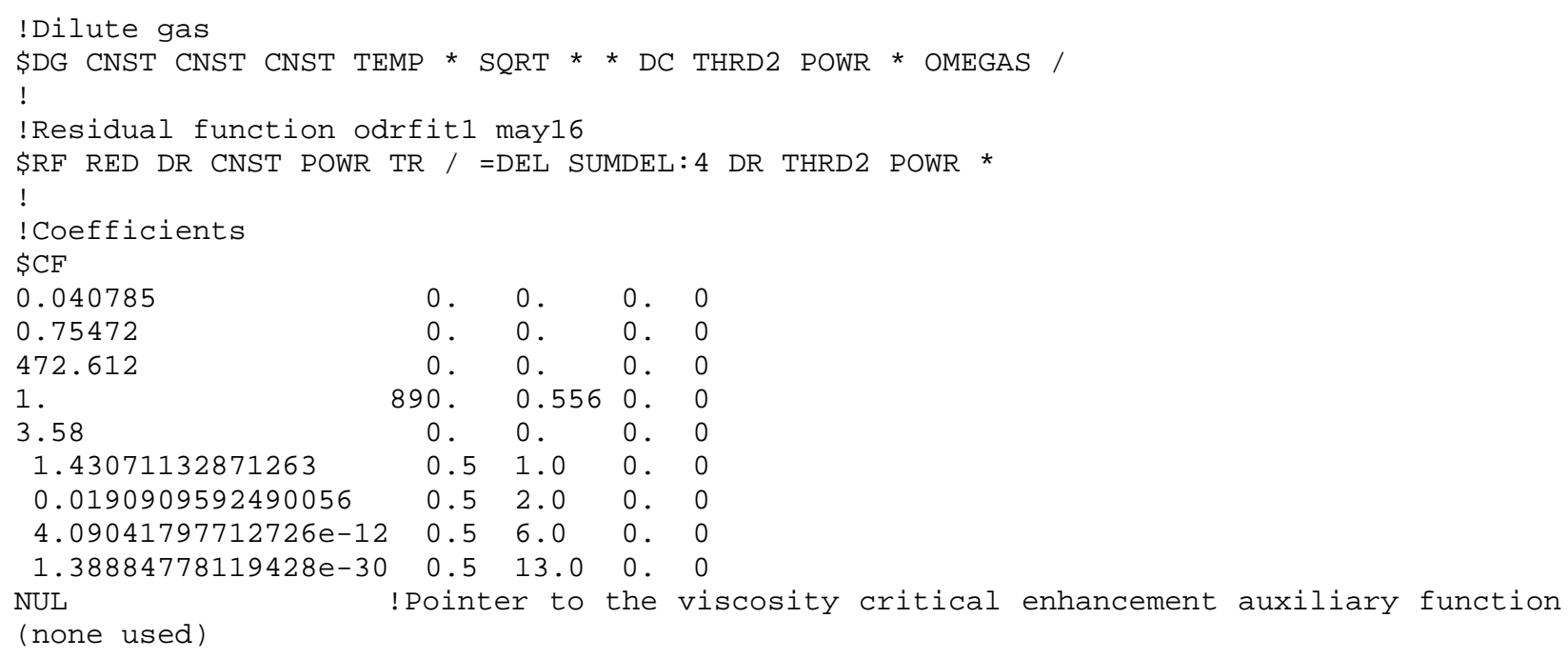


?Perkins, R.A., Sengers, J.V., Abdulagatov, I.M., and Huber, M.L.,

? "Simplified model for the critical thermal-conductivity enhancement in molecular fluids"

? Int. J. Thermophysics, 34(2):191-212, 2013. doi: 10.1007/s10765-013-1409-z

$?$

!

$\odot$.

10000 .

○.

$\odot$.

9000

$1.0 \quad 1.0 \quad 1.0$

0.63

1.239

1.02

0.063

1.0

often set to 1 )

$0.343 e-9$

$\odot .082$

1. $218 \mathrm{e}-9$

1335.0

!\# terms: c02-terms, spare, spare, spare

!Reducing parameters for $\mathrm{T}$, rho, $\mathrm{tcx}[\mathrm{mW} /(\mathrm{m}-\mathrm{K})]$

! Nu (universal exponent)

!Gamma (universal exponent)

! R० (universal amplitude)

!Z (universal exponent--not used for t.c., only viscosity)

!C (constant in viscosity eqn $=1 /\left[2-(\right.$ alpha + gamma $\left.) /\left(2^{*} n u\right)\right]$, but

! Xi@ (amplitude) [m]

! Gamo (amplitude) [-]

!Qd_inverse (modified effective cutoff parameter) [m]

! Tref (reference temperature) $=1.5^{*} \mathrm{Tc}[\mathrm{K}]$

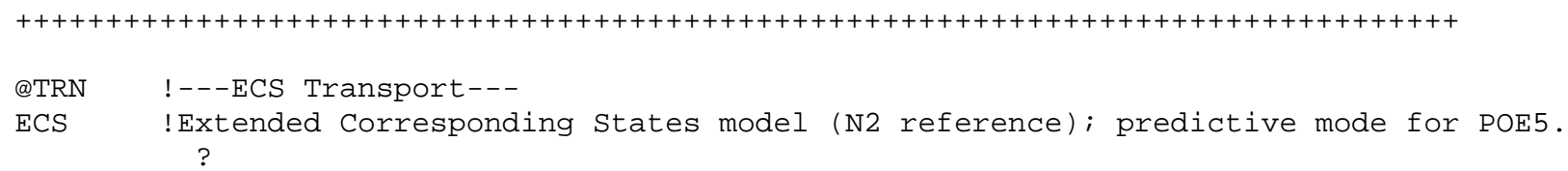

?The Lennard-Jones parameters were taken from Reid, R.C., Prausnitz, J.M., and Poling, B.E., "The Properties of Gases and Liquids," 4th edition, New York, McGraw-Hill Book Company, 1987. ?

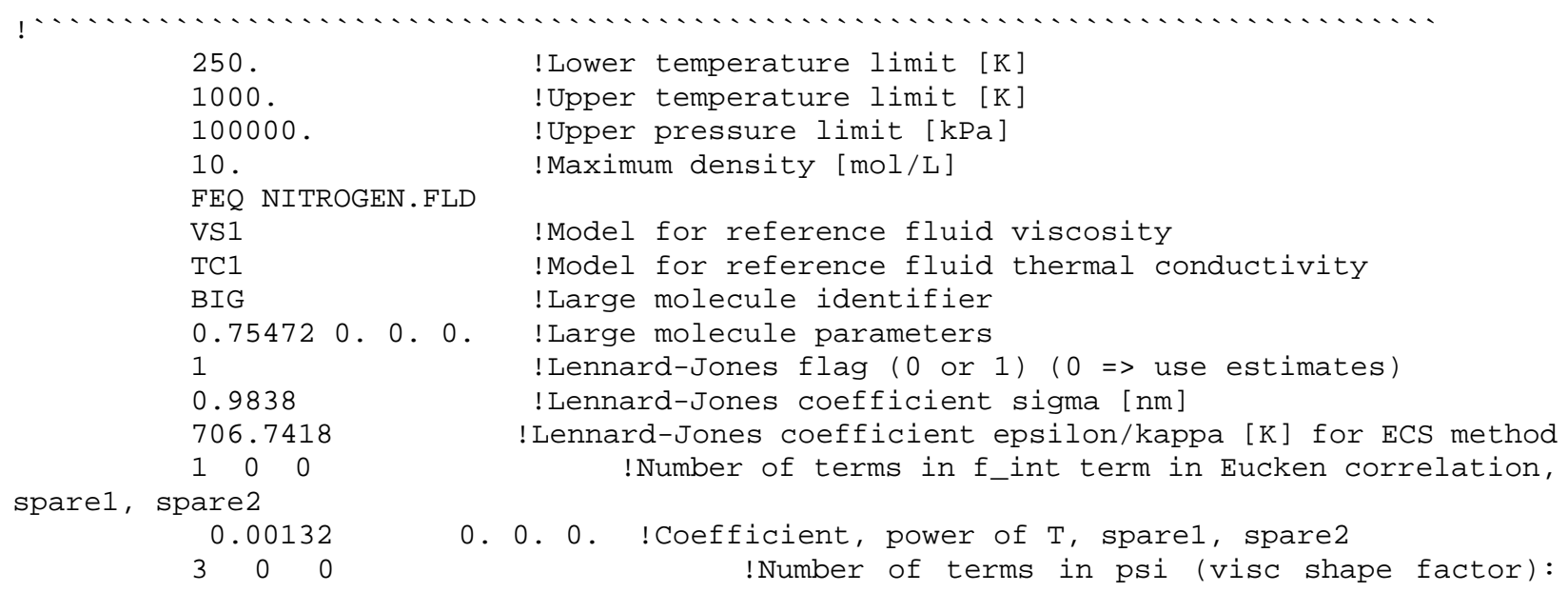

250 .

1000 .

100000 .

10.

FEQ NITROGEN. FLD

VS1

TC1

BIG

$\odot .75472$ ๑. $\odot . \odot$.

1

0.9838

706.7418

$\begin{array}{lll}1 & 0 & 0\end{array}$

spare1, spare2$$
\odot .00132
$$$$
\begin{array}{lll}
3 & 0 & 0
\end{array}
$$

! Lower temperature limit [K]

!Upper temperature limit [K]

!Upper pressure limit [kPa]

!Maximum density [mol/L]

!Model for reference fluid viscosity

!Model for reference fluid thermal conductivity

! Large molecule identifier

! Large molecule parameters

! Lennard-Jones flag ( $\odot$ or 1$)(\odot \Rightarrow$ use estimates)

! Lennard-Jones coefficient sigma [nm]

! Lennard-Jones coefficient epsilon/kappa [K] for ECS method ! Number of terms in $f_{\text {_int }}$ term in Eucken correlation,

๑. ๑. ๑. ! Coefficient, power of T, spare1, spare2

! Number of terms in psi (visc shape factor):

poly, spare1, spare2 


1.09271
-0.161324
0.0486596
1000
poly, spare1, spare 2
$1 . \Theta$
TK3

1.09271

0.0486596

TK3
๑. ๑. ๑. !Coefficient, power of $\mathrm{Tr}$, power of $\mathrm{Dr}$, spare

$\odot$. 1. $\odot$. !Coefficient, power of $\mathrm{Tr}$, power of $\mathrm{Dr}$, spare

$\odot$ ๑ 2. ๑. !Coefficient, power of $\mathrm{Tr}$, power of $\mathrm{Dr}$, spare ! Number of terms in chi (t.c. shape factor):

๑. ๑. ๑. !Coefficient, power of $\mathrm{Tr}$, power of $\mathrm{Dr}$, spare !Pointer to critical enhancement auxiliary function

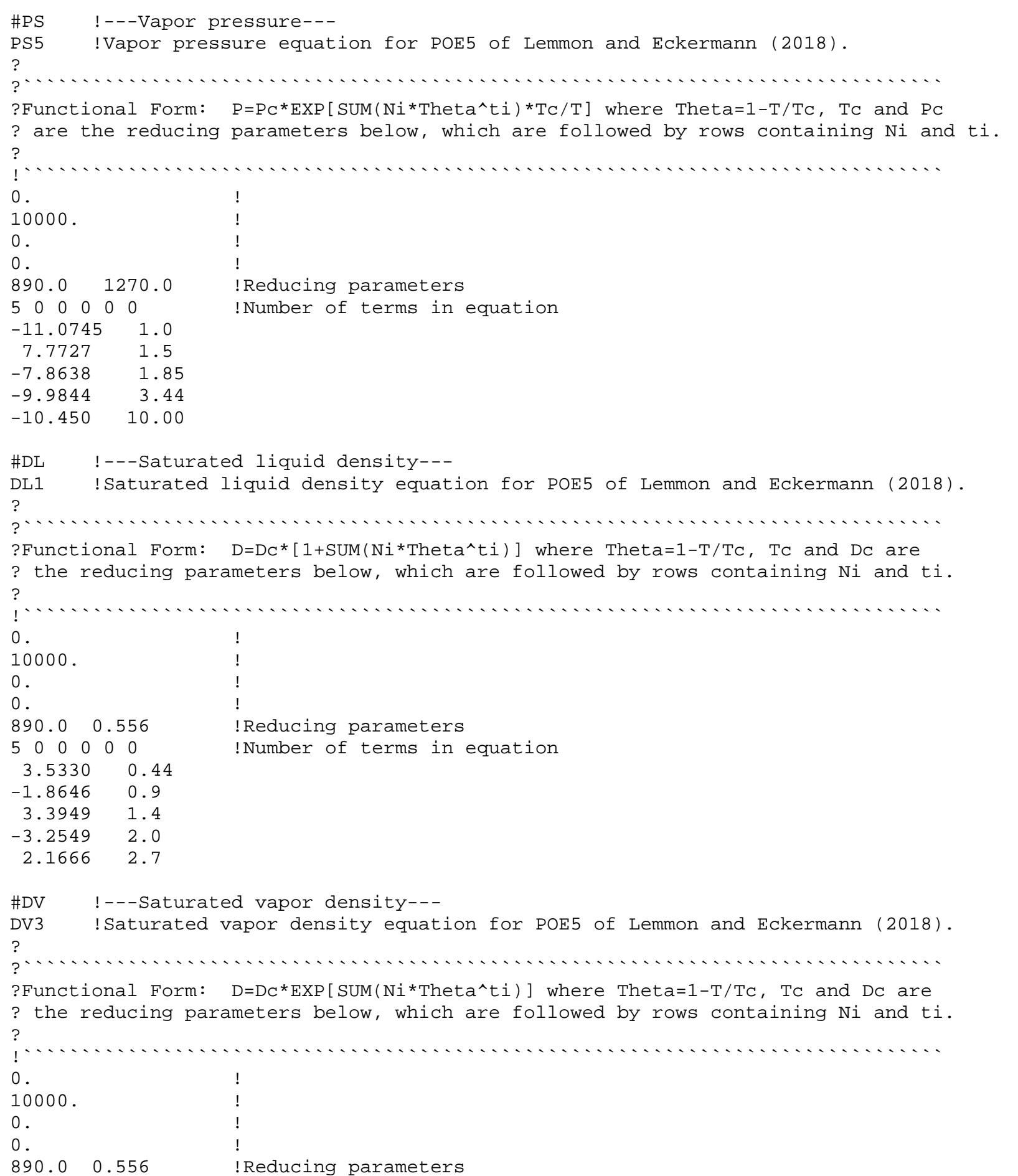




$\begin{array}{ll}5 \odot \odot \odot \odot \odot \\ -4.8478 & 0.483 \\ -9.9537 & 1.5 \\ -28.962 & 3.08 \\ -81.61 & 5.5 \\ -280.12 & 12.0\end{array}$

! Number of terms in equation

@END

$\begin{array}{lllllllll}\mathrm{C} & 1 & 2 & 3 & 4 & 5 & 6 & 7\end{array}$

c2345678901234567890123456789012345678901234567890123456789012345678901234567890

\title{
C.2. POE7.FLD
}

\author{
P0E7 !Short name \\ 25811-35-2 !CAS number \\ pentaerythritol tetraheptanoate !Full name \\ C33H6008 !Chemical formula \\ POE7 ! Synonym \\ 584.835 !Molar mass $[\mathrm{g} / \mathrm{mol}]$ \\ 250. \\ 770.561 \\ 940.0 \\ 1030.0 \\ 0.412 \\ 1.06 \\ -1 . \\ NBP \\ 10.0 \\ !Triple point temperature [K] \\ !Normal boiling point [K] \\ !Critical temperature [K] \\ !Critical pressure [ $\mathrm{kPa}]$ \\ !Critical density [mol/L] \\ !Acentric factor \\ !Dipole moment [Debye] \\ ! Default reference state \\ !Version number \\ ! compiled by E.W. Lemmon, NIST Physical and Chemical Properties Division, Boulder, \\ Colorado \\ ! 12-01-17 EWL, Original version. \\ ! ๑8-09-18 EWL, Add final equation of state. \\ ! 09-08-18 MLH, Add viscosity correlation. \\ ! ०9-๑9-18 MLH, Add thermal conductivity correlation.
}

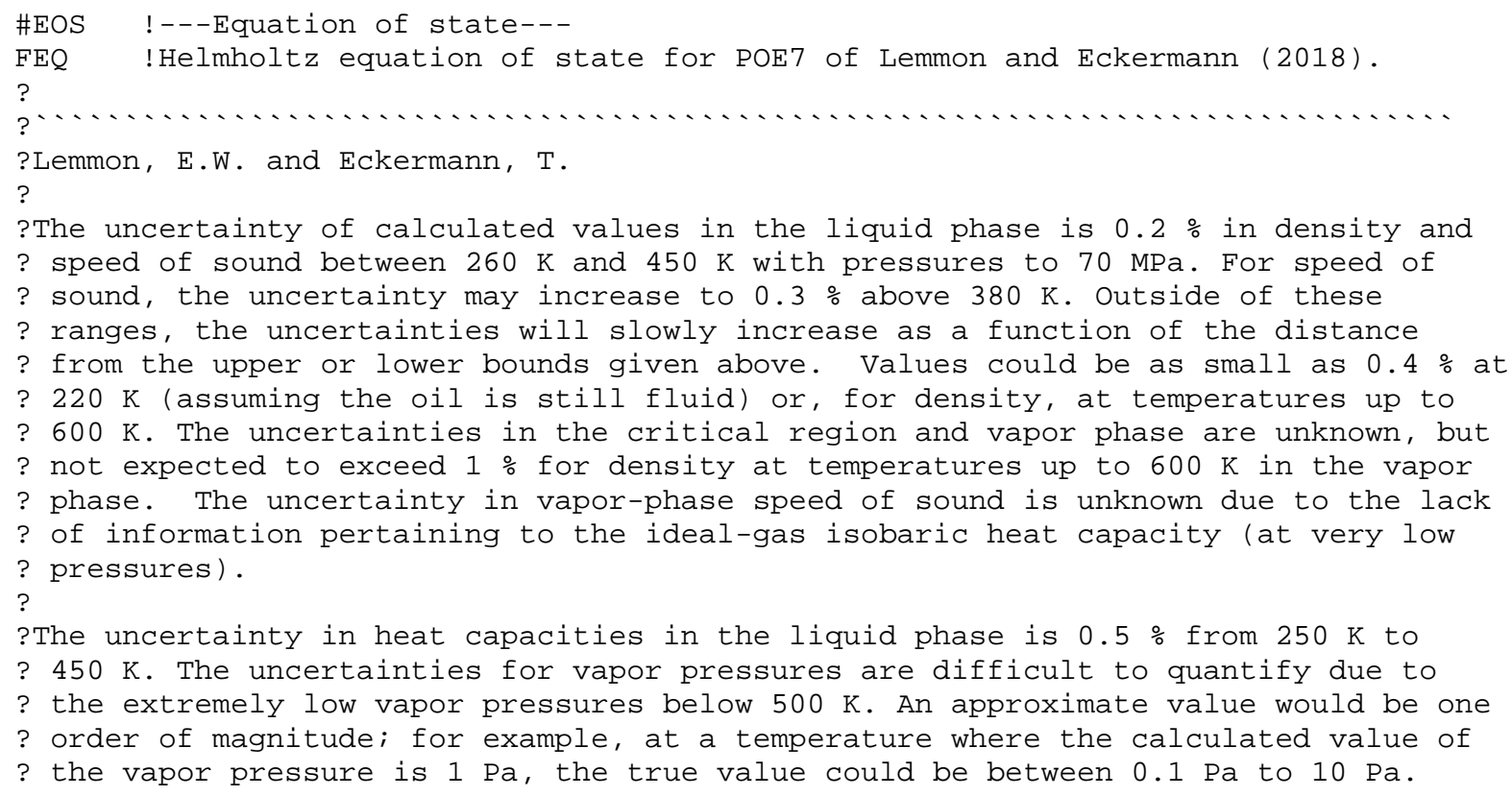


? For vapor pressures above 1 atmosphere, the same may be true, but it is ? estimated that the values would be less than $50 \%$.

?

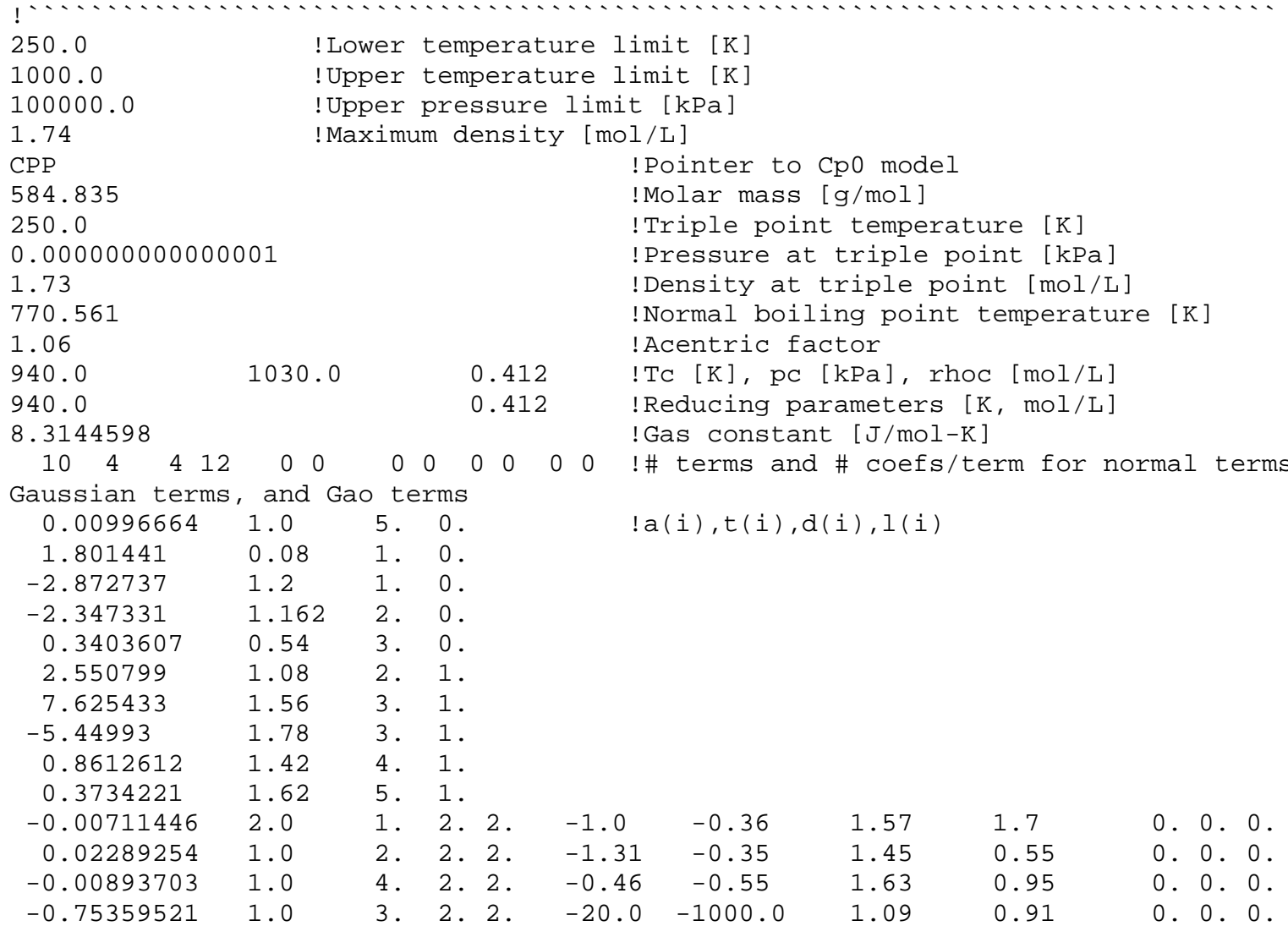

\#AUX !---Auxiliary function for cp९

CPP !Ideal gas heat capacity function for P0E7 of Lemmon and Eckermann (2018). ?

$\wedge \wedge \wedge \wedge \wedge \wedge \wedge \wedge \wedge \wedge \wedge \wedge \wedge \wedge \wedge \wedge \wedge \wedge \wedge \wedge \wedge \wedge \wedge \wedge \wedge \wedge \wedge \wedge \wedge \wedge \wedge \wedge \wedge \wedge \wedge \wedge \wedge \wedge \wedge \wedge \wedge \wedge \wedge \wedge \wedge \wedge \wedge \wedge \wedge \wedge \wedge \wedge \wedge \wedge \wedge \wedge \wedge \wedge \wedge \wedge \wedge \wedge \wedge \wedge \wedge \wedge \wedge \wedge \wedge \wedge \wedge \wedge \wedge \wedge \wedge \wedge \wedge \wedge \wedge \wedge$

\#ETA !---Viscosity---

VS7 !Pure fluid viscosity model for POE7 of Huber (2018).

?

?

? Estimated uncertainty is 5 \% for the liquid over the temperature range $280 \mathrm{~K}$ to 450

$\mathrm{K}$ at pressures to $140 \mathrm{MPa}$.

? Larger uncertainties in the gas phase; data unavailable for comparisons.

? 


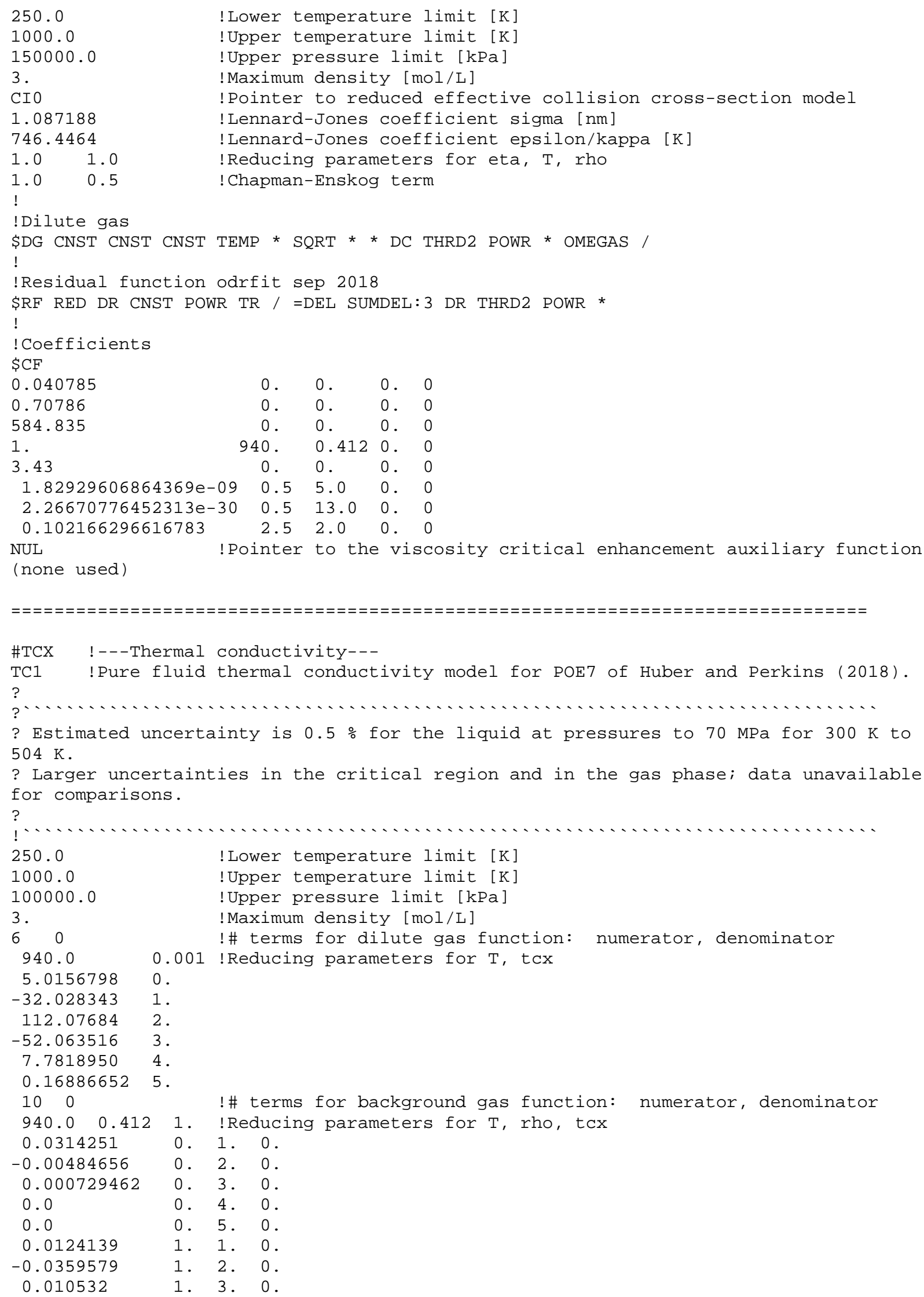




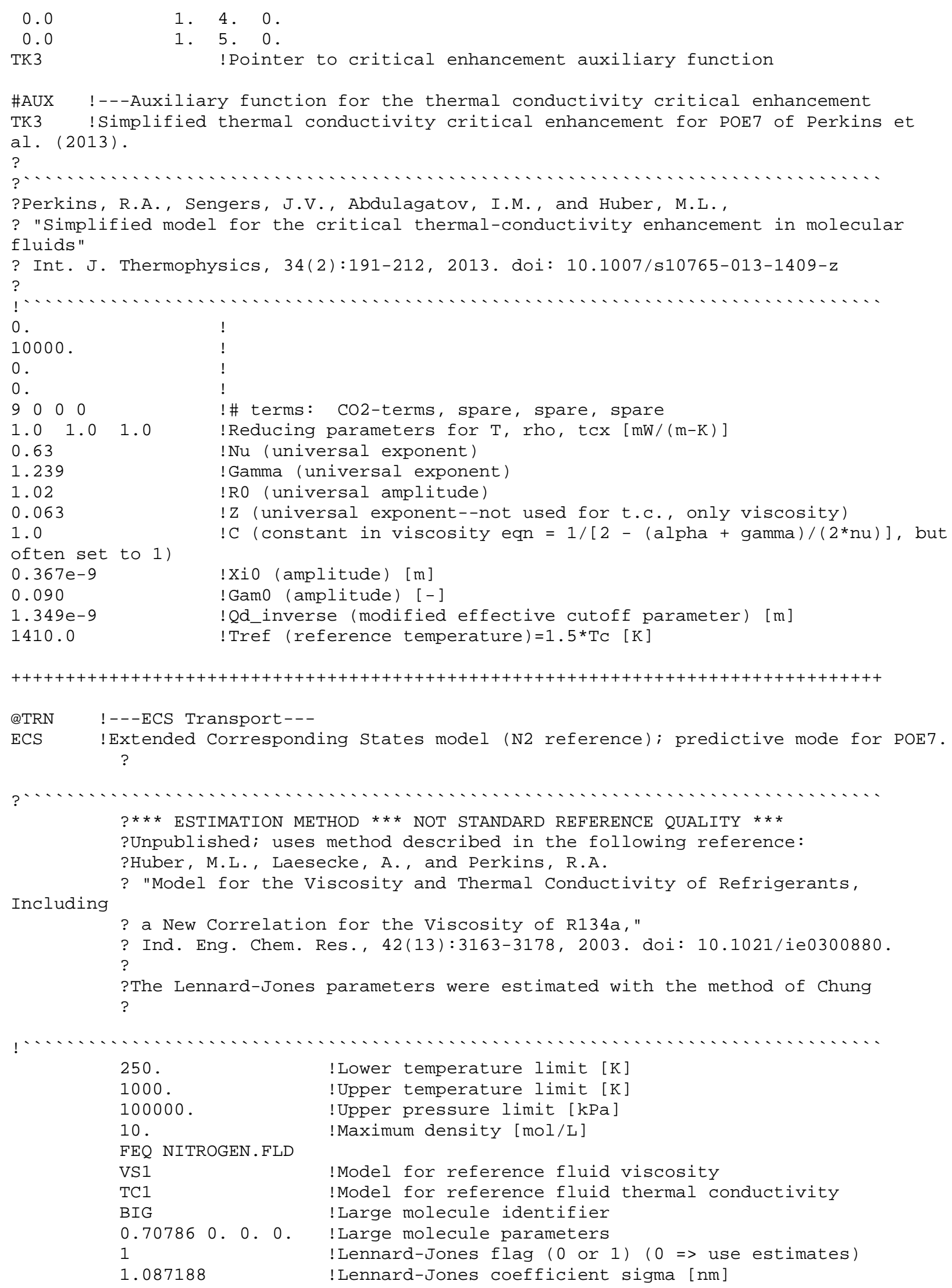

250 .

1000 .

100000 .

10.

FEQ NITROGEN. FLD

VS1

TC1

BIG

$\odot .70786$ ๑. ๑. ๑. 1

1.087188

! Lower temperature limit [K]

!Upper temperature limit [K]

!Upper pressure limit [kPa]

!Maximum density [mol/L]

!Model for reference fluid viscosity

!Model for reference fluid thermal conductivity

! Large molecule identifier

! Large molecule parameters

! Lennard-Jones flag ( $\odot$ or 1$)(\odot \Rightarrow$ use estimates)

! Lennard-Jones coefficient sigma [nm] 
method

746.4464

$1 \odot \odot$

correlation, spare1, spare2

0.00132

300

poly, spare1, spare2

1.09271

$-0.161324$

0.0486596

100

poly, spare1, spare2

1.0

TK3
! Lennard-Jones coefficient epsilon/kappa [K] for ECS

! Number of terms in $f$ int term in Eucken

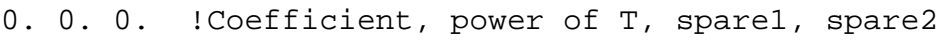

! Number of terms in psi (visc shape factor):

๑. ๑. ๑. !Coefficient, power of $\mathrm{Tr}$, power of $\mathrm{Dr}$, spare

$\odot$. 1. ๑. !Coefficient, power of $\mathrm{Tr}$, power of $\mathrm{Dr}$, spare

$\odot$. 2. $\odot$. ! Coefficient, power of $\mathrm{Tr}$, power of $\mathrm{Dr}$, spare ! Number of terms in chi (t.c. shape factor):

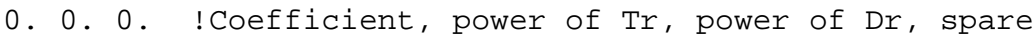
!Pointer to critical enhancement auxiliary function

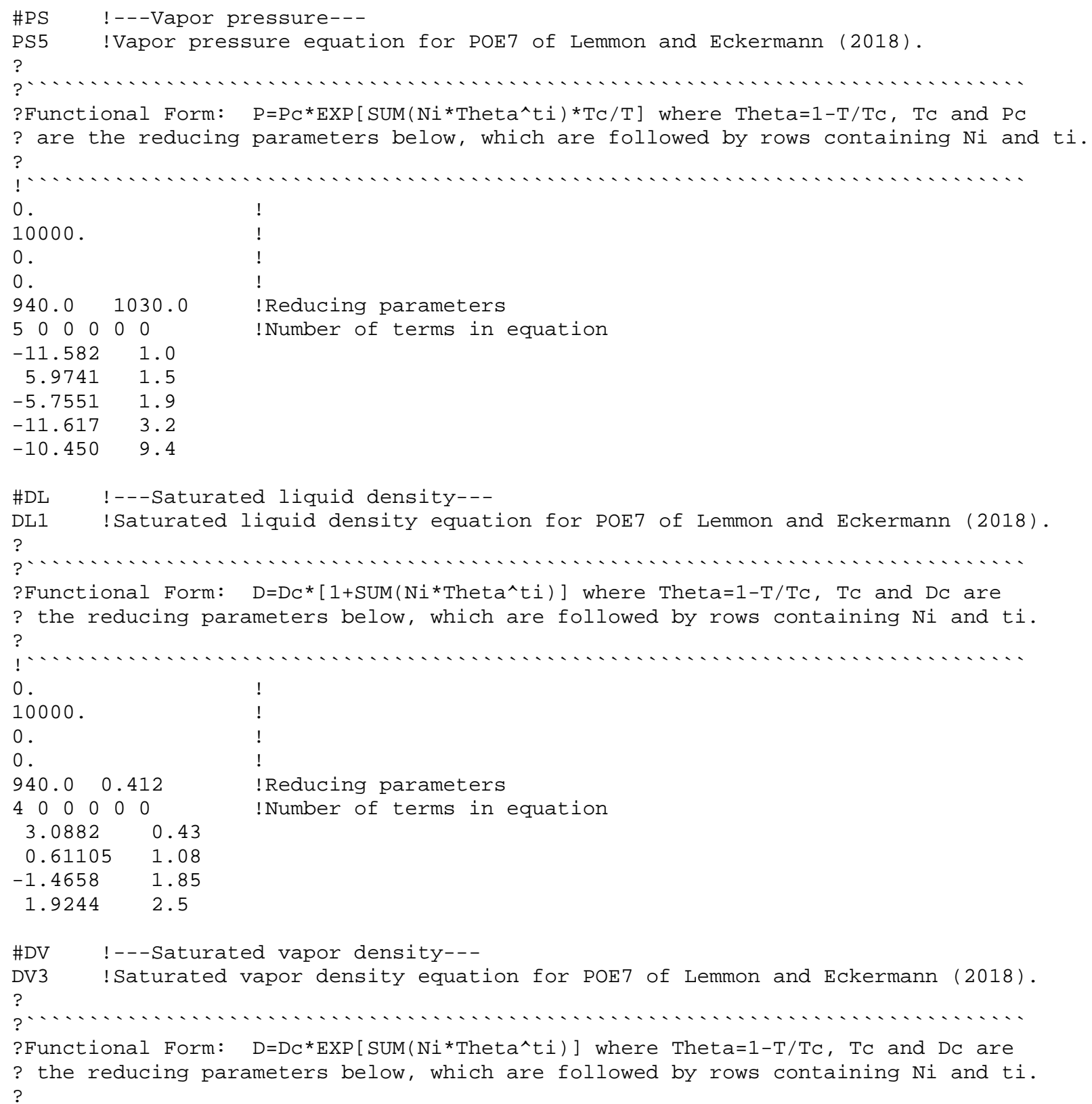




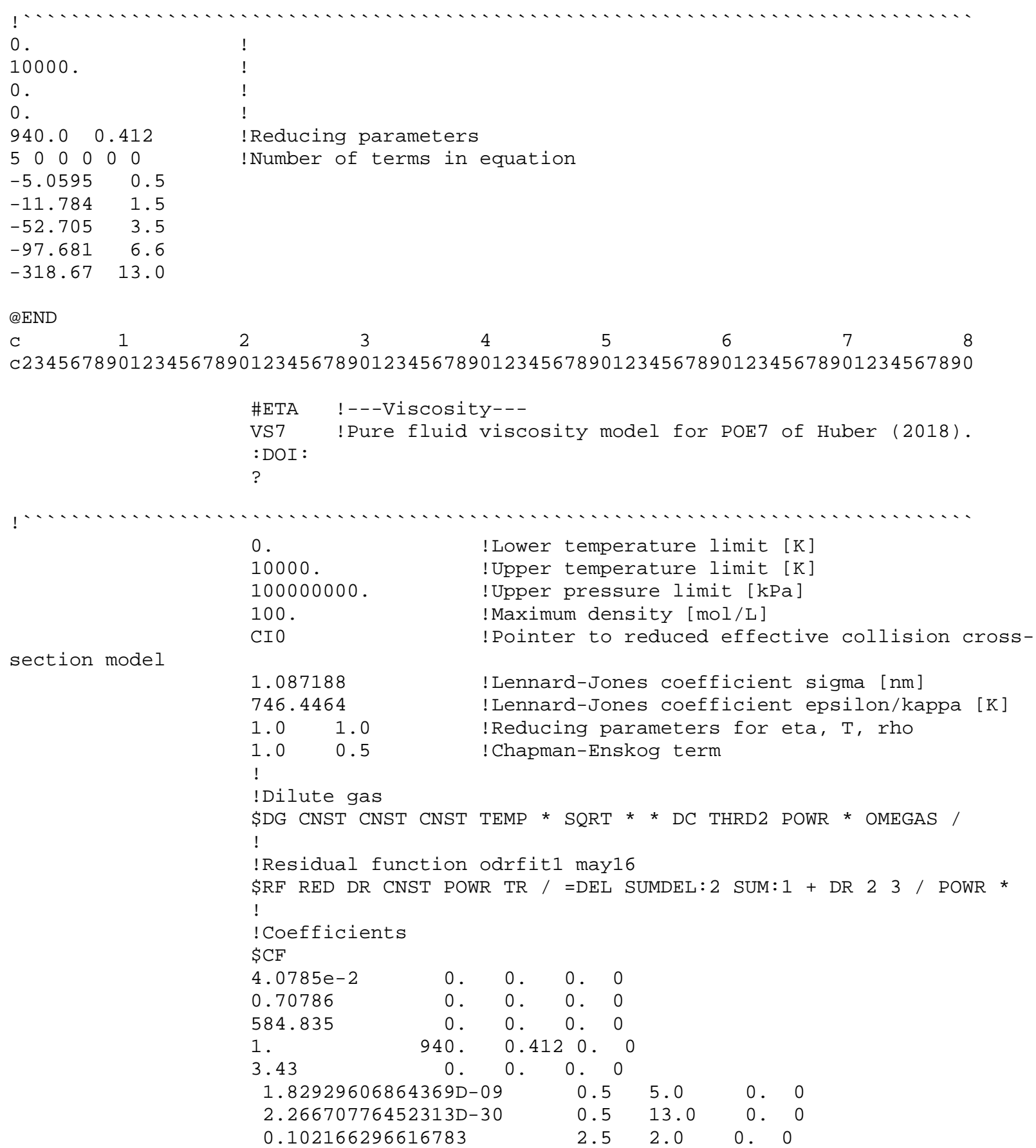

auxiliary function (none used)

!Pointer to the viscosity critical enhancement

\#ETA !---Viscosity---

VS7 !Pure fluid viscosity model for POE7 of Huber (2018).

$\operatorname{aug} 20.4 \mathrm{fit}$

:DOI :

?

$\odot$.

10000 . 100000000
! Lower temperature limit [K]

!Upper temperature limit [K]

!Upper pressure limit [kPa] 


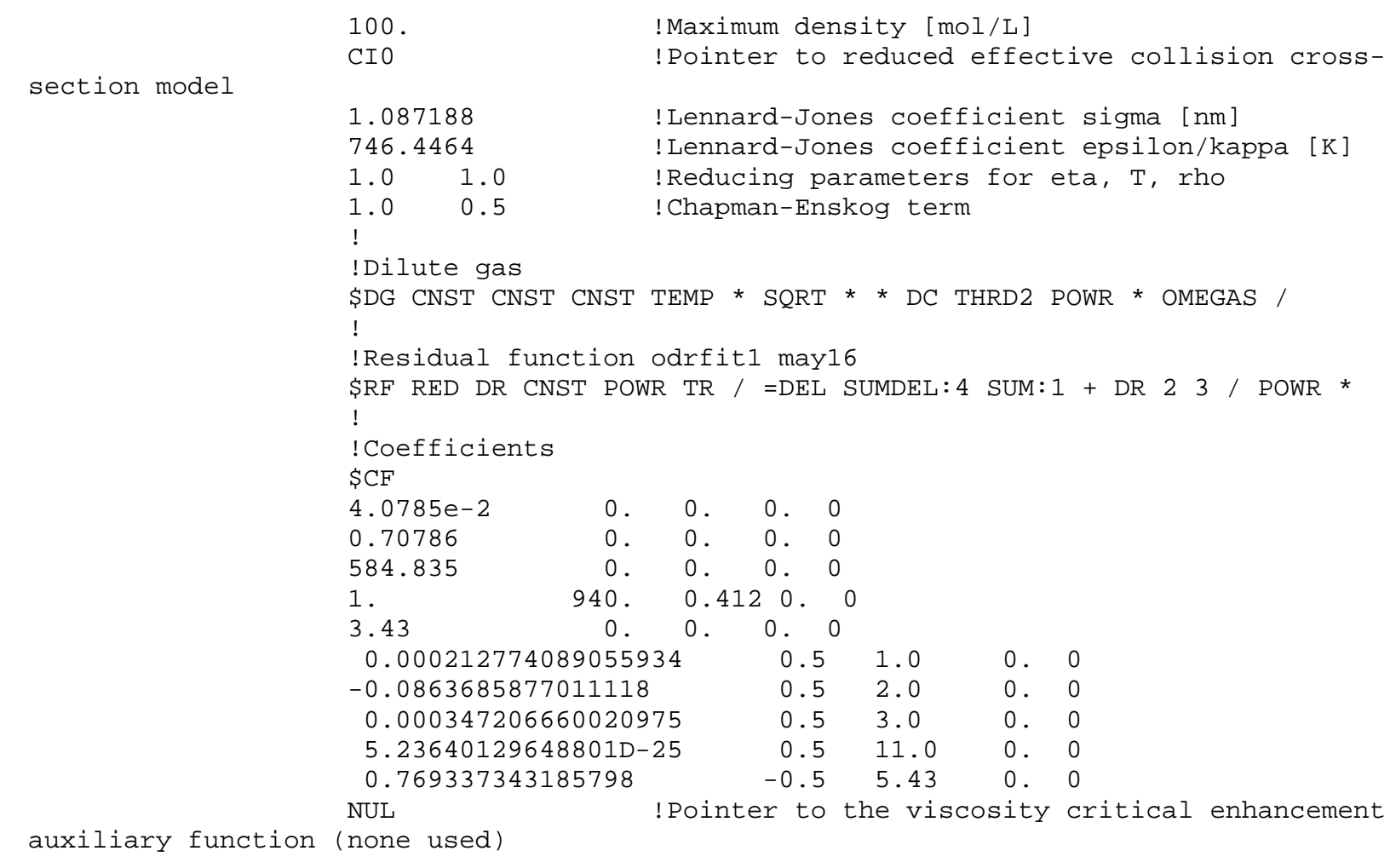

\section{C.3. POE9.FLD}

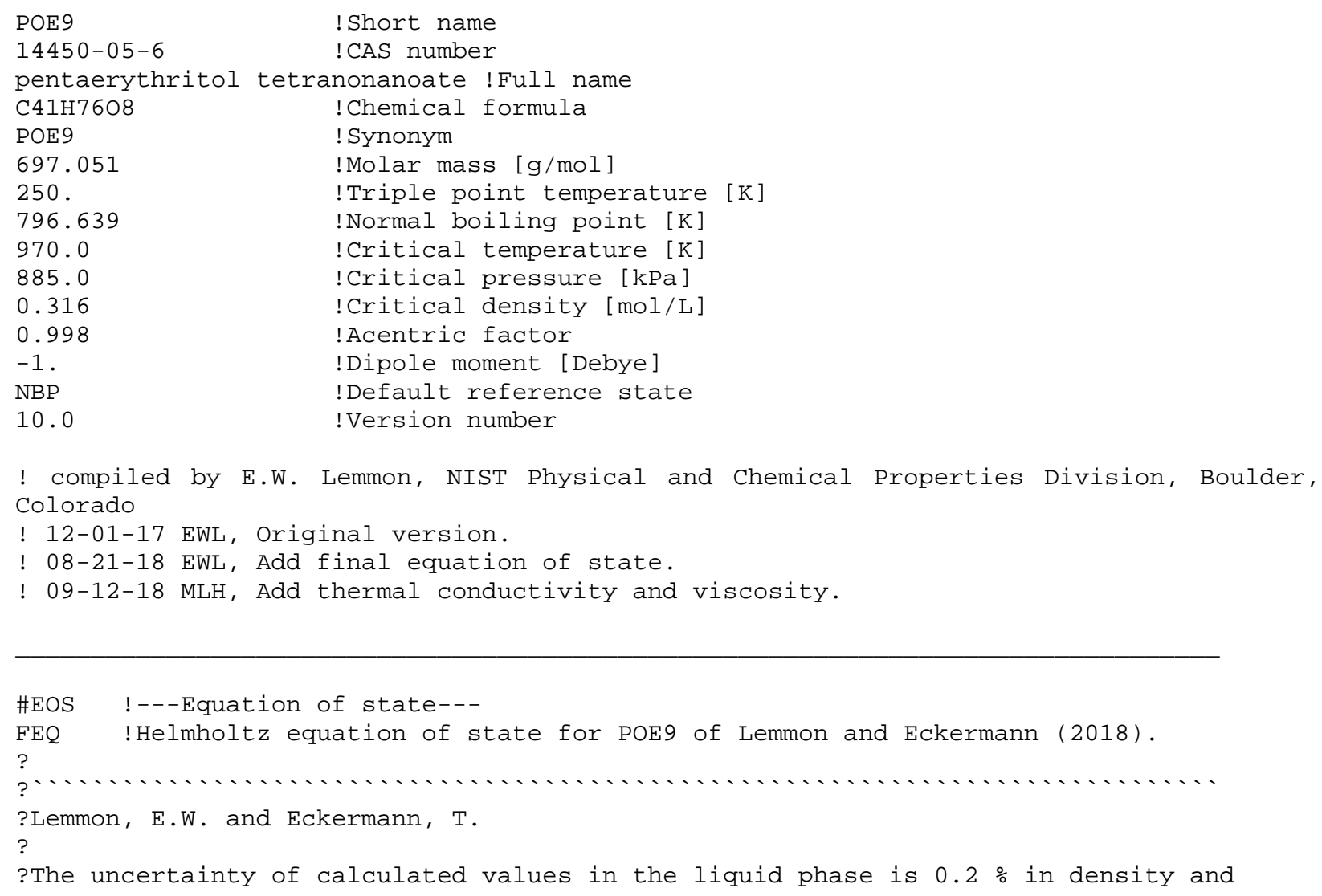

! compiled by E.W. Lemmon, NIST Physical and Chemical Properties Division, Boulder, Colorado 
? speed of sound between $260 \mathrm{~K}$ and $450 \mathrm{~K}$ with pressures to $70 \mathrm{MPa}$. For speed of ? sound, the uncertainty may increase to $0.3 \%$ above $380 \mathrm{~K}$. Outside of these

? ranges, the uncertainties will slowly increase as a function of the distance ? from the upper or lower bounds given above. Values could be as small as $0.4 \%$ at

? $220 \mathrm{~K}$ (assuming the oil is still fluid) or, for density, at temperatures up to

? $600 \mathrm{~K}$. The uncertainties in the critical region and vapor phase are unknown, but

? not expected to exceed $1 \%$ for density at temperatures up to $600 \mathrm{~K}$ in the vapor

? phase. The uncertainty in vapor-phase speed of sound is unknown due to the lack

? of information pertaining to the ideal-gas isobaric heat capacity (at very low

? pressures).

$?$

?The uncertainty in heat capacities in the liquid phase are $0.5 \%$ from $250 \mathrm{~K}$ to

? $450 \mathrm{~K}$, and increases to 1 \% or more below $330 \mathrm{~K}$. The uncertainties for vapor

? pressures are difficult to quantify due to the extremely low vapor pressures

? below $500 \mathrm{~K}$. An approximate value would be one order of magnitude; for example,

? at a temperature where the calculated value of the vapor pressure is $1 \mathrm{~Pa}$, the

? true value could be between $0.1 \mathrm{~Pa}$ to $10 \mathrm{~Pa}$. For vapor pressures above 1

? atmosphere, the same may be true, but it is estimated that the values would be

? less than $50 \%$.

?

250.0

1000.0

100000.0

1.43

CPP

697.051

250.0

0.000000000000001

1.42

796.639

0.998

970.0

970.0

8.3144598

104

Gaussian terms, and Gao terms

$0.00811 \quad 1.0 \quad 5.0$.

1. 446665

0.08

1.

$-2.303871$

$-2.320483$

0.336244

2. 383751

8. 210469

$-6.200227$

$\odot .813201$

$\odot .377317$

$\odot .0023328$

$\odot .0217847$

$-0.009014$

$-1.54251$

1.1622.

0.54 3. $\odot$.

1.08 2. 1.

1.56 3. 1 .

1.78 3. 1.

1.42 4. 1.

1.625 .1
! Lower temperature limit [K]

!Upper temperature limit [K]

!Upper pressure limit [kPa]

!Maximum density [mol/L]

! Pointer to $\mathrm{Cp} \odot$ model

! Molar mass $[\mathrm{g} / \mathrm{mol}]$

!Triple point temperature [K]

!Pressure at triple point [ $\mathrm{kPa}]$

!Density at triple point [mol/L]

! Normal boiling point temperature [K]

! Acentric factor

! Tc $[\mathrm{K}]$, pc $[\mathrm{kPa}]$, rhoc $[\mathrm{mol} / \mathrm{L}]$

! Reducing parameters $[\mathrm{K}, \mathrm{mol} / \mathrm{L}]$

! Gas constant [ $\mathrm{J} / \mathrm{mol}-\mathrm{K}]$

$\odot \odot$ !\# terms and \# coefs/term for normal terms,

\#AUX !---Auxiliary function for Cp॰

CPP !Ideal gas heat capacity function for POE9 of Lemmon and Eckermann (2018).

?

?Lemmon, E.W. and Eckermann, T.

?

!

0 .

10000 .

$\odot$.

$\odot$.

$2.0 \quad 1.2 .2 . \quad-1.0 \quad-0.36 \quad 1.57$

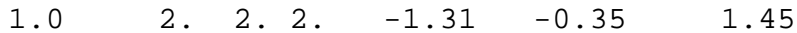

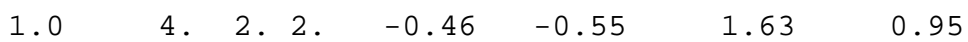

0.55

๑. ๑. ๑.

๑. 0.0 .

3. 2. 2. $-20.0-1000.0 \quad 1.09$

0.91

$\odot . \odot . \odot$.

$\odot . \odot . \odot$.

…… 


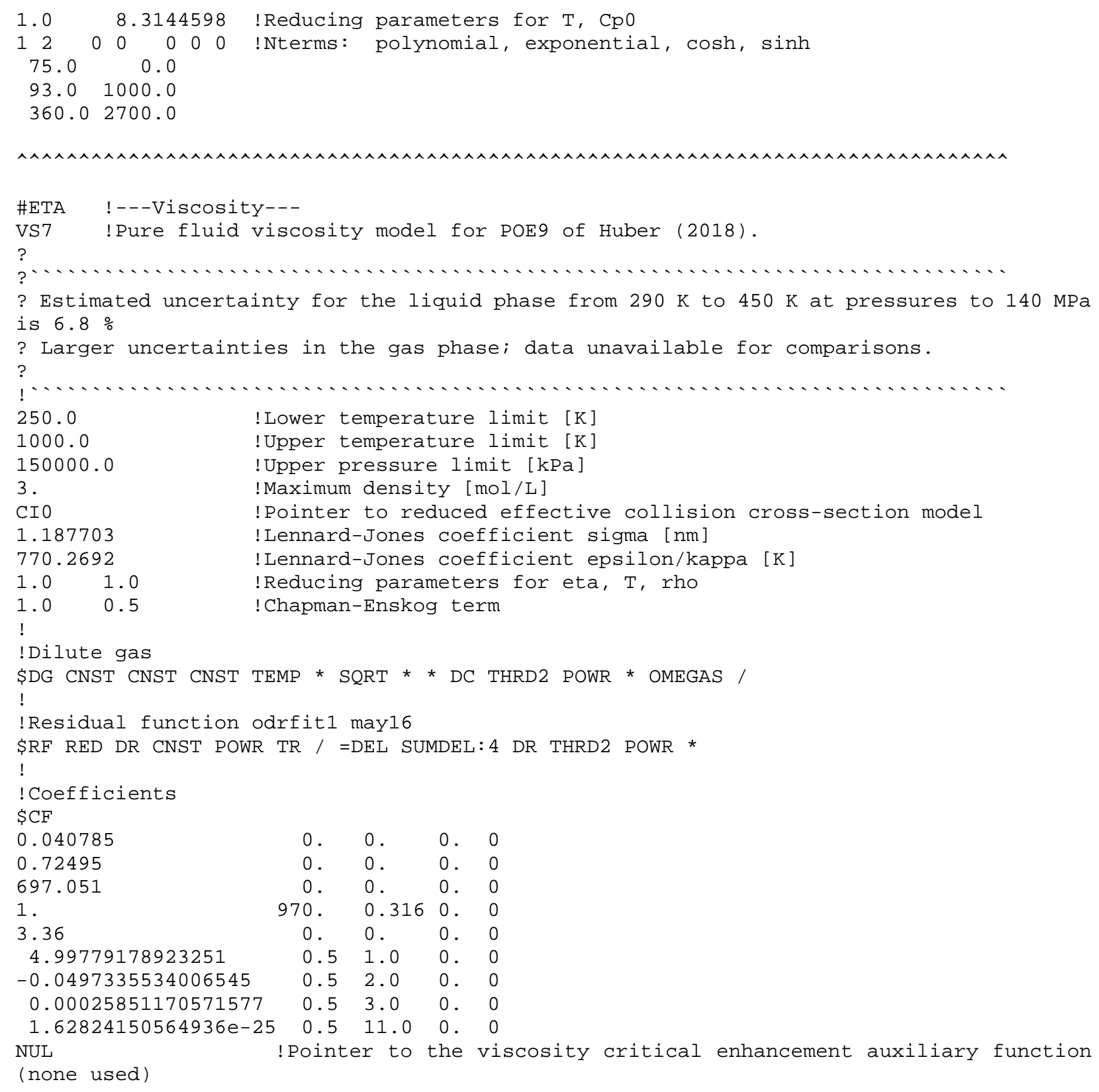




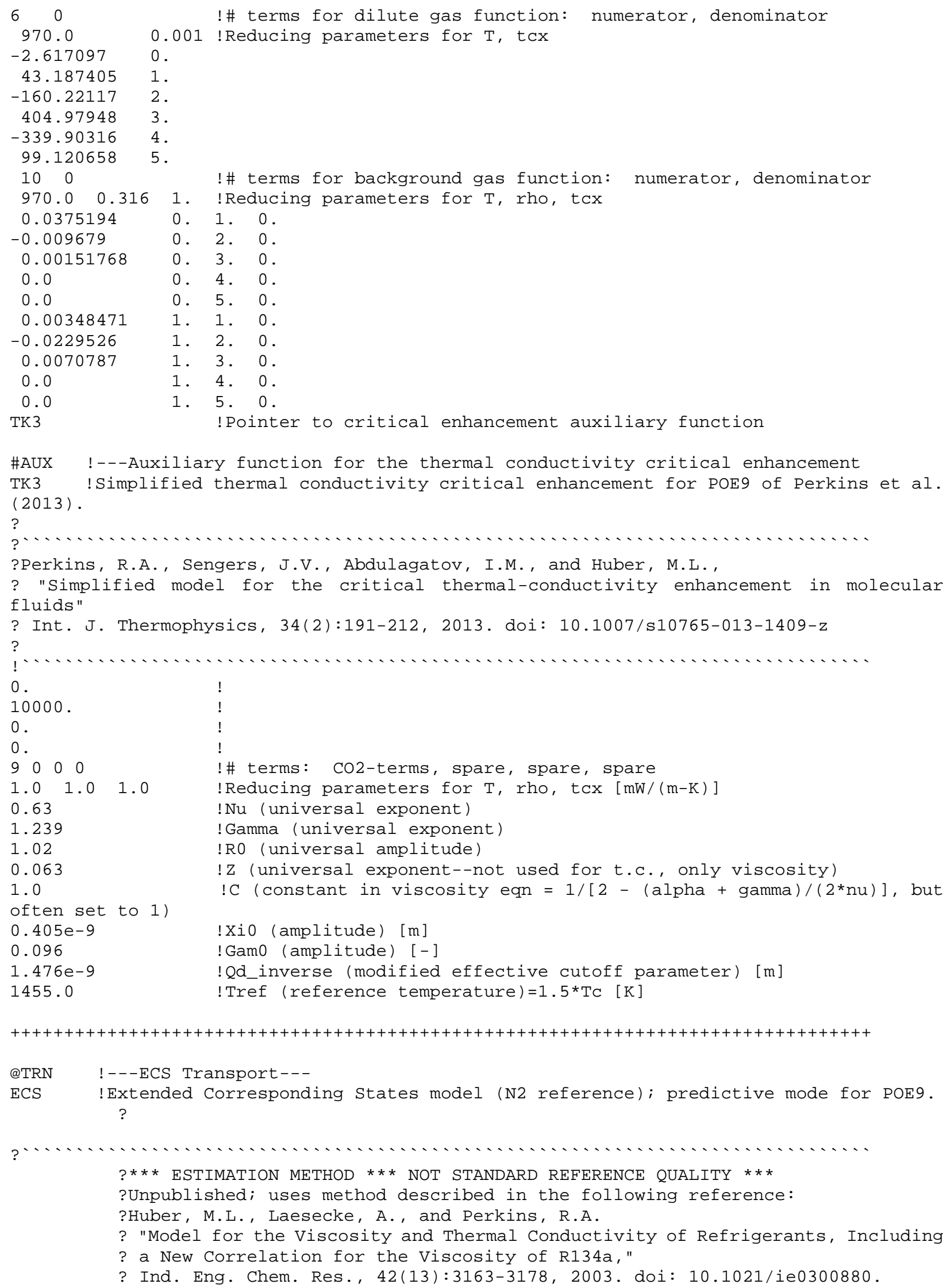


?

?The Lennard-Jones parameters were estimated with the method of Chung

?

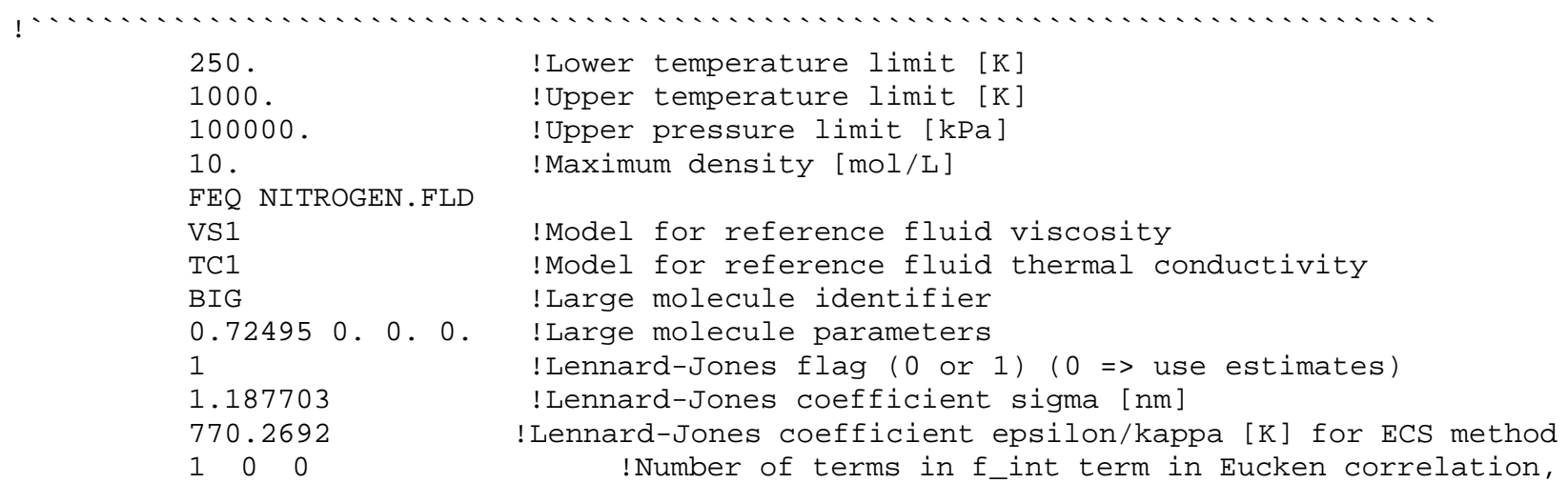

spare1, spare2
0.00132
๑. ๑. ๑. ! Coefficient, power of $T$, spare1, spare2

300

! Number of terms in psi (visc shape factor):

poly, spare1, spare2

1.09271

$-0.161324$

0.0486596

๑. ๑. ๑. !Coefficient, power of $\mathrm{Tr}$, power of $\mathrm{Dr}$, spare

$\odot$. 1. $\odot$. !Coefficient, power of $\mathrm{Tr}$, power of $\mathrm{Dr}$, spare

100

$\odot$. 2. $\odot$. !Coefficient, power of $\mathrm{Tr}^{\text {, }}$ power of $\mathrm{Dr}$, spare ! Number of terms in chi (t.c. shape factor):

poly, spare1, spare2

1.0

TK3

๑. ๑. ๑. !Coefficient, power of $\mathrm{Tr}$, power of $\mathrm{Dr}$, spare !Pointer to critical enhancement auxiliary function

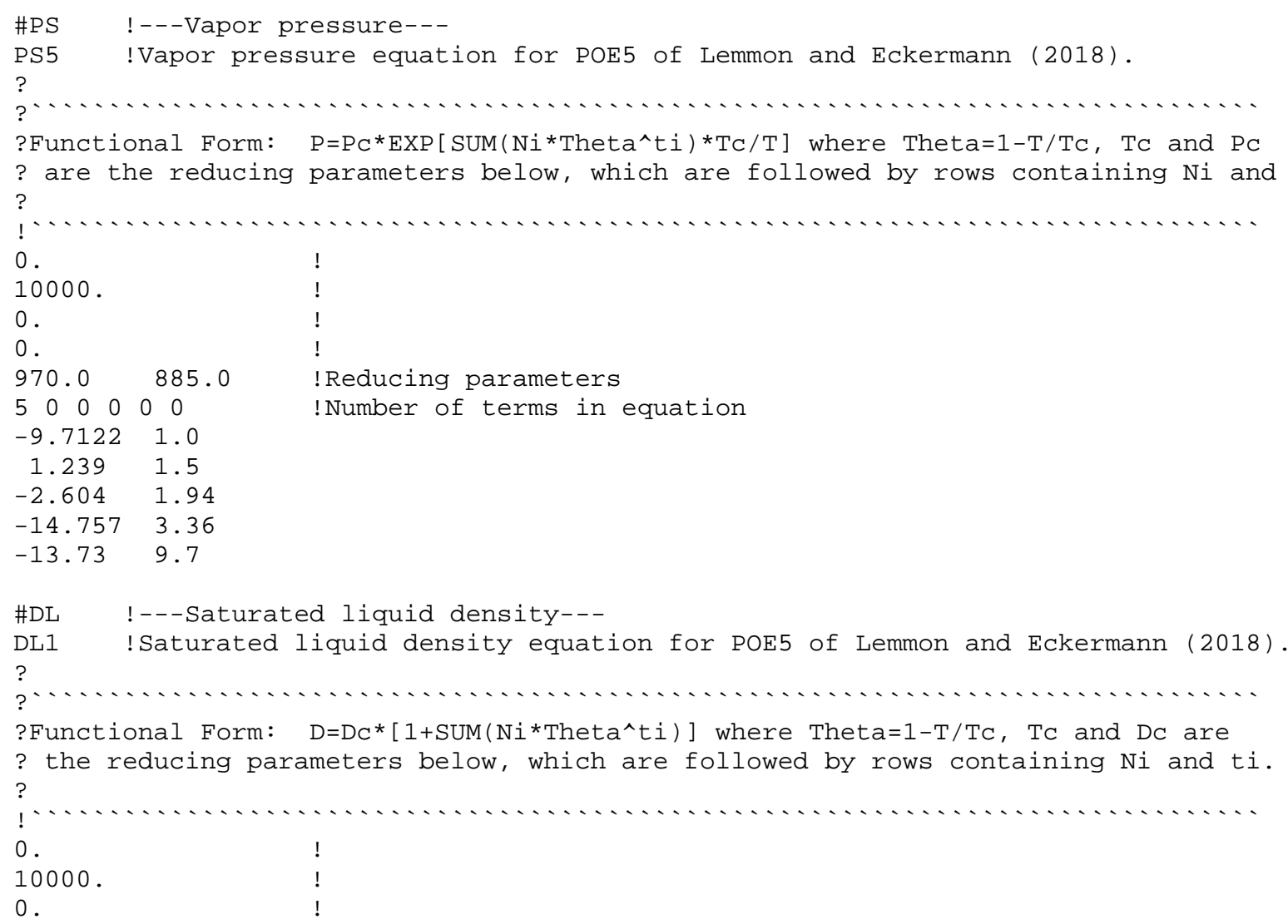


$\odot$.

$970.0 \quad 0.316$

$5 \odot \odot \odot \odot \odot$

$0.8426 \quad 0.23$

$7.992 \quad 0.8$

$-10.95 \quad 1.25$

$6.562 \quad 1.7$

$-0.02654 \quad 2.5$

\#DV !---Saturated vapor density---

DV3 !Saturated vapor density equation for POE5 of Lemmon and Eckermann (2018). ?

?Functional Form: $\mathrm{D}=\mathrm{DC}{ }^{*} \operatorname{EXP}\left[\operatorname{SUM}\left(\mathrm{Ni}^{*}{ }^{*}\right.\right.$ Theta^ti)] where Theta=1-T/TC, TC and $\mathrm{DC}$ are

? the reducing parameters below, which are followed by rows containing $\mathrm{Ni}$ and $\mathrm{ti}$. ?

\section{C.4. MILPRF23699.FLD}

MIL - PRF - 23699

1-1-1

MIL - PRF - 23699

MIL - PRF - 23699

MIL-PRF - 23699

557.6

250.

753.335

930.0

1080.0

0.439

$\odot .97$

-1 .

NBP

10.0
! Short name

! CAS number

! Full name

! Chemical formula

! Synonym

! Molar mass $[\mathrm{g} / \mathrm{mol}]$

!Triple point temperature [K]

!Normal boiling point [K]

!Critical temperature [K]

!Critical pressure [kPa]

!Critical density [mol/L]

! Acentric factor

!Dipole moment [Debye]

! Default reference state

!Version number

! compiled by E.W. Lemmon, NIST Physical and Chemical Properties Division, Boulder, Colorado

! 12-01-17 EWL, Original version.

! 08-29-18 EWL, Add final equation of state.

! 09-12-18 MLH, Add transport.

\#EOS !---Equation of state---

FEQ !Helmholtz equation of state for MIL-PRF-23699 of Lemmon (2018).

? 


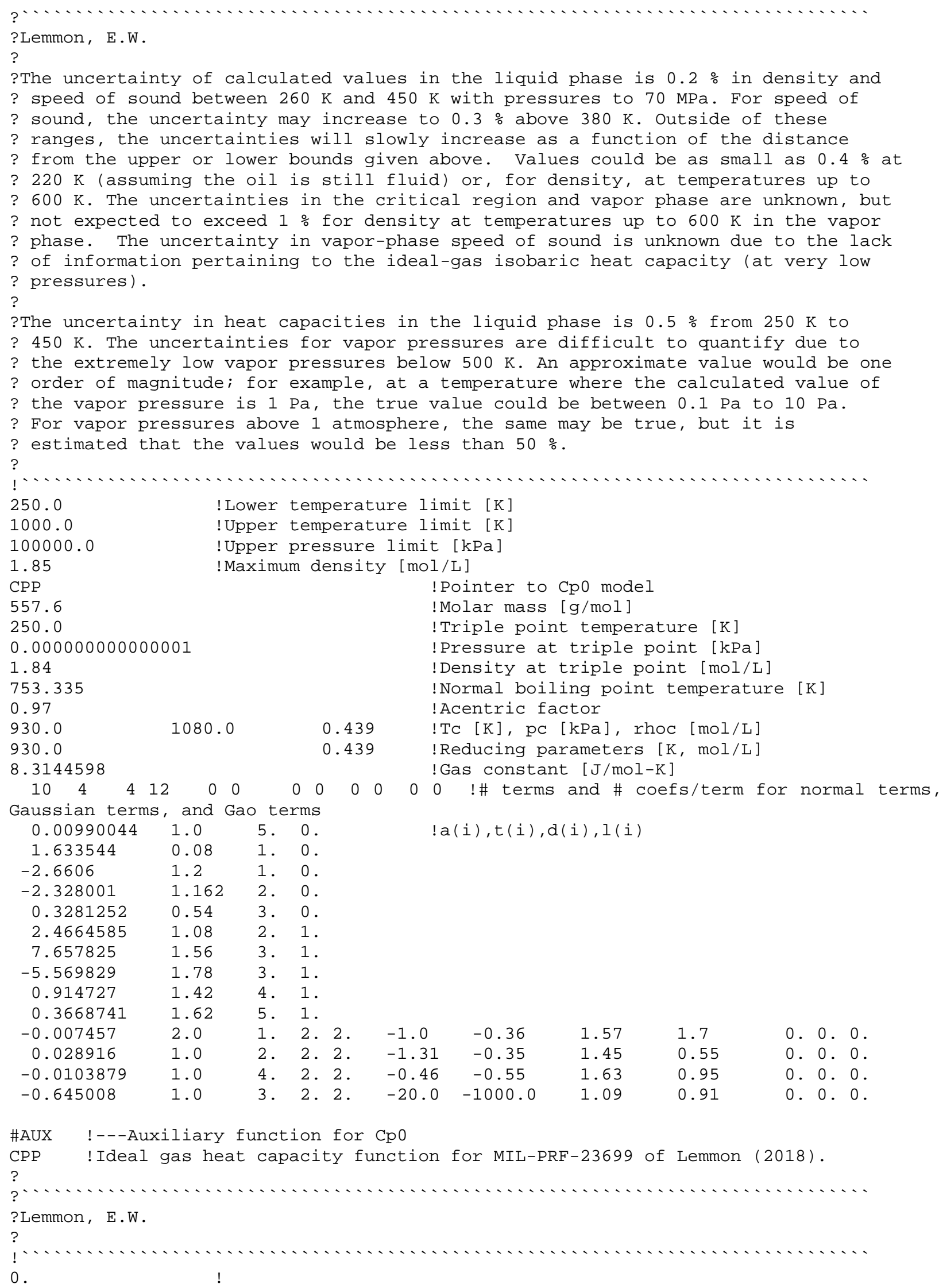




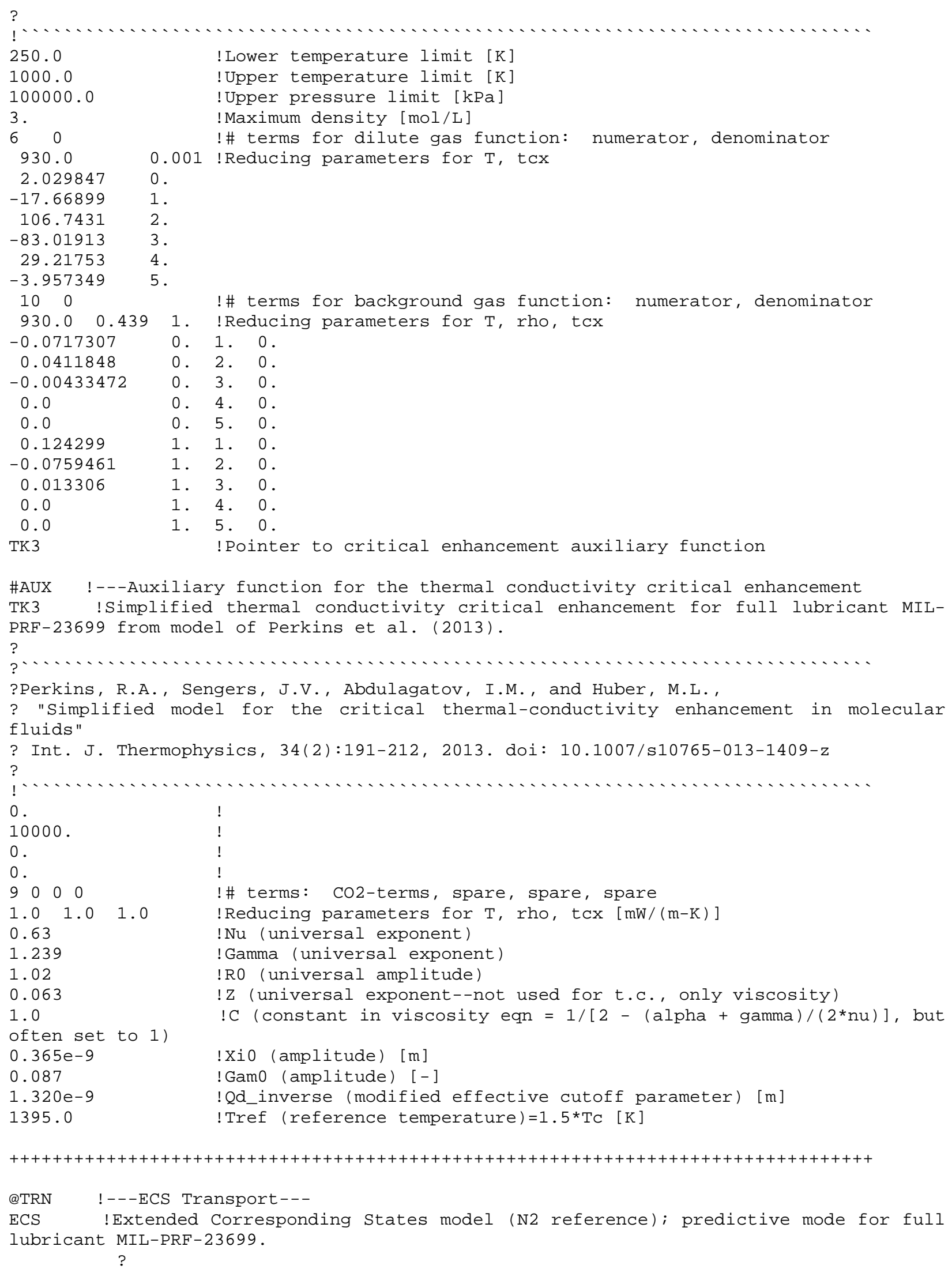




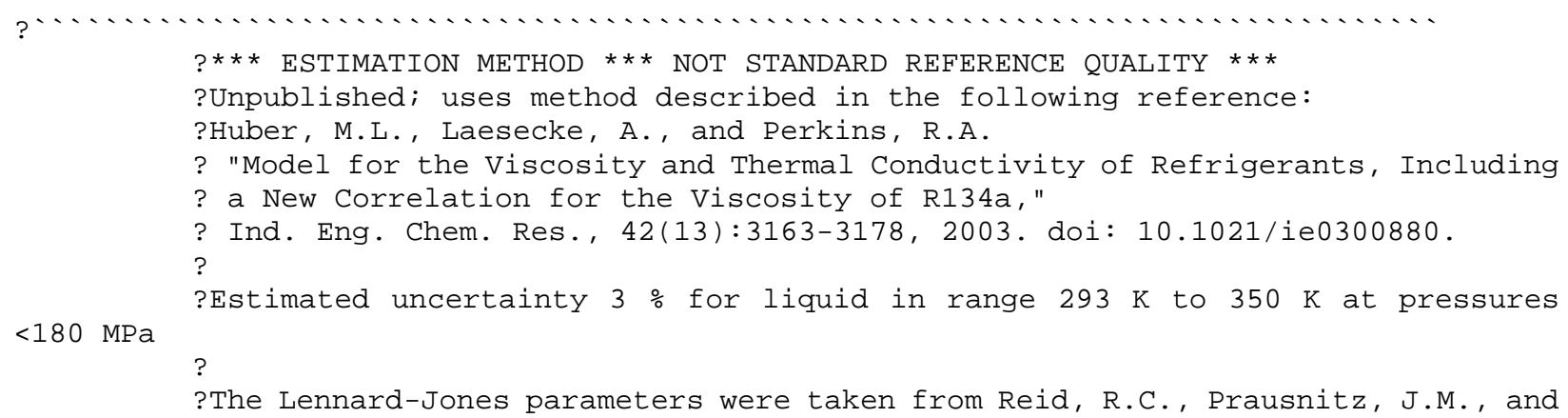
Poling, B.E., "The Properties of Gases and Liquids," 4th edition, New York, McGraw-Hill Book Company, 1987.

?

\begin{tabular}{|c|c|}
\hline $\begin{array}{l}25 \odot . \\
100 \odot . \\
1000 \odot \odot . \\
10 . \\
\text { FEQ NITROGEN. FLD } \\
\text { VS1 } \\
\text { TC1 } \\
\text { BIG } \\
\odot .73267 \odot . \odot . \odot . \\
1 \\
1.064427 \\
738.5055 \\
1 \quad 0 \quad 0\end{array}$ & $\begin{array}{l}\text { ! Lower temperature limit [K] } \\
\text { !Upper temperature limit [K] } \\
\text { !Upper pressure limit [KPa] } \\
\text { !Maximum density [mol/L] } \\
\text { !Model for reference fluid viscosity } \\
\text { !Model for reference fluid thermal conductivity } \\
\text { ! Large molecule identifier } \\
\text { ! Large molecule parameters } \\
\text { ! Lennard-Jones flag ( } \odot \text { or } 1)(\odot=>\text { use estimates) } \\
\text { ! Lennard-Jones coefficient sigma [nm] } \\
\text { ! Lennard-Jones coefficient epsilon/kappa [K] for Ecs method } \\
\quad \text { !Number of terms in } \mathrm{f} \text { int term in Eucken correlation, }\end{array}$ \\
\hline
\end{tabular}

spare1, spare2

0.00132

$3 \odot \odot$

poly, spare1, spare2

1.09271

$-0.161324$

$\odot .0486596$

100

poly, spare1, spare2

1.0

TK3
๑. ๑. ๑. !Coefficient, power of $T$, spare1, spare2

! Number of terms in psi (visc shape factor):

๑. ๑. ๑. !Coefficient, power of $\mathrm{Tr}$, power of $\mathrm{Dr}$, spare

๑. 1. ๑. !Coefficient, power of $\mathrm{Tr}$, power of $\mathrm{Dr}$, spare

$\odot$. 2. $\odot$. !Coefficient, power of $\mathrm{Tr}$, power of $\mathrm{Dr}$, spare !Number of terms in chi (t.c. shape factor):

๑. ๑. ๑. !Coefficient, power of $\mathrm{Tr}$, power of $\mathrm{Dr}$, spare !Pointer to critical enhancement auxiliary function

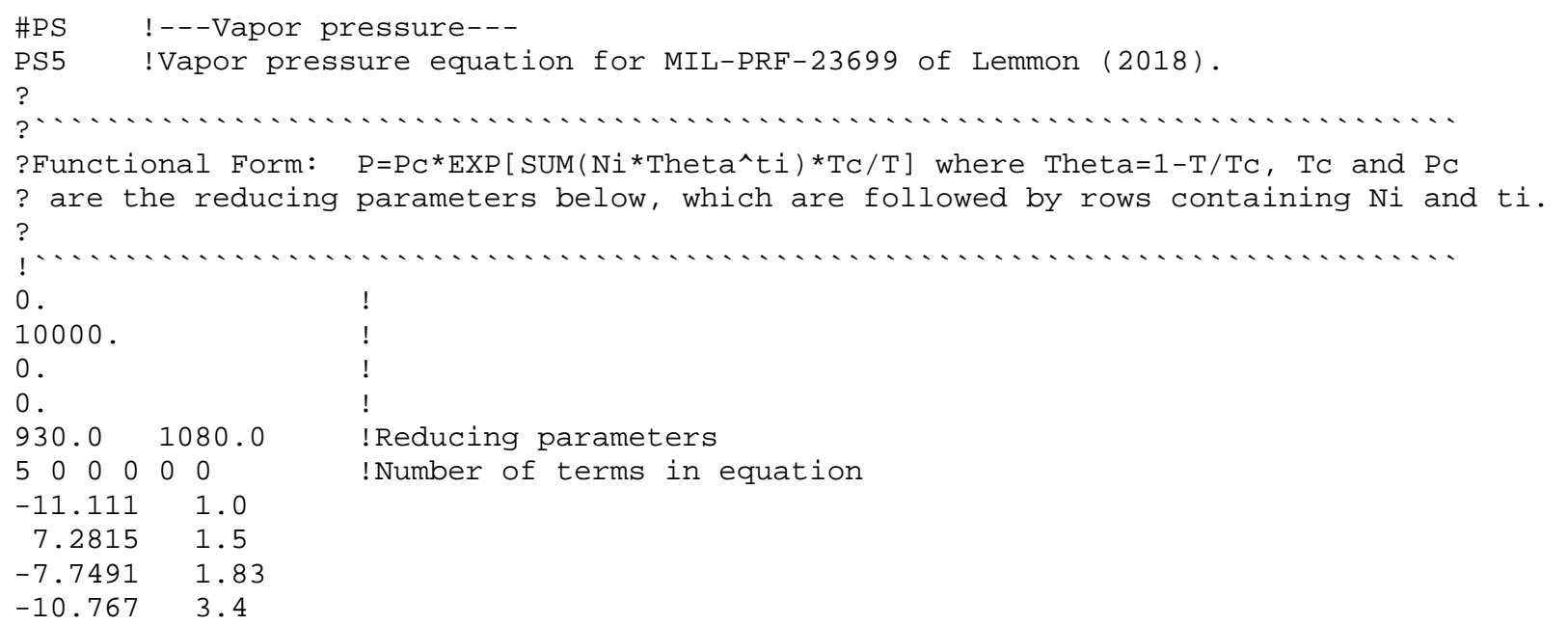




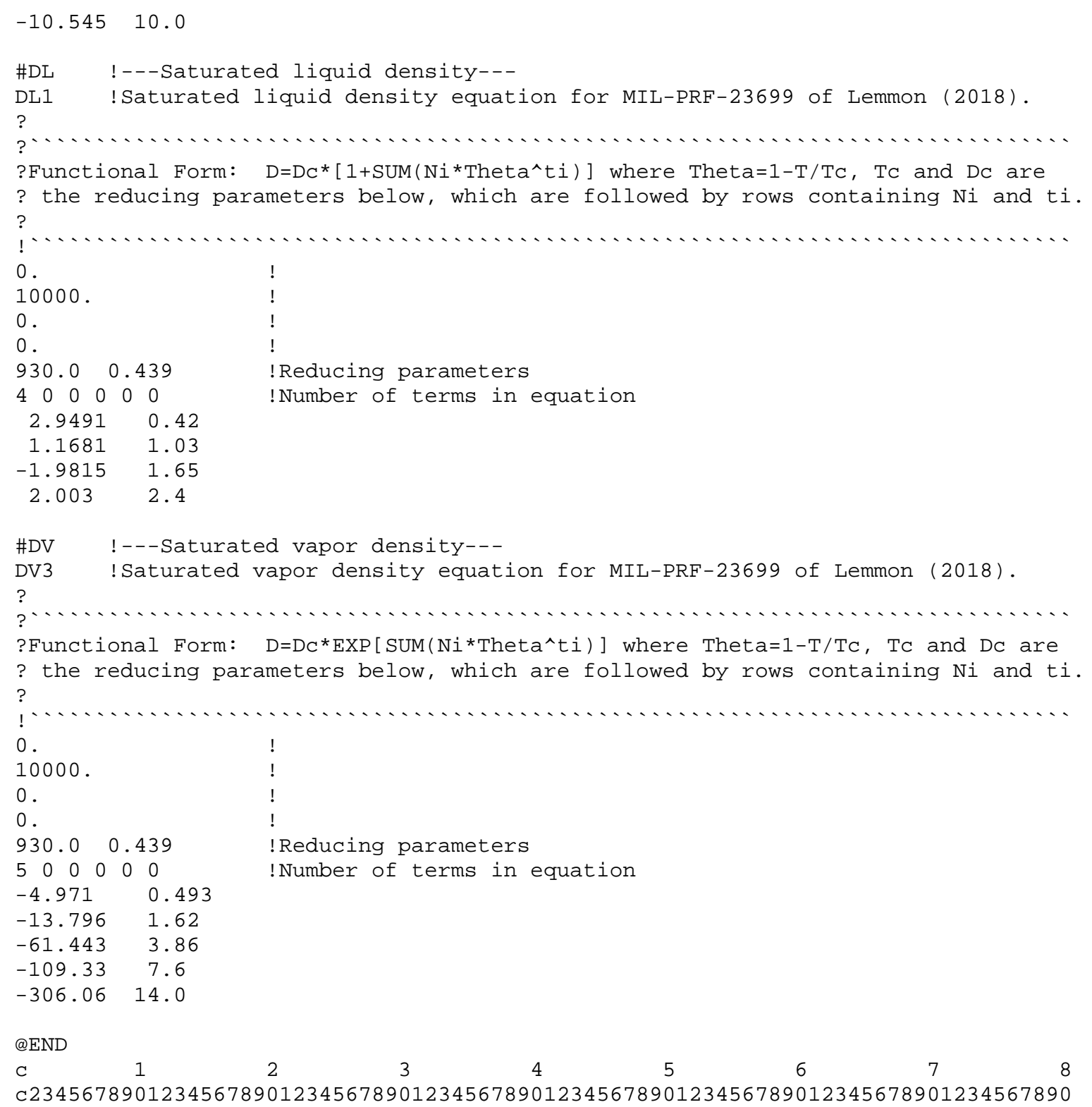

